\title{
Dynamics of HI Photodissociation
}

\section{in the $\mathrm{A}$ band Absorption via \\ H-atom Doppler Spectroscopy}

\author{
by \\ Denis Julien Gendron
}

\begin{abstract}
A thesis
presented to the University of Waterloo

in fulfilment of the

thesis requirement for the degree of

Doctor of Philosophy

in
\end{abstract}

Physics

Waterloo, Ontario, Canada, 1997

O Denis Julien Gendron 1997 
National Library

of Canada

Acquisitions and Bibliographic Services

395 Wellington Street Ottawa ON K1A ON4 Canada
Bibliothèque nationale du Canada

Acquisitions et services bibliographiques

395, rue Wellington

Ottawa ON KIA ON4

Canada
The author has granted a nonexclusive licence allowing the National Library of Canada to reproduce, loan, distribute or sell copies of his/her thesis by any means and in any form or format, making this thesis available to interested persons.

The author retains ownership of the copyright in his/her thesis. Neither the thesis nor substantial extracts from it may be printed or otherwise reproduced with the author's permission.
L'auteur a accordé une licence non exclusive permettant à la Bibliothèque nationale du Canada de reproduire, prêter, distribuer ou vendre des copies de sa thèse de quelque manière et sous quelque forme que ce soit pour mettre des exemplaires de cette thèse à la disposition des personnes intéressées.

L'auteur conserve la propriété du droit d'auteur qui protège sa thèse. Ni la thèse ni des extraits substantiels de celle-ci ne doivent être imprimés ou autrement reproduits sans son autorisation. 
The University of Waterloo requires the signature of all persons using or photocopying this thesis.

Please sign below, and give address and date. Thank you. 


\title{
Dynamics of HI Photodissociation in the A band Absorption
}

\section{via H-atom Doppler Spectroscopy}

\begin{abstract}
The technique of Doppler spectroscopy was used to measure the angular distribution of the H-atoms ejected during the dissociation of isolated $\mathrm{HI}$ molecules excited in the $\mathrm{A}$ band absorption continuum by a polarized beam of UV radiation. The observables characterizing the dynamics of HI photodissociation were obtained from the Doppler spectra at nine photolysis photon energies throughout the $A$ band. The comparison of these observables with those obtained in previous studies exhibits marked discrepancies, which are shown to be the consequence of HI clusters that were present in the molecular expansion used in a previous study.

The high level of confidence in the precise values of the observables determined in the present study warranted a formal quantum mechanical analysis leading to the determination of the electronic structure of HI in the $A$ band. The parameters of the potential energy curves were obtained for the dissociative states involved in the transitions responsible for $A$ band absorption. These parameters allowed for an extrapolation of the observables between and beyond the discrete values of photolysis photon energies at which experimental measurements were performed, over the whole $A$ band absarption. The assumption of a direct adiabatic dissociation model provided an excellent representation of the experimental observables for this primary process.
\end{abstract}




\section{Acknowledgments}

I wish to express my gratitude to my supervisor, Dr. John W. Hepburn, for providing me with the opportunity to perform this study. He has been generous of his time and energy, in addition to being a model of patience and tolerance for my shortooming; his eloquence aret his sharp wit have continually impressed me. I am very obliged to Dr. Hepburn for making this enabling experience, a positive one: thank you John!

I am truly indebted to Konstantina, my patient fiancee and dearest friend, for her moral support through this long journey, for the care she provided me during the painful last months of completion, and for proofreading the whole manuscript, even if she found it incredibly boring; I also thank George and Mary for their trust and support. I am also grateful for my brothers: Michel, Daniel and Marc, and their respective families, and my parents Claire and Gerald, for their moral support, their encouragements, and their prayers. I should not forget my cat, Mouser, for his constant playfulness has relieved many moments of stress, and provided a fresh perspective on life.

Numerous thanks also go to the members of my group: Denise Lacombe, whose energetic personality and lively character were always stimulating, to Aaron Beatty, for being such a pleasant and calm persona, and to James Martin, for his clever scientific advices. Special thanks are due to Ralph Shiell, for his professional advice on scientific writing, for proofreading the thesis, and above all, for being such a good fellow. I must also thank all the members of the science shop, and especially John Selwood, Ed Linton, Reg Graf, and Chris Gendron, who provided help of such high quality. I must also thank the members of the electronic shop: Dave Rider, Dave Bowan, and especially Ivan Frola and Jacek Szubra, for their dedicated expertise, and patience in solving numerous problem throughout the years. I am especially grateful to Jacek for being a good friend when I needed it most; I hope for more of those tennis games! But as anyone having had any dealings with him would agree on, his noble nature and professionalism are exemplary. I also appreciate working with George Dascalu for his dedicated help in fixing equipment in strategic time, when he performed beyond the call of duty. Although she left the CMBLC group, I thank Heather Hergoth, for cheering us all, with her laughs and jokes and happy ways.

Special thanks also go to Chris Mathers, for his friendship often made the basement a happier place to be, and for his help with solving computer problems in numerous instances, in addition to facilitating the technical details of my data analysis by sharing his expertise and his resources on UNIX. I also wish to thank Henry Tiedje, for being a good friend and always being there to take a break and chat with me, and to incite me to do some crosscountry skiing; I still have wet feet just thinking about it! I am also grateful for a very special character, Peter Owens, who has been a good friend, by making me laugh and sharing his positive insights and sense of humor, and Betty's company. 
Numerous thanks are also due to the professors who served on my $\mathrm{Ph}$. D. advisory committee: Robert $\mathrm{L}$. Brooks, Neil Isenor, Wing-Ki Liu, Jim Sloan. I must also thank Robert J. Le Roy, for his guidance and constructive criticism with regards to the quantum mechanical calculation of the analysis, and Mary Thompson, for discussing the statistical optimization method, and providing constructive advices toward the evolution of this achievement. The author also wish to acknowledge the dedicated work of the Ph. D. thesis committee members: Josef Paldus, Andre Bandrauk, Robert L. Brooks, Robert J. Le Roy, John W. Hepburn; their constructive comments contributed greatly to enhance the quality of this manuscript.

The author is also grateful for the help and dedication of Shannon Stokes in constructing the $I_{2}$ cell, and $A l$ Richardson for his vivacious presence in the laboratory, during his summer term with us. The help provided by Andreas Mank at the beginning of this project was critical and it is sincerely appreciated. Finally, I would like to thank Doug Milburn for his help and advice, in introducing me to $\mathrm{C}$ language programing and computer interfacing, by sharing his passion of the subject with me. 
To Konstantina, for she bears her name ... 


\section{PLEASE NOTE}

Page(e) misoing in number only; tent

follows. filmed as received.

VIII

\section{UMI}




\section{TABLE OF CONTENTS}

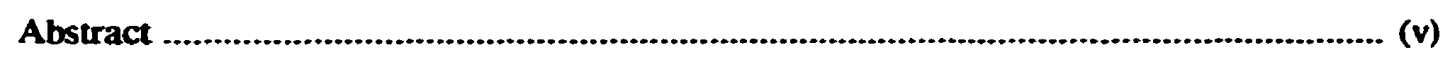

Acknowledgment …................................................................................... (vii)

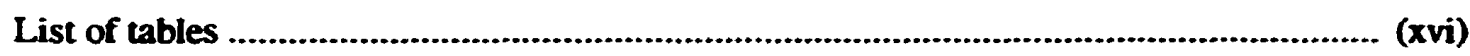

List of figures ....................................................................................................... (xvii)

\section{Chapter 1 Introduction}

1.1 Photofragment spectroscopy ............................................................................ 1

1.2 Historical perspective ............................................................................................. 3

1.2.1 General perspective ............................................................................. 3

1.2.2 Topical perspective .................................................................................. 5

1.3 Motivation for the specific topic: evolution of the perspective .................................... 6

1.3.1 Initial goal: coherent control ..................................................................... 6

1.3.2 Revision of the assumption: electronic structure of $\mathrm{HI}$............................. 6

1.4 Overview of the thesis .............................................................................................. 7

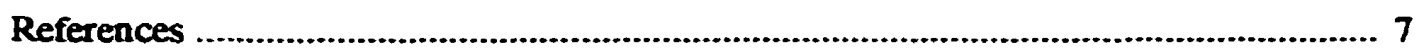

Chapter 2 The low-lying electronic states of $\mathbf{H I}$

2.1 Symmetry: coupling cases and correlation diagram ................................................. 9

2.1.1 Mulliken's prediction ............................................................................ 9

2.1.2 Angular momenta: coupling cases ................................................. 11

2.1.3 Correlation diagram: derivation ............................................................... 13

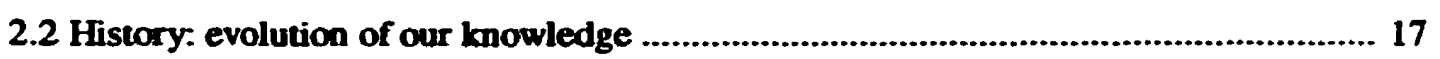

2.2.1 Early studies (1935-1955) ........................................................................ 17

2.2.2 Era of photochemical studies (1955-1975) ............................................ 18

2.2.3 Age of photofragment spectroscopy (1970-1995) ..................................... 24

2.2.4 Recent works related to our study on HI photodissociation ........................ 27

2.3 Summary: observations and discussion .......................................................................... 33

2.3.1 General observations of consistency .................................................... 33

2.3.2 Disagreements and controversy ............................................................... 34

2.3.3 Effects of the ion pair state on molecular structure ................................ 35

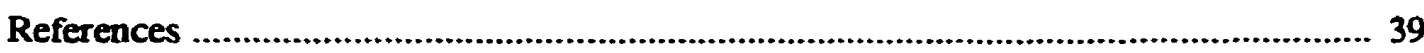


Chapter 3 Theory of the photophysical processes

3.1 Photophysical processes and observables ................................................................. 41

3.1.1 The three steps of our photofragment experiment ............................... 41

3.1.2 The observables .................................................................................... 42

3.1.3 The photophysical processes and their theoretical descriptions ........... 43

3.2 Theory for the direct photodissociation of $\mathrm{HI}$ molecules ............................................ 44

3.2.1 Basic physical concepts ..................................................................... 44

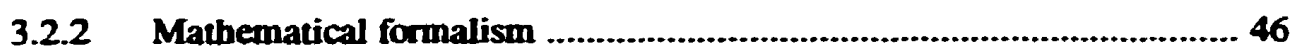

3.2.3 Discussion on the finer details of the theory of photodissociation ............. 50

3.3 Doppler spectroscopy of $\mathrm{H}$-atom photofragments ..................................................... 52

3.3.1 Background ...................................................................................... 52

3.3.2 Principles of the method ...................................................................... 52

3.3.3 Doppler profiles and angular distributions ............................................... 53

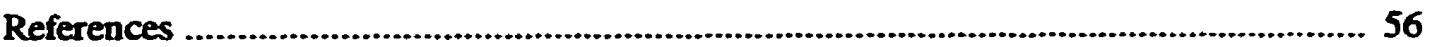

\section{Chapter 4 Experimental Method}

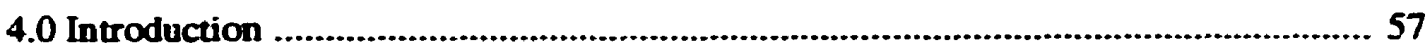

4.1 Conceptual description of our photofragment spectroscopy experiment ................... 59

4.1.1 Doppler spectroscopy detection ........................................................... 59

4.1.2 Photodissociation in collision-free condition ............................................. 59

4.1.3 Laser photodissociation dynamics ........................................................ 59

4.2 Description of the apparatus I: crossed molecular jet and lasers beams .................... 62

4.2.1 Supersonic free jet expansion source ........................................................ 62

4.2.2 Laser systems .......................................................................................... 64

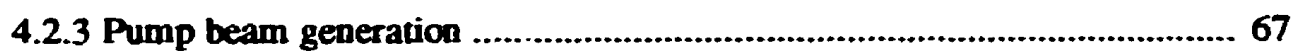

4.2.4 VUV probe beam generation .............................................................. 70

4.2.5 Wavelength calibration and linearity ................................................... 72

4.3 Description of the apparatus II: electronic control and synchronization ..................... 73

4.3.1 Detectors and signal amplification ........................................................ 73

4.3.2 Electronic synchronization ................................................................. 77

4.3.3 Computer and hardware interface ........................................................... 79

4.3.4 LABSCAN: software for data acquisition and control .............................. 80 


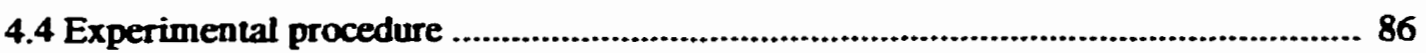

4.4.1 Alignment of the axis: lasers and jet ................................................ 86

4.4.2 Alignment of dye lasers for nonlinear optics .......................................... 86

4.4.3 Manjpulation to find the signal from photofragment LIF ....................... 87

4.4.4 Optimization of the injection laser frequency ............................................. 88

4.4.5 On the Soleil-Babinet compensator ...................................................... 91

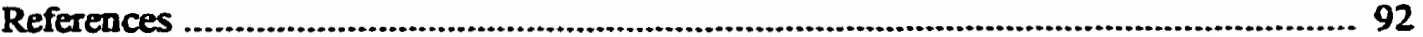

\section{Chapter 5 Experimental measurements and data reduction procedure}

5.1 Photofragment spectroscopy on HI photodissociation ........................................... 93

5.2 Data reduction procedure ................................................................................. 93

5.2.1 Power normalization ......................................................................... 94

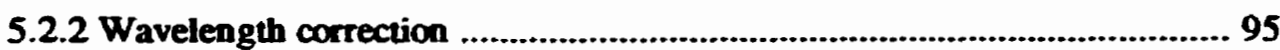

5.2.3 Statistical data averaging ................................................................ 97

5.2.4 HII LIF background subtraction .......................................................... 97

5.2.5 Illustration of the systematic procedure of data reduction ......................... 98

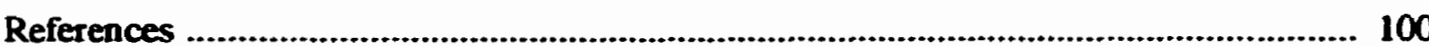

Chapter 6 Analysis, discussion and quantum mechanical calculation

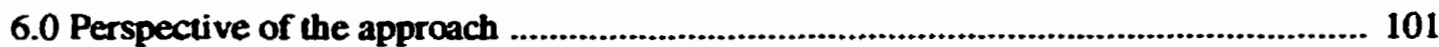

6.1 Determination of the observables from experimental measurements ....................... 103

6.1.1 Algorithm of the Doppler profile simulation ....................................... 103

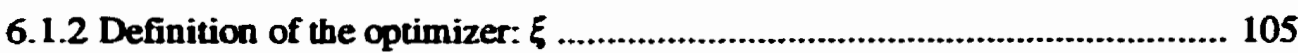

6.1.3 Iterative method to determine the observables ...................................... 105

6.1.4 Evaluation of the uncertainties ......................................................... 106

6.1.5 Results of the forward convolution procedure ...................................... 107

6.1.6 Test of the method ............................................................................. 126

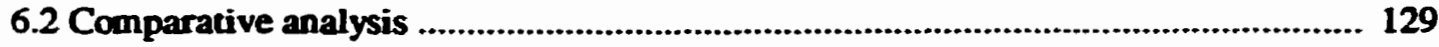

6.2.1 Comparison with previous studies .......................................................... 129

6.2.2 Explanation of the discrepancies ........................................................... 129

6.2.3 Second determination of the observables ................................................ 135

6.2.4 Summary on the determination of the observables ................................. 136 
6.3 Quantum mechanical analysis .................................................................... 148

6.3.1 Model for the dynamics of HI photodissociation in the A band ................. 148

6.3.2 Application of the model: from TACS to PACS .................................. 149

6.3.3 Description of the method for PEC determination ................................. 152

6.3.4 Application of the method for PEC calculation ................................... 156

6.3.5 Summary of the QM determination of the PEC .................................... 171

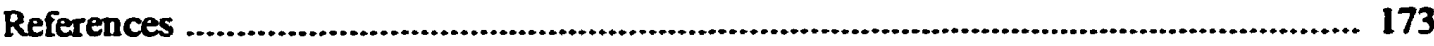

\section{Chapter 7 Conclusion}

7.1 Photodissociation dynamics of HI upon excitation in the A band .......................... 175

7.1.1 Observables from Doppler spectroscopy of H-atoms ................................ 175

7.1.2 Explanation of the discrepancies ...................................................... 175

7.1.3 Consequence for the photodissociation model ..................................... 176

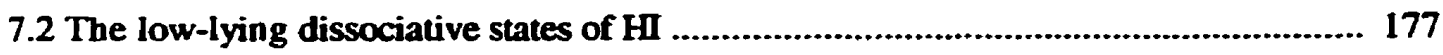

7.2.1 Quantum machanical model calculation .......................................... 177

7.2.2 Inference from the model ................................................................. 178

7.2.3 Note on the low-lying electronic structure of hydrogen halides ................ 178

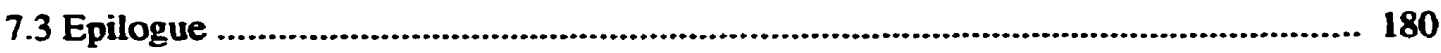

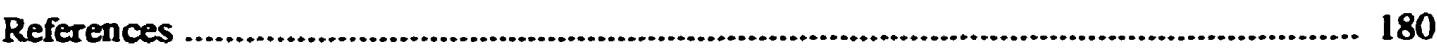

\section{APPENDICES}

Appendix A Cryostatic HI source and free supersonic jet expansion

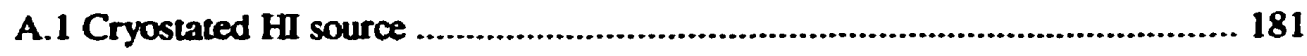

A.2 Supersonic expansion for a $\mathrm{HI}: \mathrm{He}$ mixture........................................... 183

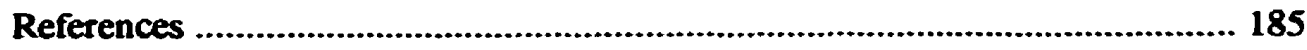

Appendix B Laser dye concentration

B.1 Optimization of F548/ $\mathrm{NaOH}$ dye laser solution for $532 \mathrm{~nm}$ pumping ......... 187

B.2 Laser dye concentrations ........................................................................ 187

Appendix C Generation of VUV by THG in phase-matched $\mathrm{Kr}$ :Ar

C.1 FWM techniques for VUV generation at Lyman $\alpha$..................................... 191

C.2 Implementation of THG of VUV ..................................................... 194

C.3 Resonant ionization of $\mathrm{Kr}$ : significance of $\chi^{5}$......................................... 196

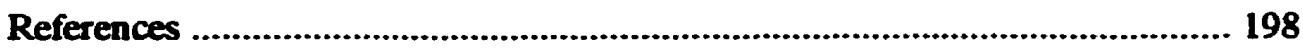


Appendix D Stability and Linearity of the dye laser wavelength tuning mechanism

D.I Stability of the tuning mechanism

D. 2 Linearity of the tuning mechanism .................................................. 201

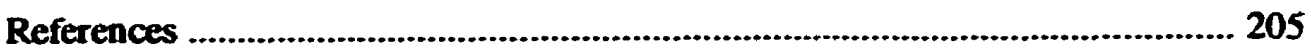

Appendix E Linearity of PMT detectors and electronic preamplifiers

E.1 Linearity of the preamplifiers .................................................................... 207

E.2 Amplification and gain saturation in a PMT detector ................................ 208

E.3 Characterization of the PMT gain saturation ......................................... 209

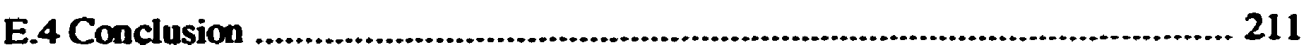

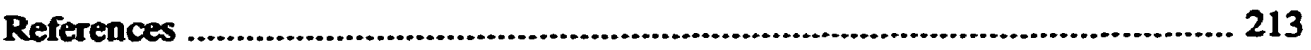

Appendix F Optimization beyond least mean squares

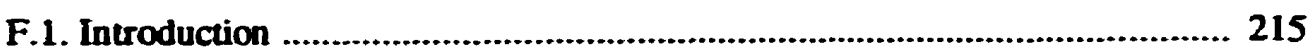

F.2. Mathematical description ........................................................... 219

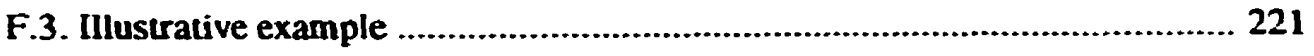

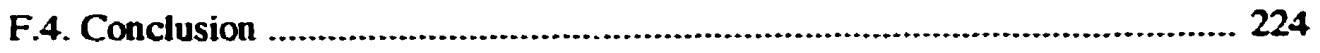

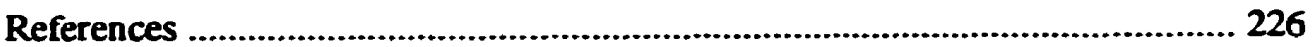

Appendix G Non-adiabatic transition in dissociation dynamics

G.1 Effect of non-crossing rule on molecular electronic structure ................... 227

G.2 Diabatic curves crossing and nonadiabatic transition ............................... 229

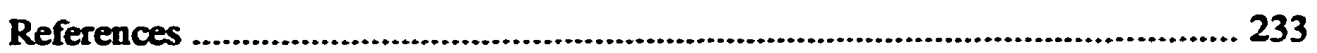




\section{LIST OF TABLES}

Table 2-1 Molar extinction data from Huebert and Martin .......................................... 20

Table 2-2 Absorption data measured by Ogilvie ............................................................... 23

Table 2-3 Electronic branching ratios obtained from photochemical methods ................ 23

Table 2-4 Results from photofragment spectroscopy studies on HI ............................... 27

Table 2-5 Molecular parameters for ground electronic state of HI .................................. 29

Table 2-6 PES parameters from previous studies ............................................................ 32

Table 2-7 Atomic and molecular properties of $\mathrm{HX}$ ion pair .......................................... 32

Table 4.1 Performance the YAG laser ..........................................................................6 66

Table 4.2 Requirements for the synchronized delays generator ..................................... 79

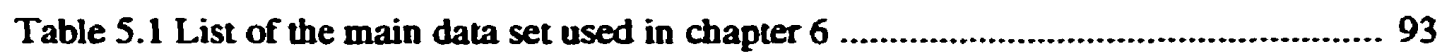

Table 6.1 Observables of $\mathrm{HI}$ photodissociation obtained from fit to $\beta\left(I^{*}\right)$ and $R$............. 107

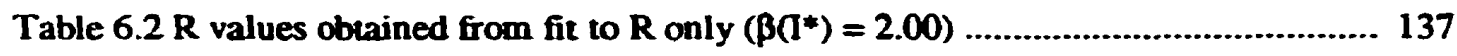

Table 6.3 Extrapolation of TACS from Ogilvie at our experimental frequencies ............. 149

Table 6.4 Experimental PACS for the $I^{*}$ product, calculated from tables 6.2 and 6.3 ..... 151

Table 6.5 PACS $\left(I^{*}\right), R$ and PACS$(I)$ calculated from the PEC $\left({ }^{3} \Pi_{0}+\right)$ and TACS ........... 163

Table 6.6 PEC parameters for states of the $A$ band, for analytical form equation (6-3) ... 171

Table B.1. Dye solutions used for the generation of the UV (PUMP) photolysis .............. 189

Table B.2. Dye solutions used for VUV generation ......................................................... 189

Table E-1 Amplification properties of the preamplifiers used in the present work .......... 208

Table F.1 Data used for the comparative example ........................................................ 222

Table F. 2 Comparison of the parameters obtained by different optimization method ...... 224 


\section{LIST OF FIGURES}

Figure 1.1 Types of dissociation mechanism

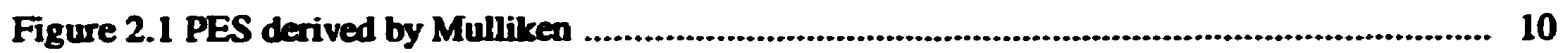

Figure 2.2 Angular momenta cases c coupling .................................................................... 12

Figure 2.3 Correspondence between atomic and molecular symmetry group ................................. 15

Figure 2.4 Tensorial product of arbital and spin representation .............................................. 15

Figure 2.5 Correlation diagram derived in section 2.1 ............................................................... 15

Figure 2.6 Absorption spectrum and theoretical decomposition by Romand et al. ....................... 19

Figure 2.7 Absorption spectra of $\mathrm{HI}$ and $\mathrm{HBr}$, in the range of 3000 to $1800 \AA$...................... 21

Figure 2.8 Theoretical decomposition HI absorption from Compton and Martin ......................... 21

Figure 2.9 PACSS and PES from Clear et al. ........................................................................... 25

Figure 2.10 PACSS and PES from van Veen et al. ............................................................. 26

Figure 2.11 Calculated and experimental dipole moment of $\mathrm{HI}$.................................................. 28

Figure 2.12 Ab initio CI calculations of the low-lying electronic states of $\mathbf{H I}$.............................. 30

Figure 2.13 PACSS and PES of HI from Levy and Shapiro ............................................ 31

Figure 2.14 Comparison between the results of previous studies .......................................... 36

Figure 3.1 Correspondence between Doppler profile and velocity distribution ............................ 55

Figure 4.1 Photodynamic processes present in the pump-probe experiment ................................. 58

Figure 4.2 Layout of the experimental setup ..................................................................60

Figure 4.3 Escape time for the H-atoms fragments vs photolysis photon energy .......................... 61

Figure 4.4 The free jet source ...........................................................................................63

Figure 4.5 Optical layout of the injection-seeded YAG laser ................................................... 65

Figure 4.6 Schematic representation of the polarized photolysis pump generation ....................... 69

Figure 4.7 Optical layout for the VUV generation at Lyman \& .............................................. 69

Figure 4.8 Tuning range of cohereat VUV radiation by THG in Kr.Ar .................................... 72

Figure 4.9 Vacuum chamber and physical description signals ................................................. 74

Figure 4.10 Snout of the LIF pichup sleeve for the LIF PMT .................................................. 76

Figure 4.11 Comparison of ICE scan with regular scan ............................................................. 81

Figure $4.12 \mathrm{I}_{2}$ LIF spectra illustrating the problems with tuning drive stability ........................... 83

Figure 4.13 Improvement brought about by the "Smooth Drive" driver ..................................... 84 
Figure 4.14 CO LIF spectrum used to adjust the LIF detection

Figure 5.1 Nonlinearity characterization and stability verification of tuning drive 96

Figure 5.2 Determination of a normalized HI LF background function 98

Figure 5.3 Example of systematic statistical averaging for a series profiles 99

Figure 5.4 Example of systematic HI LIF background subtraction 100

Figure 6.1. Flow chant of the forward convolution procedure 102

Figure 6.2. Example of simulated Doppler profile for $230 \mathrm{~nm}$ photolysis 104

Figure 6.3. 3D plot of the $\xi$-function; fit to data at $266.0 \mathrm{~nm}$ wavelength 108

Figure 6.4. Comparison of experimental and simulated ( $1^{\text {xt }}$ fit) profiles at $266.0 \mathrm{~nm}$ 109

Figure 6.5. 3D plot of the $\xi$-function; fit to data at $257.0 \mathrm{~nm}$ wavelength 110

Figure 6.6. Comparison of experimental and simulated ( $1^{\text {st }}$ fit) profiles at $257.0 \mathrm{~nm}$ 111

Figure 6.7. 3D plot of the $\xi$-function; fit to data at $248.0 \mathrm{~nm}$ wavelength 112

Figure 6.8. Comparison of experimental and simulated ( ${ }^{\mu}$ fit) profiles at $248.0 \mathrm{~nm}$ 113

Figure 6.9. 3D plot of the $\xi$-function; fit to data at $240.0 \mathrm{~nm}$ wavelength 114

Figure 6.10. Comparison of experimental and simulated ( $1^{\text {st }}$ fit) profiles at $240.0 \mathrm{~nm} \ldots \ldots \ldots \ldots \ldots . . .115$

Figure 6.11. 3D plot of the $\xi$-function; fit to data at $232.5 \mathrm{~nm}$ wavelength 116

Figure 6.12. Comparison of experimental and simulated ( $\left(^{n t}\right.$ fit) profiles at $232.5 \mathrm{~nm}$.............. 117

Figure 6.13. 3D plot of the $\xi$-function; fit to data at $225.0 \mathrm{~nm}$ wavelength ................................. 118

Figure 6.14. Comparison of experimental and simulated ( $1^{\mathrm{k}}$ fit) profiles at $225.0 \mathrm{~nm} \ldots \ldots \ldots \ldots \ldots . . . .119$

Figure 6.15. 3D plot of the $\xi$-function; fit to data at $216.7 \mathrm{~nm}$ wavelength ................................... 120

Figure 6.16. Comparison of experimental and simulated ( ${ }^{\text {ex }}$ fit) profiles at $216.7 \mathrm{~nm}$............... 121

Figure 6.17. 3D plot of the $\xi$-function; fit to data at $215.0 \mathrm{~nm}$ wavelength .................................. 122

Figure 6.18. Comparison of experimental and simulated ( $1^{\mathrm{m}}$ fit) profiles at $215.0 \mathrm{~nm}$.............. 123

Figure 6.19. 3D plot of the $\xi$-function; fit to data at $212.5 \mathrm{~nm}$ wavelength ................................ 124

Figure 6.20. Comparison of experimental and simulated ( ${ }^{* 4}$ fit) profiles at $212.5 \mathrm{~nm}$................ 125

Figure 6.21. Artificial data created for $256 \mathrm{~nm}$ photolysis wavelength ........................................ 127

Figure 6.22. Results of the forward convolution procedure for the data of figure 6.21 ................. 128

Figure 6.23. Comparison of the observables obcained with previous results ................................ 130

Figure 6.24. Experimental TOF spectra taken from van Veen et al. ......................................... 131

Figure 6.25. Results from the study of Zhang et al. HI dimers photodynamics .......................... 133

Figure 6.26. Determination of $R$ for $\beta=2.00$ at $266.00 \mathrm{~nm}$ photolysis wavelength .................. 137 
Figure 6.27. Comparison of $R$ from $2^{\text {bd }}$ fit with the results from previous studies

Figure 6.28. Comparison of experimental and simulated ( $2^{\text {oc }}$ fit) profiles at $266.0 \mathrm{~nm}$.............. 139

Figure 6.29. Comparison of experimental and simulated ( $2^{\text {Dd }}$ fit) profiles at $257.0 \mathrm{~nm} \ldots \ldots \ldots \ldots . . . .140$

Figure 6.30. Comparison of experimental and simulated ( $2^{\text {Dd }}$ fit) profiles at $248.0 \mathrm{~nm}$............. 141

Figure 6.31. Comparison of experimental and simulated ( $2^{\text {od }} \mathrm{fit}$ ) profiles at $240.0 \mathrm{~nm} \ldots \ldots \ldots \ldots \ldots 14$

Figure 6.32. Comparison of experimental and simulated ( $\left.2^{\text {Dd }} \mathbf{f i t}\right)$ profiles at $232.5 \mathrm{~nm} \ldots \ldots \ldots \ldots \ldots 143$

Figure 6.33. Comparison of experimental and simulated ( $2^{\text {nd }}$ fit) profiles at $225.0 \mathrm{~nm}$............... 144

Figure 6.34. Comparison of experimental and simulated (20 fit) profiles at $216.7 \mathrm{~nm}$.............. 145

Figure 6.35. Comparison of experimental and simulated ( $2^{\mathrm{pd}}$ fit) profiles at $215.0 \mathrm{~nm}$............... 146

Figure 6.36. Comparison of experimental and simulated (204 fit) profiles at $212.5 \mathrm{~nm}$.............. 147

Figure 6.37. TACS data from Ogilvie with extrapolated data points of table $6.3 \ldots \ldots \ldots \ldots \ldots . . . . . . . .150$

Figure 6.38. HTS H.O. radial wavefunctions and for ground electronic state HI, in $v=0 \ldots \ldots . . .152$

Figure 6.39. Determination of the PEC for the ${ }^{3} \mathrm{H}_{0}+$ state, using PACS(I*) ............................... 158

Figure 6.40. Comparison of the parameters uncertainties for their determination:

a) from visual inspection, and b) from the statistical method ........................................ 159

Figure 6.41. Comparison of a) the PACSS for b) two analytical form of the PEC ...................... 161

Figure 6.42 a) Optimizer function showing the bistable path to the maxima;

b) comparison of simulated PACSS for two local maxima, and for the optimum

Figure 6.43 a) Optimizer function for the determination of $P E C\left({ }^{1} \Pi\right)$ by a fit to the PACS(I) above

$43500 \mathrm{~cm}^{-1}$; b) Comparison of optimum simulated and experimental PACS(T) 166

Figure 6.44 Details of the optimizer function for the determination of PEC('I) by a fit to the

PACS(I) above $43500 \mathrm{~cm}^{-1}$, showing "oscillatory" behaviour 167

Figure 6.45 Plot of the optimizer function for a fit to a region of experimental PACS(I)

restricted to above $43500 \mathrm{~cm}^{-1}$ and below $52000 \mathrm{~cm}^{-1}$, a) for a low resolution

grid of the parameters domain and $b$ ) a fine grid for PEC(' $(\mathrm{I})$

parameters determination 168

Figure 6.46 Comparison of the PACSS('I) calculated from the PEC('II) with the experimental PACS(I) 169

Figure 6.47 Comparison of optimum simulated and experimental PACSS( $\left.{ }^{3} \Pi_{1}\right)$ 170

Figure 6.48 Comparison between the sum of the simulated PACSS and the TACS 170

Figure 6.49 Comparison of our calculated $R$ with the results from previous studies 172

Figure 7.1 Comparison of PEC from a) this work, and b) Mulliken's prediction (from ref. 2) ..... 177 Figure 7.2 Extrapolation based on PEC from this work for a) R, and b) PACS(I) and PACS(I*) .. 179 
Figure A.1 Model for the supersonic expansion of the HI:He mixture

Figure B.1 a) Spectral absorption and b) optimization of NaOH:F548 mixture

Figure C.I a) VUV cell and b) gas handling system for the cell 193

Figure C.2. VUV wavelength tuning range of the cell for various gas mixtures 195

Figure C.3. a) Resonant absorption and b) its explanation 197

Figure D.1 A comparison between the properties of driver 200

Figure D.2 Example of motion commanded by the smooth drive software driver 200

Figure D.3 Determination of the nonlinear deviation of the dye laser cuning machanism 202

Figure D.4 Comparison between the results for two characterization methods 202

Figure D.5 Illustration of a "mysterious" problem elucidated by this system characterization 204

Figure D.6 Suggested solution for the possible cause of the nonlinearity in the mechanism 205

Figure E.1 Linearity of the preamplifiers used in the present study 207

Figure E.2 Schematic drawing of a PMT components ............................................................ 208

Figure E.3 Output signal vs bias voltage at constant $\Phi$; EMR PMT ........................................ 210

Figure E.4 Output signal vs bias voltage at constant $\Phi$; Hamamatsu PMT ................................. 210

Figure E.5 Typical data used to characterize the EMR PMT saturation process (fig.E.6) .............. 212

Figure E.6 Characterization of the gain saturation for the EMR PMT

Figure F.1 BSO method: a) Convergence of the O-function to a well-defined b) optimu.n 223

Figure F.2 Comparison of the optimization methods 225

Figure G.1 Various cases of avoided crossing 228

Figure G.2 Example: electronic structure of LiF molecule ....................................................... 228

Figure G.3 Generic problem of nonadiabatic transition in atomic collision ................................. 230

Figure G.4 Landau-Zener curve crossing problem ............................................................ 230 


\section{Chapter 1 Introduction}

\subsection{Photofragment spectroscopy}

The term photofragment stands for photodissociation fragment. Photofragment spectroscopy addresses the following problem: to characterize the intimate mechanisms occurring during the primary photochemical events, i.e. the dynamics of the photolytic bond-breaking. In the protocype of a photodissociation process, a molecule AB is excited by the absorption of a photon hv, and then the system evolves to produce two fragments, A and B, each in their specific quantum state, denoted $\varphi_{2}$ and $\varphi_{0}$ in equation (1-1).

$$
A B+h v \rightarrow[A B]^{*} \rightarrow A\left(\varphi_{a}\right)+B\left(\varphi_{b}\right)
$$

The first step of equation (1-1) represents the absorption of the photon observed with traditional techniques of absorption spectroscopy, while the second step is the concern of molecular dynamics: the fate of the excited state, and its evolution into a velocity distribution of photofragments. Hence, this subject is concerned with three related sets of information: (1) the electronic structure of the parent molecule involved in the absorption processes, (2) the distribution of energy in the internal degrees of freedom of the nascent fragments, and (3) the correlation between the vector quantities involved in the global process, from photon absorption to fragment recoil and internal excitations. The methods of photofragment spectroscopy proceeds through the measurement of (2) and (3), and the deduction of (1) from the analysis of the experimental information.

The general case of single photon photodissociation includes many types, ${ }^{\prime}$ among which we distinguish direct and indirect dissociation mechanisms. The case of direct dissociation, shown in figure 1 a), is characterized by broad featureless absorption, and the fragments evolve on the dissociative potential following the onset of photon excitation. On the other hand, indirect dissociation processes involve an additional coupling of the initially excited bound state with another molecular state through which dissociation occurs. This more complex situation is exemplified by electronic predissociation, illustrated in figure $1 \mathrm{~b}$ ), where a rovibronic level of an otherwise bound state is coupled to a dissociative state. The nature of the bound excited state is deduced from the discrete transitions in the absorption spectrum. The coupling to the dissociative state reduces the lifetime of the excited bound state, and as a consequence, this mechanism broadens the effective width of the energy levels observed in absorption spectroscopy, in proportion to the square of the coupling strength. In larger molecules, vibrational predissociation $\alpha$ intemal vibrational energy redistribution (IVR) can occur when a bound vibronic state couples to another vibronic state above its dissociation limit. However, the present study needs only to consider the simpler case of direct dissociation to describe the dissociation of $\mathrm{HI}$ ensuing absorption in the $\mathbf{A}$ band. 
a)

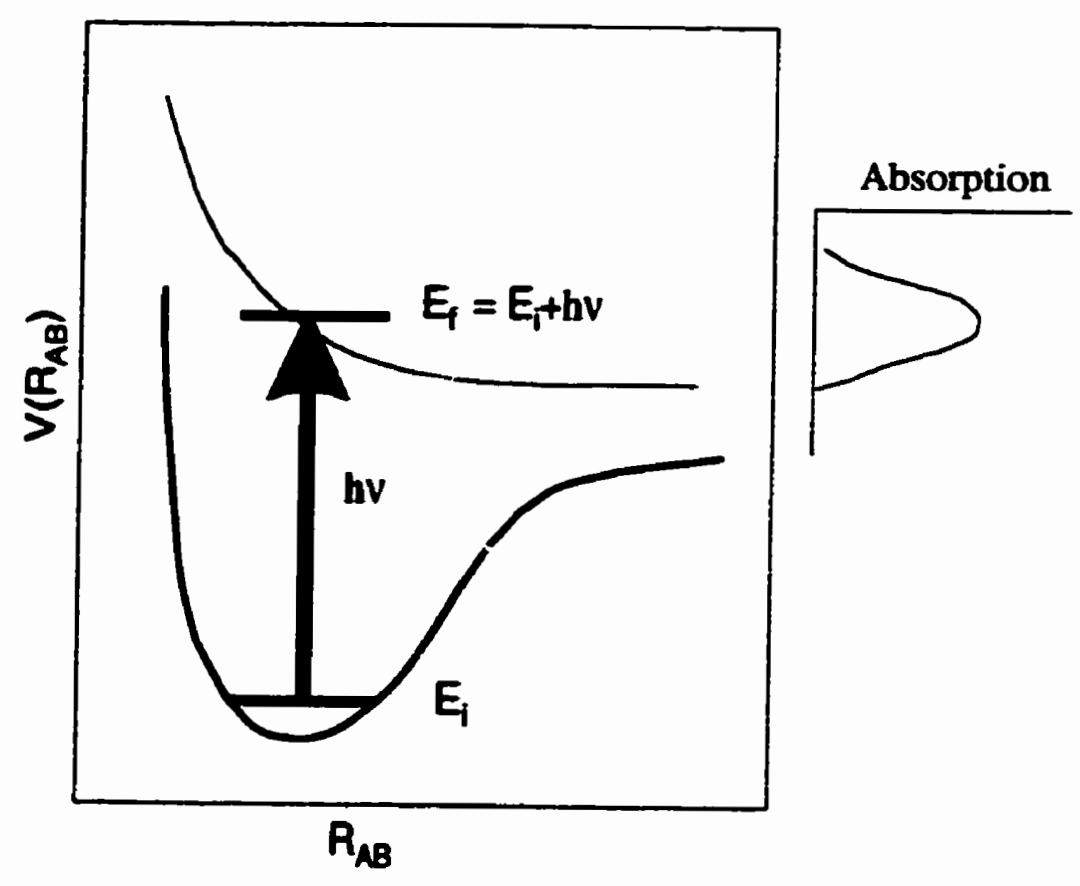

b)

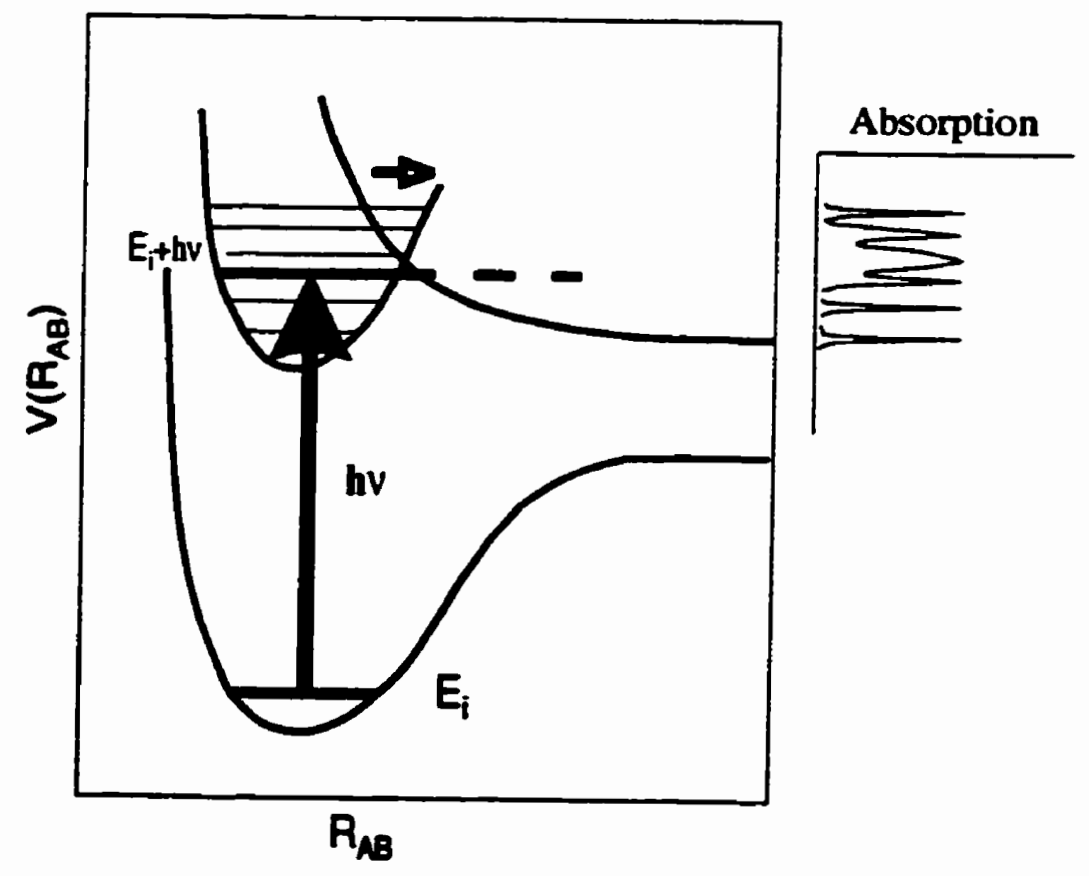

Figure 1.1 Schematic representation of a) direct dissociation, and an example of indirect dissociation is illustrated by b) electronic predissociation, where the rate of predissociation depends on the coupling between two excited states. Note the shape of the corresponding absoption specter, represented on the right of the energy diagrams. The bottom axis of the energy diagram represents the internuclear distance, while the left axis represents the potential energy of the electronic interaction between the atoms $A$ and $B$. The vertical arrow represents the energy hv transferred from the photon to the molecule, resulting in a transition between electronic states of the molecule. 
An experimental investigation of photofragment spectroscopy proceeds typically in three steps. First a beam of coherent light excites an ensemble of target molecules to an electronic state, and the excited molecules break apart. evolving rapidly to their asymptotic state. Second, the final states of the photofragments are probed by a technique best suited to the needs of the experiment. In the case of the present experimental study, the technique used was Doppler spectroscopy of the hydrogen atom fragments. Third, the data obtained by photofragment spectroscopy are juxtaposed with the results of absorption spectroscopy, and the whole set of information is analyzed with the methods of quantum mechanics. The final results are the characterization of the molecular states involved in the dissociation, and consequently, the analytical representation of the photofragmentation dynamics.

\subsection{Historical perspective}

The history of photofragment spectroscopy is naturally tied to both photochemistry and to spectroscopy, respectively from the fundamental quest it has originated and from the enabling analytical probe it provides. However, only through a detailed study of the electronic structure of the molecules, may a unifying picture arise. In the limit, this field can be linked to a large fraction of atomic, molecular and optical (AMO) physics. Thus, because the list of all the studies performed in the field is too extensive for this introduction, reference is only made to major realizations ${ }^{\wedge}$ and to published works representing milestones for the general and topical perspective of this study. .

\subsubsection{General perspective}

An early milestone in unravelling the physico-chemical effect of light upon matter has been afforded by the technique of flash photolysis, introduced in 1949 by Norrish and Porter², and refined in 1950 by Porter'; their work was recognized by the 1967 Nobel prize, "for their studies of extremely fast chemical reactions, effected by disurbing the equilibrium by means of very short pulses of energy", which was won half-jointly with Eigen from Götingen. Although great progress has been made from this technique, indirect photochemical methods used to probe the fundamental processes had a relatively low selectivity for excitation and a limited detection sensitivity. Consequently, the answers to many crucial fundamental questions were either inaccessible or impractical.

The criginal work of Alfred Kastler on optical pumping led to his discovery of double resonance, and to the 1966 Nobel prize in Physics, "for the discovery and development of optical methods for studying hertzian resonances in atoms". His work had far-reaching consequences in spectroscopy and in atomic structure, where its concepts can be linked to level-crossing spectroscopy and nonlinear optics in gases. Of specific interest here, his work led

\footnotetext{
A Below, the expressions in double quotes are the original quotes from the Nobel foundation, taken from the searchable data base on their web site (http://www.nobel.se/).
} 
to the development of masers and lasers, between 1952 and 1958, by Townes in the US, and by Prokhorov and Basov in the USSR, for which they won the joint Nobel prize in 1964. Searching for better gain medium for laser. Zare and Herschbach 4 have investigated vector correlations in the photodissociation dynamics of Nal used as a source of excited iodine atoms, with regards to the consequence of the Doppler broadened fluorescence on gain medium properties. The experimental observation of an efficient atomic iodine laser based on $C_{3} I$ photodissociation, by Kasper and Pimentel, ${ }^{5}$ have stimulated further interest for the basic mechanisms in molecular photodissociation processes.

Prior to widespread availability of lasers as a laboratory tool, the technique of photolysis mapping ${ }^{6}$ provided a rudimentary means to investigate correlations between the photolysis light polarization and the momentum of the photodissociation products. The group of Richard Bersohn also investigated photodissociation dynamics in a molecular beam using mass spectrometric detection, ${ }^{7}$ but the excitation afforded by the mercury-xenon lamp used, which spans a few tens of nancmetres around $300 \mathrm{~nm}$, was lacking selectivity, also only crude information could be obtained concerning the energetics of the process.

The discovery of lasers in the fifties, and their availability for laboratory application in the sixties, have spurred breakthrough developments, with regards to these limitations. The group of Kent Wilson initiated the field of laser photofragment spectroscopy. In their pioneering experiments, an effusive source was used to expand molecular species in vacuum, which were photodissociated with the $4^{\text {th }}$ harmonic of a YAG laser at $266.2 \mathrm{~nm}$, and the photofragments were detected by quadrupole mass spectrometry. Their apparatus yielded measurements on the distributions of translational energy, and angular distribution of velocities, for the photofragments.. They applied their methods to the investigation of the photodissociation dynamics of a few alkyl iodides ${ }^{9}$ and $\mathrm{HI}^{10}$. Now in the nineties, widespread availability of very short pulse $\left(10^{-15}-10^{-12}\right.$ second) laser sources has enabled experimental methods capable of probing the dynamics of primary photochemical processes in real-time. These sophisticated tools have permitted to enhance the knowledge on this topics, and they have confirmed the very short time scales of the dynamics which were expected from molecular structure derived from spectroscopic analysis.

Also of primary importance to the present work are some of the studies by Mulliken ${ }^{12.12 .13}$ on molecular physics, whose tremendous realization won him the 1966 Nobel prize in chemistry "for his fundamental work concerning chemical bonds and the electronic structure of molecules by the molecular orbital method".

As the present thesis will demonstrate, it can be said that photofragment spectroscopy is still in its adolescence. Although a respectable body of data exists for numerous small molecules, the understanding of the fundamental processes, and the rationalization of their physical causes, is by no extent definitive. Consequently, the challenge to unravel their nature awaits us. 


\subsubsection{Topical perspective}

Among the numerous studies of photofragment spectroscopy, the leading work of Welge and coworkers must be mentioned. They used the technique of Doppler spectroscopy to probe $\mathrm{NH}_{2}{ }^{14}$ and $\mathrm{OH}^{15}$ fragment in the UV, and they used initiated Doppler spectrosoopy of atomic hydrogen (H-atom) at the VUV Lyman $\propto$ transition. ${ }^{16} \mathrm{H}$-atom detection is a central theme in Welge's group. They developed a resonant two-photon-ionization (TPD) technique to detect H-atoms, which led to the method of translational spectroscopy of H-atom photofragments. ${ }^{17}$ These technical innovations were used to investigate the VUV photofragmentation of $\mathrm{H}_{2} \mathrm{O}^{18}$ Lately, Welge and coworkers refined the time-of-flight (TOF) mass spectroscopic detection of H-atoms by using the two-photonresonance process to promote the nascent H-atom fragments to a long lived high Rydberg state rather than directly ionizing the atoms with the TPI process. This improvement resulted in a superior energy resolution: $0.3 \%$ as opposed to $1-2 \%$ for the TPI. ${ }^{19}$

Welge's group exploited this technique to study the fundamental $\left(H+D_{2}\right)$ hydrogen exchange reaction in a crossedbeam dynamical studies. ${ }^{20}$ The method has also been used by Ashfold and coworkers to obtain a precise determination of the $\mathrm{C}$-H bond dissociation energy of $\mathrm{C}_{2} \mathrm{H}_{2}{ }^{21}$ and to further investigate the dissociation dynamics of $\mathrm{H}_{2} \mathrm{O}$ at $121.6 \mathrm{~nm}$ photolysis. ${ }^{2}$ Very recently, the group of Wodtke has demonstrated that time-of-flight of neutral atoms and others radical species, ${ }^{23}$ excited in a metastable electronic state, has the potential to unravel finer details of the dissociation dynamics in polyatumic molecules.

Doppler spectroscopy of photofragments has been utilized by many groups previously. Bersohn and his co-workers have recently employed Doppler spectroscopy of H-atoms to obtain dynamical information both on the dissociation of $\mathrm{H}_{2} \mathrm{~S}$ (used as a source of fast $\mathrm{H}$ atoms), ${ }^{24}$ and on hydrogen exchange reactive scattering experiments ${ }^{25}$. Paul Houston has studied ${ }^{26}$ and reviewed ${ }^{272}$ the topic of vector correlation within which Doppler spectroscopy plays a central role; he and his coworkers have made extensive use ${ }^{20.30 .31}$ of this technique.

Of significant importance to our study, as we will see in chapter 2 and 6, is the work performed by the group of De Vries. Their work was the first simultaneous determination of the branching ratio and angular distribution of the fragment products of HI photodissociation, at the 193, 222 and $248 \mathrm{~nm}$ excimer photolysis wavelengths. Their photofragmentation spectroscopy experiment used TOF detection ${ }^{32}$ of the fragments to probe the phocodissociation processes in $\mathrm{ICl}$ and $\mathrm{IBr}^{2}$, in $\mathrm{CK}_{3} \mathrm{I}$ and $\mathrm{CD}_{3} \mathrm{I}^{33}$ and in $\mathrm{HI}^{36}$.

Wittig and coworkers have also considerably contributed to H-atom photofragment spectroscopy. Using the technique of velocity-aligned Doppler spectroscopy (VADS), they measured the branching ratios and angular distributions of the photofragment produced by the photolysis of $\mathrm{HI}$ at the 193 and $248 \mathrm{~nm}$ wavelength, and of $\mathrm{HBr}$ at $193 \mathrm{~nm}^{35}$ They also studied photoinitiated processes in CO-HI complexes with subpicosecond excitation. ${ }^{36}$ 
Using the method developed by Welge and co-workers, i.e. high resolution time-of-llight (HRTOF) of high-n Rydberg states, they observed effects of intermolecular collisions initiated in molecular aggregates (called clusters).

Through the present study, our contribution to the field is the measurement of electronic branching ratios and angular distributions of the photofragments velocities distribution, for the dynamics of photodissociation of $\mathrm{HI}$ ensuing absorption in the $A$ band. Determinations of the partial absorption cross sections, for each electronic product, will allow a quantum mechanical calculation of the electronic structure for the direct dissociative states responsible for $\mathbf{A}$ band absorption.

\subsection{Motivation for research topic: evolution of the perspective}

\subsubsection{Initial goal: coherent control}

Before our investigation on the topic started, Levy and Shapiro had just published a study on the determination of the electronic structure of $\mathrm{HI}$ in the $\mathrm{A}$ band. ${ }^{7}$ Assumed well-understood, the $\mathrm{HI}$ molecule appeared as an ideal candidate to test a theory of quantum interference in photodissociation dynamics, developed by Asaro, Brumer and Shapiro. ${ }^{3 *}$ This theory asserts that the dynamics of molecular photodissociation can be controlled, through quantum interference between excited scattering states, via the polarization of a photolytic optical excitation. Hence the HI molecule was a model system to demonstrate quantum interference in photodissociation; this was indeed the initial goal of the present study.

Repeated efforts, aimed at observing the predicted quantum interference effects in the photodissociation dynamics, did not meet with success. Within the precision of our measurements $(<5 \%)$, it has been asserted that no interference effect between scattering matter wave producing a noticeable change of the products angular distribution, occurs as a result of varying the polarization state of the photolysis laser. This result was presented as an oral paper at a conference two years ago (OSA-ILS '95, in Dallas, Texas).

\subsubsection{Revision of the assumption: electronic structure of HI}

The complex experimental apparans, that was initially constructed for the coherent control experiment, has been employed to investigate more usual questions concerning the dynamics of $\mathrm{HI}$ photodissociation following absorption continuum, also denominated the $A$ band. There was still a lot of experimental work required to completely confirm the theory on the electronic structure of the first absorption continuum of HI, developed by Levy and Shapiro. ${ }^{37}$ Thus, the experimental apparatus and the spectroscopic method implemented were used to investigate the validity of that theory. Therefore the present study is concerned with the description of this investigation, and the analysis of the measurements that were obtained. 
Through our journey toward understanding the intricate nature of the microscopic world, discoveries are sometimes found at the end of a long winding road, and it require patience and often serendipity. This certainly applied to the broader investigation which led to the topic at hand.

\subsection{Overview of the thesis}

Chapter 2 summarizes previous studies on the low-lying electronic states of the HI molecule, and other related properties of this system. Chapter 3 introduces the basic theory describing direct photodissociation and discusses vector correlation in these processes, with the perspective of relating them to the Doppler spectroscopy technique. The technical aspect of the experimental work is presented in chapter 4. The data reduction procedure, i.e the statistical averaging and related processing of the digital measurements, is described in chapter 5 .

In chapter 6, the results of systematic measurements, aimed at testing the theory of Levi and Shapiro on the electronic structure of $\mathrm{HI}^{37}$ are analyzed. The angular distributions of the photofragments velocities, and the branching ratios for the products are obtained from the analysis of the $\mathrm{H}$-atom Doppler spectra. Then a comparative analysis exhibit the central result of this work concerning the photodissociation dynamics in this molecule. A quantum mechanical analysis yielded a quantitative determination of the electronic structure for the A band absorption, enabling extrapolation of the observable between and beyond our measurements. Chapter 7 summarizes the results and introduces a few suggestions regarding expectations for other members of the hydrogen halide family.

\section{References}

1. R Schinke, Photodissociation Dynamics, Cambridge monographs on atomic, molecular and chemical physics, Cambridge University Press (1993)

2. R.G.W. Norrish and G. Porter, Nature, 164, 658 (1949)

3. G. Porter, Proc. R. Soc. A200, 284-300 (1950)

4. R.N. Zare and D.R. Herschbach, Proc. IEEE, 51, 173 (1963)

5. J.V.V. Kasper and George Pimentel, Appl. Phys. Lett., 5, 231 (1964)

6. J. Solomon, J. Chem. Phys., 47, 889 (1967)

7. M. Dzvonik, S. Yang and R. Bersohn, J. Chem. Phys., 61,4408 (1974)

8. G.E. Busch, J.F. Cornelius, R.T. Mahoney, R.I. Morse, D.W. Schlosser and K.R. Wilson, Rev. Sci. Instrum., 41, 1066 (1970)

9. S.J. Riley and K.R. Wilson, Discuss. Faraday Soc. 53, 132 (1972)

10. R.D. Clear, S.J. Riley and K.R. Wilson, J. Chem. Phys., 63, 1340 (1975) 
11. R. S. Mulliken, Phys. Rev. 50, 1017 (1936)

12. R. S. Mulliken. Phys. Rev., 50. 1028 (1936)

13. R. S. Mulliken, Phys. Rev., 51, 310 (1937)

14. R. Schmiedl, R Boettner, H. Zacharias, W. Meier and K. H. Welge, Opt. Comm., 31, 329 (1979)

15. R. Schmiedl, W. Meier and K. H. Welge, Chem. Phys. Lett., 80, 495 (1981)

16. R. Schmiedl, H Dugan, W. Meier and K. H. Welge, Z. Phys. A., 304, 137 (1982)

17. H. Zacharias, H. Roottke, J. Danon and K. H. Welge, Opt. Comm., 37, 15 (1981)

18. H J. Krautwald, L. Schnieder, K. H. Welge and M.N.R. Ashfold, Faraday Discuss. Chem. Soc., 82, 99 (1986)

19. L. Schnieder, W. Meier, K.H. Welge, M.N.R. Ashfold and C.M. Western, J. Chem. Phys., 92,7027 (1990)

20. L. Schnieder, K. Seckamp-Rahn, F. Liedeker, H. Steuwe and K. H. Welge, Faraday Discuss. Chem. Soc., 91. 259 (1991)

21.D. H. Mordaunt and M.N.R. Ashfold, J. Chem. Phys., 101, 2630 (1994)

22. D. H. Mordaunt, M.N.R. Ashfold and R. N. Dixon, J. Chem. Phys., 100, 7360 (1994)

23. M. Drabbels, C. G. Morgan, and A. M. Wodtke, J. Chem. Phys., 102, 611 (1995), and M. Drabbels, C. G. Morgan, and A. M. Wodke, J. Chem. Phys., 103, 7700 (1995)

24. G. W. Johnston, B. Katz, K. Tsukiyama and R. Bersohn, J. Phys. Chem., 91, 5445 (1987)

25. A. Chattopadhyay, S. Tasaki, R. Bersohn and M. Kawasaki, J. Chem. Phys., 95, 1033 (1991)

26. G. E. Hall, N. Sivakumar, G. Chawla, P. L. Houston, I. Burak, J. Chem. Phys., 88, 3682 (1988)

27. P. L. Houston, J. Phys. Chem., 91, 5838 (1987)

28. G. E. Hall and P. L. Houston. Annu. Rev. Phys. Chem., 40, 375 (1989)

29. G. E. Hall, N. Sivakumar, R. Ogorzalek, G. Chawla, H.-P. Haerri, P.L. Houston, I. Burak, J.W. Hepburn, Faraday Discuss. Chem. Soc., 82, 13 (1986)

30. N. Sivakumar, G. E. Hall, P. L. Houston, J.W. Hepburn, I. Burak, J. Chem. Phys., 88, 3692 (1988)

31. R. Ogorzalek Loo, Charles E. Strauss, H.-P. Haerri, G. E. Hall, P.L. Houston, I. Burak, J.W. Hepburn, J. Chem. Soc., Faraday Trans. 2, 85, 1185 (1989)

32. M. S. DeVries, N. J. A. van Veen, M. Hutchinson and A. E. DeVries, Chem. Phys., 51,159 (1980)

33. G. N. A. van Veen, T. Baller, A. E. DeVries, Chem. Phys., 87, 405 (1984)

34. G. N.A. van Veen, K.A. Mohamed, T. Baller, A. E. DeVries, Chem. Phys., 80, 113 (1983)

35. Z. Xu, B. Kopliz, C. Wittig, J. Phys. Chem., 92, 5518 (1988)

36. C. Jacques, L. Valachovic, S. Ionov, E. Bohmer, Y. Wen, J. Segall and C. Wittig, J. Chem. Soc. Faraday Trans., 89, 1419 (1993).

37. I. Levy, M. Shapiro, J. Chem. Phys., 89, 2900 (1988)

38. C. Asaro, P. Brumer, M. Shapiro, Phys. Rev. Lett., 60, 1634 (1988). 


\section{Chapter 2 The low-lying electronic states of HI}

The goal of this chapter is to recapitulate our state of knowledge of the low-lying electronic states of molecular HI, prior to the experimental study described in this thesis. In section 2.1, a discussion of coupling cases is followed by a derivation of the correlation diagram from group theory arguments. The numerous experimental and theoretical studies that contributed to the knowledge of this system are introduced in section 2.2. The information is summarized in section 2.3, and the global picture obtained is further complemented by a discussion of the perturbation from ion pair on the electronic structure. The facts gathered in this chapter will be referred to in the comparative analysis of section 6.2 .

\subsection{Symmetry: coupling cases and correlation diagram}

To represent the eigenstates of a quantum system, it is customary to make use of invariance under rotation. In an atomic system, the total angular momentum $J$ is conserved as a consequence of the spherical symmetry of the central potential of the nucleus. In a diatomic molecule, the spatial symmetry imposed by the internuclear axis results in the conservation of $\Omega$, the axial projection of the total electronic angular momentum. Below we follow the method delineated by Herzberg' to show how the molecular states can be derived from the constraints of the molecular field on the atomic symmetry.

\subsubsection{Mulliken's prediction}

A lot of information on the structure of molecular energy levels can be obtained from group theory arguments, on the basis of symmetry considerations and correlation diagrams. Mulliken ${ }^{789}$ first demonstrated these principles for simple molecules such as $\mathrm{HI}$, as depicted by figure 2.1 taken from reference 9 . This picture illustrates only those electronic states that possess the proper symmetry to take part in a one photon absorption spectrum from the ${ }^{1} \Sigma_{0,}$ ground state, denoted $N$ by Mulliken. One can note that amongst the first group of states, the ${ }^{3} \Pi_{1},{ }^{3} \Pi_{0 *}$, and ' $I$ states denoted by Mulliken as the ${ }^{3} Q_{1},{ }^{3} Q_{a}, Q$ or the $Q$-group of states, those with $Q=1$ correlate with the ground iodine atom product, while the one with $\Omega=0$ leads to an excited iodine atom. Hence Mulliken denoted the A band absorption as the $\mathbf{N}-\mathbf{Q}$ transition.

Another important observation that Mulliken emphasized was that the $T^{3} \Sigma_{1}$ state should be too high in energy to contribute to the A band absoption, because it correlates to the second excited configuration of the united atom. Below, we will further discuss correlation in the electronic structure, but first we shall discuss the coupling of angular momenta pertaining to the electronic states involved in the dynamics of HI photodissociation. 


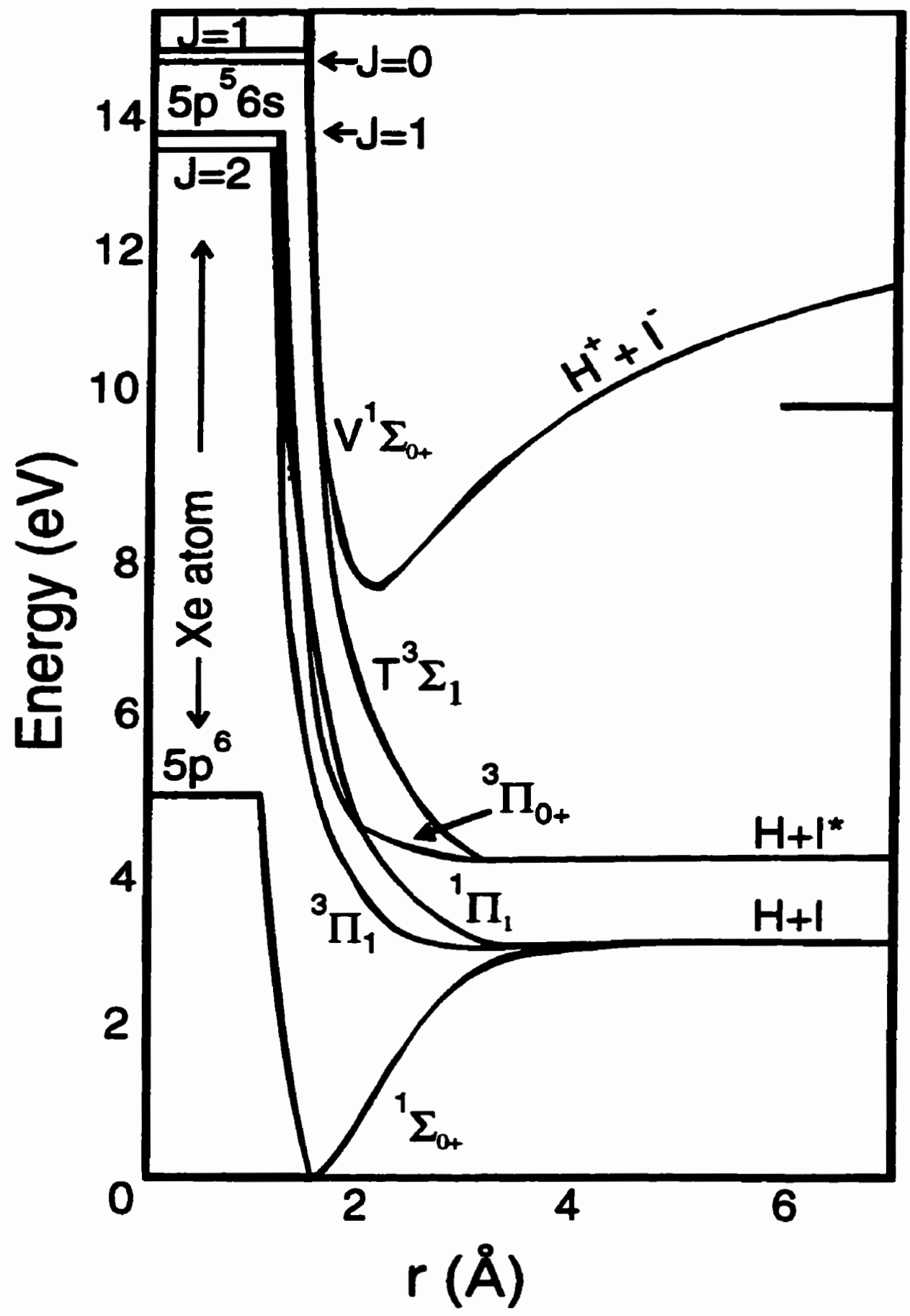

Figure 2.1. PEC derived by Mulliken from theoretical considerations (taken from ref. 9). 


\subsubsection{Angular momenta: coupling cases}

The various coupling cases to describe angular momenta in molecules can be grouped in two classes: the weak spin-arbit coupling cases (analog of atomic Russell-Saunders in an atom), and the strong spin-orbit cases (analog of atomic $j-j$ coupling in an atom).

In the limit of weak spin-orbit coupling for a molecule, the orbital electronic angular momentum of each atum ( $\left.\mathrm{L}_{\nu}\right)$ is coupled with the internuclear field; this dominates the siructure of the energy levels. The projection of the total orbital angular momentum onto the internuclear axis, $\Lambda$, is a good quantum number. The tocal spin $S$ is then coupled with $\Lambda$ and the quantization of the projection $\Sigma$ of the total spin results; this is the prescription for $\Lambda-\Sigma$ coupling, also denoted Hund's case a coupling. In this angular momentum coupling scheme, $\Omega=\Lambda+\Sigma$ is also conserved. In the consideration of the general problem, the rotation of the nuclei $N$, is added to $\Omega$ to produce $J$, the total angular momentum of the system. When $\Lambda=0$, we have the Hund's case $b$, in which case the total spin, $\mathrm{S}$, does not couple to the axis, and it is vectorially added directly to the rotation of the nuclei to produce J; the consequence is that each rotational level (with $N \neq 0$ ) is split into $(2 S+1)$ components.

Depending on the relative strength of the spin-orbit interaction within the separated atoms versus the interaction energy between the component atoms, two case c coupling schemes need to be considered: the "close nuclei" spinorbit, or Hund's case c coupling, denoted here by $\left(J_{2}, \Omega\right)$, and the "far nuclei" spin-orbit, or Mulliken's case $\left.c\right)$ coupling, denoted here by $\left(\omega_{1}, \omega_{2}\right)$.

In the Hund's case c, first the total orbital electronic angular momentum of the diatom L couples with the total spin of the diatom $S$, and then the total spin and total orbital angular momenta add to produce $J_{2}$, whose projection on the internuclear axis $\Omega$ is conserved; consequently, the possible values of $\Omega$ span the range: $\Omega=[|L-S|,|L+S|]$. The vectorial addition of the nucleus rotation $N$ to $\Omega$ gives $J$, the total angular momentum of the system, which is also an invariant of the system. In order for such a spin-orbit interaction to occur efficiently, it was deemed required that the atoms must be in close proximity, whence the "close-nuclei" denomination for this case $c$.

In the Mulliken case $c_{i}$ it is the total electronic angular momentum of each atom $J_{i}=L_{i}+S_{i}$, $i=1,2$, which each couples to the internuclear axis to result in $\omega_{1}$, and therefore $\Omega=\omega_{1}+\omega_{2}$, is the only good quantum number for this coupling scheme. Mulliken realized that interaction between diatom partners, at a large enough interatomic distance, will be such that the interaction energy of the atomic spin-orbit will prevail over the diatom interaction energy. Consequenty, he concluded that the "far nuclei" case c must always come into play, to a variable extent, when the diatom separates; the transition occurs in the recoupling region ${ }^{2}$, the region where each individual total atomic angular momentum recouples. 


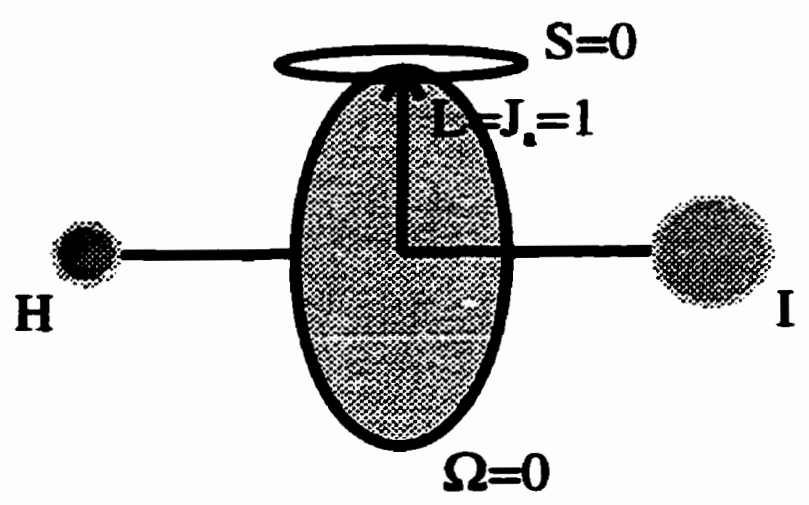

a) close nuclei case $\mathrm{c}$ for $\mathrm{HI}$ in the $\mathrm{X}{ }^{\prime} \Sigma_{0,}$ state
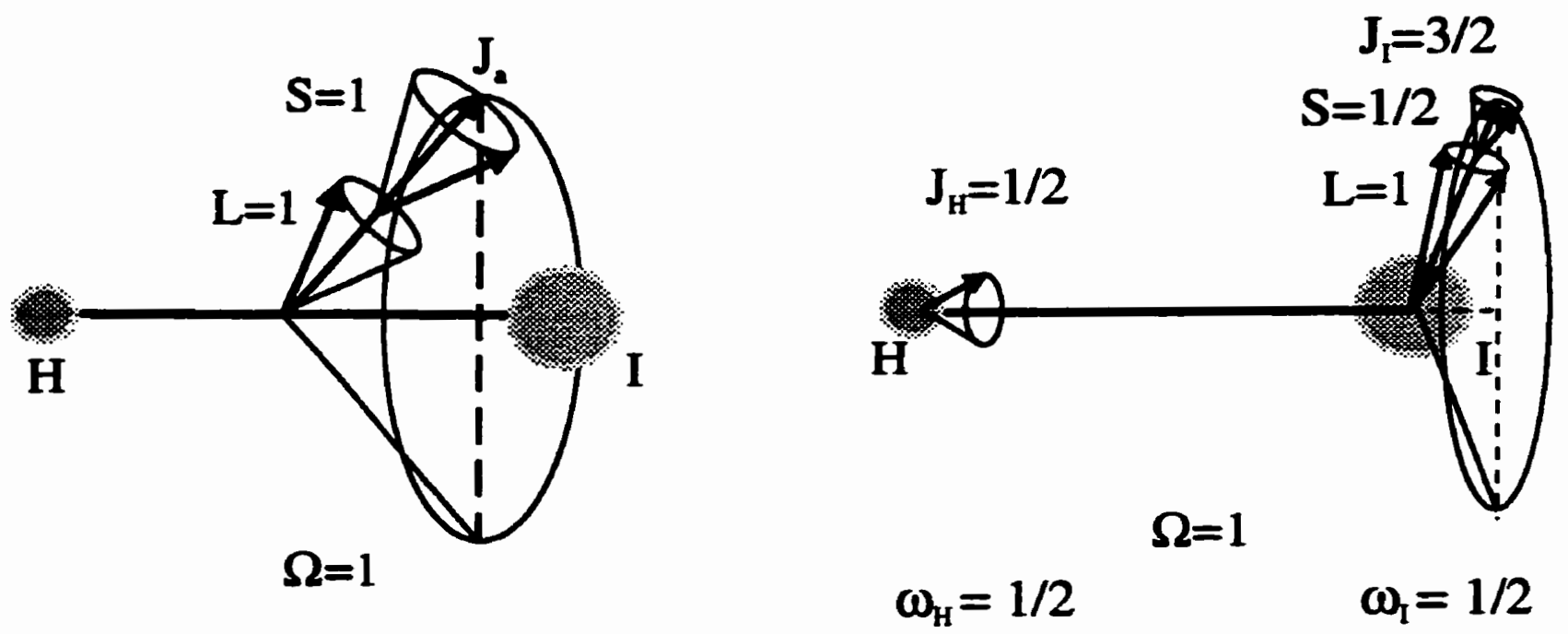

b) close nuclei (Hund's) case $c$ for $H I{ }^{3} \Pi_{1}$, and far nuclei (Mulliken's) case c for $H I{ }^{3} \Pi_{1}$

Figure 22. Angular mamenta coupling cases in $\mathbf{H I}$; a) close nuclei for the ground electronic ${ }^{2} \boldsymbol{\Sigma}_{0 *}$ state, and b) comparison of the close and far nuclei coupling in the ${ }^{3} \mathbf{H}_{1}$ dissociative state. 
This important concept can be used to guide vector coupling algebra aimed at explaining the general correlation between molecular electronic states and the asymptotic atomic states. In the ground electronic state of the HI molecule, the "close nuclei" case c should prevail, on the basis of the existence of the strong bond, $3.054 \mathrm{eV}$, compared to the iodine spin-orbit energy $0.943 \mathrm{eV}$. The total orbital angular momentum of the electrons, $L$, contributed by the p-orbital of the iodine atom, has a null projection on the internuclear axis, as shown in figure 2.2 a), so it must precess around it, averaging to zero over time.

In an excited dissociative state of $\mathrm{HI}$, the "far nuclei" case $\mathrm{c}$ will rapidly become dominant, if it is not already so, right upon excitation to the dissociative state. As an example, lets consider the ${ }^{3} \Pi_{1}$ state, for which both the "close" and "far nuclei" case c coupling are illustrated in figure $2.2 \mathrm{~b}$ ). In principle, we may assume that the excited molecule is born in close nuclei coupling; then, as the atoms repel each other and the atomic spin-orbit energy comes to dominate the electronic structure, the far nuclei coupling better represents the system.

However, it may also be that the recoupling region is, to a large extent, in the Franck-Condon region, in which case the dissociative state could be described as the correlated sum of two atomic states. The reader should note that the coupling correlation energy for the angular momenta should be very weak, of the order of $\mathrm{MHz}$ to GHz. If the separation of the atoms is sufficiently sudden, there will be no time for the correlation between the atomic states to evolve; the relatively slow natural frequency of that correlation is in the range of few tens of femtoseconds, which is more than four order of magnitude slower than the relaxation time of dissociation for this system. Consequently, the distribution of the respective angular momenta for the atomic partners will be aligned and/or oriented, depending on the polarization of the photolysis excitation. At the end of this thesis, we will elaborate on the conservation of total angular momentum in the matter-radiation system.

\subsubsection{Correlation diagram: derivation}

\section{A) Various approach to molecular orbital theory}

In the present surdy, we draw many arguments from the work of Mulliken ${ }^{789}$ to derive the diagram of figure 2.5 , illustrating the correlation between the extreme cases of unitod atoms and separated atoms. The electronic orbitals of a diatomic molecule can be derived by using the building-up principle and the atomic orbitals of the separated atcoms (method of Heitler and London, or valence-bond theory). Conversely the molecular orbital can be obtained from the molecularization of the iscelectronic united atoms orbitals (Xe atom in the case of HI), i.e. the symmetrization of an atomic orbital in the molecular field. At a higher level of sophistication, one often considers the linear combination of atomic orbital-molecular orbital (LCAO-MO); this approach uses the combination of original atomic orbitals symmetrized by the axially symmetric molecular field. 
For example, the np atomic orbital becomes divided into npo and np $\pi$ in the internuclear molecular field, while the ns orbital can only become nso. In the ground electronic state of hydrogen iodide, only the po orbital of the iodine atom enters into chemical valence with the so crbital contributed by the hydrogen atom, but the np$\pi$ orbital, due to the proximity of its energy level, also influences the structure.

Starting from the united atom, the ground state electron configuration of $\mathrm{Xe}:(5 \mathrm{~s} \sigma)^{2}(5 \mathrm{p \sigma})^{2}(5 \mathrm{p} \pi)^{4}$ corresponds to the ground state ' $\Sigma_{0,}$ in the $(\Lambda-\Sigma)$ representation, $\alpha \sigma^{*}$ in the $(\Omega-s)$ representation. The first excited configuration of molecularized atomic orbitals $(5 s \sigma)^{2}(5 \mathrm{po})^{2}(5 \mathrm{p} \pi)^{3} 6 s \sigma^{*}$ correlates to the states of the Q-group: $\left({ }^{1} \Pi_{,}{ }^{3} \Pi_{0 \times 0}{ }^{3} \Pi_{1}\right)$. The next excited united atoms configuration is the $(5 s \sigma)^{2}(5 p \sigma)(5 p \pi)^{4} 6 s \sigma^{*}$, which correlates to the T and V states illustrated in figure 2.1 .

\section{B) Heuristic arguments regarding the electronic transitions to the $Q$-group}

According to Mulliken's theory, absorption in the A band results from excitation of a non-bonding $\pi$-electron to an anti-bonding $\sigma^{*}$ orbital. Invoking molecular orbital theory ${ }^{3}$, the consequence of this electronic transition is to reduce the bond order from 1 to $1 / 2$. Consequently, excitation in the first continuum weakens the bond and dissociation occurs. Another heuristic argument is developed as follow: (1) one needs to excite a hole in the p $\pi$ shell to generate the Q-group of $I$ molecular states; (2) the $\pi$-arbital results from the contribution of the p-electron by the iodine atom to the molecular orbital; consequently: one can loosely say that the iodine is the chromophore for the $\mathrm{A}$ band absorption.

\section{C) Correspondence between atomic and molecular states}

As the atoms approach each other, the spherical symmetries of the individual atoms are altered by the interaction arising along the internuclear axis, and the invariance of the total electronic angular momentum for each separate atom is lost. However, the axial symmetry of the diatom potential results in the conservation of the projection of the total electronic angular momentum along that axis. Invoking the Ehrenfest adiabatic law', we expect a correspondence between the atomic and molecular states as the atoms approach or repel each other.

Mulliken concluded that the molecular states of the first absorption continuum are: $\left\{{ }^{1} \Pi,{ }^{3} \Pi_{0+},{ }^{3} \Pi_{1}\right\}$ in the customary $(\Lambda-\Sigma)$ representation. To generate these states from the separated atomic orbitals, the only atomic terms that need to be considered are the ground state ${ }^{2} \mathrm{~S}$ of hydrogen and the lowest ${ }^{2} \mathrm{P}$ term of iodine. Although the hydrogen $2 \mathrm{~S}$ has two hyperfine components separated by $1.4204 \mathrm{GHz}$, it is a single state at our experimental resolution. Thus the spin-arbit components of the iodine atom, ${ }^{2} \mathbf{P}_{1 / 2}$ and ${ }^{2} \mathbf{P}_{322}$ are the atomic states responsible for the symmetries of the molecular states corresponding to the first continuum of absorption. However this relation of completeness is not reciprocal, since the iodine multiplet also correlates to ${ }^{3} \Pi_{2}$ and to the $T^{3} \Sigma_{1}$ state. 


\begin{tabular}{lcl|}
\hline Atom & $S O(3)$ & $C_{\infty . v}$ \\
$H(S)$ & $S_{g}$ & $\Sigma^{+}$ \\
$I(P)$ & $P_{u}$ & $\Sigma^{+} \oplus \Pi$ \\
hence: & & \\
$\Sigma^{+} \otimes\left(\Sigma^{+} \oplus \Pi\right)=$ & $\Sigma^{+} \oplus \Pi$ \\
\end{tabular}

Figure 2.3 Correspondence between atomic and molecular symmetry group, for the electronic orbital part of the molecular state.

\begin{tabular}{|cll|}
\hline Spin & $C_{\infty, v}$ \\
0 & $\Sigma^{+}$ & \\
1 & $\Sigma^{-} \oplus \Pi$ \\
$s_{\Lambda}=R_{\text {Spin }}^{C_{-. v}} \otimes R_{\text {Orbical }}^{C_{-\infty}}$ & $=\Omega$ \\
${ }^{1} \Sigma^{+}=$ & $\Sigma^{+} \otimes \Sigma^{+}$ & $=\left(0^{+}\right)$ \\
${ }^{3} \Sigma^{+}=\left(\Sigma^{-}+\Pi\right) \otimes \Sigma^{+}$ & $=\left(0^{-}, 1\right)$ \\
${ }^{1} \Pi=\Sigma^{+} \otimes \Pi$ & $=(1)$ \\
${ }^{3} \Pi=\left(\Sigma^{-}+\Pi\right) \otimes \Pi$ & $=\left(0^{+}, 0^{-}, 1,2\right)$
\end{tabular}

Figure 2.4 Tensorial product of orbital and spin representation.

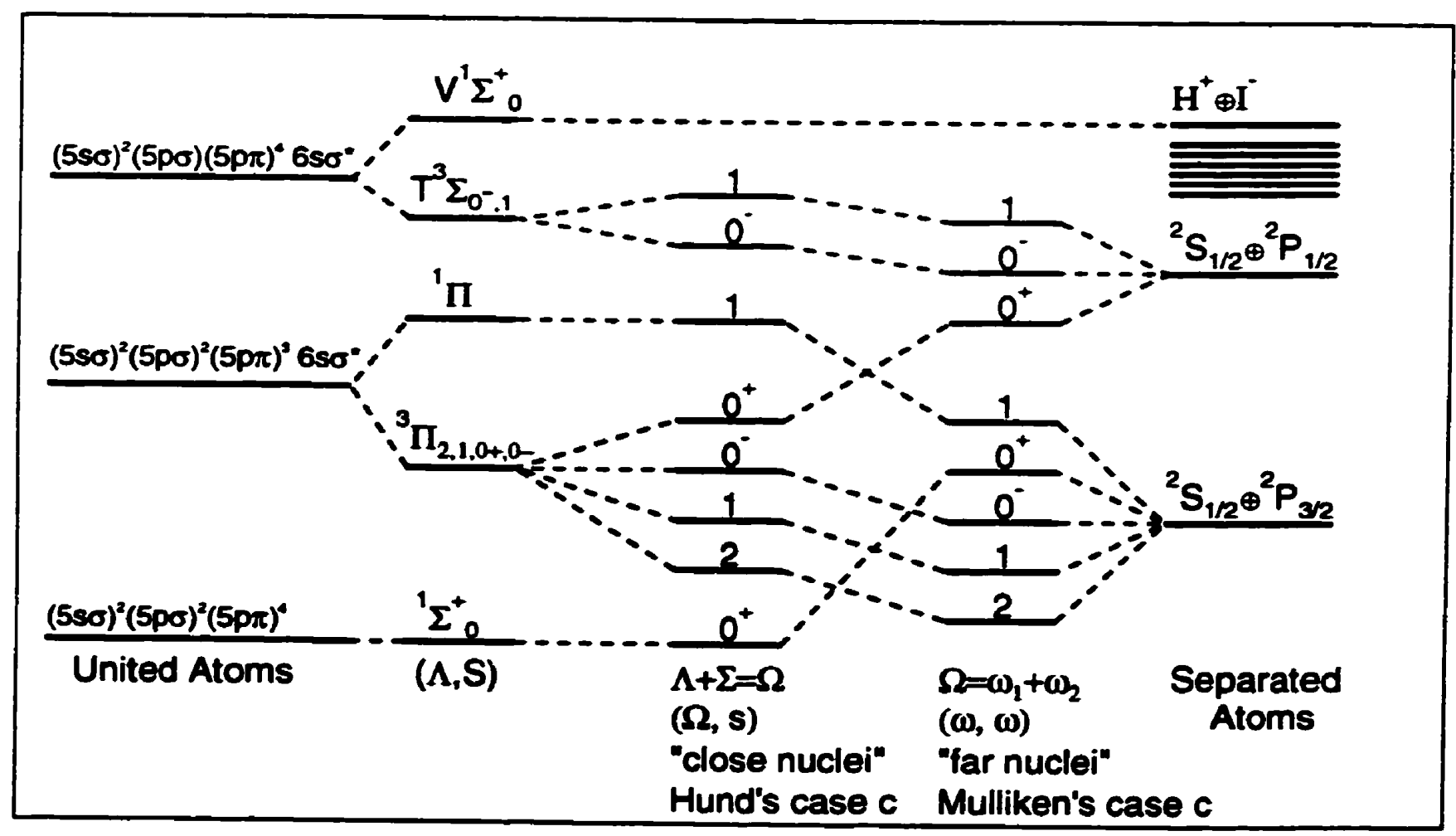

Figure 2.5 Correlation diagram derived in section 2.1. 


\section{D) Enumeration of states using group theory}

In $(\Lambda-\Sigma)$ representation, the molecular atbitals are obtained with the aid of the following group theory arguments. The representation of an atomic term, in the symmetry group of rotation SO(3), is determined by the total orbital angular momentum of the term, and the parity of each atomic term, $g$ or $u$, depends on whether $\Sigma l_{i}$ is even or odd respectively. Using the correspondence of the representation between the atomic symmetry group $S O(3)$ and the diatomic symmetry group $C_{-. m}$ their contributions result in the possible molecular orbital symmetries. Only molecular states of the type $\Sigma^{+}$or $\boldsymbol{I}$ are obcained from the tensor product of the representations for each atomic systems, as shown in the figure 2.3. When $\Lambda=0$, the resulting $\Sigma$ term may have either + or - parity; the parity is defined by': $p=\Sigma_{a} L_{i n}+L_{1}, p=0$ or 1 corresponds to a term of molecular parity denoted by + or - respectively.

If the interaction energy between the two atomic orbitals is stronger than the coupling between the $L_{\mathbf{l}}$ and $S_{\mathbf{t}}$ in each of the separated atoms, we can add the spins of the individual atoms and use the possible values of the sum to attribute a multiplicity to the spinless molecular terms obtained above. In the limit of weak spin-orbit coupling. the states are denoted by terms of the form: ${ }^{(2 \cdot 1)} \Lambda_{\alpha}$. Due to the spin-orbit coupling, the total spin couples with the field of the internuclear axis through $\Lambda \cdot \Sigma$ coupling, and $\Omega=\Lambda+\Sigma$ is a better invariant.

For HI, the total spin can be 0 or 1, the multiplicities are singlet and triplet, and we obtain the following terms: ${ }^{3} \Pi_{2},{ }^{3} \Pi_{1},{ }^{3} \Pi_{0 .}, \Pi_{0,}$ and ${ }^{1} \Pi$. For increasing strength of the spin-orbit coupling, the precession frequency of the total spin and orbital angular momenta becomes so large that they lose their meaning, and $\Omega$ is the sole good quantum number eventually left. To properly enumerate the states, we must: (I) reduce the spin wavefunction to the molecular group symmetry and, (ii) take the tensor product of the spin wavefunction ${ }^{4}$ projected in the $C_{-. v}$ group ${ }^{5}$; this procedure is represented schematically in the figure 2.4 .

Mulliken showed that the energy of the components of the ${ }^{13}$ II multiplet, or the Q-group, should increase in the order: ${ }^{3} \Pi_{2},{ }^{3} \Pi_{1},{ }^{3} \Pi_{a},{ }^{3} \Pi_{0,}$, and ' $\Pi$, for reasons similar to the one invoked by Hund's rule for the inversion of the atomic multiplet when the term configuration is more than half-full. Among these states, only ${ }^{3} \Pi_{1},{ }^{3} \Pi_{0,}$, and ${ }^{\prime} I I$ are of interest to this study, because single photon transitions to the ${ }^{3} \Pi_{2}$ and ${ }^{3} \Pi_{0}$ are symmetry forbidden. Although the large spin-arbit energy of the iodine atom affects the coupling of angular momenta in HI, such that $\Omega$ is the only good quantum number for the electronic states in this system, the $(\Lambda, \Sigma)$ representation of the electronic terms allows for an intuitive and familiar way to enumerate the states. The correlation diagram of figure 2.5 resulted from the consequence of the above considerations.

To conclude this section, the global symmetry of this dynamical system is briefly discussed, in view of its central importance in the present study. It must be noted that the A band involves two types of electronic transitions: 
parallel, $\Delta \Omega=0$, and perpendicular, $\Delta \Omega= \pm 1$. Moreover, assuming that Mulliken is right in his assertion that the A band absorption is due to the $\mathbf{N}-\mathbf{Q}$ transitions, then the correlation between atomic and molecular symmetries predict that the ground state iodine products are the results of perpendicular transitions, and excited state iodine products are the consequence of a parallel transition.

\subsection{History: evolution of our knowledge}

Most of the previous wark on the photodissociation of HI, which brought new information on the dynamics of this system pric to the present study, are reviewed below. A few spectroscopic works on HI, devoted to the region of energy above the first continuum of absorption and up to molecular ionization, are also cited whenever they were found to contribute useful arguments toward the elucidation of the low-lying electronic structure of HII.

\subsubsection{Early studies (1935-1955)}

A) Goodeve and Taylor (1935-1936)

In the mid-hirties, Goodeve and Taylo measured the earliest absorption spectrum for HI in the ultraviolet. They obtained a quantitative assessment of the absorption coefficients. They concluded that the maximum of the smooth continuum of absorption was located around $2250 \AA$.

\section{B) Mulliken (1936-1937)}

During the same period, Mulliken published a series of three theoretical papers ${ }^{78.9}$ on the low-lying electronic states of simple heteropolar diatomic molecules. In these studies, he investigated the differences and similarities of the electronic structure of uni-univalent diatanic molecules built from so-so and po-so molecular orbitals from the combination of separated atoms of hydrogen, metal and halogen. The third paper of the series focussed on univalent diatomic halides; the case of hydrogen halides (especially $\mathrm{HI}$ and $\mathrm{HBr}$ due to the availability of data) was discussed in detail. His theoretical considerations, developed on the basis of coupling cases and symmetry correlation, yielded an estimate of the low-lying electronic structure of HI shown in figure 2.1 .

\section{C) Price (1936-1938)}

Price ${ }^{10}$, who first recorded part of the $B$ band with a resolution better than $1 \AA$, observed that a spectral feature at $1762 A$ was consisting of a very sharp $Q-b r a n c h, n o t$ wider than $10-15 \mathrm{~cm}^{-2}$, with diffuse rotational structure on either side; be observed that band in the range extending from 1759-1770 $\AA$. At much higher energy above the first continuum, the discrete spectrum was also photographed for the first time by Price ${ }^{11}$ in 1938 . He recorded the VUV absorption spectra of HI from 1800-1128 A. 
Additionally, Price oblained the ionization potential by extrapolating the Rydberg series of bands existing between 1124.4 - $1346.1 \AA$. By observing that the Rydberg series corresponded to the upper component of the ${ }^{2} \mathrm{M}^{2} \mathrm{HI}^{+}$, and by assuming that the spin-arbit coupling of the $\mathrm{HI}^{+}$ion is very close to the atomic value, he estimated the two limits of the ionization potential, which correspond respectively to the $X\left(-\Pi_{32}, v=0\right)$ and to the $X\left(-\Pi_{1 / 2}, v=0\right)$ at: 10.33 and $10.99 \mathrm{eV}$. This compares very well to the more recent values obtained by Hepburn and Hart ${ }^{12}$ : 10.386 and $11.051( \pm 0.001) \mathrm{eV}$.

\section{D) Romand and Vodar (1948)}

Romand and Vodar ${ }^{13}$ have measured the spectrum from 2300 to $1500 \AA$, shown in figure 2.6 a). They observed that the first continuum, denoted the $A$ band, reaches a maximum at $2180 \AA$, after which it decreases to a minimum at $1900 \AA$. A markedly sharp rise occurs at $1800 \AA$, marking the onset of the $B$ band.

Romand ${ }^{14.15}$ has theoretically analyzed the $A$ band absorption. He has postulated a shape for the $A$ continuum, free of the presumed overlapping by the B band below $2000 \AA$. On theoretical basis, he has decomposed the A band into two partial absorptions, as shown in figure $2.6 \mathrm{~b}$ ), where curve 1 is the total $A$ band absorption, curve 2 is the partial absorption due to the ${ }^{3} Q_{a_{+}}-N$ transition, and curve 3 is the partial absorption due to the ${ }^{3} Q_{1}-N$ transition. Note that according to Romand, the photolysis of HI would produce a strongly inverted population of the electronic spin-orbit products. Moreover, he was assuming that the B-band was produced by the ' $Q-N$ transition; this was a reasonable assumption justified on the ground that this singlet-singlet transition must be the strongest of the Q-group, and that the much stronger B band appeared to be a good candidate for this assignment.

\subsubsection{Era of photochemical studies (1955-1975)}

Here are considered the studies that further illuminated the dynamics of HI photodissociation, either as a goal in itself, or as a consequence of research on a related topic. In general, photochemical studies provided instructive results, but they also suggested more questions, which partly motivated the development of photofragment spectroscopy ${ }^{2}$.

\section{A) Donovan and Husain (1962)}

Donovan and Husain have produced a series of three articles on the generation of excited lodine atoms $I\left({ }^{2} P_{1 / 2}\right)$ by flash photolysis of $\mathrm{CF}_{3} \mathrm{I}^{16}$ and $\mathrm{HI}^{17}$, and their collisional deactivation ${ }^{18}$ (or quenched relaxation) in various bath gases. These studies used the technique of kinetic spectroscopy ${ }^{19}$ of the Iodine atom products in the VUV to investigate the dynamics of photodissociation initiated by flash photolysis. The broadband photolysis source. assumed to encompass the whole absorption continuum of $H$, was observed to produce about one fifth of the Iodine atoms in the excited state. 
a)

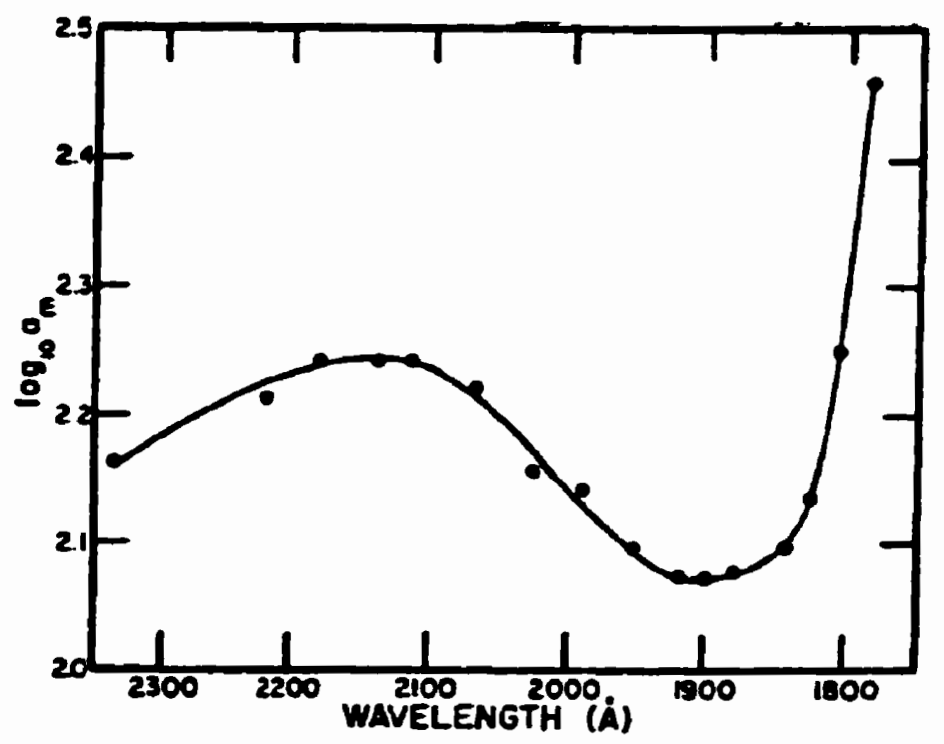

b)

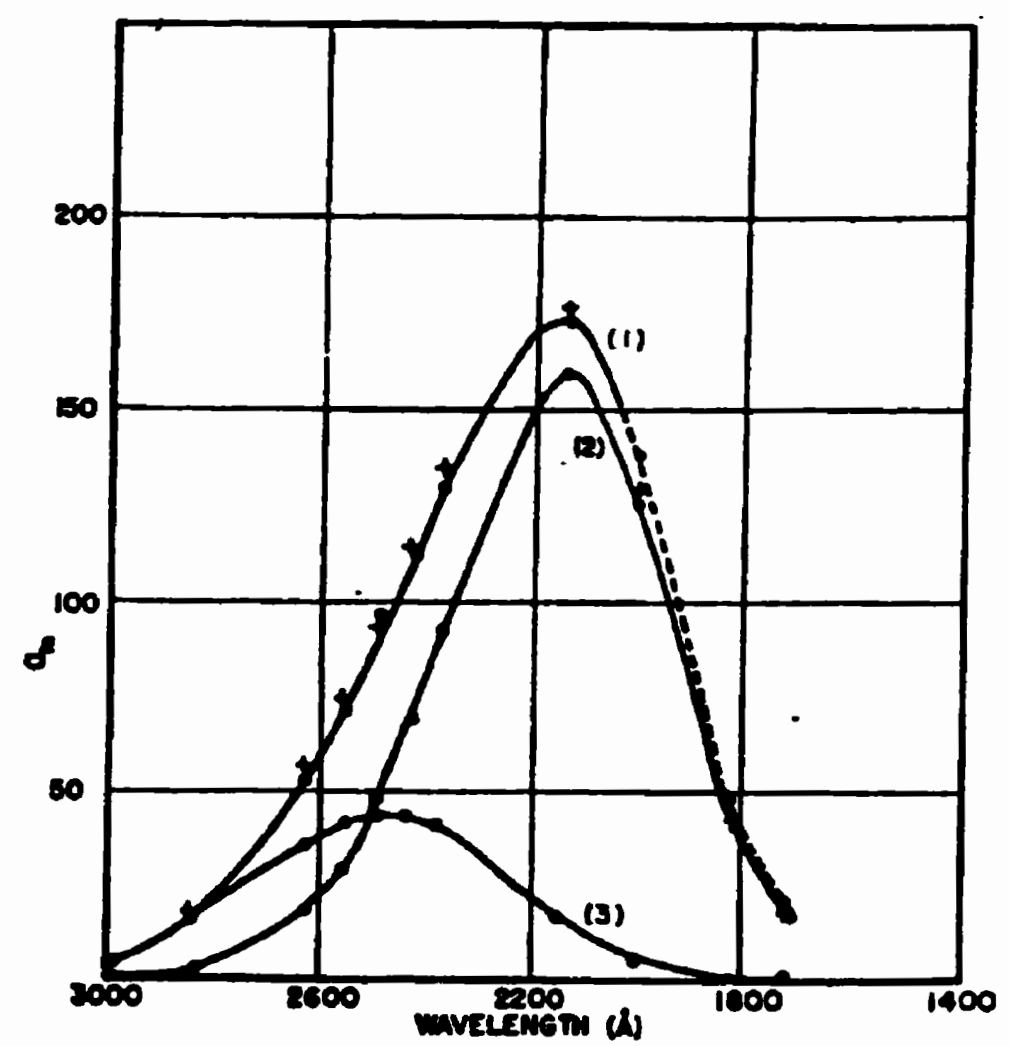

Figure 2.6. a) Absorption spectrum measured by Romand and Vodar (from ref. 13). b) Theoretical decomposition, by Romand, of the total absorption of HI into partial absorption by each dissociative states (from ref. 15). 


\section{B) Martin and Willard (1964)}

Martin and Willard ${ }^{20}$ used the photolysis of $\mathrm{HI}$ at $1849 \AA$ to produce hot H-atoms with 2.6 and $3.6 \mathrm{eV}$ of kinetic energy. Their results provided an experimental test for the theoretical model obtained from the analysis of Romand cited above. They noted that due to the effect of the selection rule $\Delta S=0$, the ' $Q-N$ must be several times stronger than the ${ }^{3} Q_{1}-N$. This points to the fact that Romand had assumed that the $B$ band corresponds to the 'Q-N transition. The mere presence of spectral structure, near the region where the $B$ band and the $A$ band overlap, indicates the involvement of a predissociating state, most likely located above the Q-group. Because of this fach, the interpretation of Romand required revision.

\section{C) Huebert and Martin (1968)}

Huebert and Martin ${ }^{21}$ measured the absorption spectrum of HI from 3000 to $1800 \AA$ shown in figure 2.7. Their result confirmed qualitatively the measurement of Romand, but provided more information and improved measurement precision. By measuring the absorption spectrum of $\mathrm{HI}$, with and without Dry Ice packed around the cell, they concluded that $I_{2}$ absorbed around $9 \%$ of the radiation in the range 1850-1900 at S.T.P., while the contribution to the absorption by $\mathrm{I}_{2}$ at longer wavelength is less than the measurement uncertainty of $0.5 \%$. The numerical values of their extinction coefficients for $H$ are shown in table 2-1.

Table 2-1. Molar extinction data from Huebert and Martin (from ref. 21).

\begin{tabular}{|c|c|c|c|c|c|}
\hline Wavelength $(\AA)$ & $\epsilon 1 . \mathrm{mol}^{-1} \mathrm{~cm}^{-1}$ & $\delta(\epsilon) 1 . \mathrm{mol}^{-1} \mathrm{~cm}^{-1}$ & Wavelength $(\AA)$ & $\epsilon 1 . \mathrm{mol}^{-1} \mathrm{~cm}^{-1}$ & $\delta(\epsilon) 1 . \mathrm{mol}^{-1} \mathrm{~cm}^{-1}$ \\
\hline 3000 & 7.4 & 0.1 & 2300 & 203.5 & 1.0 \\
\hline 2900 & 13.6 & 0.1 & 2215 & 212.9 & 0.5 \\
\hline 2800 & 24.9 & 0.2 & 2100 & 196.0 & 0.7 \\
\hline 2700 & 45.3 & 0.1 & 2000 & 164.1 & 0.4 \\
\hline 2600 & 78.1 & 0.8 & 1950 & 146.1 & 0.9 \\
\hline 2537 & 106.8 & 0.6 & 1900 & 133.5 & 0.5 \\
\hline 2500 & 124.3 & 1.2 & 1877 & 130.8 & 0.8 \\
\hline 2400 & 170.3 & 0.9 & 1850 & 134.2 & 1.2 \\
\hline
\end{tabular}

\section{D) Cadman and Polanyi (1968)}

Cadman and Polanyi investigated the reaction dynamics of $H$ with $H$-atoms generated by $2537 \AA$ gas phase photolysis $^{2}$, and by a discharge technique ${ }^{2}$. They probed the reaction dynamics via measurement of the emission intensity from the infrared chemiluminescence of electronically excited Iodine $I\left({ }^{2} P_{1 / 2}\right)$ product. From the combination of these studies, they obtained an estimated value of $55 \pm 25 \%$ for the relative yield of excited atoms from the primary photodissociation event at $2537 \AA$ photolysis. 


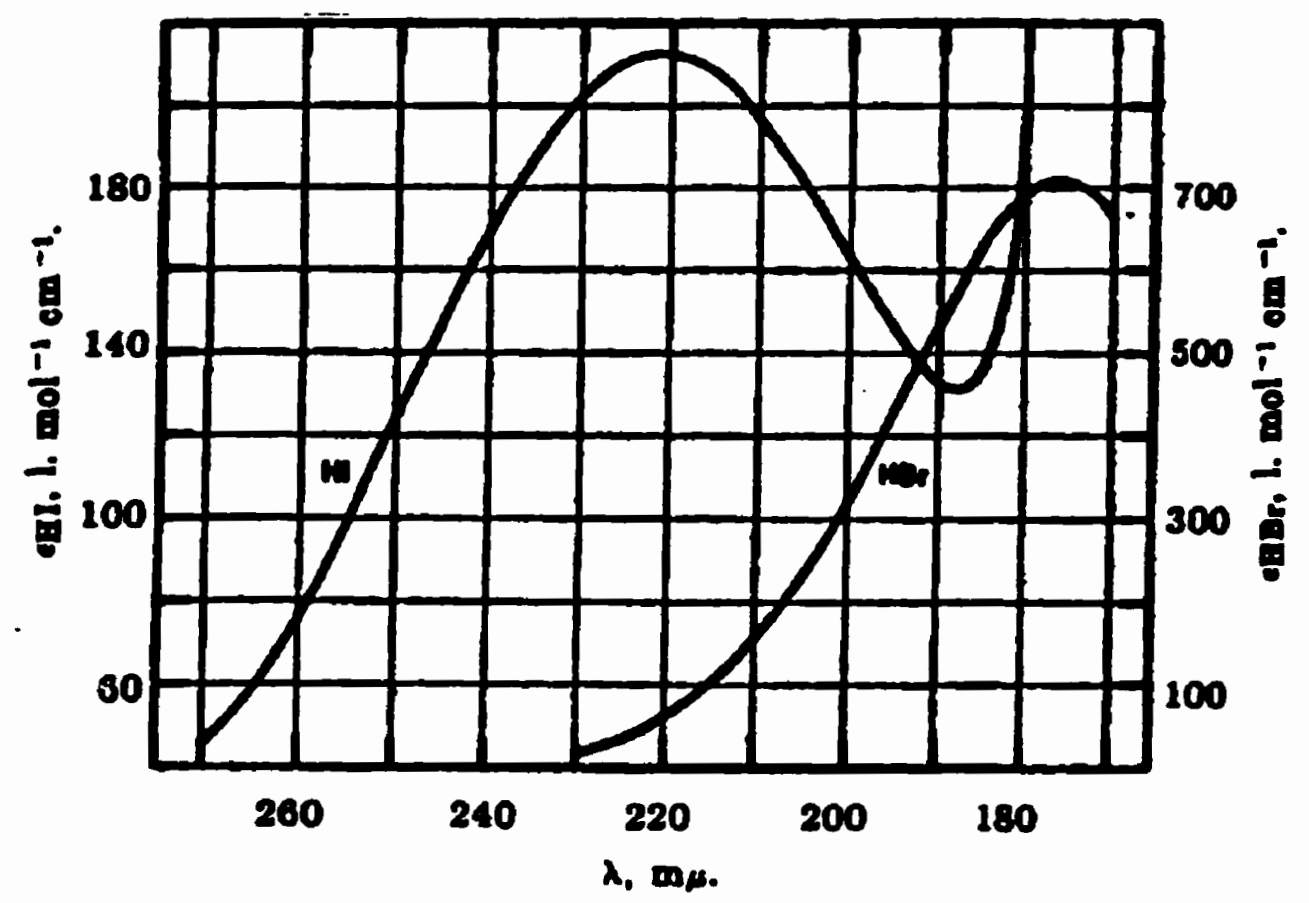

Figure 2.7. Absorption spectra of $\mathrm{HI}$ and $\mathrm{HBr}$, in the range of 3000 to $1800 \mathrm{~A}$. (taken from ref. 21 ).

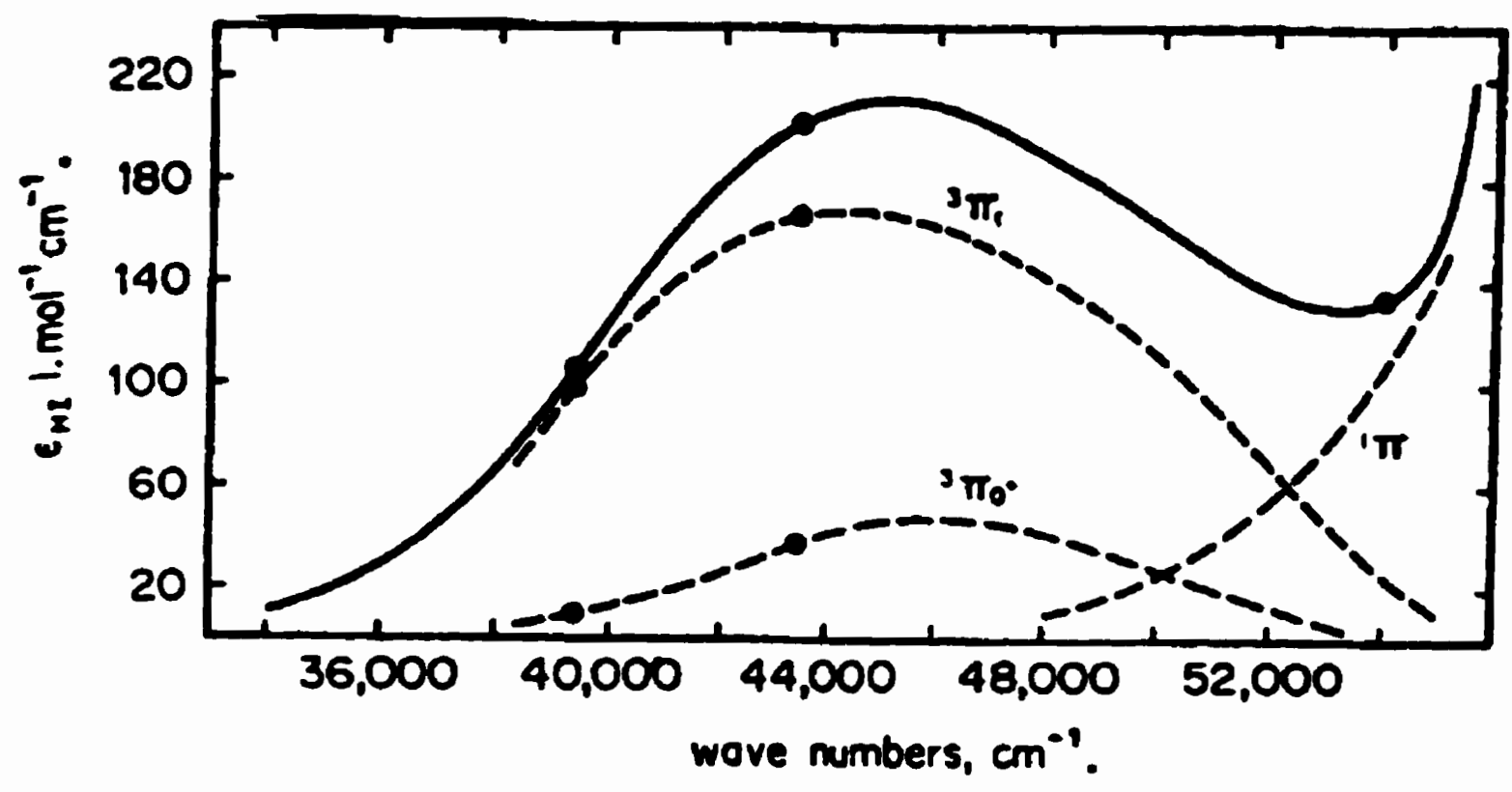

Figure 2.8. Theoretical decomposition of the total absorption of HI suggested by Compton and Martin (taken from ref. 24), on the basis of their experimental measurement. 


\section{E) Compton and Martin (1969)}

Compton and Martin ${ }^{2}$ investigated the photodissociation dynamics of HI. Their photochemical method was based on the abstraction reaction by hot $\mathrm{H}$-atoms occurring when $\mathrm{HI}$ was photolysed at the 2537,2288 and $1850 \AA$ wavelengths, in the presence of $C_{4} D_{10}$ yielding $H D$. The analysis of their results provided an estimate of the electronic branching ratio for each photolysis photon energy investigated.

Based on their values of electronic branching, they suggested that Romand's earlier theoretical decomposition of the absarption spectrum to partial absorption cross section for the states of Q-group needed reconsideration. Their experimental branching ratio, along with their estimate of the partial absorption cross section for each states of the Q-group, is shown in figure 2.8. However they followed Romand's assumption that the B band was due to the 'II of the Q-group, using the argument that this singlet-singlet transition must be much more intense than the transition to the ${ }^{3} \Pi$ states.

F) Ogilvie (1970)

Ogilvie measured the absorption spectrum of $\mathrm{HI}$ in the spectral region $32000-52000 \mathrm{~cm}^{-t}$, shown in table 2-2;.25 the uncertainty on the extinction coefficients $\epsilon$ was quoted $2 \%$. He also calculated the radial wavefunction for the lowest vibrational state of the ground electronic state $\Sigma\left({ }^{1} \Sigma_{0_{0}}\right)$ of HI represented in equation 2.1 , using the Jdependent formula derived by Herman, Tipping and Short, based on the parameters of a Dunham potential..$^{\infty}$ He showed that the radial wavefunction is insensitive to the rotational quantum number $\mathrm{J}$ from $\mathrm{J}=0$ to $\mathrm{J}=4^{\wedge}$, but at $\mathrm{J}=14$, corresponding $10 \mathrm{~T}-2000 \%$, the vibrational wavefunction differ significantly from that for the $\mathrm{J}=0$ state.

$\Psi_{(0,4)}^{\mathrm{HI}}=c_{0} \cdot \exp \left[c_{1} \cdot x+c_{2} \cdot x^{2}+c_{3} \cdot x^{3}+c_{4} \cdot x^{4}+c_{5} \cdot x^{5}\right]$

where: $\mathrm{x}=\left(r-r_{e}\right) / r_{e}$,

$c_{0}=2.7183 \quad c_{1}=1.28235 \quad c_{2}=-88.6353$

$c_{3}=75.7743 \quad c_{4}=-55.3790 \quad c_{5}=34.0135$

In the same study, Ogilvie also attempted to represent the first absorption continuum by a transition to a single dissociative potential energy curve (PEC). Because the use of a single PEC allows for only one spin-orbit component, and therefore fails to represent the two spin-orbit products previously observed in numerous studies, his calculated PEC is not useful for the present study. However we will use his measurement of extinction coeficients, and the radial function obtained for the ground vibronic state, later in chapter 6.

\footnotetext{
A $\mathrm{J}=4$ is the most populated state at room temperature.
} 
Table 2-2. Absorption data measured by Ogilvie (from ref. 25).

\begin{tabular}{|c|c|c|c|c|c|}
\hline $\begin{array}{c}\text { wavenumber } \\
\left(\mathrm{cm}^{-1}\right)\end{array}$ & $\begin{array}{c}\epsilon \\
\left(1 . \mathrm{mol}^{-1} \mathrm{~cm}^{-1}\right)\end{array}$ & $\begin{array}{c}\text { wavenumber } \\
\left(\mathrm{cm}^{-1}\right)\end{array}$ & $\begin{array}{c}\epsilon \\
\left.\text { I. mol. } \mathrm{cm}^{-1}\right)\end{array}$ & $\begin{array}{c}\text { wavenumber } \\
\left(\mathrm{cm}^{-1}\right)\end{array}$ & $\begin{array}{c}\epsilon \\
\left(1 . \mathrm{mol}^{-1} \mathrm{~cm}^{-1}\right)\end{array}$ \\
\hline 32000 & 2 & 38500 & 82 & 45000 & 220 \\
\hline 32500 & 3 & 39000 & 97.5 & 45500 & 219.5 \\
\hline 33000 & 4 & 39500 & 113.5 & 46000 & 217.5 \\
\hline 33500 & 5.5 & 40000 & 130 & 46500 & 215.5 \\
\hline 34000 & 8 & 40500 & 145 & 47000 & 209.5 \\
\hline 34500 & 11 & 41000 & 159.5 & 47500 & 202.5 \\
\hline 35000 & 15 & 41500 & 174.5 & 48000 & 197 \\
\hline 35500 & 20.5 & 42000 & 185 & 48500 & 191 \\
\hline 36000 & 27 & 42500 & 195 & 49000 & 184.5 \\
\hline 36500 & 35.5 & 43000 & 203.5 & 49500 & 177.5 \\
\hline 37000 & 45.5 & 43500 & 210 & 50000 & 170.5 \\
\hline 37500 & 55 & 44000 & 216.5 & 50500 & 163.5 \\
\hline 38000 & 68.5 & 44500 & 219.5 & 51000 & 156.5 \\
\hline
\end{tabular}

\section{G) Oldershaw, Porter and Smith (1972)}

Oldershaw, Porter and Smith investigated the energy partitioning in the photolysis of HI, by studying the product yield from reaction of the photochemical $\mathrm{H}$-atoms with $\mathrm{N}_{2} \mathrm{O}^{2}$ An estimate of $11 \pm 14 \%$ was obtained for the proportion of excited Iodine atoms produced by the $279 \mathrm{~nm}$ photolysis of $\mathrm{HI}$.

\section{H) Summary of the results from various photochemical studies}

From a glance at table 2-3, it is readily observed that values for the electronic branching ratio, obtained by photochemical methods, were plagued by large uncertainty and discrepancies. Clearly, a more accurate method to determine these physical quantities was highly desirable.

Table 2-3. Summary of the electronic branching ratios obtained by photochemical methods

\begin{tabular}{|c|c|c|}
\hline PhotolysisWavelength $(\mathcal{A})$ & Branching ratio: $R=\sigma\left(I^{*}\right) /\left[\sigma(I)+\sigma\left(I^{*}\right)\right]$ & References \\
\hline Integrated continuum & Approximately 0.20 & 19 \\
\hline 2790 & $0.11 \pm 0.14$ & 27 \\
\hline 2537 & $0.55 \pm 0.25$ & 22 \\
\hline 2537 & $0.07 \pm 0.10$ & 24 \\
\hline 2288 & $0.19 \pm 0.10$ & 24 \\
\hline 1850 & $0.00 \pm 0.10$ & 24 \\
\hline
\end{tabular}




\subsubsection{Age of photofragment spectroscopy (1970-1995)}

Experimental techniques of photofragment spectroscopy were developed to allow a detailed investigation of the dynamics of photodissociation events. Fundamental information on the dissociative states of matter, required to complement the extremely detailed knowledge of bound states obtained by traditional method of spectroscopy, can be provided by these sophisticated techniques.

\section{A) Clear, Riley and Wilson (1975)}

After having developed the photofragment spectrometer ${ }^{2}$, Wilson and coworkers studied the photodissociation dynamics of many simple molecular systems, including the photolysis of hydrogen iodide at $266 \mathrm{~nm}^{29}$ Combining the results of previous photochemical studies with their measurements at the 266 nm photolysis wavelength, they calculated the energy dependence of the partial absorption cross section for each electronic state of the first absorption continuum, as shown in figure 2.9 a).

From this information, a PEC calculation was performed using the reflection method to evaluate the radial overlap of the wavefunctions for the bound-to free transition. The limited precision of the data used in their partial cross section calculation did no warrant an exact quantum mechanical solution for the dissociative wavefunction, and they made use of the semiclassical 8 -approximation. The results obtained from this procedure indicated that the $A$ band, or the first absorption continuum, can account for all three allowed transitions to the states of the $Q$ group, which are shown in figure $2.9 \mathrm{~b}$ ). This result was in marked discrepancy with the conclusion of Romand ${ }^{17}$. but in qualitative agreement with Mulliken's prediction ${ }^{4}$ and consistent with Price's observations ${ }^{5}$, with regards to the predissociative nature of the state(s) of the B band, and the direct dissociative nature expected for the states of the Q-group.

\section{B) Schmiedl, Dugan, Meier and Welge (1982)}

The applications of the Doppler spectroscopy technique to the detection of photofragments have been reviewed by Welge and Schmied ${ }^{30}$ for both one-photon and multi-photon dissociation processes. Soon after, Schmiedl, Dugan, Meier and Welge ${ }^{31}$ applied this technique to the detection of $\mathrm{H}$-atoms by laser induced fluorescence (LIF) at the Lyman- $\alpha$ transition. In the latter work, a linearly polarized $266 \mathrm{~nm}$ photolysis pump beam dissociated HI molecules in a static cell at low pressure, and an orthogonal vacuum ultraviolet (VUV) probe beam was tuned over the range of Doppler shifted Lyman a ( $\left.{ }^{2} \mathrm{~S}-2 \mathrm{P}\right)$ transitions of the fast $\mathrm{H}$-atoms. The resulting Doppler profiles were acquired for few symmetric relative arientations of the linear polarization of the photolysis with respect to the probe beam direction. The angular distribution and electronic branching ratio of the photoproducts were obtained by a forward convolution technique. Although values for the branching ratio and anisotropy of the products were estimated from these measurements, the main results of this paper was limited to the demonstration of the proofof-principle for Lyman- $\alpha$ detection of $\mathrm{H}$-atoms angular distribution. 


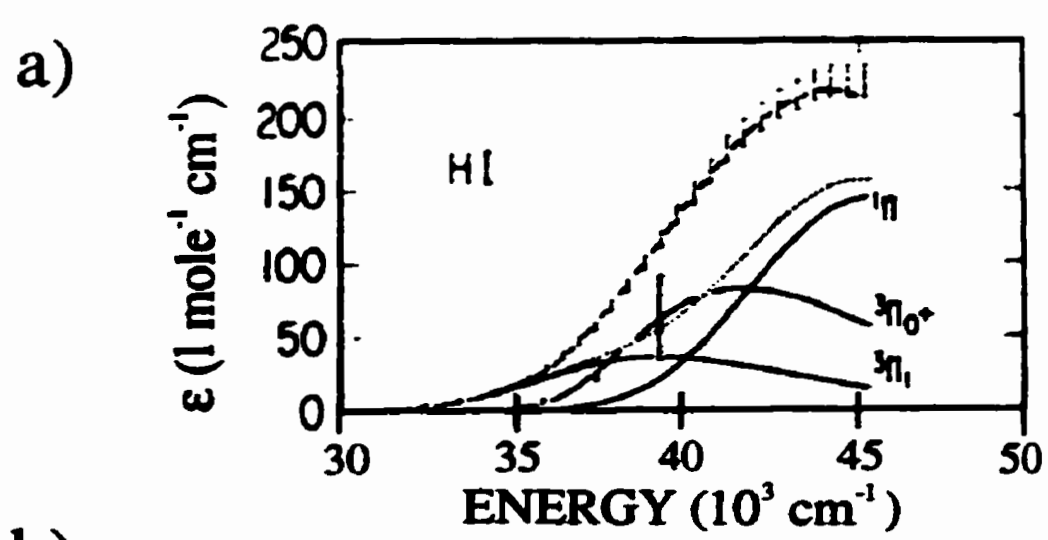

b)

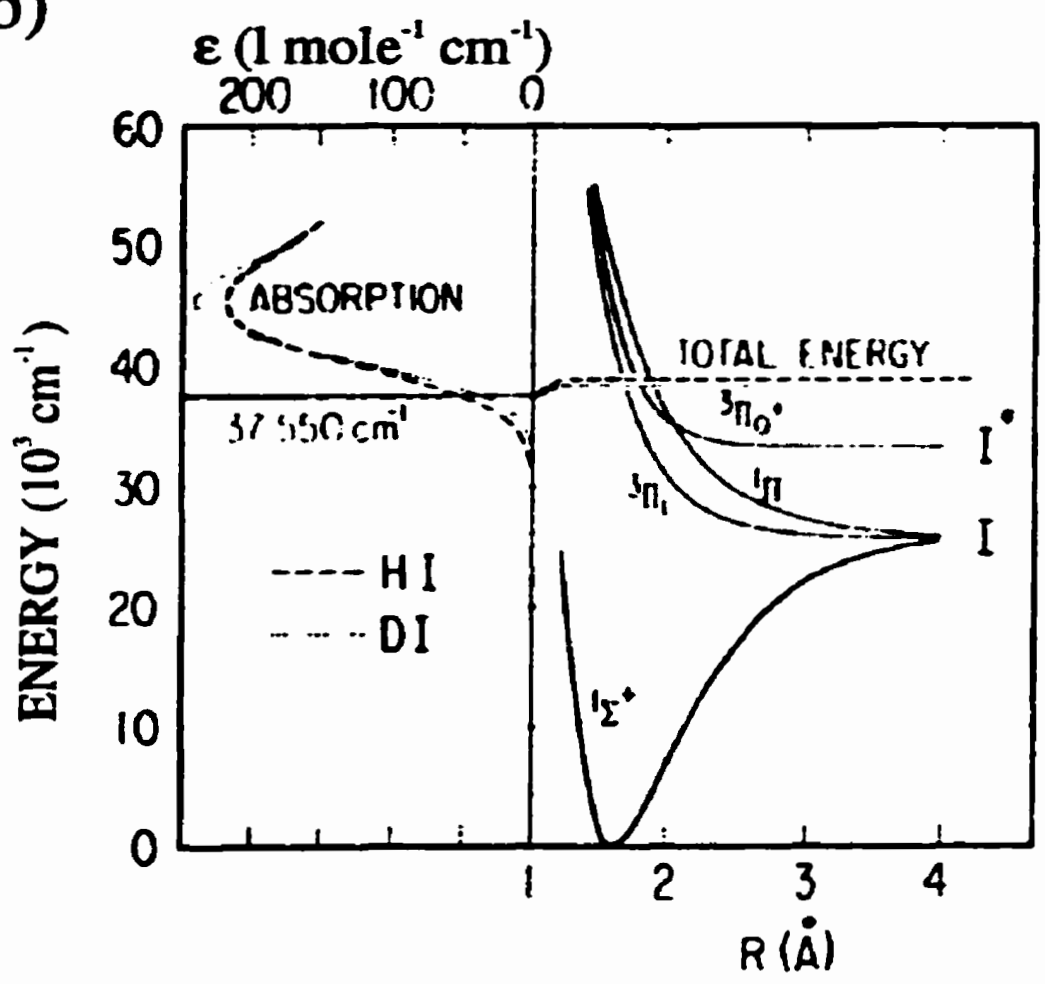

Figure 2.9. a) Partial absorption to each states of the Q-group. and b) PEC for these states (from ref. 29).

C) Van Veen, Mohamed, Baller and De Vries (1983)

Van Veen ${ }^{32}$ al al. measured the photofragment spectroscopy of HI by H-atom time-of-flight (TOF) spectroscopy, at the 248, 222 and $193 \mathrm{~nm}$ photolysis wavelengths. They observed that a perpendicular transition provided a significant cantribution to their measured cross section for the excited iodine product channel. In order to explain their results, they assumed that the $T^{3} Z_{1}$ state was located at a lower energy than Mulliken had predicted, in order to explain its contribution to the transitions of the first continuum. 
They fitted a repulsive potential for the excited dissociative states of the first absorption continuum, using a reflection method to calculate the energy dependence of the bound to free transition amplitudes, on the basis of their experimental measurements of branching ratios and angular distribution of the products. Partial extinctions to each dissociative state assumed to take part in the transitions of the first absorption continuum were obtained in the analysis of their results; these are shown in figure $2.10 \mathrm{a}$ ) and b); the PEC are showed in $2.10 \mathrm{c}$ ).
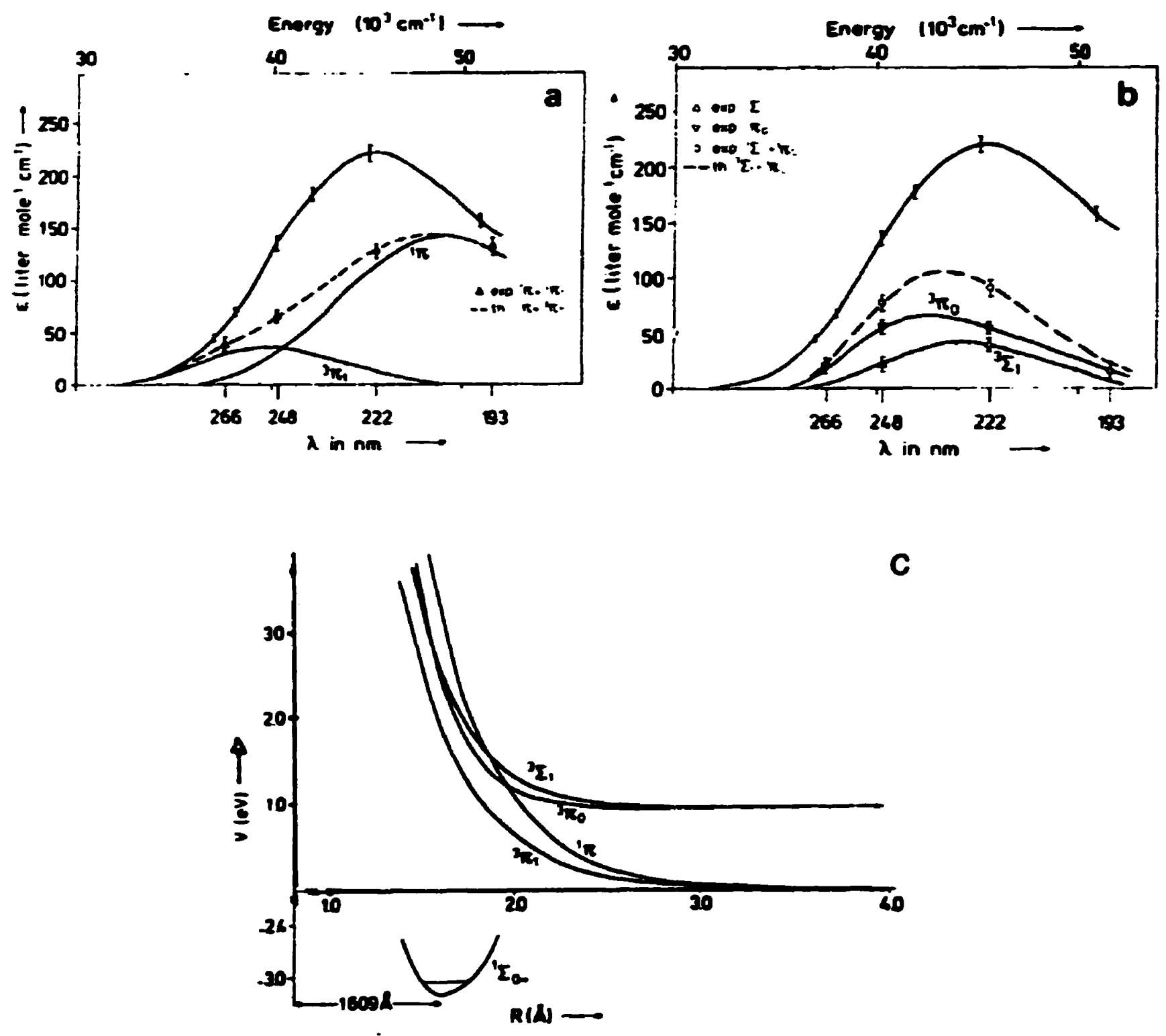

Figure 2.10. a) Partial absaption cross section by the dissociative states ('II. ${ }^{3} \mathrm{II}_{1}$ ) leading to ground state iodine atom products: b) Partial absaption cross section by the dissociative states ( $\left[{ }^{3},{ }^{3}{ }_{1}\right.$ ) leading to the excited iodine atom products: b) PEC for calculated for each of the dissociative states (from ref. 32). 
D) $X u$, Koplitz and Wittig (1988)

In this work ${ }^{33}, \mathrm{Xu}, \mathrm{Koplitz}$ and Wittig used the technique of velocity aligned Doppler spectroscopy (a variation of the technique of Schmiedl et al. ${ }^{31}$ mentioned above) to characterize the dynamics of $\mathrm{HI}$ photodissociation. They determined values of the anisotropy parameters and the electronic branching ratios of the products at the 248 and $193 \mathrm{~nm}$ photolysis wavelengths.

\section{E) Summary of results from photofragment spectroscopy on HI}

The values of the electronic branching ratios and fragments anisotropies, that were obtained by these study of photofragment spectroscopy at various photolysis wavelengths, are summarized in table $2-4$. It is readily appreciated that there is a relatively small number of wavelengths at which the dynamical variables of the photodissociation have been determined. Note that there is a disagreement between the results of $\mathrm{Xu}$ et al. ${ }^{33}$ and Van Veen ${ }^{32}$ et al. concerning anisotropy of the excited fragments, $\beta\left(I^{*}\right)$, and electronic branching, $R$, at the 248 nm wavelength photolysis.

Table 2-4. Summary of the results from photofragment spectroscopy studies on HI.

\begin{tabular}{|c|c|c|c|c|}
\hline $\begin{array}{c}\text { Photolysis } \\
\text { Wavelength (nm) }\end{array}$ & $\begin{array}{l}\text { Electronic Branching } \\
R=\sigma\left(I^{*}\right) /\left[\sigma(I)+\sigma\left(I^{*}\right)\right]\end{array}$ & $\begin{array}{l}\boldsymbol{\beta}(\mathrm{T}) \text { : Anisotropy } \\
\text { Parameter for I }\end{array}$ & $\begin{array}{l}\boldsymbol{\beta}\left(\boldsymbol{I}^{*}\right) \text { : Anisotropy } \\
\text { Parameter for } I^{*}\end{array}$ & References \\
\hline \multirow[t]{2}{*}{266} & $0.36 \pm 0.05$ & -1 & 2 & 29 \\
\hline & $0.40 \pm 0.05$ & $-1.0 \pm 0.2$ & $2.0 \pm 0.2$ & 31 \\
\hline \multirow[t]{2}{*}{248} & $0.57 \pm 0.04$ & $-0.92 \pm 0.06$ & $1.08 \pm 0.04$ & 32 \\
\hline & $0.46 \pm 0.05$ & $-1.0 \pm 0.20$ & $1.7 \pm 0.2$ & 33 \\
\hline 222 & $0.43 \pm 0.04$ & $-0.90 \pm 0.06$ & $0.78 \pm 0.04$ & 32 \\
\hline \multirow[t]{2}{*}{193} & $0.09 \pm 0.05$ & $-0.88 \pm 0.06$ & $\mathbf{N} / \mathbf{A}$ & 32 \\
\hline & $<0.10$ & $\sim-1.0$ & $\mathbf{N} / \mathbf{A}$ & 33 \\
\hline
\end{tabular}

\subsubsection{Recent works related to our study on BI photodissociation}

A few other studies, important to the argumentation developed later in the present work, are briefly summarized.

\section{A) Huber and Herzberg (1979)}

Huber and Herzberg ${ }^{34}$ compiled a list of the spectroscopic constants for HI published before 1979. They tabulated a dissociation energy of $3.054 \mathrm{eV}$, as calculated from the dissociation energies of $\mathrm{I}_{2}$ and $\mathrm{H}_{2}$ and from the heat of formation of HI. They also tabulated a synthesis of the spectroscopic studies on the electronic structure of HI in the energy range encompassing the region from above the first absocption continuum up to the ionization threshold. The most prominent contributors to this set of spectroscopic data have been Ginter and coworkers. 
B) Ginter and coworkers (1970-1982)

Ginter and coworkers have published a series of studies ${ }^{353637}$ in which VUV absorption spectra of HI were analyzed to elucidate the molecular electronic structure in the range of 55800 to $74400 \mathrm{~cm}^{-1}$. In this range of excitation energy above the first absorption continuum, the $T$ and $V$ states have been identified, along with numerous other states. We will return to this result in the third section of this chapter.

\section{C) Wermer, Reinsch and Rosmus (1981)}

Werner, Reinsch and Rosmus ${ }^{30}$ have performed $a b$ initio calculation of the $r$-dependent dipole moment for the ground electronic state of the hydrogen halides, from HF to HI. It can be noted in figure 2.11 that HI distinguishes itself from the other hydrogen halide with respect to this physical property. Their results illustrate the fact that the dipole moment of $\mathrm{HI}$ has a relatively small sensitivity to its dependence on internuclear distance around $r_{e}$. This indicates a relatively weak perturbation by the ion state.

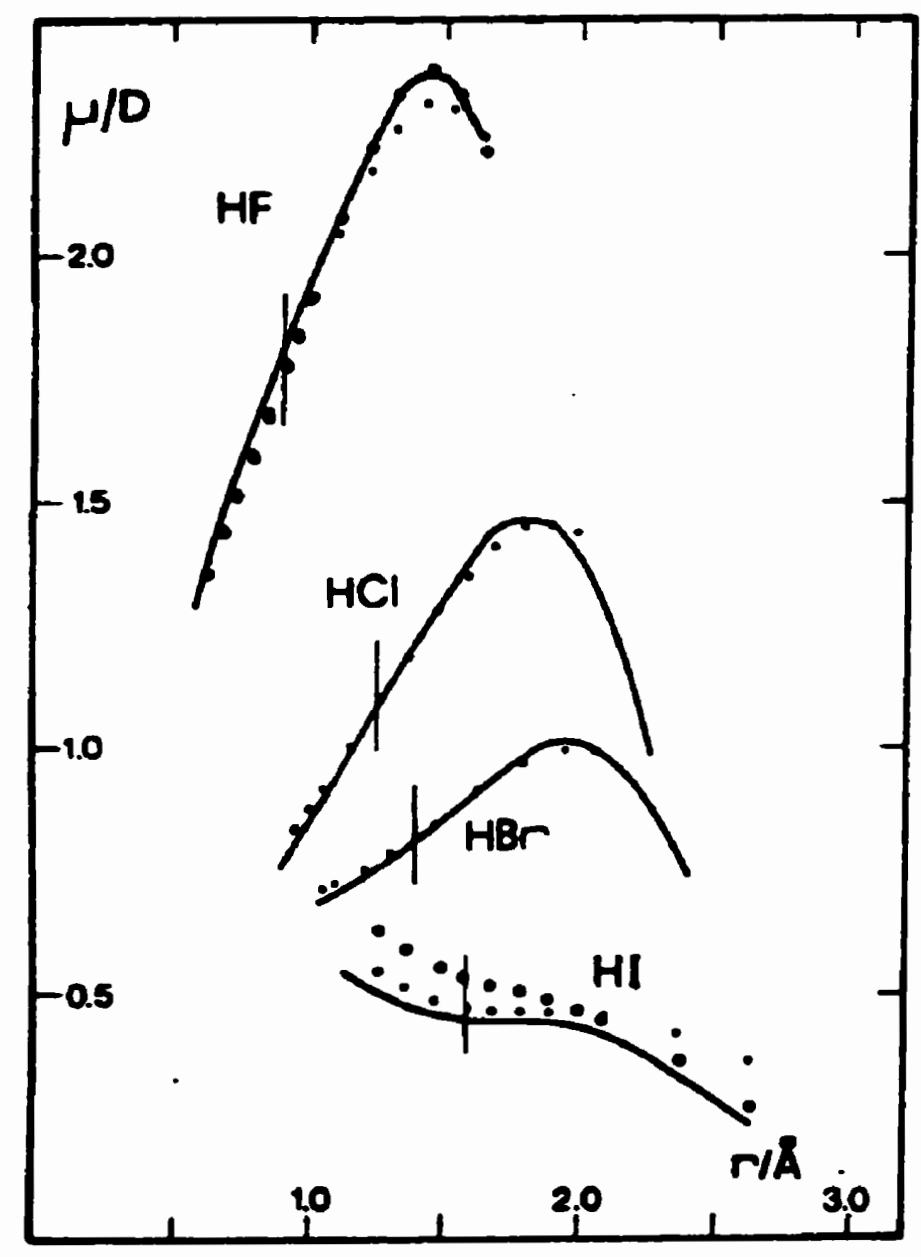

Figure 2.11. Calculated and experimental dipole moment of hydrogen halides (from ref. 38). The vertical bars indicate the equilibrium internuclear distance for each molecules, the symbols represent theoretical values, and the solid lines are obtained from experimental measurements. 
D) Chapman, Balasubramanian and Lin (1985)

Chapman et al ${ }^{39}$ performed relativistic configuration interaction calculations on the low-lying electronic states of HI. Although their calculation deviated by up to $-10 \%$ for the dissociation energy, their results agreed qualitatively well with Mulliken's predictions. The qualitative aspects of the PEC for the calculated dissociative states, shown in figure 2.12 , confirms the theoretical expectation that these dissociative states are properly described by the "far-nuclei" or Mulliken case c coupling.

\section{E) Levy and Shapiro (1988)}

Prior to the present thesis, the work of Levy and Shapiro ${ }^{\circ}$ was the latest theoretical study on the photodissociation dynamics of HI following A band excitation. It was based on a collection of experimental data obtained from photochemical and photofragment spectroscopy studies, but the data of van Veen et al ${ }^{32}$ had apparently a predominant influence on their theory, which was reasonable given the fact that it was the most detailed study on this system. As a consequence of the observation made by van Veen et al. ${ }^{32}$ regarding the anisotropy of the excited iodine fragment channel, and of their conclusion regarding the low-lying electronic structure of HI. Levy and Shapiro analyzed the dynamics of dissociation, including the nonadiabatic interactions that would ensue. A pictorial summary of their results is reproduced in figure 2.13. According to their analysis, the "truly" adiabatic potential energy curve (PEC) was determined for the four dissociative states previously assumed by van Veen et al. ${ }^{32}$ to participate in the transitions of the first absorption continuum.

\section{F) Coxon and Hajigeorgiou (1991)}

Coxon and Hajigeorgioust obtained the most recent parameters for the vibrational and rotational structure of the ground state $\left(X^{\prime} \Sigma^{2}\right)$ of $\mathrm{HI}$ from the fit of an effective nonadiabatic Hamiltonian, of a large number of line positions available from the literature. The rovibrational energy levels are given by the power series of equation (2-2). They provided accurate parameters for the ground electronic state. We listed, in table 2-5, the parameters for the five lowest vibrational levels. Their work provided an accurate description of the ground state for HI molecule.

$E(v, J)=G_{v}+B_{v}[J(J+1)]-D_{v}[J(J+1)]^{2}+H_{v}[J(J+1)]^{3}+\ldots$

Table 2-5 Parameters for the levels of the ground electronic state of HI (from ref. 36).

\begin{tabular}{|c|c|c|c|c|}
\hline $\mathrm{v}$ & $\mathrm{G}_{\mathrm{v}}$ & $\mathrm{B}_{\mathrm{v}}$ & $10^{\cdot} \cdot \mathrm{D}_{v}$ & $10^{9} \cdot \mathrm{H}_{\mathrm{v}}$ \\
\hline $\mathrm{O}$ & 1145.2436 & 6.4263643 & 2.068672 & 2.8782 \\
\hline 1 & 3374.8252 & 6.2552378 & 2.064996 & 2.6329 \\
\hline 2 & 5524.4701 & 6.0829499 & 2.066559 & 2.319 \\
\hline 3 & 7593.2780 & 5.9088108 & 2.074726 & 1.9121 \\
\hline 4 & 9579.9517 & 5.7319726 & 2.091303 & 1.3769 \\
\hline
\end{tabular}




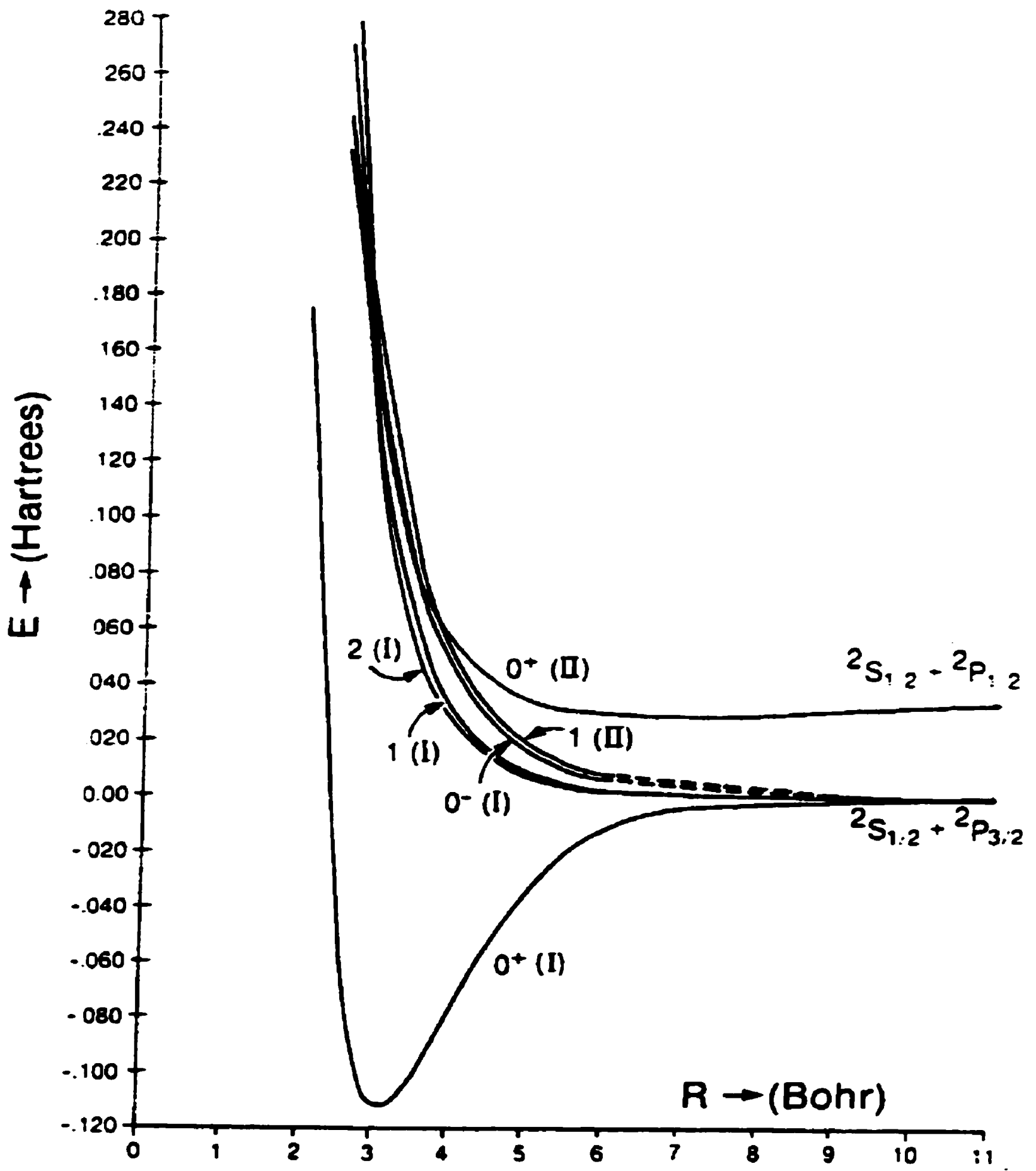

Figure 2.12. Configuration interaction calculations of the low-lying electronic states of HI (from ref. 39). 
a)

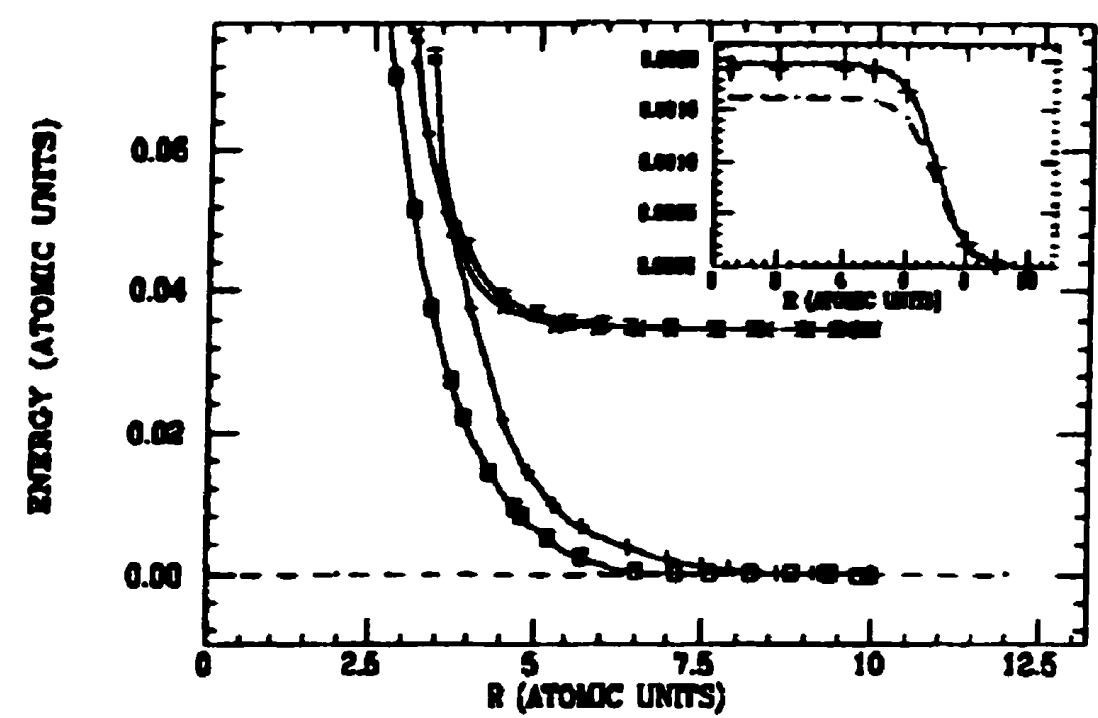

Adiabatic potentials and diabatic coupling terms for the first four excited states of HI. In the main figure are adiabotic potentials of the: ${ }^{3} n_{1}$ (D), ' $n_{1}(+),{ }^{3} n_{0}(x)$ and the ${ }^{3} \Sigma_{1}\left(^{*}\right)$ states. In the inset are coupling terms in the diabatic representation between: ${ }^{3} \Pi_{1}-n_{1}(-)_{1}{ }^{3} \Pi_{1}-{ }^{3} \Sigma_{1}(+)$. and ' $n_{1}-\Sigma_{1}(-\cdot-)$.

b)

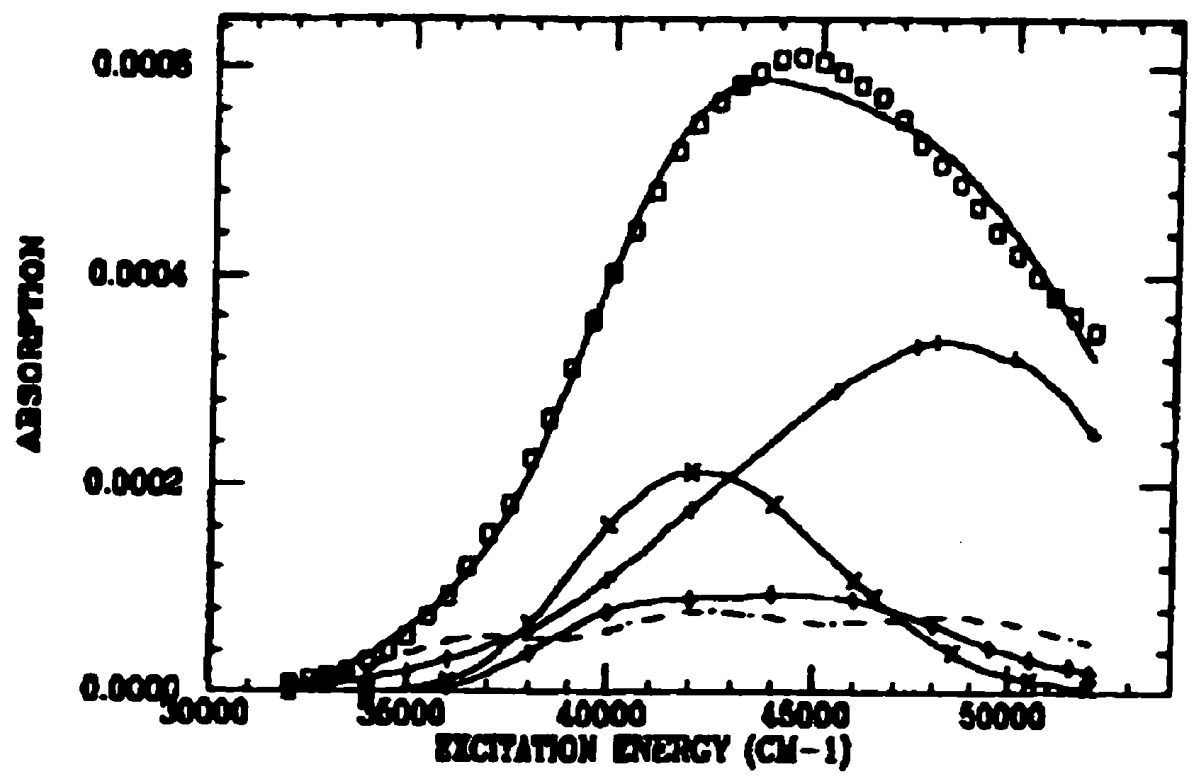

Partial and total photoabsorption croas sections for HI. The total cross section (marked as -) is compared to the experimental poiats of Ref. 10 (marked as $\square$ ). Partial cross sections due to the individual electronic states are marked as $(-\cdot-\cdot)^{\prime} \Pi_{10}(0)^{3} \Sigma_{10}(x)^{3} \Pi_{0}(+) ' \Pi_{1}$.

Figure 2.13. Summary of results from the calculation of Levy and Shapiro on the electronic states of the first absorption continuum of HI: a) adiabatic PEC, with nonadiabatic coupling in the insert; b) PACSS calculated with coupling (from ref 40 ). 
G) Zhang, Dulligan, Segal, Wen, and Wittig (1995)

In this work, Zhang et al. ${ }^{2}$ studied the photodissociation dynamics of FI dimers. The dynamic of this process has been clearly elucidated with unsurpassed sensitivity, provided by the technique of high-n Rydberg time-of-night (HRTOF) spectroscopy. They have shown many convincing spectroscopic proofs that the dissociation dynamics of photoexcited HI dimers proceeds in a concerted and predictable fashion, a somewhat surprising result; we will return to this topic in the discussion of chapter 6.

\section{H) Comparison of the PEC parameters from previous studies of $\mathrm{HI}$}

In all the determinations of the PEC for the dissociative states of the first continuum, the functionality assumed is an exponential dependence on the internuclear radius, expressed by equation 2-3. Each PEC is characterized by three parameters: a preexponential factor $A$, an exponent factor $B$, and an offset $C$ which is equal to 0 or $7603.15 \mathrm{~cm}^{-1}$, the energy separation between the spin-orbit states of the Iodine atom. ${ }^{-3}$ The value of $C$ is fixed by the asymptotic product corresponding to each dissociative state.

$V(R)=A \cdot \exp \left[-B\left(R-R_{e}\right)\right] \quad(2-3)$

A comparison of the values for the parameters obtained in previous studies is listed in table 2-6 a), b) and c), for the results of Clear et al. ${ }^{29}$, van Veen et al. ${ }^{32}$ and Levy and Shapiro* respectively. The values of the average transition dipoles, obtained by Clear et al. ${ }^{39}$ and by van Veen et al. ${ }^{32}$, are of limited usefulness because the reflection methode used to calculate the energy dependence of the transition intensity overestimates the value ofthe transition dipole, $\mu$, as it underestimate the overlap of the ground and dissociative wavefunction. Also the numerical values obtained by this method should only be regarded as an approximate relative values. In the case of Levy and Shapiro, their proper quantum mechanical calculation led to much smaller values of the transition dipole, which are in the same order of magnitude than the quantitative estimates obtained by Mulliken.

Table 2-6 a) PEC parameters from Clear et al. (taken from ref. 29).

\begin{tabular}{|c|c|c|c|}
\hline States & $\langle\mu\rangle^{2}($ debye' & $A\left(\mathrm{~cm}^{-1}\right)$ & $B\left(\AA^{-1}\right)$ \\
\hline$' \Pi$ & $0.126 \pm 0.015$ & $21850 \pm 350$ & $1.90 \pm 0.09$ \\
\hline${ }^{3} \Pi_{0}$ & $0.160 \pm 0.024$ & $11250 \pm 400$ & $4.10 \pm 0.10$ \\
\hline${ }^{3} \Pi_{1}$ & $0.038 \pm 0.004$ & $15800 \pm 250$ & $2.85 \pm 0.06$ \\
\hline
\end{tabular}

Table 2-6 b) PEC parameters from Van Veen et al. (taken from ref. 32).

\begin{tabular}{|c|c|c|c|}
\hline States & $\langle\mu\rangle^{2}($ debye $)$ & $A\left(\mathrm{~cm}^{-1}\right)$ & $B\left(\AA^{-1}\right)$ \\
\hline${ }^{\prime} \Pi$ & $0.193 \pm 0.025$ & $25900 \pm 500$ & $2.86 \pm 0.06$ \\
\hline${ }^{3} \Pi_{0_{0}}$ & $0.146 \pm 0.006$ & $12350 \pm 250$ & $4.80 \pm 0.05$ \\
\hline${ }^{3} \Sigma_{1}$ & $0.041 \pm 0.003$ & $12600 \pm 250$ & $3.75 \pm 0.06$ \\
\hline${ }^{3} \Pi_{1}$ & $0.038 \pm 0.004$ & $16300 \pm 250$ & $2.85 \pm 0.06$ \\
\hline
\end{tabular}


Table 2-6 c) PEC parameters from Levy and Shapiro. (from ref. 40).

\begin{tabular}{|c|c|c|c|}
\hline States & $\langle\mu\rangle^{2}\left(\right.$ debye $\left.^{2}\right)$ & $\mathbf{A}\left(\mathrm{cm}^{-1}\right)$ & $\mathbf{B}\left(\AA^{-1}\right)$ \\
\hline${ }^{\prime} \Pi$ & $1.0417 \mathrm{E}-04$ & 23860 & 2.081 \\
\hline${ }^{3} \Pi_{0_{*}}$ & $3.6719 \mathrm{E}-05$ & 10240 & 3.483 \\
\hline${ }^{3} \Sigma_{1}$ & $2.7908 \mathrm{E}-05$ & 9161 & 3.001 \\
\hline${ }^{3} \Pi_{1}$ & $1.7170 \mathrm{E}-05$ & 12090 & 1.971 \\
\hline
\end{tabular}

The assumption, by Levy and Shapiro, that nonadiabatic effects are important in the dissociation dynamics of $\mathrm{HI}$, had important consequences on the values of transition dipole and PEC parameters that they obtained. Yet, even with their highly sophisticated theoretical model, they only achieve partial agreement with the absorption measurements of Ogilvie, as the reader will appreciate by inspection of the figure $2.13 \mathrm{~b}$ ).

\subsection{Summary: observations and discussion}

\subsubsection{General observations of consistency}

A) Energy ordering within the Q-group of states

Following Mulliken, the ordering of the states above the Franck-Condon region, near the equilibrium internuclear

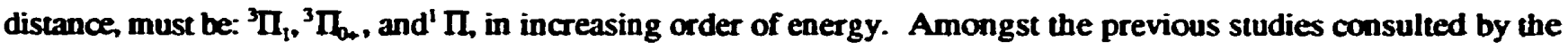
author of the present study, all agreed on this point.

\section{B) Coupling case}

Indication of the coupling case at work in a given molecular system is provided by its consequences on the structure of the energy levels and the relative strength of the optical transitions in that molecular system. For example, in $\Lambda-\Sigma$ coupling, energy separation between the pairs of states $\left({ }^{3} \Pi_{2}-{ }^{3} \Pi_{1}\right)$ and $\left({ }^{3} \Pi_{1}-{ }^{3} \Pi_{0_{3}}\right)$ are expected to be approximately equidistant and of the order of the atomic spin-orbit energy. However, in the case of "close nuclei" (Hund's) case c coupling, the position of the dissociative energy levels are expected to lie in two groups: the lower pair $\left({ }^{3} \Pi_{2}-{ }^{3} \Pi_{1}\right)$ and the higher pair $\left({ }^{3} \Pi_{0+}-{ }^{1} \Pi\right)$, which would be separated by an energy of the order of a or $\mathrm{a}^{+}$, the spin-abit constant of the halogen atom or ion respectively. The energy separation within each group is expected to be much smaller than a.

At first glance, the electronic structure of the first continuum of $\mathrm{HI}$ can be expected to be mainly described by the "close nuclei" case c coupling at $r_{e}$ the internuclear distance of the ground state equilibrium. This "close nuclei" (or Hund's) case $c$ should dominate the coupling in the ground state of the HI molecule, and in the dissociative state at $r_{e}$. However, the large energy of the spin-orbit coupling in the iodine atom implies that the "far nuclei" 
(or Mulliken's) case $c$ becomes rapidly dominant in the first continuum, and perhaps may even contribute significantly near $r_{e}$. In fact, if the recoupling of the electronic structure of the atoms begins to be important at $r_{s}$, the description of the dissociative state reduces to a repulsion of oriented atoms ${ }^{\wedge}$ in a collective state of correlated angular momentum.

A relativistic configuration interaction (CD) calculation was performed by Chapman et al.; they obtained ab initio PEC's for the low-lying electronic states of HI. Inspection of their PEC, shown in figure 2.12, suggests a qualitative agreement with Mulliken conclusion, which predicted that the "far nuclei" coupling should dominate the electronic structure in the Q-group of states.

\section{C) Relative strength of transitions}

It was observed, in early experiments on HI, that the transitions to the first absorption continuum are relatively weak compared to transitions to bound states at higher energies. To explain this observation, Mulliken pointed out that the molecular structure of the first continuum results from the molecularization of the two spin-orbit components of the ground electronic term of the iodine atom. Recalling that intramultiplet transition in atomic system are either forbidden or much weaker than intermultiplet transition, the corresponding situation in molecular system, although relaxed, explains the low relative intensity of the first continuum.

\subsubsection{Disagreements and controversy}

\section{A) Comparison of results from previous photofragmentation studies}

A comparison of the observables measured in previous photofragment spectroscopy studies is summarized in the cable 2-4. Figure 2.14 compares of these measurements with the theoretical calculation of Levy and Shapiro. ${ }^{20}$ The reader will recall that the latter calculation was based on the results of van Veen et al. ${ }^{32}$ Noteworthy is the fact that the measurements of Schmiedl et al. ${ }^{31}$ at $266 \mathrm{~nm}$ photolysis wavelength, and those of Xu et al..$^{33}$ at 248 , are in distinct disagreement with van Veen results, and hence also with the theory of Levy and Shapiro.

\section{B) Evolution of our knowledge of $H I$}

Consideration of previous investigations on the low lying electronic structure of HI listed in section 2.2 offers a global perspective on the evolution of our understanding of this system. Soon after the first measurement by Goodeve and Taylor, ${ }^{6}$ Mulliken developed the theory for the electronic structure of simple diatomic molecules, and suggested a rigorous model for the A band of HI. Ten years later, Romand and coworkers ${ }^{13.14 .15}$ attempted to

\footnotetext{
A By oriented atom. the author refers to the orientation of the total electronic angular momentum vector of the atom. Since the relative crientation of the atomic fragments is a consequence of total angular momentum conservation in the light-matter interaction. and the molecular eigenstates are characterized by a well-defined state of total angular momentum. the atomic correlation must be related to the angular momentum of the field.
} 
apply that theory to explain measurements that also included the $B$ band above $50000 \mathrm{~cm}^{-1}$. Because the $B$ band was so much more intense, he ascribed its origin as being due to a transition to the 'II state. Photochemical measurements of Martin and coworkers ${ }^{20.21 .20}$ represents an early attempt at testing the state of the theory on $\mathrm{HI}$. by a determination of the physical observables, but they were misled in the assignment of the partial cross section of the products, by following Romand's assumption regarding the causality relation between 'II state and $B$ band.

Photofragment spectroscopy, combined with the rise of the computer and the information age, has permitted clarification on this topic. Clear et al. ${ }^{29}$ used their measurements, combined with a thorough documentation of all previous works on the topic, to show that Mulliken's model was consistent with the observations. However, it must be noted, that they only obtained measurement at only one photolysis photon energy, at the then newly available $266 \mathrm{~nm}$ wavelength from the fourth harmonic of a YAG laser. Moreover, the quantum mechanical calculation was rather crude, and depended largely on the body of previous photochemical measurements, which were plagued with large uncertainties. This motivated the photofragment spectroscopy experiment of van Veen et al..$^{32}$ at other photolysis photon energies, on the basis of which Levy and Shapiro ${ }^{20}$ developed their theory.

\subsubsection{ErTects of the ion pair state on molecular structure}

In order to offer the best understanding at the end of this study, some fundamental concepts of molecular physics. developed by Mulliken and discussed by Herzberg, are recalled for the sake of completeness. The relation between the relative energy position of the ion pair state, and its consequences on the electronic structure, are discussed for hydrogen balides, with particular attention paid to the properties affected by their perturbative contributions to the electronic structure.

\section{A) Perturbation by the ion pair state}

Our understanding of the molecular structure is developed on the basis of first-order states, such as the ones mentioned in the correlation diagram of figure 2.5. However, experience in quantum mechanical calculation has showed that in order to better describe experimental observation, a coupling between the first-order states must be introduced, which correspond to the introduction of a perturbation, and a more exact quantum mechanical calculations of such states are obtained by taking into account these perturbations.

Of specific importance for the ground electronic state is the perturbation by a special state, the ion pair state, i.e. the state that corresponds to the separation of the diatomic molecule into positive and negative ions. Its importance is determined by the combination of the three following facts: 1) the ground state of a close shell diatomic molecule has a ' $\boldsymbol{\Sigma}_{0}$ symmetry type, and it is also the case for the electronic state corresponding to a pair of close shell atomic ions; 2) the perturbation is most significant between states of same symmetry type; 3) the magnitude of the perturbation depends on the energy spacing between the first order-states. 

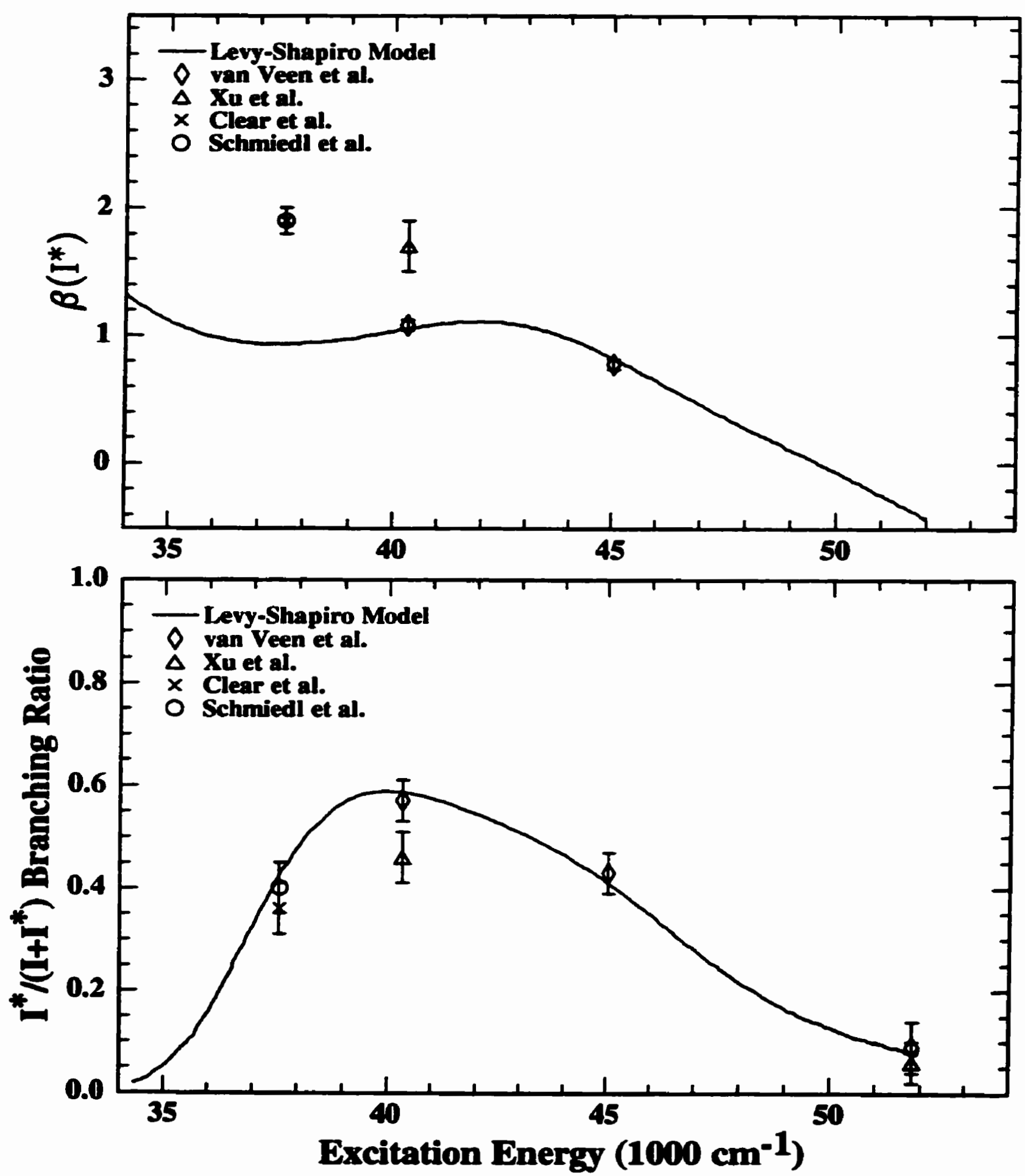

Flgre 2.14. Comparison between the results of photofragment spectroscopy prior to the present study. Note the disagreement between the predictions of the obervables values derived from the "truly" adiabatic potential obtained by Levy and Shapiro, compared with the later results of Xu et al., and Schmiedl et al. 


\section{please note}

Papo(s) misaing in number only; wat follows. Finned as recoived. 
probably be much smaller than for a heavier positive ion, since only the proton-proton repulsion can account for the repulsion. By comparing the difference between the energy positions of $D_{0}$ and $W_{x}\left(R_{v}\right)$, with the permanent dipole, $\mu$, the reader will note that the closer this zero-order ion pair state is to the true (experimental) ground state, the bigger is the value of the permanent dipole.

\section{B) Radial dependence of the permanent dipole for $\mathrm{HI}$}

In figure 2.11, the permanent dipole moment for HI was shown to have a relatively small dependence on the internuclear radius. By inspection of table 2-7, the reader will note that $E_{*}$ is lower than $E_{x}$ for all hydrogen halide molecules except for HI. This may be explained by the fact that for HI, the F ion pair, i.e. the ion pair $\mathrm{H}^{-}$ $+I^{*}$, does actually lie at lower energy than the usual $\pm\left(i . e . H^{*}+I^{-}\right)$; as a consequence, they may be mixed and effectively "balance" each other around the ground state equilibrium internuclear distance of the ground state. Because the electron-electron repulsion term can be expected to be stronger for the $E_{\bar{\tau}}=E\left(H / X^{*}\right)$ than for the $E_{z}$ $=E\left(H^{+} / X^{-}\right)$, these two ion pair states will eventually cross, for the singular case of $H$ molecule. This is a reasonable explanation for the striking difference in the radial dependence of the permanent dipole for $\mathrm{HI}$, in comparison with the other hydrogen halide molecules, as witnessed by figure 2.11 above.

\section{C) Position of the $\mathrm{T}^{3} \mathrm{\Sigma}_{1}$ state}

A final point of controversy that must be addressed is the assignment by van Veen et al. ${ }^{32}$ of the ${ }^{3} \Sigma_{1}$ state to a partial absorption cross section contributing to A band absorption. Mulliken had argued that this state will be too high to contribute to this absorption because it correlates with the second excited configuration in the united atom picture, in addition to the fact that its symmetry must be mixed with that of an ion pair state whose asymptote is relatively high. The $\mathrm{E}_{z}=\mathrm{E}\left(\mathrm{H} / \pi^{*}\right)$ asymptotic limit was shown in table 2-7 to set a lower limit for that ion pair state at $1.583 \mathrm{eV}$ above the dissociation energy. Assuming the electron repulsion term contributes an energy of the order of $1 \mathrm{eV}$, this second ion pair could in fact be involved with the $\mathrm{B}$ band.

In the spectroscopy community, Ginter and coworkers ${ }^{35,36,37}$ have been working on the electronic structure of $\mathrm{HI}$ for a few decades, and have previously assigned the $\mathrm{T}$ and the $\mathrm{V}$ states to bound electronic states. The $\mathrm{V}$ state has bands lying in the region of $68000-72000 \mathrm{~cm}^{-1}$, and a diffuse band at $64300 \mathrm{~cm}^{-1}$ has been attributed to the $T^{3} \Sigma_{1}$ state. By assigning the $\mathrm{T}^{3} \boldsymbol{\Sigma}_{1}$ state to a partial absorption contribution of the $A$ band, van Veen et al. ${ }^{32}$ implicitly assumed that Mulliken and Ginter were misled. 
References

1. G Herzberg, Molecular Spectra and Molecular structure. I. Spectra of Diatomic Molecules, 2nd ed, Krieger Publishing Co. (1989)

2. S. J. Singer, K. F. Freed, Y. B. Band, Adv. Chem. Phys., 61, 1 (1985)

3. D. A. McQuarrie, Quantum Chemisury, University Science Books, Mill Valley, California (1983)

4. C. Cohen-Tannoudji, B. Diu, F. Laloe, Mecanique Quantique, Tome I, Hermann (1973)

5. D. B. Chestnut, Finite Groups and Quantum Theory, Wiley-Interscience, John Wiley (1974)

6. C. F. Goodeve and A.W.C. Taylor, Proc. Roy. Soc. A152. 221, (1935); A154, 181 (1936)

7. R. S. Mulliken, Phys. Rev. 50, 1017 (1936)

8. R. S. Mulliken, Phys. Rev., 50, 1028 (1936)

9. R. S. Mulliken, Phys. Rev., 51, 310 (1937)

10. W. C. Price, Proc. Roy. Soc. (London) A154, 181 (1936)

11. W. C. Price, Proc. Roy. Soc. A, 167, 216 (1938)

12. D'Arcy J. Hart, Coherent VUV Spectroscopy of NO and HI, Ph. D. Thesis, U. of Waterloo, Ontario (1987)

13. J. Romand, B. Vodar, Compt. Rend., 226, 890 (1948)

14. J. Romand, Compt. Rend. 227, 117 (1948)

15. J. Romand, Ann. Phys., (Paris) 4, 527 (1948)

16. R. J. Donovan, D. Husain, Trans. Faraday Soc., 62, 11 (1966)

17. R. J. Donovan, D. Husain, Trans. Faraday Soc., 62, 1050 (1966)

18. R. J. Donovan, D. Husain, Trans. Faraday Soc., 62, 2023 (1966)

19. R. J. Donovan and Dr. D. Husain, Nature, 206, 171 (1965)

20. R. M. Martin, J. E. Willard, J. Chem. Phys., 40, 2999 (1964)

21. B. J. Huebert, R. M. Martin, J. Phys. Chem., 72, 3046 (1968)

22. P. Cadman, J. C. Polanyi, W. M. Smith, J. Chim. Phys., 64, 111 (1967)

23. P. Cadman, J. C. Polanyi, J. Phys. Chem., 72, 3715 (1968)

24. L. E. Compton, R. M. Martin, J. Phys. Chem., 73, 3474 (1969)

25. J. F. Ogilvie, Trans. Faraday Soc., 67, 2205 (1971)

26. R. M. Herman, R. H., Tipping, S. Short, J. Chem., Phys., 35, 595 (1970)

27. G. A. Oldershaw, D. A. Porter, A. Smith, Chem. Soc. Faraday Soc. Trans. 1, 68, 2218 (1972)

28. G. E. Busch, J. F. Comelius, R. T. Mahoney, R. L. Morse, D. W. Schlosser, K. R. Wilson. Rev. Scient. Instr., 41, 1066 (1970) 
29. R. D. Clear, S. J. Riley, and K. R. Wilson, J. Chem. Phys., 63, 1340 (1975)

30. K. H. Welge, R. Schmiedl, Adv. Chem. Phys., 47, part 2, 133 (1981)

31. R. Schmied, H Dugan, W. Meier and K. H. Welge, Z. Phys. A., 304, 137 (1982)

32. G. N. A. van Veen, K. A. Mohamed, T. Baller, A. E. de Vries, Chem. Ptys., 80, 113 (1983)

33. Z. Xu, B. Koplitz, C. Wittig, J. Phys. Chem., 92, 5518 (1988)

34. K. P. Huber, G. Herzberg, Molecular Spectra and Molecular Structure. IV. Constants of Diatomic Molecules, Van Nostrand Reinhold Co. (1979)

35. S. G. Tilford, M. L. Ginter, A. M. Bass, J. Mol. Spectr., 34, 327 (1970)

36. M. L. Ginter, S. G. Tilford, A. M. Bass, J. Mol. Spectr., 57, 271 (1975)

37. D. S. Ginter, M. L. Ginter, S. G. Tilford, J. Mol. Spectr., 92, 40 (1982)

38. H.-J. Werner, E.-A. Reinsch, P. Rosmus, Chem. Phys. Lett, 78, 311 (1981)

39. D.A. Chapman, K. Balasubramanian, S. H. Lin, Chem. Phys. Lett. 118, 192 (1985)

40. I. Levy, M. Shapiro, J. Chem. Phys., 89, 2900 (1988)

41. J. A. Coxon and P. G. Hajigeorgiou, J. Mol. Spect., 150, 1 (1991)

42. J. Zhang, M. Dulligan, J. Segal, Y. Wen, C. Wittig, J. Phys. Chem., 99, 13680 (1995)

43. C. E. Moore, Atomic Energy Levels, NBS Circular No 467, Vol. 1, Washington, D.C. USA (1958)

44. R. Schinke, Photodissociation Dynamics, Cambridge, University Press (1993)

45. The value of ionization energies and electron affinities are taken from CRC Handbook of Chemistry and Physics, 69 ${ }^{\text {th }}$, ed., (1988-1989), while the values of equilibrium internuclear distances and dissociation energies are taken from reference 34 . 


\section{Chapter 3 Theory of the photophysical processes}

The objective of this chapter is to provide a theoretical framework to analyse and to discuss the experimental results of this study. Before getting involved in the details of the theory, a few concepts related to this pump-probe experiment are introduced, in order to provide a better physical picture. The theoretical formalism and the experimental measurements will combine into a clear picture of the photophysical processes that occur in molecular HI excitation to the $A$ band, and in the subsequent detection of photofragments.

\subsection{Photophysical processes and observables}

Typically, photofragment spectroscopy employs an intense UV source, usually a laser or other bright optical source, to dissociate molecules into fragment products. The latter can be detected using methods of mass spectroscopy, optical spectroscopy or a mixture of both. In our study, an optical method is used to detect the photofragment products: the technique of Doppler spectroscopy.

\subsubsection{The three steps of our photofragment experiment}

The formal description of our photofragment spectroscopy experiment involves three steps: photoexcitation of the parent molecules, the dynamics of dissociation, and the product detection. Although our specific experimental study considered only these three steps, it should be mentioned that the initial preparation of the parent molecule. and a final detection of the polarized fluorescence from excited fragments, could in principle also be performed. as anticipated in the theoretical summary of Greene and Zare, and exploited in the density matrix formalism developed by Singer, Freed and Band (SFB). ${ }^{2}$ However, the scope of the present experimental study is appropriately described by the wavefunction formalism reviewed by Shapiro and Bersohn. ${ }^{3}$

\section{A) Photoexcitation of parent molecules}

The optical excitation of a parent molecule to a dissociative state, and the consequent dissociation dynamics, are the central topies of photofragment spectroscopy. The photolysis laser used to excite molecules into dissociative state(s) is referred to as the pump laser. The excitation of parent molecules by the pump laser is the first lightmatter interaction occurring in the course of our photodissociation experiment.

\section{B) Dynamics of dissociation}

Various dynamical effects can play a role during a dissociation event, as we already mentioned in chapter 1 , depending on the type of electronic states involved and the specific mechanism of dissociation that results. Dissociation processes can be separated in two types: direct dissociation, and indirect dissociation or predissociation. In a direct dissociation process an excited state of the molecule decays into photofragments very 
rapidly compared to the typical time scale of the molecular vibration. By contrash, the dynamics of indirect photodissociation is usually slower, because it proceeds via the coupling of an excited metastable state to a dissociative state. The present sudy is concerned with the dissociation of HI following absorption in the A band, for which the broad featureless absorption spectrum suggests a direct dissociation process.

\section{C)Detection of photofragments}

The detection of photofragments is performed with Doppler spectroscopy. This technique uses a tunable coherent light beam of narrow linewidth, referred to as the probe beam, to excite resonantly a selected velocity group of the photofragments. Tuning the frequency of the probe laser over the width of a Doppler broadened resonance, and collecting the consequent Laser Induced Fluorescence (LIF), yields the Doppler profile spectrum.

\section{D) Goal of the overall procedure}

To obtain Doppler profile measurements is the first stage of a procedure leading to the determination of the dissociative states. Such measurements were performed at various photon energies of the photolysis pump excitation. Analysis of these measurements yielded numerical values for the observables characterizing the photodissociation dynamics. Based on these observabies, a quantum mechanical calculation allowed the determination of potential energy curves (PEC's) for the excited dissociative states of the A band absorption. .

\subsubsection{The observables}

To describe the photodissociation of $\mathrm{HI}$ molecules following one-photon excitation in the A band, the observables used are: branching ratios between electronic products, and photofragment anisotropies. These observables were determined from the experimental Doppler profile, and they provide sufficient information to unravel the dynamics of photodissociation in this system.

\section{A) Electronic branching of the photoproducts}

Following excitation in the $A$ band at a specific energy, two product channels are possible, corresponding to the accessible electronic states of the photoproducts: the ground ( $\left.{ }^{2} \mathrm{P}_{32}\right)$, and the excited ( $\left.{ }^{2} \mathrm{P}_{12}\right)$ states of the iodine atoms. The dependence of the electronic branching ratios on photolysis photon energies characterizes the dissociation dynamics. In addition to quantifying the partition of energy among the product channels, the electronic branching is of central importance, in combination with total absorption cross section (TACS) measurements, to the determination of the PEC's corresponding to the dissociative states.

\section{B) Anisotropy of the photofragments}

Direct photodissociation of diatomic molecules with a pump laser of linear polarization results in an axially symmetric angular distribution of the photofragments with respect to the polarization direction. In the case of 
an unpolarized or circularly polarized photolysis laser, the symmetry axis is defined by the propagation axis of the pump laser, i.e. its wavevector. "The anisotropy of the photofragment distribution reveals information on the type of electronic states involved in the optical transition.

Photofragment anisctropies are of significant importance in assessing the presence of nonadiabatic effects in the dissociation dynamics, ${ }^{1}$ since large and abrupt variation of the anisotropy dependence on the photolysis photon energy is predicted to result from nonadiabatic effects. ${ }^{2}$

\subsubsection{The photophysical processes and their theoretical descriptions}

Initially the molecular ensemble is in an isotropic state: for any J-value of the total angular momentum, the $M_{\mathrm{J}}$ projections are all equally populated. The optical excitation of the pump beam induces a first anisotropy that depends on the state of the pump laser polarization, and the on symmetry of the ground state and of the excited dissociative states. Once the molecular bond is broken and the atoms are flying apart, the challenge remains to measure the dynamical states of the system.

The photofragment dynamics must be probed soon after the dissociation event occurrs, in order to avoid collision relaxation with background gas, or before the photofragments leave the experimentally accessible zone altogether. Doppler spectroscopy of the H-atom photofragments is used to perform this task. To this end, a probe beam is tuned around the Doppler-shifted resonant frequency of the $\mathrm{H}$-atoms to obtain a Laser Induced Fluorescence (LIF) signal. This second light-matter interaction involves a specific transition in the H-atom fragments; it exploits the dependence of the precise resonant frequency of a given $\mathbf{H}$-atom, on the projection of its velocity onto the probe axis. The resulting Doppler spectrum is effectively a projection of the photofragments angular distribution onto the probe beam axis. It is a 1-D projection of the 2-D angular distribution of the photofragments.

Doppler spectra were measured for two orthogonal orientations of the pump polarization versus the probe laser beam axis. The Doppler spectra, orthogonal in this vectorial space, can be inverted to retrieve the angular distribution of the photofragments using a mathematical procedure outlined below. The angular distribution results from the photodissociation dynamics, which is characterized by physical observables. The determination of the observables is the goal of the products detection performed with the Doppler spectroscopy.

Section 3.3 is devoted to the mathematical relation between Doppler spectra and physical observables. The determination of these observables from these Doppler spectra is the first goal of our analytical procedure. Once determined with sufficient accuracy, the values of these observables are used in a quantum mechanical analysis leading to the determination of the PEC's for the dissociative states of the first absorption continuum. The quantum mechanical analysis of chapter 6 is a numerical implementation of the theory described hereafter. 


\subsection{Theory for the direct photodissociation of $\mathrm{HI}$ molecules}

During the last two decades, the theoretical understanding of the photodissociation processes have evolved a great deal as a result of the increased availability of experimental observations, and of powerful computers, which spurred development for numerical implementation of quantum mechanical methods. The theory of diatomic photodissociation has been developed to a high degree of sophistication by SFB' ${ }^{2}$, whose density matrix formulation allows the systematic description of all the possible steps mentioned in section 3.1.1. However, the theoretical description of the physical processes occurring in the photodissociation dynamies of HI is developed along the lines of the wavefunction formalism ${ }^{2}$, because it satisfied the immediate requirements for the analysis of our experimental results. Only some concepts defined in the work of $\mathrm{SFB}^{2}$ will be used in discussing some finer points. The basic concepts that suffice to the operational analysis of the present study (chapter 6 ) are introduced.

\subsubsection{Basic physical concepts}

\section{A) Photodissociation reacrion}

Consider a gas of isolated HI molecules, at a density low enough to ensure that the photodissociation is a unimolecular process, i.e. that intermolecular collisions are negligible both in the photodissociation process and up to the time of photofragment detection. A molecular expansion (see chapter 4) is used to produce an isotropic ensemble of HI molecules at a relatively low temperature; i.e. the population of the rotational (N) and vibrational (v) quantum number is predominanty in their respective ground state: $\mathbf{N} \rightarrow \mathbf{0}, \mathbf{v} \rightarrow \mathbf{0}$. Optical absorption of $\mathrm{HI}$ molecules in the A band excites direct dissociative states, which relax rapidy to their corresponding assymptotic atomic products. The photodissociation reaction can be denoted by equation $(3-1)$, where $(\mathrm{HI})^{\bullet},(\mathrm{HI})^{-2}$, etc represent the indeterminacy in the number and identity of the excited states.

$H I+\hbar \omega \rightarrow\left\{\begin{array}{c}(\mathrm{HI})^{* 1} \\ (\mathrm{HI})^{* 2} \\ \ldots \\ (\mathrm{HI})^{* m}\end{array}\right\} \rightarrow\left\{\begin{array}{l}\mathrm{H}+\mathrm{I} \\ \mathrm{H}+\mathrm{I}^{*}\end{array}\right\}$

Two aspects of the reaction are explicitly undefined in the equation (3-1): (i) the exact number of excited dissociative states involved in the absorption continuum, and (ii) the correspondence between dissociative states and atomic product channels. Our gaal in writing equation (3-1) is to represent the controversy that existed when we approached this topic (see chapter 2), and that has been resolved through the experimental measurements and the corresponding quantum mechanical analysis perfomed in the present work.

While the number and symmetry type of the electronic states are intrinsic properties of the molecule, the nature of the correspondence between the dissociative state and product channel can, in general, be made complex by the 
presence of nonadiabatic interaction during the act of dissociation. Although previous studies ${ }^{5.6}$ had concluded that these interaction were significant, the present study will show, on the basis of our experimental measurements, that it is not the case; the discrepancies between ours and previous results will be explained.

\section{B) Instantaneous photoexcitation and fast recoil}

The symmetry of a dissociative electronic state determines the possible molecular orientations, at the time of excitation, relative to the polarization of the photolysis radiation field. Because the angular frequency of molecular rotation is more than three orders of magnitude slower than the angular frequency of the $U V$ radiation being absobed, a given molecule appears frozen during an electronic transition. Thus the absorption of a quantum of radiation can be approximated as being instantaneous, on the time scale of nuclear motion.

In the first absorption continuum of the $\mathrm{HI}$ molecule, the cross section is non-zero only at energies such that the fragment atoms are produced with a large kinetic energy in the centre-of-mass, in the range of 0.50 to $3.5 \mathrm{eV}$. Once a molecule is excited, the atoms recoil axially, due to their mutual interaction, within a few tens of femtoseconds. On the other hand, the period of molecular rotation is of the order of three picoseconds, two orders of magnitude longer than the fragment recoil time; this justifies the axial recoil approximation, which assumes that the trajectory of the fragments is along the direction of the vibrational motion.

\section{C) Symmetry of electronic states}

As a result of the vectorial nature of the electromagnetic radiation, two types of electronic transition may occur at low intensity of the photolysis radiation: parallel or perpendicular transition. Each corresponds to a change of the projection of the total electronic angular monentum onto the intermolecular axis equal to 0 or \pm 1 respectively.

The terms parallel and perpendicular take their meaning from the geometric relation between transition dipole and intermolecular axis, within the framework of the dipole approximation for electronic transition. For a diatomic molecule, a parallel transition is represented by a transition dipole vector that lies parallel to the molecular axis, and correspondingly for the perpendicular transition. When irradiated with linearly polarized light, molecular excitation to an excited state occurs with a probability $P$ that depends on the relative orientation of the transition dipole $\mu$ with respect to the electric field $\mathbf{E}$ as: $P=|\mathbf{E} \cdot \boldsymbol{\mu}|^{2}=\{\mathrm{E} \mu \cos (\theta)\}^{2}$.

\section{D) Global perspective of the theoretical treatment}

In brief, this work will show that the direct photodissociation of HI, following absorption in the A band, is very well represented by assuming the validity of axial recoil approximation, and that the dynamics of dissociation proceds adiabatically, i.e. excitation to a specific dissociative state is followed by evolution to fragment product 
without transition to other states. These assertions are based on the facts that: (i) there is no physical evidence that nonadiabatic transitions between the dissociative states play a role in this system, and (ii) the axial recoil approximation reproduces the measurements within experimental uncertainties. Moreover, the steep radial dependence of the excited discociative surfaces, and the rotational constant of the electronic ground state, are such that the initial rotation of the molecule could not affect significantly the angular distribution of the products in the experimental conditions used in this work. Consequently, the angular distribution of the fragments is solely determined by the symmetry of the states involved in the excitation. These assertions are concisely expressed in the mathematical formalism developed hereafter.

\subsubsection{Mathematical formalism}

\section{A) Conservation of energy}

Conservation of energy in the photodissociation reaction is expressed in equation 3-2, where hw is the photolysis photon energy, $E_{0}(H I)$ is the parent excitation prior to photon absorption, $D_{0}(H I)$ is the molecular dissociation energy, $E(H)$ and $E(I)$ represents the possible product excitation, and $K\left(m_{\mathrm{H}-1}\right)$ is the centre of mass kinetic energy.

$\mathrm{E}_{0}(\mathrm{HI})+\hbar \omega=\mathrm{E}(\mathrm{H})+\mathrm{E}(\mathrm{I})+\mathrm{K}\left(m_{\mathrm{H}-\mathrm{I}}\right)+\mathrm{D}_{\mathrm{o}}(\mathrm{HI})$

$E_{0}(H I) \cong 0 ; E(H) \equiv 0 ; E(I)=\left\{0,7603.15 \mathrm{~cm}^{-1}\right\} ; D_{0}(H I)=24630 \mathrm{~cm}^{-1}$

The first absorption continuum in HI, which span the range [32000-52000] $\mathrm{cm}^{-1}$, produces either the ground ( ${ }^{2} P_{3 / 2}$ ) or the excited ( $\mathbf{P}_{1 / 2}$ ) electronic spin-orbit state of the I-atom product, while the only electronic state energetically available for the $\mathrm{H}$-atom is the ground state. The use of a molecular beam expansion reduces the initial thermal excitation $\left(300^{\circ} \mathrm{K}\right)$ of the parents to a low level $\left(10-30^{\circ} \mathrm{K}\right)$; thus the initial parent excitation is negligible compare to the photolysis excitation and dissociation energy.

\section{B) Hamiltonian}

The energy operator is expressed by equation (3-3), which is the sum of the operators for the matter Hamiltonian $\mathrm{H}_{\mathrm{M}}$, and the operator representing the radiation-matter interaction $\mathrm{H}_{\mathrm{i}}$. The matter Hamiltonian, equation (3-4), is the energy operator in the absence of the radiation field, while the light-matter interaction is represented in terms of the semiclassical dipole approximation of equation (3-5). The energy of the radiation field is quantized, but the vector properties of the radiation field and the form of the interaction are described classically. This customary representation satisfactorily describes the measurements performed in the present study.

$\mathbf{H}=\mathbf{H}_{\mathbf{M}}+\mathbf{H}_{\mathbf{I}}$

where: $\left\{\begin{array}{l}H_{M}\left|\Psi_{i}\right\rangle=E_{i}\left|\Psi_{i}\right\rangle \\ H_{I}=\mu_{i, m} \cdot \varepsilon(t)\end{array}\right.$ 


\section{C) Basis states}

The basis is heuristically chosen as the set of molecular bound states, eigenstates of $H_{M}$, and the scattering states of the dissociative half-collision correlating asymprotically to plane waves for the atoms in electronic states $\pi$, with energy $E_{n}$, and momentum $h k_{p}$. As usual, the total wavefunction is expressed as a product of electronic and nuclear wavefimctions, whose properties ensure crthonormality of the basis in the general sense, i.e. equations 3-8.

Continuum basis: $\left\langle R, r \mid \Psi_{m} E_{\mathrm{n}}, k_{\mathrm{n}}, \mathrm{n}^{-}\right\rangle=\varphi_{\mathrm{k}}^{\mathrm{m}}(r ; R) \chi_{\mathrm{k} . \mathrm{m}-}^{\mathrm{m}}(R)$

Bound states basis: $\left\langle R, r \mid \Psi_{i}\right\rangle=\Phi_{i}(r ; R) \chi_{i}(R)$

Orthonormality: $\left\{\begin{array}{l}\left\langle\Psi_{m} E_{n}, k_{n}, n^{-} \mid \Psi_{m^{\prime}} E_{n^{\prime}}, k_{n^{\prime}}, n^{\prime-}\right\rangle=\delta\left(E_{n^{\prime}}-E_{n}\right) \delta_{n \cdot n^{\prime}} \delta_{m \cdot m^{\prime}} \\ \left\langle\Psi_{i} \mid \Psi_{m} E_{n}, k_{n}, n^{-}\right\rangle=0 \\ \left\langle\Psi_{i} \mid \Psi_{j}\right\rangle=\delta_{i, j}\end{array}\right\}$

In the last equations, $R$ is the internuclear distance, $r$ represents the electronic degrees of freedom, $n$ refers to an asymptotic electronic product, $m$ is an index denoting an excited dissociative state, and the index $i$ and $j$ are indices for bound states.

\section{D) Borm-Oppenheimer approximation and PEC}

The separability of the molecular wavefunction implicitly assumes the Born-Oppenheimer approximation, i.e. that the electronic degrees of freedom follow adiabatically the motion of the nuclei; the electron distribution rearranges itself in real time, without experiencing transitions between the eigenstates of the instantaneous electronic system. Consequenty the matter Hamiltonian is separable as a sum of nuclear and electronic Hamiltonian (3-9). Because the electronic wavefunction depends parametrically on the internuclear coordinate $R$, in equations (3-10), the eigenstates of the electronic Hamiltonian also depends on $R$. This dependence defines $V(R)$ in equation (3-11), the potential energy curve (PEC) representation for that molecular state.

$$
\begin{aligned}
& H_{M}=H_{e}+H_{N} \\
& \begin{aligned}
\left\langle\Psi_{m}\left|H_{e}\right| \Psi_{m}\right\rangle & =\left\langle\varphi_{k}^{m}(R)\left|H_{e}\right| \varphi_{k}^{m}(R)\right\rangle\left\langle\chi_{k, n}^{m} \mid \chi_{k, n-}^{m}\right\rangle \\
& =V_{m}(R) \equiv P E C
\end{aligned} \\
& V_{m}(R) \cong A_{m} \exp \left[-B_{m}\left(R-R_{e}\right)\right]+C_{m}
\end{aligned}
$$

In practice, the PEC for the ground state is obtained from a numerical fit to spectroscopic constants obtained experimentally. This was done for the ground state of $\mathrm{HI}$ by Coxon and Hajigeorgiou (see chapter 2). For the direct dissociative states, it has been customary to represent the PEC by the analytical form of equation (3-12). 


\section{E) Transition amplitude and Franck-Condon Approximation}

Within the dipole approximation, the transition amplitude from an initial bound state $\left|\Psi_{i}\right\rangle$ to a final dissociative state $\left|\Psi_{n} E_{n}, n\right\rangle$ can be calculated with equation (3-13). Integrating over the electronic coordinates in (3-14), the result is an $R$-dependent transition dipole $\mu_{i s}(R)$ of (3-15). Faced with a lack of information on the radial dependance of $\mu_{i n}(R)$, one usually invokes the Franck-Condon approximation (FCA), expressed in equation (3-16); it will be showed to describe the observation appropriately.

$$
\begin{aligned}
T_{m, i} & =\left\langle\Psi_{i}|r \cdot \varepsilon| \Psi_{m} E_{n}, k_{n-1} n^{-}\right\rangle \\
& =\left\langle x_{i} K \varphi_{i}|r| \varphi_{k}^{m}\right\rangle\left|x_{k, n-}^{m}\right\rangle \cdot \varepsilon \\
& =\left\langle x_{i}\left|\mu_{i, m}(R)\right| x_{k, n-1}^{m}\right\rangle \cdot \varepsilon \\
& \cong\left[\left\langle\mu_{i, m}\right\rangle \cdot \varepsilon\right]\left\langle x_{i} \mid x_{k, n-1}^{m}\right\rangle
\end{aligned}
$$

\section{F) Partial and total absorption cross sections}

The calculation of a transition amplitude, from the ground state $|i\rangle$ to a given dissociative state $|\mathrm{m}\rangle$, allows for the definition of a partial absorption cross section to state $m$ (PACSS(m), or $\sigma_{m}$ ), as shown in (3-17). Note that we denoted $\sigma_{m}$ the partial absorption cross section to a state $m$ (PACSS(m)), and $\sigma^{\mathrm{a}}$ the partial absorption cross section for the product $\mathbf{n}$ (PACS(n)). Note also that rigorously speaking, $\mathbf{R}$ is the relative yield for excited product; although the branching ratio is sometime defined as the ratio of the relative yield, $R$ defined in the present work is a ratio, and it clearly quantifies the product branching, so it is a proper qualifier of the branching ratio. The occurrence of nonadiabatic transitions in the dissociation dynamics is discussed in appendix $\mathbf{G}$. Because no indication of the presence of nonadiabatic effect was observed in the results of our experimental study of HI photodissociation, a direct relation is expected between dissociative state and product, as expressed by (3-18).

The total absorption cross section (TACS) is calculated by summing all PACSS(m) in equation (3-19), while the electronic branching ratio $R_{n}$ for the product $n$, is simply the ratio of the partial absorption cross section for the product $n\left(P A C S(n)\right.$ or $\sigma^{2}$ ) divided by the TACS, as expressed in equation (3-20).

$\operatorname{PACSS}(m): \quad \sigma_{m}=\left[\frac{8 \pi^{3} \omega}{c}\right]\left|T_{m, i}\right|^{2}$

$\operatorname{PACS}(n): \sigma^{n}=\left[\frac{8 \pi^{3} \omega}{c}\right] \sum_{m}\left|T_{m, i}^{n}\right|^{2} \quad$ (adiabatic case) $(3-18)$

Total absorption cross section: $\quad \sigma_{\mathrm{T}}=\sum_{\mathrm{n}} \sigma^{\mathrm{D}} \quad(3-19)$

Electronic branching: $\quad R_{n}=\sigma^{n} / \sigma_{T} \quad(3-20)$ 
To calculate the TACS in terms of a sum of PACSS(m), using the wavefunction of the bound ground state and the wavefunction of the dissociative excited state, the expression (3-21) was taken from Le Roy' et al. This formula is used, to determine the PEC for each state of the $A$ band in chapter 6 . In $(3-21), \epsilon_{T}$ is the decadic molar absorption in ( 1. mole $\left.^{-1} \mathrm{~cm}^{-1}\right), \epsilon_{0}=8.43946 \cdot 10^{-4}, m$ is the reduced mass of the photofragment in amu, $v$ is the photon frequency in $\mathrm{cm}^{-1}, \mathrm{~K}_{\mathrm{p}}$ is the asymptotic kinetic energy $\left(\mathrm{cm}^{-1}\right)$ of the fragment product $\mathrm{n}$, and $\mathrm{g}_{\mathrm{m}}$ is the electronic degeneracy of the dissociative state: 1 if $\Omega=0$, and 2 if $\Omega=1$.

$\varepsilon_{T}(v)=\varepsilon_{0} m^{1 / 2} v \sum_{n}\left\{K_{n-}^{-1 / 2} g_{m}\left|\int_{0}^{\infty} d R \chi_{i, n-}^{m}\left(R ; v, A_{m}, B_{m}, C_{m}\right) \mu_{i, m}(R) \chi_{i}(R)\right|^{2}\right\}$

Note that in equation (3-21), the unbound radial wavefunction $\chi^{(1-5-)}(r ; A, B, C)$ is determined by the same parameters that defines the PEC. In principle, the goal of the photofragment spectroscopy is to determine the parameters of the PEC for each dissociative state of the absorption continuum, along with their respective transition dipole. These physical quantities characterize the photodissociation dynamics for this system.

In the present work, the electronic branching ratios were determined experimentally for the products of $H$ photodissociation following absorption in the $A$ band. The anisotropy of the product velocity distributions, and their dependence on excitation photon energy, were also determined experimentally, as they are crucial parameters to characterize the symmetry of the dissociative states. These physical observables were in turn used to calculate the PACSS using equations (3-12) to (3-21) in chapter 6.

\section{G) Anisotropy of the fragments angular distribution}

The angular distribution of photofragments produced by weak^ radiation of a linearly polarized field can be represented by the relation (3-22). The anisotropy parameter, $\beta_{m}$ expressed in (3-23), is determined experimentally. The angle $\eta_{m}$, between the transition dipole and the molecular axis, is constrained by symmetry to either $0^{\circ}$ or $90^{\circ}$, corresponding respectively to parallel and perpendicular transition. The deflection of the fragment recoil velocity, due to initial parent rotation, defines the angle $\alpha$ which is approximated by the superposition of asymptotic axial recoil, and initial tangential speed of molecular rotation.

$I_{m}(\theta)=\left(\sigma_{m} / 4 \pi\right)\left[1+\beta_{m} P_{2}(\cos \theta)\right]$

$\beta_{m}=2 P_{2}\left(\cos \eta_{m}\right) P_{2}(\cos \alpha) g(\omega \tau)$

$g(\omega \tau)=\frac{\left[1+(\omega \tau)^{2}\right]}{\left[1+4(\omega \tau)^{2}\right]}$

\footnotetext{
A By weak. it is meant that second order process induced by the field are negligeable.
} 
In the case of HI, the axial velocity of the $\mathrm{H}$-atoms recoil is of the order of $15 \cdot 10^{3} \mathrm{~m} / \mathrm{sec}$, while the orthogonal tangential velocity, due to initial parent rotation, is of the order of $60 \mathrm{~m} / \mathrm{sec}$. This corresponds to $\alpha=0.0043 \mathrm{radian}$, and $P_{2}(\cos \alpha)=0.99999$, i.e. a negligible deflection, and consequently the axial recoil approximation is justified.

After excitation to a repulsive state and rapid dissociation of the diatomic molecule, the angular distribution of recoiling fragments reflects the possible molecular orientations at the time of excitation. Measurement of the anisotropy of the fragments velocities permits a carrelation between the vector properties of the radiation field and of the molecular dynamics. If the dissociation could proceed instantaneously upon excitation to the dissociative state, the angular distribution of the fragments produced would reflect exactly the molecular symmetry of the corresponding transition dipole. The finite time required for the atoms to recoil into their asymptotic atomic states, combined with possible initial parent rotation, may effectively blur the angular distribution of photofragments.

To describe the extent of the blurring effect due to rotation of parent molecules, Jonah" has defined a lifetime function $g(\omega \tau)$ expressed in equation (3-24), for which $\omega$ is the angular frequency of molecular rotation, and $\tau$ is the effective time required to reach the asymptotic state. For the case of prompt decay, $\omega \tau-0$ and $g(\omega \tau)-1$, while for slow decay, $\omega \tau) \mathrm{I}$ and $\mathrm{g}(\omega \tau)-1 / 4$. For the case of the HI molecule, $\omega=4-10^{\prime \prime} \mathrm{rad} / \mathrm{sec}, \tau=20 \cdot 10^{-15} \mathrm{sec}$, and $g\left(\omega \tau=8 \cdot 10^{-3}\right)=0.9998=1.0$; hence, the blurring due to initial parent rotation is negligible. Therefore in the case of such a fast direct dissociation, if the products emerge from a dissociative state of pure symmetry, the anisotropy parameter $\beta$ can only take one of two values: $\beta=\{2,-1\}$, corresponding to a parallel or a perpendicular electronic transition respectively. In principle, if a mixture of states having different symmetries contributes to the formation of a product, the angular distribution anisotropy for that product can have any value within the range $[-1,2]$.

\subsubsection{Discussion of finer detalls of the theory of photodissociation}

\section{A) Recoupling of the atomic structure}

$\mathrm{SFB}^{2}$ distinguished three internuclear regions to describe the dissociation of a diatomic molecule: (i) the molecular region around the equilibrium position of the ground electronic state, usually refered to as the Franck-Condon region, (ii) the recoupling region, where the electronic structure rearranges itself from molecular into atomic states, and (iii) the atomic region, which extends to infinity from the point of negligible interatomic interaction.

The electronic structure of $\mathrm{HI}$ calculated in chapter 6 suggests that recoupling of the atomic orbitals occurs relatively early after the electronic excitation to the dissociative states. Also, the fast dissociation dynamics could be described as the results of the electronic repulsion of atoms in an incompatible, but correlated orientation of their respective angular momenta. In the case of $\mathbf{H I}$, the recoil is so sudden that reorientation of the electronic angular momenta of the atoms cannot take place. Tremendous repulsive force and limited time precludes such 
rearrangement, which, if they could occur, would correspond to nonadiabatic transitions between states corresponding to the same asymptotic product. Consequenty the dissociation dynamics is constrained to its simplest case: dynamics on a single PEC.

\section{B) Absence of rotational transition}

Excitation to a direct dissociative state can be viewed as the "ionization" of the bond's orbital. The bonding character of the ground electronic state of a molecule is the result of electron exchange between the atoms, associated with an increased probability of the electron distribution being centred between the nuclei, and a distribution of electron exchange frequencies. If the bond is "ionized" upon excitation to a dissociative state, no torque can be applied to the bond by the radiation, since the bond no longer exists.

Because the HI molecule falls apart within few tens of femtoseconds upon electronic excitation to a dissociative states of the A band, no vibrational or rotational state is established on the repulsive surface. For the free system of atoms, the corresponding state is described by a radial scattering wave in the continuum, and a quantized impact parameter customary to quantum scattering theary.' While the the vibrational degree of freedom in the bound state corresponds to the radial motion in the scattering state, a rotational level in the bound state corresponds to a quantized impact parameter in the scattering state.

While the radial wavefunctions of the ground and excited state are used to calculate the energy dependence of the relative transition intensity between ground and dissociative state, the slower rotational degree of freedom, and the corresponding inpact parameter of the unbound state, can be considered as a "spectator" in the process. As a consequence, the impact parameter is a direct consequence of the rotational quantum number of the corresponding bound state. Chapter 7 discusses conservation of angular momentum in this process.

An additional argument in favour of the absence of rotational transition in fast recoil process is that there is a finite time required for the system to undergo a rotational transition, in term of the bound state, or to effectuate a change of impact parameter, in term of scattering photofragments in the dissociative state. On account of the large momentum of the photofragments, this would require the excited molecule to "wait" a time of the order of the rotational period, before the photofragments separate. Such a transition is physically impossible, because there is no mechanism that could prevent the repulsive potential from acting. This provides another argument to justify the axial recoil approximation. As a consequence, electrcanic transitions to direct dissociative states of $H I$ can be said $t o$ be insensitive to rotation, at least within the limits of the present investigation (i.e. Js4), and the propensity for rotational transition can be expected to be negligible for excitation to direct dissociative state. If the rotational structure was observable, this would correspond to Q-branch only in the absorption spectrum. Note, however, that only a broad single Q-branch is observed in the B-band of HI, lying at higher energy (see chapter 2 ). 


\section{C) Abser:ce of nonadiabatic transition}

In general, nonadiabatic transitions between dissociative states may occur during the dissociative half-collision. The physical causes of these interactions can be Coriolis coupling ( $\Delta \mathrm{J}= \pm 1$ ), spin-orbit interaction and avoided crossing ( $\Delta \mathrm{J}=0$ ). Coriolis effects can become significant when the kinetic energy of fragment translation is comparable to the kinetic energy of initial parent rotation, averaged over the time of dissociation; according to the above considerations, our experimental conditions precludes these effects for $\mathrm{HI}$. According to SFB ${ }^{2}$, nonadiabatic transitions are expected to occur predominantly near the energetic threshold for product formation in photodissociation; they are favoured by relatively long time spent by the photoproducts in the region where interatomic forces are significant. Appendix $\mathrm{G}$ further discuss this topic for the interested author.

\subsection{Doppler spectroscopy of $\mathrm{H}$-atom photofragments}

\subsubsection{Background}

The starting point of our experimental method is the Doppler spectroscopy technique. Application of the Doppler spectroscopy technique to the detection of photofragments bas been reviewed by Welge and Schmiedl ${ }^{10}$ for both one-photon and multi-photon dissociation reactions. Soon after, they developed its application to the detection of H-atom $s^{11}$ by laser induced fluorescence (LIF) at the Lyman $\alpha$ transition. In the latter work, a linearly polarized pump beam, at $266 \mathrm{~nm}$ wavelength, was used to dissociate HI molecules in a low pressure static cell, and an orthogonal vacuum ultraviolet (VUV) probe beam was tuned around the Doppler shifted Lyman $\alpha$ transitions ( ${ }^{2} \mathrm{~S}-\mathrm{P} \mathrm{P}$ ) of the fast $\mathrm{H}$-atoms. The resulting Doppler profiles were acquired for few symmetric alignments of the linear photolysis polarization, with respect to the probe beam direction. The anisotropy of the photofragment angular distribution was obtained using a forward convolution method.

We improved the method by cartying out the experiment in a seeded free jet expansion; this minimized scattering losses and background for fluorescence detection, in addition to reducing the probability of intermolecular collisions during the unimolecular photodissociation process.

\subsubsection{Principles of the method}

In order to define a mathematical relation between Doppler profile and angular distribution of photofragments velocities, an explicit expression of the angular distribution must first be provided. Each product fragment is identified by the spin-arbit state $\left\{{ }^{2} \mathrm{P}_{32,}{ }^{2} \mathrm{P}_{1 / 2}\right\}$ of the I-atom fragment; therefore a distinct magnitude of the H-atom fragment velocities and a corresponding symmetry of their angular distribution can be attributed to each I-atom spin-orbit product. 


\section{A) Energy and momentum conservation}

The kinetic energy available in the centre of mass of the photofragments, $\mathrm{K}\left(m_{\mathrm{H}-1}\right)$, is given by equation (3-2). Conservation of linear momentum in the direct dissociation results in most of the available kinetic energy, $M_{\mathrm{P}}\left(\mathrm{M}_{1}+\mathrm{M}_{\mathrm{H}}\right)=99.21 \%$ that is, being systematically imparted to the $\mathrm{H}$-atom fragment in all half-collision events. This fraction of $\mathrm{K}\left(m_{\mathrm{H}-1}\right)$ determines the magnitude of the velocity for each $\mathrm{H}$-atom product. Thus, momentum conservation results in most of the available kinetic energy being channelled into the $\mathrm{H}$-atom fragments.

Taking into account the PACS of each spin-orbit product at the various photon energies of the first absorption continuum, i.e. in the photon energy range of $[3.97,6.45] \mathrm{eV}$, the photolysis of $\mathrm{HI}$ can produce $\mathrm{H}$-atoms in the range of kinetic energy of $[0.91,3.39] \mathrm{eV}$ for the ground state spin-orbit $\left.\mathrm{I}^{(} \mathrm{P}_{3}\right)$ products, and in the energy range of $[0.35,2.45] \mathrm{eV}$ for the excited state $I\left({ }^{2} \mathrm{P}_{1 / 2}\right)$ product. Note that the kinetic energy spread for a given product depends solely on the initial energy distribution of the parents prior to photon absorption, and on the energy resolution of the photolysis laser.

\section{B) Angular distribution of photofragment velocities}

For each dissociative state, the angular distribution of the photofragments can be represented with equation (3-22). The strength of the optical transition determines $\sigma_{\mathrm{m}}$. The symmetry type of the electronic states involved in the transition is reflected through $\beta_{m}$, the anisotropy parameter. The transition dipole $\boldsymbol{\mu}_{i m}(3-15)$ defines $\beta_{m}$, via its vectorial property, and $\sigma_{\mathfrak{m}}$, via its magnitude.

\subsubsection{Doppler profiles and angular distributions}

For a given experimental value of the photolysis photon energy, the experimental determination of anisotropies and branching ratio of the products ensues from the link between angular distribution and Doppler profiles.

\section{A) Doppler shift}

When a fast moving atom interacts with a laser beam, the apparent frequency "perceived" in the moving frame of reference is shifted by the quantity $w$ given in equation (3-25). In that expression, $w_{n}=\Delta v_{D}$ is the Doppler shift of the product $n, k_{p}$ is the probe wavevector $v_{n}$ is the atomic velocity of the product $n_{,}$and $v_{n}$ and $v_{0}$ are the resonant frequencies of the H-atom in motion $v_{a}$ and at rest respectively. The vectorial dependence of a Doppler shifted resonance is expressed by equation $(3-25)^{16}$; note that if $v_{b}$ is in $\mathrm{cm} / \mathrm{sec}$, and $k_{p}$ is in $\mathrm{cm}^{-1}$, the $w_{a}$ is in Hertz.

$w_{n}=\Delta v_{D}=v_{n}-v_{P}=k_{P} \cdot v_{n}$

Because the velocities of the bydrogen atoms produced in our study are of the order of $2.10^{\circ} \mathrm{m} / \mathrm{s}$. the last expression of the Doppler shift is accurate to within 1 part in $10^{8}$ with respect to the exact relativistic expression. 
This more than suffices for our needs, since the resolution of our laser system has only a precision of 3 parts in $10^{6}$ at best, while maintaining 1 part in $10^{5}$ routinely. In our experimental arrangement (see chapter 4), the molecular velocities in the free jet at the experimental volume are nearly perpendicular to the plane defined by the pump-probe lasers, and the angular spread of the velocity at the experimental volume spans a half-angle $\Theta_{1 / 2} \leq 3^{\circ}$; consequently, the initial molecular velocities in the jet are perpendicular to the laser, to a good approximation. Because the effects of perpendicular velocity on the Doppler shift are second-order $\sim(v / c)^{2}$, they can be safely neglected.

\section{B) Doppler profile of the angular distribution}

To appreciate the nature of this physical measurement, we consider the angular distribution of the velocity vectors for an ensemble of $\mathrm{H}$-atoms corresponding to a given spin-orbit product, i.e. having a single velocity magnitude, illustrated in figure 3.1. A spectrum of Doppler shifted frequencies, or Doppler profile, corresponds to the probability distribution for the possible projection of the $\mathrm{H}$-atom velocity vectors onto the probe axis orientation. Because the Doppler shift depends on the projection angle $\theta$ of the velocity vector onto the probe axis, a given Doppler shifted frequency corresponds to a cone in velocity space. The fraction of the $\mathrm{H}$-atom velocity distribution excited by the linewidth of the probe laser corresponds to that fraction of the velocity distribution included between two cones $(\delta \theta)$ in velocity space. This is illustrated by figure 3.1, where a) displays the angular distribution for a single product, and b) depicts the corresponding Doppler profiles which would be measured with an ideal probe (zero laser linewidth), and a realistic probe (finite laser linewidth). Therefore the relative intensity in a Doppler spectrum, at a given value of Doppler shifted frequency, is proportional to the sum of the probabilities that an $H$ atom has its velocity pointing in all the symmetric directions corresponding to that value of Doppler shifted frequency. The general mathematical expression ${ }^{16}$ for the Doppler profile can be written as in (3-26), where the function $F$ represents the vectorial field of velocities. The velocity field $F$ can be expressed as a sum of two components of radial velocity in equation (3-27), where the indices 1 and 2 refer to each electronic product.

$D_{n}(w)=\int_{v_{n}} d v^{3} F(v) \cdot \hat{e} \delta\left(w \cdot k_{P} \cdot v_{n}\right)$

$F(v)=\left\{v_{1} I_{1}(\theta, \phi)+v_{2} I_{2}(\theta, \phi)\right\}$

The angular distribution of the velocity field $I_{\text {ap }}$ contains the dependence on $\theta$ and $\phi$. Since the mathematical relation between the angular distribution and the Doppler profile is linear, the simulated Doppler profile is obtained by summing the contributions of angular distributions corresponding to each product channel. In order to simplify the task of calculating the partial Doppler profiles corresponding to the angular distributions of each product, the polar axis of the spherical frame of reference is defined along the probe wavevector $k_{p}$. The calculation of each partial profile reduces simply to a $2 \pi$ integration over the azimuthal angle in equation (3-28). 


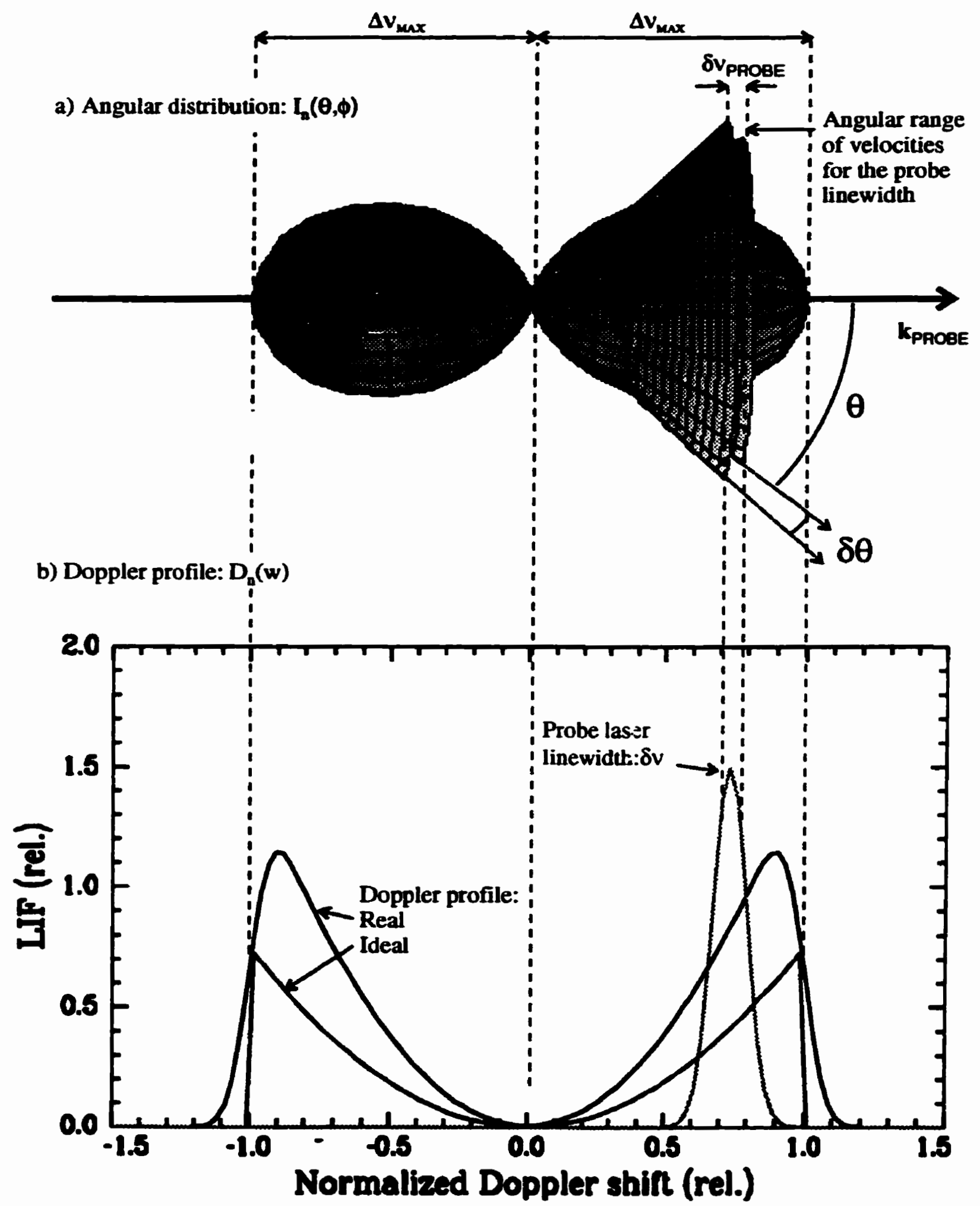

a) Angular distribution: $I_{n}(\theta, \phi)$

Figure 3.1 Graphical definition of a Doppler profile spectrum illustrating the correspondence between Doppler shifted frequency and velocity group. for a given angular distribution of fragment velocities probed by LIF. The conical surface represent the boundary of an angular danain corresponding to a realistic value of the probe linewidth; an ideal and a realistic spectra. that can be measured with such a probe linewidith, is simulated in the lower part of the figure. 
$D_{n}(w)=\left\{\begin{array}{l}\text { substitution of: } \\ \cos \theta=w / w_{0, n}\end{array}\right\} \rightarrow \int_{0}^{2 \pi} d \phi I_{n}(\theta, \phi) \cos \theta(3-28)$

$D_{t}=\sum_{n} D_{n}$

In the last equation, $w_{n}=k_{p} \cdot v_{n}, w_{\infty}=\left|k_{p}\right|\left|v_{p}\right|$, and $\cos \theta=\left[k_{p} \cdot v_{p}\right] /\left[\left|k_{p}\right|\left|v_{p}\right|\right]$, where $k_{p}$ is the probe wavevector, $v_{a}$ is the H-atom velocity, and $w_{a}$ is the correponding Doppler shift; the same quantities with an additional index " 0 " correspond to the maximum value of the accessible physical range. Hence, the numerical calculation of the simulated Doppler profiles, for each velocity group, proceeds by (i) a rotation of the spherical frame of reference such that the polar axis lies along the probe laser, followed by (ii) a $2 \pi$ integration over the azimuthal angle, and (iii) the substitution of $w_{v} / w_{\infty}=\cos \theta$ for the trigonometric expressions.

Thus, the selected fragment must possess a velocity sufficientiy large to ensure that the range of Doppler shifted frequencies is much larger than the probe linewidth, in order to provide information on the fragments angular distribution with this technique. It must be enphasized that the applicability of this Doppler spectroscopy technique to the present measurement of branching ratios and anisotropy parameters is a consequence of three properties present in the dynamics of $\mathrm{HI}$ photodissociation: (i) the large kinetic energy release in the H-atom fragments, (ii) the relatively large spin-abit energy splitting in the ground state configuration of the iodine atom, and (iii) the inherent symmetry in this process. This fortunate combination allows the possibility to distinguish experimentally between the velocities of the electronic products, and consequently this permits the measurements of anisotropies and product branching ratios in this photodissociation process. These appropriate conditions result from the nature of the electronic system, i.e. the energetics of the electronic states excited in the $A$ band and the lightness of the hydrogen atom. A narrow bandwidth probe laser was made available (see chapter 4) to execute this experiment, and it allowed for the practical implementation of this technique.

\section{References}

1. C.H. Greene and R.N. Zare, Ann. Rev. Phys. Chem., 33, 119 (1982)

2. S. A. Singer, K. F. Freed and Y. B. Band, Adv. Chem. Phys., 61, 1 (1985)

3. M. Shapiro, R. Bersohn, Ann. Rev. Phys. Chem., 33, 409 (1982)

4. R. N. Zare, Mol. Photochem., 4, 1 (1972)

5. G. N. A. van Veen, K. A. Mohamed, T. Baller, A. E. De Vries, Chem. Phys., 80, 113 (1983)

6. I. Levy, M. Shapiro, J. Chem. Phys., 89, 2900 (1988)

7. R. J. Le Roy, R.G. Macdonald and G. Burns, J. Chem. Phys., 65, 1485 (1976)

8. C. J. Jonah, Chem. Phys., 55, 1915 (1971)

9. C. Cohen-Tannoudji, B. Diu, F. Laloe, Mecanique Quantique, Tome II, Hermann ed., Paris (1986)

10. K. H. Welge, R. Schmiedl, Adv. Chem. Phys., 47, part 2, 133 (1981)

11. R. Schmiedl, H Dugan. W. Meier and K. H. Welge, Z. Phys. A., 304, 137 (1982) 


\section{Chapter 4 Experimental Method}

\subsection{Introduction}

The specific questions tackled by the central experiment of this work, and the general method to answer them, are introduced before embarking on the detailed description of the experimental equipment and procedures. When photoexcited in the first absorption continuum, in the range of $31000-52000 \mathrm{~cm}^{-1}(3.85-6.45 \mathrm{eV})$, HI molecules undergo direct dissociation. The determination of the dynamics of this process is the problem at hand. Two product channels are open, as illustrated in equation 4-1. The nature of the excited state of $H$ has been discussed in chapter 2 and will be discussed further in chapter 6 , in the light of the results obtained in the present work.

$H I+h v_{\text {diss }} \rightarrow(H I) *\left\{\begin{array}{l}H_{\text {slow }}+I^{*}\left({ }^{2} P_{1 / 2}\right) \\ H_{\text {fast }}+I\left({ }^{2} P_{3 / 2}\right)\end{array}\right\}$

A salient feature of this unimolecular process is that $99.2 \%$ of the available kinetic energy in the centre-of- mass is directed into the $\mathbf{H}$-atom fragment, as a result of momentum conservation. Thus, the H-atoms possess large velocities which differs significantly between the two product channels. These properties of the HII molecular system allows the characterization of the photodissociation dynamics to be made via measurements of the velocity distribution of the $\mathbf{H}$-atom photofragments.

Following dissociation with linearly polarized light, the angular disuribution for each photofragment channel is generally described ${ }^{\prime}$ by equation (4-2), where $\theta$ is measured with respect to the linear polarization vector of the photolysis light beam. The value of $\beta_{i}$ characterizes the anisotropy of the velocity distribution for the fragment product ' $i$ ', and it is prescribed by the symmetry of the excited dissociative state. The branching ratio for the product ' $i$ ' is defined as: $R_{i}=\sigma_{i} /\left(\sum_{0} \sigma_{b}\right)$, where the indices $n$ and $i$ can take values representing either the $I *\left({ }^{*} P_{1 / 2}\right)$ or the I ( $\left({ }^{2} P_{32}\right)$ product channel, and $\sigma_{i}$ is the total cross-section for a given product channel.

$I_{i}(\theta)=\left(\sigma_{i} / 4 \pi\right)\left[1+\beta_{i} P_{2}(\cos \theta)\right] \quad(4-2)$

The goal of the present experimental study was to probe the photofragmentation dynamics by measuring the anisotropy parameter $\beta_{1}$ and the protuct branching ratio: $R$. The technique of Doppler spectroscopy of the $H$-atom fragment was selected $t 0$ perform this task at various photolysis wavelengths throughout the first absorption continuum. Compared to other techniques developed during the last two decades (re. chap. 2), Doppler spectroscopy offered a balance of measurement precision and data throughput appropriate for our investigation. In the following, the photofragment spectrometer constructed in our laboratory is described. Essential aspects of the procedure required to execute the experimental measurements conclude this chapter. 


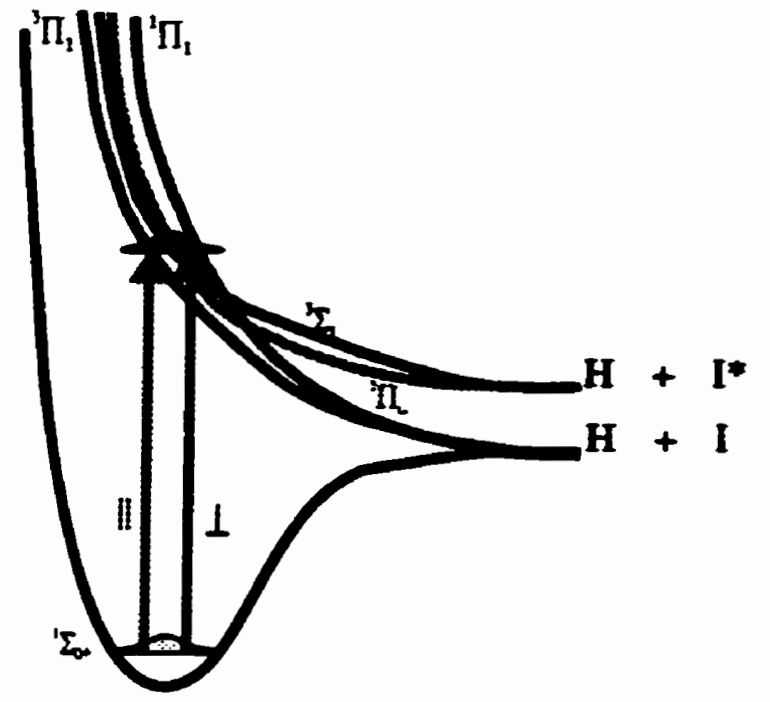

a) HI Dissociation

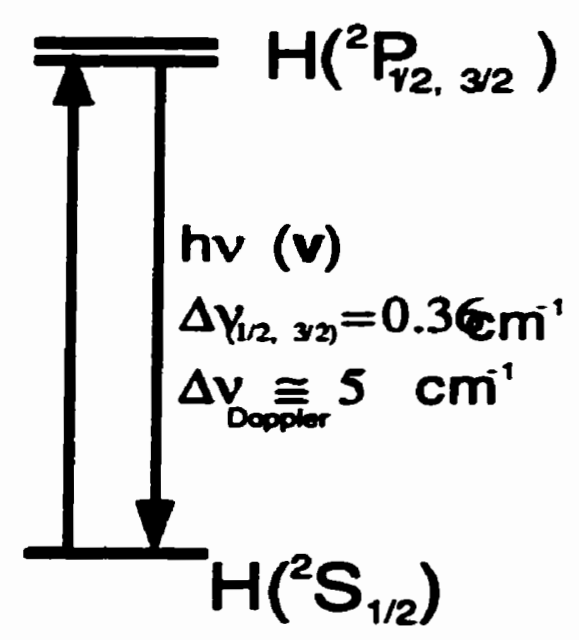

b) H-atom LIF

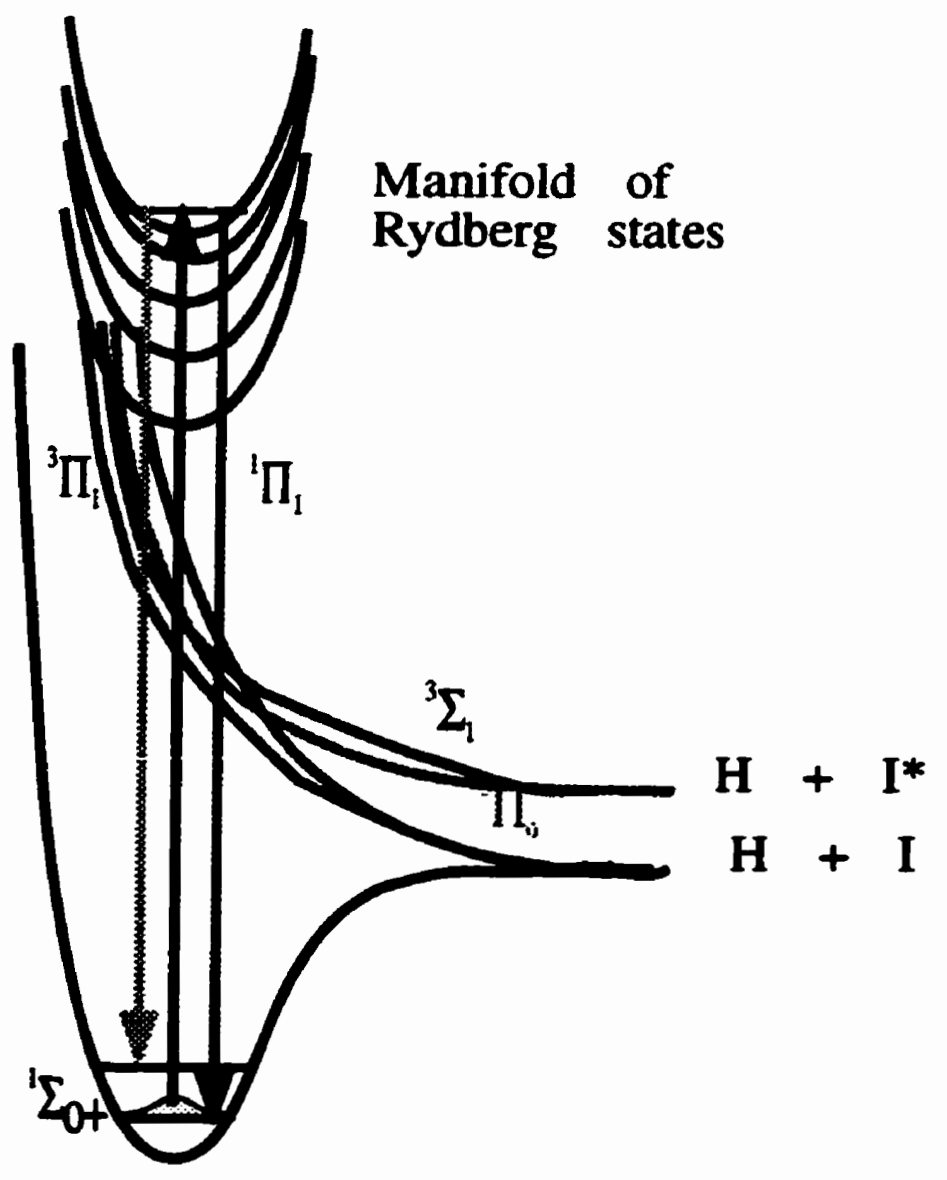

Figure 4.1. Pbotodynamic processes present in the pump-probe experiment described in this thesis. a) The pump laser excites HI molecules to an ensemble of dissociative states. b) The probe laser excites resconantly the H-atoms, whose resonance flucrescence is collected. c) Conocmitant resonant absorption of the probe by HI molecule, and the consequent resonance and red-shified fluorescence, results in a spurious signal on the LIF detector. 


\subsection{Conceptual description of our photofragment spectroscopy experiment}

\subsubsection{Doppler spectroscopy detection}

Doppler spectroscopy, a lineshape spectroscopy method, is enabled by the generation of a coherent narrow-band $\left(0.65 \mathrm{~cm}^{-1}\right)$ VUV probe beam, and the symmetry present in the photodissociation dyaamics of HI ensuing excitation in the A band. Correlation of Doppler spectra, for symmetric orientations of the pump polarization with respect to the probe beam axis, enables one to retrieve the differential cross section for producing the $H$-atom photofragments.

Figure 4.1 illustrates the photon dynamics (photonics) in the overall pump-probe system. The photonic processes, intended by the experimental design, are the photodissociation of HI by the pump laser beam, and the excitation of the H-atom photofragments by the probe laser beam. A concomitant absorption of the probe laser by $\mathrm{HI}$ molecules, due to an accidental resonant excitation of HI to Rydberg states, results in both red-shifted and resonance fluorescence, which contribute to spurious signals on the H-atom LIF detector. Therefore, a filter described below was inserted in front of the LIF detector, in order to minimize the background signal due to this strong red-shifted fluorescence.

\subsubsection{Photodissociation in collision-free conditions}

A common trait of photofragment spectroscopy methods is the use of a supersonic expansion to prepare target molecules. This was done with a pulsed source of gas expanding in high-vacuum. The primary effects of the expansion are to cool the internal degrees of freedom, and to provide a collision-free state of the target molecules. in order to simplify the study of the photodissociation dynamics initiated by a pump laser pulse.

\subsubsection{Laser photodissociation dynamics}

a) Global laboratory setup

A diagram of the overall layout of the laboratory is shown in figure 4.2. The experimental laboratory space required to realize the setup described in this work is approximately $10 \times 10$ square metre. The vacuum chamber, where the photophysical processes ocaur, is located in the upper left part of the figure 4.2. Lasers and other optical devices are discussed in the next section, along with the molecular jet source. The signal detection, electronic amplification, and computer acquisition and control are the subject of the following section.

\section{b) Specific details of HI photodissociation in the A band}

Microsocpically, the molecular dissociation occurs within a few tens of femtoseconds, from photon absorption to fragments separation. Upon dissociation, the H-atom fragments acquire a speed of the order of $1.5 \cdot 10^{4} \mathrm{~m} / \mathrm{sec}$., and the asymptotic limit of the interaction occurs at internuclear separation of approximately 4-5 $\AA$. 


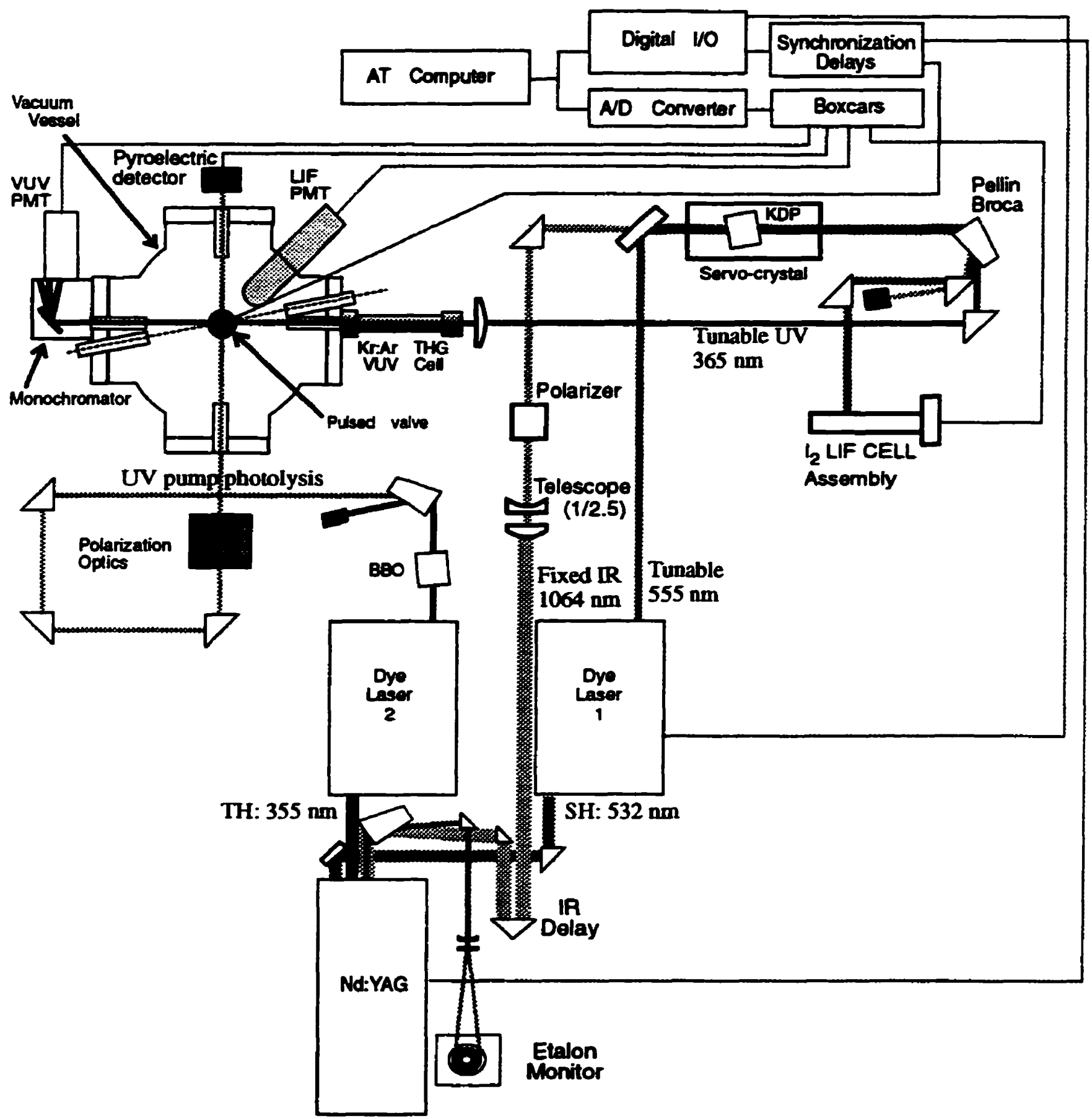

Figure 42 Layout of the experimental setup. The YAG laser pumps two dye lasers and a nonlinear mixing process in $\mathrm{KD}^{\circ} \mathrm{P}$. The chains of optical processes to generate the pump and probe shown here are described in the text. along with the computer for data acquisition and various other electroaic system components. 


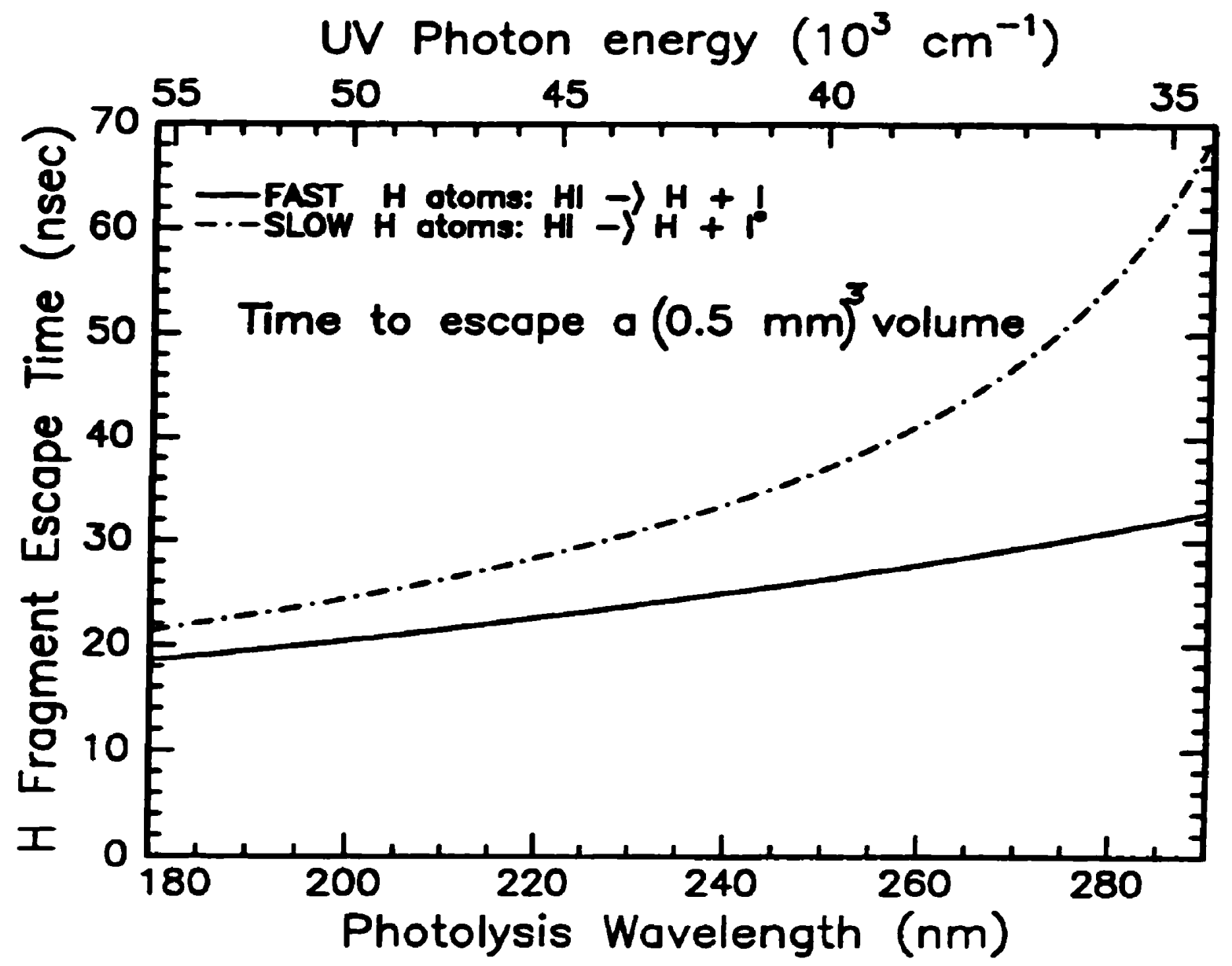

Figure 4.3 Escape time for the $\mathbf{H}$-atom fragments at various photolysis photon energies of the $A$ band absorption.

\section{c) A pulsed pump-probe spectroscopy experiment in free supersonic jet}

The experimental volume is defined by the intersection between pump and probe beams, and measures approximately 5 cubic millimetres. In the middle of each $200 \mu \mathrm{sec}$ gas pulse of the molecular free jet, a 10 nanosecond pulse of polarized UV radiation pumps the HI molecules to dissociative states; after a delay of 10 nanoseconds, the photofragments have already reached their asymptotic scautering states, and they are probed by a beam of pulsed VUV of 10 nanosecond duration. Energy conservation ensures that the fast H-atoms have moved only a small distance during the pump-probe delay in comparison to the dimension of the experimental volume, and consequently the large majority of the $\mathrm{H}$-atoms are still within the experimental volume.

Figure 4.3 shows the escape time at various photolysis energy across the absorption continuum; the escape time is quantified as the time required for a $0.5 \mathrm{~mm}$ displacement. The pulsed photodissociation experiment is performed at a 10 Hertz repetition rate, determined by the YAG laser at the base of the optical train. 


\subsection{Description of the apparatus I: crossed molecular jet and lasers beams}

The generation of the free molecular jet used to produce the gas target is briefly discussed below. An operational description of the intense lasers, related optical devices and nonlinear optics component used to generate the pump and probe beams is reported. The laser system and the methods of nonlinear optics are briefly outlined, along with the signal measured for wavelength calibration and reference.

\subsubsection{Supersonic free jet expansion source}

\section{a) Vacuum vessel}

The molecular expansion occurs in the experimental chamber, which is a vacuum vessel containing the pulsed valve, represented by the cylindrical shape in the lower left of figure 4.4. The chamber was partly inherited from Dr. Scoles, and partly built at the machine shop of the University of Waterloo for Dr. Hepburn. The dimensions of the vacuum vessel are $50 \mathrm{~cm}$ height and $40 \mathrm{~cm}$ diameter. The flanges at the top and bottom ends of the cylinder are made to fit the Alaria 400 Italian standard. The two sets of diametrically opposite flanges are arranged on two orthogonal axis, contained in a plane nomal to the cylindrical axis of the chamber; these flanges are of the Alaria 250 type, and were installed on its side, such that the distance between the internal faces of opposite flanges is 45 $\mathrm{cm}$. Optical ports for 1 inch diameter window were installed on each Alaria 250 flanges, in order for the pump and probe beam to intersect under the free molecular jet expansion in vacuum, as shown in figure 4.4. The experimental apparatus has been constructed to allow the pump and probe beams to cross in an orthogonal geometry, approximately in the middle of the cylinder.

The vacuum vessel was coupled to a cryogenic trap which was joined to a diffusion pump (NRC. Model VHS-6, type No. 184) having a pumping speed of 3000 litres per second. A mechanical pump (Alcatel, Model ZM2008AC), having a pumping speed of $5 \mathrm{cfm}$, was backing the diffusion pump at $5 \cdot 10^{-3} \mathrm{Torr}$. The cryogenic trap was filled with liquid nitrogen, in order to prevent oil backstreaming from the mechanical pump into the vessel, hence providing clean optical windows for up to 60 hours of operation. Ten to fifteen litres of liquid nitrogen was required every 6 hours to maintain proper operation of the trap. The ultimate pressure of the vacuum vessel was $2 \cdot 10^{-7}$ Torr and the typical background pressure during free jet operation was $2 \cdot 10^{-6}$ Torr.

\section{b) Cryostatic gas source}

In order to deliver a well-defined concentration of gaseous HI:He solution to the vacuum expansion source, we have developed a cryogenic HI:He source, illustrated in the upper part of figure 4.4. This design exploits the constant equilibrium vapour pressure produced in a thermostated HI source. The source is thermostated in an 


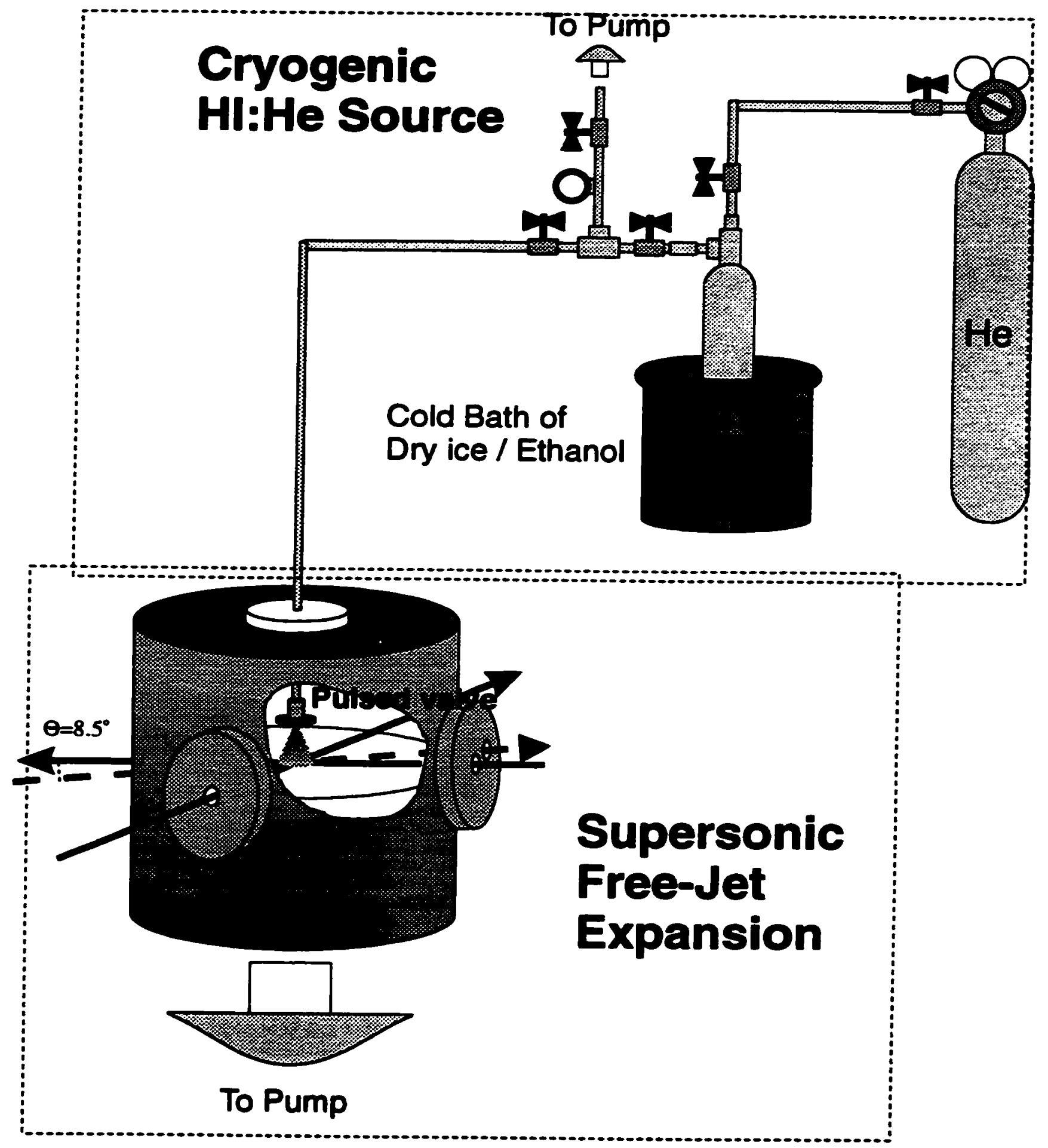

Figure 4.4. The free jet source is described as four parts: the cryostated HI:He source, the pulsed valve, the free-jet expansion. and the vacuum vessel. 
ethanol/dry ice slush bath, which provides a temperature at $-72^{\circ} \mathrm{C}$, at which the partial pressure of $\mathrm{HI}$ is $96 \mathrm{Torr}$. Applying a quieccent back pressure of Helium at 2 atmospheres absolute, and maintaining a very slow flow ( $\approx 10$ $\mathrm{ml} / \mathrm{min}$ ) of He gas, thermal equilibrium can be maintained in the $500 \mathrm{ml}$ source of $\mathrm{HI}$, which results in the production of a very stable HI:He mixture at $=5 \%$ concentration. See appendix $A$ for more details concerning this system and the flow determination of the gas mixture.

\section{c) Pulsed valve}

A high speed pulsed valve^, with a $0.5 \mathrm{~mm}$ orifice was used to produce a pulsed supersonic free jet expansion at a position $10 \mathrm{~mm}$ above the experimental volume. The length of the gas pulse was typically $200 \mu \sec _{\text {, and }}$ the quiescent pressure of a HI:He mixture used behind the pulsed valve was consistently 2 atmosphere. The electronic driver that powered the pulsed valve has been designed and constructed by Jacek Szubra, from the Electronics Shop of the University of Waterloo. This driver allows a variable width of the gas pulse, an adjustment for the amplitude of the valve opening, and can be triggered either internally or externally.

\section{d) Free supersonic expansion of a seeded molecular jet}

In his review, Levy ${ }^{2}$ has clearly illustrated the numerous advantages of jet expansions for laser spectroscopy. Supersonic expansion of the gas mixture emerging from a pulsed valve results in adiabatic cooling of the internal degrees of freedom in a gas mixture. The characterization of this process is of interest to the present study, to the extent of its use to estimate both the concentration and the temperature for the internal degrees of freedom of $\mathrm{HI}$ molecules in the target region, i.e. the experimental volume. Further consideration of the details of this topic are discussed in appendix $A$. The results of these considerations indicated that in the typical conditions used in the present study, i.e. the expansion of a $5 \% \mathrm{HI}: \mathrm{He}$ mixture, the concentration of the $\mathrm{HI}$ molecules $10 \mathrm{~mm}$ below the nozzle could vary between $1.0 \cdot 10^{-6}$ and $1.0 \cdot 10^{-7} \mathrm{~mole} / \mathrm{ml}$, which correspond to a number density of $6 \cdot 10^{15}-6 \cdot 10^{16}$ molecules $/ \mathrm{cm}^{3}$, and the rotational temperature was estimated to be in the range of 1 to $15^{\circ} \mathrm{K}$, and most likely around $10^{\circ} \mathrm{K}$.

\subsubsection{Laser systems}

This portion of the experimental setup is described in two parts: (i) the YAG laser system, which initiates the series of optical events, and (ii) the dye lasers pumped by the YAG laser. Using methods of nonlinear optics, the visible laser beams produced by the dye lasers were employed to generate, respectively, the probe beam at a tunable frequency, and the pump beam at selectable frequency. The layout of the optical beams in our experimental setup is illustrated in figure 4.2 .

A Series 9 high speed solenoid valve manufactured by General Valve Corparation. 202 Fairfield. N.J. 07006. 
The Nd:YAG laser produces the initial coherent light at a fixed infrared (IR) wavelength of $1.064 \mu \mathrm{m}$. The IR beam is partially converted into a visible light beam at $532 \mathrm{~nm}$ (GREEN) and a near-ultraviolet light beam at 355 $\mathrm{nm}$ (BLUE) by nonlinear processes in $\mathrm{K}^{*} \mathrm{DP}$ crystal; these processes are referred to as harmonic generation. These BLUE and GREEN harmonics were used to excite tunable dye lasers, on the basis of which the pump and probe beam were generated. The BLUE harmonic of the YAG pumped the dye laser that was used to generate the UV photolysis pump beam, while the GREEN harmonic pumped the dye laser used to generate of the VUV probe beam. We elaborate on a few aspects of the laser system that were crucial for our laser spectroscopy experiment.

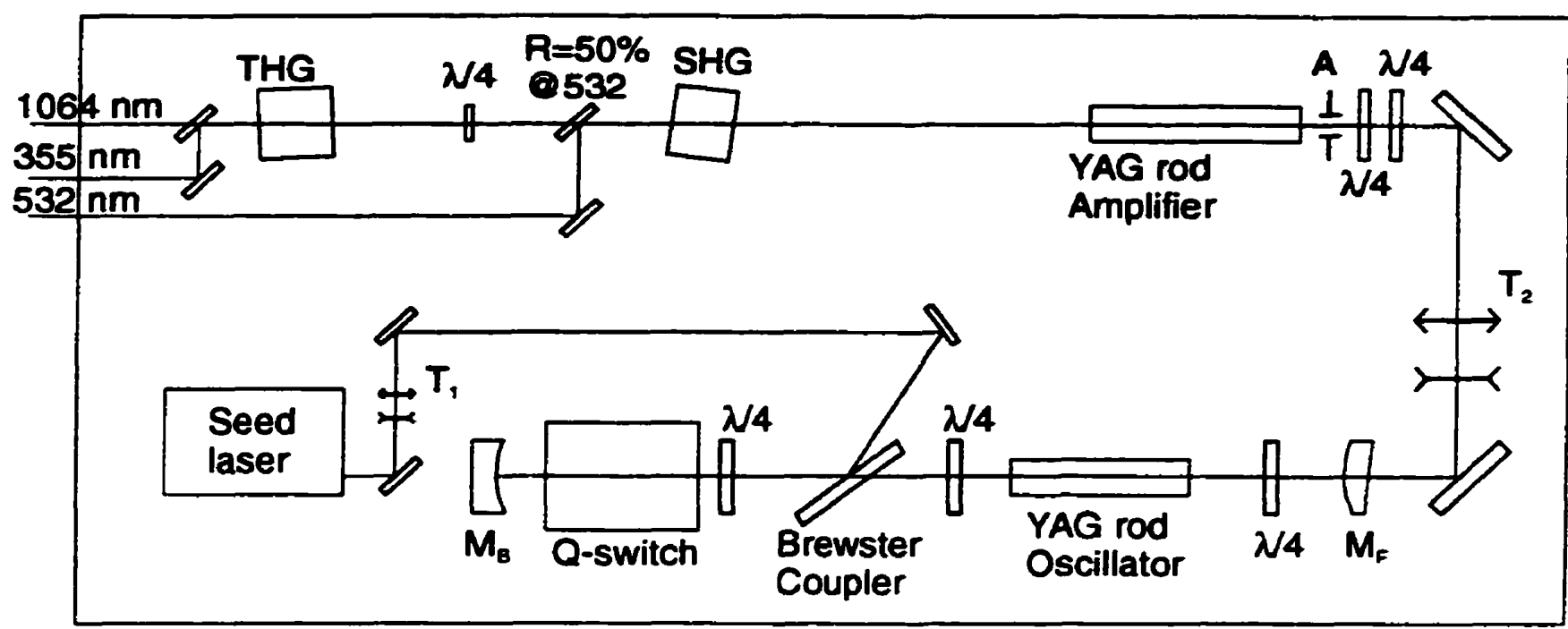

Figure 4.5. Schematic representation of the YAG laser. $M_{R}$ : back mirror. T: telescope, $\lambda 4$ : quarter waveplate, $M_{F}:$ froot mirror, A:apodizer. Other components are described in the text.

\section{a) Injection-seeded YAG laser}

Figure 4.5 shows a schematic of the YAG laser system. A Quantel^ YAG laser, model YG581CSF-10, stands at the base of the optical system. It produces pulses of $10 \mathrm{nsec}$ (FWHM) at a repetition rate of $10 \mathrm{Hertz}$, with a time jitter between pulses of 5 nanoseconds with respect to the Q-switch trigger, and a IR frequency bandwidth of 0.4 $\mathrm{cm}^{-1}$. The YAG system includes $\mathrm{KD}^{*} \mathrm{P}$ crystals for $2^{\text {ted }}$ harmonic generation (SHG=GREEN) and $3^{\text {rd }}$ harmonic generation (THG=BLUE); the harmonic crystals are an integral part of the laser head assembly.

The addition of a seed injection system improves the performances of the laser, by reducing the time jitter to 0.5 nanosecond, and producing a bandwidth of $0.013 \mathrm{~cm}^{-1}$. The seed injection system is essentially an isolated cw laser made simultaneously resonant with a single mode of the host cavity and the centre of the gain medium. In

A The American operation of the Quantel company has now changed its name to Continuum after it became independent of the French mother company. 
addition to greatly improving pulse-to-pulse stability in these system, seed injection can also enhance the generation of $3^{\text {rd }}$ harmonic by up to $40 \%$, under proper operating conditions.

The maximum and the typical output power used are shown in table 1 . The maximum powers are measured before the laser beams proceed through the next stage (e.g., IR was measured before the SHG crystal, etc.); hence, they illustrated the maximum power available from this system, as measured by the author. The typical powers were measured during normal operation conditions, with proper seed injection.

Table 4.1 Typical and maximum performance the YAG laser used in this work

\begin{tabular}{|l|c|c|c|}
\hline $\begin{array}{c}\text { Power output at } \\
\text { various barmonics }\end{array}$ & $\begin{array}{c}\text { Fundamental: } \mathbf{R} \\
\text { (mJ/pulse @ 1064 nm) }\end{array}$ & $\begin{array}{c}2^{\text {nd }} \text { Harmonic: GREEN } \\
\text { (mJ/pulse @ 532 nm) }\end{array}$ & $\begin{array}{c}3^{\text {rd }} \text { Harmonic: BLUE } \\
\text { (mJ/pulse @ 355 nm) }\end{array}$ \\
\hline Maximum power $^{\wedge}$ & 1100 & 500 & 190 \\
\hline Typical power used $^{8}$ & 700 & 400 & 100 \\
\hline
\end{tabular}

The parameters of the YAG laser configuration that can be optimized are briefly outlined. Each rods of our YAG laser is pumped by two high power flashlamps. The power ${ }^{c}$ delivered in the flashlamp pulse, and the delay between the pulses of amplifier and oscillator flashlamps, determine the maximum output of the YAG laser. In our typical operating condition, the voltage controlling the electrical power delivered to the flashlamp was reduced to $1.40 \mathrm{kV}$ from the maximum value of $1.44 \mathrm{kV}$ allowed by the Q-switch hold-off ( $1.54 \mathrm{kV}$ in our case).

The set of two waveplates placed before the amplifier rod, combined with the tilt angle of the SHG crystal, determine the fraction of fundamental power converted into GREEN $532 \mathrm{~nm}$. The THG power also depends on crystal tilt angle and on the power and polarization of the $1064 \mathrm{~nm}$ and $532 \mathrm{~nm}$ light beam; to adjust or optimize the $355 \mathrm{~nm}$ power, the polarization of the IR light can be varied by a waveplate placed before the THG crystal.

The fraction of the SHG injected into the THG crystal can be made less than unity by using a dichroic mirror with a $30 \%$ or $50 \%$ reflectivity, as opposed to the $100 \%$ value used in the default configuration. We used a dichroic mirror with a reflectivity of $50 \%$ to send that part of the GREEN output to dye laser 1, as indicated in figure 4.2. the rest of it being coupled with the remaining fundamental $\mathbb{R}$ radiation in the THG crystal to generate the BLUE harmonic. After conversion in the THG crystal, the remaining GREEN light was used to monitor the proper

\footnotetext{
^ Maximum power that can be produced independently for each harmonic by the laser system.

B Powers produced with the system in our typical configuration.

c The limit of the maximum flasblamp power that can be safely bandle by the laser depends on the quality of the alignment of the YAG laser oscillator, specifically the Q-switch lasing hold-off.
} 
behaviour of the seed-injection process by observing the interference pattern from a Fabry-Perot etalon, also shown in figure 4.2. The remaining IR power, approximately $200 \mathrm{~mJ} / \mathrm{p}$, was used for frequency mixing with the output of dye laser 1 in a KD'P crystal, as described below. Under these conditions, $200 \mathrm{~mJ} / \mathrm{p}$ of GREEN power was produced, out of the $50 \%$ split $532 \mathrm{~nm}$ beam, to pump ${ }^{\wedge}$ dye laser 1 , and $110 \mathrm{~mJ} / \mathrm{p}$ of BLUE radiation was produced to pump dye laser 2.

\section{b) Dye lasers}

Two FL2002E ${ }^{\mathrm{TM}}$ Lambda Physiks dye lasers were used in this experiment. Dye laser 1 used a solution of Fluorescein $548^{\mathrm{B}}$ (F548) diluted in a solution of sodium hydroxide ( $\mathrm{NaOH}$ ) in ethanol; the optimization of the solution is discussed in appendix B. The optimum concentrations of F548, for oscillator and amplifier solutions, are respectively 550 and $155 \mathrm{mg} /$ in ethanol solvent containing $80 \mathrm{mg} / \mathrm{l}$ of $\mathrm{NaOH}$.

Using these solutions pumped with a power of $150 \mathrm{~mJ} /$ pulse of GREEN harmonic $(532 \mathrm{~nm})$, a conversion of $33 \%$ was obtained for the $555 \mathrm{~nm}$ output. The laser linewidth was smaller than $0.2 \mathrm{~cm}^{-1}$, as measured with narrow lines of the iodine LIF calibration spectrum. The output of the F548 dye can be tuned in the 540-565 nm range. The other dye laser was pumped at $355 \mathrm{~nm}$ with a typical power of $100 \mathrm{~mJ} / \mathrm{p}$. The table b.1 of the appendix B lists the laser dye solutions used in that laser. A laser pumped at $355 \mathrm{~nm}$ exhibited a conversion efficiency of 12-20\%.

\subsubsection{Pump beam generation}

The generation of the photolysis pump beam laser is generated by doubling a dye laser beam in a BBO crystal. The fundamental frequency of the dye laser was filtered out, and then the state of polarization was controlled with the aid of a Soleil-Babinet compensator. Figure 4.6 is a schematic representation of the process.

\section{a) Frequency mixing in crystal}

Sum-frequency mixing of the wavelength $\lambda_{1}$ with $\lambda_{2}$ in a nonlinear crystal can generate, under proper conditions, radiation at a wavelength $\lambda_{3}$. For the usual collinear beam geometry , the requirements of energy and momentum conservation are expressed by equations 4-3 and 4-4 respectively.

\footnotetext{
A The maximum pump power specification for the Lambda physics dye laser, $120 \mathrm{~mJ} / \mathrm{pulse}$, is limited by damage threshold of the grating due to nonlinear effects such as self-focussing in the oscillator gain medium. However, the preamplifier and the amplifier can bandle a much higher pump power, given some additional precaution. By inserting a Neutral Density (ND) filter in front of the cacillator cuvette to reduce the oscillator pump power within specification, up to $225 \mathrm{~mJ} /$ pulse of $532 \mathrm{~nm}$ could be injected in the dye laser without damage; the ND filter was selectewd to safely handle a total power of $200 \mathrm{~mJ} / \mathrm{p}$. B Fuorescein 548 is a trademark of Exciton. Inc., Dayton, OHIO, USA
} 
$\frac{1}{\lambda_{1}}+\frac{1}{\lambda_{2}}=\frac{1}{\lambda_{3}}$

$\frac{n\left(\lambda_{1}\right)}{\lambda_{1}}+\frac{n\left(\lambda_{2}\right)}{\lambda_{2}}=\frac{n\left(\lambda_{3}\right)}{\lambda_{3}}$

In (4-4) $n(\lambda)$ is the refractive index at the wavelength $\lambda$. If the birefringence of a nonlinear crystal allows one to simultaneously satisfy both of the above relations for the three wavelengths, efficient frequency mixing can occur. This is usually done by tilting a specific crystal axis, with respect to the $k$ vector of the light, around the $\mathbf{E}$ vector of one of the optical frequency components of the sum; however, fine adjustment can also be done by thermal control of the crystal, which relies on the anisotropy of thermal expansion in these materials, and the associated change of refractive index ellipsoid.

The output of the dye laser pumped with $355 \mathrm{~nm}$ produced pulses of 12-20 mJoule/pulse of energy in the blue region of the visible. The light beam was sent through a BBO crystal ${ }^{\wedge}$ to generate $0.5-5 \mathrm{~mJ} / \mathrm{pulse}$ of UV, second harmonic generation, to be used as the photolysis pump laser. The case of second harmonic generation (SHG) corresponds to $\lambda_{1}=\lambda_{2}$. This UV output, produced collinearly with the fundamental beam of the dye laser, is linearly polarized in the horizontal plane, perpendicular to the vertical polarization of the fundamental.

The fundamental and second harmonic are separated by a Pellin-Broca prism ${ }^{\mathrm{B}}$, and the fundamental is sent to a beam dump, as shown in figure 4.2. The Pellin-Broca prism and beam dump combination is effectively a perfect filter to reject the fundamental frequency, as schematically depicted in figure 4.6. The axis of the photolysis pump is defined beforehand by a pair of iris placed on each side of the vessel. Using three $90^{\circ}$ prisms ${ }^{c}$, the second harmonic beam was steered through the windows of the vacuum vessel, onto the photolysis pump axis.

\section{b) Polarization optics}

A Soleil-Babinet compensator (Optics for Research, Model SB-10) was used to control the state of polarization of the UV photolysis light, once a beam of the right frequency has been produced and aligned onto the pump axis. The Soleil-Babinet compensator was inserted between the last $90^{\circ}$ prism and the entrance window of the vessel. The operation of this optical device is described in the section 4.4 .5 below.

A BBO: B-Barium Borate crystal, obtained from INRAD Co.

- Fused silica prism fabricated by Interoptics, Ontawa, Canada.

c $90^{\circ}$ prism made of fused silica. from Esco Products Inc., Oak Ridge, NJ, 07438-0155,USA 


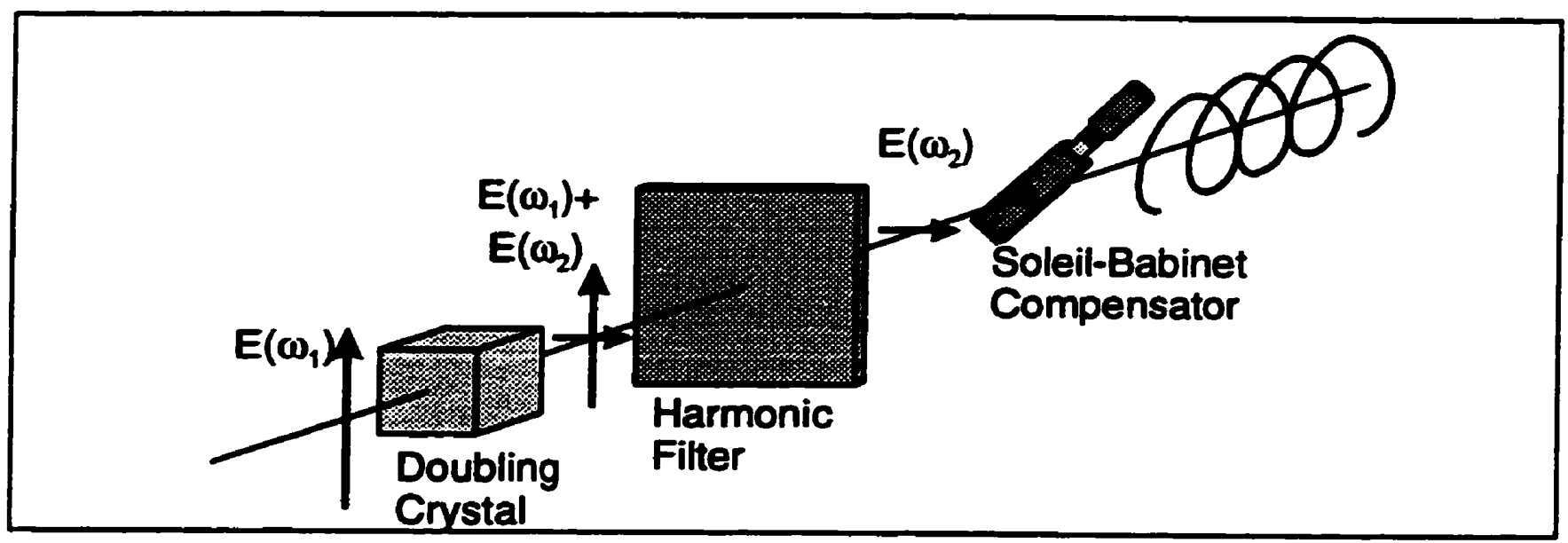

Figure 4.6 Schematic for the generation of a pump beam with an adjustable state of polarization..

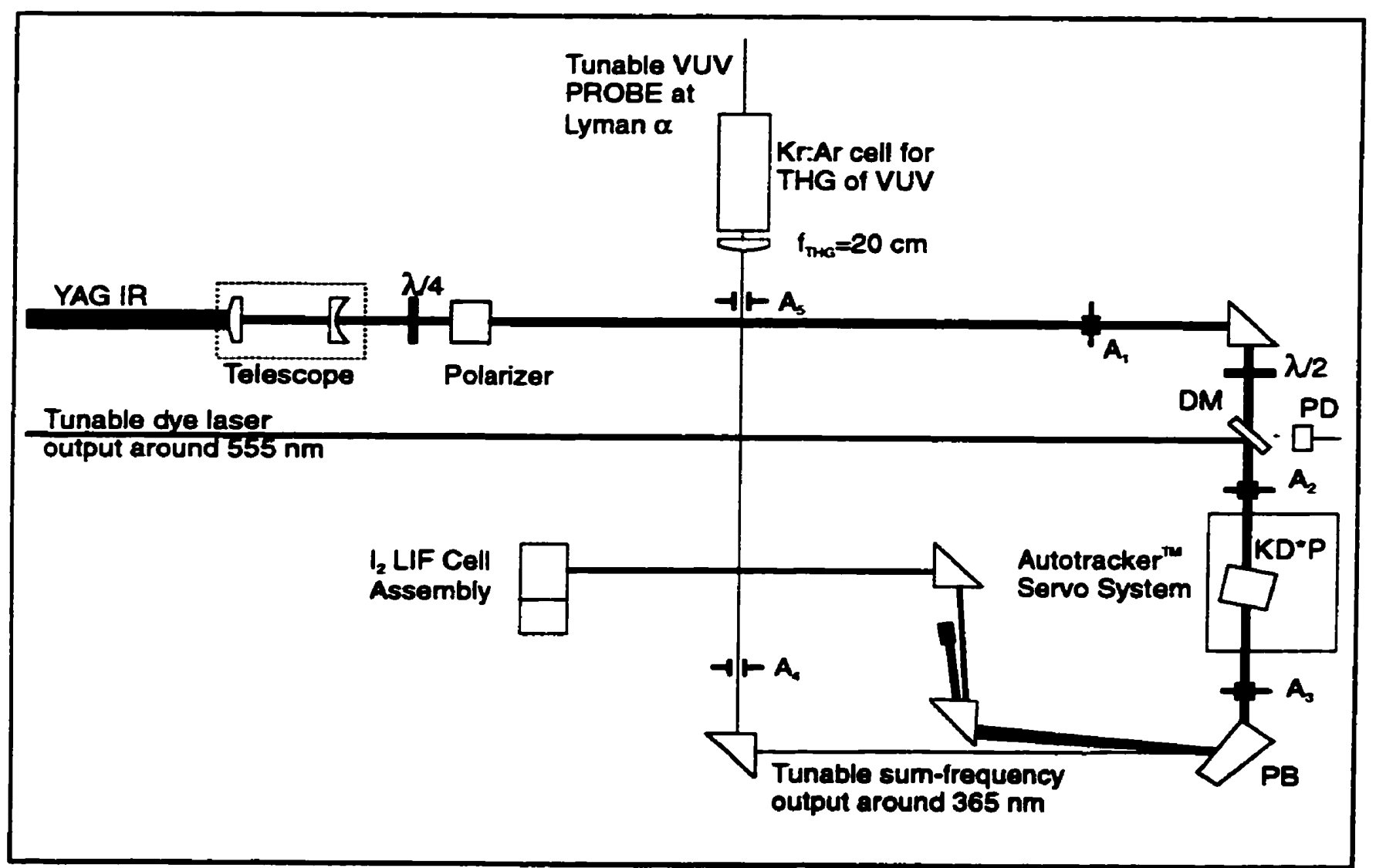

Figure 4.7 Represeatation of the optical layout for the $365 \mathrm{~mm}$ generation used for VUV generation at 121 am. $A_{1}$ aperture for alignment, DM: dichroic mirror, PD: photodiode, $\lambda / 4$ and $2 / 2$ : quarter-and balf-waveplates, PB: Pellin Broca prism. 


\subsubsection{VUV probe beam generation}

a) YAG and dye lasers beam mixing

i) IR beam shaping

The $1064 \mathrm{~nm}$ and the $532 \mathrm{~nm}$ pulses are simultaneously emitted from the YAG laser. As shown in figure 4.2, the $1064 \mathrm{~nm}$ beam is first passed through a delay line, to compensate for the delay acquired by the $532 \mathrm{~nm}$ pump inside the dye laser, such that both the fundamental IR pulse of the YAG and the output pulse of the dye laser 1 are synchronized. Then the $1064 \mathrm{~nm}$ beam propagates to a reducing telescope, composed of a converging lens $\left(f_{c}=17 \mathrm{~cm}\right.$ ) and a diverging lens $\left(f_{d}=-5.0 \mathrm{~cm}\right)$; the $-5 \mathrm{~cm}$ lens was mounted on a $X-Y-Z$ translation stage, to allow optimization of the resulting beam divergence and its overlap with the axis for $365 \mathrm{~nm}$ generation. This 3.4:1 telescope reduces the cross section of the $\mathbb{R}$ beam, initially $1 \mathrm{~cm}$ in diameter, to about $3 \mathrm{~mm}$, so that it better matches the dye laser beam cross section, which has an elliptical profile of $2 \times 1 \mathrm{~mm}$.

Before insering any following elements into the optical train, great care was taken to optimize the divergence of the $1064 \mathrm{~nm}$ beam by adjusting the distance between the lens of the telescope. This delicate operation was crucial, because there is great risk of damaging the following optics by inadvertent focussing of the $100 \mathrm{~mJ} / \mathrm{p} \mathrm{IR}$ beam. Also, the convergence of the $\mathbb{R}$ was varied to make the light beam as parallel as possible: the beam divergence was adjusted and the beam size monitored at few points along a $6 \mathrm{~m}$ long beam path, in a iterative process.

\section{ii) IR polarization control and axis definition}

After the telescope, the polarization of the $1064 \mathrm{~nm}$ beam was optimized with a $\lambda / 4$ waveplate for its transmission through a linear polarizer, this provides a maximum power in linear polarization state, and decouples the effect of adjustment of the YAG's harmonic generator crystal onto the polarization of the IR output. After this, the 1064 $\mathrm{nm}$ beam was aligned on the mixing axis with the aid of a $90^{\circ}$ glass prism mounted on a horizontal translation mount, to allow for fine adjusement of the $\mathbb{R}$ beam into the mixing axis. Two apertures installed along the $\mathbb{R}$ axis defined the mixing axis for $365 \mathrm{~nm}$ generation. After the $90^{\circ}$ turning prism, a $\lambda / 2$ waveplate was installed in the IR beam path to orient the linear polarization horizontally, as required for frequency mixing process in the KD'P.

\section{iii) Generation of tunable $365 \mathrm{~nm}$ radiation}

Sum-frequency mixing of $100 \mathrm{~mJ} / \mathrm{p}$ of $1064 \mathrm{~nm}$ light with $50 \mathrm{~mJ} / \mathrm{p}$ of tunable radiation around $555 \mathrm{~nm}$, in a KD'P crystal (Inrad, model M1) generates $15 \mathrm{~mJ} / \mathrm{p}$ of unable radiation around $365 \mathrm{~nm}$. A schematic layout of the probe beam generation emphasizes the representation of the optical components, as seen in figure 4.7. Each mirror and prism of the optical layout are mounted on a kinematic double-hinge mount to allow vertical and horizontal tilt adjustment of the light beam. 
To spatially overlap the two beams, a dichroic mirror^ reflects the $\sigma$-wave of the vertically polarized dye laser beam with an efficiency of more than $99.5 \%$, over a $50 \mathrm{~nm}$ range centred on the $555 \mathrm{~nm}$ wavelength; an additional antireflection coating, deposited on the second surface, permits $>95 \%$ transmission for the $\pi$-wave at $1064 \mathrm{~nm}$. Vertical spatial overlap of the two beams was achieved by using the internal telescope of the dye laser for the transverse adjustment, such that it intersects the dichroic mirror at the height of the mixing axis, set at 18 cm above the optical table. Horizontal spatial overlap of the IR with the $555 \mathrm{~nm}$ beam was provided by translation stages at the base of the kinematic mounts holding the dichroic mirror and the IR prism located at the beginning of the axis for the frequency mixing of the $\mathrm{IR}$ with the dye laser output.

Due to the large birefingence of the KD'P cystal, the spatial beam overlap of the IR and dye laser has to be done without the crystal in place. Once the two beams are collinear, the crystal is installed, and the sum frequency mixing process is optimized by tuning the tilt angle of the crystal with a motorized Autotracker ${ }^{\mathrm{TM}}$ system, a servocontrolled unit from INRAD. At the optimum tilt angle, 8-15 mJ/pulse of $365 \mathrm{~nm}$ UV radiation was produced, depending primarily on the divergence of the $\mathrm{IR}$ beam, the linewidth and divergence of the dye laser, and the quality of the overlap between the two laser beams. The optimum tilt angle for mixing depends on wavelength; the angle must be adjusted every time the wavelength of the dye laser beam is changed. This is done by the servo mechanism of the Autotracker ${ }^{\mathrm{TM}}$ system. The principle of operation of this device relies on the symmetry of the sum-mixing beam, and the way this beam comes into shape as the crystal is tuned in and out of the optimum angle. In practice, one has to "teach" the servo where to "look" for the optimum tilt, and the algorithm of the servo is devised to maintain the optimum tilt as the wavelength varies. The optimum angle is sought by using a dither of adjustable amplitude on the tilt angle, and then measuring its influence on both beam symmetry and power. Under proper operation, this device maintains better than $90 \%$ of the sum frequency signal adjusted initially. After the sum-mixing process, the 365, 555 and $1064 \mathrm{~nm}$ radiations are separated by a Pellin-Broca prism. The beam profile of the UV radiation was nearly Gaussian, with a FWHM diameter of $1.0 \mathrm{~mm}$.

\section{b) Generation of tunable radiation for VUV probe}

A four-wave-mixing (FWM) technique, called nonresonant third harmonic generation (THG) in phased-matched Kr.Ar gases ${ }^{3}$, was used to generate the tunable VUV radiation in the region of the hydrogen Lyman $\alpha$ transition, by frequency tripling the $365 \mathrm{~nm}$ radiation. This technique has been used in most optical detection scheme for the H-atoms." After comparing with other FWM techniques, THG was also found most appropriate to generate the coherent VUV probe beam. The optical cell and gas manifold to handle the Kr:Ar gas are illustrated in appendix C. Using $12 \mathrm{~mJ} / \mathrm{p}$ at $365 \mathrm{~nm}$ and a lens with a focal of $20 \mathrm{~cm}$, an optimum $\mathrm{Kr}$ :Ar mixture of $300: 785$

^ CVI Laser Corporation East. Putnam. CT. 06260. (203) 928-1928; Part \#: LWP(45)RS555/TP1064-PW1025C. 
mbars was used to generate VUV radiation around the Lyman $\alpha$ transition, within a tuning range sufficient to encompass the Doppler broadened lineshape of the fast H-atoms, as seen in figure 4.8. Appendix E discusses this technique in more detail.

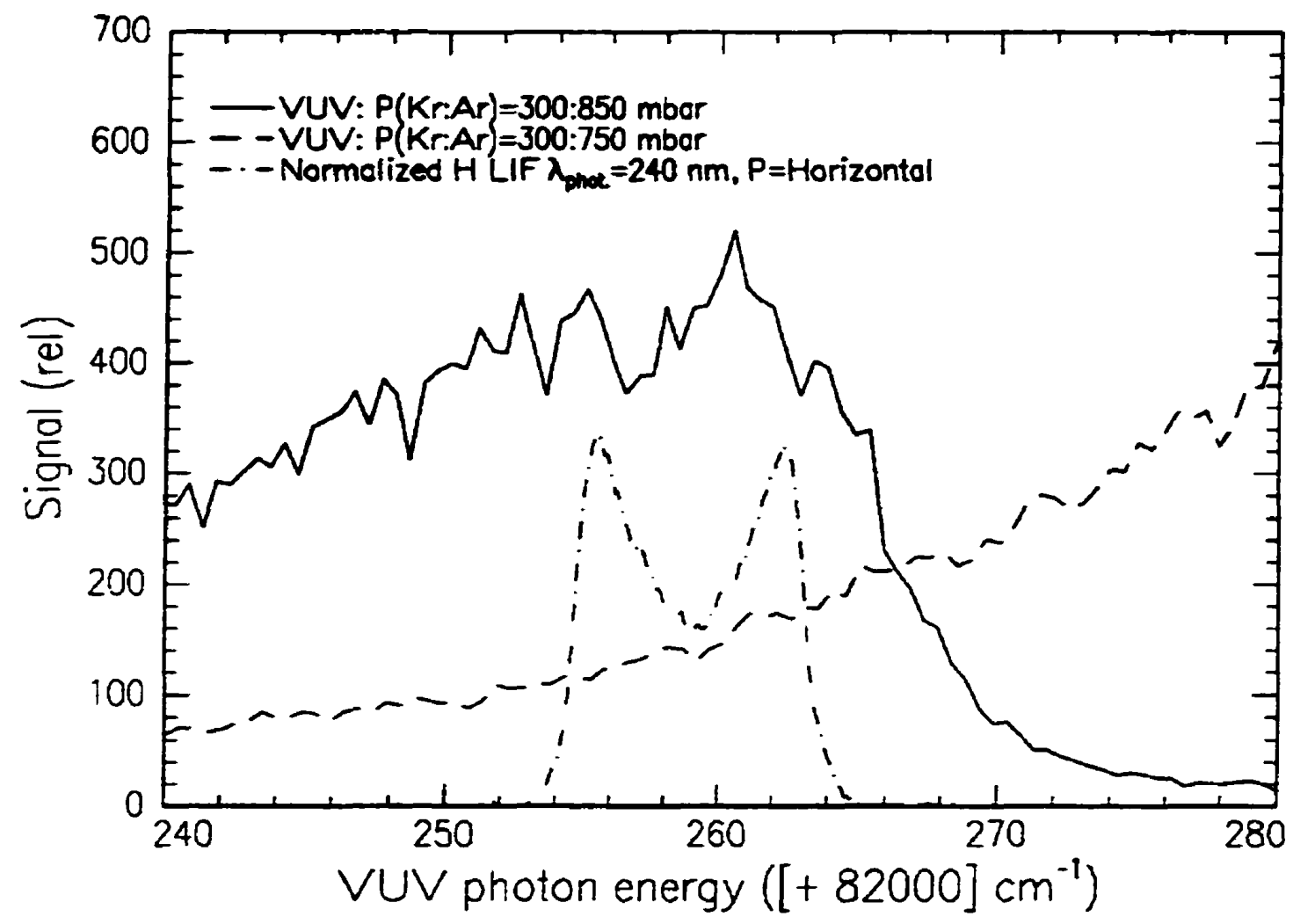

Figure 4.8 Tuning range of coherent VUV radiation by THG for a gase mixture of $\mathrm{Kr}: \mathrm{Ar}=300: 785 \mathrm{mbars}$.

\section{c) Polarization optics}

A polarizer was added to the $\mathbb{R}$ beam after the reducing telescope, as seen in figure 4.7. We must mention the subule rationale for this device. If the IR polarization varies, as a result of an adjustment of the YAG harmonic crystal, an elliptical state of $\mathbb{R}$ input polarization may translate into a frequency dependent $\mathrm{UV}$ polarization of the $365 \mathrm{~nm}$ laser beam. Because the resonance fluorescence is polarized ${ }^{6}$, and the photofragment fluorescence was detected only at a specific angle, the LIF detection was sensitive to relatively small changes of the probe polarization. Consequently, installing a polarizer in that position ensures that the $\mathbb{R}$ polarization will be constant. and it avoids the possibility of that inadvertent problem altogether.

\subsection{Wavelength callbration and linearity}

a) $I_{2} U F$ wavelength reference

A wavelength reference for the probe laser was obtained by recording an $\mathrm{I}_{2}$ LIF spectrum along with the data. lodine calibration spectra were measured on the tunable visible frequency that was mixed with the fixed $\mathbb{R}$ beam 
used to produce the tumable UV at $365 \mathrm{~nm}$. The wavelength reference spectrum was always recorded, along with the other signals of interest, using the $I_{2}$ LIF assembly illustrated in figures 4.7 and 4.2 . This measurement, in combination with tabulated wavelengths for the $I_{2}$ molecule, provided both a vacuum wavelength reference and a reference of linearity for the tuning mechanism, which was vital for the present study. The availability of the latter measurement was of crucial importance for the data reduction procedure, which is delineated in chapter 5.

\section{b) Monitor etalon reference}

Linearity measurements to characterize the wavelength tuning mechanism of the Lambda Physik FL2002E dye laser have also been acquired from the fringes produced with a monitor etalon included with this dye laser. Comparison with wavelength linearity measurement obtained from the $I_{2}$ LIF calibration confirmed the observation of a problem with nonlinear deviation in the wavelength tuning mechanism of this laser. This imperfection of the dye laser has been characterized, and as a result of its reproducibility, appropriate correction was possible. This problem and its characterization are described in appendix D. At this point, it should only be said that the two methods used to characterize linearity of uning mechanism provided very consistent information.

\subsection{Description of the apparatus II: electronic control and synchronization}

In this pump-probe experiment, there are three physical quantities that are measured during each pulsed experiment: the pump laser intensity, the probe laser intensity, and the laser induced fluorescence (LIF) from resonantly excited species in the experimental volume. The physical origins of these three signals are represented schematically in figure 4.9, an discussed below. After describing the detection of the signals and their amplification, this section proceeds with the description of the electronic synchronization between the various components of the experimental apparatus.

\subsubsection{Detectors and signal amplification}

a) Pump intensity signal

The pump is a coherent beam of UV, in the range of 200 to $300 \mathrm{~nm}$. Its is generated with a fluence in the range of 0.5 to $5 \mathrm{~mJ} /$ pulse, as measured before the entrance window of the experimental chamber, and after the steering and polarization optics. The absolute intensity of the beam was measured with a bimetal thermopile detector (Ophir, Model AN, detector head model 10A-P) during alignment and cptimization of the nonlinear optics devices used to generate the photolysis UV. However, the slow response time of the thermopile makes it impractical for measurement on each pulse, which is required for power normalization of the measurements. Thus the relative intensity for each pulse of the UV pump beam was measured with a pyroelectric detector (Molectron, P1-31) positioned after the exit window of the vacuum chamber, on the pump beam axis. A reflection of the UV beam from the surface of a quartz window was used to cut the intensity by a factor of 5-10 (depending on the angle 


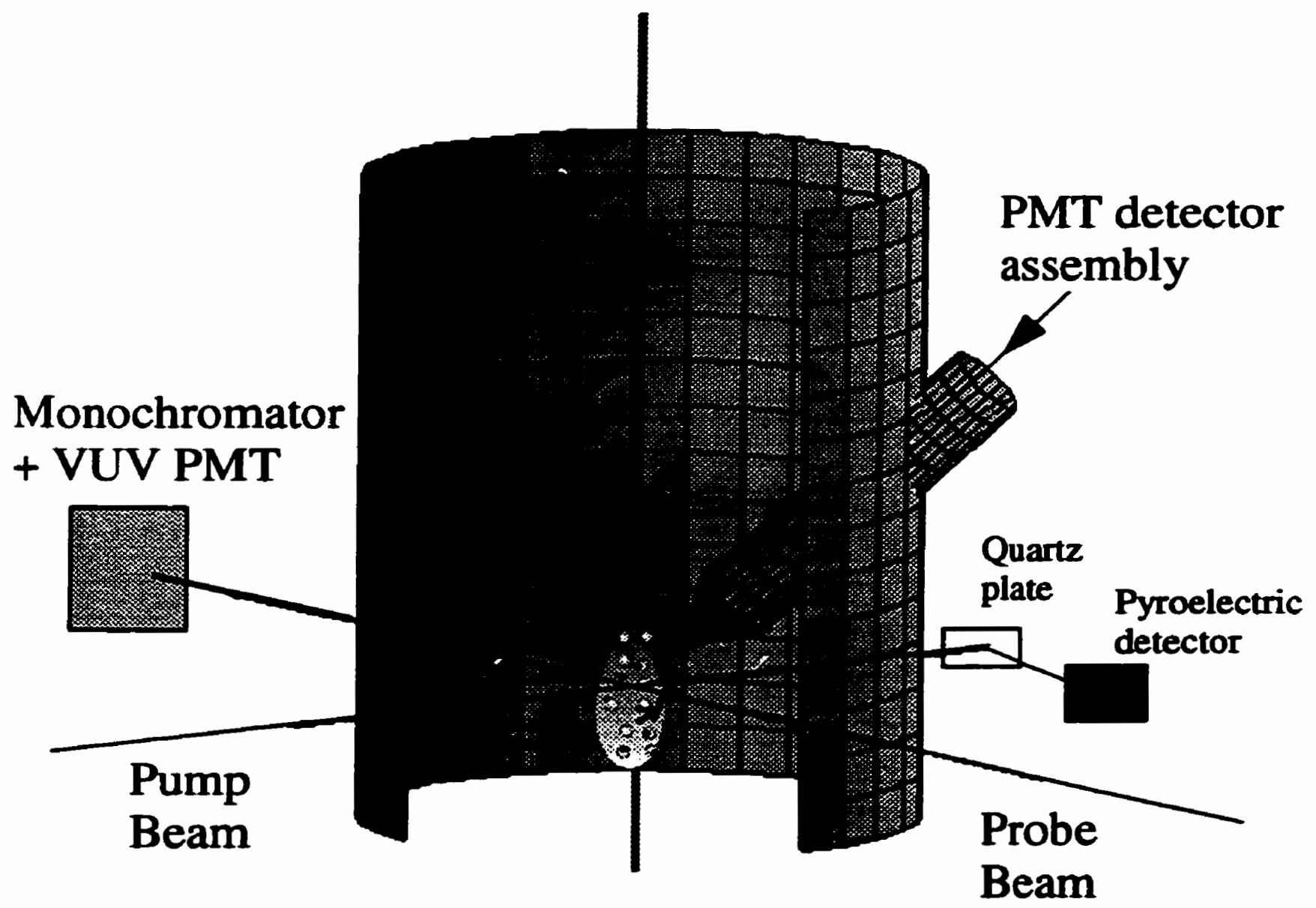

Figure 4.9. Vacuum chamber and sleeve of the PMT to pick up the LIF signal 
of reflection used), to avoid saturation of the detector, which occurs around $0.3 \mathrm{~mJ} / \mathrm{p}$ for our beam size. An integrated opamp within the PI-31 detector provides an output signal in the range of $100 \mathrm{mV}$ and a width of few microseconds, such that no further amplification was required prior to the input of the gated boxcar averager (Stanford Research Systems, SRS, model SR250). The output of the boxcar was connected to the AD converter.

\section{b) Probe intensity signal}

Because the VUV probe beam is generated by THG, the collinear UV radiation at the fundamental frequency must first be separated prior to VUV detoction. A 0.2 metre vacuum monochromator (Acton Research, model VM-502) was used to separate the $365 \mathrm{~nm}$ UV and $121 \mathrm{~nm}$ VUV radiation; the monochromator was evacuated with a mechanical pump through a liquid nitrogen trap to prevent oil backstream to the monochromator. The VUV beam exited the monochromator and was sent to a VUV PMT (Hamamatsu, model R1459)^. The signal output of the PMT was sent to the input of a fast preamplifier (Phillips Scientific, model 6954, B-10) having a gain of 7.47 for a 10 nanoseconds pulse for 50 ohms load. The output of the preamplifier was sent to the input of another Gated Integrator and boxcar averager (SRS, model SR250), to integrate the amplified pulsed signal, and to provide a DC output to the $A / D$ converter. Appendix $E$ discusses the linearity of PMTs and electronic preamplifiers. An electro-mechanical shutter was installed before the entrance window of the THG cell, to allow zeroing of the probe and LIF signals in a systematic fashion, by a software command at the beginning of each spectral measurement.

\section{c) LF signal from $H$-atoms photofragment}

The LIF radiation emiued from the species excited by the VUV probe beam was detected with a VUV PMT (EMR photoelectric) ${ }^{\text {; }}$; the PMT was inserted in the LIF detection sleeve illustrated in figure 4.9. The sleeve was evacuated with a mechanical pump through a liquid nitrogen trap. The LIF PMT was secured inside the sleeve via an insert machined out of Nylon, which also served as a bolder for an optical filter (Acton Research) ${ }^{c}$ installed in front of the PMT to block spurious optical signals. This filter was crucial for the detection of the H-atom LIF, by blocking scattered radiation from the pump beam, and discriminating out the red-shifted fluorescence resulting from absorption by accidentally resonant Rydberg states of $\mathrm{HI}$.

The sleeve holding the LIF PMT, illustrated in figure 4.9, is terminated by a conical "snout" which was instrumental in optimizing the Signal-to-Noise (S/N) ratio of the LF signal from H-atoms. The details of this snout, designed and machined by the author, are showed in figure 4.10. This shape was optimized for three

\footnotetext{
A Hamamatsu photomultiplier tube, model R1459, with a LiF window and a solar blind CsI photocathode of quantum efficiency: $16 \%$ at $121.6 \mathrm{~nm}, 13 \%$ at $147 \mathrm{~nm}, 1 \cdot 10^{-4} \%$ at $253.7 \mathrm{~nm}, 5 \cdot 10^{-2} \%$ at $365 \mathrm{~nm}$.

B EMR photoelectric, Scblumberger, model 541G-08-17, solar blind (CsI) photocathode and a LiF window.

C Acton Research. part no. 122-N-1D; 20 nm FWHM band-pass around 122 nm, peak transmission of 14\%.
} 


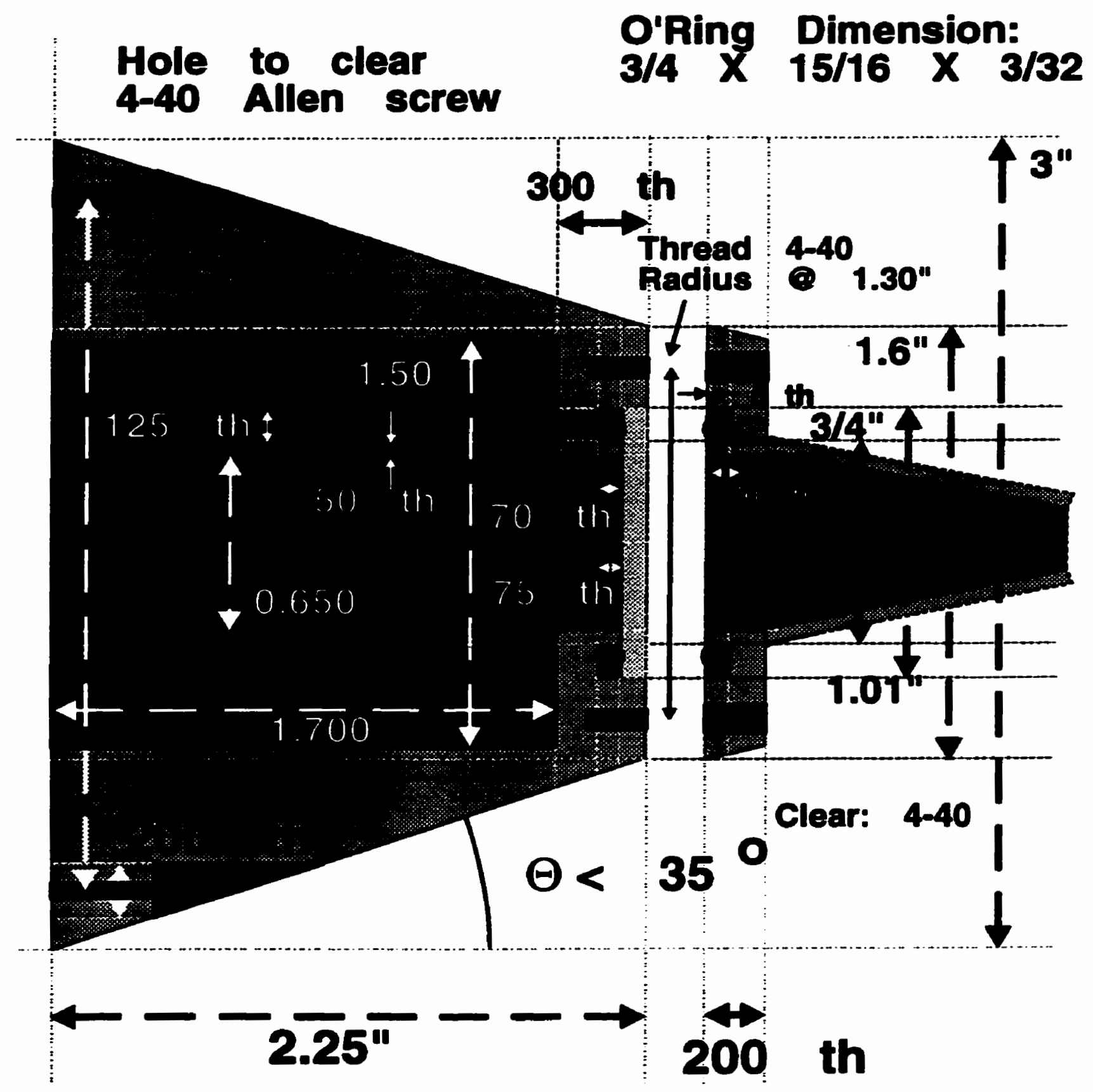

Figure 4.10. Snour of the UF pickup sleeve holding the PMT detoctor as close as passible to the experimental volume. The conic shape terminating the snout was optimized for the best SN LIF detection, by maximizing the collection of the fluorescence from the experimental volume, while minimizing the scattered light from reflective surfaces (windows, metal). 
purposes: 1) to bring the PMT as close as possible to the experimental volume while minimizing the pickup of spurious signal, 2) to hold an optical window transmitting VUV, and 3) to limit the field-of-view to the experimental volume. The flange terminating the snout was cushioned with an o-ring to carefully hold a $2 \mathrm{~mm}$ thick LiF VUV windown. A conic blinker, indicated in figure 4.10, was machined as a part of the flange, to block the intense near-specular back scattering from the probe exit window and other elastic VUV scattering processes, which is effectively a source of noise. The blinker reduced the field-of-view of the PMT to the experimental volume. The signal from the PMT was sent to a preamplifier ${ }^{8}$, whose output was fed to boxcar (SR250) and ADC channel.

\section{d) $L F$ signal from $I_{2}$ cell}

The numerous resonance lines of the iodine molecule enable the use of an iodine cell as a wavelength calibration reference for the dye laser 1 , which was tuned around $555 \mathrm{~nm}$. This device is based on a sealed silica cell containing $I_{2}$ vapour which was irradiated by the visible laser beam, after the $I R+$ dye frequency mixing process generating the UV beam at $365 \mathrm{~nm}$. The beam was attenuated by 2-3 orders of magnitude with a Neutral Density filter, prior to irradiating the iodine vapour. The cell was enclosed in a tube assembly with a spherical reflector to collect the fluorescence in one end, and a PMT at the other end. A pass-band filter, inserted between the PMT and the cell, was selected to anenuate the elastic scattering from the pump by at least 1000 , while transmitting as much as possible the fluorescence at slightly lower frequency. The signal due to fluorescence from resonant excitation could easily be made 5 times stronger than the one due to non-resonant elastic scattering, which was sufficient for our needs. No further amplification was required prior to boxcar gating and AND conversion.

\section{e) Monitor etalon insensity}

Another signal of interest was obtained by recording the interference fringes produced by sending the dye laser beam onto a monitor etalon. The arrangement used to execure this measurement used a reverse-biased PIN silicon photodiode (Motorola, MRD-510) behind an aperture whose size is set to be smaller than the fringe width at that particular location. The free spectral range (FSR) of the solid-state monitor etalon is $0.67 \mathrm{~cm}^{-1}$, and the typical bandwidth of the laser is $0.2 \mathrm{~cm}^{-1}$. The details related to this measurement, used to confirm results of linearity measurements obtained from the $\mathrm{I}_{2}$ cell, are described in appendix $F$.

\subsubsection{Electronic symchronization}

a) System requirements

There are three levels of synchronization: the pump-probe radiation-matter interaction and the consequent LIF

\footnotetext{
^ Engelhard/Harshaw Optical crystal. part no. 02502V; 1.0" dia $\times 2 \mathrm{~mm}$ Lithium Fluoride VUV window.

${ }^{8}$ Model IF $934 * 2$. designed and built by Ivan Frola, UW electronics shop; gain of $4.53 @ 400 \mathrm{MHz}$ in $50 \Omega$.
} 
signal, the pulsed valve opening and the kinetics of the gas, and the HV electronics for the YAG laser, i.e. capacitor charging and flashlamp excitation of the YAG rods. The electronic units that must be synchronized are: the pulsed valve driver, the YAG laser and the gated boxcar integrators, which were used to store the signal from the detectors, until they were digitized by the AD converter and stored in the RAM of the computer. The whole experimental apparatus is synchronized by a master clock provided by the AT computer.

A TSR (terminate and stay resident) routine was installed at the interrupt' 0x IC to set the timer 0 of the 8253 for an internal clock at $20 \mathrm{Hertz}$, which was used to generate a $10 \mathrm{Hertz}$ square wave at the bit 8 of the Parallel Input/Output interface (PI/O, described in the next section) of the AT computer running the acquisition and control software. The 10 Hertz square wave is sent to trigger a synchronized delay generator (SDG) unit that triggers the other electronic units.

\section{b) Description of the cycle of events}

The cycle of the synchronized events in the pulsed experiment is described in the following. The chain of events begins with rising edge of the square wave produced at the pin 8 of the PI/O, which triggers the SDG unit. A first (Charge) signal triggers the charging of the YAG capacitor banks; when the capacitors voltage reached a predefined level, an end-of-charge level is set to zero by the YAG control unit. Then the flashlamp discharge of the capacitor bank can be safely triggered by the Fire pulse. The flashlamp discharge pulse has an approximate duration of $100 \mu \mathrm{sec}$ FWHM.

The pulsed valve driver is then triggered. It takes a few hundreds of microseconds to mechanically open the valve, and few tens of microsecond for the gas to reach the experimental volume. An adjustable delay is set to have the gas pulse synchronized with the pump and probe laser at the experimental volume; the duration of the gas pulse is typically $200 \mu \mathrm{sec}$ FWHM. A Q-switch pulse is provided with an adjustable delay that permits to trigger lasing of the YAG cavity when energy starage is maximum in the rods. This is usually adjusted for maximum YAG laser power. Approximately 50 nanoseconds after triggering the Q-switch, a pulse of the photolysis pump hits the target in the experimental volume. Ten nanoseconds later, a pulsed probe interrogates the fragments produced; the delay between pump and probe pulse is defined by a difference of optical path.

Nearly simultaneous to the probe excitation, the fluorescence of the excited fragment is detected by the LIF PMT, while the pump and probe intensities are measured by the detectors described above. A PIN photodiode, excited by the scattering from the first optics on the table, is used to trigger the boxcars for each pulsed signal. Each

\footnotetext{
^ Delays Generator Unit, design and built by Jacek Szubra. UW Electronics Shop.
} 
boxcar output is connected to an AD channel. The computer software uses the falling edge of the $10 \mathrm{Hertz}$ clock to trigger the $\mathrm{AD}$ conversion and data storage.

\section{c) Timing requirements}

Every 100 millisecond, a sequence of electronic signals are used to trigger drivers at specific time, and a set of delays encompassing 8 orders of magnitude in time need to be generated. The stability of each electronic delays has a limit of relative precision of $10^{-4}$, as a consequence of the thermal stability of the resistors and capacitors components used in the RC loops of the monostable multivibrators integrated circuit (National Semiconductor, part no.: 74HC221A) used to build the SDG unit. Therefore, precision at shorter time scale relies on triggering on a light pulse of the system, by using a MRD-510 PIN photodiode, reversed biassed at 15 volts; a 0.1 nanosecond risetime is specified for this device when used in these conditions. The signal of a photodiode is used to trigger the gated boxcar integrator for the fast signals of the various detectors. The table 4-1 lists the units and the synchronization delays prescribing the required performance of the SDG unit.

Table 4.2 Specific requirements for the synchronized delays generator

\begin{tabular}{|l|l|l|l|l|l|}
\hline Units & triggering edge & Amplitude & Width & Min. delay & Max. delay \\
\hline Pulsed valve & rising & TTL & $1.7 \mathrm{msec}$ & $100 \mu \mathrm{sec}$ & $3.5 \mathrm{msec}$ \\
\hline Charge & rising & TTL & $2.2 \mathrm{msec}$ & N/A & N/A \\
\hline End-of-charge & sink (by laser) & TTL & N/A & N/A & N/A \\
\hline Fire & rising & TIL & $150 \mu \mathrm{sec}$ & $55 \mu \mathrm{sec}$ & $760 \mu \mathrm{sec}$ \\
\hline Q-switch & rising & $8-10$ Volts & $2 \pm 0.5 \mu \mathrm{sec}$ & $2.9 \mathrm{msec}$ & $6.5 \mathrm{msec}$ \\
\hline Extra & rising & TTL & $1.7 \mathrm{msec}$ & $100 \mu \mathrm{sec}$ & $3.5 \mathrm{msec}$ \\
\hline
\end{tabular}

\subsubsection{Computer and hardware interface}

a) Computer

The experimental setup was controlled by a PC, (Tatung TCS-7000) based on a 286 processor. Two interface cards were used for communication to and from the computer: a parallel port and an $A / D$ converter.

\section{b) Parallel inputoutput (PLO) card}

The parallel port was provided by a 32-line digital I/O subsystem (Data Translation Inc., model DT2817), to transmit data between the host processor and external devices.

C) Analog-to-digital converter (ADC) card

Digital conversion of the DC signal from the boxcar was accomplished by a 12-bits monolithic converter (Data Translation Inc., DT2814) capable of conversion at a rate of $25 \mathrm{kHz}$. 
4.3.4 LABSCAN: software for data acquisition and control

A general description of the software developed for data acquisition and control is outlined. LABSCAN has been developed in 'C' language, extending the template structure initially developed by Dr. Doug Milburn. The author is indebted to Doug for his generous helping tips and advice, at the beginning of LABSCAN development. The robustness of this software lies in its well-defined structure; it is designed in a modular fashion, for ease of use and upgrading. First, the various functions of the main menu are outlined. Then some finer details of the system are highlighted, and suggestions for improvement are mentioned for the benefit of an eventual upgrade developer.

\section{a) Mode of data acquisition: types of scan}

LABSCAN performed data acquisition against two types of variables: the time, in increments of laser shots (100 msec), or the wavelength of dye laser. Prior to doing wavelength scan, a wavelength initialization must be performed, after which the wavelength position is tracked in memory, and the "smooth drive" concept ensures consistency of repeated measurements. The time scale is refreshed for each time scan. Whenever a user initiates a given scan type, ditto functions suggest the parameters currently stored in the active register (RAM) of the computer, from either the last data acquisition, or last data read from the disk. This allows the convenience of template definitions for parameters setup.

\section{i) Grating scan and intracavity etalon scan}

Two types of wavelength scans can be performed: a normal grating scan, or an intracavity etalon (ICE) scan which takes advantage of the maximum possible resolution for the dye laser system. Typically, one can use a grating scan to measure a given spectrum at nomal resolution, then an ICE scan can be used to look at finer spectroscopic details; the former allows a resolution of $0.18-0.25 \mathrm{~cm}^{-1}$, while the latter offers a resolution of $0.06-0.08 \mathrm{~cm}^{-1}$. Grating scans only requires driving the stepper motor of the grating. On the other hand, an ICE scan also requires slaving the grating to the etalon motion, and the master-slave relationship has to be calibrated every time the intracavity etalon is installed or a new wavelength range is selected for measurement. A systematic procedure has been designed and implemented in LABSCAN to ease greatly the setup of ICE scans.

By design,' the tracking of the etalon-grating master-slave relationship should be maintained over a scanning range of approximately $4 \AA$ around $5550 \AA$. The consistency of the relationship is illustrated in figure 4.11 a), by comparing the same spectral measurement for a regular grating scan and for an ICE scan: proper tracking is obeyed over 1.5 A. However in $4.11 \mathrm{~b}$ ), a suspicious dramatic drop in the intensity of the ICE scan is observed around 5536 , in comparison to the regular grating scan. Detailed considerations showed that the cause of this imperfection in the grating etalon relationship is the nonlinear deviation of the tuning mechanism of the grating. as discussed in appendix D. Finally, it should be noted that data acquisition requires longer time at higher resolution; hence the photofragment spectroscopy data of this study were acquired with regular grating scans. 
a)

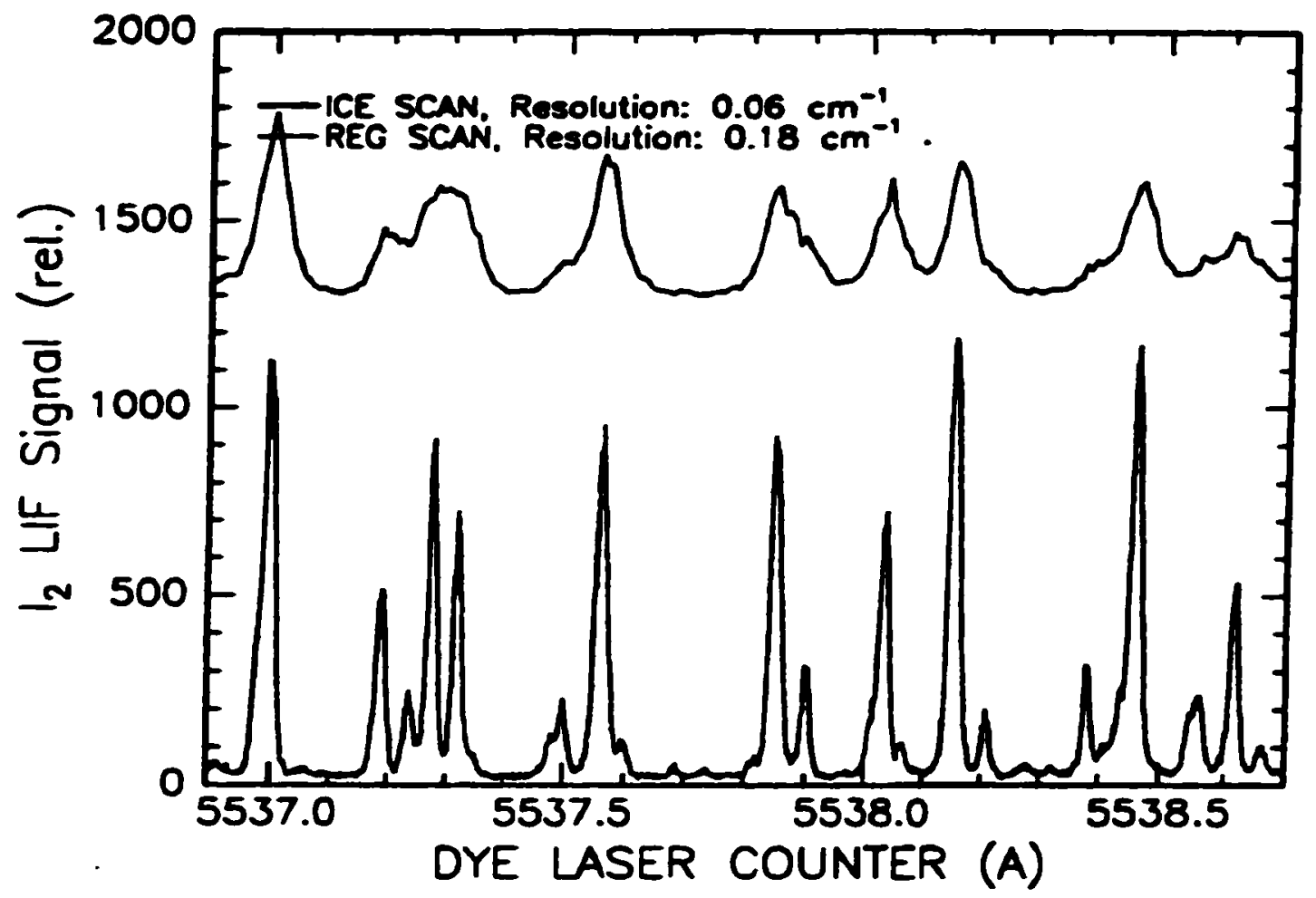

b)

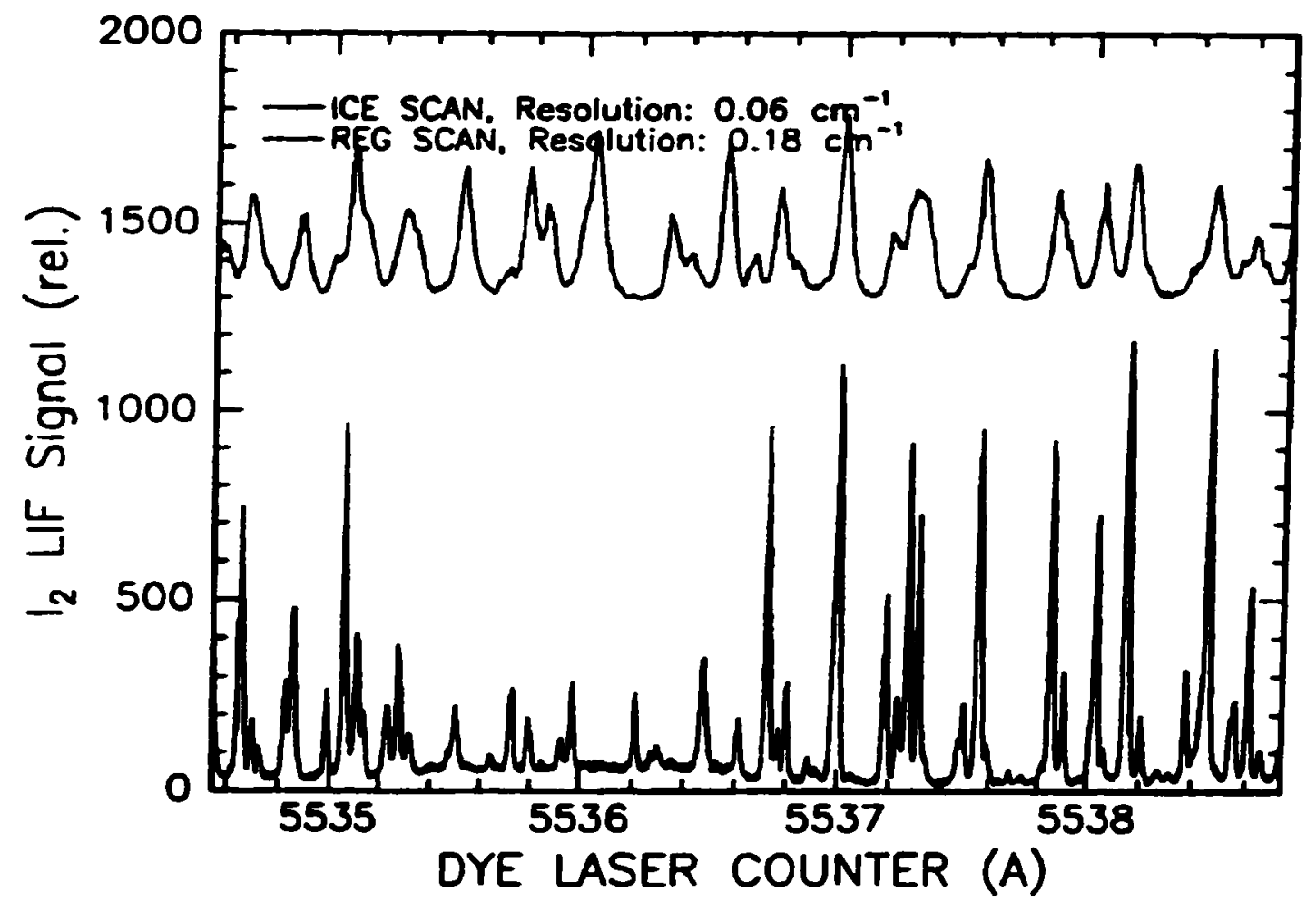

Figure 4.11. Ilustration of the ICE scan capability; in each graph. the higher curve is the ICE scan while the lower curve is the regular scan. In a) proper tracking is observed. while in b) a diminution of the relative intensity in the ICE scan around $5536 A$ resulted from a tracking problem. due to grating drive noalinearity. This has been characterized in appendix $D$. 


\section{b) Graphic Uuser interfaces (GUI): real-time and afterward}

Inspection of real-time data acquisition requires the ability to vary the magnification scales of the data for visual checking of the digital information being acquired. After acquisition, the requirements for display are different, as they are driven by the need of coarse analysis. For example, they are likely to require comparative overlap of data from different files and cursor operation. Additionally, capability for basic data manipulation is a needed tool for first hand data validation. As a results, two GUIs were designed and implemented in this program.

\section{c) Basic data manipulation capability}

As soon as experimental data have been acquired, some basic data manipulation must be done in order to evaluate the information obtained, and to appropriately assess the next course of action. To satisfy this requirement, basic operations are implemented in the data manipulation sub-menu of LABSCAN, such as pump and probe intensity normalization of the LIF signal and data averaging.

\section{d) Binary data format}

The format used to save the data file on disk is the same binary format used in GENPLOT ${ }^{10}$; it was originally defined by the authors of the Ryan-McFarland F77 compiler. This is a very robust format with respect to error checks, and it handles an unlimited number of blocks, each having a maximum size of 4 Kbytes.

\section{e) Peculiar properties of the overall system \\ (i) Effect of ineria on the grating drive}

As a result of the stringent requirement for wavelength resolution imposed by the Doppler spectrosacopy method. a great deal of care was paid to ensure repeatability of the spectral measurement. It has been observed that, when the dye laser grating was controlled by another previously written program, spectral measurement were not repeatable, within the precision of the laser resolution: the position of the spectral features were randomly, yet continuously, shifted on the wavelength axis, by three to six time the wavelength resolution. Figure 4.12 illustrates the problem: iodine LIF spectra were taken at different step size of the dye laser grating motion; the spectra measured with 1 motor step and 2 motor steps resolution did not coincide.

Detailed investigation of this problem revealed that it was caused by the software controller, which exceeded the maximum step rate specification for the stepper motor driver of the grating, and accelerated the tuning mechanism without consideration for mechanical inertia in the system. The general result from these considerations have resulted in the "Smooth Drive" algarithm for real-time control of the motor drive. This concept bas been included in the software control for the stepper motor driver of the grating. The effectiveness of the concept is clearly demonstrated by figure 4.13: the spectra measured are independent of the step size. Appendix D further discusses this concept, both in the specific context of this study, and in the general context of real-time control optimization. 

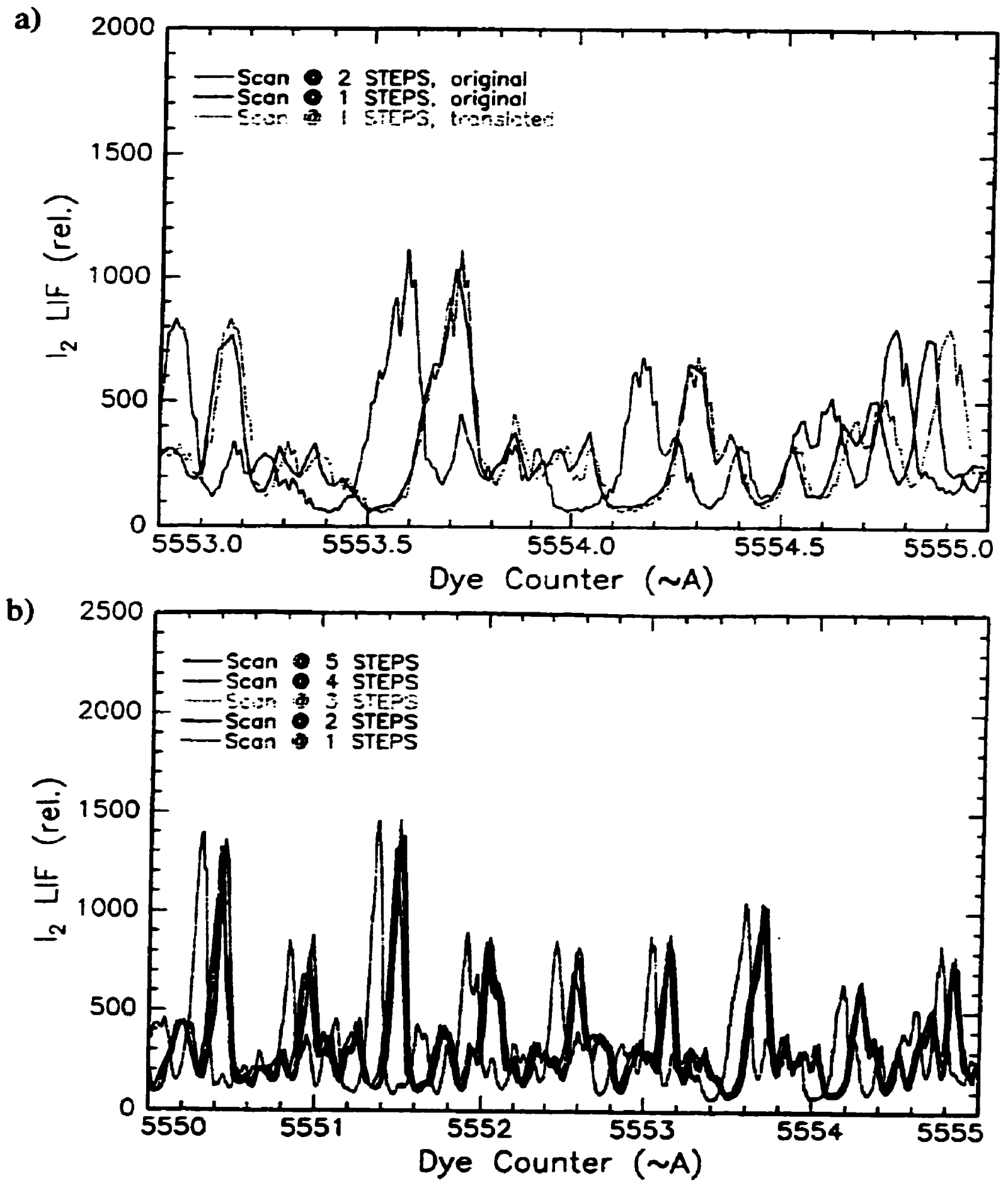

Figure 4.12. Measurement of $L_{2}$ LF spectra illustrating the problem solved by the "Smooth drive" algorithm. In a) a narrow range of wavelength shows that when the grating was stepped with one or two steps, the spectrum was shifted. In b) similar scans roquired with an increasing number of steps, shows that the difference is related to either moving one step, or any small number of numerous steps. Analysis of these results suggested the hypothesis of inertial limitation for the mechanical system 
a)

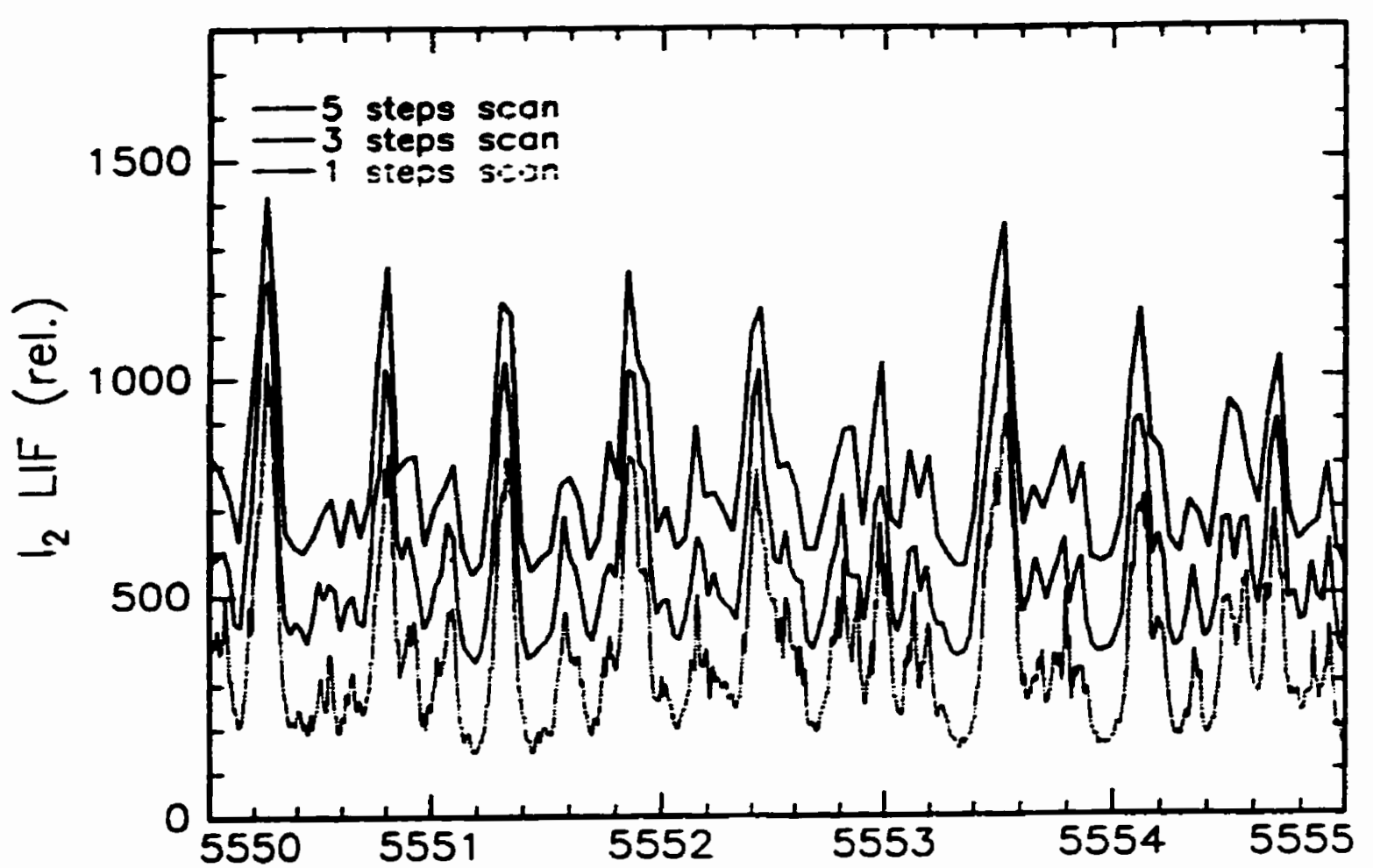

b)

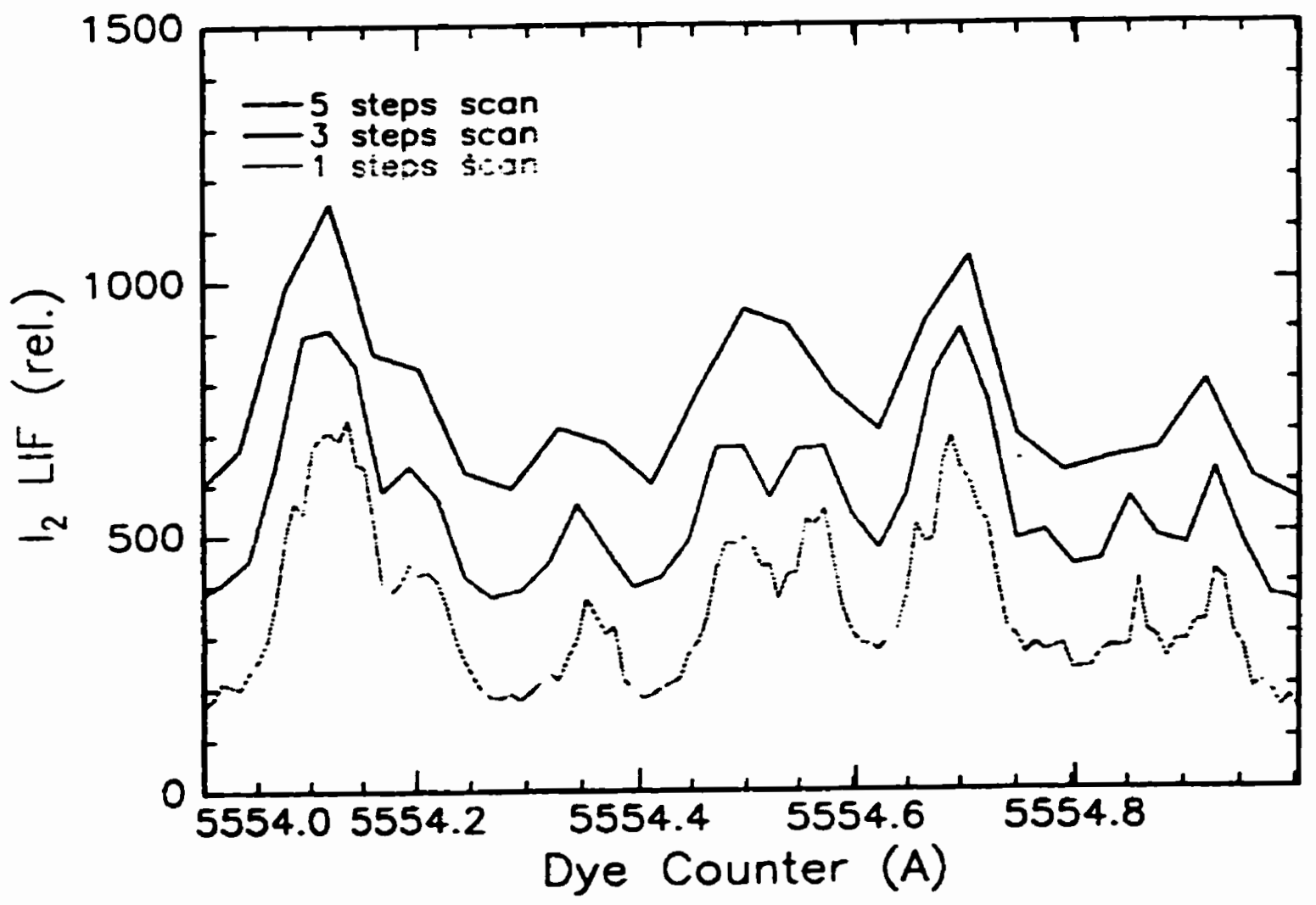

Figure 4.13. Similar measurement as in figure 4.12, but acquired after the implementation of the "Smookh Drive" driver. 


\section{(ii) ADD conversion: channel cross talk}

The data acquisition system used prior to LABSCAN was also plagued with cross-talk between ADDC channel; using a precise $D C$ source to test the $A D C$ card, it was observed that there was about 3-6\% cross talk between the input channels of the ANDC. It was found that proper handling of the status flags of the ADC card, and two to three additional successive read operations to the same channel prior to storing its value, solved this problem.

\section{f) Suggested improvements for eventual upgrade of the LABSCAN program}

In the following, the author suggested some small yet practical improvements that could be implemented in the LABSCAN program, with significant enhancement of the system for operation in a laser spectroscopy laboratory.

\section{(i) Automatic characterization of the grating drive nonlinearity}

In its present status, the program can store straightforward measurements of fringes from a monitor etalon. As it was discussed in appendix $D$, these measurements could be used to determine the wavelength nonlinearity, and this information could be stored in the computer memory, such as to allow for real-time correction of the nonlinear deviation. Recalling that this nonlinearity is reproducible, after this procedure has been completed, the grating could be scanned with a linearity better than its bandwidth without intracavity etalon, and consequently etalon tracking could be reliable over the designed bandwidth: $4 \AA$ @ $5550 \AA$. Implementation of these features would greatly enhance the capability and effectiveness of the dye laser as a laboratory instrument.

\section{(ii) Variable rate of time scan}

The time scan option could be made to store data point at a variable interval, so that temporal stability of experimental measurement could be assessed over few hours, or even days, without creating huge data files.

\section{(iii) Numerous $A$ DDC readout}

An assumption that is almost always made, when using an experimental system such as this one, is that the output level of the boxcar is a perfect DC level, which is proportional to the boxcar input. While this is mostly true, the presence of HV discharges in the YAG laser and high current discharge in the solenoid pulsed valve can introduce temporarily noise burst during each $100 \mathrm{msec}$ cycle between laser pulse. Also, it would be useful to have the option to measure the output signal of the boxcar at the maximum ADC rate, between two laser pulses, and to display the output of such a measurement for visual inspection.

If a time dependent noise structure is present, it could be then possible to select the optimum time window to collect the signal at the output of the boxcar, or to collect a greater number of reading cycles within the time available, in order to average out this hypothetical noise. 


\subsection{Experimental procedure}

The experimental procedure of this pump-probe experiment involved a considerable number of operations. To avoid overwhelming the reader with the complete details of the experiments, only few essential manipulations. that led to the central resuits presented in this work, are reported hereafter.

\subsubsection{Alighment of the axis: lasers and jet}

Prior to sending the pulsed laser beams into the experimental chamber, the axis of the pump and probe laser were aligned in such a way as to cross under the pulsed valve. The experimental volume is defined by the intersection of pump and probe laser beams. An additional constraint for the pump-probe alignment was for the location of the experimental volume be within the field of view of the LIF PMT detector. The pulsed valve had X-Y adjustment that permits motion in a plane parallel to the pump-probe plane, and which allowed one to optimize the overlap of the free jet with the experimental volume.

The prescription for this procedure is to permit the laser beams to travel through their respective set of optical ports, diametrically positioned on each side of the vacuum chamber. To achieve this goal in a systematic fashion, optical apertures were installed in the path of each optical axis, with the aid of two He-Ne lasers. The He-Ne beams, which were used to simulate the pump and probe beams, were used to install the apertures collinearly with the optical ports of the vacuum chamber, in such a way as to define the overlap of the pump and probe laser an optimum position. The optimization of the LIF detection was monitored, through the empty sleeve of the LIF PMT, by observing the light scattering from a needle tip installed at the intersection of two He-Ne beams. The location of the experimental volume was optimized when the needle's tip was centred in the field of view of the LIF PMT. Once the LIF detection was optimized, i.e. the He-Ne laser beams were defining the experimental volume at an optimal location for LIF detection, each axis position was located in space by placing two variable optical apertures (iris) centred on the He-Ne beam, and arranged on both side of the vacuum chamber.

\subsubsection{Alignment of dye lasers for nonlinear optics}

To achieve efficient processes of nonlinear optics, it is essential to pay special care to the quality of the input laser beam at the fundamental frequency. Thus, while proceeding with the alignment of the pulsed dye laser, a trade-off between maximum power on one hand, and optimum beam quality and narrowest linewidth on the other hand, must be made. It has been observed that, when the latter predominates, better nonlinear conversions are obtained. Good beam quality is beneficial for nonlinear optical processes both in gas phase, through a smaller spot size obtained at the focus and the consequently higher peak power, and in a crystal, where the divergence of the beams is critical due to sensitive frequency dependence of the optimum crystal tilt angle. The latter also defines the spread of the angular acceptance for that crystal. 
a) The beam profile is determined by the oscillator output; the laser linewidth was typically $0.17-0.22 \mathrm{~cm}^{-1}$.

b) In the preamplifier stage, the tilt and height of the cylindrical pump lens allowed for trade-off between power and linewidth. A perfect overlap of the pump beam with the optical axis of the preamplifier increased the propensity for broadband amplified spontaneous emission (ASE) feedback to the oscillator, which may compete with the narrowband wavelength selected in the oscillator cavity, this situation translates into degradation of both beam profile and linewideh, although it is likely to result in highest output power. Also, when a trade-off is solely made toward higher power, very poor conversion efficiency often results, in addition to increasing the propensity for the occarrence of self-focussing, which can be harmful to the laser system, and to the following devices in the optical train.

c) The final divergence of the dye laser beam results from the combined effect of the preamplifier beam expander telescope, and from the spatial inhomogeneity of the gain and refractive index of the amplifier medium; this homogeneity is related to line focussing of the amplifier pump used to couple pump to gain medium. The final laser beam divergence bas a determining influence on frequency doubling efficiency, as discussed above.

d) The telescope adjustment of the preamplifier beam also affects the laser beam profile and linewidth in an unexpected way: while adjusting the transverse degree of freedom of the telescope, in order to align the preamplifier beam onto the amplifier axis, the first element of the telescope (a diverging lens) can often be made to accidentally send a reflex of the broadband ASE from the preamplifier or oscillator gain region back into the oscillator, due to the finite angular acceptance of its axis. This can degrade linewidth and beam profile significantly or even completely, self-focussing in the amplifier may result from this inadvertent situation. This phenomenon can often be observed when relatively high output power is obtained from the dye laser, yet unusually small power can be produced from frequency doubling or mixing in crystal.

\subsubsection{Manipulation to find the signal from photofragment LIF}

The signal acquisition uses boxcar gating to produce DC levels, which are then easily read and stored in the computer. Initial gate adjusument for the 10-20 nanosecond pulse from the LIF signal of the photofragments can be tricky, because it depends on the overlap of the two laser beams with the free jet, on the laser frequency of the probe, and on the relative delay of the pulsed valve with respect to the laser pulses. Thus the spatial alignment of the pump and probe laser were based on the irises installed prior to optimization of the nonlinear optics processes for pump and probe generation. Then, wo adjust the timing gate for the PMT detection of the LIF signal, it is simpler to use a reference signal that does not depend on pump photolysis, yet has electronic transitions sufficiently close to the energy region of Lyman $\alpha$, such that it can be probed by the VUV generated by the same THG method; carbon monoxide (CO) was used for such a reference signal. 


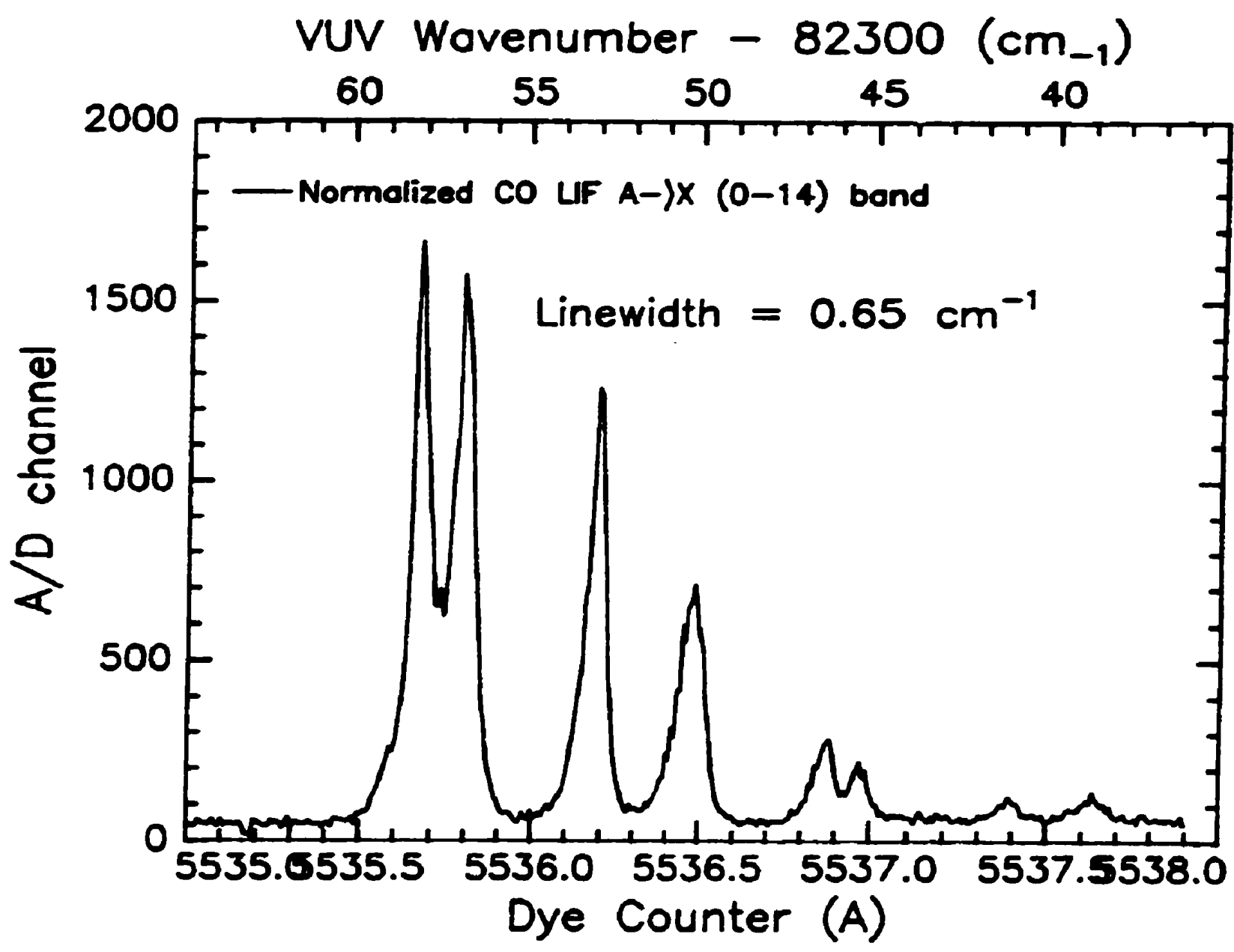

Figure 4.14. CO LF spectrum used to adjust the LIF detection electronic, measured the probe linewidth. and test the proper operation of the free supersonic jet.

The CO LIF reference signal has three additional uses. First, it permits the experimental assessment of the linewidth of the VUV probe, and second, to qualitatively ascertain that the supersonic jet expansion behaves properly, i.e. that a decent cooling of the rotational population is achieved. These last two points are illustrated in figure 4.14. The intensity of the rotational energy levels indicates a rotational temperature around $10{ }^{\circ} \mathrm{K}$, and the VUV probe linewidh is approximately $0.65 \mathrm{~cm}^{-2}$. The third use is described in the following subsection.

\subsubsection{Optimization of the injection laser frequency}

Another crucial aspect of the global experimental system was the stability of the injection laser (seed) that is used to precisely establish the frequency of the Nd:YAG laser (host); the system was illustrated in figure 4.5. The injection laser system^ is a monolithic, continuous wave (cw), Nd:YAG laser, pumped by a diode laser.

\footnotetext{
A Lightwave Electronics, model: $\mathbf{S 1 0 0}$
} 
The resonant frequency of the injection laser can be adjusted via the temperature of the YAG crystal, through both the thermal dependence of the electronic properties of Nd:YAG determining gain bandwidth, and the thermal expansion of the Nd:YAG material afiecting the modes of monolithic laser. A thermoelectric cooler/heater system controls the temperature of the injection YAG laser, so that it can be adjusted to match the host laser bandwidth; this defines the goal of the optimization for the combined injection and host systems, also referred to as masterslave relation between the systems.

The gain bandwidth of the Nd:YAG material is about $4 \mathrm{~cm}^{-1}$. The modes of the main (or iost) YAG laser are separated by $0.0083 \mathrm{~cm}^{-1}$. When the host laser is pumped by flashlamp, and Q-switched into lasing without seed injection, the distribution of lasing frequencies span about $1 \mathrm{~cm}^{-1}$ around the peak of the gain medium, as a result of the properties of the unstable resonator and the random nature of the centre frequency for each pulse. This is due to the stochastic nature of the initial competition between the mode of the host cavity excited into lasing by amplified spontaneous emission (ASE). A cavity built-up time (BUT) of 10 nanosecond, associated with lasing initiated by natural ASE, is observed following Q-switching.

The role of seed injection is to replace the natural ASE with a fixed, controllable, $\mathrm{cw}$, frequency reference. Hence pulse after pulse, the same frequency of electromagnetic radiation dominates the host oscillator. An additional consequence of proper laser injection is a dramatic BUT reduction; the BUT is all the more reduced for good overlap between injection beam and host cavity axis, and position of the seeder frequency at the centre of the host gain bandwidth.

The host gain bandwidth encompasses numerous modes of the host oscillator cavity. A piezoelectric element, on which is mounted the back mirror of the host oscillator cavity, allows for dithering the length of the host cavity by a minute amount, in principle smaller than the distance carresponding to the difference of wavelengths between two successive modes of the host oscillator cavity. Heterodyne signal detection, at the dither frequency, aims at minimizing the BUT of the host lasing. The feedback loop of the heterodyne circuit produces an analog signal driving the average position of the piezoelectric element, which eventually locks onto a specific mode of the host cavity, cptimum for the given injection laser frequency. However, the injection laser frequency must be adjusted, to match the centre of the gain bandwidth at the equilibrium temperature of the Nd:YAG in host oscillator cavity.

In order to temperature-tune the frequency of the injection laser to overlap the centre of the host gain bandwidth, a very effective spectroscopic method was found, which corresponds to the third use for the CO LIF reference signal mentioned above. The VUV frequency, used to probe the experimental volume, equals three times the UV frequency around $365 \mathrm{~nm}$. The UV frequency at $365 \mathrm{~nm}$ is the sum of the tunable dye frequency, and the fixed IR frequency of the YAG laser. Consequently, the bandwidth and centre frequency of the VUV is directly related 
a)

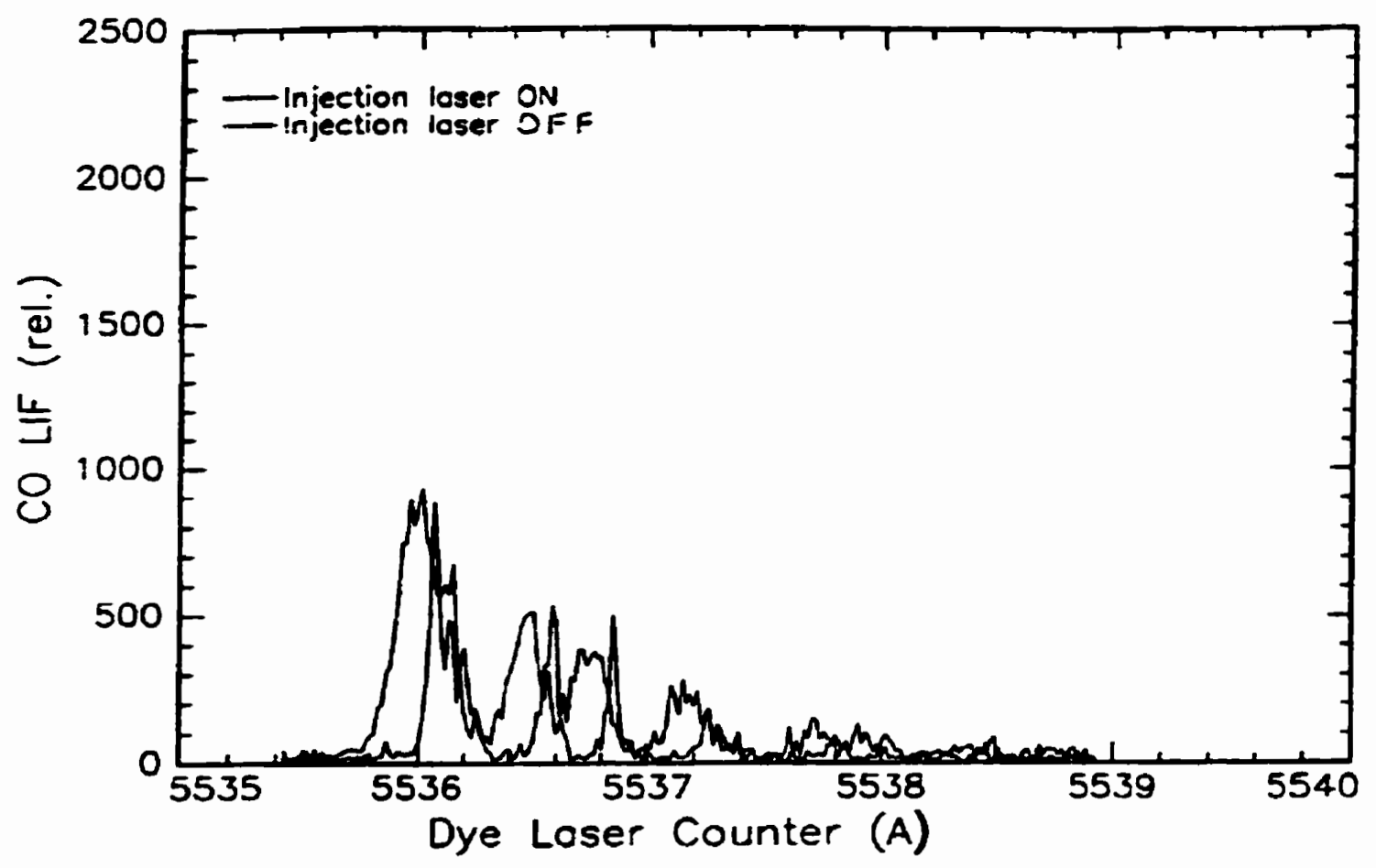

b)

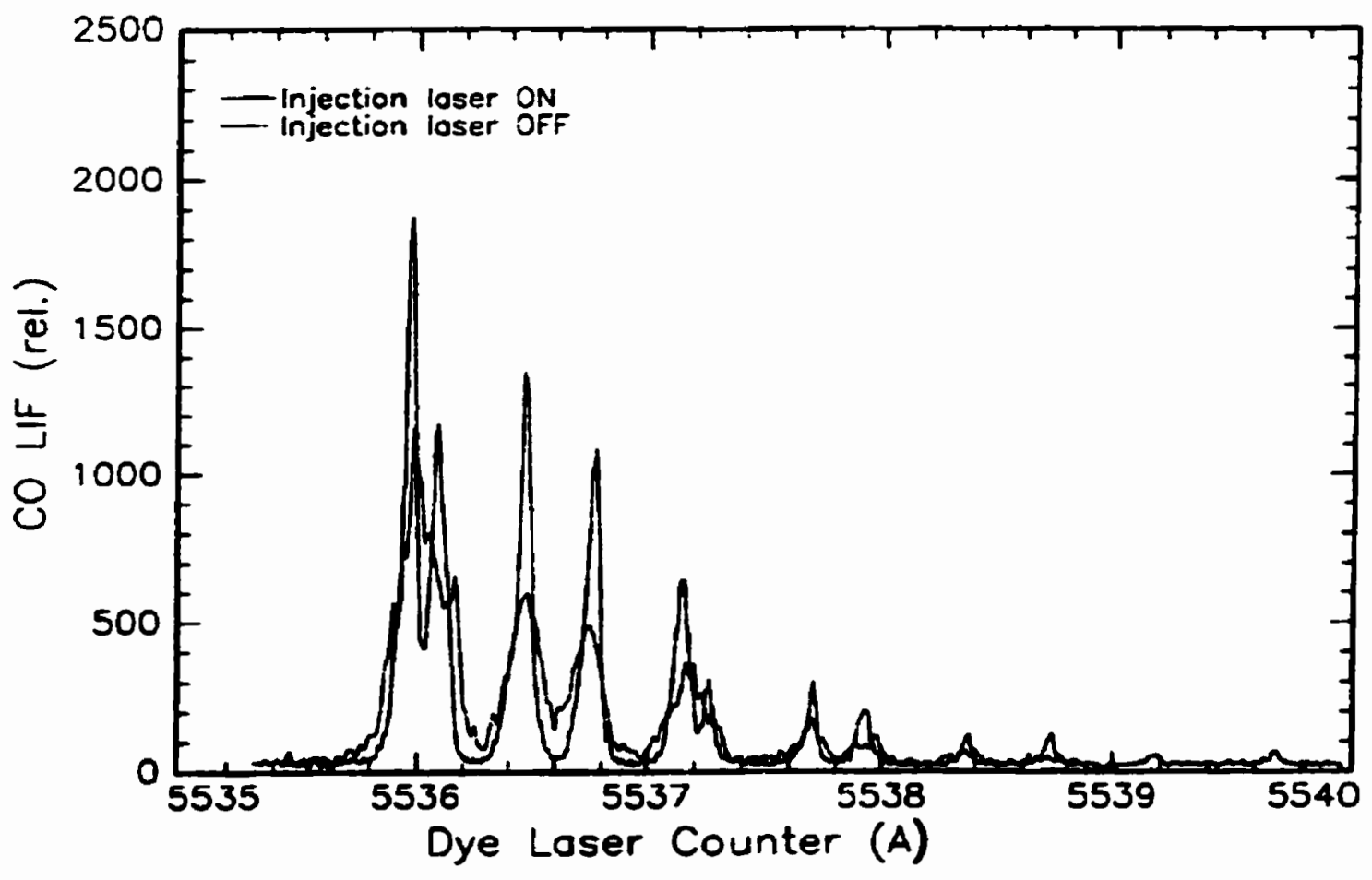

Figure 4.15. Impartanoe of matching the seeder frequency to the centre of the bost bandwidth. a) Seeder frequency off-centre: b) Seeder frequency matching the centre of the host gain bandwidth. 
to the bandwidth and centre frequency of the seed laser. If the seed laser is off-centre of the host gain bandwidth, very poor performance of the VUV bendwidth is observed, as seen in the figure 4.15 a). However, when the seed frequency is matched with the bost gain centre, an optimum improvement of the VUV bandwidth results, as shown by figure 4.15 b). Henceforth, through successive adjustment of the seed frequency, this spectroscopic method permits a precise frequency overlap for optimization of the master-slave relation between injection and host laser.

\subsubsection{On the Soleil-Babinet compensator}

The Soleil-Babinet compensator was described in 4.2 .3 b). It is a variable waveplate, that can be calibrated for any wavelength that has a reasonable transmission through pure crystalline quartz. This device is composed of two quartz wedges, made of left-handed and right-handed quartz, respectively. Basically, it relies on the circular dichroism property of quartz material for its retardation properties at a given wavelength. This device is a multiorder waveplate of adjustable retardation, and whose optical axis can be oriented with a divided circle rotating mount. These two variables, retardation and orientation, allows a completely arbitrary control of the state of polarization over the aforementioned range of wavelength.

Before using the retarder at any given wavelength, it must be calibrated, using a polarized source and an analyser, by the following procedure. First, the polarizer is installed on the axis of the polarized source, and it is aligned for extinction of the source. Then the compensator is installed between the polarized source and the analyser. Set the micrometer so that the digital display reads 4.00. The compensator is fine-tune to achieve optimum extinction, by careful rotation of the divided circle rotator. Lock the rotator release. The retarder axis is rotated by $\pm 45^{\circ}$ by releasing the "click stop" release and rotating the compensator to the $\pm 45^{\circ}$ detent. Now by slowly varying the micrometer of the variable retarder, and one can note the values at which total extinction of the source occurs; those are the values of retardance that corresponds to the successive $(n+1 / 2) \lambda$ waveplate. A linear regression can be used to obtain a relation between waveplate retardance and digital display of the retarder.

Because the exact angle of incidence of the beam onto the wedge determines the retardance of the compensator, the calibration must be executed each time the pump beam is realigned. Also, a slight deviation of the beam was observed when the compensator was introduced in the optical path. Therefore, the calibration of the retarder and the alignment of the pump axis had to be done a few successive times in a recursive fashion, the first time that the Soleil-Babinet compensator was introduced in the pump beam. 
A clear account of the theory of polarization optics and algebra ${ }^{12}$, is given in reference 12, (Polarized light: production and use); this book contains a good description of Stokes parameters and illustrates the use of the Poincarre sphere as a tool to solve problems with retarders and other polarization optics.

\section{References}

1. R. N. Zare, D. R. Herschbach, Proc. IEEE, 51, 173 (1963)

2. D. H. Levy, Laser Spectroscopy of Cold Gas-Phase Molecules, Ann. Rev. Phys. Chem., 31, 197 (1980), and D.H. Levy, L. Wharton, R. Smalley, Laser spectroscopy in Supersonic jets, in Chemical and Biochemical Applications of Lasers, Ed. by C.B. Moore. Academic press, Vol 2. p. 1 (1977).

3. H. Langer, H. Puell, H. Rohr, Optics Comm., 34, 137 (1980)

4.J. W. Hepburn, Generation of Coherent Vacuum Ultraviolet radiation : Application to high resolution photoionization and photoelectron spectroscopy, in Laser Techniques in Chemistry, A. Myers and T.R. Rizzo, (1994), Wiley. J. W. Hepburn, Israel J. Chem., 24, 273 (1984).

5. R. Wallenstein, Optics Comm., 33, 119 (1980)

6. G. Moruzzi, F. Strumia, The Hanle Effect and Level-Crossing Spectroscopy, Plenum Press, New York (1991)

7. See the interrupt vector assignment of the IBM PC, in Murray Sargent III and Richard L. Shoemaker, The IBM Personal Computer Inside Out, Addison-Wesley (1984)., p.65.

8. See appendix $\mathbf{H}$.

9. Lambda Physics Dye laser FL 2002E Operation Manual.

10. GENPLOT manual, Computer Graphic Service, 52 Genung Circle, Ithaca, NY, 14850-8716, (607) 277-4913

11.W. A. Shurcliff, Polarized light: production and use, Harvard University Press, Cambridge, (1962) 


\section{Chapter 5 Experimental measurements and data reduction procedure}

The main body of spectral measurements presented in this thesis was acquired to determine the electronic structure of molecular $H I$ in the energy range of the low-lying dissociative states excited by absorption in the $A$ band. Section 5.1 defines the ranges of the experimental variables at which the Doppler spectra were acquired for this purpose. Section 5.2 elaborates on the data reduction procedure, which must be operated on the measurements prior to extraction of meaningful information.

\subsection{Photofragment spectroscopy on $\mathrm{HI}$ photodissociation}

The purpose of these measurements was to elucidate the dynamics of HI photodissociation. The components of the data set are identified by specific values for the wavelength and polarization states of the photolysis pump beam. The experimental apparatus allowed Doppler spectroscopy measurements to be performed with the relative alignment of the pump-probe axis in orthogonal geometry. In chapter 6, the analysis of these Doppler spectra will allow definite assertions to be made with regards to the dynamics of HI photodissociation.

In table 5.1, the photolysis wavelengths and the polarization states are listed along with the statistical signal-tonoise $(S / N)$ ratios that have been attained through the procedure of data reduction and averaging of successive Doppler spectnum measurements. The statistical SN ratio of a given Doppler spectrum is calculated on the power normalized spectrum, in term of the relative fluctuation with respect to the average signal, and this quantity is averaged over the spectral lineshape. Note also that measurements were acquired for polarized photolysis laser in both vertical (V) and horizontal (H) states of linear polarization, at each photolysis wavelength; here vertical and horizontal is defined with respect to the horizontal plane of the intersecting pump-probe axis.

Table 5.1 List of the main data set used in chapter 6

\begin{tabular}{|l|c|c|c|c|c|c|c|c|c|}
\hline$\lambda(\mathrm{nm})$ & 266.0 & 257.0 & 248.0 & 240.0 & 232.5 & 225.0 & 216.7 & 215.0 & 212.5 \\
\hline S/N (V) & 18 & 22 & 14 & 28 & 21 & 29 & 25 & 6 & 21 \\
\hline S/N (H) & 18 & 21 & 16 & 26 & 20 & 31 & 28 & 3 & 22 \\
\hline
\end{tabular}

\subsection{Data reduction procedure}

Meticulous care was taken to reduce the data in a systematic manner. The procedure to do so involves: power nomalization of the LIF signal with respect to the power of pump and probe beams, correction for the nonlinearity of the tunable wavelength, statistical averaging and integral normalization for each power-normalized $H$ LIF Doppler spectrum, and finally, subtraction of the HI LIF background. 


\subsubsection{Power normalization}

The experimental LIF signal from the $H$-atoms, $S_{\text {exp }}(v)$ is given by equation (5-1). It is a product of HI molecular density, pump and probe intensity (propartional to the power measured for these beams), and the convolution of $D(v)$, the Doppler lineshape function, with $g(v)$, the probe lineshape function. The Doppler lineshape function. and its dependence on the relative orientation between the probe axis and the pump polarization vector, contains the information on the photodissociation dynamics. Also, the power normalized Doppler profiles, $S_{\text {bomm }}(v)$, to be obtained from experimental measurements, correspond to the integral term in the curly bracket.

$S_{\exp }(v)=I_{\text {pump }} I_{\text {probe }} n_{\text {HI }}\left\{\int_{\Delta v^{\prime}} d v^{\prime} D\left(v^{\prime}\right) g\left(v-v^{\prime}\right)\right\}=I_{\text {pump }} I_{\text {probe }} n_{\text {HI }} S_{\text {norm }}(v)$

Consequently, the first step in the procedure of data reduction is the shot-to-shot power-normalization of each $\mathrm{H}$ atom Doppler spectrum by the corresponding pump and probe intensity signals. Note that the number density of $\mathrm{HI}$ in each pulse was not measured, and it is assumed constant. The stability of the HI number density has been assessed separately, and its fluctuations will be considered as an additional source of noise in this experiment. Also a great deal of effort was invested in constructing a fairly stable HI source for this study (see appendix A).

Due to the finite dynamic range of detectors and their electronic amplification, a validation check for the range of each signal was performed prior to power normalization. Precise characterization (see appendix E) of electronic gain and saturation limits was performed for the detectors and their preamplifiers, which was necessary to automate validation checks on the vast amount of data processed in this study. For each set of spectra acquired under identical conditions, the detection setup information (PMT bias, identity of preamp used) were input to the normalization program, and the user was notified if any recorded signal accidentally exceeded its respective range of validity during data acquisition. This allowed selection of valid data, and rejection of data affected by the pernicious effects of signal saturation', which may not be obvious in an individual spectrum, due to their finite signal-to-noise ratio. Once the individual signal intensities were validated, the shot-to-shot normalization was operated, to yield the power-normalized LIF signal of the H-atoms, i.e. the experimental Dopplet profile.

An additional test was performed periodically on a few sample spectra, in order to determine the proper behaviour of the normalization procedure, and to further ensure linearity of the overall measurement. The premises of the test were as follows: if the data normalization behaves properly, both the short term and long term fuctuations of the LIF signal should be correlated to the product of the VUV probe and UV pump fluctuations. This implies that the normal statistical fluctuation for these signals must be correlated. Consequently the power-normalized LIF signal, i.e. the ratio of the LIF signal divided by the signals from VUV and UV detectors, must exhibit smaller relative fluctuations. 
To verify this assumption, the normal fluctuations were quantified for each signal, and the inequality 5-2 was tested. Equation 5-2 simply states that the normal fluctuations, $\sigma_{N}$, of the power-normalized signal, $S_{\text {nomm }}(v)$, must be less than the normal fluctuations, $\sigma_{\mathrm{Lr}}$ of the experimental LIF signal, $S_{\text {exp }}(v)$. The symbol $\sigma_{\text {ov }}$ represents the

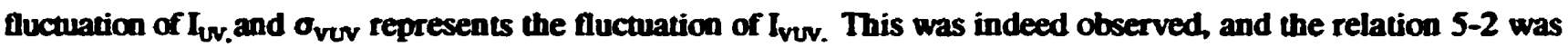
confirmed. The equality limit would be reached for ideal noiseless electronics and perfectly repeatable pulses of HI:He gas mixture, where the fluctuations would only be caused by absorption and fluorescence of radiation.

$\sigma_{\mathrm{N}}^{2} \leq \sigma_{\mathrm{LIF}}^{2}-\left(\sigma_{\mathrm{VUV}}^{2}+\sigma_{\mathrm{UV}}^{2}\right)(5-2)$

It is interesting to note the dependence of the LIF signal fluctuations on those of the YAG laser. Equations (5-3) shows that because the VUV probe depends on the $9^{\text {ta }}$ power of the fundamental IR of the YAG laser, and the UV pump depends on the $6^{\text {ma }}$ power of this fundamental $\mathbb{R}$, the normalized fluctuations of the LIF signal can actually be up to 15 times larger than those of the intensity of the YAG fundamental IR frequency, as a result of equation (5-1). Recalling that the amplitude of the fluctuation reduces the effective dynamic range of the electronic amplification, this fact provides an independent rationale to use seed injection during operation of the YAG laser, since it greatly reduces the shot-ko-shot fluctuations for the intensity of the fundamental IR frequency of the YAG laser output.

$$
\begin{aligned}
\sigma_{\mathrm{LIF}}^{2} & =A+\sigma_{U V}^{2}+\sigma_{\mathrm{VUV}}^{2}=A+2 \cdot \sigma_{\mathrm{IH}}^{2}+3 \cdot\left(\sigma_{\mathrm{SH}}^{2}+\sigma_{\mathrm{IR}}^{2}\right) \\
& =A+15 \cdot \sigma_{\mathbb{R}}^{2}
\end{aligned}
$$

\subsubsection{Wavelength correction}

In order to ensure the reliability of the information obtained from spectroscopic measurements, three operations were performed: linearization of the tuning drive, calibration with a spectroscopic reference, and verification check on repeatability. As it will be appreciated below, racking of the dye laser wavelength was required to characterize the nonlinearity ${ }^{2}$ of the tuning mechanism, which was crucial for the success of this experimental study. A LIF spectrum from iodine molecules was systematically acquired, during data acquisition of the Doppler profile, for the visible wavelength region corresponding. through the frequency mixing processes, to the H-atom VUV LIF spectra. In addition to permitting a correction for the noalinearity of the dye laser tuning wavelength, this signal provided an absolute vacuum wavelength calibration.

An iodine LIF reference spectrum was acquired and stored along with each H-atom LIF spectrum. Using the correction procedure described in appendix $D$, the experimental $I_{2}$ LIF spectrum was compared with a reference spectrum from tabulated lines, to obtain the correction for the nonlinearity of the tuning drive. The importance on this nonlinearity is illustrated by figure 5.1 a). 
a)

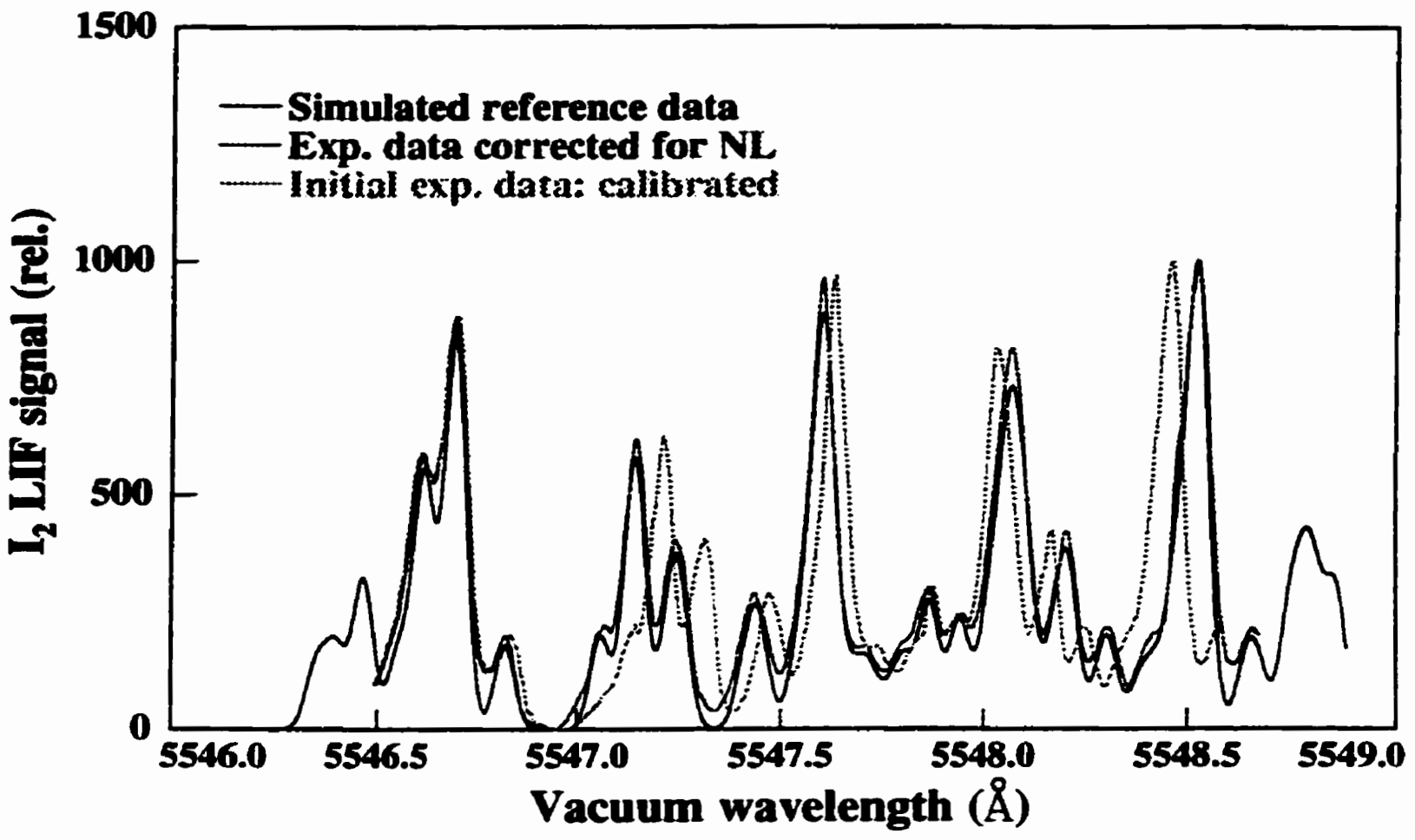

b)

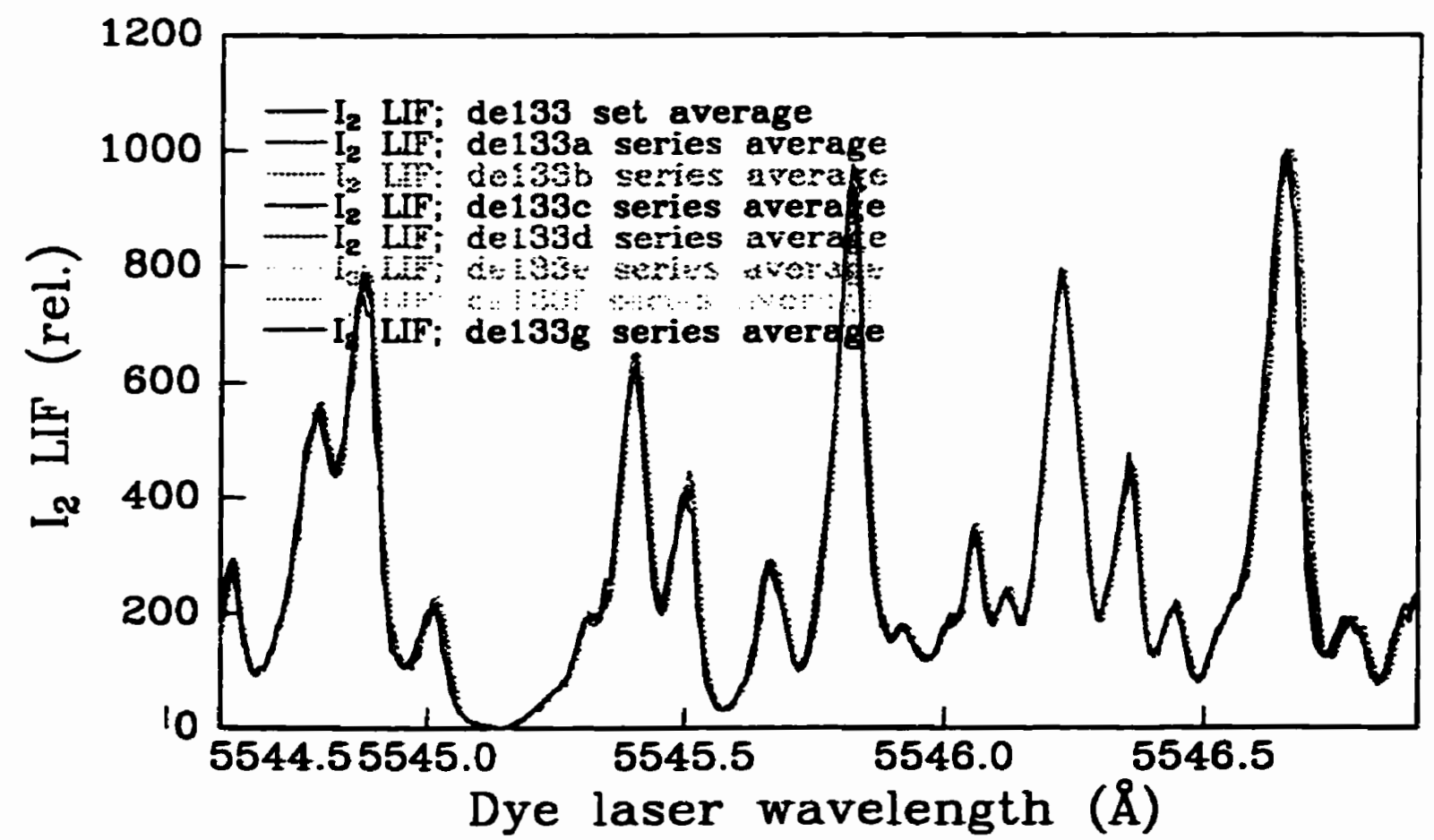

Figure 5.1. a) I/ LIF spectra used to characterized the nonlinearity of the tuning drive of the dye laser. b) illustration of the stability of the tuning drive for consecutive scan of Doppler profiles during 6 hours. 
However, the reproducibility of the wavelength tuning, demonstrated by the figure $5.1 \mathrm{~b}$ ), permitted us to process each series of Doppler spectra in batch during the data reduction procedure. After a visual inspection for repeatability of the wavelength tuning, the nonlinearity was characterized and corrected for, and finally the linearized wavelength axis was calibrated with respect to the vacuum wavelength reference afforded by the tabulated spectral lines ${ }^{3}$ of $I_{2}$ molecules.

\subsubsection{Statistical data averaging}

The nature of statistical fluctuation is such that the signal-to-noise (S/N) ratio squared is proportional to $n$, the number of equivalent samples, i.e. $n \propto(\mathrm{S} / \mathrm{N})^{2}$. Consequently, it takes typically four times as many samples to improve the $S / N$ by a factor of two. This intrinsic property of statistics was taken into account in our data averaging procedure, by using a statistical weight, defined as $\{\mathrm{S} / \mathrm{N}\}^{2}$, consistently for each power normalized Doppler spectrum, where $[S / N\}$ is calculated as the average $S / N$, at one standard deviation, over the power normalized Doppler spectrum. Once the statistical weight was calculated for a power-normalized Doppler spectrum, it was integral-normalized to have an area proportional to the $\{\mathrm{S} / \mathrm{N}\}^{2}$, consistent with the above statistical argument. The addition of integral-normalized spectra, acquired in equivalent experimental conditions, is then statistically correct: in other words, the statistical averaging, defined in this way, made the wisest use of the information contained in the individual spectra, by weighting them in proportion to their respective information content.

\subsubsection{HI LIF background subtraction}

Ideally, the LIF detector should only pick up the fluorescence from the photofragments excited by the probe. In practice, the HI molecule also has resonances in the same energy region. Although a Lyman $\alpha$ filter reduces the LIF detection sensitivity for this spurious signal, a fraction of the HI molecules produce resonance fluorescence that must be subtracted. However, this resonant HI scattering must first be characterized, prior to being subtracted from the H-atom LIF. The characterization of the HI LIF was made through the Lyman $\alpha$ filter. For each experimental run, the HI LIF was measured by scanning the wavelength range used for H-atom LIF, but without the photolysis laser being present. Figure 5.2 shows the shape of the background function obtained by averaging a few spectra, and also after Fourier filtering the noise out; note that the HI LIF spectra obtained without the Lyman $\alpha$ filter was about 20 times stronger, and it shares similar features but it has different relative intensities. Fortunately, the LIF of HI around Lyman $\alpha$ has two spectral features lying outside the range of Doppler broadened H-atom absorption, around $-7.5 \mathrm{~cm}^{-1}$ and $8.0 \mathrm{~cm}^{-1}$ respectively. These features were used to scale the HI LIF background function, in order to assess the contribution of the HI LIF background "hidden" in the H-atom LIF signal. The suberaction of the HI LIF background was only executed once at the end of the data averaging procedure, for each combination of wavelength and polarization state on the final component of each set of spectra. 


\section{HI background on the LIF detector through Lyman $\alpha$ filter}

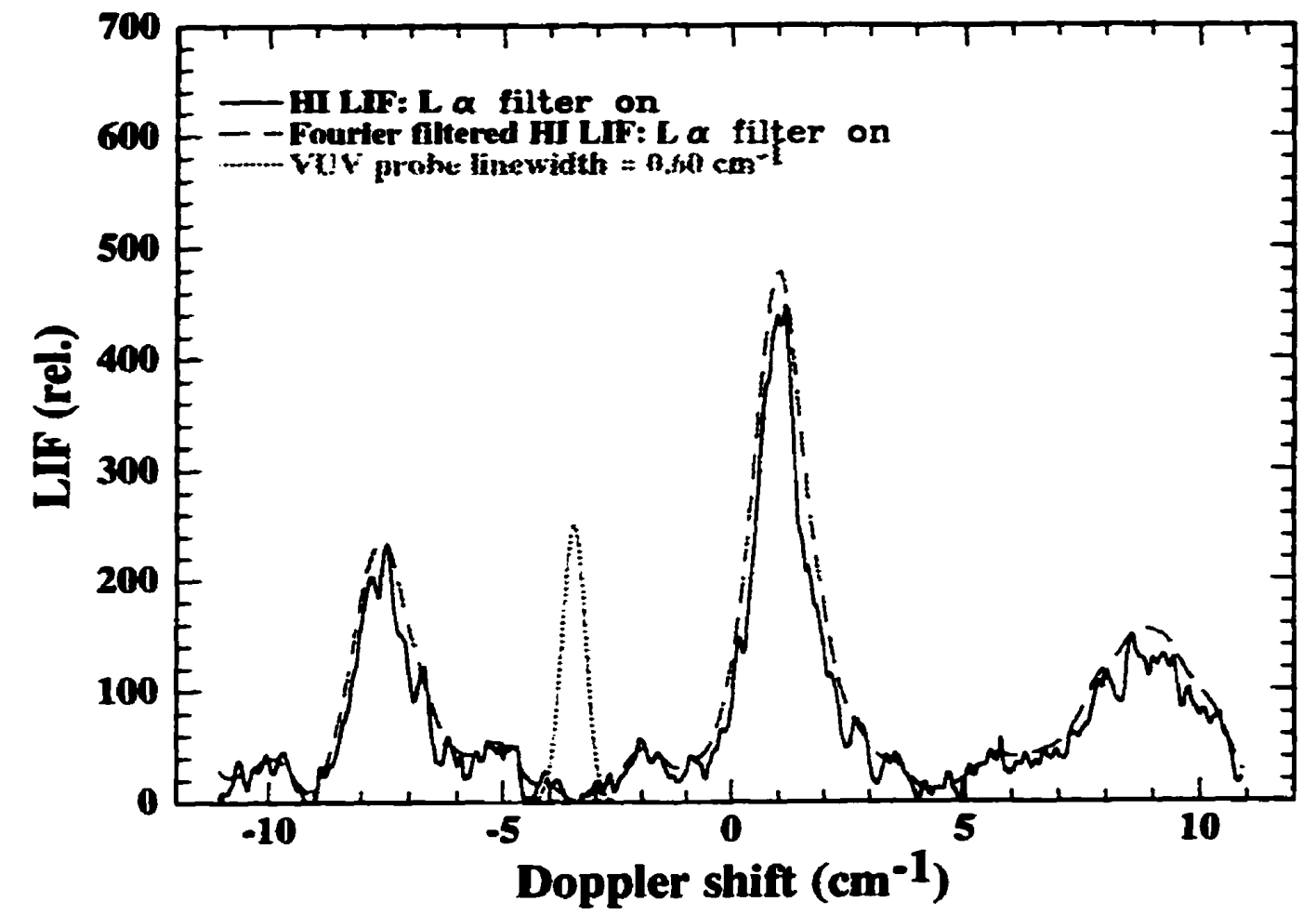

Figure 5.2. Determination of a normalized HI UF background function. The average of few power normaliazed HI LIF spectra is Fourier filtered for structure at higher frequency that can be detected by the smallest probe linewidth. i.e. $0.6 \mathrm{~cm}^{-1}$.

\subsubsection{Illustration of the systematic procedure of data reduction}

To apply this procedure systematically, a program was written in the macto command language of GENPLOT^, in order to monitor and control the quality of the data. Graphical outputs were generated at the end of each step of the process, for the sake of transparency and for direct visualization of results, which was especially useful during the development stage of the procedure.

An example of the data averaging and subtraction of the HI LIF background is illustrated in the figure 5.3 and 5.4, for a set of Doppler spectra acquired with vertical polarization of the photolysis beam at $240 \mathrm{~nm}$ wavelength. In figure 5.3, integral normalized Doppler profiles from a series of measurements were statistically averaged. Each member of the series were statistically weighted on the basis of the experimental fluctuations, (S/N ratio); after averaging the numerous points acquired at a given value of the probe frequency, the spectrum was area normalized in proportion to the square of the experimental SNN ratio used to quantify the information content.

^ GENPLOT: A Data Analysis and Graphical Plotting Program for Scientist and Engineers, Computer Graphic Service, 52 Genung, Circle, Ithaca, NY. 14850-8716, (607) 277-4913. 

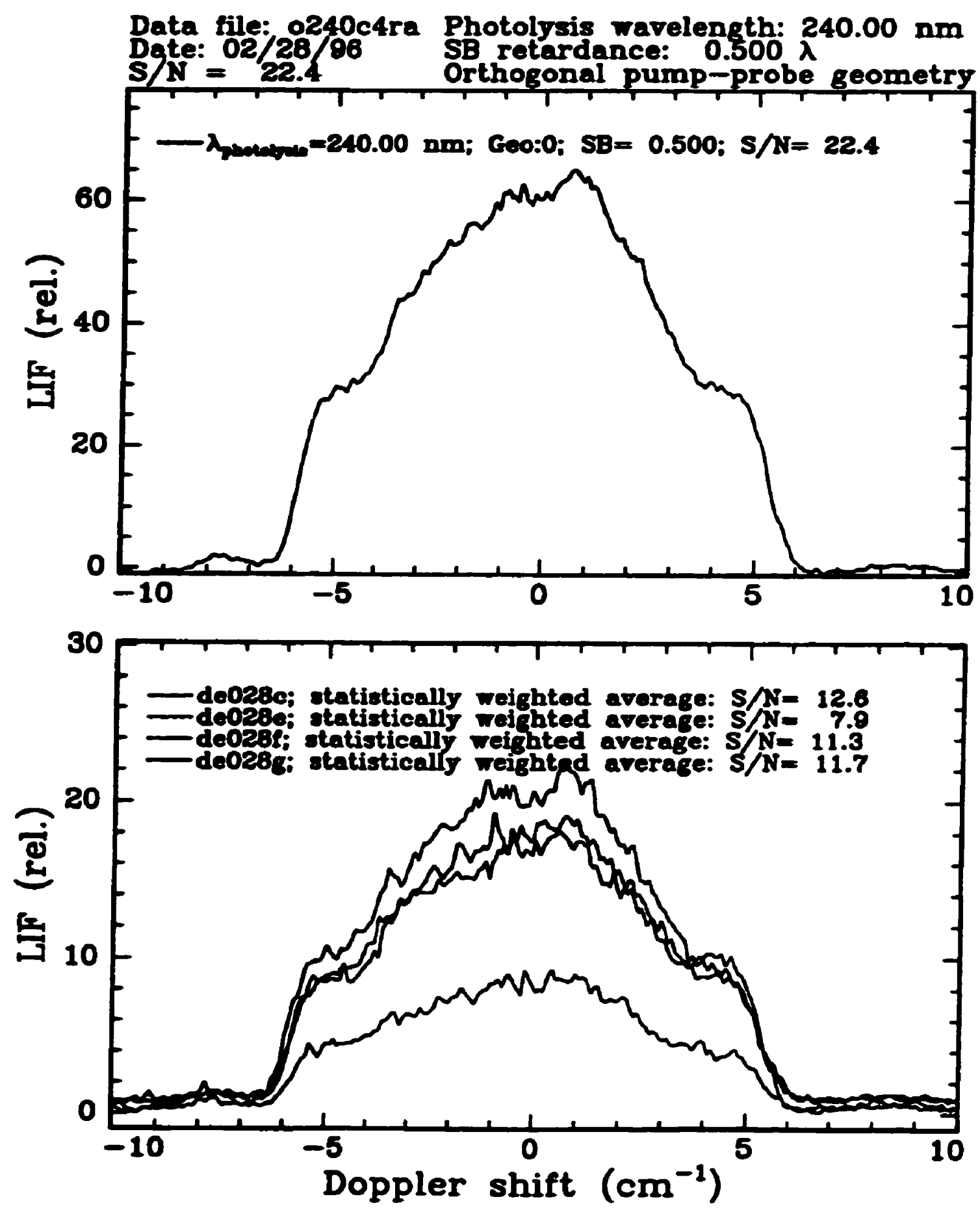

Figure 5.3. Example of systematic statistical averaging of a series of averages Doppler spectra. The terms in the legend not self-evident are explained: SB retandance is the Soleil-Babinet retardance inposed on the photolysis beam incoming with horizontal polarization, and the optical axis of the retander being ariented at $45^{\circ}$ from the vertical, therefore $S B=0 \lambda$ implies

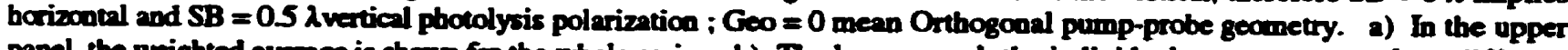
panel, the weighted average is stown for the whole series. b) The lower panel, the individual average spectra from different experimental run are weighted in ecoundance of their statistical content, through their respective area. This plot was done for each series average, in order to monitor each data set. See text for the definition of the legend in the graph. 


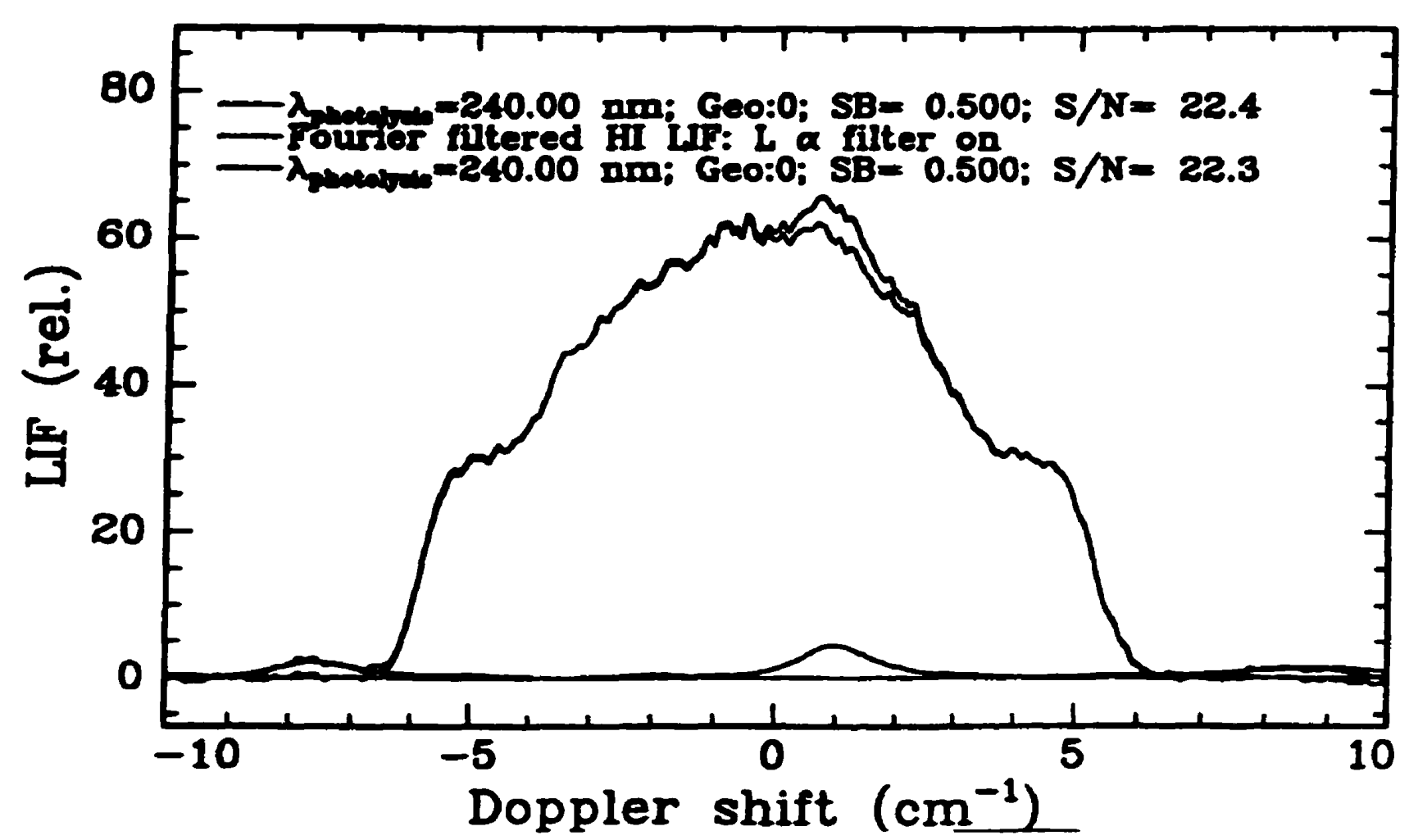

Figure 5.4. Example of systematic HI LIF background (re fig 5.2) subtraction from the average spectrum from figure 5.3.

In figure 5.4, the contribution from the HI LIF background present in the averaged H-atom signal was assessed by scaling the background HI LIF function to spectral features at $-7.5 \mathrm{~cm}^{-1}$ and $8.0 \mathrm{~cm}^{-1}$ Doppler shift, near the wings of the Doppler spectra; then the scaled background was subtracted, and the final Doppler profile was obtained. These final Doppler profiles, for each combination of photolysis wavelength and polarization investigated, are the final product of the experimental measurement and data averaging. The analysis of chapter 6 is developed on the basis these average measurements.

\section{References}

1. As it can be appreciated after consultation of appendix $E$, the creeping saturation of the electron multiplier in PMT can easily go undetected for S/N 3-7, typical of individual spectrum recorded in the laboratory.

2. See appendix $D$.

3. S. Gerstenkorn and P. Luc, Atlas du spectre d'Absoption de la Molecule D'Iode, Vol. I I4800-20000 $\mathrm{cm}^{\prime l}$ (Laboratoire Aime Cotton, CNRS II, Orsay, 1977) 


\section{Chapter 6 Analysis, discussion and quantum mechanical calculation}

\subsection{Perspective of the approach}

The goal of this chapter is to elucidate the photodissociation dynamics of HI, following absorption in the first continuum. This physical process is characterized by two observables, the anisotropies and the electronic branching of the photofragment products, which an be defined as functions of the photolysis photon energy. The values for these observables will be obtained at nine photolysis photon energies from our spectroscopic measurements on $\mathrm{H}$-atom photofragments, and they will enable a determination of partial absorption cross sections for each electronic product.

These experimental results will be compared with those from previous studies, and the discrepancies that will be found will be rationalized. Based on these experimental results, a quantum mechanical (QM) determination of the potential energy curve (PEC) for the dissociative states will substantiate the theory of direct photodissociation in HI, which has been summarized in chapter 3. This model, however simple, provides correct predictions of the observables for the photoproducts across the range of photolysis wavelengths over which we obtained experimental observations, and it suggests an extrapolation of the observables over the whole $A$ band absorption.

The analysis of our experimental measurements proceeded in four steps: (i) the determination of product branching ratios $(R)$ and anisotropy parameters $(\beta)$ at various photolysis wavelengths by inversion of the Doppler profiles, (ii) a comparative analysis of our results with those from previous studies and a return to the profile inversion, (iii) a calculation of the partial absorption cross section (PACS) for the electronic products, and (iv) a QM determination of the PEC. The first step yielded values for the observables of the photodissociation dynamics process. However, discrepancies existed amongst the values of these observables, which bad only been measured at a few photolysis photon energies. Consequently, a comparison of our results with those obtained in previous studies was necessary, and turned out to be crucial for the present study, as it will be shown in section 6.2.

Once the observables of the photodissociation dynamics were determined with a relatively high degree of confidence, a QM determination of the PEC was performed. To this end, the total absorption cross section (TACS) from Ogilvie ${ }^{1}$ was decomposed into partial absorption cross section (PACS) of the electronic products, by using our experimental values of branching ratios R. Finally, these experimental PACS were used as the input data for the QM determination of the PEC. 


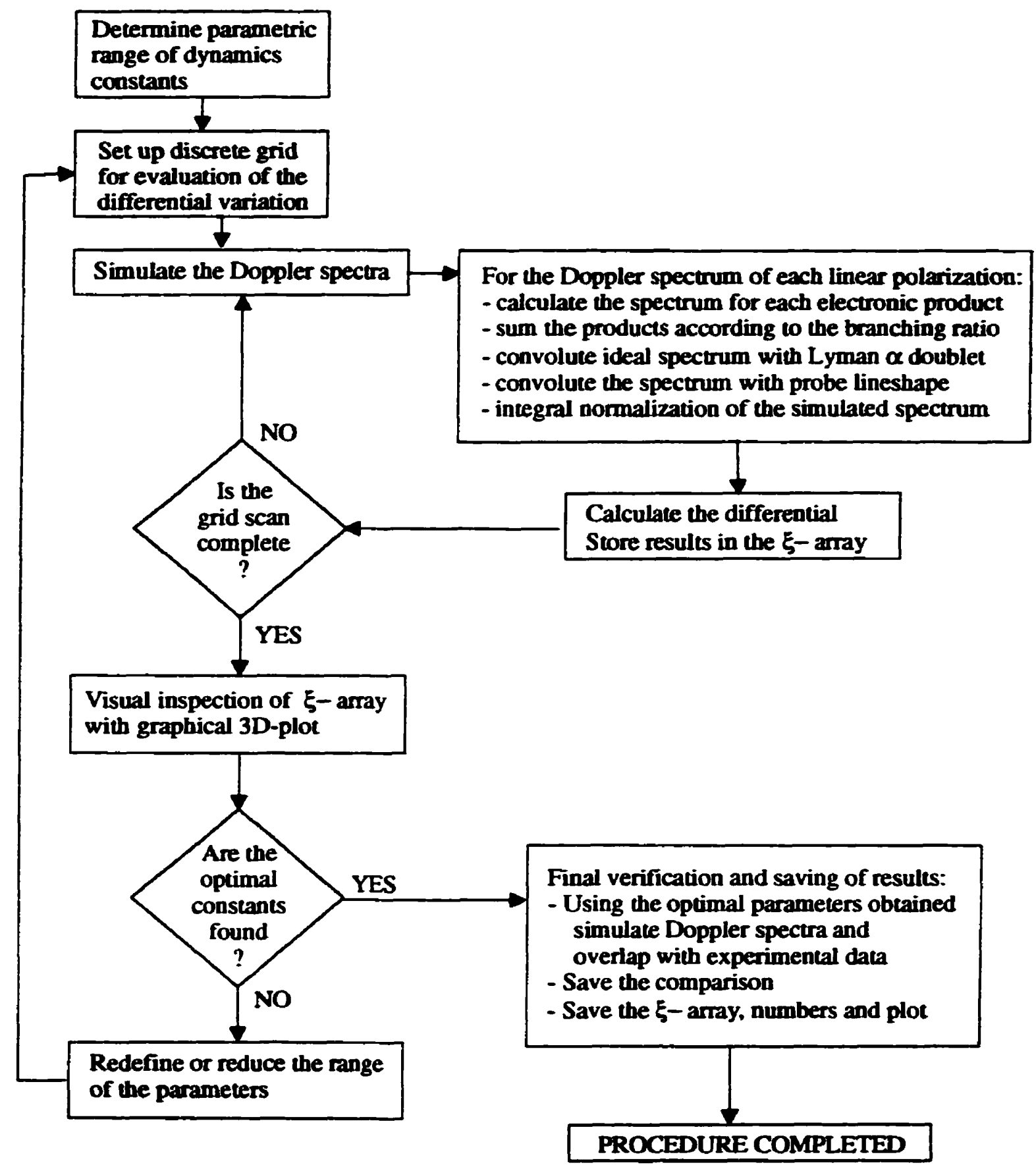

Figure 6.1. Flow chart of the forward convolution procedure to extract the observables of the photodissociation dynamics from the Doppler profile spectra. Two parts can be distinguished: Doppler profile simulation. and iterative optimization of the dynamical constants, whose optimum values determine the experimental observables. 


\subsection{Determination of the observables from experimental measurements}

The Doppler profiles were measured with a photolysis beam in a state of either vertical or horizontal linear polarization, and with an orthogonal arrangement of the pump-probe gecmetry. These profile measurements provided sufficient information to extract the two constants characterizing the photodissociation dynamics. These constants, $R$, the product branching ratia and $\beta$, the anisotropy parameter, will be referred below as the dynamical constants. At each photolysis wavelength and photolysis polarization, repeated measurements were statistically averaged in the manner outlined in the chapter 5.

A fituing routine was devised to extract the values of the dynamical constants from the averaged Doppler profiles. The routine was essentially a forward convolution technique, in which trial values for the dynamical constants were varied within a range, and the simulated Doppler profiles were calculated for each polarization state, at the trial value of the dynamical constants. The values of these constants were varied until the corresponding simulated profiles best agreed with the experimental spectra.

In the following section, the algorithm devised to simulate the Doppler profile, and the method to optimize the fit of the constants, are described. This section closes with a discussion on the evaluation of the uncertainties for the optimum values of the dynamical constants. The flow chart of figure 6.1 represents the forward convolution procedure used to obtain the observables from the Doppler profile measurements.

\subsubsection{Algorithm of the Doppler profile simulation}

For a given set of dynamical constants, the Doppler profiles corresponding to vertical or horizontal polarization of the photolysis laser can be calculated using the formulas derived in chapter 3. The calculation involved the following steps: (i) a partial Doppler profile was simulated for each of the two velocity groups, each corresponding to a product channel, according to the anisotropy assumed for each product channel: (ii) these two partial profiles, were summed with a weight determined by the branching ratio; the result was an ideal Doppler profile. carresponding to a probe laser of zero linewidth for an infinitely narrow transition; (iii) the fine-structure splitting $\left(0.36 \mathrm{~cm}^{-1}\right)$ of the Lyman $\alpha$ transition in the hydrogen atom was taken into account by adding two replicas of the ideal profile, weighted by the respective multiplicity of the doublet's components ${ }^{2}$, offet by the energy splitting, and positioned at the centre of gravity of the doublet. The result was a Doppler profile for an ideal probe laser, i.e. a probe laser of zero linewidth; (iv) the latter profile was then convoluted with the finite linewidth of our experimental probe. The latuer was determined from VUV calibration measurement (CO LIF) described in chapter 4; (v) the simulated profile obtained in this way was normalized to unit area. 
Figure 6.2 illustrates the calculation steps to simulate a Doppler profile, for a photolysis beam in horizontal polarization at $\mathbf{2 3 0} \mathrm{nm}$ wavelength. The steps include the calculation of the partial profile contribution for each product channel, their sums convoluted with the H-atom's Lyman $\alpha$ doublet which results in an ideal Doppler profile, the convolution of the latter with the finite probe linewidth which results in the simulated profile, and its comparison with an experimental Doppler profile. The Doppler profile simulation was an intermediate step of the forward convolution algorithm. This algorithm was repeated for both photolysis polarization components at each set of values of the adjustable photodissociation constants.

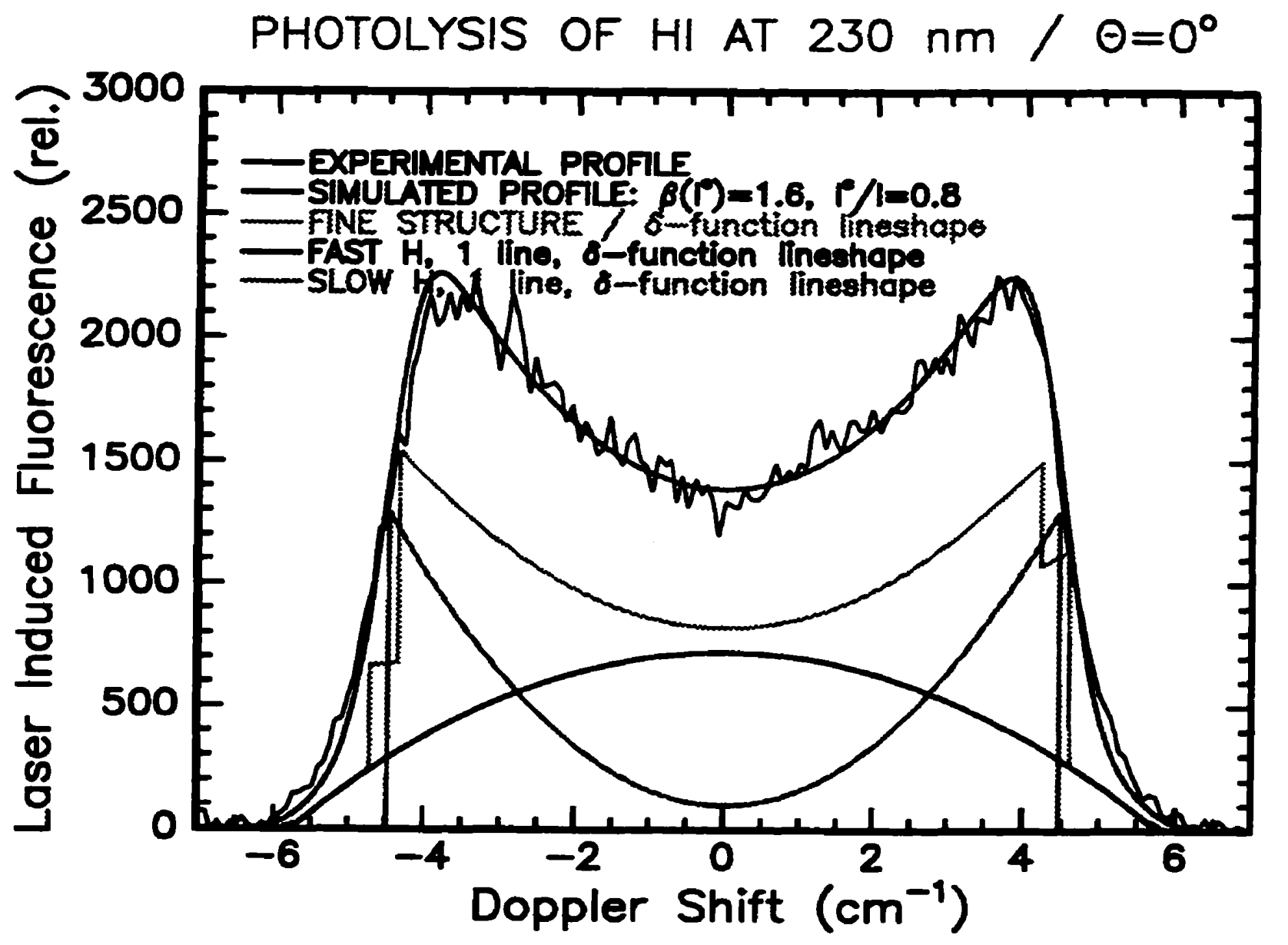

Figure 6.2. Example of simulated Doppler Profile, including the two product channels, the fine structure doublet, and the convolution with a finite probe linewidth, compared to an experimental measurement for photolysis at 230 nm wavelength.

Note that prior to the present study, the anisotropy parameter of the ground state iodine product, $\beta\left(I^{2} \mathrm{P}_{32}\right)$, was experimentally observed and theoretically predicted to be purely perpendicular. Therefore, $\beta\left({ }^{2} P_{32}\right)=-1.0$ was initially set fixed in the fit procedure, and the final results obtained in this way showed excellent consistency with our experiment, after optimization of the two adjustable constants, $R$ and $\beta\left(I^{*}{ }^{2} P_{1 / 2}\right)$. Thus $\beta\left(1{ }^{2} P_{32}\right)=-1.0$ is not discussed further below, and the following, reference to adjustable anisotropy parameter $\beta$ will refer to $\beta\left(1^{*}{ }^{2} P_{1 / 2}\right)$. 


\subsubsection{Definition of the optimizer: $\xi$}

For every set of adjustable dynamical constants, the above algorithm was repeated. The experimental data were nomalized to unity at the beginning of the fit procedure. The differences between the simulated and experimental profiles were calculated and squared point-by-point, and the squared differences were integrated for the profiles of each photyssis polarization state. The reciprocal of the integral of the squared difference was summed, for both polarization spectra, with a statistical weight in proportion to their respective $(\mathrm{S} / \mathrm{N})^{2}$, this weight was used to quantify the relative information content for each component. This calculation is summarized in equation (6-1).

$\xi(R, \beta)=\left\{\left[\int_{\Delta v} d v\left(D_{\exp }(v)-D_{\operatorname{sim}}(v ; R, \beta)\right)^{2}\right] / \Delta v\right\}^{1}$

In this equation, $D_{\text {ap }}$ is the experimental Doppler profile, $D_{\text {min }}$ is the simulated profile, and the integral of the difference squared is taken over the range of Doppler shifted frequency $\Delta v$. The resulting quantity was denoted $\xi$ and named the optimizer, because it was the quantity to optimize here. Defined in this way, the optimization is a search for the maximum value of $\xi$, to which corresponds the optimal values of the dynamical constants, i.e. which best represents the experimental Doppler profiles. Section 6.1.4 will discuss the practicality of this $\xi$ definition regarding the determination of the correlated uncertainties for the optimum values of the constants.

\subsubsection{Iterative method to determine the observables}

The numerical values of the observables $\beta$ and $R$ are only physically meaningful over the ranges $\beta \in[-1.0,2.0]$ and $R \in[0.0,1.0]$, respectively, where the branching ratio is defined as: $R=\sigma\left(I^{*}\right) /\left(\sigma\left(I^{*}\right)+\sigma(1)\right)$, and $\sigma$ is the total cross section for an electronic product channel. The dependence of the $\xi$-function on the adjustable constants allows for a smooth convergence to the set of optimal values for the dynamical constants, which correspond to the maximum value of $\xi$. This property suggested a straightforward method to be devised, for the determination of the optimum constants of the photodissociation dynamics.

Initially, the entire domain of the possible values for the dynamics constant was searched. Typically, a grid size

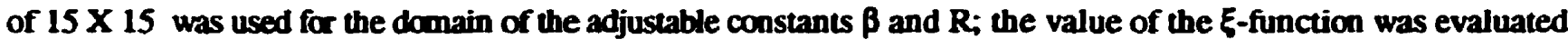
for each points of the grid, resulting in a numerical $\xi$-function. The optimal values of the adjustable constants were obtained by locating the maximum value of $\xi$, and by identifying to the corresponding values of the domain. The domain was reduced by a factor of the order of 2 and centred around these optimum values for the constants. The grid of the domain was redefined, and the $\xi$-matrix was calculated again. The process of $\xi$-function calculation and domain reduction was repeated until the grid size was smaller than the uncertainty of the adjustable constants. The iterative procedure was repeated until the region of the maximum was clearly located; typically 46 cycles sufficed to attain the required precision for the constants. The precision of the optimal values for the adjustable constants is discussed further below. It was found empirically that, as long as the ranges of the domain are made to diminish slowly enough (i.e. by a factor $\leq 2$ ), convergence to the optimum was obtained. 
This technique was preferred over the more sophisticated gradient method ${ }^{3}$ because of its ease of implementation and for the graphical monitoring of the fit convergence. An algorithm developed in the macro language of GENPLOT, based on a UNDX platform. The choice of GENPLOT macros was selected because this numerical analysis program includes powerful graphics capability, which avoid the time-consuming development of a graphical interface, which was required to monitor the fit routine. The iterative process was monitored graphically by plotting the E-fubction as a 3D surface against the domain of adjustable constants. The final iterations of the forward convolution procedure, for the fit of the dyamical constants at each photolysis wavelength of the main data set, are represented in figures of odd numbering 6.3 to 6.19 .

\subsubsection{Evaluation of the uncertainties}

A definite rationale for the definition of $\xi$ as the quantity to optimize is provided by the fact that the maximum

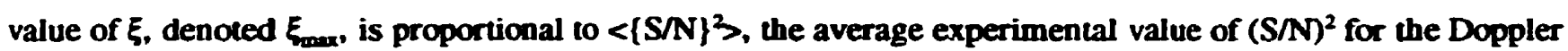
profiles, on the basis of which the dyamical constants were calculated; note that the (S/N) is calculated on the basis of the statistical fluctuation, for one standard deviation. The limit of the precision of the experimental measurements must be reflected in a corresponding limit for the precision of $\xi_{\max }$ having a physical significance.

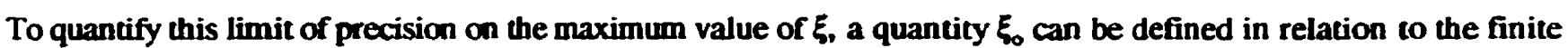

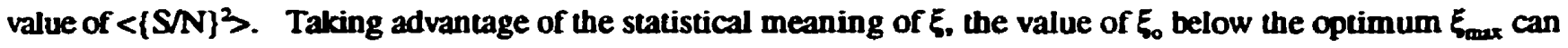
be defined by the equation (6-2), where $\Gamma$, a statistical measure of the effective $S / N$ for the combination of the experimental Doppler profiles, is defined as: $\Gamma=<\{S / N\}>=\left[\left\{(S / N)_{m}\right\}+\left\{(S / N)_{\text {poni }}\right\}\right] / 2$. Recalling (equation $(6-1)$ ) that $\xi$ bas the unit of $\{N / S\}^{-1}$, defining the symbolic quantities $S / N=\Gamma$, the statistical meaning of $\xi_{0} / \xi_{\max }=[S$. $N]$ / S, i.e. the uncertainties of optimum determination due to experimental $<S / N\rangle$.

$\xi_{o} / \xi_{\max }=1-1 / \Gamma$

The uncertainties on the dynamical constants were determined via the determination of the complementary quantities, i.e. range of certainty, which is described graphically as follow. In a final 3D plot of the $\xi$-function, a value of $\xi_{0}$ can be defined with equation (6-2). A horizontal plane, defined at $\xi_{0}$, will intersects with the plot of the $\xi$-function. The region of the domain defined by the locus of this intersection includes the correlated uncertainties in the determination of the optimal values for the dynamical constants. Although the position of the maximum $\xi_{\max }$ may often not lie exactly in the centre of the range for the domain of $\beta$ and $R$, it is convenient to redefine the best estimate of the dynamical variable as the centre of this domain in each variable dimension; is is also reasonable to do this, since the closer to the optimum dynamics constant we are, within one standard deviation, the lescer is our improvement in the exactness of the optimum parameter value within that range. This is a consequence of the ubiquitous nature of the gaussian (normal) distribution and its derivative. Therefore, we 
can take advantage of this definition to link the uncertainties of the optimum values for the dynamical constants to the experimental uncertainties of the Doppler profile used in the forward convolution. These estimates for the uncertainties of the dynamical constants and the $<S / N>$ of the data are listed in table 6.1.

Table 6.1 Observables of $\mathrm{HI}$ photodissociation obtained from inversion of the Doppler profiles

\begin{tabular}{|c|c|c|c|c|}
\hline Wavelength (nm) & $S / N$ & $\beta\left(I^{*}\right)$ & $\left.R=\sigma\left(I^{*}\right) / \sigma\left(I^{*}\right)+\sigma(I)\right\}$ & Pump Power $(\mathrm{mJ} / \mathrm{p})$ \\
\hline 266.0 & 18 & $1.76-2.00$ & $0.395-0.435$ & $2.0-3.0$ \\
\hline 257.0 & 21 & $1.78-2.00$ & $0.456-0.498$ & $3.0-5.0$ \\
\hline 248.0 & 15 & $1.96-2.00$ & $0.490-0.510$ & $3.5-4.2$ \\
\hline 240.0 & 27 & $1.92-2.00$ & $0.453-0.475$ & $1.5-2.5$ \\
\hline 232.5 & 20 & $1.84-2.00$ & $0.398-0.432$ & $1.0-2.0$ \\
\hline 225.0 & 30 & $1.80-2.00$ & $0.348-0.378$ & $1.5-2.5$ \\
\hline 216.7 & 25 & $1.70-2.00$ & $0.281-0.329$ & $1.0-2.0$ \\
\hline 215.0 & 5 & $1.66-2.00$ & $0.220-0.290$ & $1.0-2.0$ \\
\hline 212.5 & 21 & $1.86-2.00$ & $0.232-0.256$ & $1.5-2.5$ \\
\hline
\end{tabular}

In order to illustrate the uncertainties of the dynamical constants, the experimental Doppler profiles were compared with four simulated Doppler profiles, calculated for the $(2 \times 2)$ possible combinations of the extremum parameters values corresponding to each uncertainty range. The graphical comparison for each photolysis wavelength, which is illustrated through figures 6.4 to 6.20 of even number, confirmed that the values of the uncertainties evaluated via the topographical method delineated above are indeed reasonable. In these profiles comparison, the legends in the spectra indicate the photolysis wavelength: $\lambda_{\text {pbocotyss }}$ the pump-probe geometry: Geo:0 indicates perpendicular pump-probe axis orientation, the retardance of the Soleil-Babinet compensator controlling the pump polarization: $\mathrm{SB}=0.0$ for horizontal (lower graph), and $\mathrm{SB}=0.5$ for vertical (higher graph), and the statistical fluctuation of the averaged experimental data ( $S / N$ values).

\subsubsection{Results of the forward convolution procedure}

The procedure outlined above was applied to each set of Doppler profiles, obtained from averaged measurements acquired at 9 photolysis wavelengths. The plot of the numerical $\xi$-function for each photolysis polarization are depicted in figures 6.3 to 6.19 of odd numbering. Table 6.1 lists the fitted values obtained for the dynamical observable, and their range of uncertainties; within the experimental uncertainties, the anisotropy parameters determined at all photolysis wavelengths investigated suggest that the excited products emerges parallel transition. For the excited channel, the deviation from $\beta\left(I^{*}\right)=2.00$ has the tendency to be greater for lower $S / N$. An additional factor that must be taken into account in correlating the tendency toward $\beta\left(I^{*}\right)=2.00$ and $<S / N>$ is the slight degree of saturation in the Doppler spectra and the slight imperfection of the correction for the nonlinearity 
Photolysis at $266 \mathrm{~mm}$
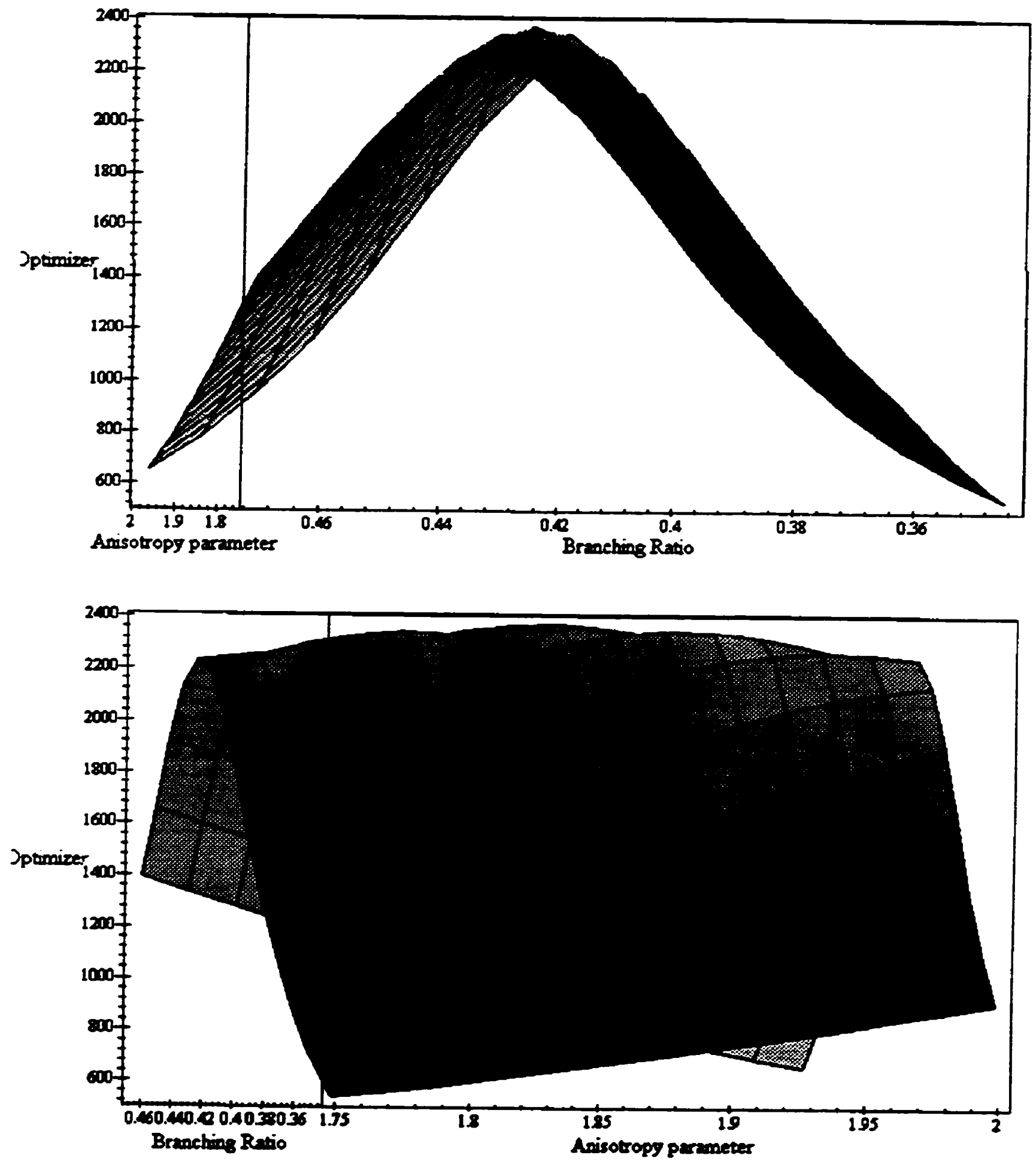

Figure 6.3. 3D plot of the optimizer- o $\xi$-function, for the forward convolution procedure. operated on the averaged experimental spectra measured at $266.0 \mathrm{~nm}$ photolysis wavelength. 

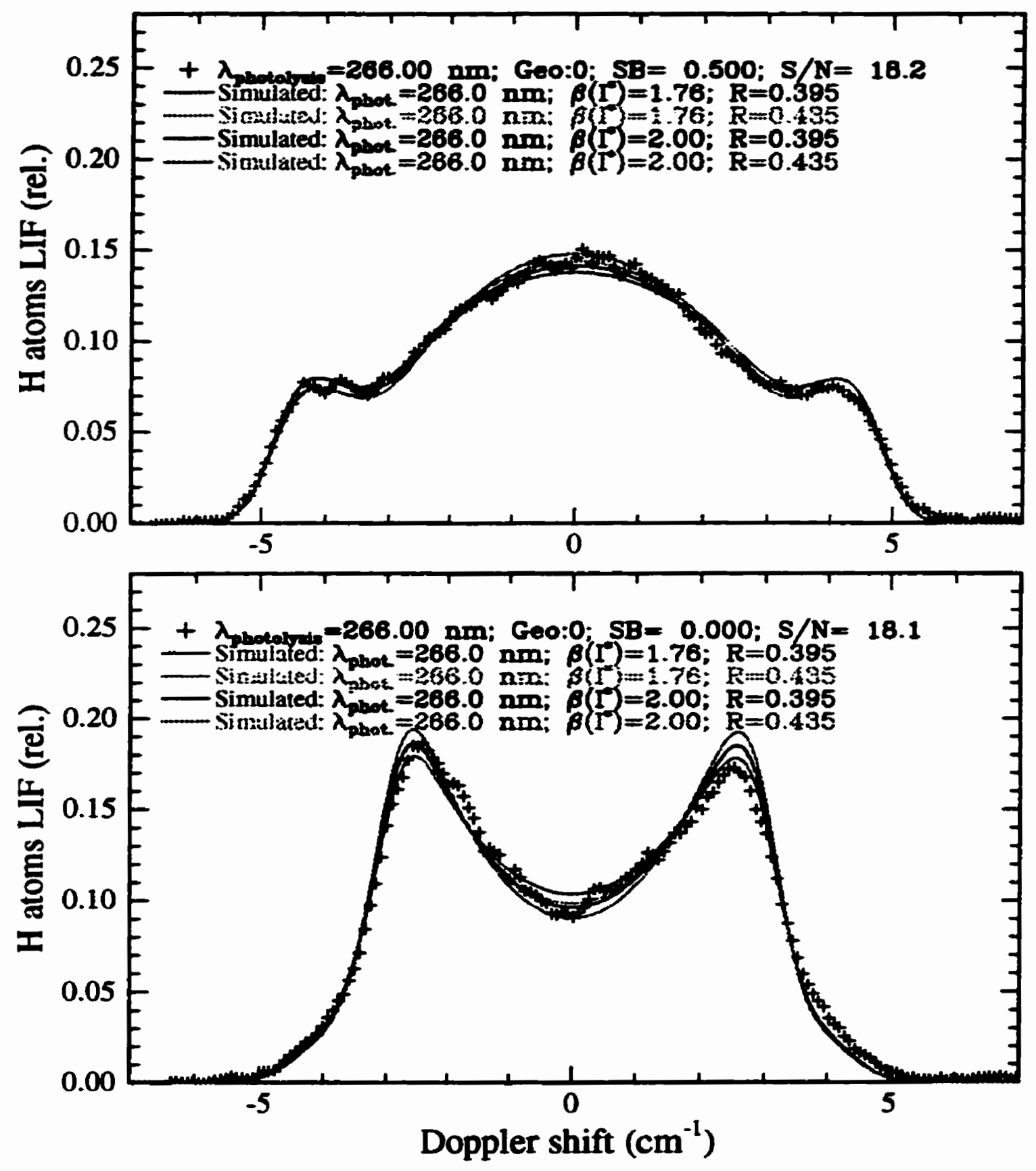

Figure 6.4. Comparison of experimental Doppler profiles, for $266.0 \mathrm{~nm}$ photolysis wavelength. with simulated profiles corresponding to the extremes of the uncertainties on the optimum values of the parameters (table 6.1). 


\section{Photolysis at $257 \mathrm{~nm}$}
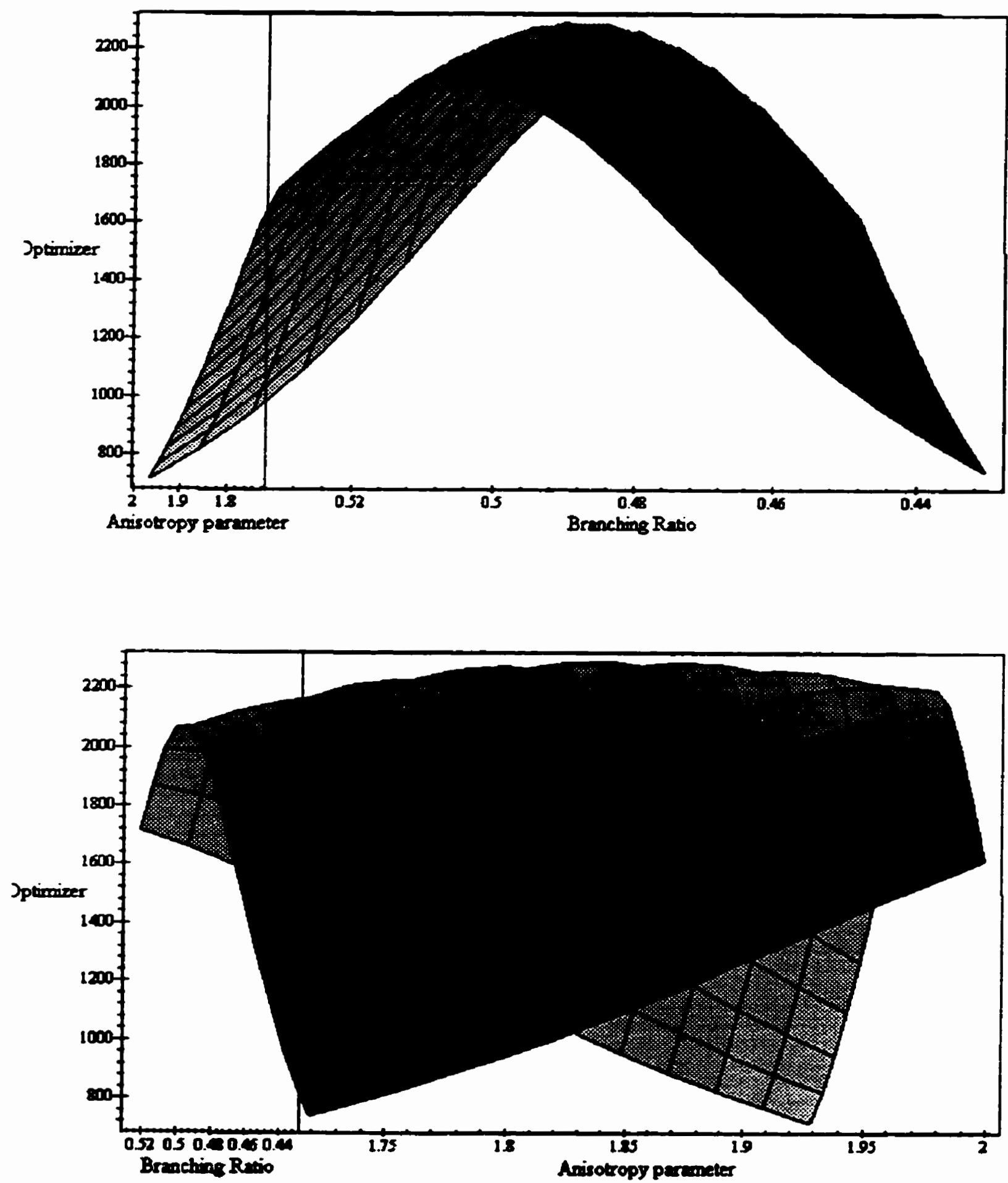

Figure 6.5. 3D plot of the optimizer- $\alpha$ E-function, for the forward convolution procedure, operated on the averaged experimental spectra measured at $257.0 \mathrm{~nm}$ photolysis wavelength. 

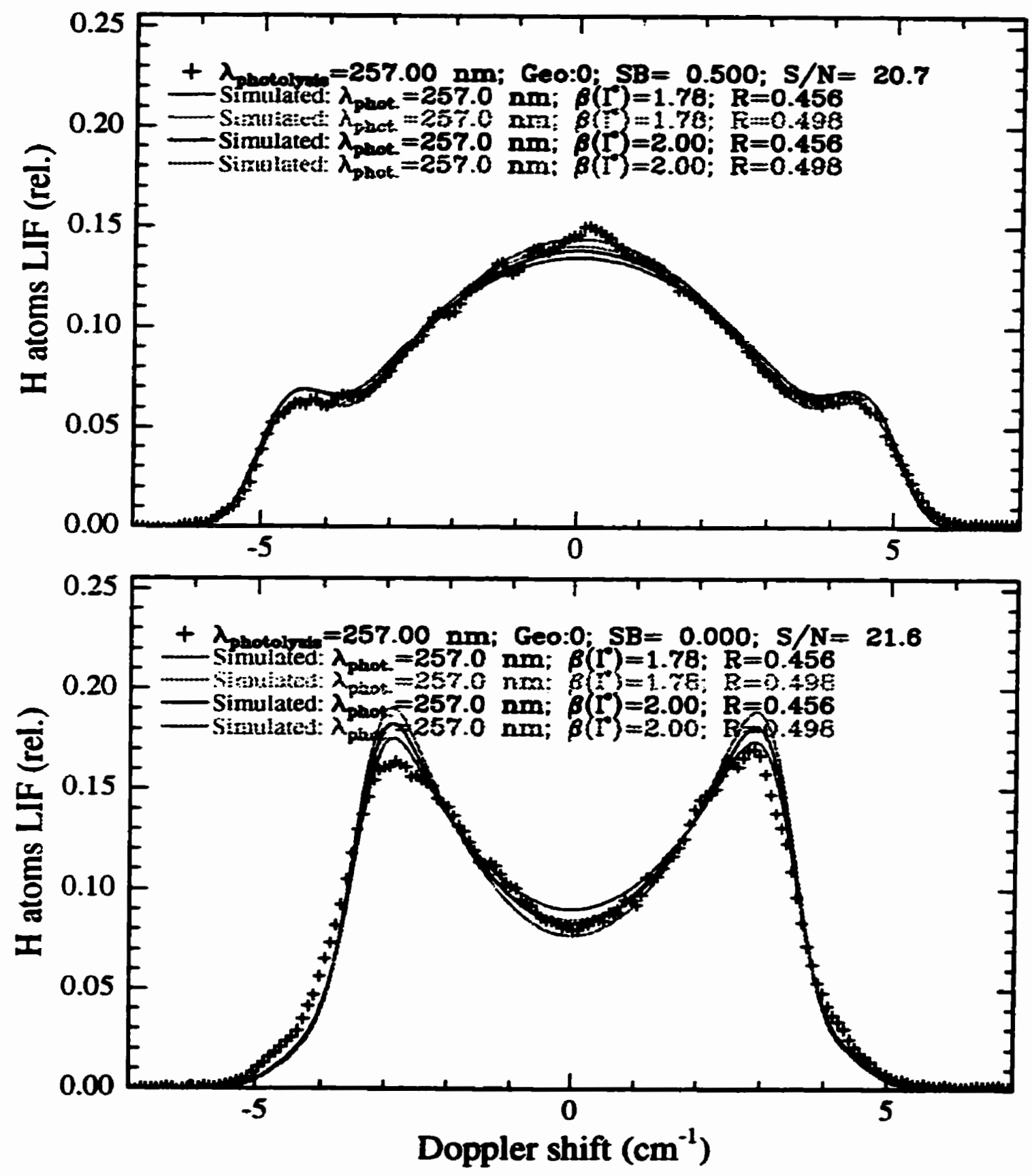

Figure 6.6. Comparison of experimental Doppler profiles, for $257.0 \mathrm{~nm}$ photolysis wavelength, with simulated profiles corresponding to the extremes of the uncertainties on the optimum values of the parameters (table 6.1). 

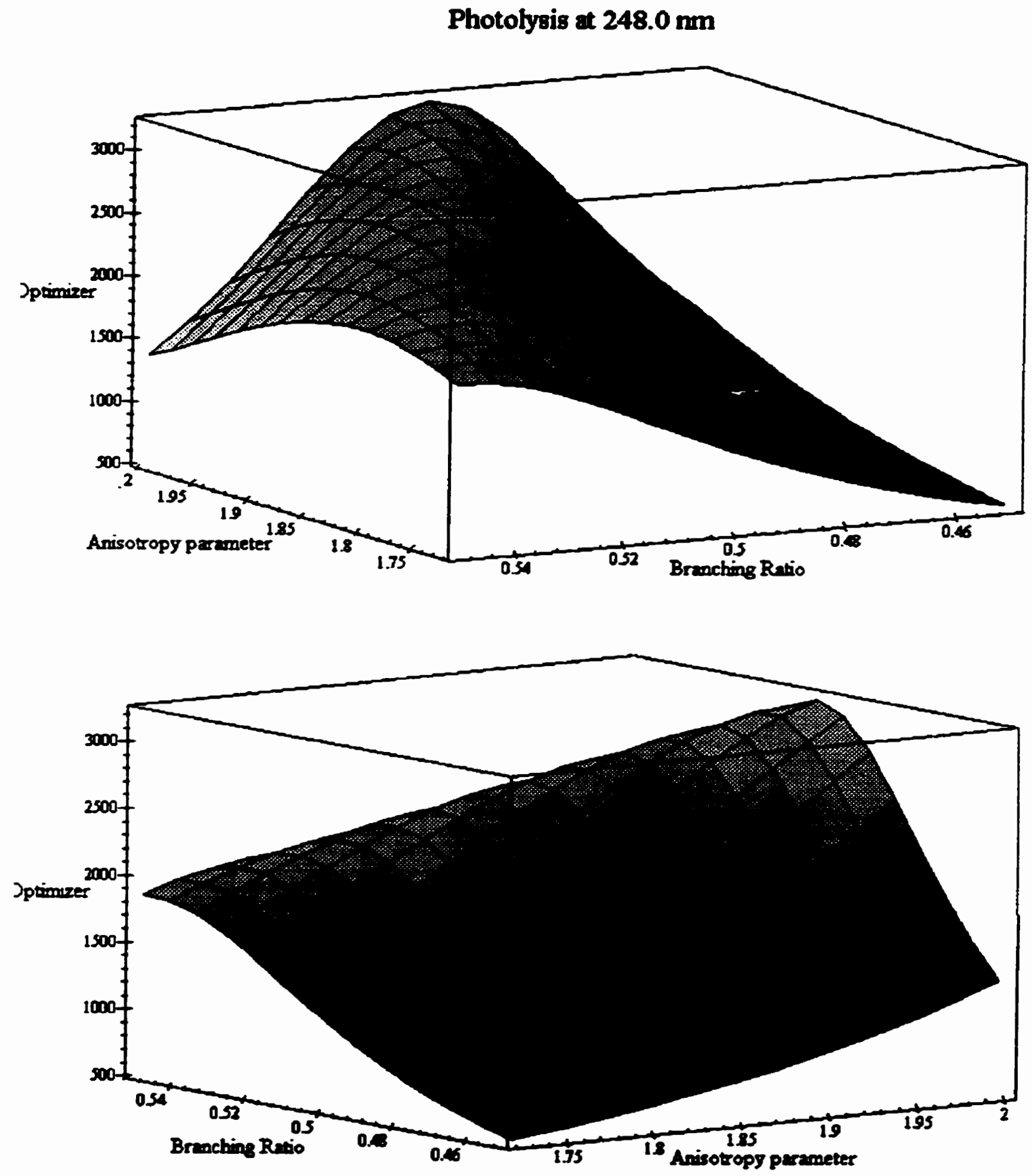

Figure 6.7. 3D plot of the optimizer- or E-function. for the forward convolution procedure, operated on the averaged experimental spectra measured at $248.0 \mathrm{~nm}$ photolysis wavelength. 

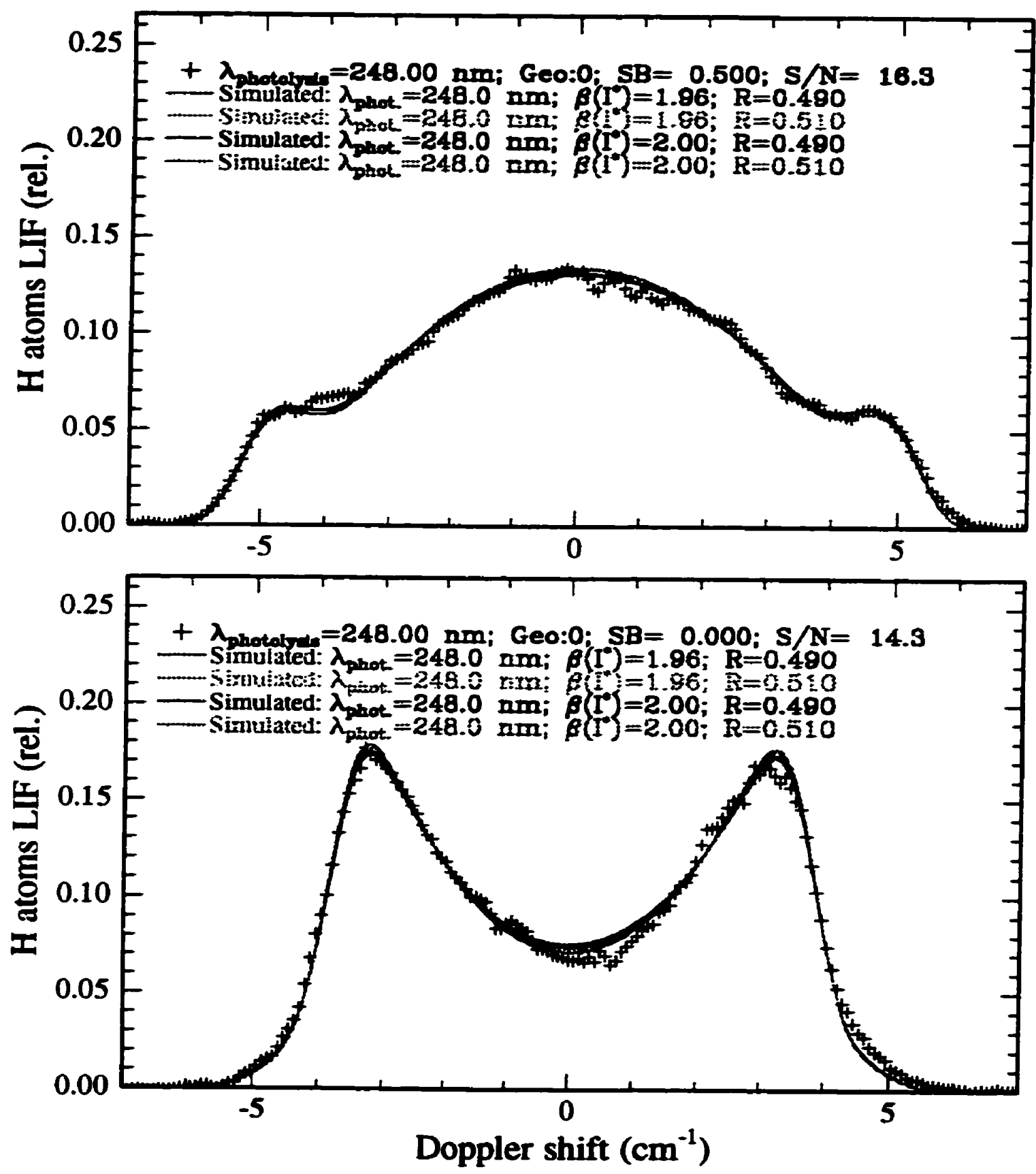

Figure 6.8. Comparison of experimental Doppler profiles, for $248.0 \mathrm{~mm}$ photolysis wavelength, with simulated profiles corresponding to the extremes of the uncertainties on the optimum values of the parameters (table 6.1). 


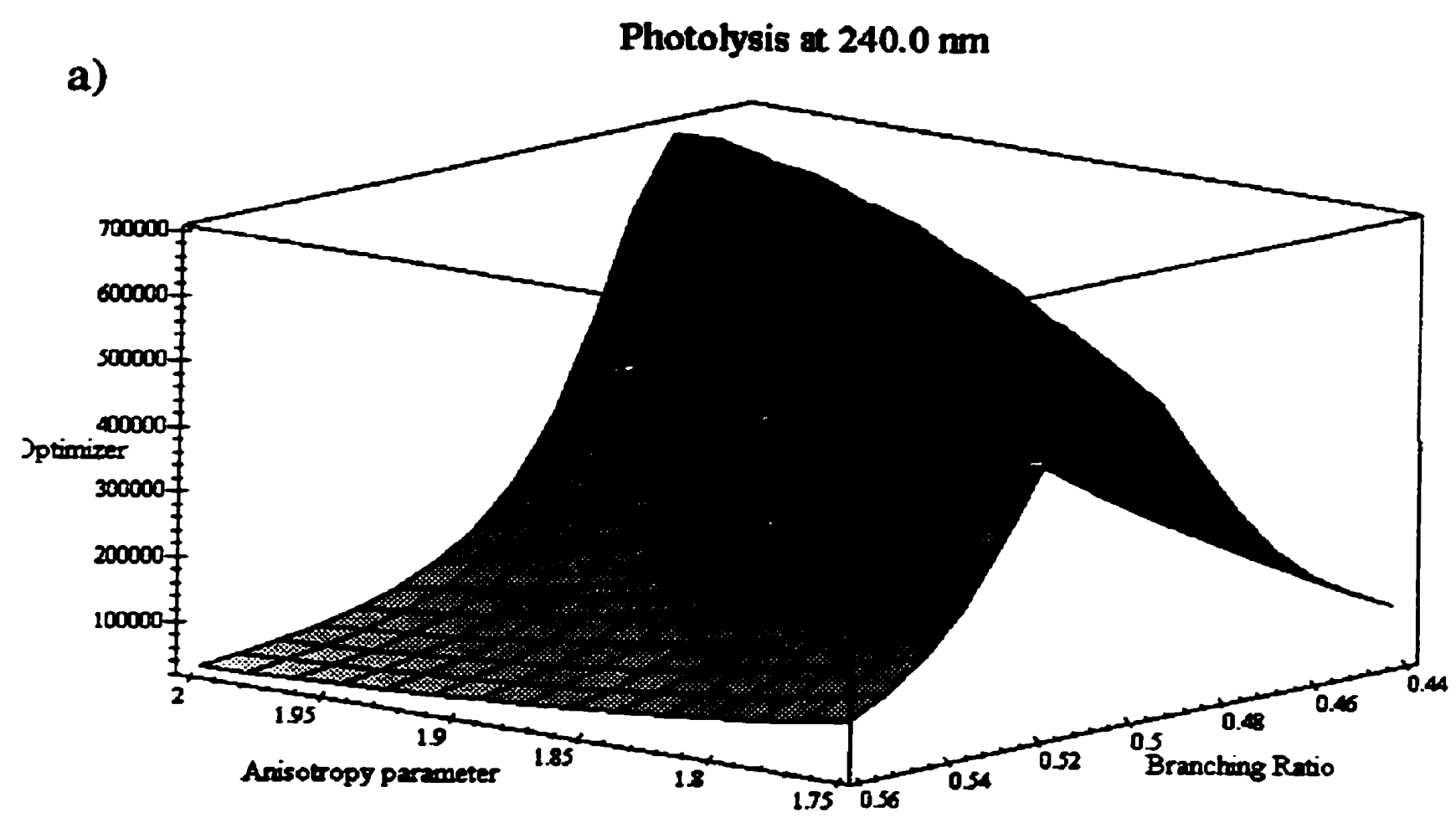

b)

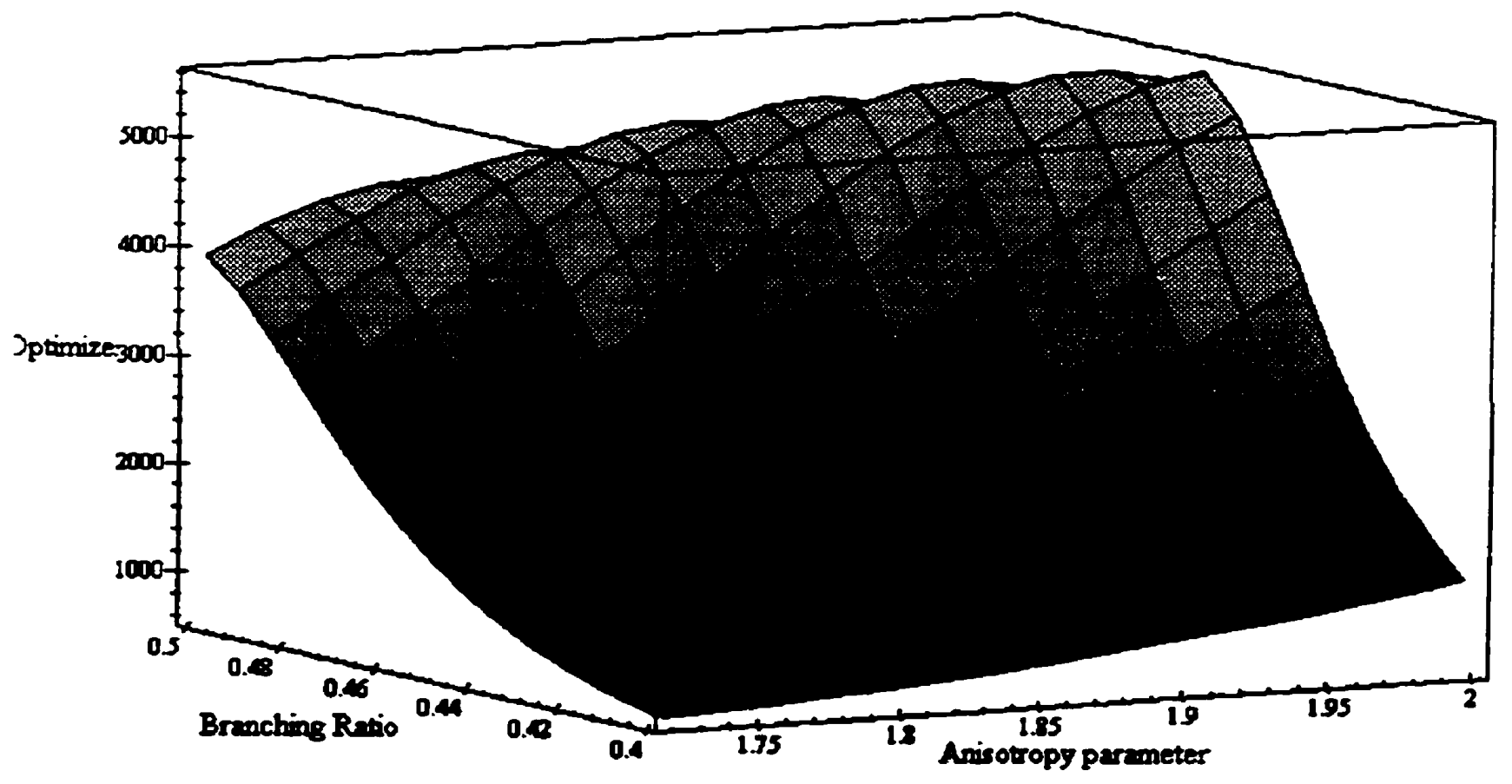

Figure 6.9. 3D ploc of the optimizer- or $\xi$-function. for the forward convolution procedure, operated on the averaged experimental spectra measured at $240.0 \mathrm{~nm}$ photolysis wavelength. 

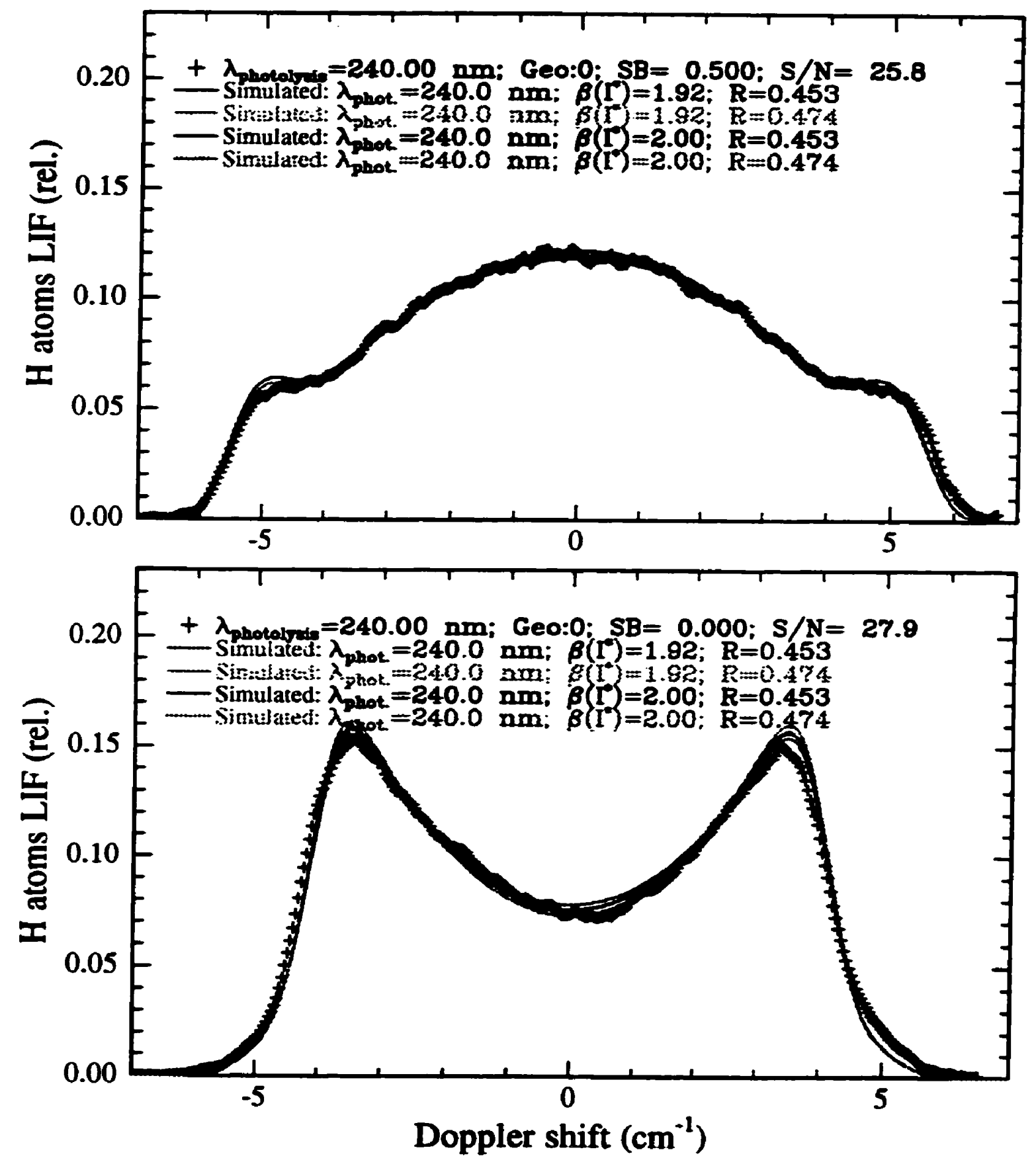

Figure 6.10. Comparison of experimental Doppler profiles, for 240.0 nm photolysis wavelength, with simulated profiles corresponding to the extremes of the uncertainties on the optimum values of the parameters (table 6.1). 


\section{Photolysis at $232.5 \mathrm{~nm}$}
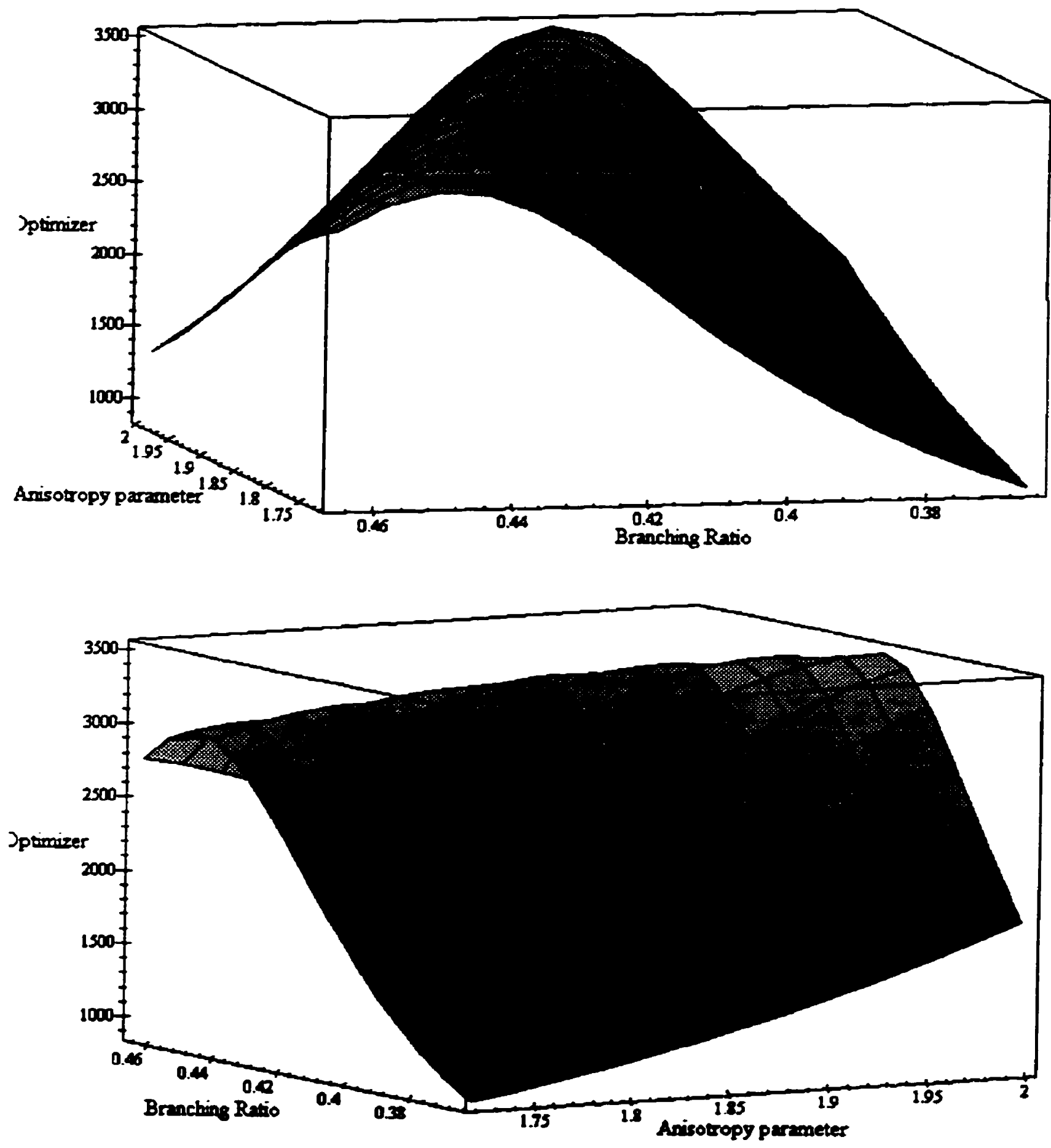

Figure 6.11. 3D plot of the optimizer- or $\xi$-function. for the forward convolution procedure, operated on the averaged experimental spectra measured at $232.5 \mathrm{~nm}$ photolysis wavelength. 

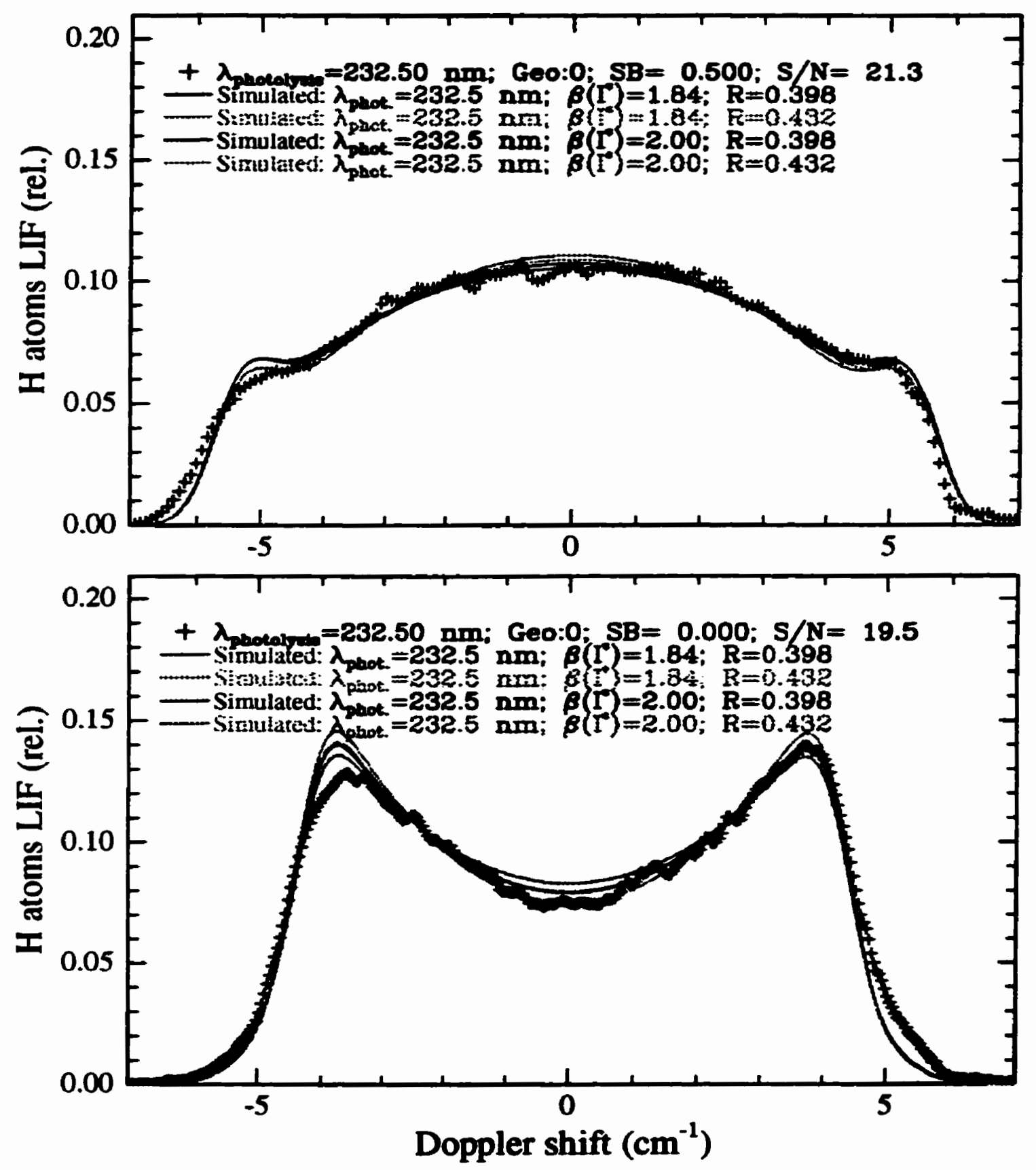

Figure 6.12. Comparison of experimental Doppler profiles, for $232.5 \mathrm{~nm}$ photolysis wavelength, with simulated profiles corresponding to the extremes of the uncertainties on the optimum values of the parameters (table 6.1). 


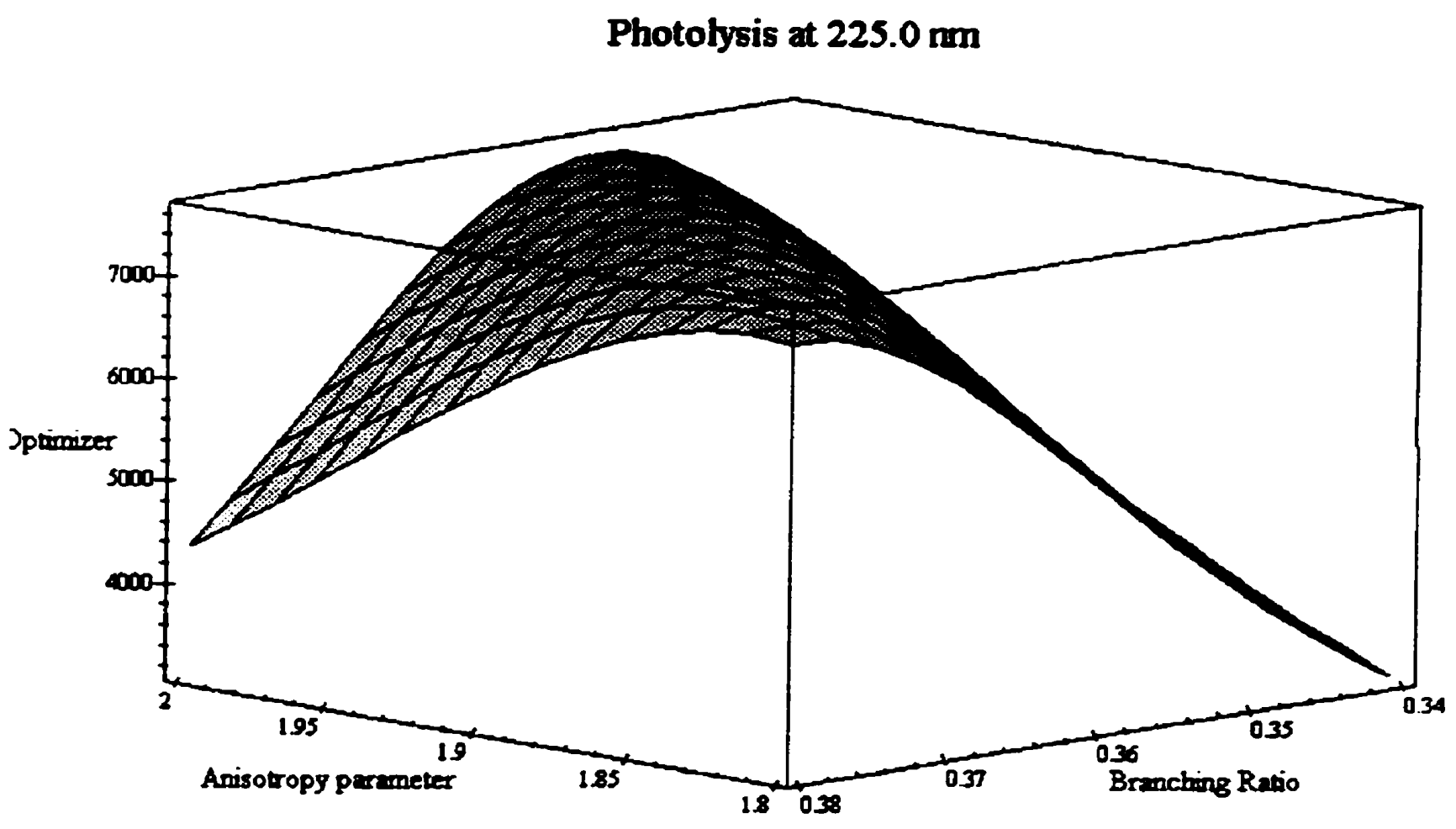

Figure 6.13. 3D plot of the optimizer- or $\xi$-function, for the forward convolution procedure, operated on the averaged experimental spectra measured at $225.0 \mathrm{~nm}$ photolysis wavelength. 

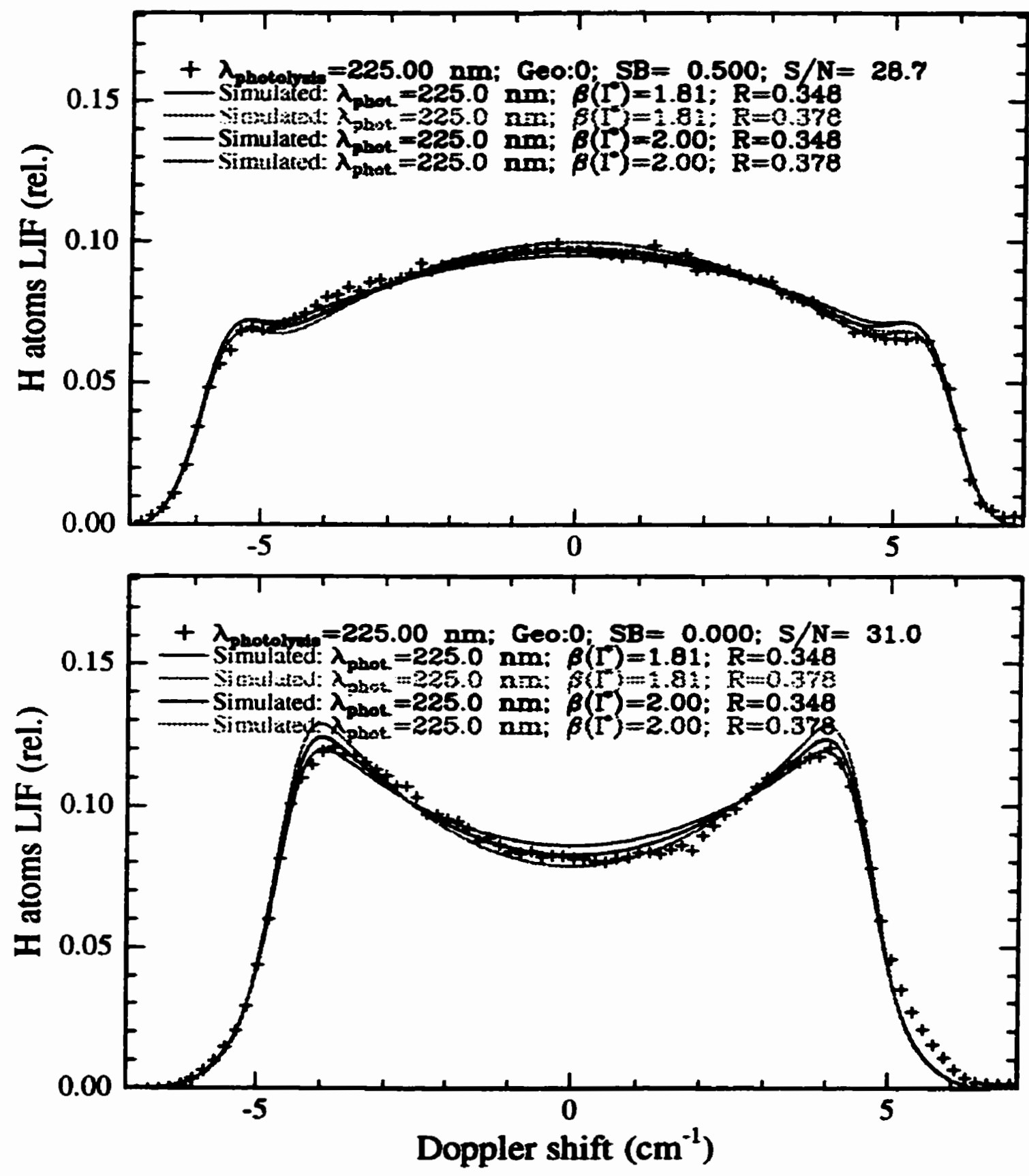

Figure 6.14. Comparison of experimental Doppler profiles, for $225.0 \mathrm{~nm}$ photolysis wavelength, with simulated profiles corresponding to the extremes of the uncertainties on the optimum values of the parameters (table 6.1). 


\section{Photolysis at $216.6 \mathrm{~nm}$}

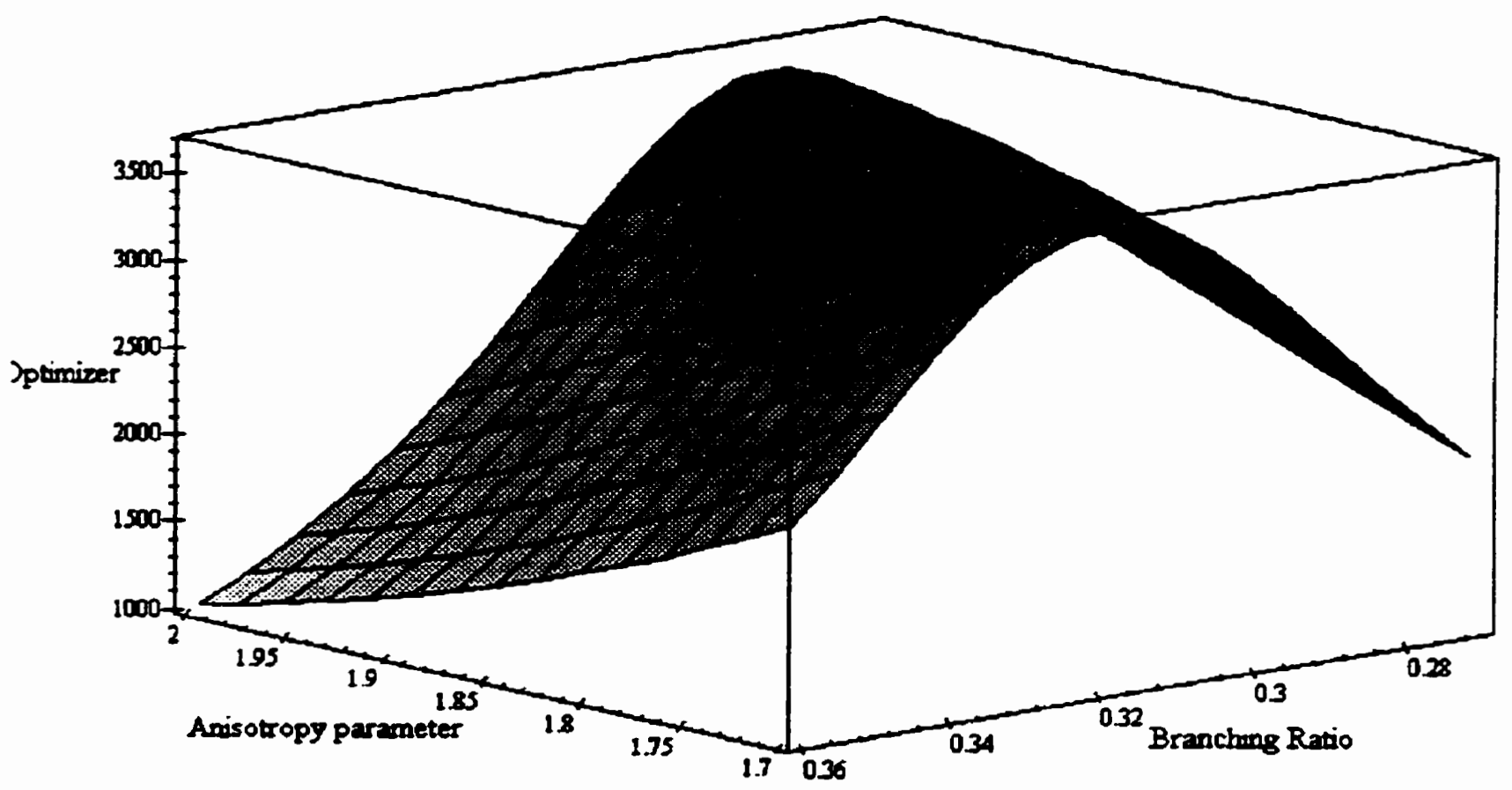

Figure 6.15. 3D plot of the optimizer- or $\xi$-function, for the forward convolution procedure, operated on the averaged experimental spectra measured at $\mathbf{2 1 6 . 7}$ nm photolysis wavelength. 

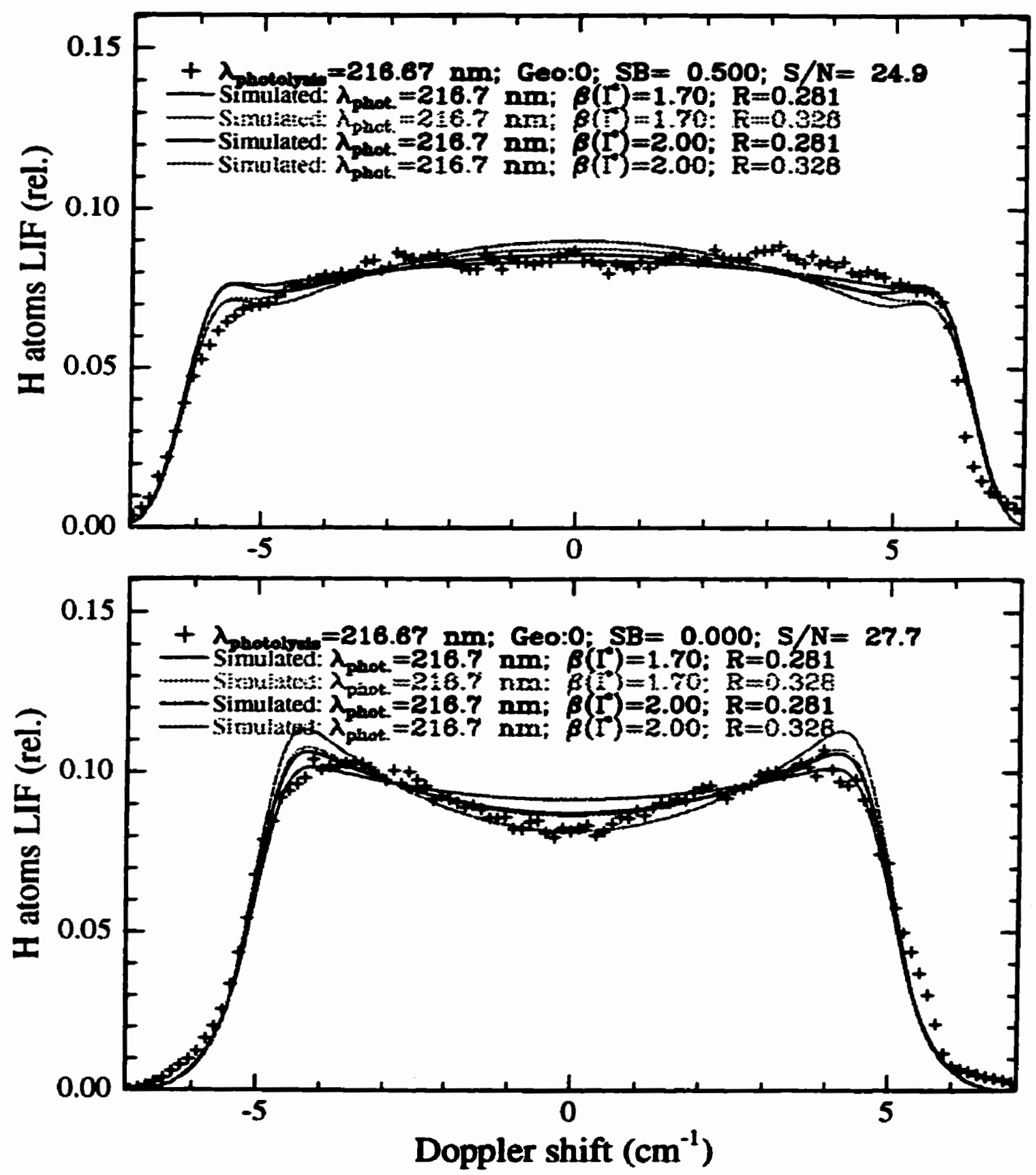

Figure 6.16. Comparison of experimental Doppler profiles, for $216.7 \mathrm{~nm}$ photolysis wavelength, with simulated profiles corresponding to the extremes of the uncertainties on the optimum values of the parameters (table 6.1). 
Photolysis at $215.0 \mathrm{~nm}$

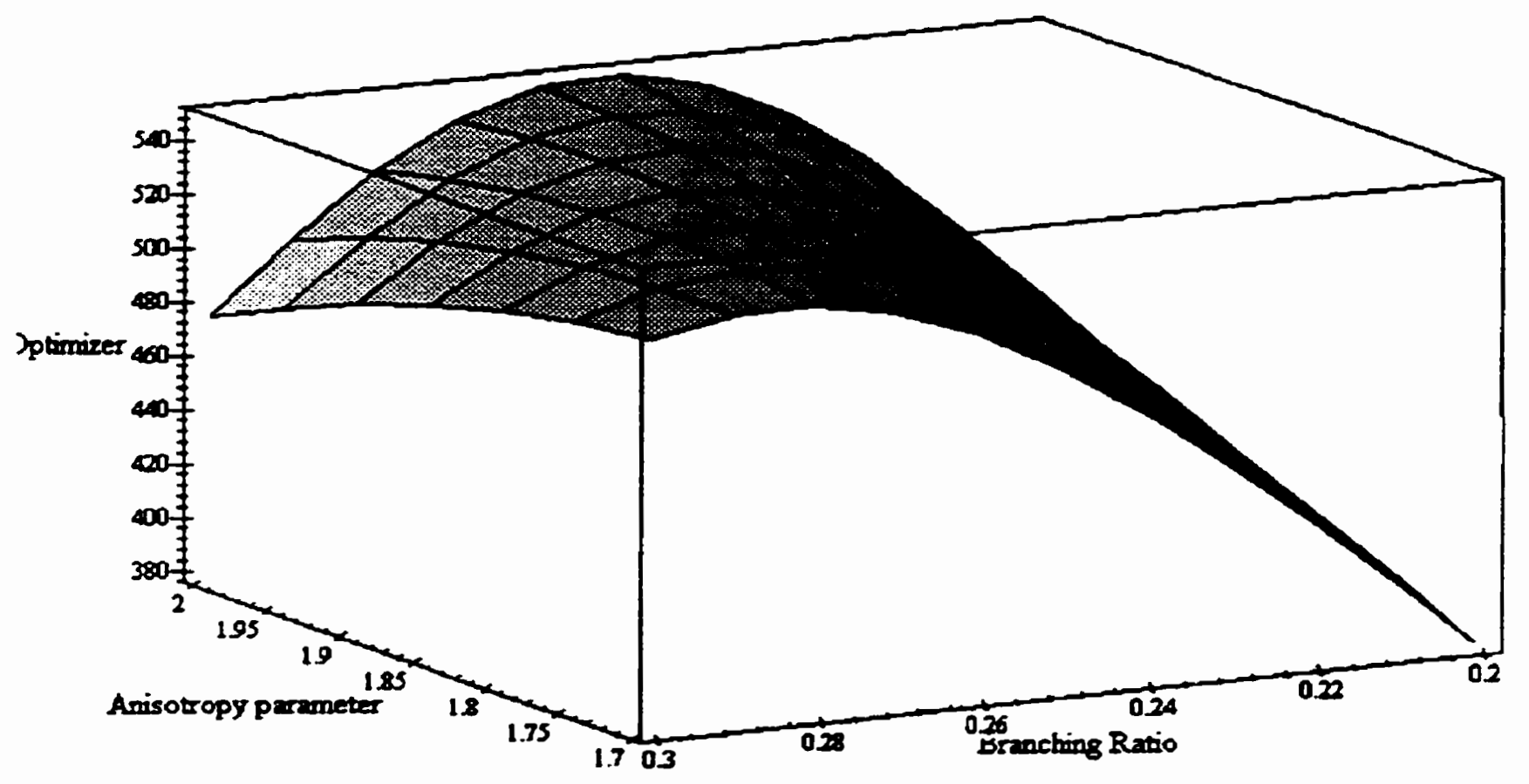

Figure 6.17. 3D plot of the optimizer- or $\xi$-function, for the forward convolution procedure, operated on the averaged experimental spectra measured at $215.0 \mathrm{~nm}$ photolysis wavelength. 

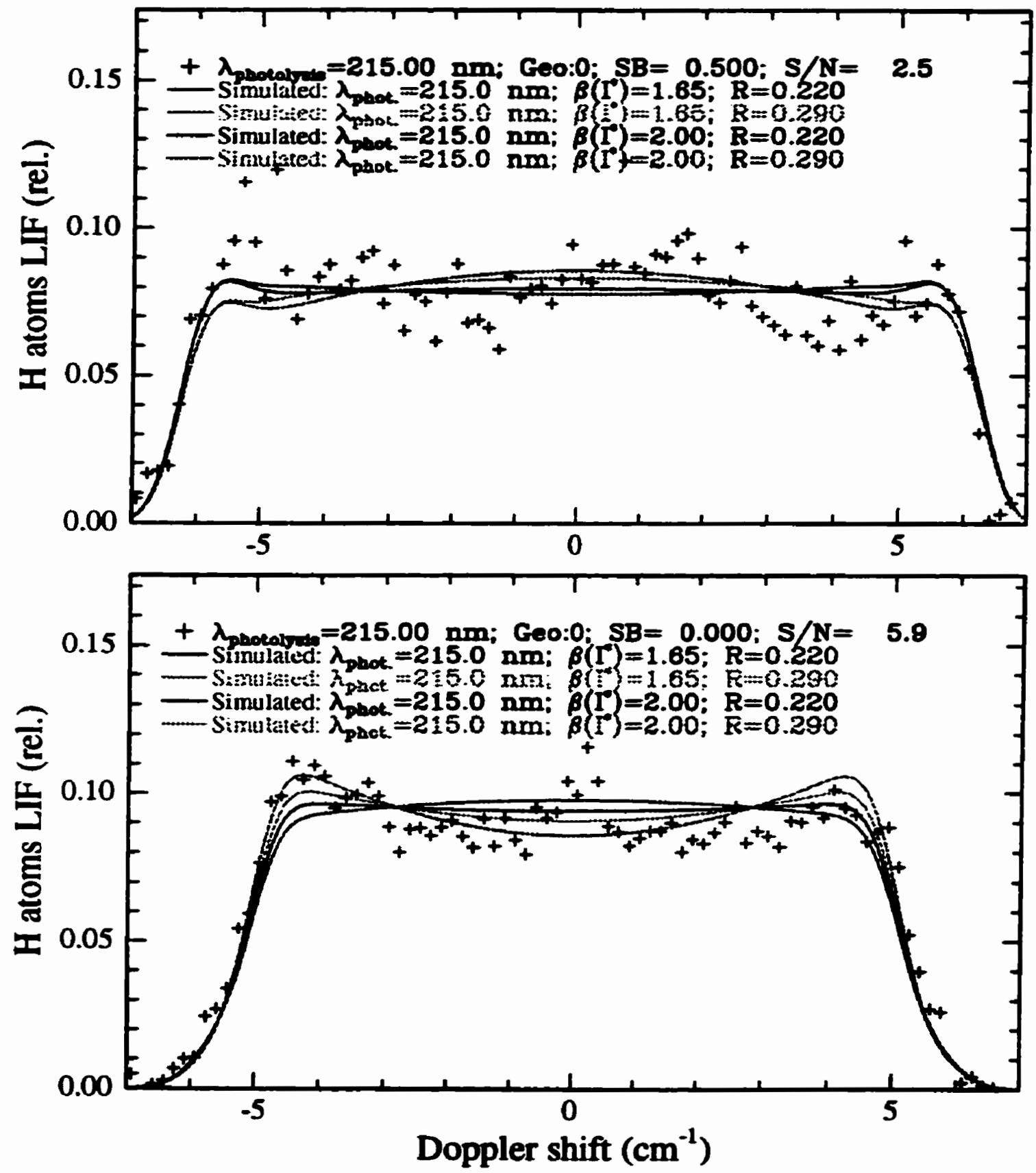

Figure 6.18. Comparison of experimental Doppler profiles, for $215.0 \mathrm{~mm}$ photolysis wavelength, with simulated profiles corresponding to the extremes of the uncertainties on the optimum values of the parameters (table 6.1). 


\section{Photolysis at $212.5 \mathrm{~mm}$}

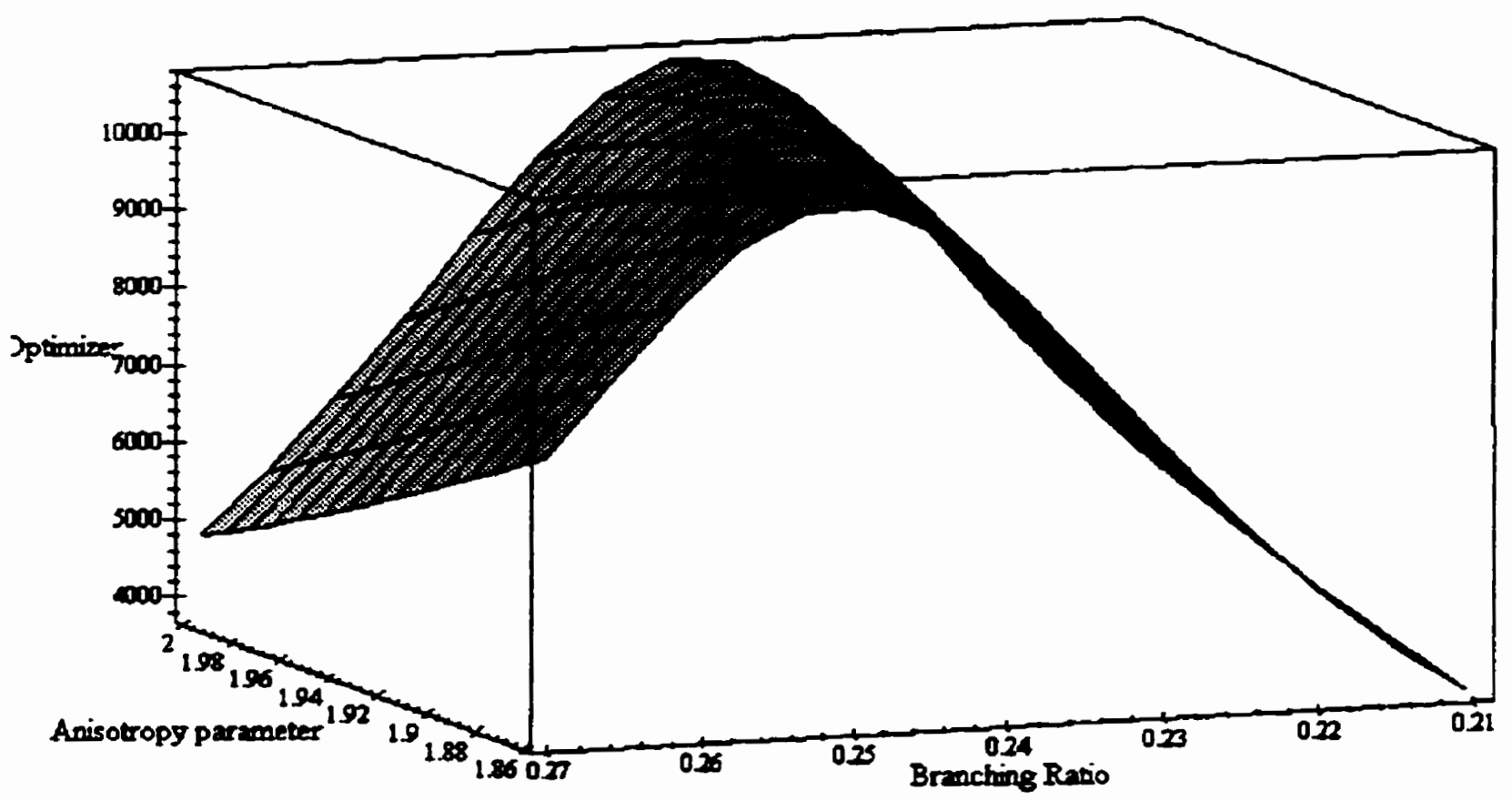

Figure 6.19. 3D plot of the optimizer- or \&-function, for the forward convolution procedure, operated on the averaged experimental spectra measured at $212.5 \mathrm{~nm}$ photolysis wavelength. 

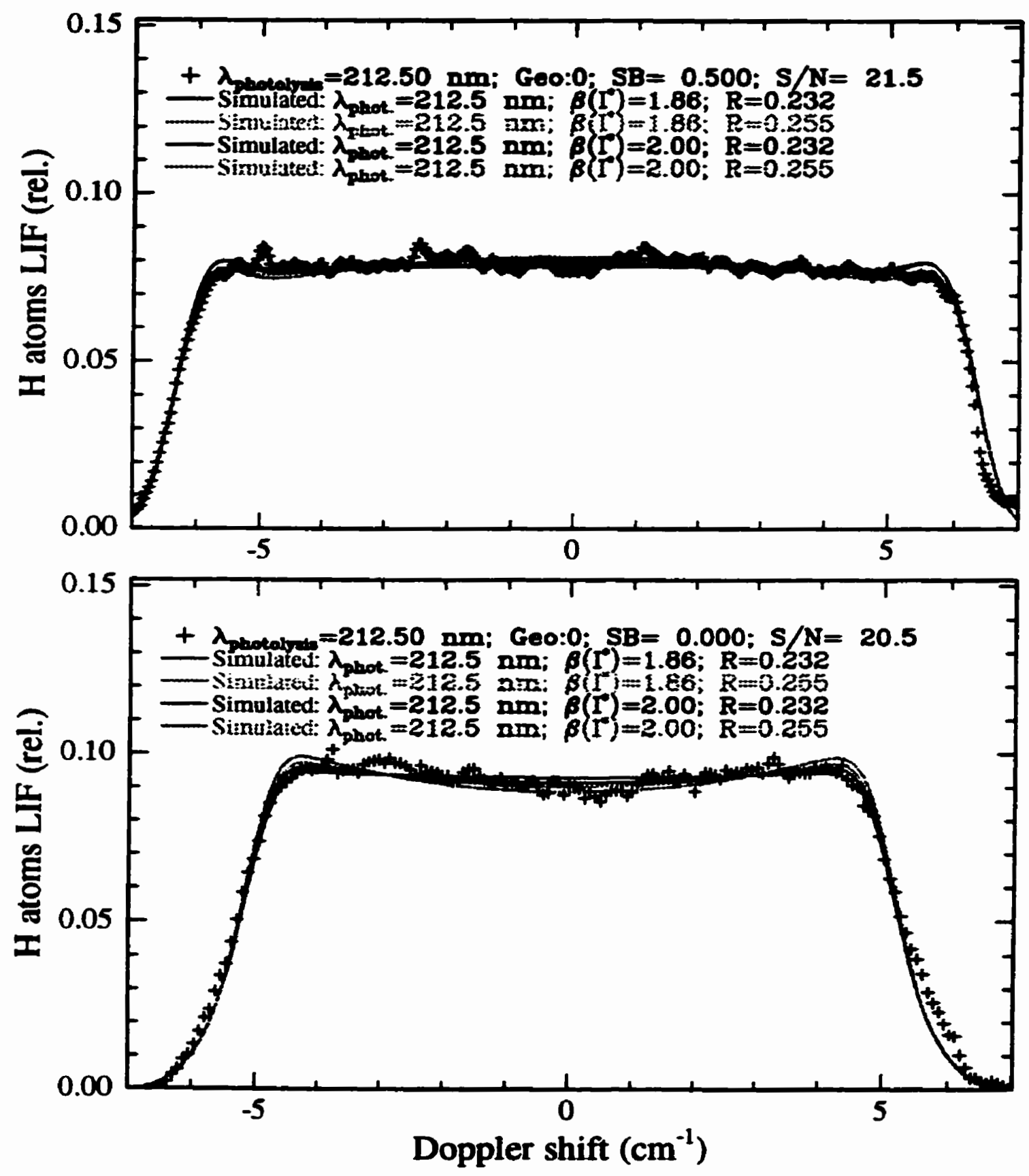

Figure 6.20. Comparison of experimental Doppler profiles. for $212.5 \mathrm{~nm}$ photolysis wavelength, with simulated profiles corresponding to the extremes of the uncertainties on the optimum values of the parameters (table 6.1). 
of the wavelength tuning drive, which contribute in reducing the effective $\mathrm{S} / \mathrm{N}$ from the purely statistical value.

It must be recalled that this symmetrical characteristic of the dissociation dynamics in the A band of $\mathrm{FI}$ was indeed predicted by Mulliken", on the basis of symmetry correlation between atomic and molecular properties. Assuming that Mulliken was right, the anisotropy parameters may be viewed as differing from $\mathbf{2 . 0 0}$ only as a consequence of the imperfection of our experimental measurements (finite $S / N$ and small systematic errors). However, before exploring this possibility further, a question raised in the following section must be answered.

\subsubsection{Test of the method}

A question that need to be investigated is: assuming that the anisotropy parameter $\beta\left(I^{*}\right)$ would in fact be different from, but close to 2.00 , say $\beta=1.80$, will the method used here permit us to distinguish its value from 2.00 ? The answer to this question is provided by a numerical test of the forward convolution procedure described hereafter.

We simulated Doppler profiles, for horizontal and vertical polarization, that would be obtained at $256 \mathrm{~nm}$ photolysis wavelength, if the true dynamical constants were hypothetically equal to: $R=0.500, \beta=1.80$; random fluctuations were added to these simulated Doppler spectra, to mimic a $S / N=25$. These artificial spectra are represented in figure 6.21. It will be noted that these data have a rather smooth appearance, when compared with the experimental Doppler profiles shown in the figure 12 to 20. This is due to the fact that in the experimental Doppler profile, the imperfections related to the correction of the nonlinearity, and the sporadic occurences of slight saturation of detection and amplification electronics, are always present to a small extent. No efforts were invested in simulating these imperfections in the present example, since they are not necessary to illustrate the main point, i.e. the capability to distinguish between $\beta$ values of 2.00 and 1.80 .

The forward convolution procedure was employed to find the optimum values of the dynamical constants for these artificial spectra. The resulting $\xi$-function from this procedure is illustrated in figure 6.22 . Firstly it can be noted that the sharpness of the maximum is much better defined here than it was the case for the experimental data, due to the aforementioned inperfections. Secondly, the maximum of the optimizer function at $R=0.500, \beta=1.80$ indicates that the method used in the present work is able to determine a $\beta$ value relatively close to 2.00 , would it be the case. Consequently, it is asserted that the Doppler spectroscopy technique, coupled with this forward convolution procedure, can indeed distinguish between $\beta=1.80$ and $\beta=2.00$, for $S / N \approx 25$.

As a corollary of the last statement, it can be argued that it is not a limitation of the present method that $\beta=2.00$ is encompassed by our measurement. Granted that the slight imperfections of real data results in somewhat less defined maximum, nevertheless it can provide a reasonable estimate of $\beta$. In the following section, a comparison with results obtained in previous studies will be discussed prior to further develop the analysis of our results. 

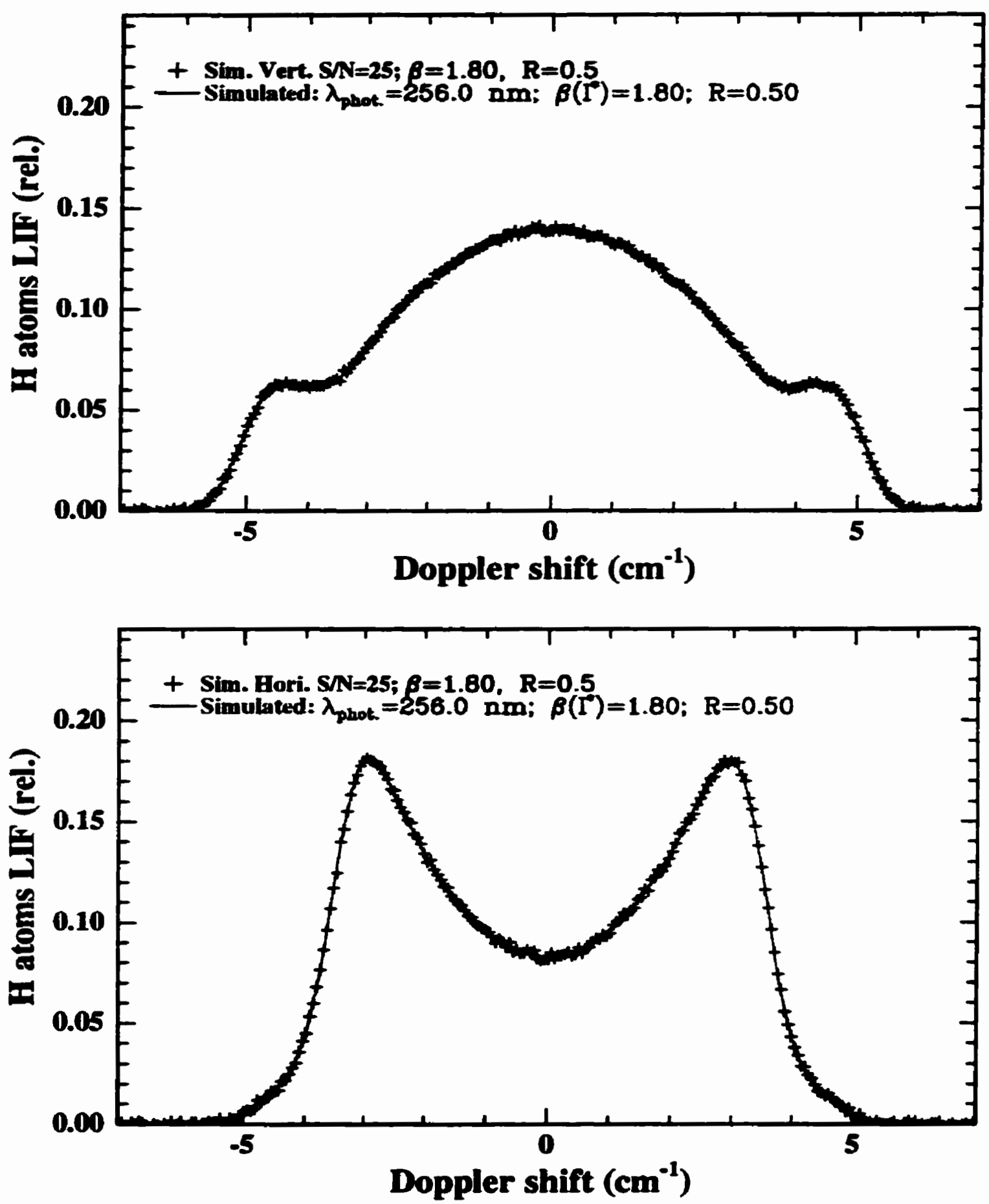

Figure 6.21. Artificial data created by the simulation of Doppler profiles for $256 \mathrm{~nm}$ photolysis wavelength, assuming the hypothetical values of $\beta=1.80$ and $R=0.500$, and adding random fluctuation with an amplitude such as to simulate $S / N=25$. 
Simulated at $256 \mathrm{~nm}$
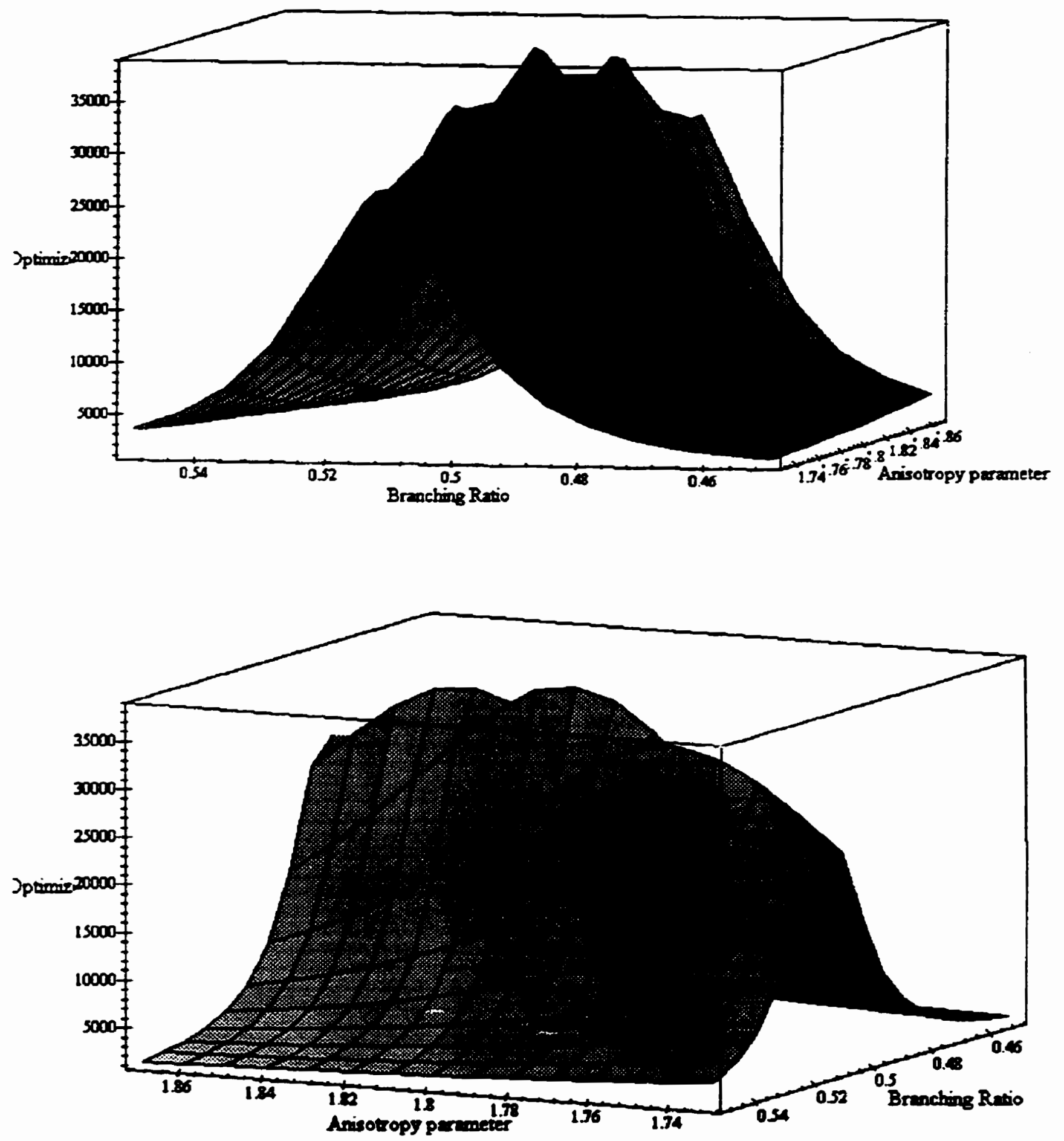

Figure 6.22. Results of the forward convolution procedure used to determine the dynamical constants for the artificial data of agure 6.21. Note that a marked maximum is obtained corresponding to the value of $\beta=1.80$. 


\subsection{Comparative analysis}

\subsubsection{Comparison with previous studies}

In the section 2.3.2 of chapter 2, we already indicated that discrepancies were exhibited among previous studies of photofragment spectroscopy performed on the A band of HI. The experimental observables, determined in the previous section, are now used to establish our position in that controversy. Figure 6.23 exhibits a comparison, for the dynamical constants, between our results and those from previous studies on this topic.

The higher panel of figure 6.23 shows a marked inconsistency between the various results, concerning the anisotropy parameters $\beta\left(I^{*}\right)$ for the excited electronic product. The results from these studies can however be classified into two groups, with regards to this disagreement. One group includes: our experimental results, the result of Xu et al. for $248 \mathrm{~nm}^{5}$ and the result of Schmiedl et al. for $266 \mathrm{~nm}$ photolysis. ${ }^{6}$ The other group includes the results from the experimental study of van Veen et al. $7^{7}$ the theoretical work of Levy and Shapiro, ${ }^{8}$ which was based on the results of van Veen et al., is also plotted to guide the eyes. It is noteworthy that the theory of Levy and Shapiro ${ }^{8}$ was published the same year as the results of the measurement of $\mathrm{Xu}$ et al. for $248 \mathrm{~nm}$, ${ }^{5}$ and consequently, the latter was not considered by the former. The lower panel of figure 6.23 indicates corresponding discrepancies amongst the previous works, with regard to the branching ratios of the electronic products, with the exception that all the the results at the $266 \mathrm{~nm}$ photolysis wavelength overlap within their respective uncertainties.

\subsubsection{Explanation of the discrepancies}

It is demonstrated that the discrepancies are explained by the hypothesis that clusters were present in the molecular expansion used by van Veen et al., ${ }^{7}$ and as a consequence, that their conclusions were tainted by an experimental artifact. The argumentation is based on factual information from $\mathrm{H}$-atom time-of-flight (TOF) measurements.

\section{a) Suspiciously broad TOF peak in the van Veen results}

In chapter 2 , we already observed that the results of van Veen et al. ${ }^{7}$ were in marked disagreement with the results of Xu et al. ${ }^{5}$ and Schmied et al. ${ }^{6}$ After noticing these discrepancies, the experimental studies from which the various results had originated were scrutinized to seek an explanation for the discrepancies. I observed that van Veen et al., who had done their experiment in the early eighties, were using a molecular expansion of pure HI.? During the eighties, a great deal of experimental work has been done on clusters. Now in the nineties, it is a widely accepted fact that a gas of high polarizability, such as $\mathrm{Xe} \sigma \mathrm{K} \mathbf{r}$, expanded in vacuum through a small nozzle $(0.1 \mathrm{~mm})$ from a stagnation pressure of a few atmospheres, has a large propensity to condense. Because $\mathrm{HI}$ is isoelectronic to $\mathrm{Xe}$, and that it possesses a permanent dipole of $\sim 0.4$ Debye, clustering is also favoured. Thus, the experimental conditions under which van Veen et al. ${ }^{7}$ conducted their experiment are prone to clustering, and they must have generated a large fraction of clusters and their vacuum expansion source. 
a)
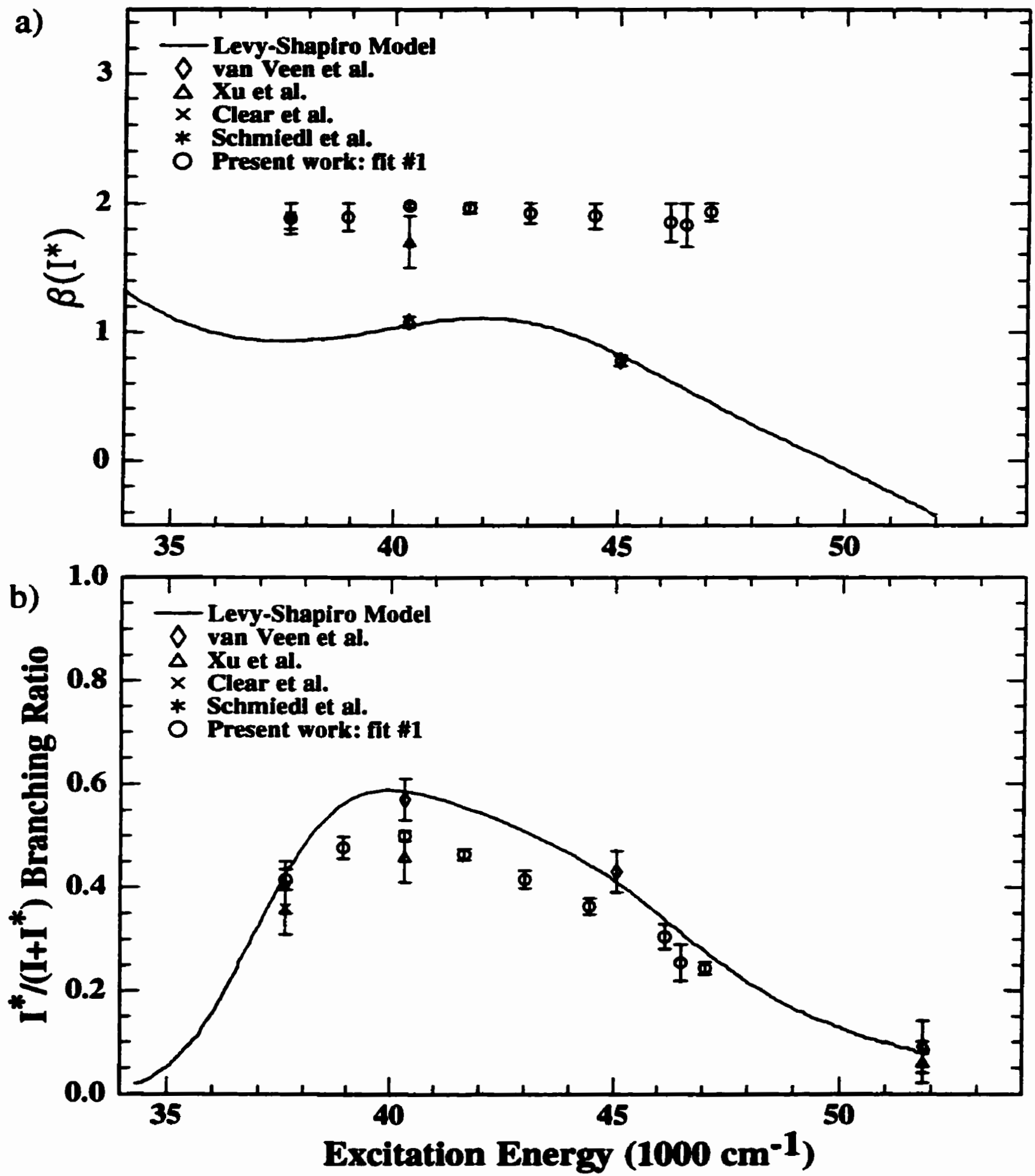

Figure 6.23. Comparing the values of the observables determined in the present study with previous results. for a) the anisotropy parameters., and b) the branching ratio for the electronic products. 

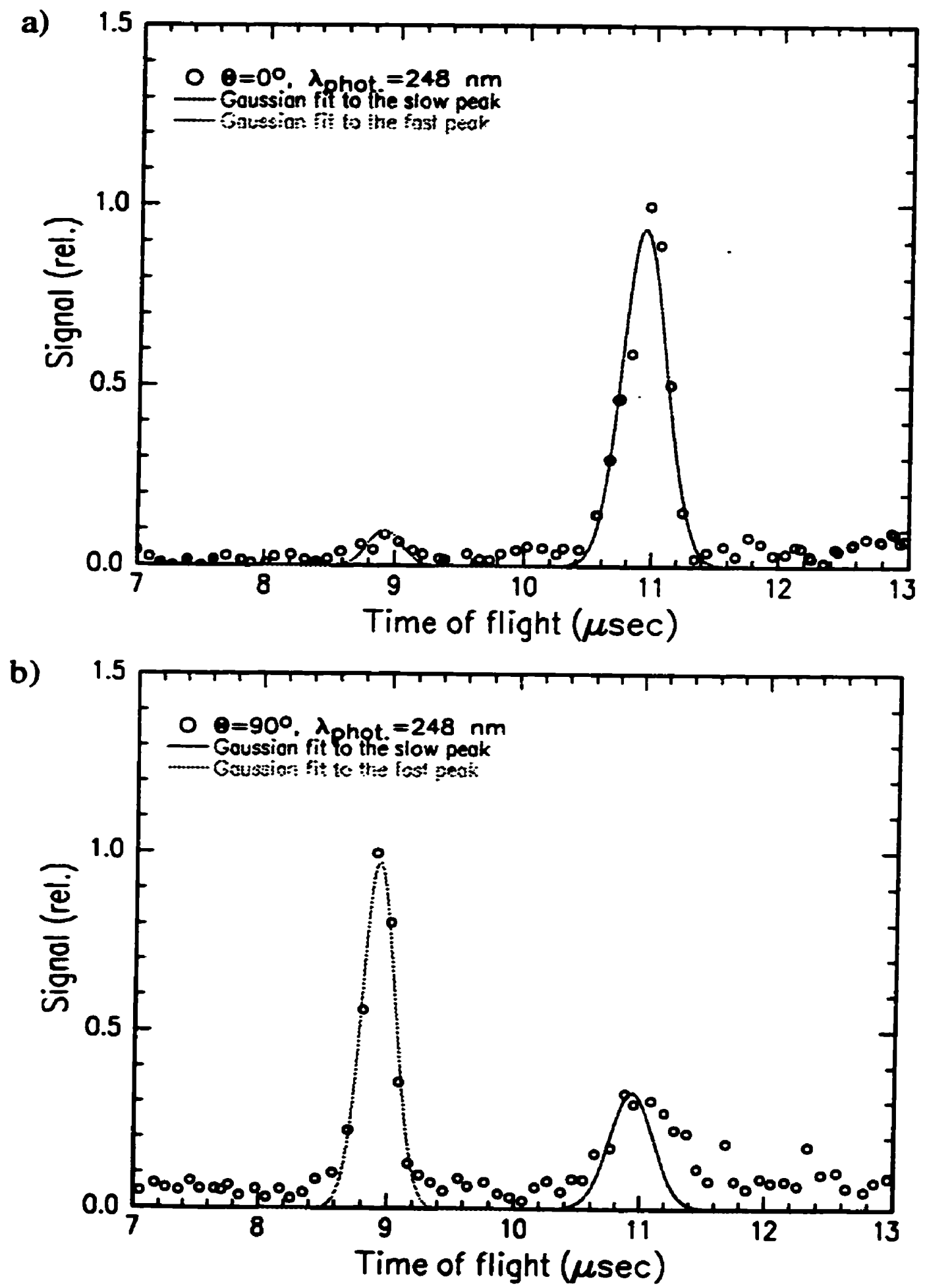

Figure 6.24. Experimental TOF spectra taken from van Veen et al.(ref. 7). a) In the TOF detecting fragments velocities measured along the photolysis polarization axis, the slow H-atom channel dominates the spectrum. b) In the direction perpendicular to the polarization axis, the fast $\mathbf{H}$-atoms are predominant, but an important signal is contributed by the slow channel. Note that the slow channel is significantly broader in the TOF spectrum measured in the perpeodicular direction. 
Once I started to suspect this problem with the experimental results of van Veen et al., an a posteriori analysis of their published H-atom TOF spectra was deemed appropriate. In figure 6.24, the TOF spectra taken from the study of van Veen et al. ${ }^{7}$ are reproduced. In the H-atom TOF spectrum measured in the direction perpendicular to the photolysis polarization, figure $6.24 \mathrm{~b}$ ), it can be seen that the slow H-atom peak, which corresponds to the excited iodine product and arrives at the detector after $11 \mu \mathrm{sec}$ flight time, has a significantly broader width than the corresponding peak measured in the parallel direction that is reproduced in figure 6.24 a).

To illustrate this point more precisely, a Gaussian function was fit to the slow peak in the parallel direction, and a function of identical width and position was scaled and transposed on the slow peak of the perpendicular measurement, and it is shown in the figure 6.24. This operation clearly showed that the peak was broadened toward longer time, or lower kinetic energy. On the basis of this comparison, the author bad suggested that clusters may bave been present in their molecular expansion target, because intracluster collision would result in energy losses, and would broaden the TOF peak toward lower energy. Thus these observations led the author to assume that condensation may have been the cause of the observed discrepancies.

\section{b) Photodissociation dynamics of dimers}

By an opportune coincidence. Zhang et al. ${ }^{9}$ have very recently published the results of an experimental study on the photodissociation dynamics of HI dimers. Their results provides supporting arguments consistent with the present hypothesis. Their experimental observations, discussed below, are supporting our argumentation.

In their work, Zhang et al. "engineered" the molecular expansion such that a minor fraction ( $2 \%)$ of the HI molecules were in the form of dimers, while the remaining were free monomers; by using a mixture of $4-5 \%$ HI in He or Ar diluent, they observed that 1-2\% of the integrated $\mathrm{H}$-atom photofragments signal was caused by the dimer fraction, the rest being clearly produced by monomer, or molecular HI. The results from their highresolution TOF (HRTOF) spectra measured on H-atom fragments are illustrated in figure 6.25 a) and b), for measurement in respectively parallel and perpendicular orientation of the detector, with respect to the linear polarization of the photolysis beam.

In these figures, the lower line, corresponding to the signal observed for the dominant HI monomer, indicates a high degree of symmetry in the dynamics, which corroborates the results of the present study. Moreover, magnification by $X 40$ and by $X 80$ of their signals, also indicated in these figures, have provided enlightening information with regards to the photodissociation dynamics of $\mathrm{HI}$ dimers. The high resolution and high sensitivity of their technique have permitted the observation of mechanisms of energy loss. 
a)

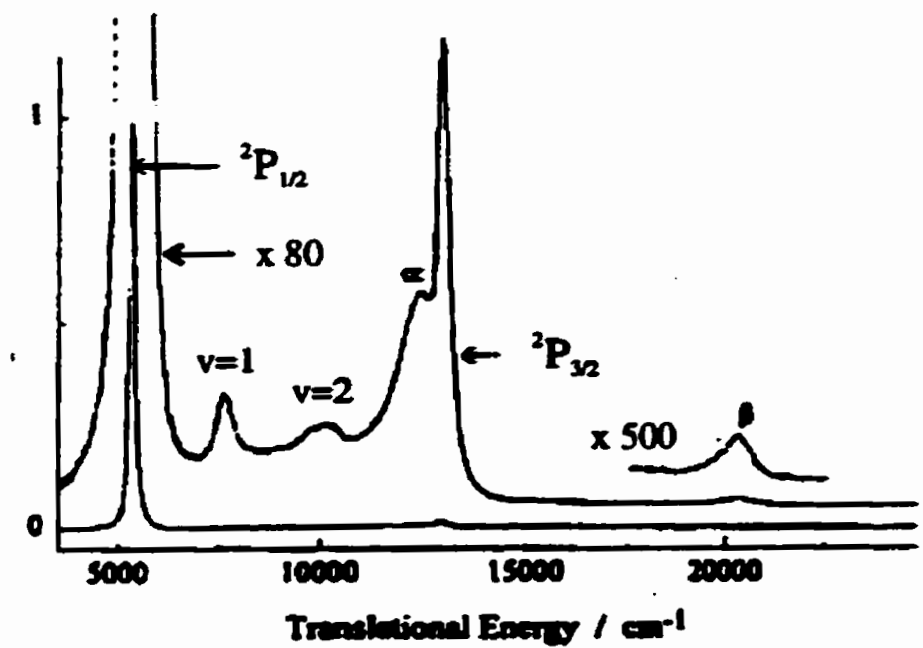

c) Dimer geometry

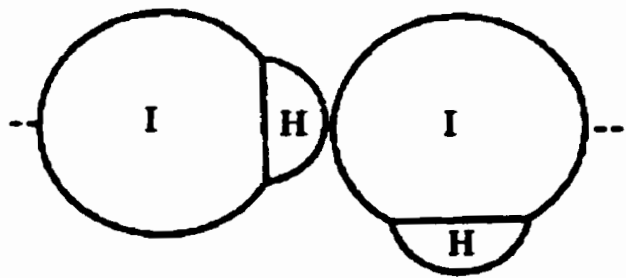

d) Dimer dynamics

b)
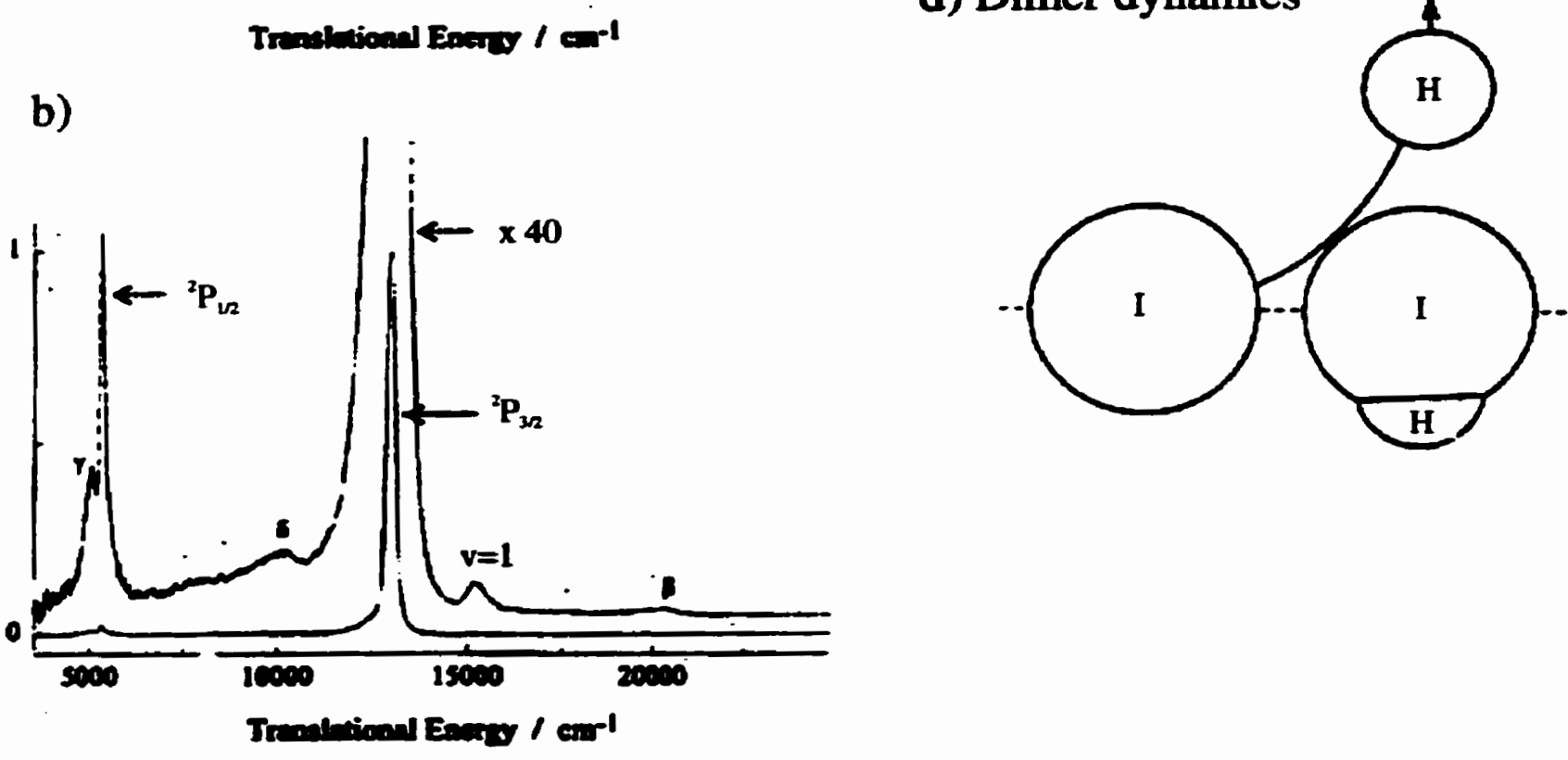

Figure 625. Ilustration of the results from the study of Zhang et al. ${ }^{9}$ on the photodissociation dyamies of HI dimers; a) the HRTOF spectrum measured parallel to the photolysis polarization; b) the HRTOF spectrum measured perpendicular to the polarization; c) equilibrium gecmetry of the dimer derived from their study, d) an illustration of a case of photodissociation dynamics in a HI dimer. which results in the "perpendicular appearance" of an cherwise parallel transition. The essential points that can be derived from their results are: (i) far froe molecules each electronic product is produced by transition of pure symmetry, and (ii) for HI dimers: an energy loss mechanism, caused by intracluster collisions, produces a noticeably broader energy distribution for these $\mathrm{H}$-atom fragments, and also increases the relative yield of the slow $\mathrm{H}$-atoms in perpendicular symmetry, in comparison to the monomer dynamics 
The TOF peaks, labelled $v=1$ and $v=2$ in these figures, correspond respectively to one quantum and two quanta of vibrational excitation imparted by a departing $\mathrm{H}$-atom, to its partner molecule, at the onset of its departure from the dimer. In their paper, Zhang et al.' also resolved rotational energy loss in the H-atom HRTOF spectra, which corresponds to the lost of a quantum of energy by the H-atom, suffered through the intracluster collision and deposited in the rotation of the co-molecule. The observation of rotational and vibrational energy loss in an $H$ atom TOF was a major breakthrough in their work.

\section{c) Supporting evidence for the condensation hypothesis}

Zhang et al.9 observed that photodissociation of HI dimers results in an apparent perpendicular contribution to the differential cross section for the slow $\mathrm{H}$-atom products, in terms of the monomer transition dipole. This apparent perpendicular transition is characterized by a broader energy spread of the kinetics energy of the products, reflected in the spectral width of the TOF peak, which was resolved by their HRTOF method. Note thas this could not have been done with usual TOF method, such as the one used by with van Veen et al. ${ }^{7}$

Figure $6.25 \mathrm{c}$ ) illustrates the equilibrium geometry of the dimer. proposed in the study of Zhang et al.' In figure $6.25 \mathrm{~d})$, a process of dimer photodissociation dynamics is depicted, in which a H-atom that would be produced in a transition of parallel symmetry in the free molecule, is ejected from the dimer in the perpendicular direction as a result of colliding with its partner molecule within the dimer.

The broader TOF features initially drew my attention, and led to the initial formulation of the present hypothesis in order to explain the discrepancies at hand. The high sensitivity and resolution of the HRTOF technique used by Zhang et al. ${ }^{9}$ allowed them to elucidate the aforesaid microscopic process responsible for the perpendicular contribution to the slow $\mathrm{H}$-atom channel. They clearly demonstrated that this process results from intermolecular collision, occurring upon photodissociation of a $\mathrm{HI}$ molecule in $\mathrm{HI}$ dimers. Moreover, they resolved the energy loss structure in the HRTOF spectra. and they were able to show that the quantum of energy loss corresponds to rotational and vibrational excitation of the undissociated partner of the dimer by the departing H-atom.

From the measurements of Zhang et al. 9 at the $266 \mathrm{~nm}$ photolysis wavelength, the value of $\beta\left(I^{*}\right)=1.98 \pm 0.02$ can be obtained; this corresponds essentially to a pure parallel transition for the production of electronically excited iodine atoms. This last study was performed with the awareness that $\mathrm{HI}$ has a high propensity to cluster in the vacuum expansion of a supersonic jet. This value of $\beta\left(I^{*}\right)$ agrees with our observation, i.e. in the absence of cluster, the photodissociation dynamics of $\mathrm{HI}$ produces excited iodine atoms through transition of pure parallel symmetry, and ground state iodine atoms through transition of perpendicular symmetry. The combination of these facts support our argument regarding the presence of clusters being indicated by: (i) broadening of the energy distribution toward lower energy, and (ii) contribution from the slow peak in perpendicular geometry. 


\section{d) Understanding the discrepancies amongst previous results}

Prior to van Veen et al. ${ }^{7}$, the first absorption continuum of the HI molecules was understood as made up of the $Q$ group of states introduced by Mulliken: $\left\{{ }^{\top} \Pi_{0 .,}{ }^{3} \Pi_{1}, ' I I\right\} . "$ The results from the TOF photofragment spectroscopy studies made by Clear et al. ${ }^{10}$ at the $266 \mathrm{~nm}$ wavelength photolysis, and the Doppler spectroscopy measurements by Schmied et al. ${ }^{6}$ at $266 \mathrm{~nm}$ both found agreement with this central assertion of Mulliken's derivation.

To understand discrepancies and agreements between the various studies mentioned above, one must notice that the molecular temperature in the supersonic jet experiment of Clear et al. ${ }^{10}$ was $205 \mathrm{~K}$, and for the gas cell temperature in the experiment of Schmiedl et al., ${ }^{6}$ it was between 230 and $300 \mathrm{~K}$; at these temperatures, there is no clustering. No estimate of the molecular beam temperature was provided by van Veen et al., but the characteristic of their specific setup (nozzle diameter: $0.1 \mathrm{~mm}$, quiescent pressure: $1.5-2.0$ atm.) allows for an estimate of their beam temperature as being in the range of $10-30 \mathrm{~K}_{i}$ in these conditions, a pure HI produces clusters efficiently.

The effect of clustering in the molecular expansion, that explains the large values of $\beta\left(1^{*}\right)$ in the work of van Veen et al. ${ }^{7}$, is also likely to affect the values of $R$. On the basis of the results from Zhang $e t$ al., ${ }^{9}$ it can be seen that the intracluster collisions between a fast departing $\mathrm{H}$-atom and a $\mathrm{HI}$ molecule also affects the branching ratio between the electronic product channels. The different photodissociation dynamics occurring in HI clusters is suggested to explain the discrepancies among the branching ratio measurements illustrated in the lower panel of figure 6.24. Note that the theoretical work of Levy and Shapiro ${ }^{8}$ was fitted to the experimental results of van Veen et al. ${ }^{7}$

The study of Zhang et al. ${ }^{9}$ at $266 \mathbf{n m}$ photolysis substanciates our explanation of the discrepancies amongst the results obtained in the various study on the photolysis of $\mathbf{H I}$ in the $A$ band, which stipulates that each electronic channel are characterized by a pure type of transition symmetry, the curves without magnification in the HRTOF spectra of figure 6.25 clearly indicate this fact; their observation provides crucial arguments to understand these discrepancies amongst the results in previous works. By resolving inelastic intracluster collision processes initiated by photodissociation, they clearly showed that the $\mathrm{H}$-atoms energy distribution of the photoproducts was broader for HI clusters than for single HI molecules. The combination of these facts confirms our hypothesis that clustering significantly affected the results of van Veen et al. ${ }^{7}$, and it suggests that the Mulliken's model discussed earlier in this work may be appropriate.

\subsubsection{Second determination of the observables}

The global picture obtained from the above consideration of the dynamics of HI photodissociation indicates that electronically excited products emerge from a pure parallel transition. In our results, the anisotropy parameters encompass the value of 2.00 , within the experimental uncertainties, at all photolysis wavelength investigated. 
The observation that the uncertainties of the observables determined by the forward convolution technique are correlated, combined with the results of the comparative analysis, suggests an enhancement of the precision with which $R$ can be obtained. If indeed the anisotropy parameter is absolutely known, the uncertainty in the remaining parameter $R$, determined by the forward convolution routine, should be reduced.

Consequently, we returned to the last iteration of the forward convolution procedure, and consider the array of the optimizer $\xi$-function that corresponded to the value $\beta=2.00$, i.e. which effectively constrains the parameter $\beta\left(I^{*}\right)$ to that value. To identify the optimum value of $R$, a quadratic fit was applied to the corresponding array of the numerical $\xi$-function; the fit was restricted to the region in the neighbourhood of the maximum of the smoothly varying numerical $\boldsymbol{\xi}$-function. The uncertainties were determined in relation to the statistical $S / N ;$ a typical result of this operation is presented in figure 6.26 . The assumption that the optimizer values always varies smoothly was verified by increasing the grid size over a constant domain of the parameters. Table 6.2 shows that the optimization of $R$ for $\beta=2.00$ either reduces the uncertainties, or keeps it the same for excellent data $(S / N>30)$.

The quality of the parameters determined in this second procedure has been evaluated through a graphical comparison, illustrated in figure 6.27 to 6.35. In these graphs, the experimental Doppler profiles are plotted along with simulated profiles calculated for the optimum value of $R$ and its minimum and maximum values, defined by the corresponding uncertainties on this optimum value. It will be observed that the optimization of $R$, for $\beta$ constrained at 2.00, resulted in simulated profiles matching the experiment very well. Visual inspection of the graphical comparisons for this second determination of $R$, figure 6.28 to 6.36, reveals that this constrained fit effectively discriminates against the slight systematic errors present in the experimental profiles, which is a positive indication of the appropriateness of the model.

The systematic errors affecting the Doppler profiles are well-understood, and they may result from the following imperfections of our experimental apparatus. (I) A lack of wavelength reference from the $I_{2}$ spectrum, corresponding to the VUV probe around $+5.5 \mathrm{~cm}^{-1}$ Doppler shift, affects the correction of the wavelength nonlinearity at that energy. (II) A slight saturation of the LIF detection, resulting from the finite dynamic range and creeping saturation of the electron multiplier devices, may also have sporadically affected some measurements. (III) Imperfection of the HI LIF background subtraction may create some noise around $+1 \mathrm{~cm}^{-1}$ Doppler shift. where the strongest spectral feature of the HI LIF is observed, especially for spectra of poor ( $S / N<10)$ S/N ratio.

\subsubsection{Summary on the determination of the observables}

The observables characterizing the photodissociation dynamics at nine photolysis wavelengths were determined from Doppler profile measureinents, by using a forward convolution. A comparative analysis of the present and previous works has demonstrated that the excited electronic products from HI photolysis in the A band are 


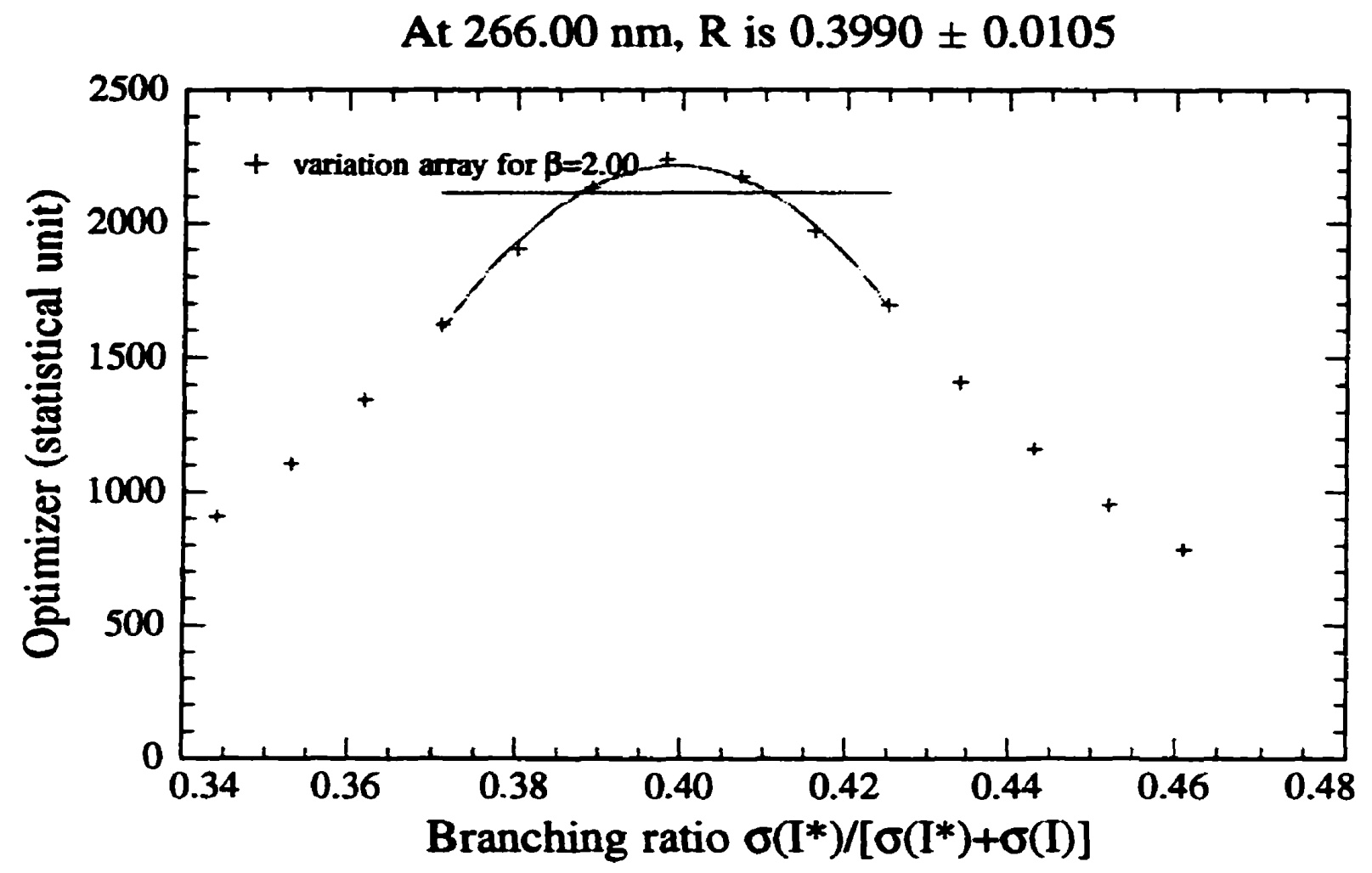

Figure 6.26. Example of $R$ determination for the constrained value $\beta=2.00$, at $266.00 \mathrm{am}$ photolysis wavelength.

Table 6.2 Observables of $\mathrm{HI}$ photodissociation resulting from constraining $\beta\left(I^{*}\right)=2.00$ in the fit

\begin{tabular}{|c|c|c|}
\hline Wavelength (nm) & $\left(\sum<\{S / N\}^{2}\right)^{1 / 2}$ & $R=\sigma\left(I^{*}\right) /\left\{\sigma\left(I^{*}\right)+\sigma(I)\right\}$ \\
\hline 266.0 & 22 & $0.399 \pm 0.010$ \\
\hline 257.0 & 25 & $0.459 \pm 0.011$ \\
\hline 248.0 & 18 & $0.500 \pm 0.010$ \\
\hline 240.0 & 32 & $0.459 \pm 0.007$ \\
\hline 232.5 & 24 & $0.407 \pm 0.009$ \\
\hline 225.0 & 36 & $0.353 \pm 0.005$ \\
\hline 216.7 & 31 & $0.285 \pm 0.009$ \\
\hline 215.0 & 6 & $0.254 \pm 0.047$ \\
\hline 212.5 & 25 & $0.239 \pm 0.006$ \\
\hline
\end{tabular}


produced in pure parallel symmetry, while the ground state iodine atoms are produced from perpendicular transitions. Moreover, the knowledge of the photodissociation of HI dimers, brought about by the study of Zhang et al.,' provided solid arguments supporting this intermediate result.

In the light of the facts presented above, the author asserts that the conclusions arrived at by van Veen et al. should be reconsidered; it was demonstrated that the mixed symmetry type, observed by van Veen et al. at $248 \mathrm{~nm}$ and $222 \mathrm{~nm}$ photolysis wavelength.' ${ }^{7}$ are the results of experimental artifacts caused by cluster formation in the vacuum expansion. In the theory of Levy and Shapiro, the nonadiabatic effects were introduced to explain the results of van Veen et al., ${ }^{7}$ but since their results were affected by experimental artifacts, their analysis was misled.

Consequently, we reconsidered the forward convolution procedure, but with the constraint of $\beta\left(I^{*}\right)=2$. This extra piece of information obtained from the comparative analysis, permitted a slight yet important reduction of the uncertainties for the branching ratio of the electronic products, as showed in figure 6.27 . The fact that the constrained fit effectively discriminates against small systematic errors, as illustrated by the comparison of figures 6.28-6.36, is a positive indication that this second determination of the branching ratios is more accurate, and therefore more likely valid.

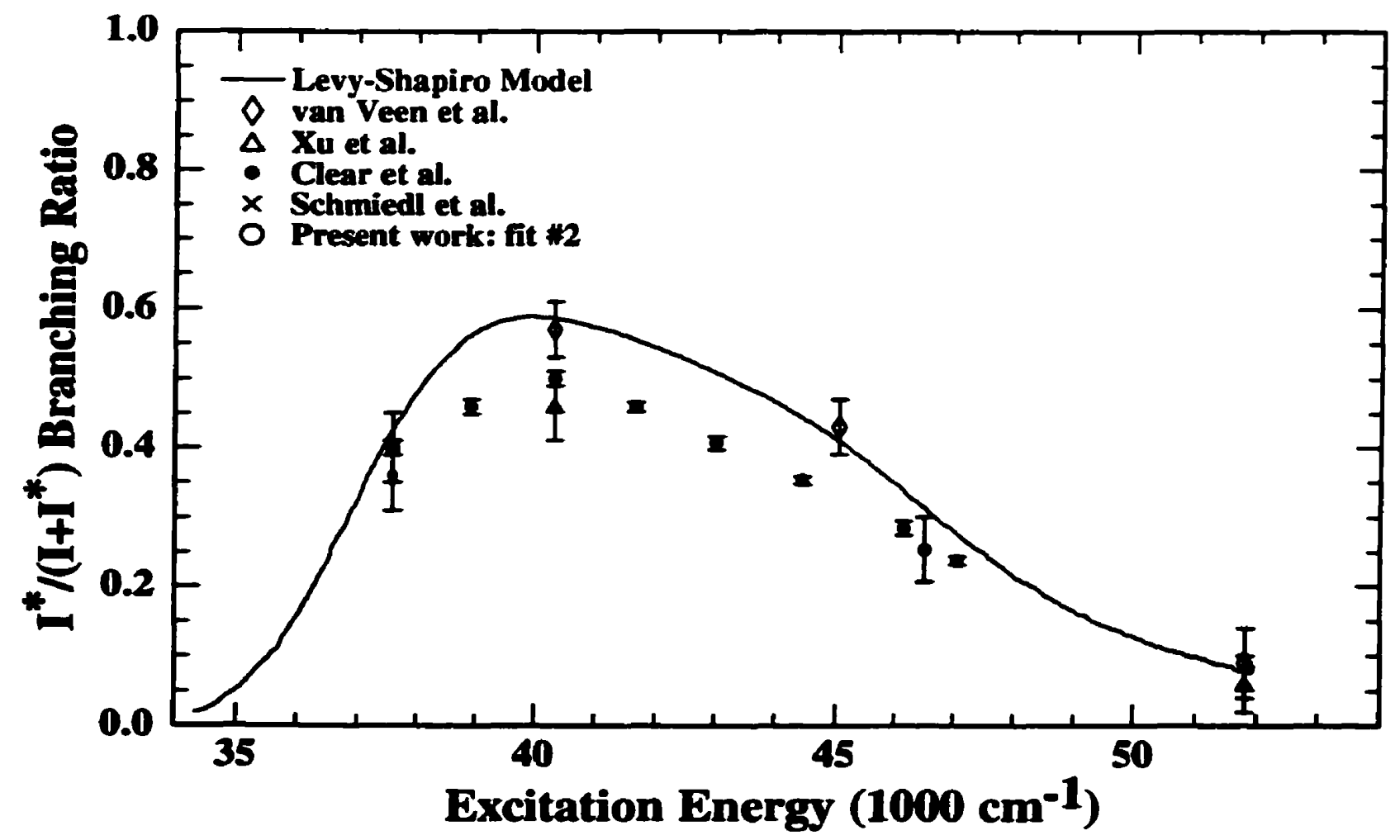

Figure 6.27. Comparison of our second branching ratio determination. with the results from previous studies. 

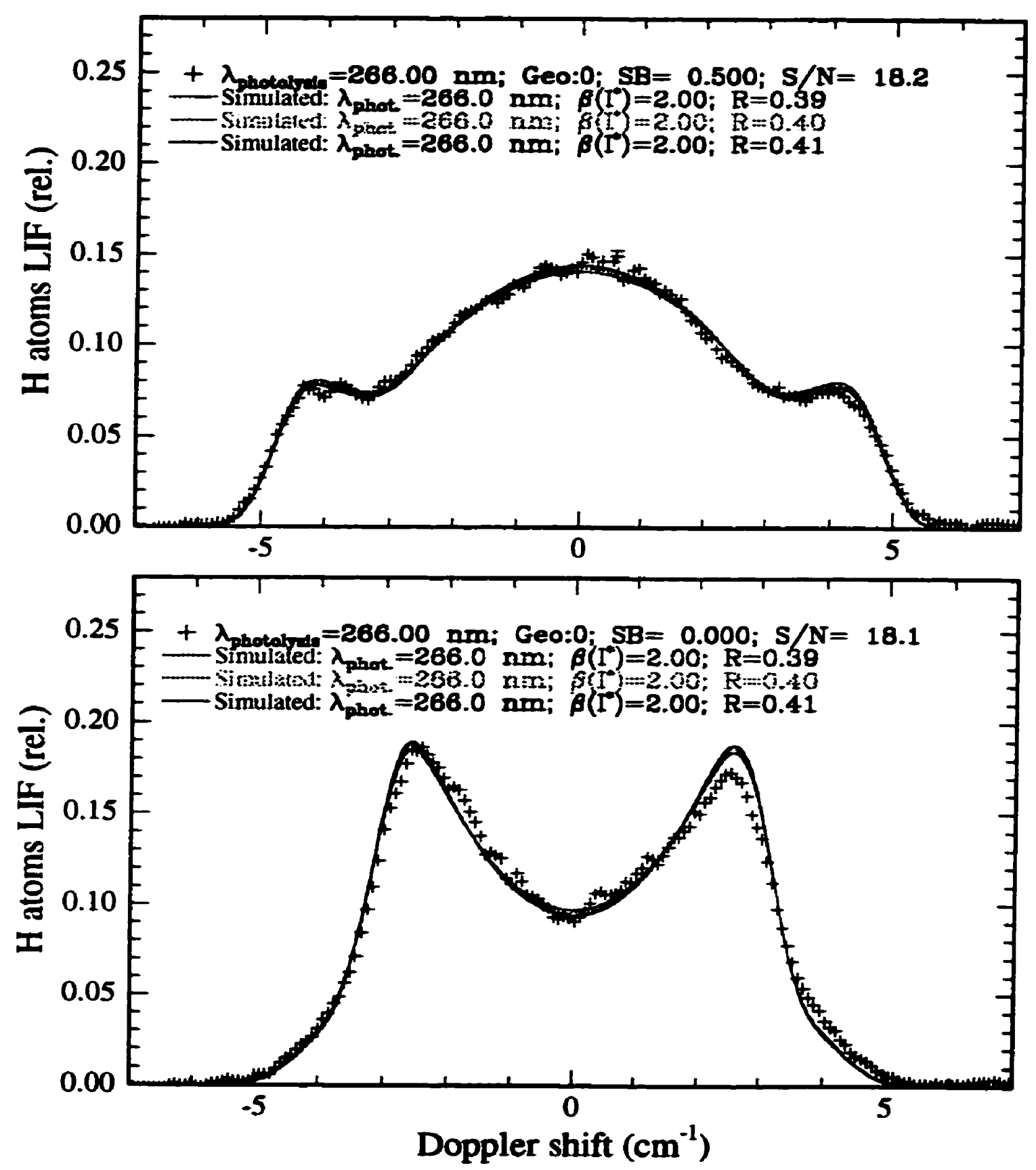

Figure 6.28. Comparison of simulated and experimental profiles for the $2^{\text {ac }}$ parameter fit at $266.00 \mathrm{~nm}$ photolysis. 

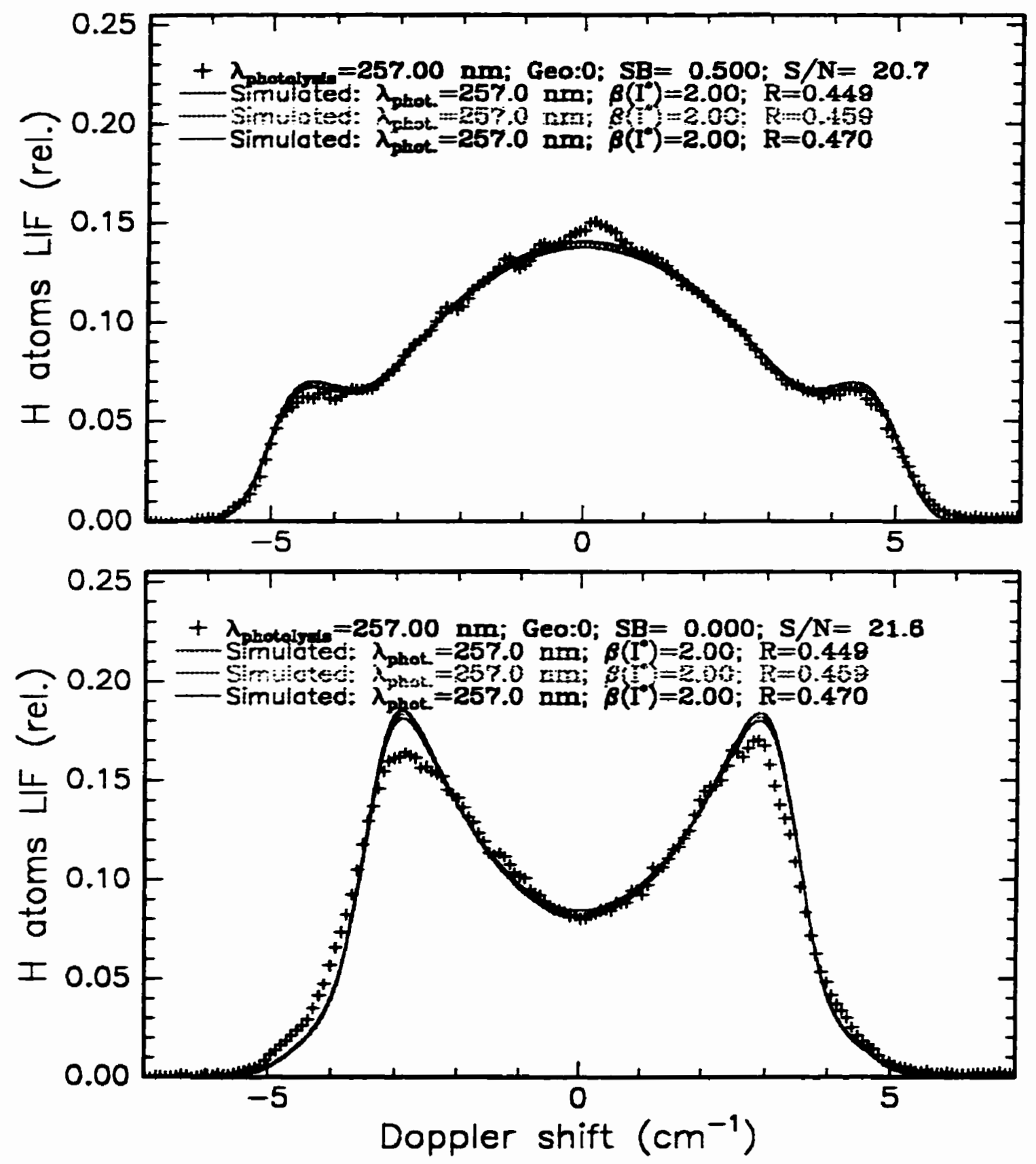

Figure 6.29. Comparison of simulated and experimental profiles for the $2^{\infty}$ parameter fit at $257.00 \mathrm{~nm}$ photolysis. 

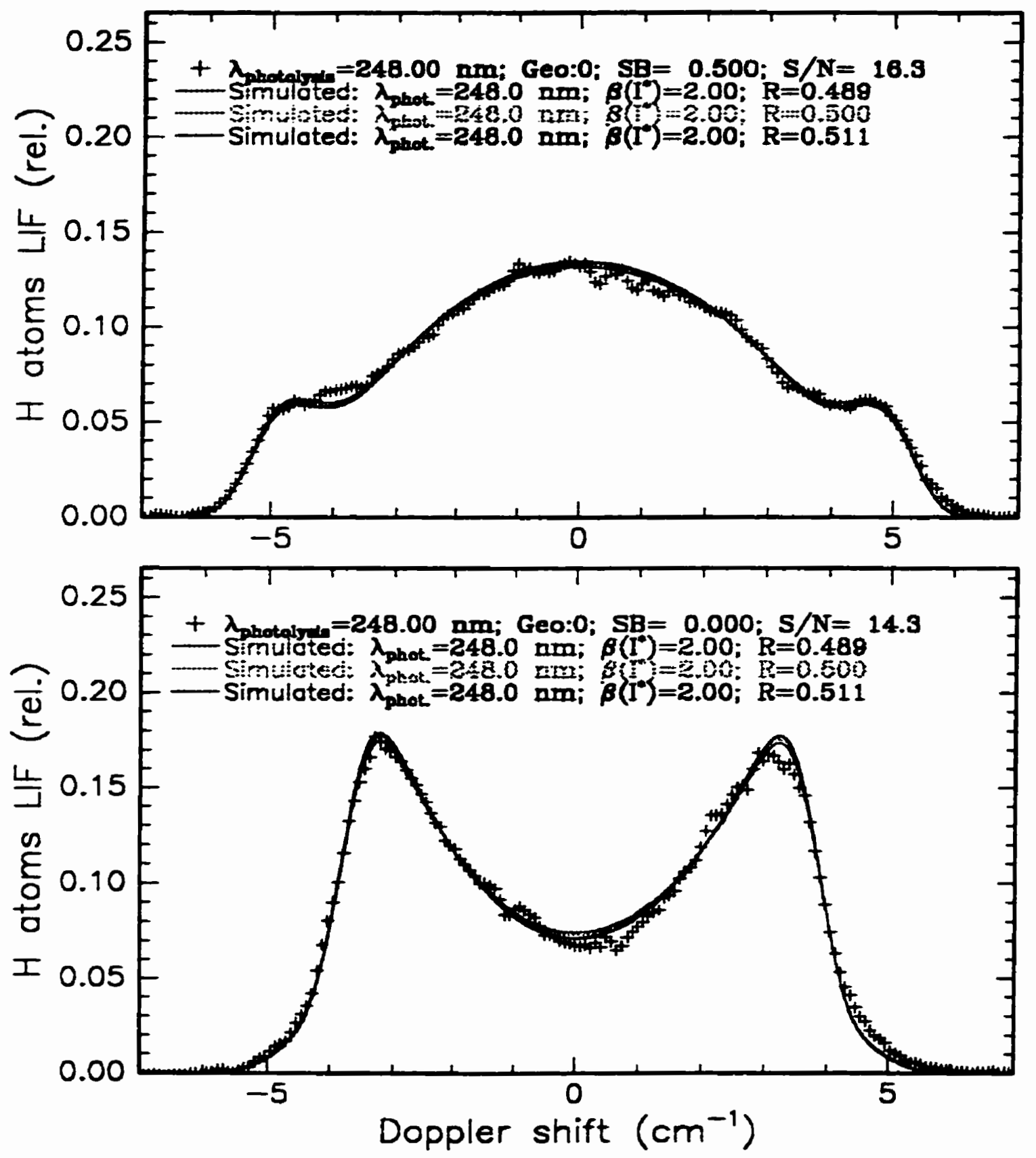

Figure 6.30. Comparison of simulated and experimental profiles for the $2^{\text {ed }}$ parameter fit at 248.00 am photolysis. 

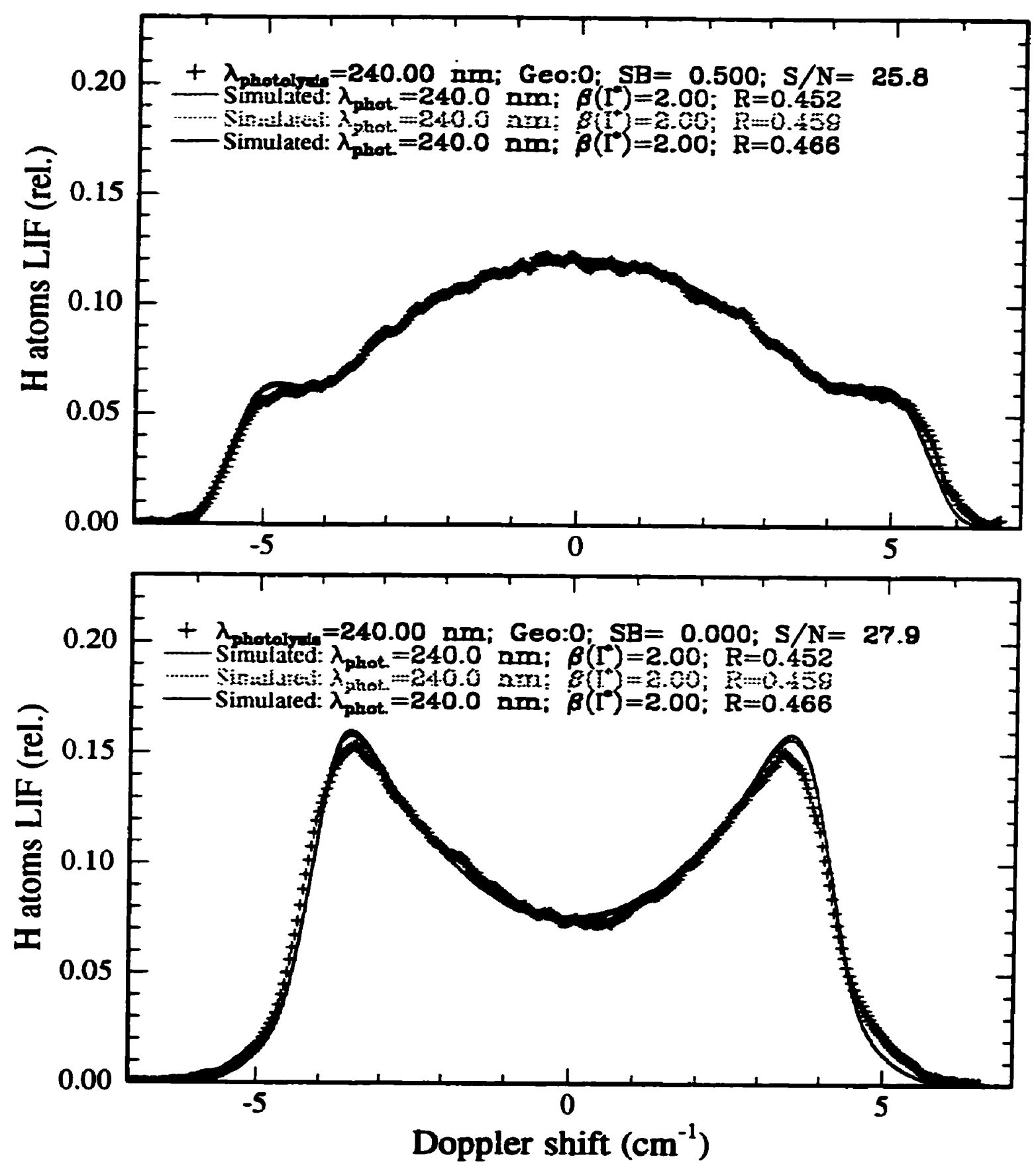

Figure 6.31. Comparison of simulated and experimental profiles for the $2^{\text {sed }}$ parameter fit at $240.00 \mathrm{~nm}$ photolysis. 

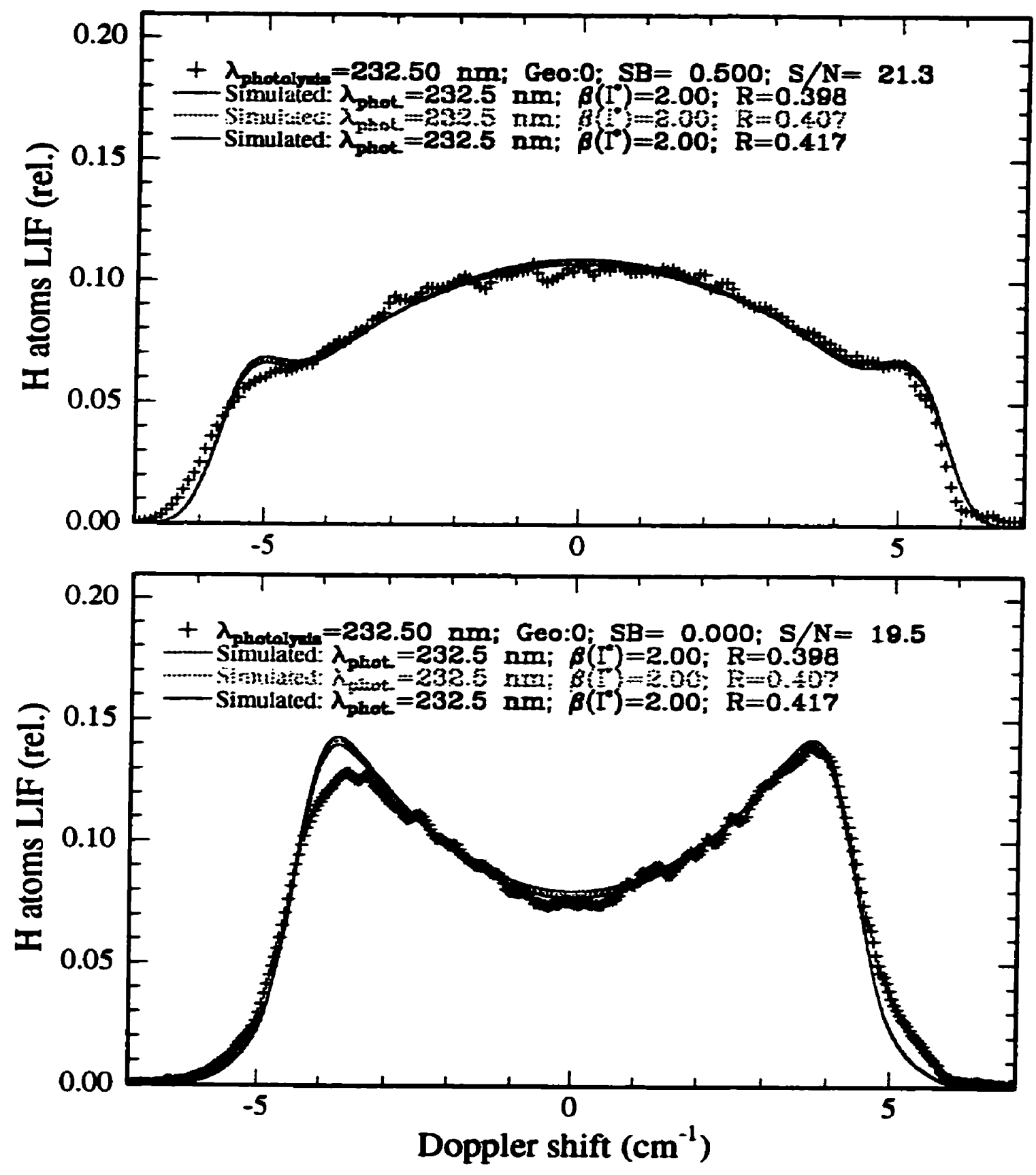

Figure 6.32. Comparison of simulated and experimental profiles for the $2^{\text {od }}$ parameter fit at 232.50 nm photolysis. 

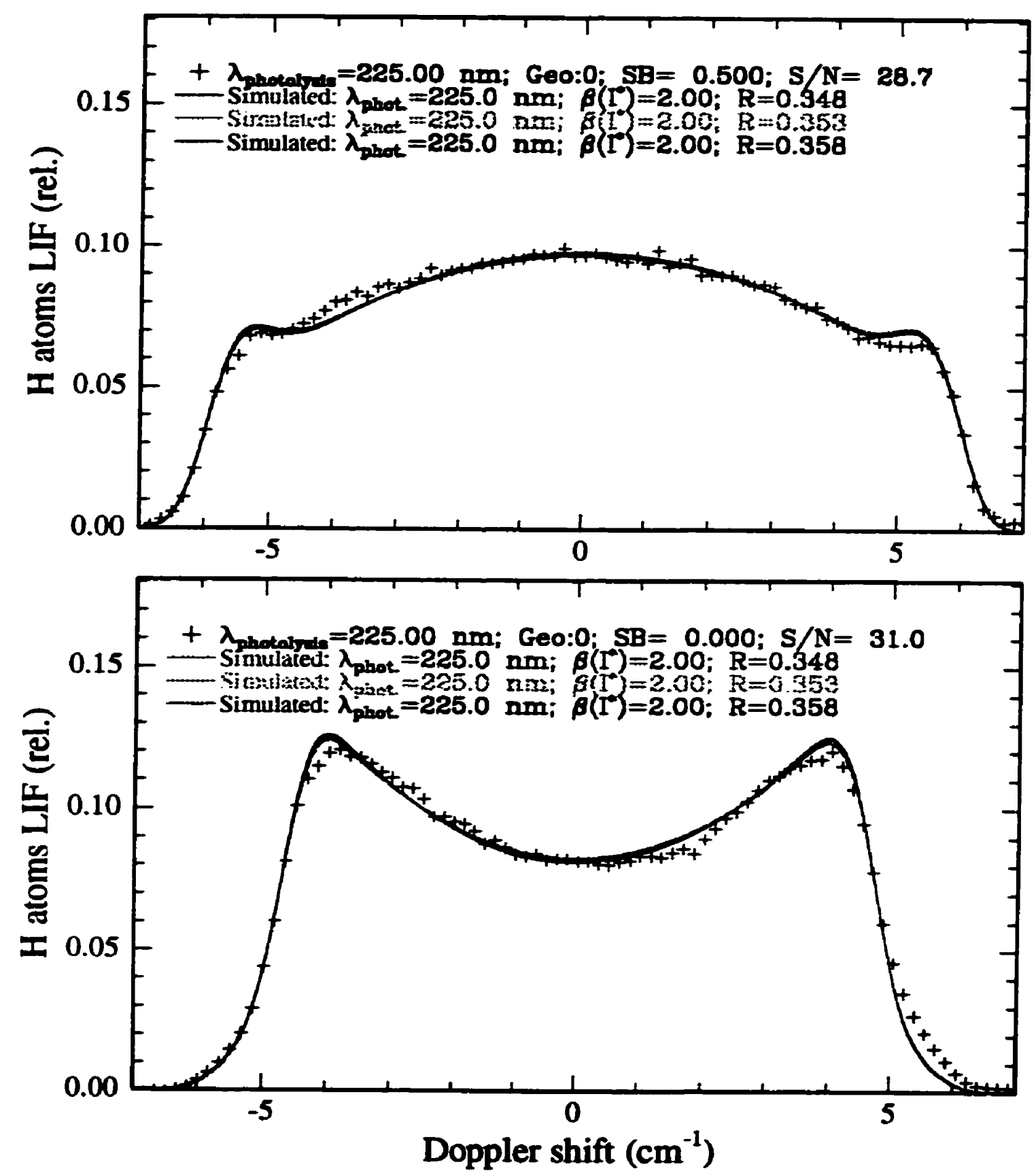

Figure 6.33. Comparison of simulated and experimental profiles for the $2^{\text {od }}$ parameter fit at $225.00 \mathrm{~nm}$ photolysis. 

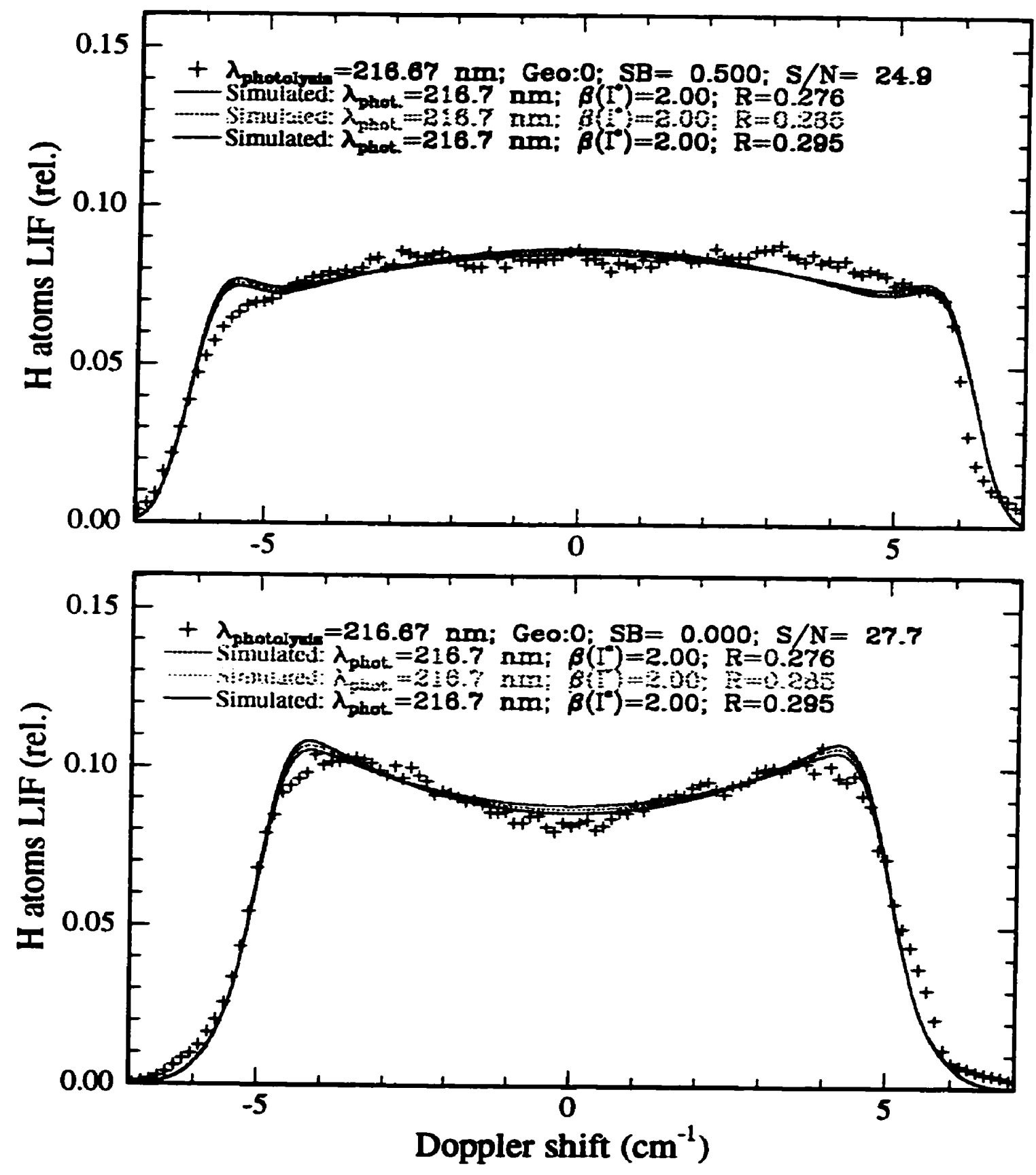

Figure 6.34. Comparison of simulated and experimental profiles for the $2^{\text {od }}$ parameter fit at 216.67 nm photolysis. 

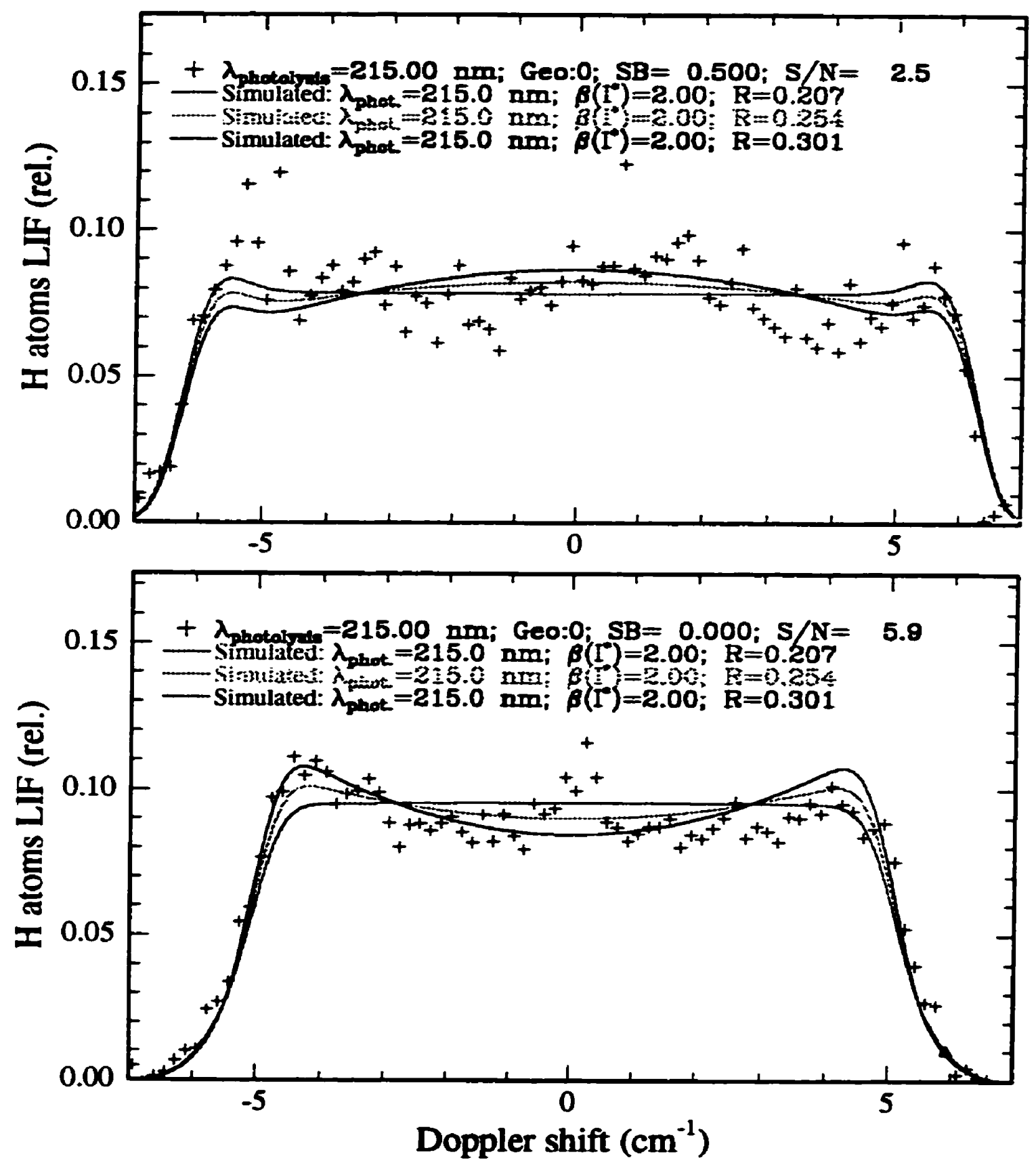

Figure 6.35. Comparison of simulated and experimental profiles for the $2^{\text {od }}$ parameter fit at $215.00 \mathrm{~nm}$ photolysis. 

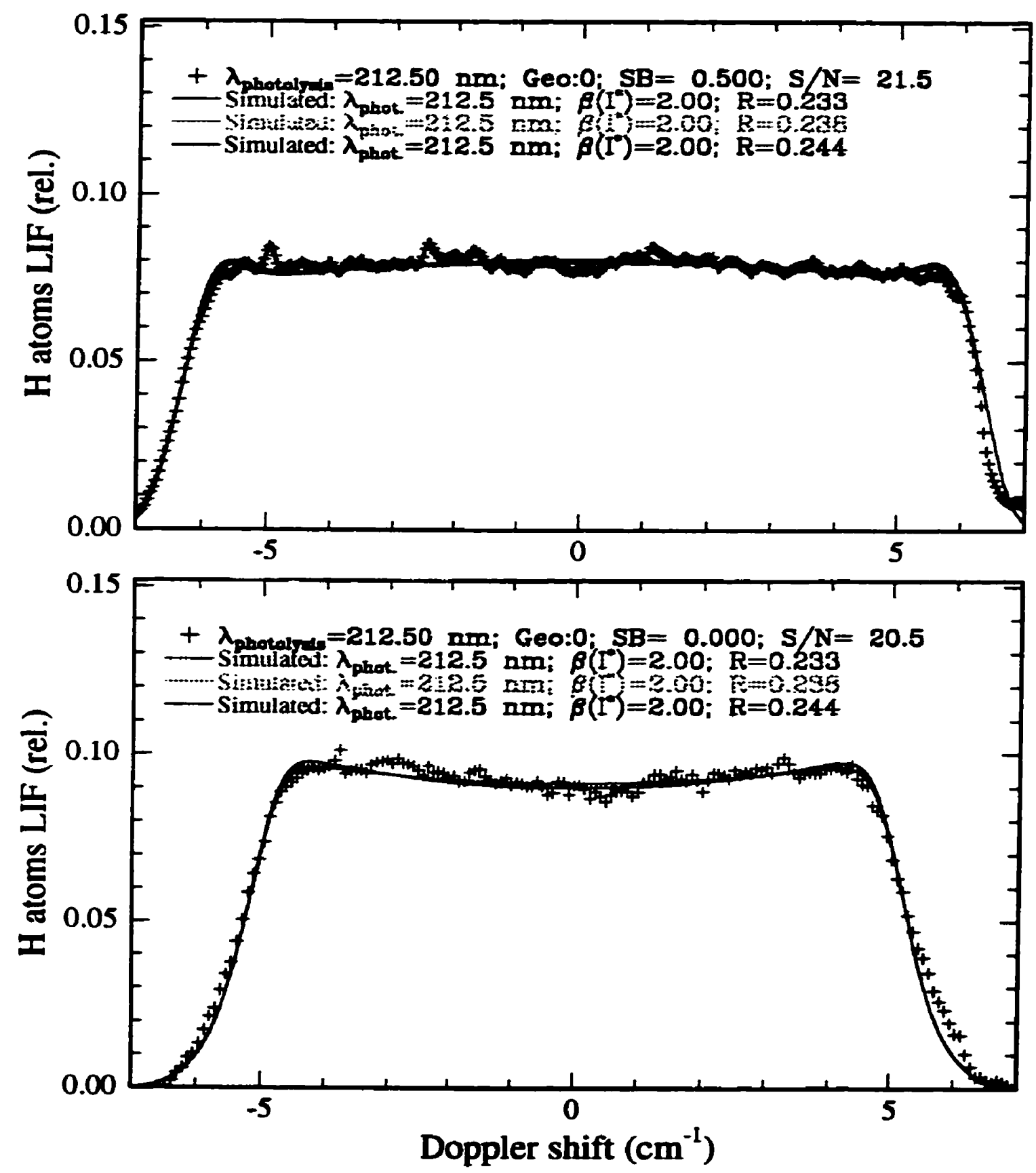

Figure 6.36. Comparison of simulated and experimental profiles for the $2^{\text {met }}$ parameter fit at 212.50 nm photolysis. 


\subsection{Quantum mechanical analysis}

At this point, the most important result obtained in the present study is the experimental observation regarding the symmetry of angular distribution for the electronic product channel. This suggested that the photodissociation dynamics of HI may possibly be appropriately described by Mulliken's model. "It must be recalled that this model was deemed appropriate until the results from van Veen et al.' were reported. Because the model of Levy and Shapiro $^{8}$ was based on the results from van Veen et al.,' the valididity of their conclusion is doubtful, to say the least. Nevertheless, because the model of Levy and Shapiro for HI expressed concerns with regards to nonadiabatic effects in the photodissociation process, they are briefly addressed here.

As discussed in chapters 2 and 3, respectively, the electronic structure and the dissociation dynamics for HI excitation in the $\mathbf{A}$ band is a dynamical system in which one least expects to encounter nonadiabatic transitions during dissociation. The symmetry of the electronic structure in the first absorption continuum is such that the only possible non-adiabatic interaction playing a role in the photodissociation dynamies is the weak Coriolis effect. Also, the relatively small average value for rotational quantum number of the parent molecules in our experiment $\left(<\mathrm{J}>=0.4\right.$ at $T_{\max }=30 \mathrm{~K}$ ) precludes the need for considering non adiabatic interaction altogether.

\subsubsection{Model for the dynamics of $\mathrm{BI}$ photodissociation in the $\mathrm{A}$ band}

The present quantum mechanical calculation attempted to use the most conventional assumptions usually applied in this field, i.e. (1) the use of the Franck-Condon approximation (FCA) to model the electronic transition, and (2) the assumption of adiabatic dissociation on a single repulsive potential energy curve (PEC). If this model had not satisfactorily performed in representing the experimental observations, further refinements could bave been developed. Since this first attempt met with success, its validity may be assumed, until further experimental tests confirm or disprove its general validity.

Due to the narrow range of internuclear distance sampled by the ground state nuclear wavefunction, the FranckCondon approximation (FCA) can be expected to be reasonably valid to model the electronic transition. Another assumption made here is that a repulsive PEC can be represented by the analytical form of equations (6-3).

$V(R)=A \exp \left[-B\left(R-R_{e}\right)\right]+C$

Three parameters, A, B and C, characterize each state, but the $C$ parameter is determined by the asymptotic product channel, hence it is known by definition. An additional parameter associated with each PEC is the average transition dipole, denoted $\mathrm{M}_{\mathbf{2}}$ in this chapter. Note that the representation of a PEC by the expression (63) can be assumed to be valid in the Franck-Condon region, for which the parameters are optimized. while its validity is uncertain beyond that range of internuclear distances. The latter point is of limited concern to us, since 
optical excitation of the molecule only involves that range of internuclear distance for which the ground vibrational state wavefunction is not zero, i.e. the Franck-Condon region. These assumptions are summarized under the label: direct adiabatic photodissociation in the FCA.

\subsubsection{Application of the model: from TACS to PACS}

The total absorption cross section (TACS) measured by Ogilvie', combined with our experimental branching ratios $R$, allows for the determination of the partial absorption cross section (PACS) for each product. The latter quantities are inputs in the quantum mechanical calculation leading to PEC determinations, which in turn will permit a calculated extrapolation for $R$ values beyond the range of our measurements. Hence the relations (6-4) link our experimental product branching ratios $R$ with the TACS data of Ogilvie ${ }^{1}$ to obtain the PACS for each product channel.

PACS(excited state product: $\left.I^{*}\right)=R \cdot T A C S$

PACS(ground state product: $I)=(1-R) \bullet$ TACS

TACS measurements and $\mathbf{R}$ measurements were taken at different frequencies; however this represented a small inconvenience, due to the relatively high density of the energy grid over which the TACS data were available, and to its smooth energy dependence. This important point is illustrated in figure 6.37, where the TACS data of Ogilvie' are reproduced, along with the extrapolated TACS values at the experimental energies of our $R$ values. These extrapolated TACS values are symbolized by circles in figure 6.37, and they are listed in table 6.3. The reciprocal operation could be applied to the branching ratios, i.e. a spline function can extrapolate between the experimentally determined values of $R$ at the energies values for which TACS values were measured. However. it is preferable to derive these extrapolated $\mathbf{R}$ values from a rigorous quantum mechanical model based on the determination of PEC for the dissociative states, as will be done through this section.

Table 6.3 Extrapolation of TACS from Ogilvie at our experimental frequencies.

\begin{tabular}{|c|c|}
\hline Wavenumber $\left(\mathrm{cm}^{-1}\right)$ & TACS $\left(1 \mathrm{~mol}^{-1} \mathrm{~cm}^{-1}\right)$ \\
\hline 37594 & $57.28 \pm 1.15$ \\
\hline 38911 & $94.64 \pm 1.89$ \\
\hline 40323 & $139.86 \pm 2.80$ \\
\hline 41667 & $178.40 \pm 3.57$ \\
\hline 43011 & $203.65 \pm 4.07$ \\
\hline 44444 & $219.34 \pm 4.39$ \\
\hline 46153 & $217.05 \pm 4.34$ \\
\hline 46512 & $215.41 \pm 4.31$ \\
\hline 47059 & $208.64 \pm 4.17$ \\
\hline
\end{tabular}




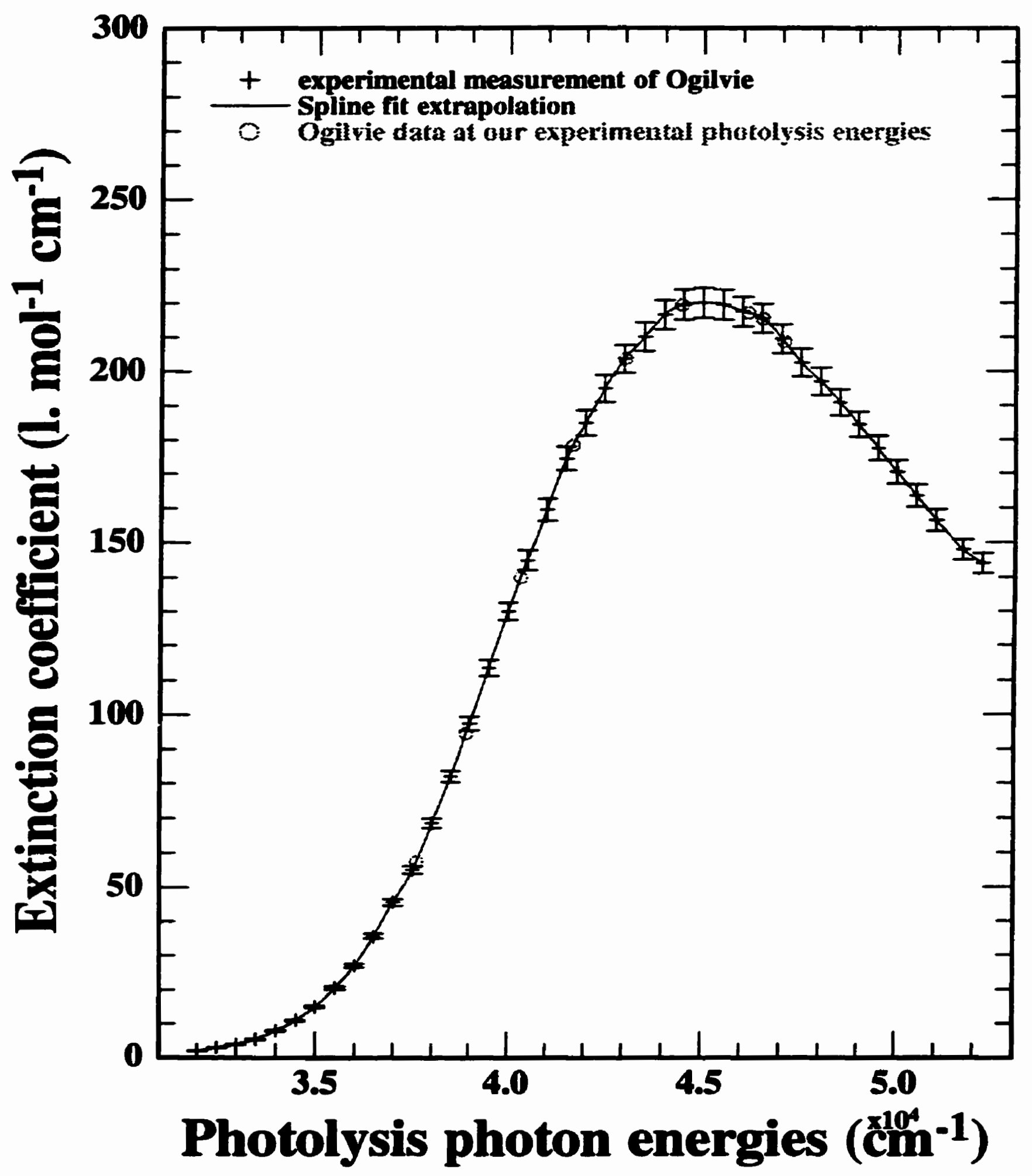

Etgure 6.37. TACS data from Ogilvie (ref. 1), along with a spline fit to extrapolate TACS values at our photolysis energies; the extrapolated data points are indicated by circles and listed in table 6.3 . 
Once the values of the $R$ and the TACS are determined over a set of common energy values, the relations (6-4) can be employed to determine the PACS for each product, since TACS = PACS(I*) + PACS(I). These determinations were performed at the energies of our $\mathbf{R}$ measurements, for which the experimental values of the PACS $\left(I^{*}\right)$ are given in table 6.4. Uncertainties on the TACS values of Ogilvie ${ }^{1}$ are quoted $2 \%$, while our $R$ values also have $2 \%$ nominal uncertainties; thus the consequent uncertainties of the PACS are approximately $3 \%$.

Table 6.4 Experimental PACS for the I* product, calculated from tables 6.2 and 6.3 .

\begin{tabular}{|c|c|}
\hline Wavenumber $\left(\mathrm{cm}^{-1}\right)$ & PACS $\left(I^{*}\right)\left(1 \mathrm{~mol}^{-1} \mathrm{~cm}^{-1}\right)$ \\
\hline 37594 & $22.85 \pm 1.03$ \\
\hline 38911 & $43.48 \pm 1.86$ \\
\hline 40322 & $69.93 \pm 2.80$ \\
\hline 41667 & $81.87 \pm 2.81$ \\
\hline 43011 & $82.93 \pm 3.61$ \\
\hline 44444 & $77.47 \pm 2.69$ \\
\hline 46153 & $61.90 \pm 3.32$ \\
\hline 46512 & $54.74 \pm 11.22$ \\
\hline 47059 & $49.76 \pm 2.25$ \\
\hline
\end{tabular}

It should be noted that the measurements of Ogilvie were made at $300 \mathrm{~K}^{\prime}$ for which the rotational population distribution is different from the one present in a free jet expansion at few tens of degree Kelvin. The effect of temperature on continuum absorption spectra were investigated for $\mathrm{Br}_{2}$ experimentally by $\mathrm{Passchier}^{12}$ et al. and explained theoretically by Le Roy ${ }^{13}$ et al.; excellent agreement between model and experiment was obtained for the range of $300-700 \mathrm{~K}$. That study illustrated the theoretical model, which explains the temperature dependence of the absorption cross section as primarily due to a corresponding change in the vibrational and rotational population of the ground state, and a consequent change in the overlap of the radial nuclear wavefunctions, between the ground and the excited states.

By inspection of the change in vibrational population at two temperatures, an upper limit for the corresponding change in the TACS can be ascessed for HI. The ratios of the populations for the first two vibrational levels, $P(v=1) / P(v=0)$ at $300 \mathrm{~K}$ and at $25 \mathrm{~K}$ are respectively: $10^{-5}$ and $10^{-6}$. Consequently less than $0.1 \%$ change is estimated for the TACS in this temperature range, owing to the large vibrational quantum of the ground state $\left(2309.5 \mathrm{~cm}^{-1}\right)^{17}$ in $\mathrm{HI}$ molecule.

There is an additional effect of remperature on the ground vibrational state, due its slight J-dependence ${ }^{14}$. Ogilvie ${ }^{1}$ examined this effect for $\mathrm{HI}$ and a comparison for $\mathrm{J}=0$ and $\mathrm{J}=4$, corresponding to $\mathrm{T}=0$ and $\mathrm{T}=300 \mathrm{~K}$ respectively, shows a negligible difference in the vibrational wavefunction of ground state for these two temperatures, as 
illustrated in figure 6.38. Alsa, the use of $300 \mathrm{~K}$ data for TACS and 10-30 K data for $\mathrm{R}$ is not a cause of concern in the present work. As a consequence of the above considerations, the effect of temperature on the TACS and on the PACS is asserted to be insignificant within the accuracy of our experimental data.

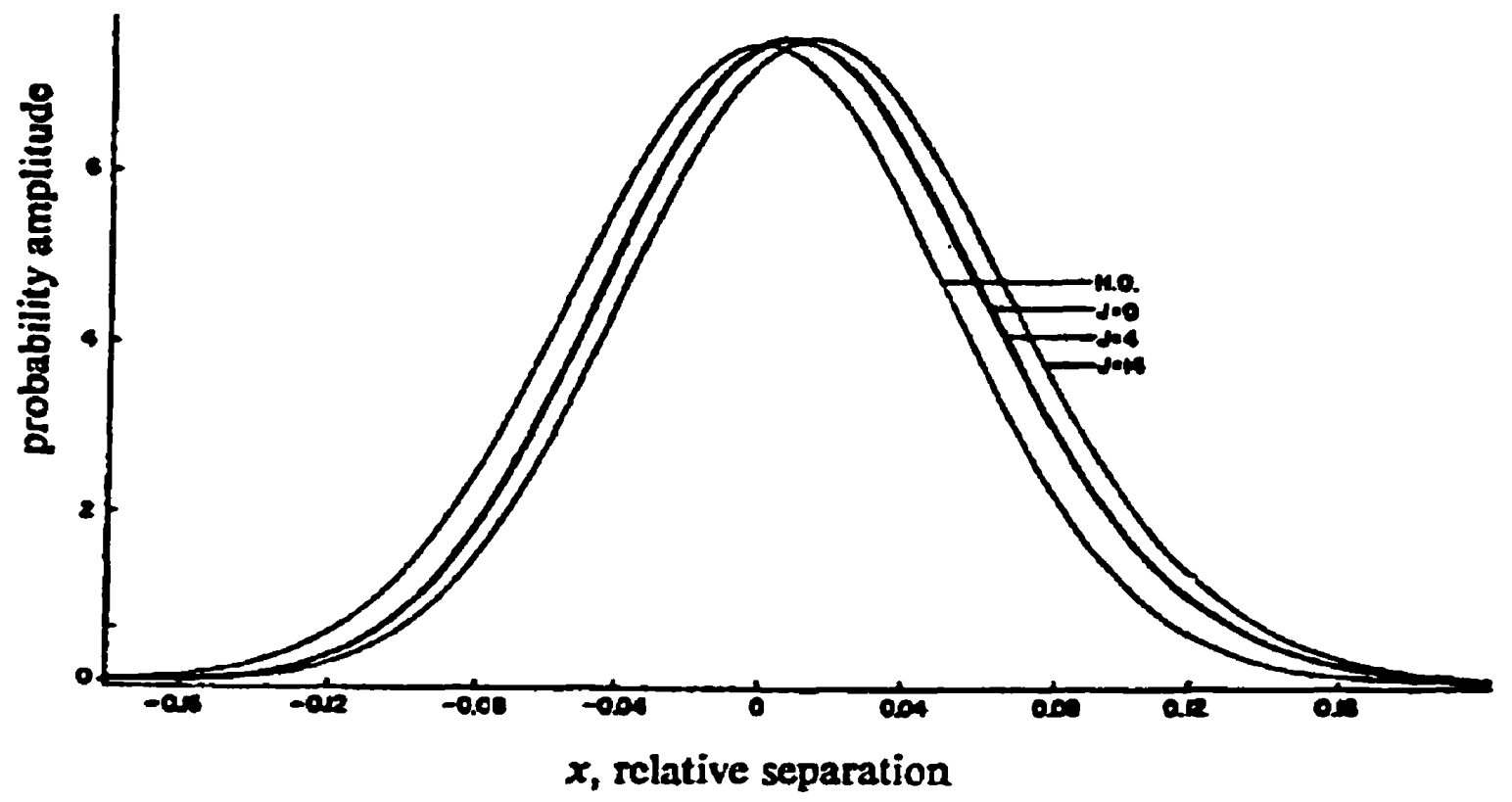

Figure 6.38. Vibrational probability amplinude function for HI in v=0, of the ground electronic state. HO: harmonic oscillator. anbarmonic oscillator for $\mathrm{J}=0,4$ and 14 are shown (taken from ref. 1 ).

Once the PACS are determined, the quantum mechanical (QM) determination of the PEC remains to be executed. Conceptually, the QM determination of a PEC for an excited state provides an operational relation between partial absorption cross section by that state (PACSS) and the PEC of the ground electronic state, on the basis of which the dissociation dynamics can be predicted for an arbitrary photolysis photon energy. A concise description of the computational method is now delineated, after which we shall report the specifics of the procedures that were performed and the results that were obtained.

\subsubsection{Description of the method for PEC determination}

To determine the parameters carrespanding to the dissociative PEC that best correspond to the PACSS, a forward convolution routine was designed. For equation (6-3), only the parameters $A$ and $B$ are free to vary, while $C$ is determined by the product channel. Ranges of trial values were selected for $A$ and $B$ parameters, for which a grid array was defined: each array's entry corresponds to a pair of parameter values, for which a PEC was calculated and the Schrobinger wave equation (SWE) was solved. The wavefunction solution, in conjunction with the ground state wavefunction, was used to calculate a simulated PACSS(A, B). The simulated PACSS(A, B) was statistically normalized to the experimental PACSS, and the optimization procedure was formulated in terms of their differences. 


\section{i) the ground state}

The PEC of the ground state for HI has been recently calculated by Coxon and Hajigeorgiou ${ }^{15}$ from a compilation of experimental data, as described in chapter 2. Their result represents the best PEC available for the ground state of HI. This very accurate potential can be used in conjunction with the program LEVEL $6.0^{16}$ from Robert J. Le Roy to obtain exact numerical radial wavefunction for the ground state. In a slightly more approximate but straightforward method, one can apply the formula of Herman, Tipping and Short ${ }^{14}$ (HTS) to obtain a close form expression for the J-dependent radial wavefunctions of the ground state, based on the parameters of a Dunham potential. These HTS anharmonic wavefunctions are plotted in figure 6.38, where it is apparent that the Jdependence is negligible for $\mathrm{J}=0$ to $\mathrm{J}=4$. The validity of this wavefunction was evaluated ty substitution in the SWE, in which the potential of Coxon and Hajigecrgiow ${ }^{15}$ was used, and by comparing the left and right side terms of SWE. A relative agreement better than $0.5 \%$ was observed, which was deemed sufficient for our purpose, so the HTS wavefunction was used below.

\section{ii) the excired state}

The excited PEC was investigated using the analytical form of equation (6-3). In equations 6-3, the value of the $C$ parameters distinguishes two asymptotic products: $\mathrm{C}=0$, which corresponds to the ground state product channel, and $C=7603.15 \mathrm{~cm}^{-1}$, which is the spin-arbit energy of an excited iodine atom. The values of $A$ and $B$ determined the shape of the PEC for each excited dissociative state and the associated radial wavefunction, as a consequence of the SWE. The radial wavefunction must be obtained by solving the SWE for each set of PEC characterized by the parameters $\{A, B, C\}$. The SWE is expressed by (6-5), where $E_{\text {a }}$ is the energy of the molecule w. r. t. the dissociated fragment at rest in their respective ground state, and $\chi$ is the radial part of the nuclear wavefunction.

$$
\begin{aligned}
& \left.\frac{\partial^{2}}{\partial r^{2}}+k^{2}(r)\right] \phi(r)=0 ; \phi(r)=\frac{\chi(r)}{r} ; k^{2}(r)=\frac{2 \mu}{\hbar^{2}}\left[E_{\mathrm{v}}-V(r)\right] \\
& \frac{\partial \phi}{\partial r}=f ; \phi=\int d r f ; \quad(6-6) \\
& \frac{\partial f}{\partial r}=-k^{2}(r) \int d r f \quad(6-7) \\
& f_{n+1}=f_{n}-k^{2}\left(r_{n+1 / 2}\right)\left(\partial r_{n}\right)^{2} \sum_{n^{\prime}=0}^{n} f_{n^{\prime}} ; f_{n}=f\left(r_{n}\right) ; \partial r_{n}=r_{n+1}-r_{n} \\
& \rho\left(E_{0}\right)=R_{0}\left(8 \mu c / h E_{v}\right)^{1 / 2}
\end{aligned}
$$


The SWE of equation (6-5) considers only the radial problem, since the effect of the electronic degrees of freedom are implicitly included in the average transition dipole. Using the substitution (6-6), the last equation is rearranged into an integro-difierential equation (6-7), which is subsequently solved for the first radial derivative $(f(r))$ of $\phi(r)$. A discrete algarithm, described in equations (6-8), was implemented numerically, and it was solved using the boundary condition conveniently defined as: $\phi^{\prime}(r)-0$ as $\left[\left(r-r_{e}\right) \cdot k(r)\right] \propto-1, \wedge$ where $r_{e}$ is the equilibrium internuclear distance in the ground state. Then $\phi(r)$ is obtained by numerical integration, and it is subjected to

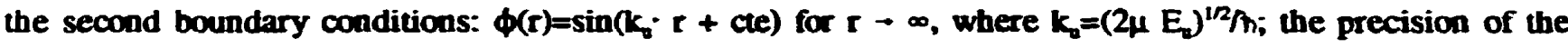
numerical solution is determined by the numerical grid and 1:10 accuracy of the assymptotic amplitude. A scale normalization condition, consistent with the density of states for a free particle ${ }^{13}$, (unit: $1 / \mathrm{cm}^{-1}$, for $R_{0}$ in $\AA$ and $E_{0}$ in $\mathrm{cm}^{-1}$ ) is given by equation (6-9), where $R_{0}$ is arbitrarily set to $1 \AA$, and $\mu$ is the reduced mass of the fragments.

We bave described a procedure to obtain the radial nuclear wavefunction, for an arbitrary energy, on an excited PEC represented by the analytical model of equation (6-3) parametrized by the values of A. B and C. Also, we can refer to the corresponding wavefunction for that excited dissociative state as: $\phi=\phi\left(r ; A, B, C, E_{s}\right)$.

\section{iii) the absorption cross section formula}

The PACSS can be related to the wavefunctions of the ground and excited states, and to the transition dipole $\mathbf{M}_{u}$, by using the numerical relation expressed in equation (6-10), taken from Le Roy et al. ${ }^{13}$ The absorption cross section is expressed in terms of the decadic molar absorption coefficient, $\epsilon_{T}$ unit of $\left(1 \cdot \mathrm{mole}^{-1} \cdot \mathrm{cm}^{-1}\right)$. In equation $6-10, \mu^{\prime}$ is the reduced mass in amu, $v$ is the photon energy in $\mathrm{cm}^{-1}$, the index $u$ refers to the unbound or dissociative state corresponding to that PACSS, $\phi_{\mathbb{R}}$ is the wavefunction of the ground state, $\phi_{\mathrm{a}}$ is the wavefunction of the dissociative state, and $g_{a}$ is its degeneracy: 1 for $\Omega=0$, and 2 for $\Omega=1 . M_{v}(r)$ is the electric dipole for the transition to state $u$, and it is assumed independent of $r$ here, following the FCA $\phi_{v}\left(r ; A, B, C, E_{v}\right)$ in 6-10 is understood as having an asymptotic amplitude of unity, consistent with a normalisation defined by (6-9).

$\varepsilon_{T}(v)=8.43946 \cdot 10^{-4}(\mu)^{1 / 2} v \Sigma_{u}\left(E_{u}\right)^{-1 / 2} g_{u}\left|\int_{0}^{\infty} d r \phi_{u}(r ; A, B, C, E) M_{u}(r) \phi_{g}(r)\right|^{2}$

iv) the formulation of the optimization procedure

The iterative procedure used to determine the PEC carresponding to a PACSS is similar to the forward convolution of section 6.1 used to determine the observables from the Doppler profile, yet it is more refined. A set of experimental values with uncertainties are compared to a model characterized by specific parameters. The goal of the procedure is to determine the values of the parameters, and their uncertainties, in relation to the experimental measurements.

\footnotetext{
A The boundary condition for the asymptotic limit at small intermuclear distance is the mathematical expression of the fact that atoms experience a large repulsive force at close distance, or simply due to: $V(r) \rightarrow \infty$, as $\left(r-r_{e}\right) \rightarrow-\infty$.
} 
The usual technique used for parameters optimization is a least-mean-square (LMS) deviation between the experimental data and a parametrized model. This technique works well in many cases where the data are abundant, and the uncertainties on the parameters are defined by the statistical fluctuations of the numerous data points around the parametrized model. However, there is potential problems in cases such as ours, where the number of experimental data (the dynamical constants at 9 energies) is relatively small, and for which the uncerainties are well-defined (from statistical averages of numerous repeated measurements), but for which the possible occaurence of small spurious systematic errors in some of the points (outlier) cannot be totally dismissed. The inherent problem of the LMS optimization technique is its lack of robustness against outliers, which in the case typified by our data set, is potentially most detrimental. This robustness problem is usually addressed by adding a correcting function (denoted influence function) to the fit ${ }^{17}$, but the arbitrariness of these influence functions leads to a fit procedure lacking the guidance provided by a physical rationale.

I have addressed this problem, and the result of careful considerations led to a procedure that represents major improvement of robustness for the statistical optimization of parametrized model, in the case of small number of data point having well defined statistical uncertainties. The derivation of the method, denoted best statistical overlap (BSO), and the demonstration of its robustness, is the object of appendix F. The validity of the technique has been asserted by a profescicnal academic statistician. ${ }^{18}$

The essence of the BSO formulation rests on an appropriate definition for the "sensitivity" of the fitting algorithm for the deviation between model and data. Assertion of this definition in terms of specific requirements will in fact prescribe the BSO formulation for the optimization method. As usual, the first requirement is a minimization of the deviation $\left(\Delta_{i}\right)$ between experiment and model for any data point. The second requirement demands that the finite uncertainties $\left(u_{i}\right)$ for each data point must be taken into account in such a way that the optimization sensitivity must vanish as $\Delta / 4-0$, i.e. the improvement in the goodness of the fit must be decreasingly important as the deviation becomes much smaller than the experimental uncertainty for any point. The rationale for this second requirement lies simply in the statistical meaning of the uncertainty: as the ratio $\Delta_{i} / u_{i}$ decreases beyond the value of one, the quality of the fit must become increasingly less sensitive to further reduction. To satisfy this second requirement, a sensitivity function can be defined for each data point; assuming the fluctuations are truly random, the Gaussian distribution, i.e.: $s_{i}=\exp \left[-(1 / 2)\left(\Delta, / u_{i}\right)^{2}\right]$ fulfills this requirement appropriately. A third requirement ensues from the possible different relative significance of each point, in account of their difierent information content, which is quantified via a relative weight $w_{i}$ in the following description.

It most be noted that there is a subtle difference between the notion of relative weight in the present case. compared to its usual meaning in LMS method. The relative weight $w_{i}$ in BSO refers to the relative importance 
of a data point compared to the ensemble of the other data points, independently of the model, while in LMS, the notion of weight refers to the relative difference od a data point with the model.

The BSO formulation of the optimization procedure is defined as the maximization of the optimizer $O$. defined as: $O=\Sigma w_{i}$ s. It will be noted that the optimizer $O$ conveniently spans the range of values: $[0,1]$, where 1 would correspond to a perfect fit. i.e. a model for which the predicted value would fall exactly at the centre of the measured range of uncertainties, for the measurements at all values of the independent variable (which in the present case is the photolysis photon energy).

In our present analysis, it was found empirically that a normalized weight $\left(\sum w_{i}=1\right)$, defined as $w_{i} \propto v_{i} / u_{i}$ where $v_{i}$ is the value of point $i$ and $u_{i}$ is its uncertainties, provides a proper representation for the relative significance of each data point; note that this weight is proportional to the inverse of the relative uncertainty of the data point. The later point may come as no surprise when one realizes that these relative weights are statistically equivalent to signal-to-noise ratios, which is a measure of the information content. It must be said that in general, the expression for $w_{i}$ will differ from the simple one used here, but its physical meaning of information content will be implemented in the statistics by an appropriate expression. It is up top the user to quantify the relative information content of the experimental data.

In practice, the maximum value of $O$ is almost never exactly one, but the closer it gets, the better the model can be asserted; the deviation of the maximum $O$ indicates that either the model is inappropriate, or that there is outlier point(s) due to sporadic spurious errors. This property is an additional property that make this statistical tool a convenient one.

\subsubsection{Application of the method for PEC calculation}

(i) The iterative forward convolution procedure

The forward convolution is a set of operations including the quantum mechanical calculation (equation 6.5 to 6.10) used to link a parametrized PEC(R; A,B,C) model with an experimental PACSS. The BSO technique was applied to determine the PEC parameters that best represent the experimental data.

The parameter $C$ of $V(r)$ was set to the value corresponding to the product channel. A range of values and a number of increments $\left(N_{A}\right.$ and $N_{B}$ ) were defined for each parameters $A$ and $B$. Then, for each one of the $\left(N_{A} X\right.$ $\mathrm{N}_{\mathrm{B}}$ ) parameters combinations, a PEC was defined and the Schrodinger equation was solved. The resulting radial wavefunction was used to calculate the energy dependence for the relative transition amplitude, within the FranckCondon approximation ${ }^{19}$ (FCA). The partial absorption cross section for that state (PACSS) was calculated using equation 6-10. The process was repeated at each photon energy for which the PACSS was determined. 
This simulated numerical PACSS was integral-normalized to the experimental PACSS, in order to provide a first guess of the average transition dipole $M_{a}$. Then a statistical optimization of $M_{v}$ was performed via the maximization of $\left.\mathrm{O}_{2} ; A, B\right)$, i.e. for these fixed $A$ and $B$ parameters, $M_{2}$ was varied and the difference between simulated and experimental PACSS (defining $\Delta_{j}$ ) was used in combination with the experimental uncertainties $u_{i}$, to calculate the optimizer $O$. The optimal value of $M_{3}$ (denominated $M_{\text {op }}$ ). corresponding to the maximum of $O$, defines $O(A, B) \equiv O\left(M_{o p t} ; A, B\right)$. The latter quantity was stored for each $\left(N_{A} X N_{B}\right) A$ and $B$ parameter combination on the grid; this defines the optimizer function. The maximum $O$ value in the optimizer function corresponds to that combination of A and B values which best represents the real PEC, within the precision of the grid of parameters used in the iterative procedure.

After initially readjusting and eventually shrinking the ranges over which the optimum values of the parameters $A$ and $B$ are sought, the application of this forward convolution technique is repeated until a satisfactory precision of the A and B parameters is achived. Typically, 6-12 successive evaluations of the optimizer function were required to determine the values of the parameters for the PEC that best represent the experimental PACSS. The computation was performed on a UNIX platform, using our algorithm developed in the GENPLOT macto language.

(ii) Specifics of the calculation procedure

a) $T h e^{3} \Pi_{0}+$ state

The assumptions used here follows the derivation of chapter 2, which was based on group theory and correlation diagrams. That derivation showed that the $I^{*}$ product can only result from transition to the ${ }^{3} \Pi_{0}+$ state. Therefore, we can take the PACS $\left(I^{*}\right)$ as being the partial absorption cross section for the state ${ }^{3} \Pi_{0}+\left(\operatorname{PACSS}\left({ }^{3} \Pi_{0}+\right)\right)$, or briefly put: $\operatorname{PACS}\left(I^{*}\right) \equiv \operatorname{PACSS}\left({ }^{3} \Pi_{0}+\right)$. The above procedure was first applied to a calculation of the $\operatorname{PEC}$ for the ${ }^{3} \Pi_{0}+$ state, using PACS $\left(I^{*}\right)$ defined by table 6.4. The results of this first application is illustrated in figure 6.39, which shows that an excellent agreement was reached between the model and the measurements.

The extrapolation of PACS(I*) over the range of TACS frequencies, calculated from this PEC, can be considered with reasonable confidence, since it only invoives PEC defined over the Franck-Condon region, for which the PEC is quite reliable. Nevertheless, a quantification of the uncertainties for the PEC parameters is desirable. Initially, a visual estimate of the uncertainties can be obtained by varying cach parameter one by one, and by estimating the quality of the consequent PACSS with the optimum one and with data, from visual inspection of a graphical representation. Figure 6.39 illustrates these visual estimates of parameters uncertainties; the curves illustrating the effect of the parameters uncertainties are intentionally plotted with the same symbol, for the idea is to create a scatter around the optimum $\mathrm{PACS}\left(\mathrm{I}^{*}\right)$ as a result of a scatter around the optimum value of each parameters. 


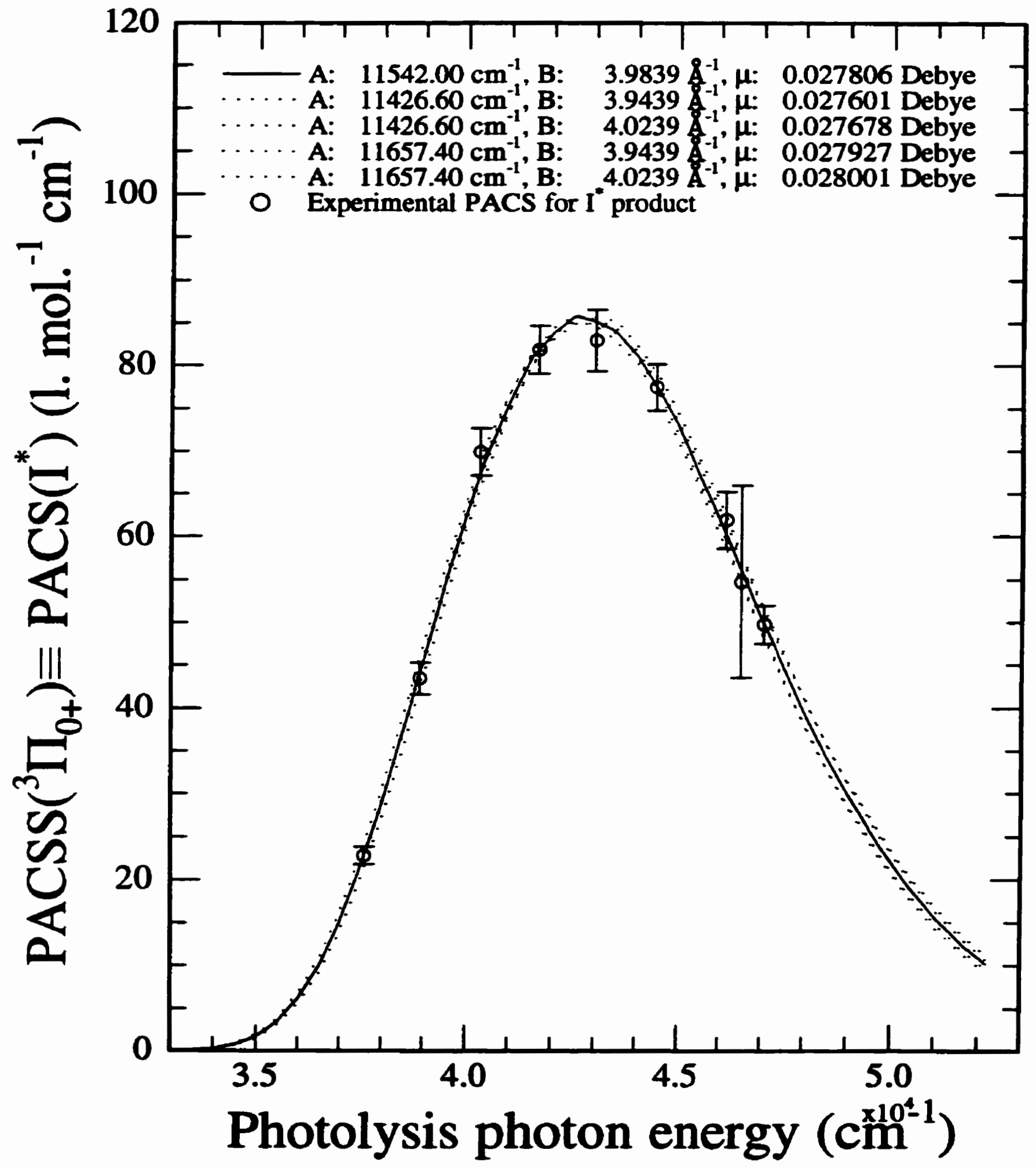

Figure 6.39. Determination of the PEC for the ${ }^{3} \Pi_{0}+$ state, using PACS( $\mathbb{I}^{*}$ ). Comparison of calculated (bold curve) and experimental PACS, along with a visual estimates (dotted curves) of the effect of the parameter uncertainties. 
a)

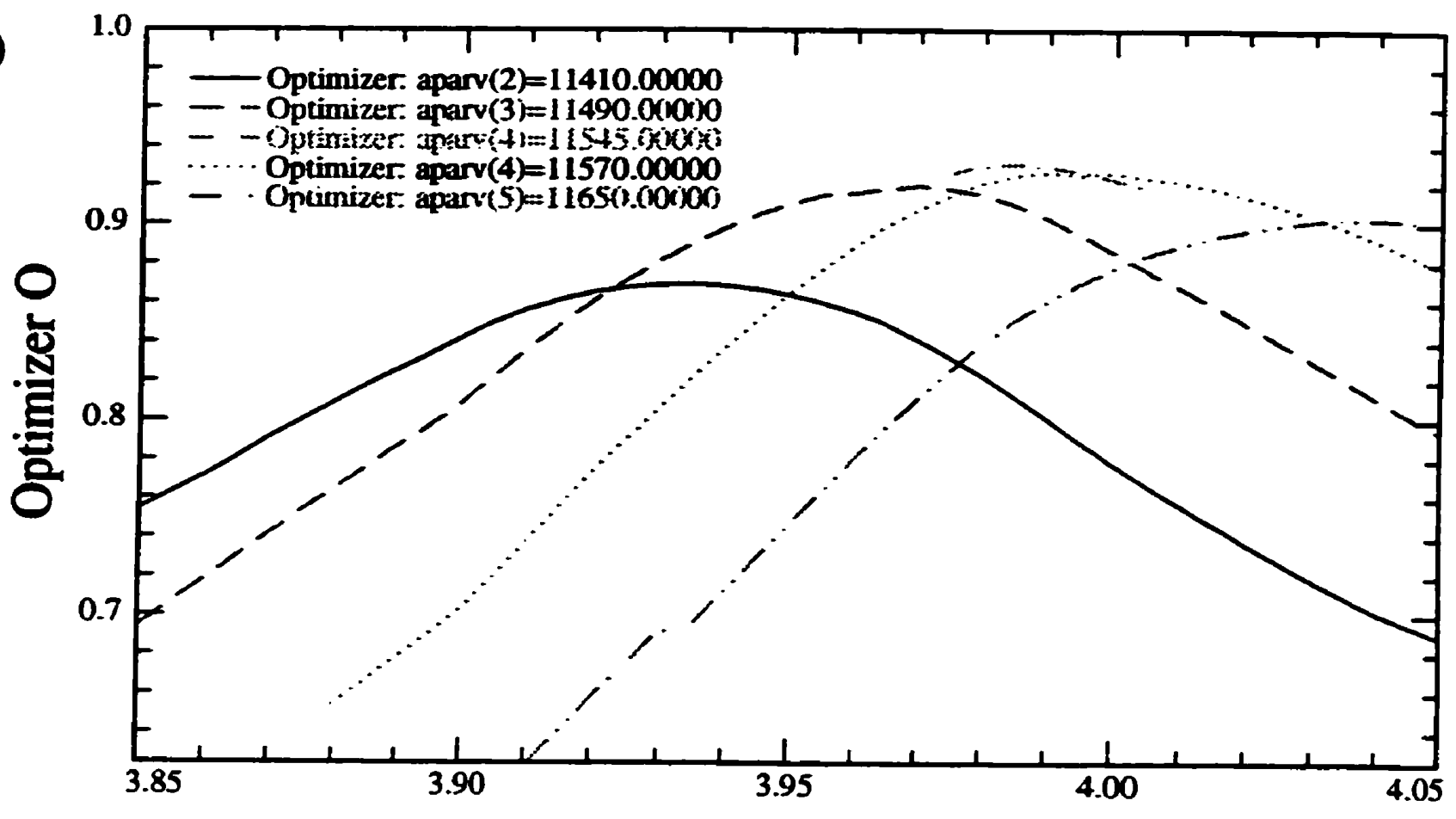

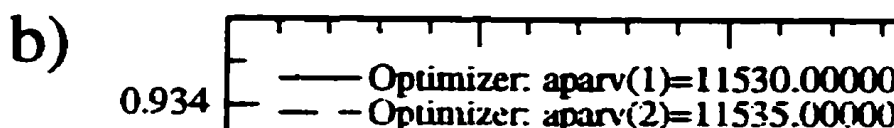

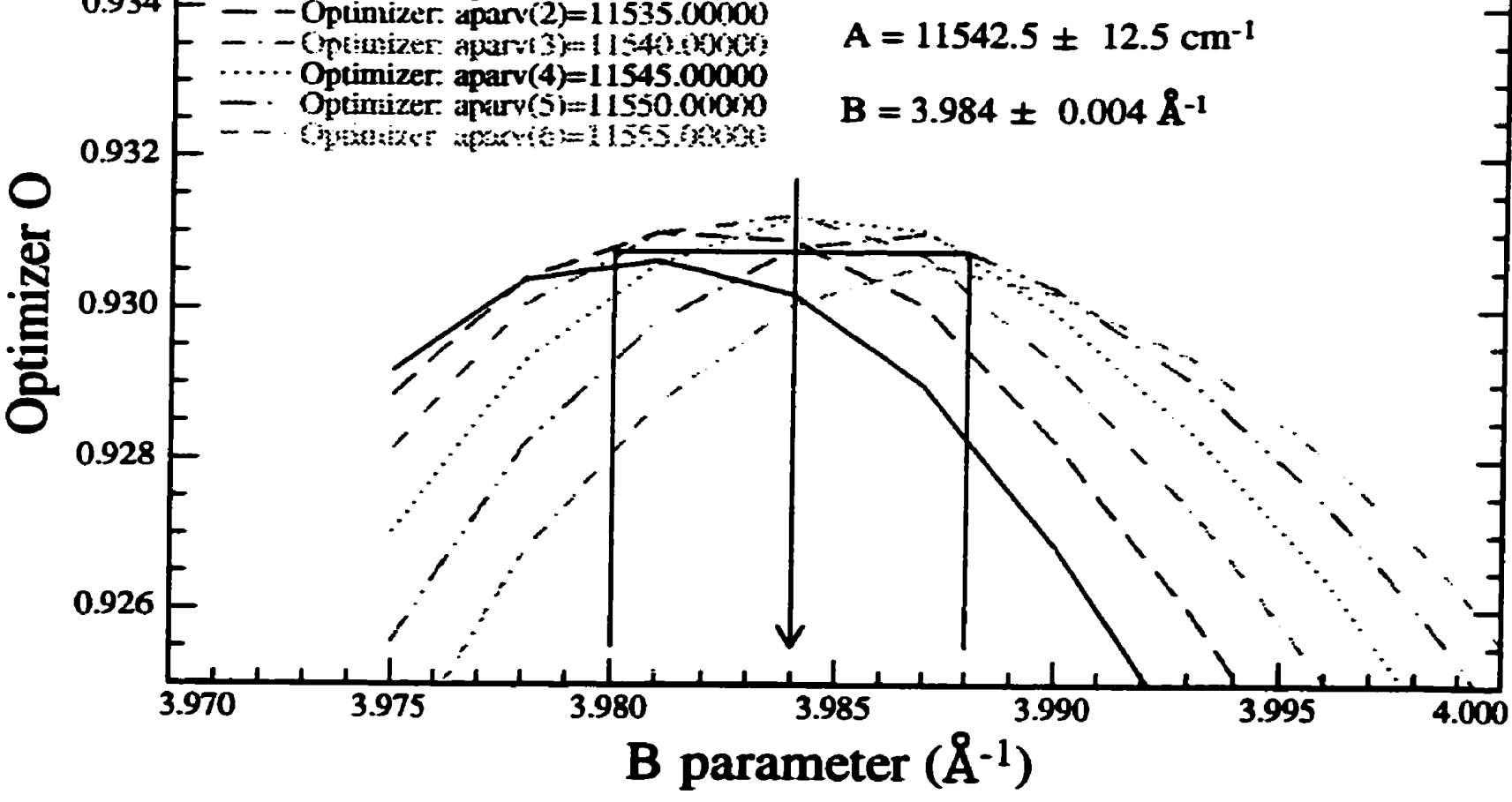

Figure 6.40. Uncertainties in the determination of the model parameters. a) Calculated Optimizer functions for the limits values of uncertainties obtained from visual inspection, and b) from the statistical method described in the text. 
The statistical method employed here allows a rigorous definition for the uncertainties of the PEC's parameters. It is defined in relation to the relative uncertainties of the data through a relative optimizer value $O_{\text {uxc }}$ calculated from equation 6-11 (see appendix F). For the sake of comparison, both the visual method and the statistical evaluations of the uncertainties are quantified in figure 6.40. In a) we calculate the Optimizer functions for the limit values of uncertainties obtained from the aforementioned visual method, while in b) we proceeded with the formal determination of the uncertainties, between $O_{\max }$ and $Q_{m}$ defined in equation (6-11). In the latter equation, $N_{\text {exp }}$ is the number of data point in the optimization, and $w_{i}$ is the statistical weight for the point $i$, which represent the relative information content among the data set, as discussed above.

$O_{\mathrm{UNC}}=O_{\mathrm{MAX}}\left[1-\frac{1}{\left.\sqrt{\sum_{i}\left(w_{i}\right)^{2} \exp \left[\frac{-1}{2}\left(\frac{\mathrm{f}^{0}\left(x_{i}\right)-y_{i}}{\sigma_{i}}\right)^{2}\right.}\right]}\right]$

This comparison showed that the parameter's uncertainties obtained from the statistical evaluation related to the experimental uncertainties is roughly ten times smaller than the one that would have been guessed from a qualitative visual estimate. This is a consequence of the high quatity of the experimental data, and the smoothness of the PEC functionality.

\section{b) Validity test for the PEC: dependence on analytical form}

The analytical form (6-3) is known to provide a satisfactory representation of the PEC over the Franck-Condon region. However, it is also expected to be valid somewhat beyond that range of internuclear distance. Thus, if it can be showed that the numerical PEC is independent of the analytical form, at least over the Franck-Condon region, this shall increase confidence in the consequence of the model, namely the extrapolation of the PACS( $\left.{ }^{*}\right)$ over the energy range of the $A$ band, and the consequent extrapolation of the branching ratios.

In order to test the dependence of the extrapolated PACS $\left(I^{*}\right)$ on the analytical model, we employed another functional form to model the PEC: $V_{2}(R)=A_{2} \cdot R \cdot \exp \left[-B_{2}(R-R e)\right]$. The optimum parameters were calculated and the results of the simulated PACS( ${ }^{*}$ ) for each analytical potential are compared. Obviously, the numerical values of the optimum A and B parameters were different for each analytical form, but both produced the same fit quality, i.e. the same $\mathrm{O}_{\operatorname{ma}}$ value. Moreover a comparison of the numerical PEC and PACSS values, shown in figure 6.41. indicates that they were indistinguishable in the Franck-Condon (FC) region, for the two analytical forms of PEC. Hence the result of this test is that the optimum PEC and the consequent simulated PACS are independent of the analytical form used for the PEC model in the (FC) region of interest; consequently, the conceptual role of the PEC model was essentially "to smoothly link" the experimental data. 

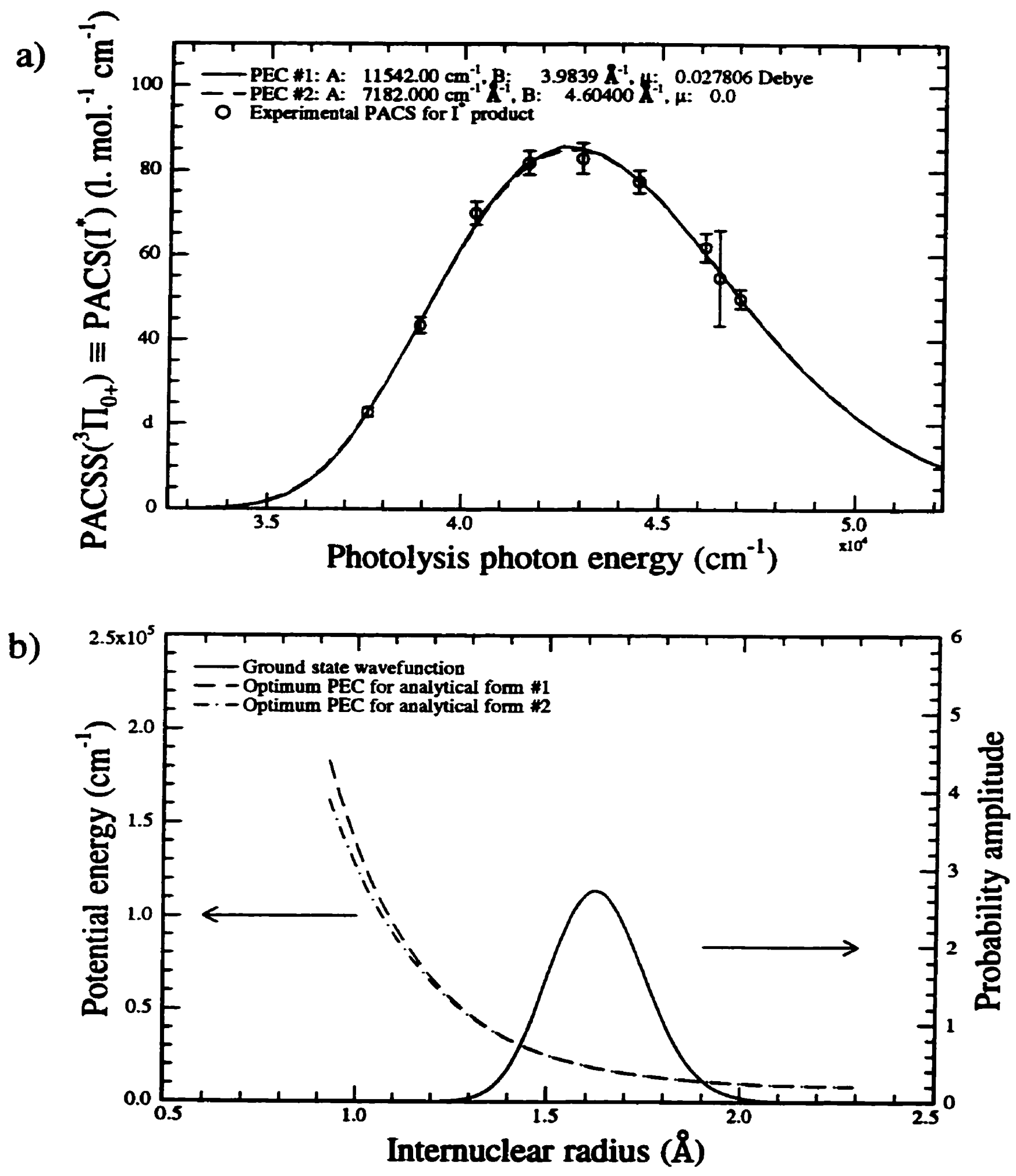

Figure 6.41. Comparison for two analytical form of the PEC: a) numerical PACSS values, and b) numerical PEC, and the ground state wavefunction, to illustrate relative spatial extension. Within the FC region, they are indistinguishable. 
Taking few steps back, one realizes that we have obtained a fairly good representation of the experimental PACS $\left(I^{*}\right)$ measurement from the calculation of a PEC for the ${ }^{3 I_{0}}+$ state. Because the virtue of the model is to represent the PACS $\left(1^{*}\right)$ over the range of energy corresponding to the A band absorption, and that the TACS is known from Ogilvie', we can evaluate the PACS(I) over this energy range with reasonable confidence, at least at the energy values for which the TACS were determined. Briefly said, we can use the $\operatorname{PEC}\left({ }^{3} \mathrm{II}_{0}+\right)$ to calculate the PACS(I) from the combination of the TACS, the PACS( $\left.I^{*}\right)$, and the set of equations (6-4). Moreover the reader will note that a calculation for the values of $R$ is within reach through equations $(6-4)$. The result of this calculation is listed in table 6.5 below, and illustrated in chapter 7.

The next logical step is to explore the possibilities of PACS(I) representation by PEC for the other states of the Q-group, for the sake of completeness and consistency. In principle, two states can be involved for PACS(I): the ${ }^{3} \Pi_{1}$ and ${ }^{\prime} \Pi$ electronic states. From chapter 2 , we recall that the ${ }^{3} \Pi_{1}$ state will lie lower in energy than ' $\Pi$ state; the latter should carry the predominant part of the cross section, while the former can contribute weakly to the ${ }^{3} \Pi_{1}$ state, owing to the propensity for electronic spin conservation in the electronic transition; however, the strong spin-arbit coupling of the lodine atom may very well give out a finite cross section for a transition to the ${ }^{3} \Pi_{1}$ state.

Following a suggestion from Le Roy ${ }^{20}$, we must nevertheless address the question regarding wether one or two states need to be used to represent the PACS(D), because a single state may suffice to represent PACS(D). The test of this hypothesis was performed, and it is described in the following paragraphs.

\section{c) The 'II state}

In order to determine whether one or two states contribute to the PACS(I), we first attempted to fit the whole PACS(I) with one PEC(' $I)$, i.e. we explored the possibility PACS( ()$\equiv P A C S S(' M)$. First, two different local maxima were observed as the overall fit improved toward a better combination of parameters; for each local maximum, either the low end or the high end of the PACS(I) tends to fit better, as illustrated in figure $6.42 \mathrm{~b}$ ).

Second the best parameter combination, corresponding to the peak in the optimizer plot in figure $6.42 \mathrm{a}$ ), only differs a litule from the one of the two aforementioned local maxima; moreover, this best simulated PACS(I) fits well only the high energy part of the experimental PACS(D), while a deficiency of PACS(I) was obtained at the low energy end of the PACS(I), as shown in figure 6.42 b). Additionally, the overall quality of the fit, quantified by $O_{m}=0.62$, is rather poor. The sum of these observations warranted the explocation of a second possibility. namely that the lower ${ }^{3} \Pi_{1}$ state may contribute significantly to the PACS(I), in keeping with the model suggested by Mulliken" 
Table 6.5 PACS( $\left.\Omega^{*}\right)$, R and PACS(I) calculated from the calculated PEC(' $\left.\Pi_{0}+\right)$ and TACS from Ogilvie ${ }^{2}$.

\begin{tabular}{|c|c|c|c|}
\hline Wavenumber $\left(\mathrm{cm}^{-1}\right)$ & $\operatorname{PACS}\left(I^{*}\right)\left(1 \mathrm{~mol}^{-1} \mathrm{~cm}^{-1}\right)$ & $\overline{\mathbf{R}}$ & PACS(I) $\left(1 \mathrm{~mol}^{-1} \mathrm{~cm}^{-1}\right)$ \\
\hline 32500 & $0.0009342 \pm 0.06$ & $0.0003114 \pm 0.000012$ & $2.999 \pm 0.06$ \\
\hline 33000 & $0.01244 \pm 0.08$ & $0.003110 \pm 0.00012$ & $3.987 \pm 0.08$ \\
\hline 33500 & $0.07179 \pm 0.11$ & $0.01305 \pm 0.00052$ & $5.428 \pm 0.11$ \\
\hline 34000 & $0.2655 \pm 0.16$ & $0.03318 \pm 0.0013$ & $7.735 \pm 0.16$ \\
\hline 34500 & $0.7423 \pm 0.22$ & $0.06748 \pm 0.0027$ & $10.258 \pm 0.22$ \\
\hline 35000 & $1.709 \pm 0.3$ & $0.1139 \pm 0.0046$ & $13.291 \pm 0.3$ \\
\hline 35500 & $3.415 \pm 0.41$ & $0.1666 \pm 0.0067$ & $17.085 \neq 0.41$ \\
\hline 36000 & $6.136 \pm 0.54$ & $0.2273 \pm 0.0091$ & $20.864 \pm 0.54$ \\
\hline 36500 & $9.933 \pm 0.71$ & $0.2798 \pm 0.011$ & $25.567 \pm 0.71$ \\
\hline 37000 & $15.03 \pm 0.91$ & $0.3303 \pm 0.013$ & $30.471 \pm 0.91$ \\
\hline 37500 & $21.497 \pm 1.1$ & $0.3909 \pm 0.016$ & $33.503 \cap 1.1$ \\
\hline 38000 & $28.771 \pm 1.37$ & $0.4200 \pm 0.017$ & $39.729 \pm 1.37$ \\
\hline 38500 & $37.159 \pm 1.64$ & $0.4532 \pm 0.018$ & $44.841 \pm 1.64$ \\
\hline 39000 & $45.639 \pm 1.95$ & $0.4681 \pm 0.019$ & $51.861 \pm 1.95$ \\
\hline 39500 & $54.399 \pm 2.27$ & $0.4793 \pm 0.019$ & $59.101 \pm 2.27$ \\
\hline 40000 & $62.485 \pm 2.6$ & $0.4807 \pm 0.019$ & $67.515 \pm 2.6$ \\
\hline 40500 & $70.010 \pm 2.9$ & $0.4828 \pm 0.019$ & $74.991 \pm 2.9$ \\
\hline 41000 & $75.920 \pm 3.19$ & $0.4760 \pm 0.019$ & $83.572 \pm 3.19$ \\
\hline 41500 & $80.967 \pm 3.49$ & $0.4640 \pm 0.019$ & $93.533 \pm 3.49$ \\
\hline 42000 & $83.760 \pm 3.7$ & $0.4528 \pm 0.018$ & $101.24 \pm 3.7$ \\
\hline 42500 & $85.782 \pm 3.9$ & $0.4400 \pm 0.018$ & $109.22 \pm 3.9$ \\
\hline 43000 & $85.190 \pm 4.07$ & $0.4186 \pm 0.017$ & $118.31 \pm 4.07$ \\
\hline 43500 & $83.860 \pm 4.2$ & $0.3993 \pm 0.016$ & $126.14 \pm 4.2$ \\
\hline 44000 & $81.030 \pm 4.33$ & $0.3743 \pm 0.015$ & $135.47 \pm 4.33$ \\
\hline 44500 & $77.167 \pm 4.39$ & $0.3516 \pm 0.014$ & $142.33 \pm 4.39$ \\
\hline 45000 & $73.031 \pm 4.4$ & $0.3320 \pm 0.013$ & $146.97 \pm 4.4$ \\
\hline 45500 & $67.395 \pm 4.39$ & $0.3070 \pm 0.012$ & $152.10 \pm 4.39$ \\
\hline 46000 & $61.943 \pm 4.35$ & $0.2848 \pm 0.011$ & $155.56 \pm 4.35$ \\
\hline 46500 & $56.290 \pm 4.31$ & $0.2612 \pm 0.010$ & $159.21 \pm 4.31$ \\
\hline 47000 & $50.470 \pm 4.19$ & $0.2410 \pm 0.0096$ & $159.03 \pm 4.19$ \\
\hline 47500 & $45.016 \pm 4.05$ & $0.2223 \pm 0.0089$ & $157.48 \pm 4.05$ \\
\hline 48000 & $39.60 \pm 3.94$ & $0.2010 \pm 0.0080$ & $157.40 \pm 3.94$ \\
\hline 48500 & $34.56 \pm 3.82$ & $0.1809 \pm 0.0072$ & $156.44 \pm 3.82$ \\
\hline 49000 & $30.05 \pm 3.69$ & $0.1629 \pm 0.0065$ & $154.45 \pm 3.69$ \\
\hline 49500 & $25.74 \pm 3.55$ & $0.1450 \pm 0.0058$ & $151.76 \pm 3.55$ \\
\hline 50000 & $21.96 \pm 3.41$ & $0.1288 \pm 0.0052$ & $148.54 \pm 3.41$ \\
\hline 50500 & $18.66 \pm 3.27$ & $0.1141 \pm 0.0046$ & $144.84 \pm 3.27$ \\
\hline 51000 & $15.68 \pm 3.13$ & $0.1002 \pm 0.0040$ & $140.82 \pm 3.13$ \\
\hline 51500 & $12.20=2.96$ & $0.08246 \pm 0.0033$ & $135.80 \pm 2.96$ \\
\hline 52000 & $10.18 \pm 2.88$ & $0.07068 \pm 0.0028$ & $133.82 \pm 2.88$ \\
\hline
\end{tabular}


Before attempting to quantify this second hypothesis, note that it has been suggested by Le Roy ${ }^{20}$ that two other physical possibilities may be able to acoount for the discrepancies in representing PACS(I) by a single state; it was suggested that either a more complex functionality of the repulsive curve, or an R-dependence of the transition dipole $\mathbf{M}_{\mathbf{z}}$ should be able to represent the PACS(I) with a single PEC. However, there is added complexity imposed by the consideration of these hypothesis. Moreover because we developed and quoted many arguments concerning the symmetry of the electronic structure for Mulliken's model (see chapter 2), the author planned to first try the laner model initially, because it was simpler. Then, in the eventuality that Mulliken would not suffice. we would bave applied this second suggestion from Le Roy ${ }^{20}$; the results of the test indicated that this was not necessary.

We now consider the determination of the PEC parameters assuming that these two states contribute to the PACS(I). In principle, there is a region of energy over which both states can contribute to the TACS, and ideally one could fit two PEC by simultaneously varying five parameters (two set of $A$ and $B$ 's and a relative transition strength) in the optimization procedure. However, a more affordable approach, aiming to first fit a PEC to a reasonably high energy part of PACS(I), proved to be more sensible, and produced stable results.

The strategy to determine the two PEC contributing to the PACS(I) was as follow: the contribution of the weaker ' $\Pi_{1}$ state, lying at the low energy end of the ground state PACS(I), must vanish at sufficiently high photon energy, where absorption by the ' $\Pi$ state dominates the PACS(I) entirely. Empirically we found that if the portion of PACS(I) above $42500 \mathrm{~cm}^{-1}$ was fitted to PEC('I), a much better optimizer value $\left(O_{\max }=0.89\right)$ was obtained for an unambiguous combination of model parameters. although the relatively good quality of the data suggests that a better fit may be achievable. Although the overall convergence of the parameter fit was quite smooth for this restricted region, oscillations of the Optimizer values around the maximum can be observed using a finer grid for the parameters domain, as seen in figure 6.44. These oscillations show that the best parameters combination do not smoothly converge to the optimum set, but it hopped, from a combination of parameters optimum for a subset of experimental point, to another combination, which is then more suitable for another subset of experimental points.

Referring to figure 6.43 b), the reader will appreciate that this second fit, to a region of PACS(I) restricted to a photolysis energy above $37000 \mathrm{~cm}^{-1}$, overestimates the PACS(I) between 37000 and $43000 \mathrm{~cm}^{-1}$. Another observation can be appreciated in this figure: the two points at the highest energy in the PACS(I) values indicates a point of inflexion. These two observations have a common physical rationale. One must recall that both Romand and Vodar ${ }^{2}$, and Huebert and Martin ${ }^{22}$, whose results were reproduced in chapter 2, have observed that absorption by the strong B band, which becomes obvious at $190 \mathrm{~nm}$, must extend to longer wavelength up to 200 $\mathrm{nm}$, or to energy as low as $50000 \mathrm{~cm}^{-1}$. 
a)
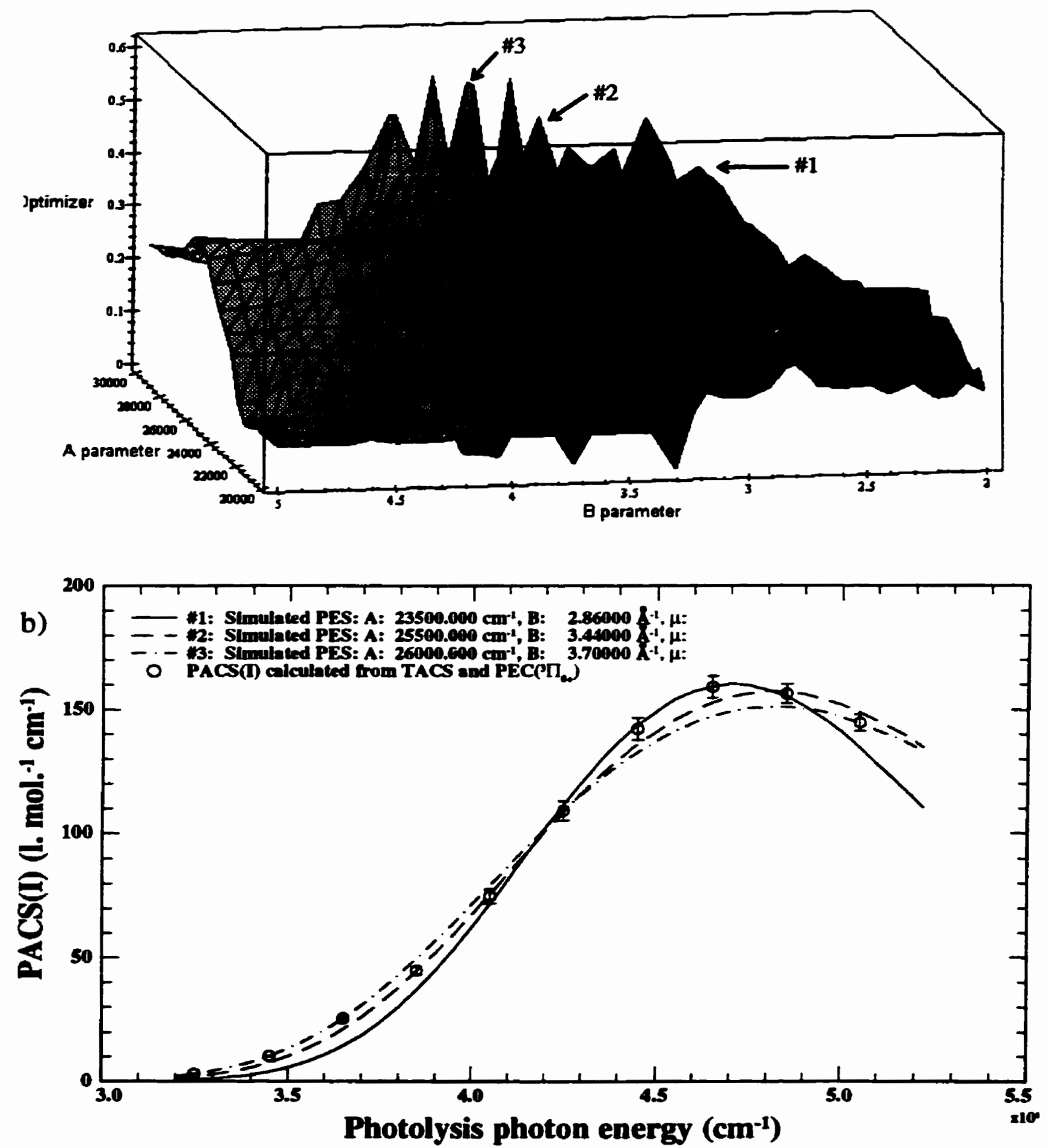

Figure 6.42 a) Plot of the optimizer function showing the bistable path to the maximum, which is itself of poor quality: b) Comparison of simulated PACSS for two points near the optimum. in each bistable path. and for the optimum. 
a)

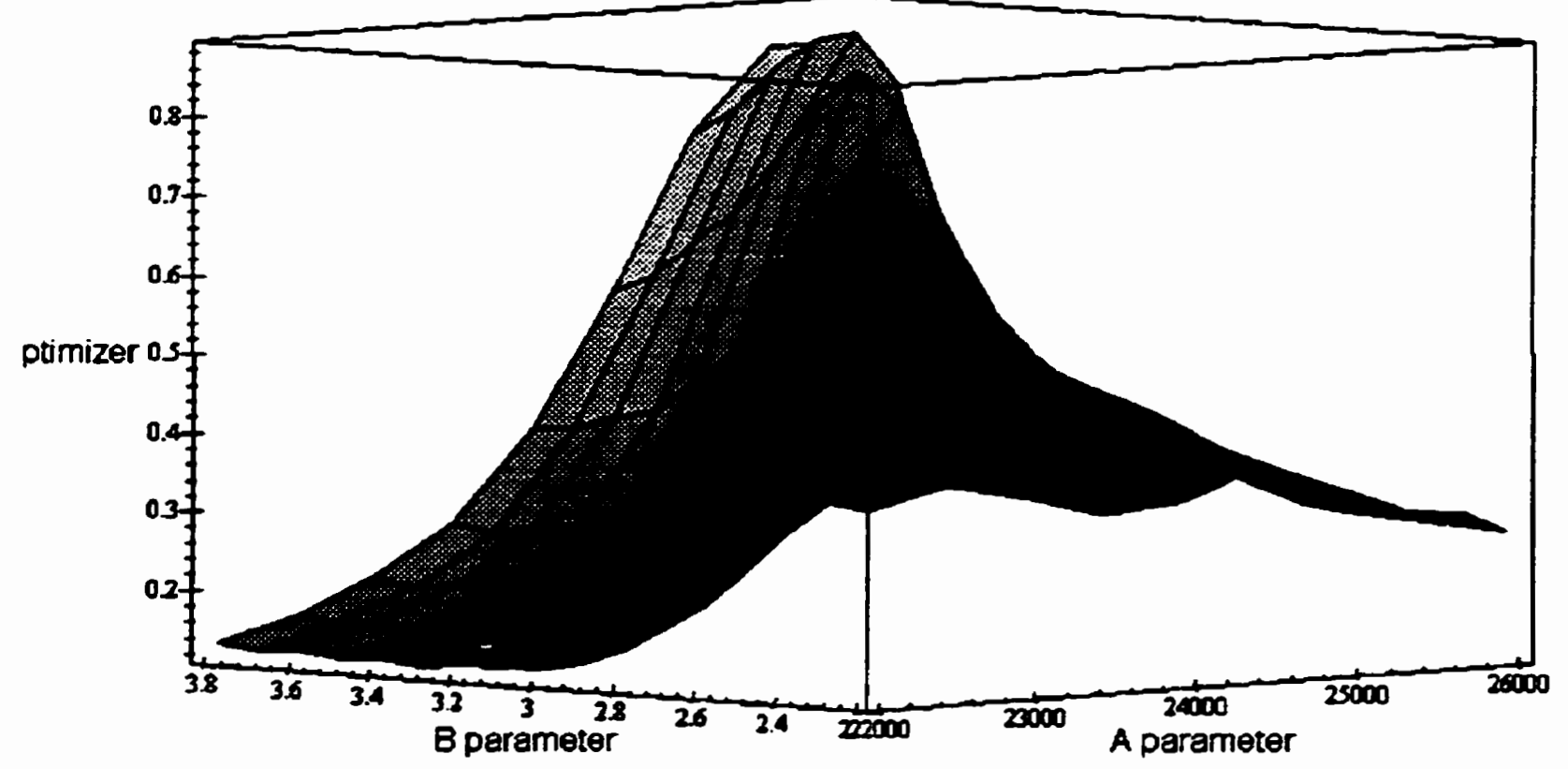

b)

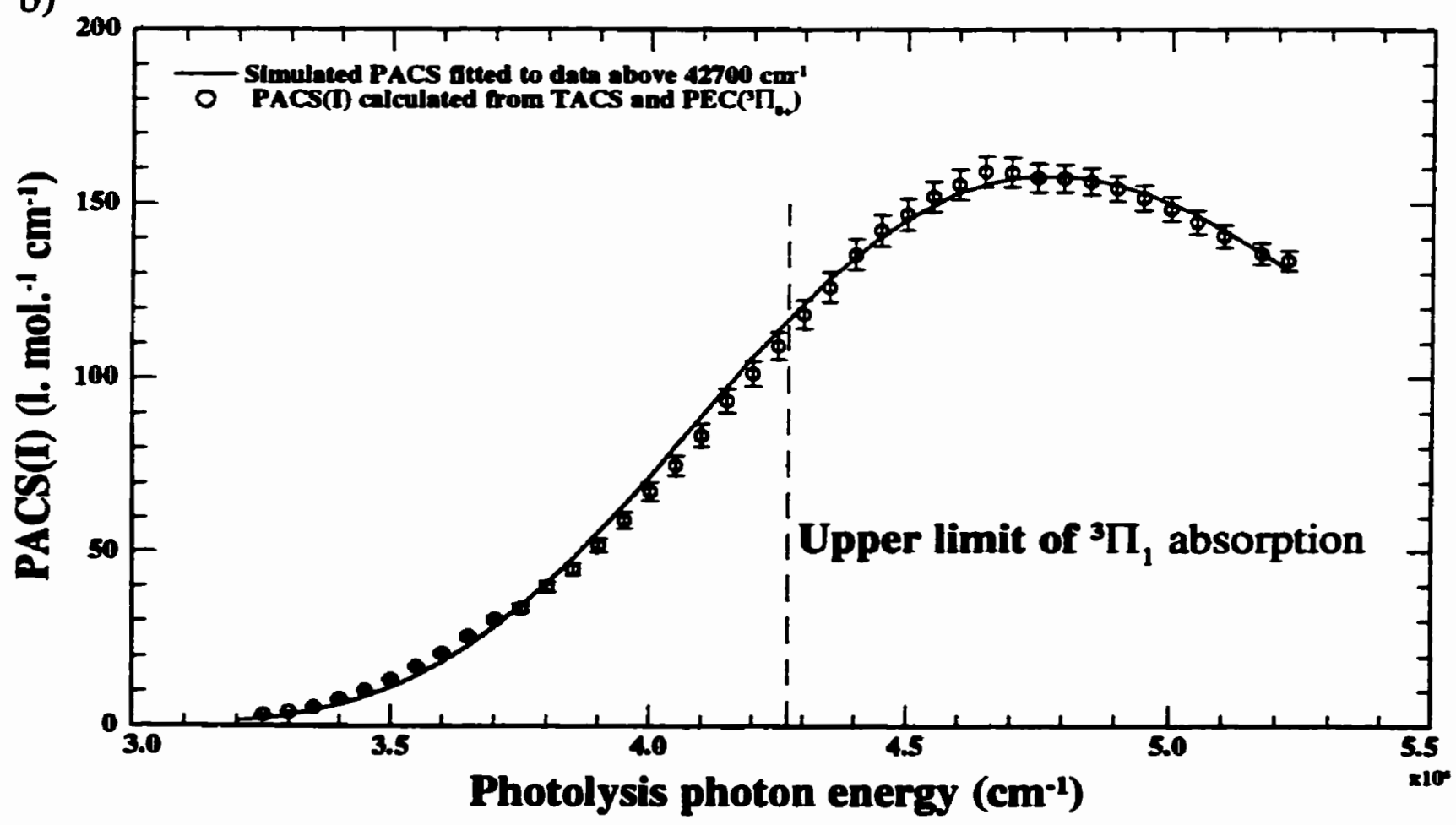

Figure 6.43 a) Plot of the optimizer function for the determination of PEC('II) by a fit to the PACS(I) above $43500 \mathrm{~cm}^{-1}$; b) Comparison of optimum simulated and experimental PACS(I). 


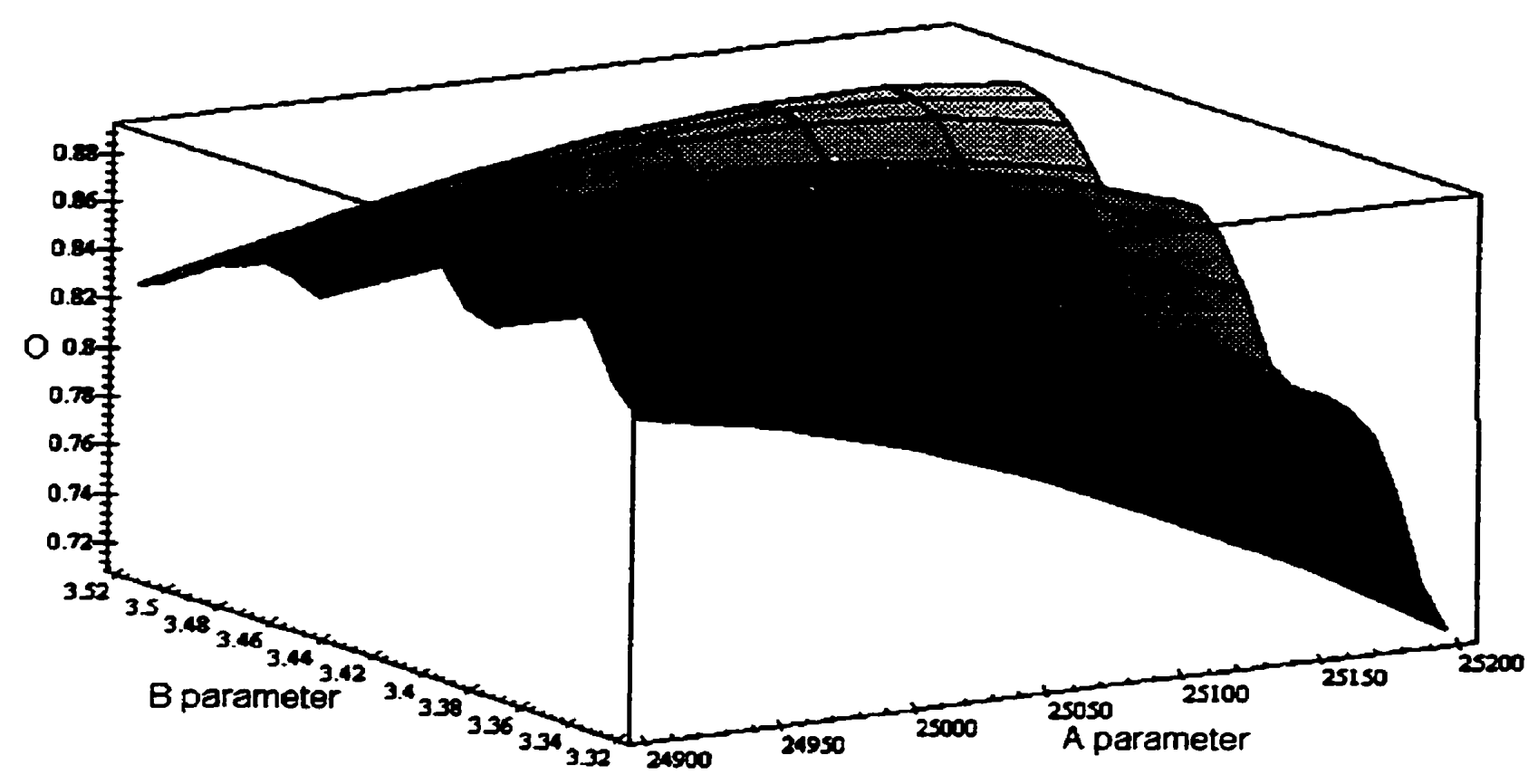

Figure 6.44 Plot of the optimizer function for the determination of PEC('II) by a fit to the PACS(I) above $43500 \mathrm{~cm}^{-1}$; detailed look at the behaviour of the optimizer function around the optimum: the "oscillatory" behaviour is discussed in the text.

Returning to $6.43 \mathrm{~b}$ ) one can appreciate that the point of inflexion suggests the onset of an absorption band, the $B$ band. Consequently, the region of the PACS(I) data fitted to $\operatorname{PEC}\left({ }^{\prime} I\right)$ should also be restricted to the energy range below $50000 \mathrm{~cm}^{-1}$, because above this energy it is very likely that another state pertaining to the $B$ band system also contributes to absorption.

Armed with this knowledge, a third cycle of optimization was performed using the experimental values of PACS(I) having photolysis photon energy above $42000 \mathrm{~cm}^{-1}$, and below $50000 \mathrm{~cm}^{-1}$; the results of the optimization are plotted in figure 6.45 a) for a low resolution grid, and in figure $6.45 \mathrm{~b}$ ) for the finest grid to identify the values of the optimum parameters. The optimizer function converged very smoothly to the optimum parameters combination, which indicates a very good certainty in determining that the model represents the reality well. The goodness of the model's representation is quantified by the maximum value of the optimizer function, which reaches the value of $O_{\max }=0.98$ for this third fit; note that this is very close the maximum possible value of $O \equiv 1$. A further set of optimizer calculation was used to obtain an estimate of the parameters uncertainties, as we did for $\operatorname{PEC}\left({ }^{3} \mathrm{II}_{0}+\right)$. 
a)

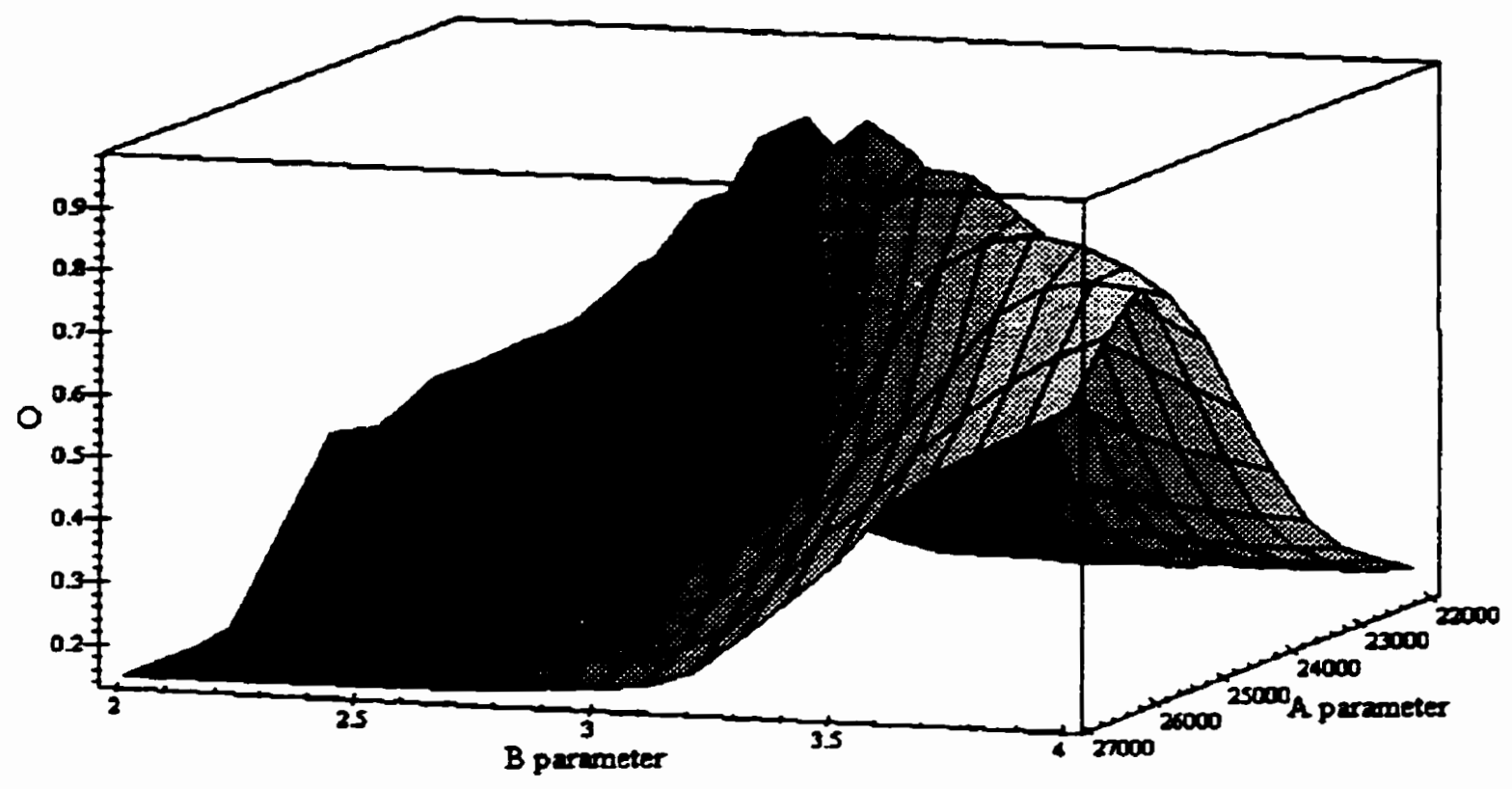

b)

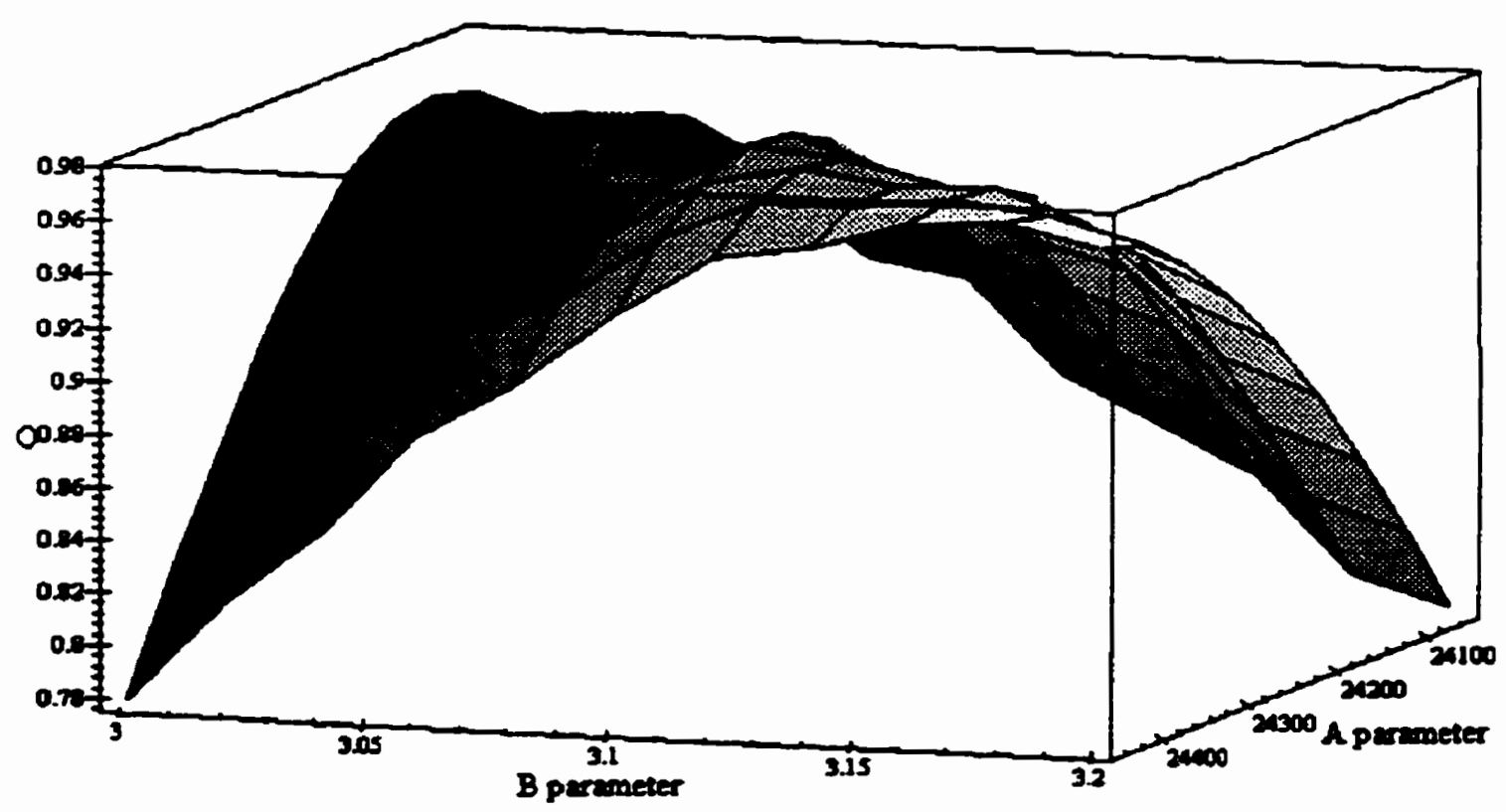

Figure 6.45 Plot of the optimizer function for a fit to a region of experimental PACS(I) restricted to above $43500 \mathrm{~cm}^{-1}$ and below $52000 \mathrm{~cm}^{-1}$, a) for a low resolution grid of the parameters domain, and b) a fine grid for PEC('II) parameters determination. 
d) The ${ }^{3} \Pi_{l}$ stase

These optimum parameters are used to calculate an extrapolation of PACSS('II) at all values of the energies for which Ogilvie has determined the TACS, which is then compared to the experimental PACS(I) in figure 6.46. The difference between PACSS('II) and the experimental PACS(I) is also plotted in the latter figure, where the low energy bump is assigned to PACSS $\left({ }^{3} \Pi_{1}\right)$, while the difference above $50000 \mathrm{~cm}^{-1}$ is due to the onset of the $B$ band absorption. Consequently it is concluded that: $\operatorname{PACS}(I)=\operatorname{PACSS}\left({ }^{(} I\right)+\operatorname{PACSS}\left({ }^{3} \Pi_{1}\right)+\operatorname{PACS}(B$ band), within the energy range of figure 6.46 .

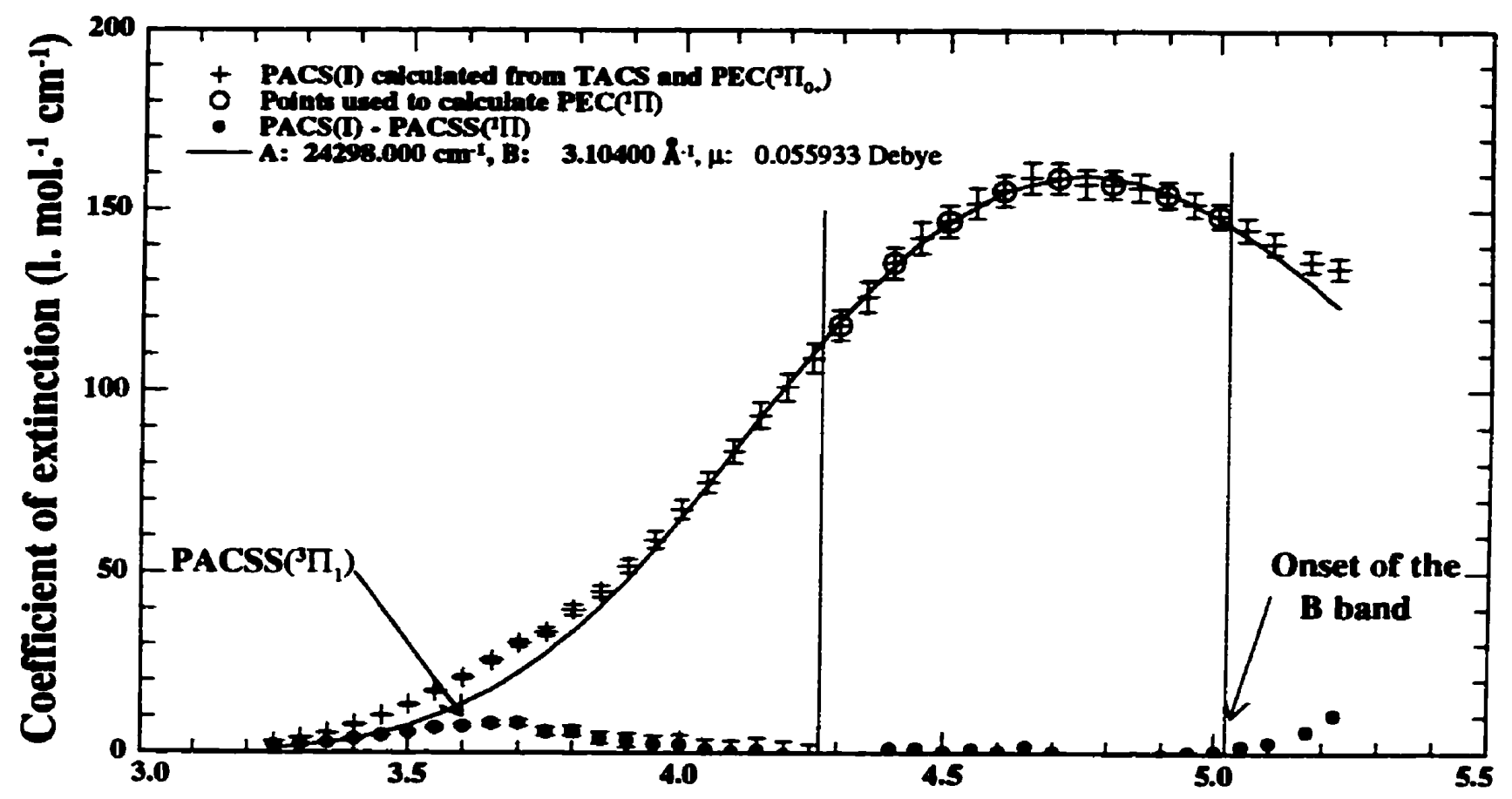

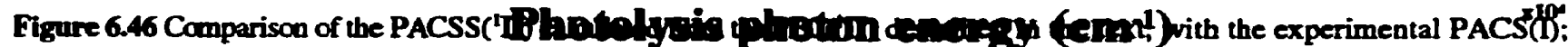
note that the parameters for the PEC('II) were determined from the range of experimental PACS(I) included between the vertical lines. Note the onset of the band consistent with previous spectroscopic observations (see chapter 2).

The reader will note that the "experimental" $\operatorname{PACSS}\left(\mathrm{JH}_{\mathrm{i}}\right.$ ), determined from the previous relation, has a wellbehaved shape, i.e. its energy dependence has the qualitative attribute of a partial absorption cross section from a single state. Once again the parameters of the $\operatorname{PEC}\left({ }^{3} \Pi_{1}\right)$ can be calculated using the optimization procedure described above, for which the results are illustrated in figure 6.47. The maximum optimizer value for the fit to this third state was: $\mathrm{O}_{\max }=0.72$; although it is not excedingly high, this is quite good considering the additive errors that accumulate along the successive subtractive steps of the procedure. 


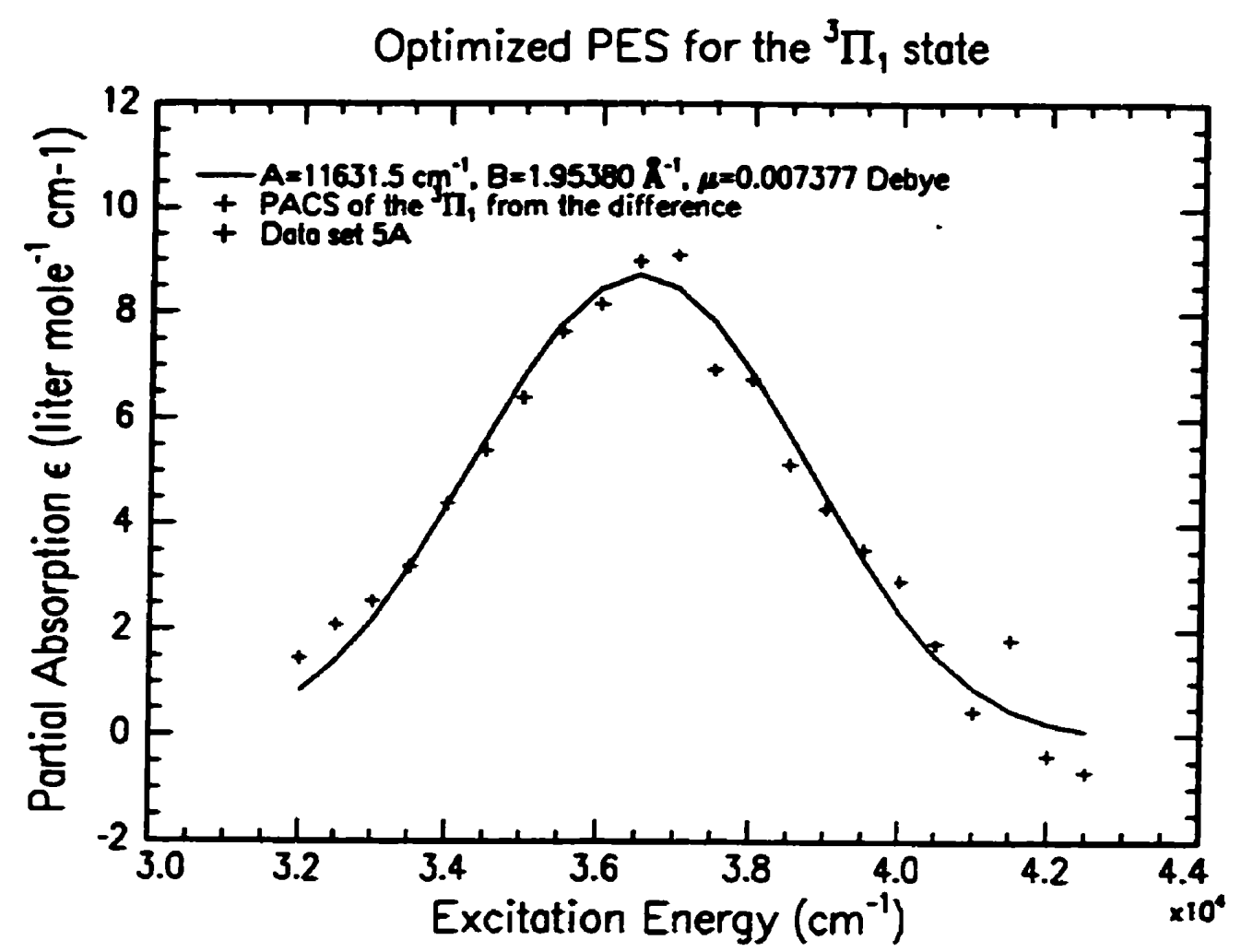

Figure 6.47 Comparison of optimum simulated and experimental PACSS $\left({ }^{\top} \Pi_{1}\right)$.

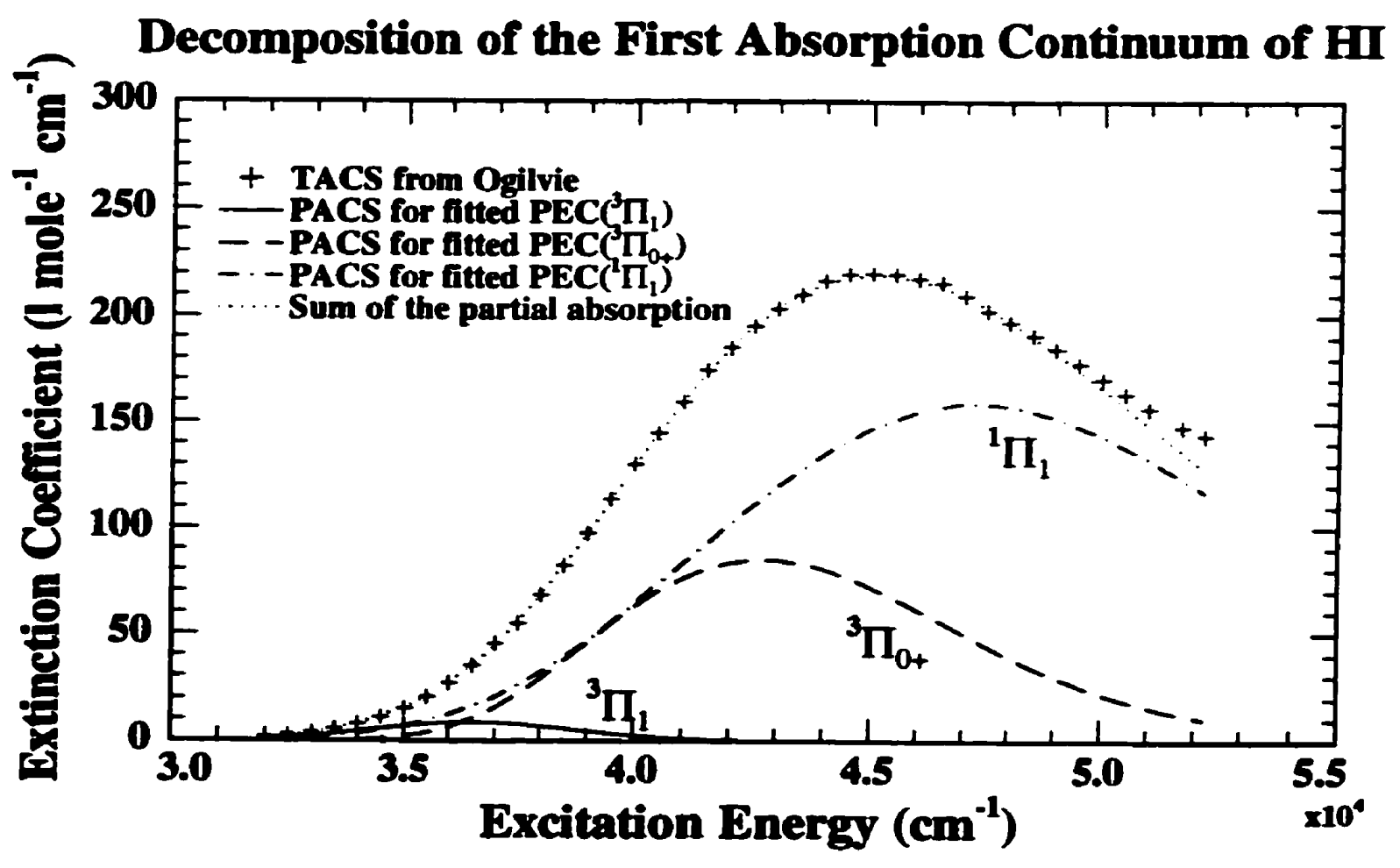

Figure 6.48 Comparison between the sum of the simulated PACSS and the TACS 


\subsubsection{Summary of the QM determination of the PEC}

The present model of the first absorption continuum involves three dissociative states: two of which $\left\{{ }^{3} \Pi_{1}\right.$ ' $\Pi$ \} correspond to the production of the ground state iodine $\left\{{ }^{2} \mathrm{P}_{3 / 2}\right\}$ products, while the other one $\left\{{ }^{3} \Pi_{0_{*}}\right\}$ is responsible for the production of the exciled state iodine $\left\{{ }^{2} \mathrm{P}_{\nu_{2}}\right\}$ product. The procedure described above was used to determine the PEC parametrized by three constants, and characterized by a value of the transition dipole.

Using three PEC, one for each state of the Q-group predicted by Mulliken", the A band absorption can be decomposed into three partial absorption cross section for each state (PACSS). The PEC parameters, for the states of the Q-group that best corresponds to our observables, have been obtained. By using equation (6-3) as an analytical representation of the PEC, the numerical values of the parameters obtained are listed in table 6.6. Figure 6.48 illustrates this by comparing the sum of the calculated PACSS with the experimental TACS. The results of this QM model provides reasonable extrapolation to predict the products branching ratios $R$ between and beyond our measurements; in fact, the prediction of $\mathbf{R}$ could be already obtained after the determination of $\operatorname{PEC}\left({ }^{3} \Pi_{0}+\right)$.

Table 6.6. PEC parameters for states of the A band, determined through the analytical form equation (6-3).

\begin{tabular}{|c|c|c|c|c|}
\hline State & $A\left(\mathrm{~cm}^{-1}\right)$ & $B\left(\AA^{-1}\right)$ & $C\left(\mathrm{~cm}^{-1}\right)$ & $M_{u}$ (Debye) \\
\hline${ }^{3} \Pi_{1}$ & $11550 \pm 80$ & $1.92 \pm 0.03$ & 0 & $0.0067 \pm 0.0007$ \\
\hline${ }^{3} \Pi_{0_{*}}$ & $11542.5 \pm 12.5$ & $3.984 \pm 0.004$ & 7603.15 & $0.0278 \pm 0.0003$ \\
\hline${ }^{2} \Pi$ & $24298 \pm 20$ & $3.104 \pm 0.008$ & 0 & $0.0559 \pm 0.0014$ \\
\hline
\end{tabular}

We mentioned that other explanations, such as a more complex PEC, or a radial dependence of the transition dipole, could modify the explanation for the PACS(I) and PACS(I*). However more experimental information, not presently available, are required to quantitatively test these hypothesis, if they are to contribute in an enhancement of our understanding for this system. Nevertheless, our procedure rests on simple but consistent ground. To further enhance the knowledge on this topic, the author suggests an experimental test of the prediction of $R$ obtained from the extrapolation afforded by the model calculation listed in table 6.5 .

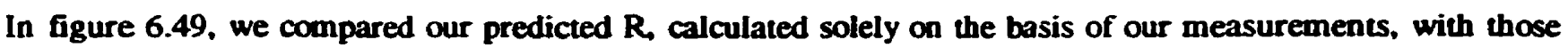
obtained in previous experimental measurements. The agreement is encouraging, since our calculation predicts a value in agreement with the value of $R$ around $51800 \mathrm{~cm}^{-1}$. observed by $X u$ et al., 5 and which was not included in the input data of this analysis. 


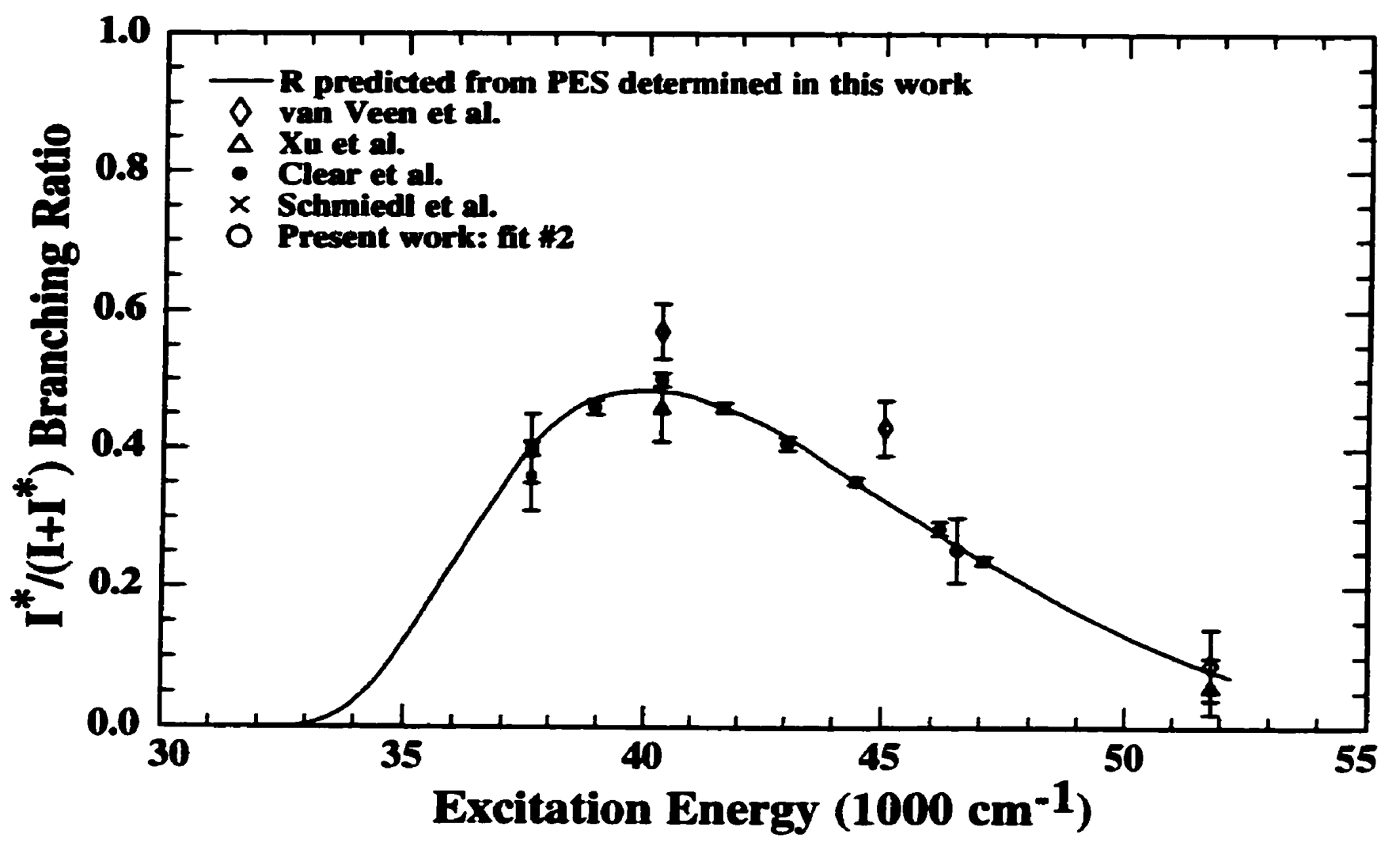

Figure 6.49 Comparison of our calculated $R$ with the results from previous photofragment spectroscopy studies and ours. 


\section{References}

1. J. F. Ogilvie, Trans. Faraday Soc. 67, 2205 (1971)

2. R. L. Kelly, Atamic and Ionic spectrum lines below 2000 Angstroms: Hydrogen through Krypton, Part I (H-Cr), J. Phys. and Chem. Ref. Data, Vol. 16, Suppl. No. 1 (1987)

3. W.H. Press, S.A. Teukolsky, W.T. Vetterling, B.P. Flannery, Numerical recipes in $C, 2^{\text {pd }}$ ed., Cambridge (1992)

4. GENPLOT, A data analysis and Graphical plotting program for scientist and engineers, Computer Graphic Service, 52 Genung Circle, Ithaca, NY 14850-8716, (607)-277-4913

5. Z. Xu, B. Koplitz, and C. Wittig, J. Phys. Chem., 92, 5518 (1988)

6. R. Schmiedl, H. Dugan, W. Meier, and K. H. Welge, Z. Phys., A, 304,137 (1982)

7. G. N. A. van Veen, K. A. Mohamed, T. Baller, A. E. de Vries, Chem. Phys., 80, 113 (1983)

8. I. Levy, M. Shapiro, J. Chem. Phys., 89, 2900 (1988)

9. J. Zhang, M. Dulligan, J. Segall, Y. Wen, C. Wittig, J. Phys. Chem., 99, 13680 (1995)

10. R.D. Clear, S.J. Riley, K.R. Wilson, J. Chem. Phys., 63, 1340 (1975)

11. R. S. Mulliken, Phys. Rev., 51, 310 (1937)

12. A.A, Passchier, J.D. Christian, and N.W. Gregory, J. Phys. Chem., 71, 937 (1967)

13. R. J. Le Roy, R.G. Macdonald and G. Burns, J. Chem. Phys., 65, 1485 (1976)

14. R. M. Herman, R. H. Tipping, S. Short. J. Chem. Phys., 53, 595 (1970)

15. J. A. Coxon, P. G. Hajigeorgiou, J. Mol. Spect, 150, 1 (1991)

16. R. J. Le Roy, University of Waterloo Chemical Physics Research Report, CP-555 (1995)

17. F.R. Hampel, E.M. Ronchette, P.J. Rousseeuw and W.A. Stahel, Robust statistics: The approach based on influence functions. Wiley, New York (1986)

18. Private communication. Mary Thompson, the chairman of Department of Statistics and Actuarial Sciences at the University of Waterloo, has confirmed the validity of the method. The author is grateful for the help of Dr. Thompson, who demonstrated the properties of this optimization technique that provide its robustness.

19. G. Herzberg, Molecular Spectra and Molecular Structure. I Spectra of Diatomic Molecules, (reprinted by) Krieger Publishing Co, Florida (1989)

20. Private communication.

21. J. Romand, B. Vodar, Compt. Rend., 226, 890 (1948)

22. B. J. Huebert, R. M. Martin, J. Phys. Chem., 72, 3046 (1968) 


\section{please note}

Page(s) misaing in number only, toxt follows.

Findod as recoived.

\section{UMI}




\section{Chapter 7 Conclusion}

The central results of this thesis are: (i) the experimental determination of the anisotropies and the electronic branching ratios for the photofragments from $\mathrm{HI}$ photolysis at nine photons energies throughout the $\mathrm{A}$ band, (ii) the corresponding determination of the partial absorption cross section (PACS) for each electronic product. and (iii) the determination of the parameters for the potential energy curves (PEC's) representation of the dissociative states, obtained by a quantum mechanical (QM) calculation done within the Franck-Condon approximation (FCA), and the consequent extrapolation of the branching ratios over the $A$ band absorption.

\subsection{Photodissociation dynamics of $\mathrm{HI}$ upon excitation in the $\mathrm{A}$ band}

\subsubsection{Observables from Doppler spectroscopy of H-atoms}

The analysis of Doppler spectra from H-atom photofragments yielded precise values for the characteristic observables of this photodissociation process. The observables obtained from the inversion of the Doppler profile measurements demonstrated that there is a one-to-one correspondence between the electronic product and the transition symmetry in this photodissociation process. The electronic branching ratios, obtained from the averaged measurements of Doppler profile at nine photolysis wavelengths, were listed in table 6.2.

The values obtained for the anisotropy parameters indicates that the excited iodine atoms result from a transition of parallel symmetry, while the ground state iodine atoms were known to result from perpendicular transitions, in agreement with our observation. However, this result was found to be in marked disagreement with those obtained in a previous photofragment spectroscopy study by van Veen et al., yet it was in good agreement with the theoretical model first derived by Mulliken ${ }^{2}$ on the basis of symmetry correlation. The author demonstrated that these discrepancies originated from clustering in the molecular expansion, which affected the results obtained in the earlier study of van Veen et al. ${ }^{1}$

\subsubsection{Explanation of the discrepancies}

First it was hypothesized that the measurements from van Veen et al.' may have been significantly affected by the presence of a relatively large concentration of clusters in their supersonic expansion of pure $\mathrm{HI}$. This suggestion was prompted by the observation of a conspicuously wider energy spread in the TOF peak for the slow perpendicular peak, which had neither been observed nor predicted before. By an opportune coincidence, Zhang et al. ${ }^{3}$ have very recently published the results of an experimental study on the photodissociation dynamics of $H I$ dimers. Their results were consistent with the present hypothesis, and they also supported the evidence of condensation regarding the broadening of the kinetic energy distribution. Moreover, they observed that the conditions under which the experiment of van Veen et al.' was conducted favour clusters formation. 
According to their paper, van Veen et al.' were seemingly not aware of the presence of clusters in their pure HI expansion. On the basis of their results, they concluded that Mulliken ${ }^{2}$ was mistaken regarding his prediction of the ${ }^{3} \boldsymbol{\Sigma}_{\mathrm{t}}$ state. They assumed that this state, the lowest electronic state that both has the appropriate symmetry and correlates to the excited iodine product, was in fact at a lower energy than Mulliken ${ }^{2}$ predicted. Van Veen et al.' also did a quantum mechanical calculation, which was based on a modified 8 -approximation, or reflection method, for the calculation of the overlap integral between the ground and excited state wavefunction.

On the basis of the same experimental results, Levy and Shapirot performed a detailed quantum mechanical calculation, and they followed the interpretation of van Veen et al. ${ }^{1}$ concerning the presence of the ${ }^{3} \Sigma_{1}$ state. In order to provide a quantitative explanation for the observations of van Veen et al., Levy and Shapiro found that nonadiabatic coupling effects between the various states of perpendicular symmetry had to be included in the photodissociation dynamics. This further complicated the physical picture for this process, prior to our study.

A reasonable explanation for the discrepancies present amongst these studies have been presented, which brings consistency among the results of all previous experimental studies. The observation of van Veen'. concerning the perpendicular component of the slow channel, should not bave been attributed to the photodynamics of the monomer, but rather to the photodynamics of an undetermined mixture of monomer, dimer, and other heavier clusters^. Moreover, in all experimental studies on HI photofragment spectroscopy performed after 1987, when general awareness of clustering had became more generally recognized, the anisotropies of the excited electronic product has been observed to be of pure parallel symmetry type..

\subsubsection{Consequence for the photodissoclation model}

The assumption of nonadiabatic interaction in this dissociation process was a consequence of the electronic structure hypothesized by van Veen et al.' This hypothesis was a consequence of the observed perpendicular contribution of the excited electronic iodine product, which was showed to be an experimental artifact. Therefore, there is no need to take account of nonadiabatic effects in the HI photodissociation.

The realization of this point, combined with the high velocity of the H-atom fragments, suggested the validity of the axial recoil approximation for this system, i.e. in this dissociation process, the molecular rotation merely witness the bond breaking. Moreover, the observables obtained in this work indicates a remarkably symmetric picture for the dynamics of $\mathbf{H I}$ photodissociation in the $\mathbf{A}$ band, which further substantiates these findings.

\footnotetext{
A Note that in the present study, experiments were carried out with an HI concentration around 5\%, for which Zhang et al. warking in very similar condition, bas showed that the dimer dynamies was contributing to less than $2 \%$ of the signal. the rest being clearly due to the HI monomer.
} 


\subsection{The low-lying dissociative states of $\mathrm{HI}$}

\subsubsection{Quantum mechanical model calculation}

The values of the experimental observables indicated that the model based on symmetry correlation, which was derived in chapter 2, is indeed valid. The quantum mechanical calculation of section 6.3 has applied the theoretical concepts introduced in chapter 3, in order to quantify the appropriateness of this model for this system.

Three dissociative states were required to best reproduce the experimental observations. The PEC parameters, and the corresponding average transition dipoles obtained for these dissociative states, are listed in table 6.6. A comparison between the final results of our calculation and Mulliiken's prediction ${ }^{2}$ is reproduced in figure 7.1. It can be appreciated that this model, derived on the basis of formal symmetry arguments, describes the physical process with reasonable agreement.
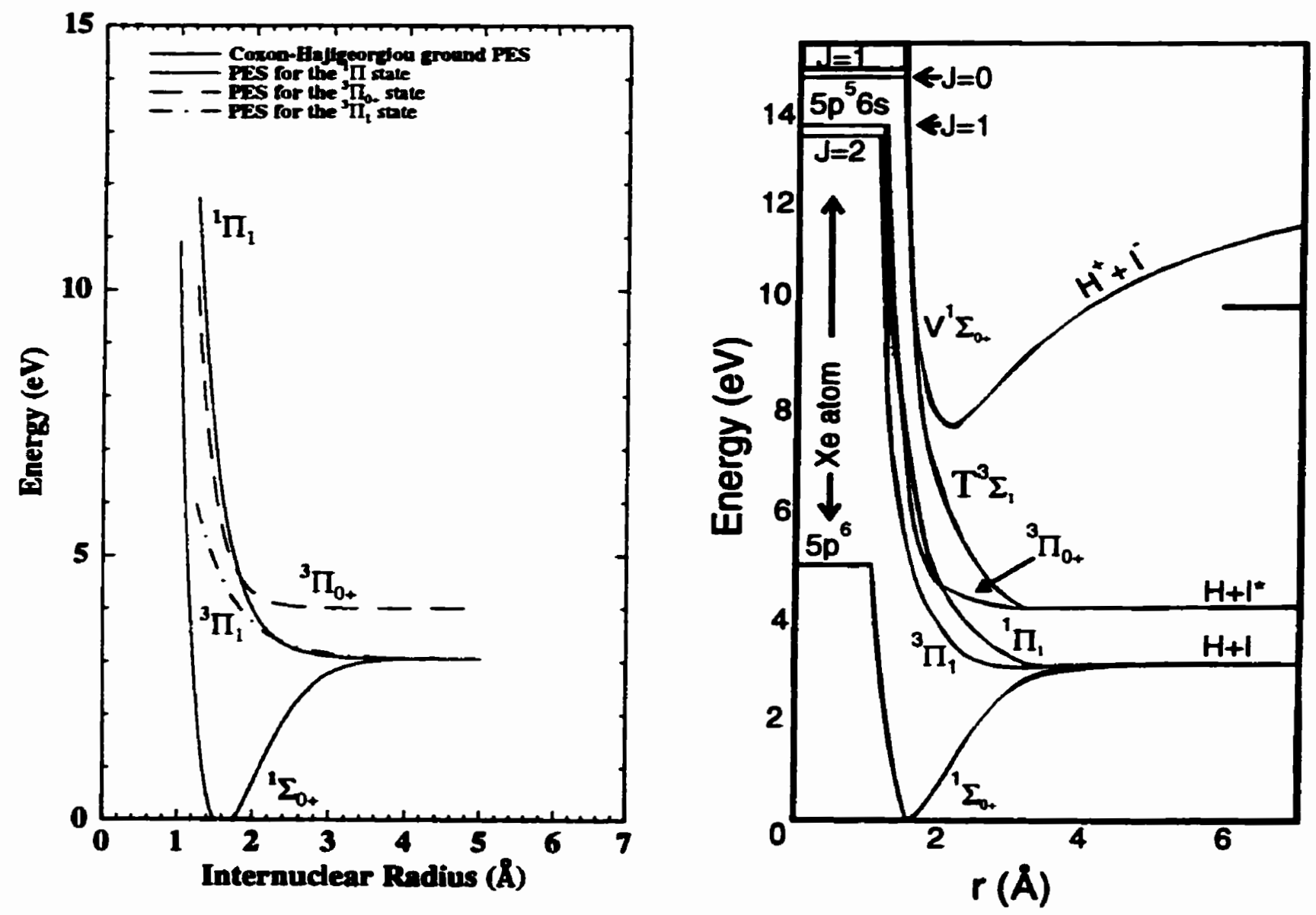

Figure 7.1. Comparison of a) the PEC determination based on the experimental measurements obtained in the present study, with b) the prediction of Mulliken'. 
This thesis demonstrated experimentally that the assumption of Mulliken ${ }^{2}$, regarding the representation of the A band absorption by the ( $Q$ - $N$ ) transitions from the ground state $(N)$ to the $Q$-group of dissociative state, does indeed provides a valid physical representation for this process.

\subsubsection{Inference from the model}

Because the model calculations are based on optical transitions from the ground state, the validity for the PEC of the dissociative states, represented by the analytical form of equation (6-3), can only be ascertained within the Franck-Condon region. However, this limitation does not limit the use of the PEC to infer PACS at arbitrary energy through the $A$ band absorption.

Moreover, it was illustrated in chapter 6 that, over the Franck-Condon region, the numerical values of the PEC obtained from the quantum mechanical calculation, are independent of the analytical model employed. The reason is simply that optical transitions from the ground state only sample a very narrow range of internuclear distances on the dissociative PEC, and this function is only characterized over that narrow range by the observable measured in a photofragment spectroscopy experiment, when the direct dissociation proceeds adiabatically.

Nevertheless. the electronic structure for the dissociative states of $\mathrm{HI}$ can in turn be used to interpolate and extrapolate the values of the ratio for the electronic branching, and the PACS for each product, beyond the energy range of our measurements. This calculation was done in chapter 6; the results, listed in table 6.5, are plotted in figure 7.2. In 7.2 a), the uncertainties over the branching ratio are also represented, as estimated from the uncertainties on the PEC parameters obtained from the quantum mechanical calculation.

\subsubsection{Note on the low-lying electronic structure of hydrogen halides}

In itself, the hypothesis regarding the possibly lower $\mathrm{T}$ state, made by van Veen et al. ${ }^{\prime}$, was a reasonable assumption, in light of their observation; but it was due to an experimental artifact in their case. However, because the $\mathrm{I}$ state is mixed with the ion pair configuration at short internuclear range, an argument can be developed, regarding the comparative probability for the $\mathrm{T}$ state to contribute to $\mathrm{A}$ band absorption, in the hydrogen halide family.

In table 2-7 of chapter 2, the atomic properties that determine the asymptotic values of the PEC for the ion pair in the hydrogen halide family of molecules were compared. The main trend illustrated through this comparison, was the relative position of the asymptotic energy levels for the ion pair state, which can be seen to lie at increasingly high energy, from HF to $\mathrm{HI}$. In the section 2.3.3, it was indicated that the ion pair can affect the electronic structure of the ground state. Here, similar arguments are used to suggest our expectation for the electronic structure of the first dissociative continuum of outer hydrogen halides, in comparison to HI molecule. 

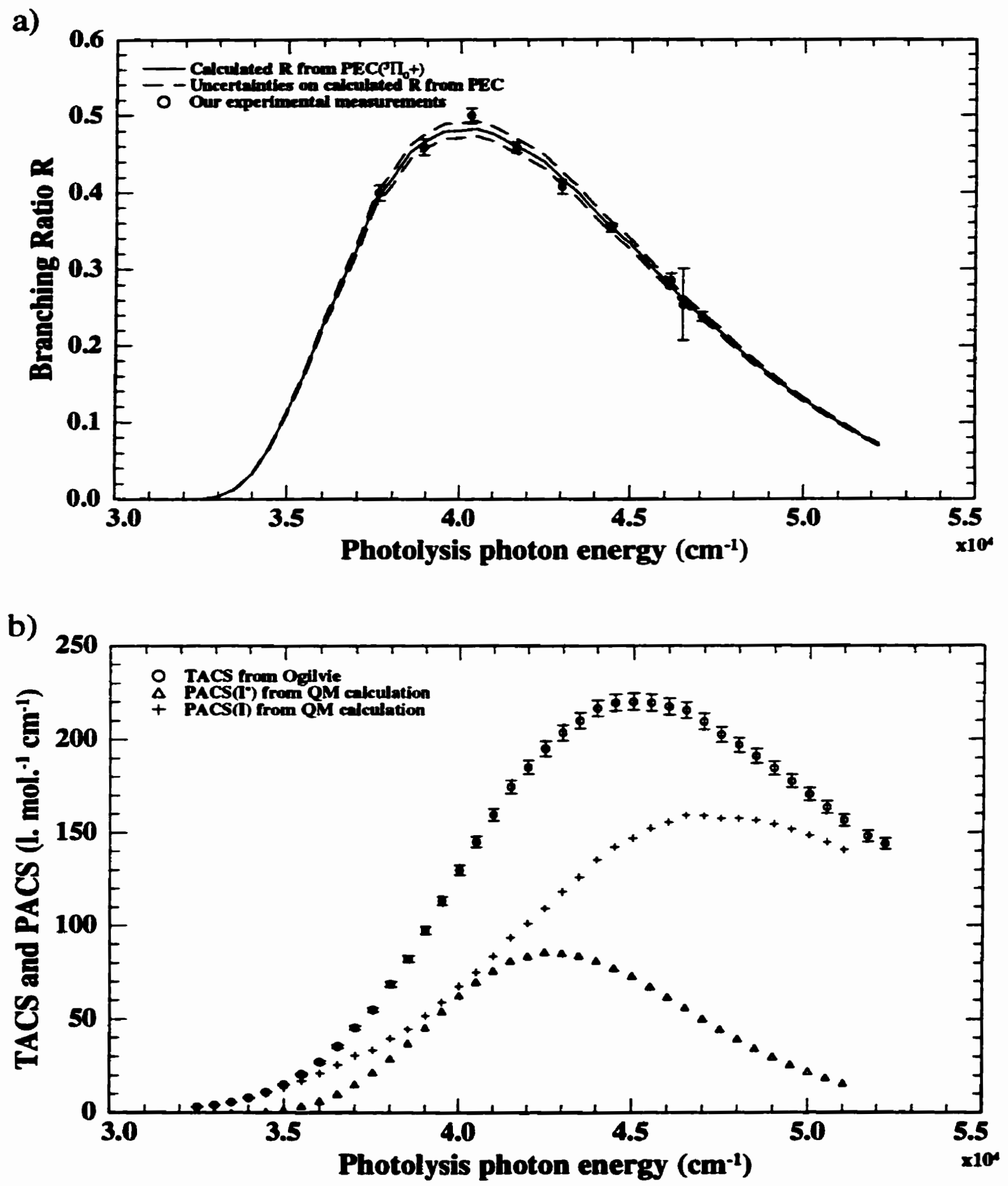

Figure 7.2 a) Extrapolated R values based on PEC calculated in this work; b) the TACS and the PACS for each product. 
Referring to figure 2.1 and 2.5, it can be observed that because the ion pair (V state) is mixed with the ${ }^{3} \Sigma$ (T state) in the united atom symmetry, these states can perturb each other. Thus, the location of the $\mathrm{V}$ state may possibly affect the $T$ state, in appropriate conditions favoured by closer interatomic distances in the molecule.

In the present study, it was concluded that the $\mathrm{T}$ state does not contribute to absorption in the $\mathrm{A}$ band. On the basis of the aforementioned arguments, it is expected that, as the ion pair state gets lower and lower from HI to HF, the contribution from the ion pair to A band absorption may become significant. Moreover, because the average internuclear distance gets shorter for lighter hydrogen halides, the mixing between ion pair and $\mathrm{T}$ states becomes more favoured by the increased contribution of the united atom symmetry, from which the mixing originates. These arguments suggest that the propensity for the involvement of the $T$ state in the first absorption band increases from HI to HF. As a consequence, if the optically accessible $T^{3} \Sigma_{1}$ is either dissociative or predissociated, the product channel corresponding to the excited halogen atom may show a perpendicular contribution from the angular distribution of the fragments velocities, for the lighter members of the HX family.

\subsection{Epilogue}

It was attempted to verify a previously established model of $\mathbf{H I}$ photodissociation, in which nonadiabatic transition was playing a major role. The final results of the present investigation indicates a much more straightforward picture for the photodissociation dynamics of $\mathrm{HI}$, yet predictable and consistent with the fundamental concepts of molecular physics. Within the precision of our measurements, we observed that the standard model of direct dissociation on a single PEC, derived on the basis of symmetry correlation from the atomic structure, does indeed describe the dissociation of $\mathrm{HI}$ in the $\mathrm{A}$ band very well.

\section{References}

1. G.N.A. van Veen, K.A. Mohamed, T. Baller, A.E. de Vries, Chem. Phys., 80, 113, (1983).

2. R. S. Mulliken. Phys. Rev., 51, 310, (1937).

3. J. Zhang, M. Dulligan, J. Segall, Y. Wen, C. Wittig, J. Phys. Chem., 99, 13680, (1995).

4. I. Levy, M. Shapiro, J. Chem. Phys., 89, 2900, (1988). 


\section{Appendix A Cryostatic HI source and free supersonic jet expansion}

A cryostatic HI:He source was constructed to deliver a constant concentration of gaseous HI:He solution to the free supersonic jet expansion; the purpose of this section is to describe both these systems in sufficient detail to estimate the gas flow from the source, and the concentration and temperature of $\mathrm{HI}$ molecules in the interaction region of the photofragmentation experiment. After a description of the HI:He source, a brief account of the theoretical concepts and analytical formula used to describe the supersonic expansion will be made.

\section{A.1 Cryostated HI source}

a) Description of the device

The cryostatic HI:He source was illustrated in the upper part of figure 4.4. The design of this source is based on the constant equilibrium vapour pressure of a thermostated HI source. The source is thermostated at cryogenic temperature in an ethanol/dry ice slush, which provides a constant temperature bath at $-72{ }^{\circ} \mathrm{C}$. At this temperature, the partial pressure of $H I$ can be calculated from equation $(A-1)$, where $K$ is the temperature in Kelvin, and $P$, the pressure is in Torr.

$\log _{10} P=(-0.2185 A / K)+B$

Using the values ${ }^{1}$ of $A=5058.2$ calories/mole and $B=7.475631$ in equation $(A-1)$, valid from -123 to $127^{\circ} \mathrm{C}$, the partial pressure of $\mathrm{HI}$ at $\mathrm{T}=-72^{\circ} \mathrm{C}$ can be calculated to be $\mathrm{P}=95.8 \mathrm{Torr}$. Applying a quiescent pressure of 25 PSIA of He gas, and maintaining a very slow flow determined below, permits to maintain the equilibrium in the $500 \mathrm{ml}$ source of $\mathrm{HI}$, and this results in a $-6 \% \mathrm{HI}: \mathrm{He}$ mixture. As illustrated in figure 4.4, the SS lecture bottle was fitted with two gas ports: one at the top, to inject a constant pressure of He used as the carrier gas, and one at the side, from which the equilibrium mixture flowed out to the puised valve.

b) Flow measurement

To calculate the gas flow through the vacuum expansion, the volume of the gas that flows out of the botele was determined through a pressure decay experiment, i.e. we recorded the lifetime for the pressure of a preset volume to decrease by $e^{-1}=0.368$. The preset volume defined by a section of the gas delivery system between the shut-off valve at the exit part of the HI source up until and including the pulsed valve, has been estimated to be $11.5 \mathrm{~cm}^{3}$. By observing the rate of the decreasing pressure in the gas manifold, after the shut-off valve is closed, an estimate of the flow can be obtained by recalling that gas flow from high pressure to vacuum depends only on the high pressure side; the gas flow: $\partial N / 2 t=K_{p} P$, where $K_{p}$ (mole/[sec-PSIA]) is a constant that depends on gas viscosisty and on the dimension of the restriction to flow. Assuming the ideal gas, i.e. $P=C N$ (using $P V=N R T$ ), where: $C=3030$ (PSIAmole) at roam temperature. One obtains $\partial N / \partial t=K_{p} C N$, which is solved to find: $N=N_{0} \exp (-V \tau)$. 
It has been observed that $\tau=1 /\left(K_{p} C\right)=600 \mathrm{sec}$, which implies: $K_{p}=1 /(\tau C)=5.5 \cdot 10^{-7}$ moles $/$ (PSI-sec). However, in normal operating condition, the number density on the high pressure side is kept constant, which implies, that: $\mathrm{N}=\mathrm{P} / \mathrm{C}=25 / 3030=8.25 \cdot 10^{-3} \mathrm{~mole}$, and therefore $<\partial \mathrm{N} / \partial \Delta=1.38 \cdot 10^{-5} \mathrm{~mole} / \mathrm{sec}=0.19 \mathrm{~cm}^{3} / \mathrm{sec}$, at 25 PSIA. Hence the steady-state flow of the HI:He mixture was around $10 \mathrm{ml} / \mathrm{min}$ or $1.4 \cdot 10^{-5} \mathrm{~mole} / \mathrm{sec}$, at stagnation pressure of 25 PSIA. During a gas pulse, the average amount of HI:He gas mixture released in the vacuum expansion is $1.4 \cdot 10^{6}$ mole/pulse or $2 \cdot 10^{-2} \mathrm{~cm}^{3}$, at 25 PSIA.

c) Notes on peculiarities of the HI source

1) On the chemical reactivity of SS 316 with hydrogen iodine

Caution regarding the notorious reactivity of $\mathrm{HI}$ suggested to use Monel alloy to build the gas delivery system that supplies the HI gas mixture to the vacuum expansion. Subsequent modifications in the layout of the laboratory required the gas delivery system to be rebuilt; for the sake of simplicity and economy, we constructed the new gas delivery system out of regular SS 316 alloy. Through operation of this gas delivery system with HI gas mixture of 5 to 100\% HII:He, it was observed that if proper care is taken to avoid trace water in the gas line, the SS 316 metal shows no sign of surface degradation even after more than two years of usage, as observed from the inside flange of the pulsed valve. On the other hand, a gas line of the same material exposed to $\mathrm{HI}$ and air consecutively, for few days at a time, showed surface damage within weeks. Therefore we observed that the water-free SS 316 metal is chemically stable with respect to HI, while the opposite is often assumed to be true.

2) On initial induction time of the HI source, due to reactive passivation of the SS surface by hydrogen iodine The reactivity of HI on metal surfaces, common to the family of hydrogen halides, is such that a fraction the HI molecules partially decompose on fresh metal surfaces. This results in an apparent induction time, before the steady-state value of HI target ar centration is reached, when one introduces the gas in the line for the first time. and to a lesser extent, after it has been exposed to the laboratory atmosphere. However, steady-state conditions are observed to recover after about 20-40 minutes of pulsed flow, depending on $\mathrm{HI}$ concentration of the source. This observation is explained by a passivation of the SS surface of the gas delivery system. A similar effect is also observed in excimer laser systems using halogen and bydrogen balides.

\section{3) On the thermal equilibrium in the cryostatic HI source}

Another peculiarity of this source was observed, with regard to stability: if the SS HI botule was surrounded with the dry ice/ethanol mixture up to the top of the bottle, while the level of $\mathbf{H I}$ was approximately at $1 / 5$ of its full volume, the partial pressure of $\mathrm{HI}$ was reduced $t 030 \%$ of the steady state conditions, within a period of 30 minutes, after 3-4 hours of having kept the bottle at low temperature. It was found that if the 1/8 inch O.D. SS tubing feeding the He pressure was heated at 60-70 ${ }^{\circ} \mathrm{C}$, the problem was remediated, and the steady-state equilibrium flow condition of the mixture were stable over the longest time observed, i.e. 20 hours. 
To explained these observations, we first recall that the $\mathrm{HI}$ is solid at $72^{\circ} \mathrm{C}$, so that heat conduction through mechanical convection of the liquid is hindered. Secondly, the latent heat of evaporation, $A=5058$ calories/mole or approximately $0.2 \mathrm{eV}$, is quite large. The flow rate calculated above implies that $0.8 \mathrm{mWatt}$ of heat must be leaked to the solid $\mathrm{HI}$ in order to keep a steady-state flow at thermal equilibrium. The heat flow depends on thermal gradient, and it will be small if the foot long SS lecture bottle is well surrounded by the ethanol/dry ice slush, SS being a rather poor heat conductor. It was deduced that the solid HI ends up being cooled down by the constant evaporation of the solid HI. Considerations on a similar problem with $\mathrm{HBr}$ were mentioned in the $\mathrm{Ph}$. D. thesis ${ }^{2}$ of Dr. Hepburn.

\section{A.2 Supersonic expansion for a $\mathrm{HI}: \mathrm{He}$ mixture}

In a supersonic expansion. the short-range intermolecular potential ${ }^{3}$ acts to couple the internal degree of freedom of the HI seed molecules to the colder translational degree of freedom of the He carrier gas. In proper conditions, a reduction of the temperature to few Kelvin is typically reached. The basic mechanism of supersonic expansion for a pure monoatomic gas has been well understood for more than thirty years, but the finer details concerning the cooling of the vibrational and rotational degree of freedom of seed molecules are still object of investigations $\mathrm{s}^{5.6}$. A synopsis of the model' for the supersonic jet is summarized below. Formula from the theory are only invoked to the extent required to obtain an estimate of temperature and number density of our seeded supersonic free jet expansion.

\section{a)Theoretical concepts}

Because the number density $n_{s}$ of the seed gas is of the order of a few percents of the carrier gas $n_{C}$, it is generally assumed that it is the carrier gas whose relaxation drives the expansion. The carrier-carrier molecular collision frequency, $\omega_{\mathrm{cc}}$ is roughly the same as the seed-carrier molecular collision frequency, $\omega_{\mathrm{sc}}$ and these are much larger than the seed-seed collision frequency $\omega_{s s}$. This argument justifies the seed gas contribution to the expansion cooling. Consequently, the problem of the seeded supersonic expansion can be treated, at least in first approximation, as the expansion of a nearly-pure carrier gas, and the thermalization of the seed gas in the surrounding flow field of the carrier gas.

\section{b) Theoretical formula}

Since the exact description of the cooling of a seeded gas is beyond the scope of this work, we will content ourselves with the simpler calculation of the local temperature of the carrier gas to provide a lower limit for the temperature of the seed. The local temperature of the carrier gas and the local number density of the seed gas are expresced below as a finction of the reduced distance from the nozzle on the flow stream axis, defined as: $z=Z / d$, where $Z$ is the distance from the nozzle and $d$ is the nozzle diameter. The dependence of local equilibrium 


\section{Seeded Supersonic Expansion}

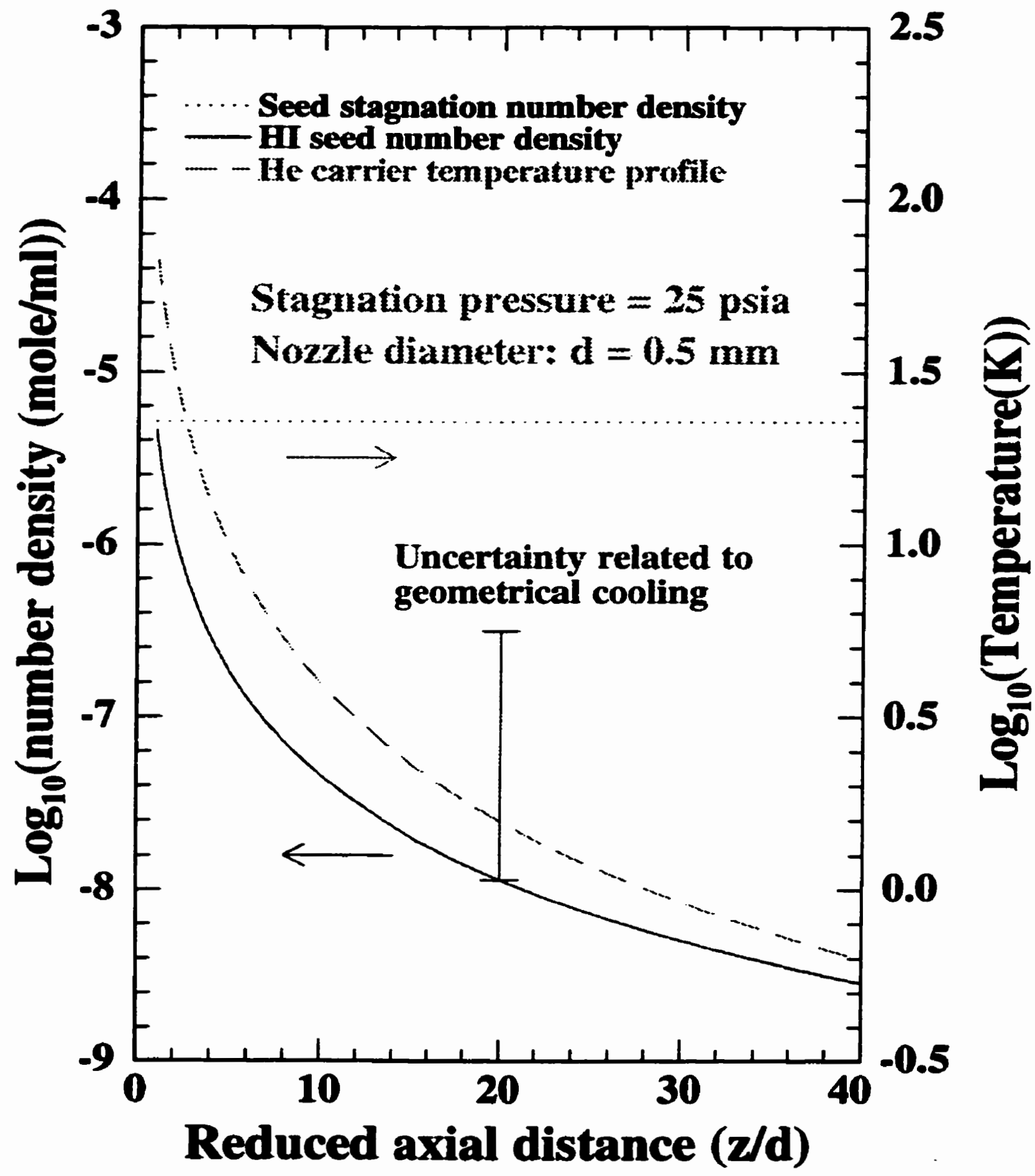

Figure A.l Predictions of the model for the supersonic expansion of the HI:He mixture. At the experimental position, z/d= 20, the translational temperature of the He carrier gas is reaching $1.6 \mathrm{~K}$. and the HI number density of the seed. $\mathrm{n}_{\mathrm{s}}$. can be estimated by using the gas ratio at the stagnation pressure. 
temperature of the carrier gas on the Mach number is expressed by equation (A-2). At large distance from the nozzle, $2>5$, where the flow is radial, the Mach number is given by (A-3).
$M(z)=C_{1} z^{\gamma-1}$
(A - 2)
$T_{c}(M)=T_{0, c}\left[1+M^{2}((\gamma-1) / 2)\right]^{-1} \quad(A-3)$
$\mathrm{n}_{c}(\mathrm{z})=\mathrm{n}_{0 . c^{2}} \mathrm{z}^{-2}\left[\left(2 / \mathrm{C}_{\mathrm{l}}(\gamma-1)\right)\right]^{1 /(\gamma-1)} \quad(A-4)$

\section{c) Numerical application}

For a monatomic gas, $\gamma=5 / 3$ and $C_{1}=3.23$. We are interested in the local number density of the seed gas in the expansion. The local number density of the carrier gas, at large distance from the nozzle, is given by (E-3). Assuming a quiescent pressure of 25 PSIA, the formula developed above are applied to our HI:He mixture. The result is shown in figure A.1.

A lower limit for the number density of the HI seed is provided by assuming: $n_{s} / n_{c}=n_{0, s} / n_{a c}$, i.e. the number density ratio remains constant through the expansion. The effects of Mach number focussing, discussed in reference 4, tends to increase the heavier seed concentration. For our seed concentration, an estimate of the effect of Mach number focussing is represented by an upper limit factor of 5 for the uncertainty in seed concentration. This was obtained by using arguments of momentum conservation and angular dependence of the free inviscid tow for the independent gas. Inspection of figure E.1 indicates that in the typical conditions used in the present study, the number density of the $\mathrm{HI}$ molecules in the experimental volume were approximately between $1.0 \cdot 10^{-8}$ -

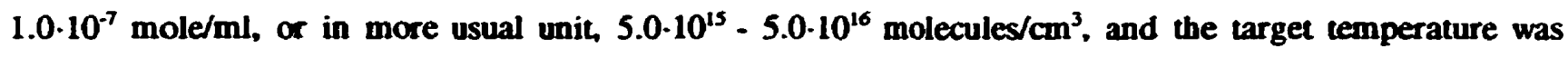
somewhere between 1 and $15^{\circ} \mathrm{K}$. This theoretical estimate is in good agreement with the temperature observed through the rotational distribution of CO molecules, measured with VUV LIF (see chapter 4), and cooled in the same conditions.

\section{References}

1. CRC, Handbook of Chemistry and Physics, 54th ed., CRC Press (1973-1974)

2. John William Hepburn, VUV LIF studies in chemical dynamics, Ph. D. Thesis, University of Toronto (1980)

3. DL. Goodstein, States of Matter, Dover (1985)

4. H. Ashkenas, F. S. Sherman, Rarefied Gas Dynamics, 4 th symp., ed. J.H. de Leeuw, 2:84, Academic (1966)

5. D. Pullman, B. Friedrich, D. R. Herschbach, J. Chem. Phys., 93, 3224 (1990)

6. P. K. Sharma, E. L. Knuth and W. S. Young, J. Chem. Phys., 64, 4345 (1976)

7. S. DePaul, D. Pullman and B. Friedrich, J. Phys. Chem., 97, 2167 (1993) 


\section{please note}

\section{Pago(o) miasing in number only; toxt follows.}

Finned as recsived.

\section{UMI}




\section{Appendix B Laser dye concentration}

\section{B.1 Optimization of $F 548 / \mathrm{NaOH}$ dye laser solution for $532 \mathrm{~nm}$ pumping}

The absarption cross section for the Fluorescein $548^{\wedge}$ (F548) laser dye depends strongly on the concentration of sodium hydroxide (NaOH) present in the ethanol solution. Alhough this fact was known, no systematic characterization of this binary system has been made. Because the $\mathrm{NaOH}$ has a reactive nature, potentially magnified by the presence of long-lived triplet excited state in the dye solution excited by the exposure to intense radiation, it was deemed wise to find the minimum $[\mathrm{NaOH}$ ] that optimize the optical properties of the gain medium for the binary system F548: $\mathrm{NaOH}$, in order to minimize the dye degradation and maximize the gain medium solution lifetime.

To achieve this goal, a set of F548 solutions in ethanol were made, with varying concentrations of NaOH. Each solution was characterized by its absorption spectrum, measured with a HP diode array spectrometer, using a quartz absorption cell with a $1 \mathrm{~mm}$ optical path. The results of the measurements are presented in figure B.1 a), for seven concentration of $\mathrm{NaOH}$, at $20 \mathrm{mg} /$ increment. Then the absorbance at $532 \mathrm{~nm}$ was noted, and it was ploted against the $\mathrm{NaOH}$ concentration in figure $\mathrm{B} .1$ b), where a clear maximum is observed. A quadratic fit was used to determine the optimum concentration, which was found to be $74.6 \mathrm{mg} / \mathrm{l}$. This optimum value was used to make laser dye solution. The conversion efficiency for the dye laser was found to be $33 \%$, at $150 \mathrm{~mJ} / \mathrm{pulse}$ pump power at $532 \mathrm{~nm}$. Moreover, the lifetime of the dye solution was observed to be more than 500 hours, which is extremely long for a chemical exposed to such intense radiation.

\section{B.2 Laser dye concentrations \\ a) GREEN (532 nm) pumped dyes}

These laser dyes solutions were used in various FWM scheme to generate VUV. In general, the solution for the amplifier are $1 / 3$ of the concentration for the oscillator, unless indicated in the table B.1.

\section{b) BLUE (355 nm) pumped dyes}

The dye solutions listed in table B.2 were employed in the dye laser used for the generation of the UV pump laser. The relatively high cross section of these dyes, and the concomitant low saturation threshold for absorption of the pump power (bleaching), results in the fact that these solutions have to be optimized for both oscillator and amplifier. Moreover, the optimum concentration can be slighdy difierent in the high and low limit of pump power for a given dye; the following concentrations were optimized for a lasers pumped with nominally $100 \mathrm{~mJ} /$ pulse.

A. Exciton inc., Dayron. OHO, USA. 
Optimization of $\mathrm{NaOH}$ concentration for F548 (550 mg/l)
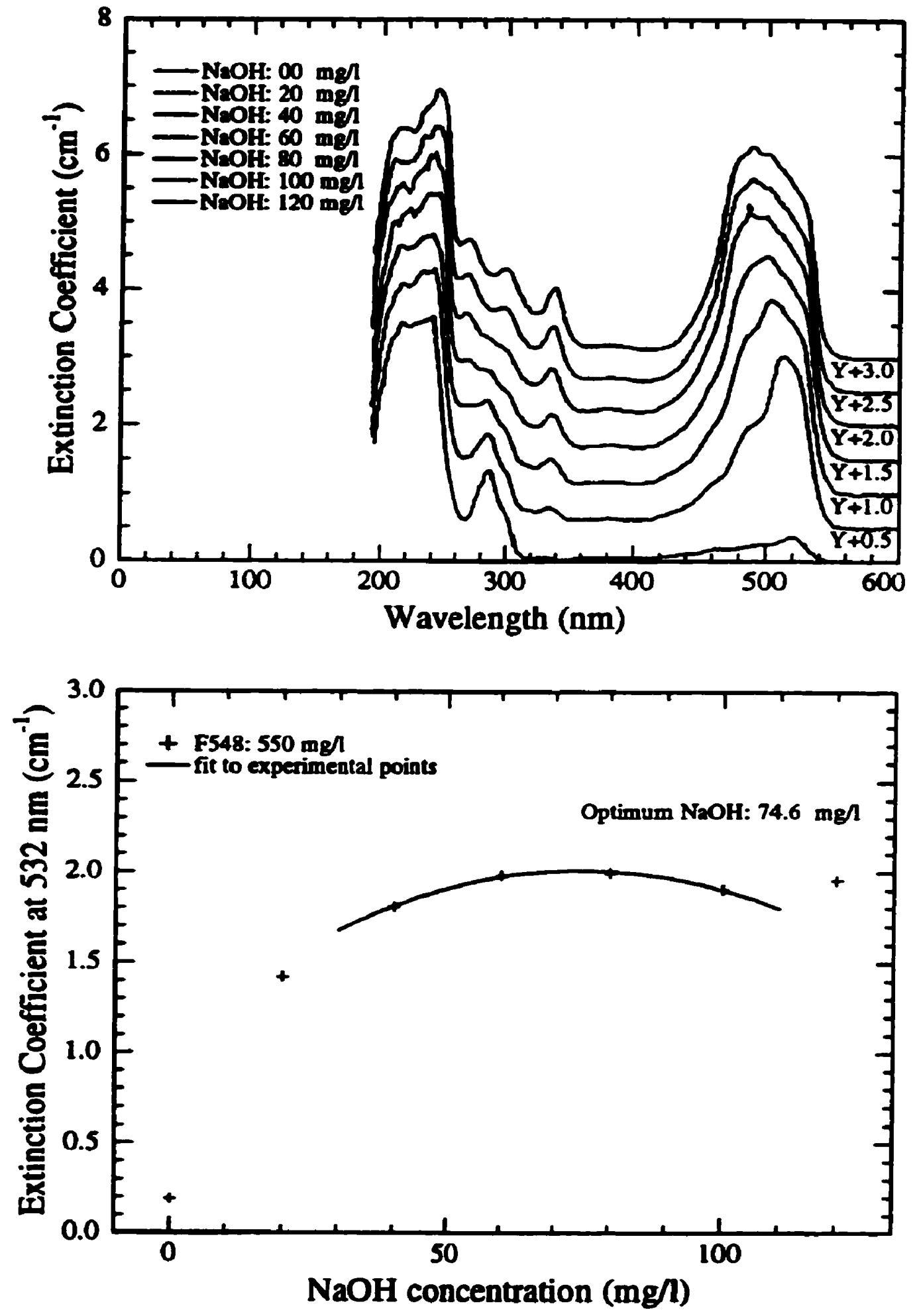

Figure B.1 a) Spectral absorption measurement; b) optimization of $\mathrm{NaOH}$ concentration for $532 \mathrm{~nm}$ absorption. 
Table B.1. Dye solutions used for the generation of the UV (PUMP) photolysis

\begin{tabular}{|c|c|c|c|c|c|c|c|}
\hline $\begin{array}{c}\text { Photolysis } \\
\lambda(\mathrm{nm})\end{array}$ & $\begin{array}{c}\text { Dye } \\
\text { Counter }\end{array}$ & order & $\begin{array}{c}\text { Typical } \\
\text { Power }(\mathrm{mJ} / \mathrm{p})\end{array}$ & Dye & $\begin{array}{l}\text { Pump } \\
\lambda \text { (nm) }\end{array}$ & $\begin{array}{l}\text { Conc.(mg/h) } \\
\operatorname{osc}(/ \mathrm{amp})\end{array}$ & Solvent \\
\hline \multirow[t]{2}{*}{212.5} & 5100 & 6 & $1.2-2.5$ & $S 420$ & 355 & $\begin{array}{l}175(/ 60) \\
185(/ 65)\end{array}$ & $\begin{array}{c}\text { MeOH } \\
\text { EtOH }\end{array}$ \\
\hline & & & & $C 440$ & $4 e+05$ & $\begin{array}{l}200(/ 67) \\
185(/ 60)\end{array}$ & $\begin{array}{l}\text { MeOH } \\
\text { EtOH }\end{array}$ \\
\hline \multirow[t]{2}{*}{225} & 5400 & 6 & $2.0-2.5$ & $\mathrm{C} 450$ & 355 & $225(175)$ & $\mathrm{MeOH}$ \\
\hline & & & & $\mathrm{C460}$ & 355 & $275(/ 90)$ & $\mathrm{ELOH}$ \\
\hline 240 & 5760 & 6 & 2 & C480 & 355 & $510(/ 170)$ & MeOH \\
\hline $\begin{array}{c}248 \& \\
257\end{array}$ & $\begin{array}{c}4960 \& \\
5140\end{array}$ & 5 & 4.2 & $\mathrm{C500}$ & 355 & $610(/ 205)$ & $\begin{array}{c}\mathrm{EtOH}: \mathrm{MeOH} \\
=1: 1\end{array}$ \\
\hline 266 & 5320 & 5 & 3 & C540A & 355 & $2300(/ 770)$ & $\mathrm{MeOH}$ \\
\hline
\end{tabular}

Table B.2. Dye solutions used for VUV generation

\begin{tabular}{|c|c|c|c|c|c|}
\hline $\begin{array}{c}\text { Lasing range } \\
\lambda(\mathrm{nm})\end{array}$ & order & Dye & $\begin{array}{l}\text { Pump } \\
\lambda(\mathrm{nm})\end{array}$ & $\begin{array}{c}\text { Conc.(mg/l) osc } \\
\text { (/ amp) }\end{array}$ & Solvent ${ }^{\wedge}$ \\
\hline $540-575$ & 5 & F548 & 532 & $\begin{array}{c}550(/ 155) \\
O D=2.12\end{array}$ & $\begin{array}{l}\text { EtOH with } \mathrm{NaOH} 80 \mathrm{mg} / 1 \\
\text { (See B.I above) }\end{array}$ \\
\hline $570-595$ & 5 & $\mathrm{R590Cl}$ & 532 & $\begin{array}{l}1.10 \mathrm{~g} / 1 \\
0.85 \mathrm{~g} / 1\end{array}$ & $\mathrm{E} \mathbf{} \mathrm{OH}$ \\
\hline $590-620$ & 5 & R610 & 532 & $\begin{array}{c}1.40 \mathrm{~g} / \\
-0.70 \mathrm{~g} / 1\end{array}$ & $\mathrm{EtOH}$ \\
\hline $610-650$ & 4 & R640 & 532 & $400(/ 133)$ & $\mathrm{E} \mathrm{EH}$ \\
\hline $660-730$ & 4 & LDS 698 & 532 & 358 & $\mathrm{MeOH}$ \\
\hline $690-750$ & 4 & LDS 722 & 532 & $\begin{array}{c}235 \\
\mathrm{OD}=1.88 / \mathrm{mm}\end{array}$ & $\mathrm{MeOH}$ \\
\hline $695-750$ & 4 & LDS 750 & 532 & 195 & $\mathrm{MeOH}: \mathrm{PC}=10: 1$ \\
\hline $720-780$ & 4 & LDS 751 & 532 & 175 & $\mathrm{MeOH}$ \\
\hline $790-845$ & 3 & LDS 821 & 532 & 285 & MeOH:PC $=10: 1$ \\
\hline $835-900$ & 3 & LDS 867 & 532 & $368 \mathrm{OD}=2.0 / \mathrm{mm}$ & MeOH:PC $=10: 1$ \\
\hline $835-850^{B}$ & 3 & $\begin{array}{l}\text { LDS821 } \\
+ \text { LDS } 867\end{array}$ & 532 & $\begin{array}{c}268 \\
70\end{array}$ & $\begin{array}{l}\text { MeOH:PC } \\
\quad=10: 1\end{array}$ \\
\hline
\end{tabular}

A. MeOH: methanol; ElOH: ethanol.

8. Optimized @ $843 \mathrm{am}$ for four-wave difference-mixing (FWDM) in $\mathrm{Kr}$ at $\mathrm{L} \alpha$ 
please note

Pape(o) miseing in number only; bxt follows. Finnod as recoived.

\section{UMI}




\section{Appendix C Generation of VUV by THG in phase-matched Kr:Ar}

The tochnique of non-resonant third harmonic generation (THG) in phased-matched krypton and argon (Kr:Ar) gases mixture is described. This technique was selected among others as the most appropriate to generate an intense coherent light beam in the Lyman a region. The experimental setup used to generate VUV for H-atom LIF spectroscopy is described, along with the tuning range that was observed. Then the observation of VUV depletion at specific energies indicates resonant multi-photon ionization, which suggested the importance of $\chi^{5}$ near-resonance in this process, as discussed in section C. 3 below.

\section{1 FWM techniques for VUV generation at Lyman $\alpha$}

\section{a) The various technique}

Four-wave-mixing (FWM) are nonlinear optics techniques of frequency mixing in atomic gases, which can be employed to generate tunable frequency in the VUV region of the electromagnetic spectrum, i.e. $115-180 \mathrm{~nm}$. These techniques rely on resonance or near-resonance of the nonlinear susceptibility, and on the relative dispersion of a gas, or mixture of gases, to phase-match the different frequencies involved in the process. These techniques aim at generating tunable narrow-band VUV light beam, with an efficiency varying in the range of $10^{-8}$ to $10^{-3}$.

To generate the VUV probe beam, three FWM techniques were compared experimentally: four-wave-sum-mixing (FWSM) in Mercury (Hg) vapour, four-wave-difference-mixing (FWDM) in Kr, and nonresonant THG in phasematched Kr:Ar. It must be appreciated that the efficiency of these processes is dependent on the intensity of the input beam. FWSM in mercury is most efficient near specific resonance lines, which are not conveniently located for Lyman a generation. Mareover, the problem of metal deposition on the optical window was very detrimental to rugged operation for this system.

It should be noted that atomic resonance in $\mathrm{Xe}$, such as the $6 \mathrm{~s}[1 / 2]^{\circ}$ component with $\mathrm{J}=2$, at $67068.047 \mathrm{~cm}^{-1}$ offers convenient two-photon resonance which allow to apply the same FWSM technique that can be done in $\mathrm{Hg}$, but without the problem of metal deposition on the window. Resonant FWDM in pure Kr has the highest efficiency for low input power (< $1 \mathrm{~mJ} /$ pulse for $10 \mathrm{nsec}$ pulse), and it has been shown ${ }^{2}$ to generate VUV at Lyman $\alpha$ with

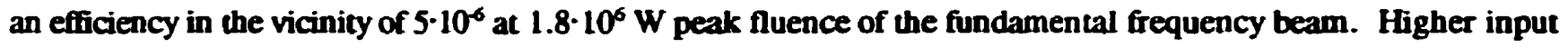
beam intensity produces efficient multiphoton ionization, which rapidly degrades the conversion efficiency.

On the other hand, the lechnique of THG in phase-matched Kr:Ar mixture has a much lower efficiency at low input intensity, but the lack of direct resonance results in the dielectric breakdown occurring at much higher input intensity. The THG technique has been shown ${ }^{3}$ to produce VUV radiation at Lyman $\alpha$ with a conversion 
efficiency of $1.4 \cdot 10^{-4}$ by using a laser intensity of $5 \cdot 10^{9} \mathrm{~W} / \mathrm{cm}^{2}$ at $365 \mathrm{~nm}$. A theoretical limit to the conversion efficiency, due to self-focussing, predicts a conversion efficiency of $1 \cdot 10^{-3}$, assuming a threshold intensity for gas breakdown of $1 \cdot 10^{1 t} \mathrm{~W} / \mathrm{cm}^{2}$.

The technique of THG in phase matched Kr:Ar gas is the FWM technique that was selected to generate a VUV probe beam in the vicinity of the Lyman $\alpha$ transition in hydrogen atoms. Further indications that this was an excellent choice was corroborated by the fact that the EURATOM project had also selected this technique to execute Dopplet broadening measurement of the H-atoms temperature in the plasma of their tokamak. Moreover, recent experiment on spin polarized H-atoms in magneto-optic trap" also used the technique of nonresonant THG in phase-matched Kr:Ar.

\section{b) Nonresonant THG in phase-matched Kr:Ar}

The following brief account of this theory is taken from Bjorklunds. This process relies on the third order susceptibility of $K_{r y p t o n,} \chi^{(3)}\left(K_{r}\right) \equiv \chi_{k r}$ for the sake of notation clarity. When a TEM $M_{\infty}$ laser beam, with a power $I_{L}$, is focussed into a gas having negative dispersion for the fundamental and third harmonic frequency, the VUV intensity produced can be expressed by equation (C-1).

$I_{\text {VUV }} \propto\left(\mathrm{N} \chi_{\mathrm{Kr}_{\mathrm{r}}}\right)^{2} \mathrm{I}_{\mathrm{L}}^{3} \mathbf{F} \quad(\mathrm{C}-1)$

In the latter equation, $\mathbf{N}$ is the number density of the gas, and $F$ is a phase matching factor that depends on $b$ - $\Delta k$, where $b$ is the confocal parameter, and $\Delta k=k(\lambda)-3 k(3 \lambda)$, for which, $k(\lambda)=2 \pi n(\lambda) / \lambda$, and $n(\lambda)$ is the refractive index. Note that $\Delta \mathbf{k}$ is defined so as to be negative in the domain of interest. In the limit of tight focussing of the input laser beam (spot size of the focus $\leq 10^{3} \lambda$ ), the phase-matching factor $F$ is given by $F=(\pi b \Delta k)^{2} \exp (b \cdot \Delta k)$; in the gas, $\Delta k=C N$, where $C$ is the wavevector mismatch per atom. Although $F$ has a maximum at $b \cdot \Delta k=-2$, since $I_{v u v} \propto N^{2} F$, the maximum $V U V$ is obtained for $b \cdot \Delta k=-4$, which corresponds to a maximum gas pressure $p_{m}=-46 / C b$, where 6 $=p / N=3.036-10^{-17}$ at $20^{\circ} \mathrm{C}$. At Lyman $\alpha(1216 \AA)$, this corresponds to $\mathrm{Kr}$ pressure of $p_{m}=54$ Torr; higher pressure of $\mathrm{Kr}$ results in decreasing VUV power, as a result of decrease phase-matching by the $\mathrm{Kr}$ negative dispersion. In order to produce higher VUV intensities, phase-matching can be recovered by using Argon gas, which has a positive dispersion, yet negligible third order susceptibility at these wavelengths. Using this method, the VUV intensity can be enhanced by more than two orders of magnitude, in comparison with the intensity produced at the optimum pure $\mathrm{Kr}$ pressure. Using $10 \mathrm{~mJ} / \mathrm{p}$ at $365 \mathrm{~nm}$ and a lens with a focal of $20 \mathrm{~cm}$. a Kr:Ar mixture of 300:785 mbar was found to be optimum for the generation of VUV radiation at Lyman $\alpha$.

\section{b) Gaussian optics of the laser beam}

Gaussian optics must be used in order to evaluate the intensity of the radiation field in the focal region. The confocal beam parameter, $z_{01}$ in equation (C-2), is calculated by taking the value of the beam waist of the 

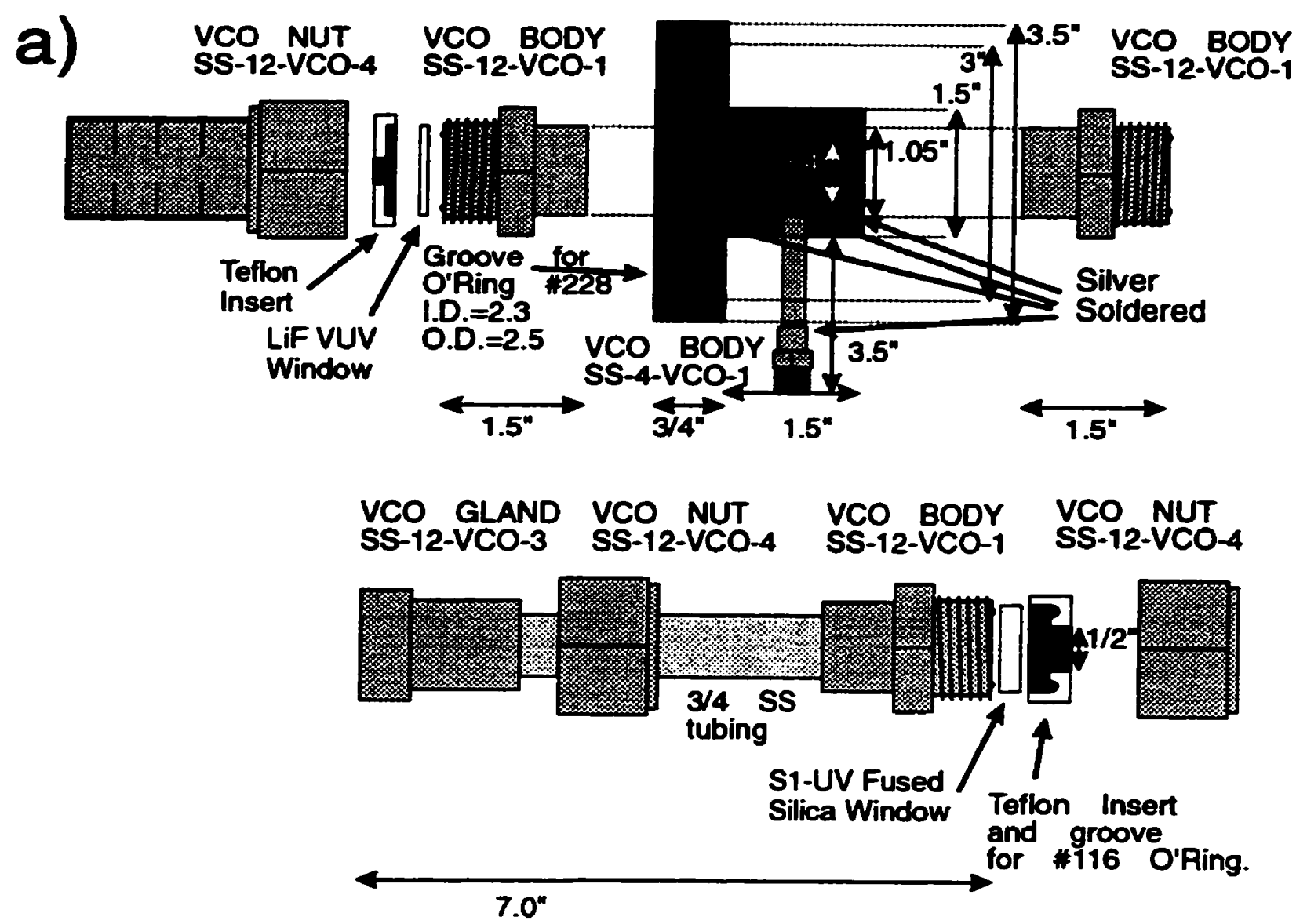

b)

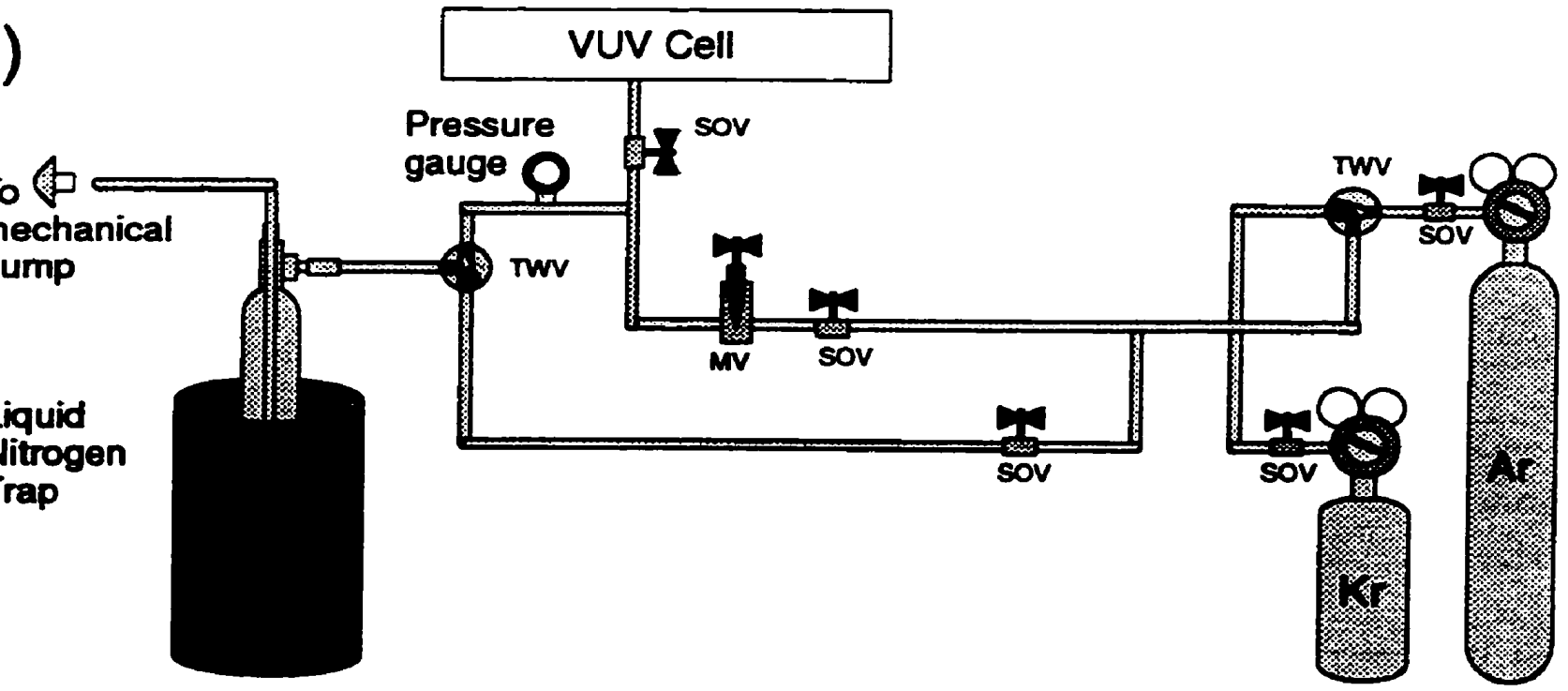

Figure c.1 a) Exploded view of the VUV cell designed for our experiment. b) Gas handling system to fill the cell with accurate gas mixture: TWV: three-way valve. SOV: shut-off valve, MV: metering valve. 
incoming $365 \mathrm{~nm}$, defined as the value of radius at which the intensity has reduced to $1 / \mathrm{e}$ of the value on the axis, estimated as: $\omega_{01}=0 . .65 \mathrm{~mm}$. Using the formula (C-3) for the propagation of Gaussian beam ${ }^{6}$, we can calculate the spot size at the focus, which can be used to obtain an estimate of the power intensity in the focal region.

$\mathrm{z}_{01}=\frac{\pi \omega_{01}^{2} \mathrm{n}}{\lambda} \quad(\mathrm{C}-2)$

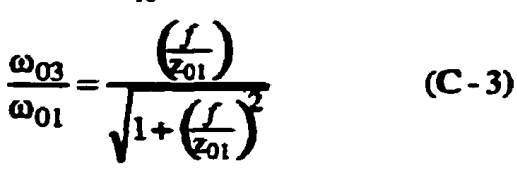

$I=\frac{\text { Power }}{\text { Area }}=\frac{\text { Fluence } / \tau_{\text {pulse }}}{\pi \omega_{03}^{2}}(C-4)$

We find $z_{01}=3.64$ meter for our incoming $365 \mathrm{~nm}$ beam. Using a lens with a focal of $20 \mathrm{~cm}$, the resulting spot size at the focus $\omega_{03}$ is calculated to be equal to $36 \mu \mathrm{m}$. Using a typical value of $F=10 \mathrm{~mJ} / \mathrm{pulse}$ for the laser fluence during the $10 \mathrm{nsec}$ pulse, the intensity at the focal spot can be estimated at $2.5 \cdot 10^{10} \mathrm{Watt} / \mathrm{cm}^{2}$ with relation(C-4).

An order of magnitude for the dielectric breakdown of the gas can be evaluated by setting the electric field of the electromagnetic radiation equal to the electric field inside a hydrogen atom. An intensity of $5 \cdot 10^{15} \mathrm{Wat}_{\mathrm{cm}} \mathrm{cm}^{2}$ is obtained. Because this simple minded picture does not take into account the multiphoton resonances occurring in such high field, this is by far an upper limit to the intensity required for dielectric breakdown; in fact it is observed to occur already in the range of $10^{11}-10^{12} \mathrm{Wat} / \mathrm{cm}^{2}$. Note that although we are operating in the region approaching the observed intensity limit for gas breakdown, we observed a third order power law dependence of the VUV fluence generated as a function of the UV fluence. It will be showed however that $\chi^{5}$ resonance already produce ionization for specific photon energy, even at the intensities used in this work.

\section{C.2 Implementation of THG of VUV}

a) Experimental setup

The optical layout to generate the $365 \mathrm{~nm}$ UV radiation pumping the THG of VUV was illustrated in figure 4.7. The mixing cell and the gas manifold to handle the $\mathrm{Kr}$ :Ar gas are illustrated in figure c.2. The flexible design of the optical port of the VUV cell takes advantage of a standard fitting. whose dimensions match the standard 1 inch diameter window, in addition to providing the o-ring seal which acts as both a rugged seal for vacuum/high-pressure operation and a soft seat ensuring mechanical stability of the window. As shown in figure c. 2 a), by adding a Teflon insert to the standard Cajon^ VCO fituing, turns the vacuum fitting into a flexible and economical optical port.

Figure c.2 b) shows the optimal design for the gas handling manifold used to meter each component of the $\mathrm{Kr}$ :Ar gas mixture. The gas handling system allows one to deal with high purity rare gases of Krypton and Argon. The 

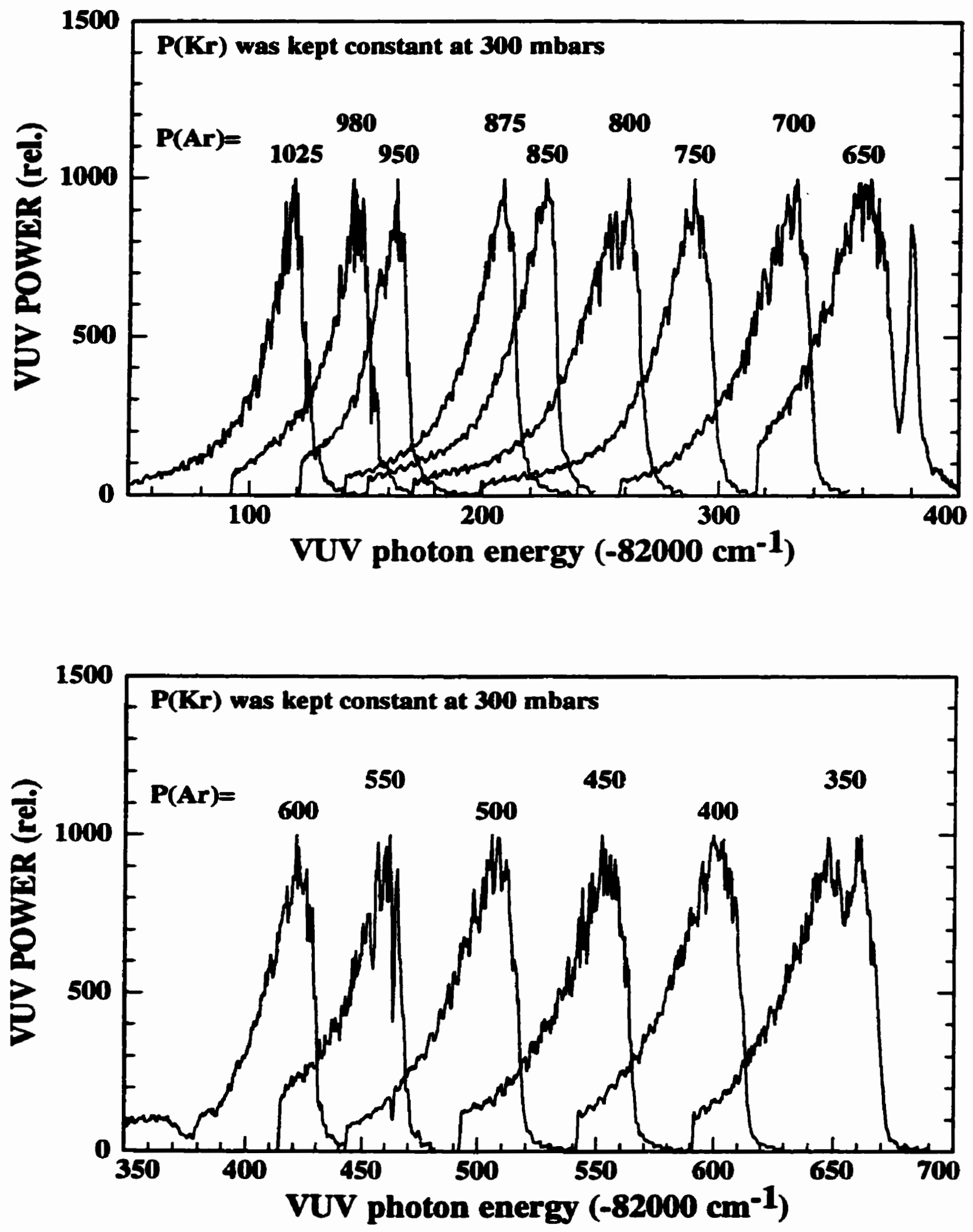

Figure c.2. VUV wavelength tuning range of the cell for various gas mixture 
best shut-off valves for prolonged leak-tight operation are the Whitey" "40" series ball valves. The company also manufactures 3-way valve that help to simplify the design of the gas handling manifold shown in c.2b). To meter each component of the gas mixture, a Nupro's "S" series metering valve was used to control the flow such that the cell pressure can be varied at a rate adjustable between 0.2-10 mbar/sec, with the gas regulator of the gas delivery adjusted at 10 PSIG. The cell pressure was measured with a piezoresistive pressure gauge, (from ICSensor, model 13A-050 A) having a precision of 1 mbar and a linearity of 0.5\%, installed on the gas manifold before the cell shut-off valve.

In typical operation, the cell was first evacuated by a mechanical pump; a liquid nitrogen trap was used between the cell and the pump to condense the backstreaming oil vapour from the pump. Then, 300 mbars of $\mathrm{Kr}$ was introduced, after what the Ar pressure was slowly adjusted for maximum VUV output at the desired wavelength. Because the timescale for the gas mixture to become completely homogeneous is about 5-10 minutes, few trials were typically required to get the mixture having the desired tuning range.

\section{b) Results: gas mixures and tuning range}

A study of the VUV uming ranges was conducted, by introducing 300 mbars of krypton in a clean cell with a fixed amount of argon. After an induction time of 10-20 minutes, the gas mixture was bomogenised, as witnessed by the constancy of its nonlinear optical properties. The tuning range was measured, for incremental values of the partial argon pressure, ranging from 350 to 1025 mbars. For increasing partial pressure of the argon component. contributing positive dispersion in this range of VUV frequencies, the peak of the tuning curve shifts to lower energy, as illustrated by figure c.2. Using $10 \mathrm{~mJ} / \mathrm{p}$ at $365 \mathrm{~nm}$ and a lens with a focal of $20 \mathrm{~cm}$, an optimum $\mathrm{Kr}$ :Ar mixture of 300:785 mbars was found to be optimal for VUV generation in the vicinity of Lyman $\left(82260 \mathrm{~cm}^{-1}\right)$.

\section{C.3 Resonant ionization of $\mathrm{Kr}$ : significance of $\chi^{5}$}

In figure c.2, one can observe that there are peculiar dips in the tuning curve at specific VUV photon energies, at $82380 \mathrm{~cm}^{-1}$ and at $82650 \mathrm{~cm}^{-1}$.

Detailed observation of the behaviour of the THG process in a range of energy spanning $150 \mathrm{~cm}^{-1}$ around 82350 $\mathrm{cm}^{-1}$, indicates that the dip in the VUV tuning curve is located at $82380 \mathrm{~cm}^{-1}$. To investigate this spectral feature systematically, we expanded the dip, as plotted in figure c.3 b), and compared its position with all the electronic states of the two atoms involved having the proper symmetry of total angular monentum, for the possible multiphoton transitions. Figure c.3 a) indicates that resonant multiphoton ionization of $\mathbf{K r}$ atoms is the physical

A. Cajon. Whitey and Nupro are Swagelok companies. 

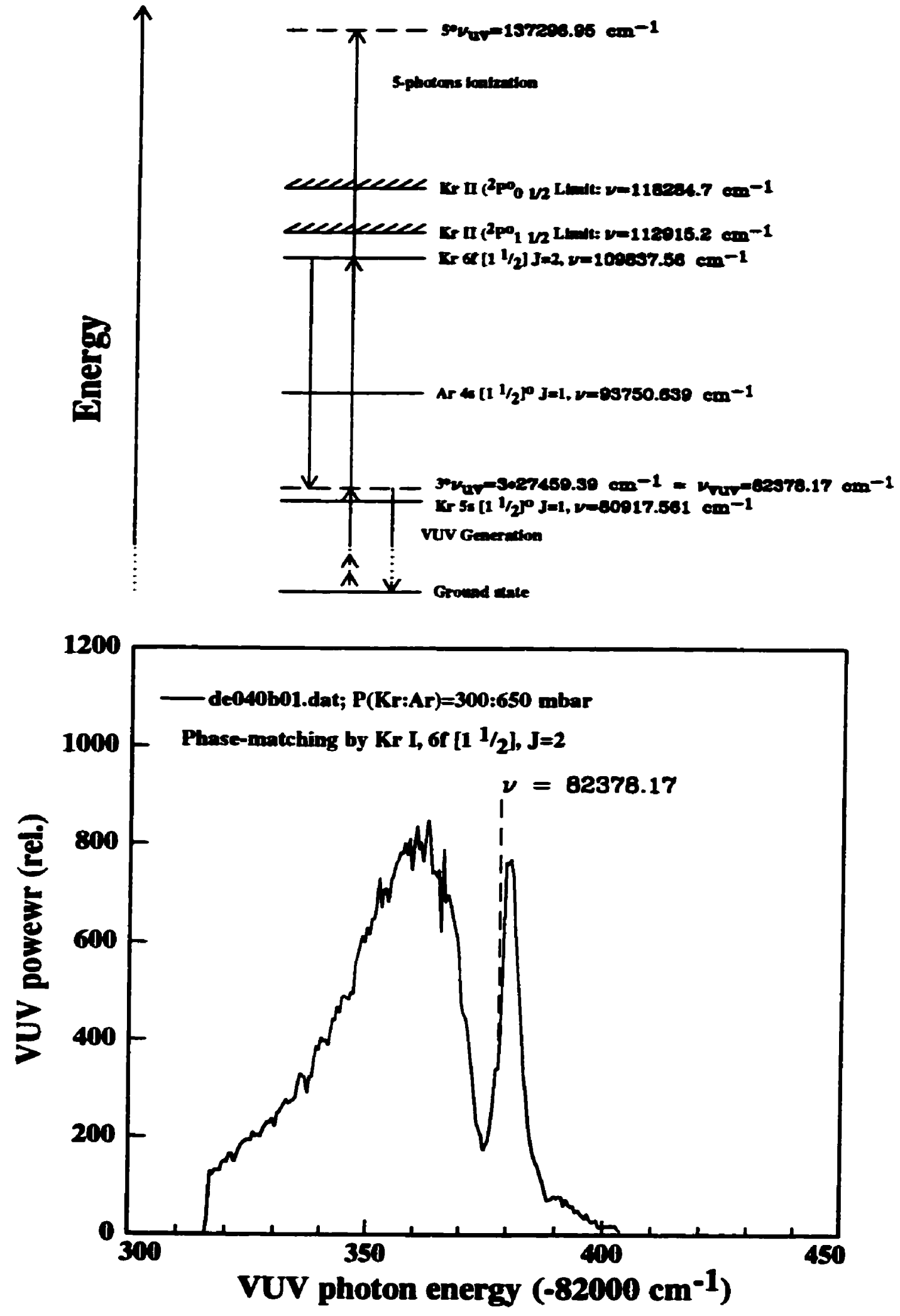

Figure c.3. a) Absorption resonance observed and b) their explanation by four-photon resonant five-photon ionization. 
cause explaining this feature. Because a great care has been taken to ensure the highest purity of gas handling system, and that this signal was repeatable and stable, it is a deemed a property of the atomic system under high intensity radiation. The dip at $82650 \mathrm{~cm}^{-1}$ can be readily explained in similar way.

It has been found that structure observed in the VUV tuning curve can be explained by a four-photon resonant. five photon ionization. As a consequence of the present consideration, if the four photons resonance can be so efficient to produce ionization, it is suggested that the importance of its nearby presence may then have been overlooked in the explanation of the observed behaviour for the "nonresonant" THG FWM process.

This process has been observed in Xe by Lukasik and Vallee in the early 80'. A rescnant fifth-order process was observed in VUV generation around 1400 A. Because the scheme goes as: $\omega_{1}+\omega_{1}+\omega_{1}+\omega_{1}-\omega_{1}=\omega_{3}$, no energy is extracted from the field for the fourth and fifth photon, and consequently they would not affect the third order dependence of the VUV intensity on the UV intensity which is verified experimentally.

As a conclusion, our observation indicated that the nonresonant THG process might indeed have to be renamed "near resonant five-photon phase-matched, THG generation". A test of this hypothesis would be best afforded by application of the present assumptions in designs to improve the effectiveness of other nonlinear optics processes.

\section{References}

1. J.W. Hepburn, Generation of Coherent Vacuum Ultraviolet radiation: Application to high resolution photoionization and photoelectron spectroscopy, in Laser Techniques in Chemistry, A. Myers and T.R. Rizzo, (1994), Wiley. J. W. Hepburn, Israel J. Chem., 24, 273 (1984)

2. R. Wallenstein, Optics Comm., 33, 119 (1980)

3. H. Langer, H. Puell, H. Rohr, Optics Comm., 34, 137 (1980)

4. O. J. Luiten, H. G. C. Werij, M. W. Reynolds, I. D. Setija, T. W. Hijmans, J. T. M. Walraven, VUV spectroscopy of magnetically trapped atomic hydrogen, Appl. Phys. B, 59, 311 (1994)

5. G.C. Bjordlund, "Effect of focussing on third-order nonlinear process in isotropic media". IEEE, J. Quantum Electron., vol QE-11, pp287-296 (1975)

6. A. Yariv, Quantum electronics, 3', Wiley (1989) 


\section{Appendix D Stability and Linearity of the dye laser wavelength tuning mechanism}

The dye laser FL2002E from Lambda Physik is a very good instrument, but as with any instrument, it is not perfect. The relatively demanding requirements of the present study have provided the opportunity to be confronted with the limitations of this instrument, although at the time when the author was struggling with this seemingly unsolvable problem it may not have appeared to be such a wonderful opportunity! Nevertheless, these problems have been solved, and due to the relatively high popularity of this instrument, these solutions were deemed warthy of being communicated. Two main problems have been observed with this instrument: (i) motion reproducibility of the tuning mechanism, and (ii) quasi-periodic nonlinearity of the wavelength tuning. These problems have been characterized, and solutions have been implemented in both cases. They are described below.

\section{D.1 Stability of the tuning mechanism \\ a) Observation of the problem}

The lack of reproducibility for spectral measurements, obtained when different step sizes where used to move the dye laser grating, was mentioned in section 4.3.4 e) of chapter 4, and illustrated in figure 4.12. After detailed consideration and numerous tests of hypotheses for probable cause of this problem, it was found that the problem was due to the carelessness of the driver generating the electrical pulses (steps) controlling the stepping motor which actuates the grating. It is meant that a sharp force was resulting from the abrupt change of frequency of the step driver, which translated into abrupt velocity change for the motion of the motor.

\section{b) Analysis of the system}

The precise requirement for the mechanical motion of this system was apparently not satisfied by a driver operating at a few preselected driver frequencies. Nevertheless this situation is customary for stepper motor controller, as electronic design of typical controller includes an oscillator and few frequency dividers. However. the availability of a computer to generate a software driver for the stepper motor control allows greater flexibility. By seeking the smoothest motion for this mechanical system, the "smooth drive" software controller was developed. The concepts emerged from the solution of the following constraints: to generate a well-defined number of pulses, in a sequence of pulses in the least amount of time, at a rate smaller than a preset frequency. and at a change of rate below a preset value. It can be shown, that there is no analytical solution to be found for such sequence of pulses, as a result of the limit of simultaneous precision on time and frequency (Fourier limit); surely, a sotware routine can simulate the pulse sequence, and can store them in the memory, but it is simpler and faster to calculate them in real time, since the solution to this problem has an iterative nature. Figure D.l presents a conceptual comparison between the customary method of analog driver, and this software driver. 
Inertia constrined control (Smooth drive):

- Control over instantaneous force

- Control over instantaneous velocity

- No mechanical overshoot

- Require real-time calculation of period

\section{Set maximum frequency}

Set number of steps

Set maximum force

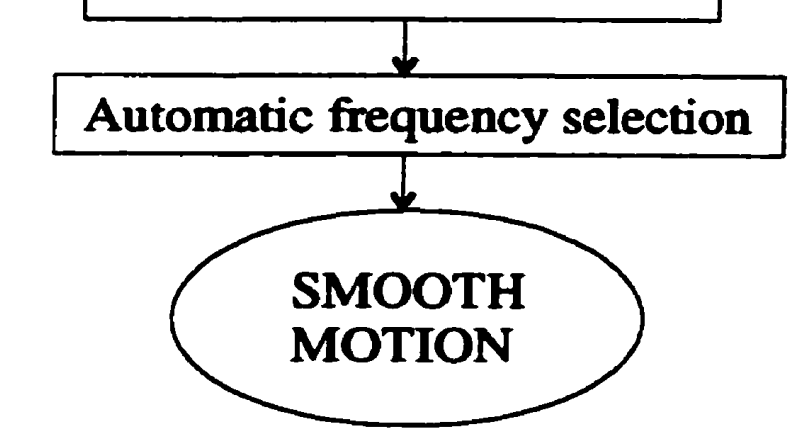

Frequency constrained control (customary):

- No control over instantaneous force

- Mechanical backlash due to inertial overshoot

- A priori calculation of period and frequency

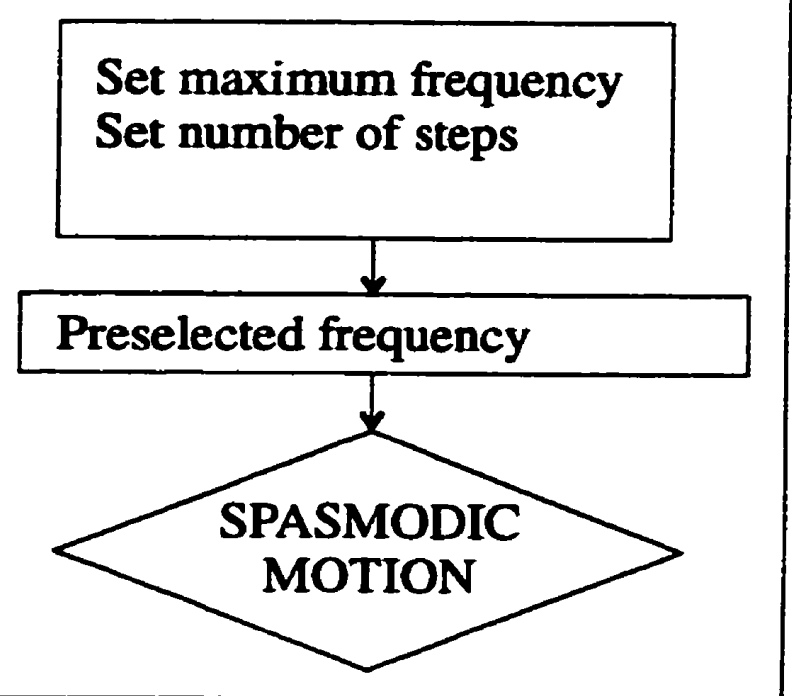

Figure D.1 A comparison between the properties of customary electronic driver and the "smooth drive" software driver.

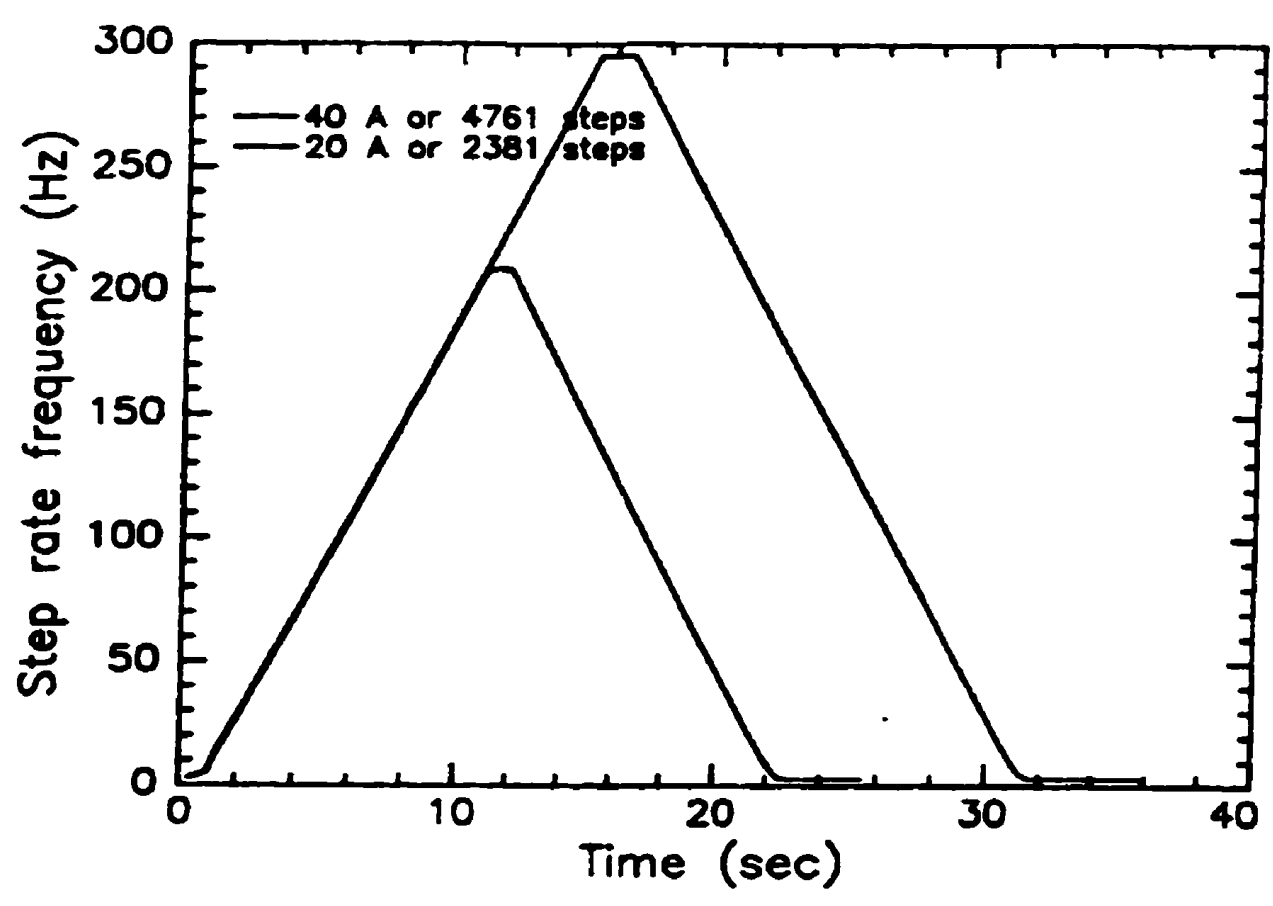

Figure D.2 Example of motion commanded by the smooth drive software driver. 


\section{c) Solution and implementation}

This "smooth drive" concept was implemented in the software driver controlling the motion of the grating, and reproducible measurement were obtained. Because the controller use dummy loop of the computer to generate pulses train of well-defined period, the computer clock was calibrated prior to implement the software driver. The only information given to the algorithm is a maximum frequency or velocity, a maximum acceleration and a number of steps. The iterative algorithm computes the sequence of periods in real-time.

The strongest argument in favour of this software driver method is its results, which are witnessed by the consistency amongst the spectral measurement of figure 4.13. To illustrate the motion resulting from this driver. we ask the computer to store the instantaneous frequency values used during the execution of a grading motion. The plot of instantaneous stepper frequency versus time is illustrated in figure D.2, for a displacement of $20 \AA$ and $40 \AA$. The smooth ramping of the frequency can actually be heard by a user standing in the laboratory.

\section{D.2 Linearity of the tuning mechanism}

\section{a) Observation of the problem}

Whenever a calibration of the Lambda physics dye laser was obtained from a spectroscopic reference, a residual was always obtained, which is 2-3 times broader than the experimental linewidth obtained from the very same measurement. Dr. Lewis, from Australian National University, has communicated to us that he also bas observed a quasi-periodic nonlinear deviation from a wavemeter measurements on a laser from Lambda Physik.

Although a wavemeter was not available for the present study, a reference spectrum, having an appropriate line density, can provide quantitative information on the nonlinear deviation of the wavelength drive. The absorption lines of an iodine cell were found to satisfy the requirements of our study. Moreover, the same nonlinearity measurement could also be obtained from fringe measurement of a Fabry-Perot etalon used to monitor the dye laser quality.

\section{b) Analysis of the problem}

The signal from an $\mathrm{I}_{2}$ cell permits linearity characterization for the tuning wavelength of the dye laser, in addition to providing an absolute vacuum wavelength calibration. This operation was afforded by the fact that the spectrum of the $\mathrm{I}_{2}$ molecule has been measured by interfercmetry, and the line positions are tabulated over this range of the visible spectrum. Comparison with this reference spectrum enables calculation of the nonlinear deviation from the wavelength tuning of the dye laser. A typical measurement of the nonlinear wavelength deviation from linearity is illustrated in figure D.3 


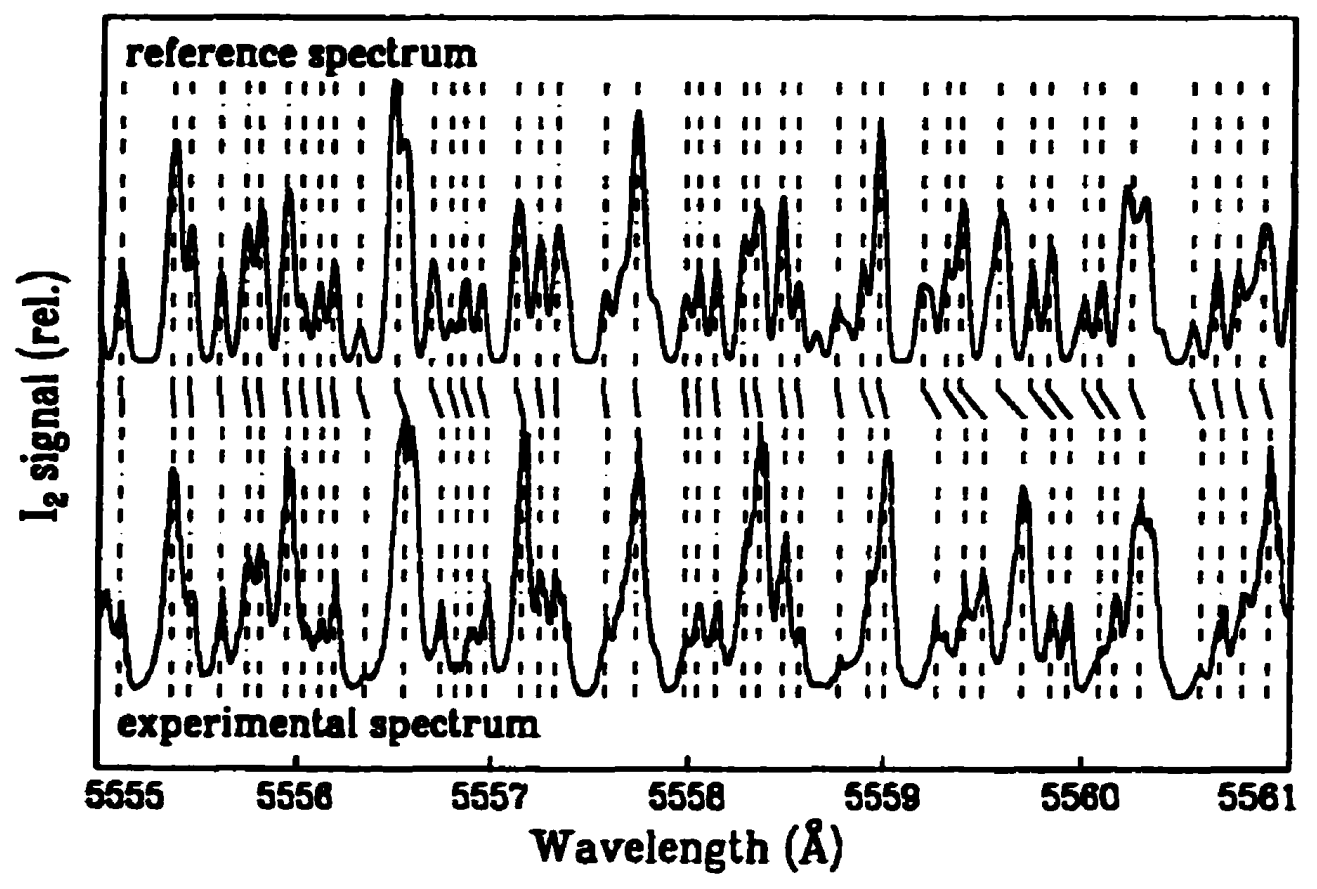

Figure D.3 Determination of the nonlinear deviation of the tuning mechanism by matching the corresponding peaks of a reference spectrum with an experimental one.

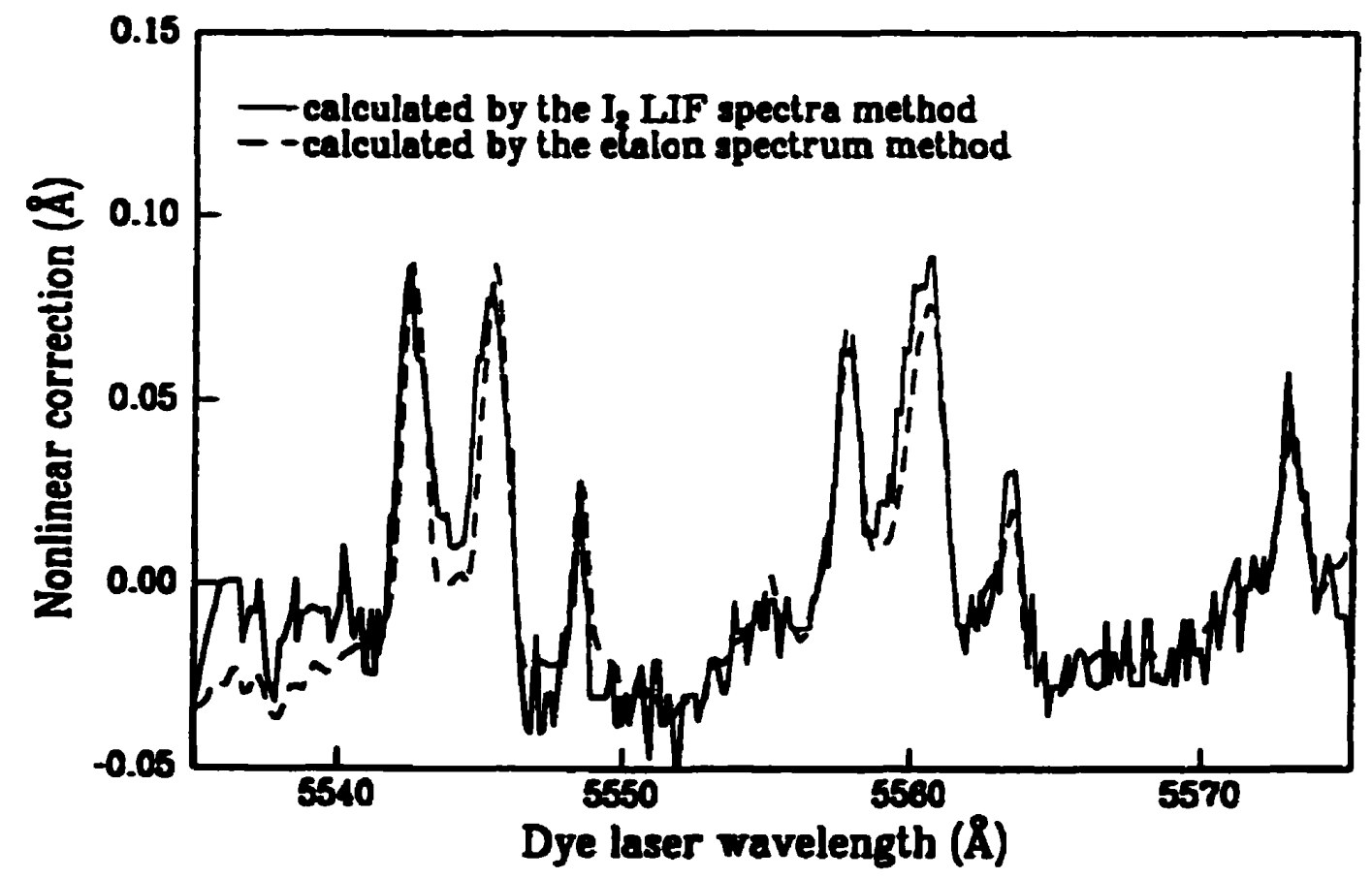

Fhure DA Comparison of the nonlinear deviation measured with two unrelated metbods: i) the nonlinear deviation from 12 LIF and ii) from the fringes of a monitor etalon. 
An equivalent determination of the nonlinearity function can be measured in a totally independent way by recording peaks produced with the fringes of a monitor etalon and a PDN diode. Figure D.4 shows that the two numerical functions determined by these completely independent methods agree very well. The nonlinear wavelength deviation exceeds the linewidth $(0.04-0.05 \AA)$ by a factor of 2 , while the amplitude of the deviation exceeds the typical linewidth by a factor greater than 3. The amplitude of the nonlinear deviation of the tuning mechanism has been observed before by other users of similar instruments made by Lambda Physiks ${ }^{2}$, but the details of its behaviour are clearly illustrated bere.

The linearization, i.e. the correction of the nonlinearity produced by the mechanical drive, relies on a prior characterization of this imperfection. Questions such as: (i) is the nonlinearity reproducible or random? (ii) can it be modelled analytically ? (iii) what is the physical cause of this nonlinear deviation ? are of interest to best solve this problem. The issues of the cause of this imperfection are only hypothetical, since we did not attempt to rebuild the laser. The important results of these considerations are that the nonlinear deviation is reproducible within a finite time. Figure D.3 and D.4 showed that the nonlinearity can be measured and characterized.

\section{c) Solution implemented}

A correction procedure for this nonlinearity is essential to obtain significant information from the spectral measurements. In the present study, $\mathrm{I}_{2}$ LIF spectra of iodine molecules were simultaneously measured with data acquisition, by using an $I_{2}$ cell assembly represented in the optical layout of figure 4.2. The typical measurements, and the associated correction procedure are outlined in the section 5.2 of chapter 5 .

Implementation of the correction for the nonlinear wavelength deviation solved a few mysteries, with respect to long-standing problems in our study. As an example, prior to understand the problem of wavelength drive stability and nonlinearity, the author of this study was very concerned with a spurious signal in the Doppler spectra. Although we knew the width of the Doppler spectra, based on energy conservation, the experimental widths were always too narrow, as illustrated in figure D.5. Moreover, a spurious signal was present on the high energy side of many spectra. This mystery puzzled us until after the realization of the fact outlined in this appendix, i.e. the grating drive was sometimes overshooting, and nonlinear wavelength deviations could be as much as three times the linewidth.

For the sake of future upgrades for this system, it should be noted that because a correction of the wavelength nonlinearity from the grating drive can be implemented on the basis of etalon fringe measurements, other possibility ensue. As a matter of fact, integration of etalon fringe measurement and expert system would allow real-time correction of spectral measurement, and rugged operation of the intracavity etalon and grating tracking. 

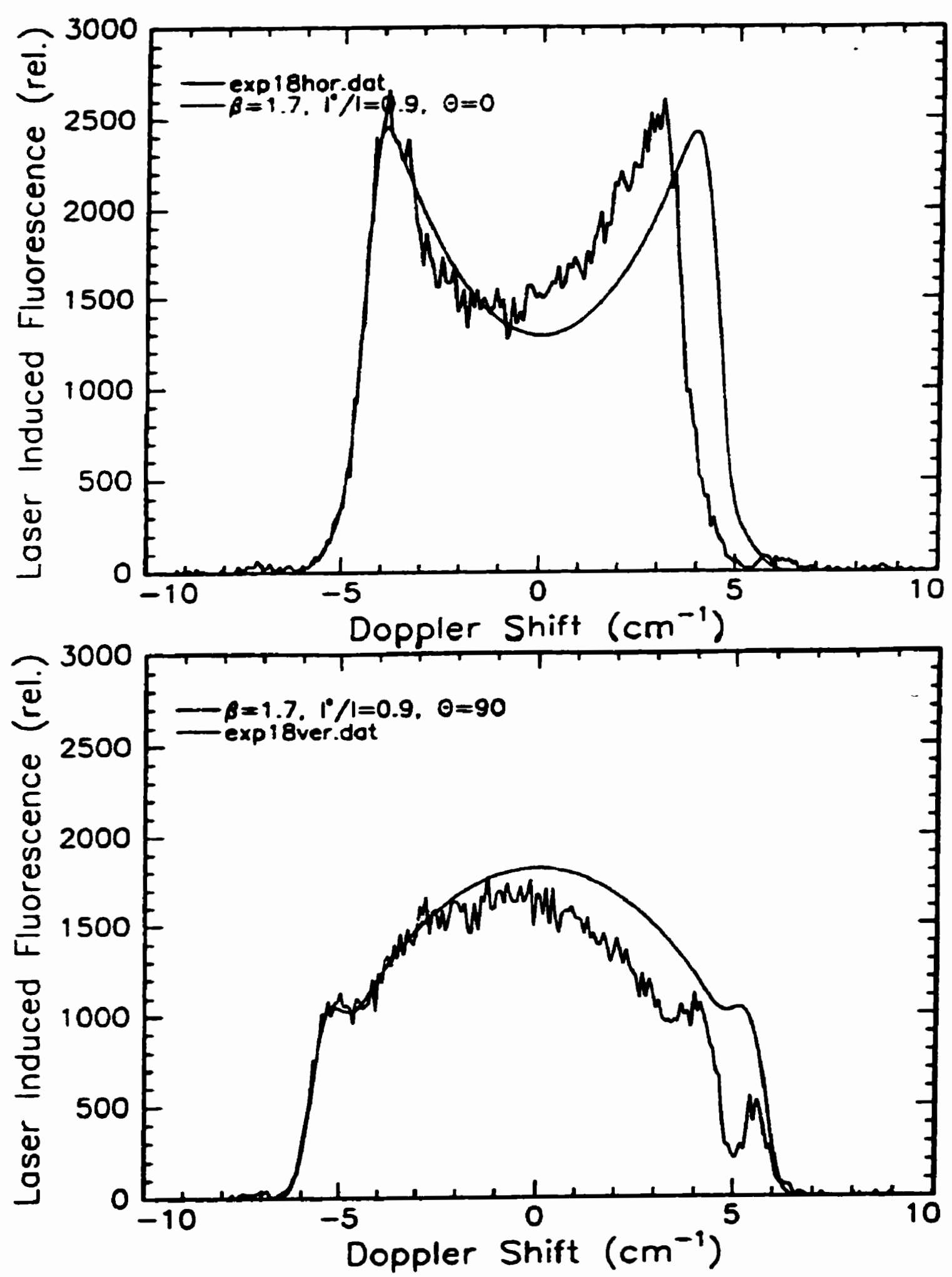

Figure DS Illustration of "mysterious" problem with "spurious" signal and energy conservation: the solid line represents the corresponding Doppler spectrum whose width were calculated from energy conservation. 


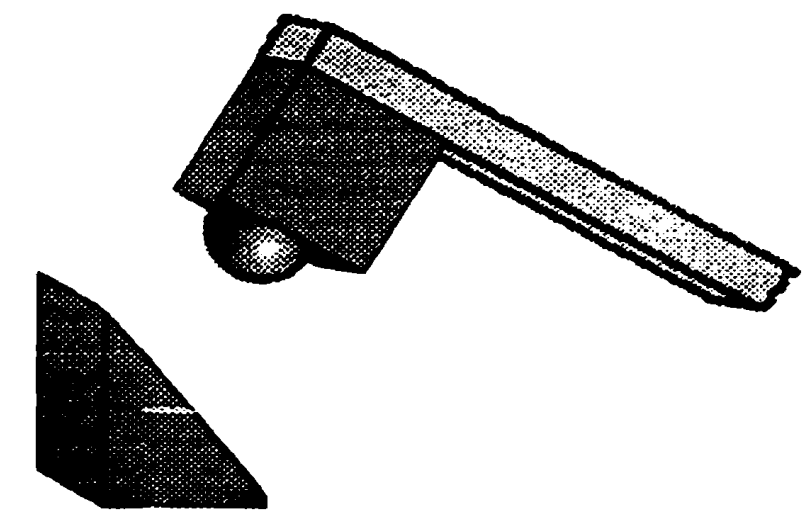

a) Present sliding mechanism

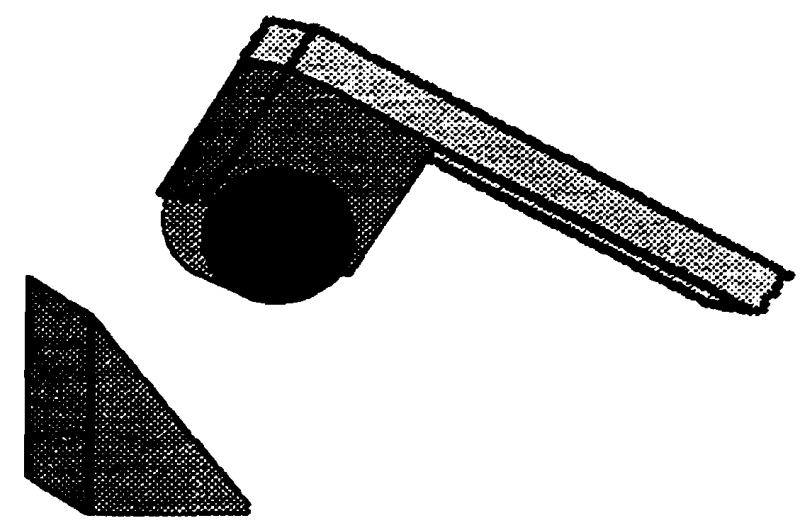

b) Proposed sliding mechanism

Figure D.6 Illustration of a suggested solution for the hypothetical cause of the nonlinearity of the grating mechanical drive.

\section{d) Solution for the hypothetical friction cause}

The grating mechanism is a sine-drive, i.e. a lever pushed up by a screw, which is connected on a stepper motor through a geared-down transmission. Because the period of the quasi-periodic nonlinearity corresponds to an integral multiple of the rotating motion of the screw pushing onto the lever of the grating, it is reasonable to assume that the nonlinearity originates from an effective wobbling motion of the screw. This wobbling may originate from a warped screw; however the minute value of the mechanical imperfection, coupled with the fact that there is a finite friction at the point of contact between the lever and the grating lever, suggests that the effective warp could indeed criginate from the friction at the point of contact. Because the point of contact is just that, a point between a sapphire ball and a shinny plate of hard metal, improvement could be achieved by replacing the ball by a cylinder, hence reducing the friction by orders of magnitude. Figure D.6 was made to provide the reader with a clear picture of what is envisioned.

\section{References}

1.S. Gerstenkorn and P. Lue, Atlas du Spectre d'Absorption de la Molecule d'Iode, VoL II. 14,800-20,000 cm' (Labocatoire Aime Cotton, CNRS II. Orsay, 1977).

2. Private communication with James J. Martin here at Waterloo, and Dr. B. R. Lewis, Australian National University. 


\section{please note}

Papo(s) misaing in number only; wat follows. Finned as recoived. 


\section{Appendix E Linearity of PMT detectors and electronic preamplifiers}

The effective dynamic range for signal detection is determined by the noise level and the saturation limit, for a detector and the following signal processing electronics. While the saturation of a transistorized amplifier results in a clear plateau of the output signal level, the saturation behaviour of an electron multiplier is not as trivial. Indeed, the latter typically exhibits a creeping saturation, to be described below, which can easily go undetected if linearity tests are only performed within a small dynamic range of output signal. In the present study, it was found critical to meticulously characterize the saturation behaviour of the photomultiplier tubes (PMT) and electroaic preamplifiers, in order to ensure the linearity of the measurements, because the signal from the PMT detectors are first sent to the preamplifier. The methods to characterize the range of linearity, for both the electronic preamplifiers and the PMT detectors, are described below.

\section{E.1 Linearity of the preamplifiers}

The maximum input level that can be fed to the fast preamplifiers (preamp) have to be determined if the overall system PMT/preamplifier is to be properly characterized, because the preamps were used to amplify the output PMT signals. There was three preamps used in this study, and whose amplification properties are plotted in picture E.1 and listed in table E-1, for 10 nanoseconds pulsed input signal. The amplification properties of these

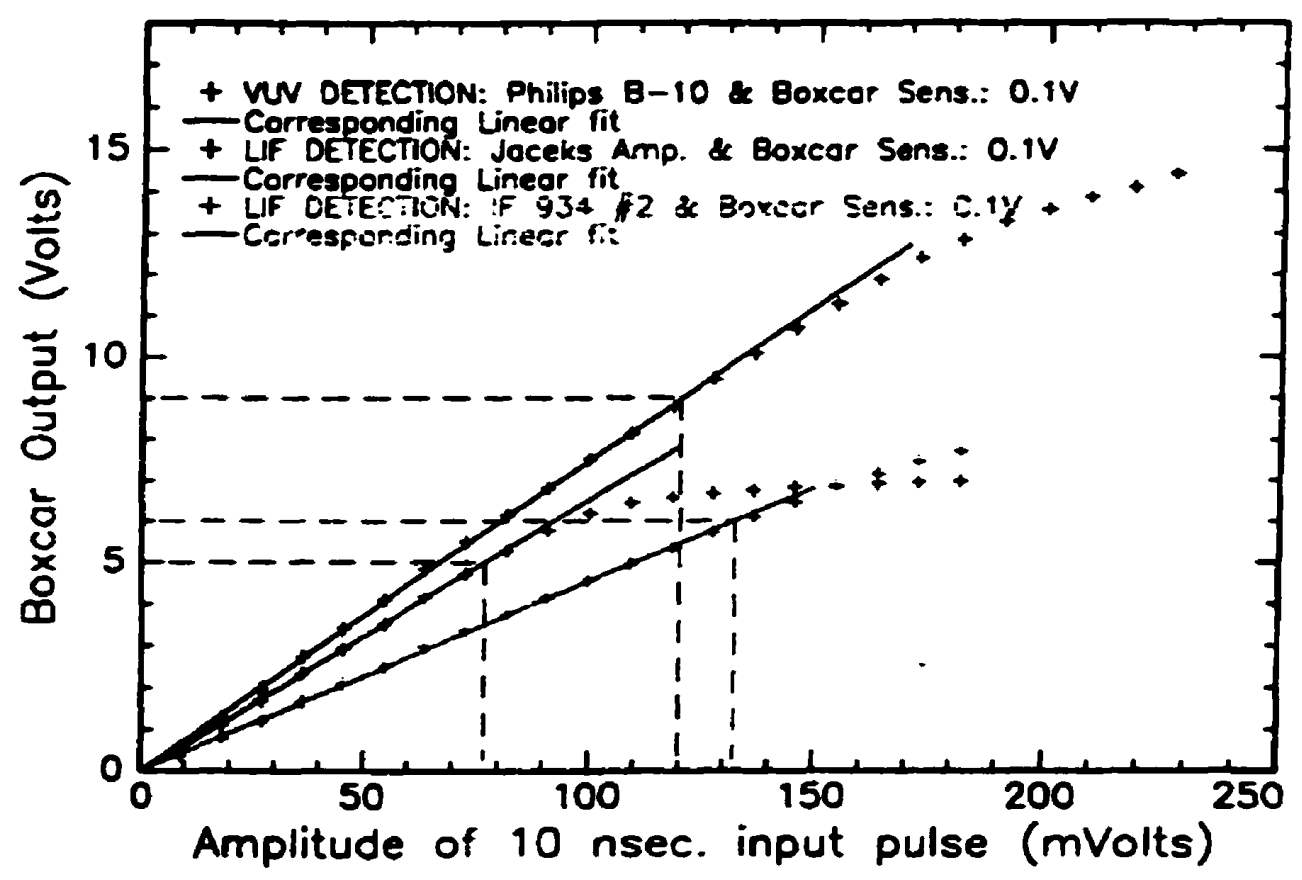

Figure E1 Linearity of the preamplifiers used in the present study. The preamplifiers named Jacek \#1 and IF 934 *2 have been built by the electronic engineers of our electronic shop, namely Jacek Szubra and Ivan Frola, respectively. 
devices were determined with a digital pulse generator (Stanford Research Instrument, Model SRS-DG535), and a $100 \mathrm{MHz}$ oscilloscope (Tektronix, Model 2245A).

Table E-1 Amplification properties of the preamplifiers used in the present work.

\begin{tabular}{|l|c|c|c|}
\hline \multicolumn{1}{|c|}{ Preamp I Properties } & Gain & Input saturation limit (mV) & Output saturation limit (V) \\
\hline Phillips B-10 & 7.49 & 120 & 0.9 \\
\hline Jacek \#1 & 6.58 & 77 & 0.5 \\
\hline IF 934 \#2 & 4.53 & 132 & 0.6 \\
\hline
\end{tabular}

\section{E.2 Amplification and gain saturation in a PMT detector}

\section{A) Amplification in a PMT detector}

A photomultiplier tube can be conceptualized in two parts: a photocathode, converting a photon signal into an electron signal, and a electron multiplier tube. In figure E.2, a typical PMT assembly is illustrated, in which the numerous dynodes are connected to a voltage divider. The process of amplification results from the following facts: (i) free electrons are accelerated between the dynodes, and they suffer energetic collisions upon impinging onto the dynode metallic surfaces; (ii) few electrons are backscattered from the metallic surface, as a result of the energetic collision, which in turn are accelerated until the next dynode; (iii) the energy of the collision being determined by the bias on the voltage divider. Within linear response, the dependence of the gain on bias voltage is typically given by equation ${ }^{1}(E-1)$ for photomultiplier tube.

\section{$G_{O}\left(V_{B I A S}\right)=\mathrm{A}\left(V_{B I A S}\right)^{\mathrm{n}} \quad(\mathrm{E}-1)$}

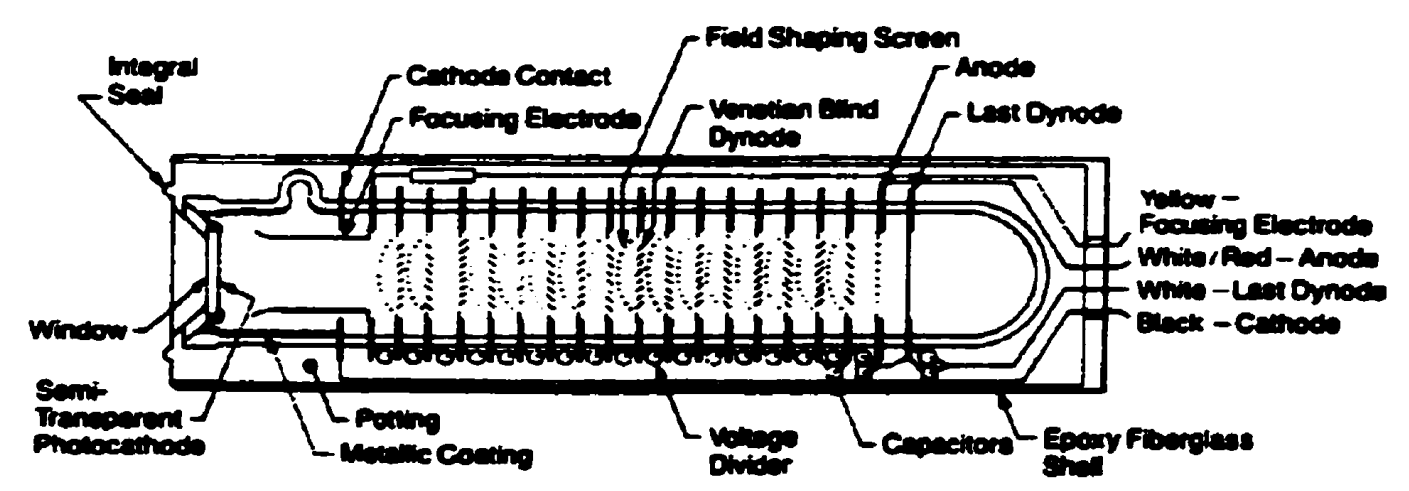

Figure E.2 Schematic drawing of a PMT, showing the photocathode, the voltage divider, and the dynodes². 


\section{B) Gain saturation in a PMT detector}

Saturation in a PMT is a charge effect; it occurs when the pulse of electrons begin to effectively produce a significant voltage on the dynode capacitance. Because the current growth, along the dynodes chain, follows a power law, the effect of temporary accumulation of charge is mainly produced on the last few dynodes. Therefore the operational cause of gain saturation is large output signal $\mathrm{V}_{\mathrm{O}}$, which in turn may result from either large gain and/or large input signal. As it is indicated by equation (E-1), the gain increases with voltage; however, at increasingly higher gain, high output will evenually result in gain saturation. Therefore, as will be demonstrated experimentally below, the critical physical quantity determining the saturation behaviour is the ratio: $V_{\mathrm{Bus}} / \mathrm{V}_{\mathrm{O}}$.

$$
G\left(V_{O}\right)=G_{O} /\left[1+K_{\text {sar }}\left(V_{O} / V_{\text {bias }}\right)^{2}\right]
$$

Through this characterization work, it was found empirically that the relation (E-2) describes properly the onset of saturation for our PMT devices, as showed in figure E.6. The nature of this relation is such that the onset of gain saturation depends effectively on the precision aimed at by the overall measurement procedure.

\section{E.3 Characterization of the PMT gain saturation}

\section{A) Experimental setup}

The onset of saturation was characterized for the two PMTs described in the chapter 4: Hamamatsu and EMR. The saturation behaviour was characterized as a function of PMT bias, and input photon irradiation, from which the dependence of this process on output signal level was deduced. The gain and limit of linearity of the EMR PMT were characterized using the following setup: (i) a coherent beam of VUV radiation was send through the photofragment spectrometer (see chapter 4) and it was detected after a monochromator (Acton Research, model VM-52) by a Hamamatsu PMT, whose limit of response linearity had been characterized prior to that measurements; (ii) a fine SS mesh was installed at the position of the target to provide a scattering source for the EMR PMT LIF detector in proportion to the incoming VUV radiation. The intensity of the VUV beam and the bias of the PMTs could be varied in a range over which both linear behaviour and onset of saturation could be observed.

\section{B) Dependence on PMT bias voltage}

The photocathode irradiation was kept constant, and the bias of the PMT was varied in a range such that the PMT gain encompasses both a linear range and the onset of gain saturation. By varying the PMT bias voltage, and recording the signal output, equation (E-1) was verified for each PMT. The measurements are reported in the figure E.2 for the EMR PMT, and in the figure E.3 the Hamamatsu PMT. The value of the exponential power was obtained by the slope of a $\log -\log$ plot of the gain versus bias voltage; the values of the fit are $n(E M R)=9.58$ and n(Hamamatsu) $=7.77$. 


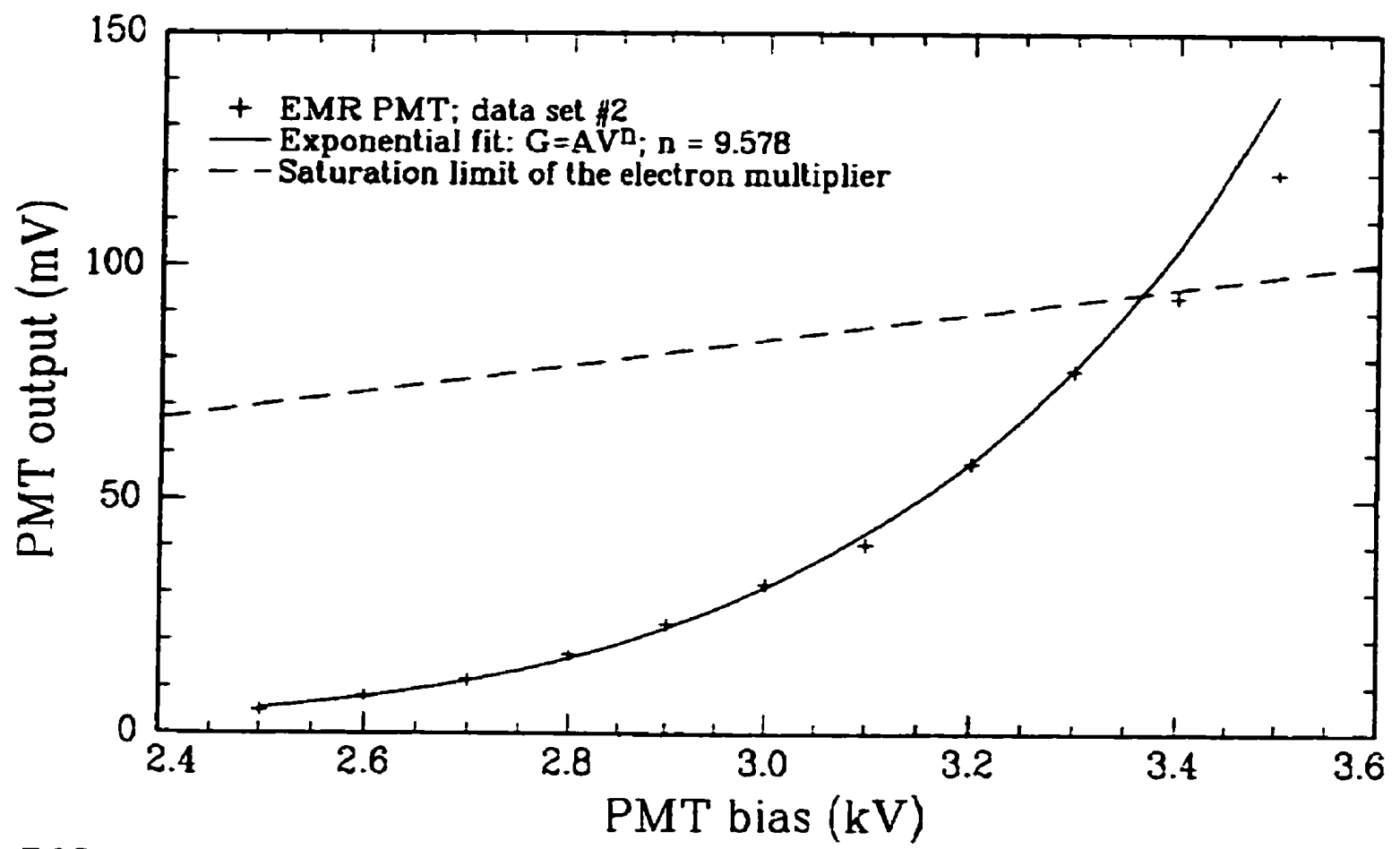

Figure E.3 Dependence of output signal on bias voltage, at constant photocathode irradiation. for the EMR PMT.

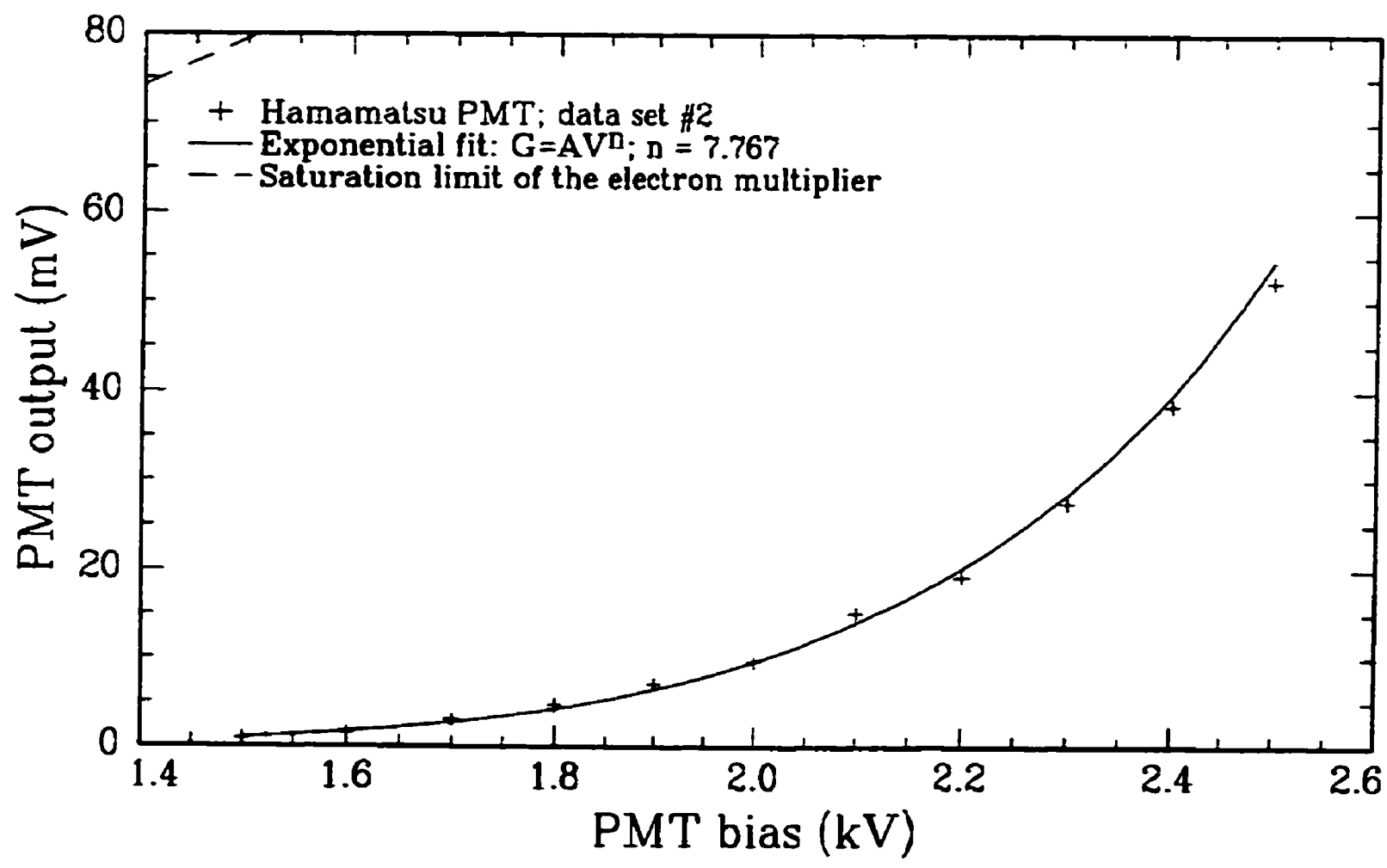

Figure E.4 Dependence of output signal on bias voltage, at constant photocathode irradiation. for the Hamamatsu PMT. 
From the above illustrations, it will appreciated that the Hamamatsu PMT was well within linear response for all measurements, while gain saturation of the EMR PMT is evident for the two highest experimental values of PMT bias, and consequently, they were not included in the fit. Also plotted in the figure E.3 and E.4 are the saturation levels as a function of the bias voltage, which were determined by the following method.

\section{C) Dependence on input photon irradiation}

Measurement of the EMR PMT response, near the onset of saturation, allowed for the characterization this gain saturation process. The measurements were carried out by varying the intensity of the VUV beam and recording the relative signal of the power monitor PMT and the LIF scattering (EMR) PMT. The VUV intensity was varied by tuning the VUV wavelength over the narrow tuning range afforded by phase-matched THG in a Kr:Ar mixture.

The measurements are illustrated in figure E.5. At first glance, the two signals seems to vary in constant proportion, but careful examination reveals that the LIF signal starts to deviate from linearity at a relatively low value of output voltage. Data manipulation produced a plot of output signal versus scattered light input on the PMT photocathode, which is showed in figure E.6. Further analysis has demonstrated that the analytical model provided by equation E-2 describes appropriately the gain saturation of our EMR PMT. Few sets of data were analyzed and compared to the model. For the EMR PMT, the value of $K_{S A T}=3.43 \cdot 10^{-5}$, with $V_{B I A s}$, the bias voltage, in $\mathrm{kV}$ and $\mathrm{V}_{\mathrm{o}}$ in $\mathrm{mV}$.

The gain dependence on the output signal level $\mathrm{V}_{\mathrm{O}}$ is such that it decreases significantly beyond a precision dependent threshold. By setting an arbitrarily value of $3 \%$ deviation from linearity as a threshold value, a limit of $30 \mathrm{mV} / \mathrm{kV}$ of linear output signal is obtained. By plotting this result on figure E.6, a consistent picture of the gain saturation process was obtained for the electron multiplier of this device.

\section{E.4 Conclusion}

Saturation in electron multiplier is caused by the accumulation of charges on the last dynodes, through a reduction of both electron multiplication and collection efficiency. Because the dynode voltage is proportional to the PMT bias, it is intuitively reasonable that the dependence of the gain saturation on the output signal should be scaled in proportion of the PMT bias. It was showed that PMT gain saturation depends on the ratio of PMT output to bias voltage, according to the equation (E-2), as showed in figure E.6 for the EMR PMT. It is concluded that this model is a suitable representation of the observations. Consequently, the range within which the linear response of a photomultiplier tube can be expected depends somewhat on the precision within which linearity is required. For example, if one plans to obtain measurements up to a Signal-to-Noise (S/N) ratio of 30, the response linearity of the detection electronics should be smaller than $3 \%$, in order to be consistent. 


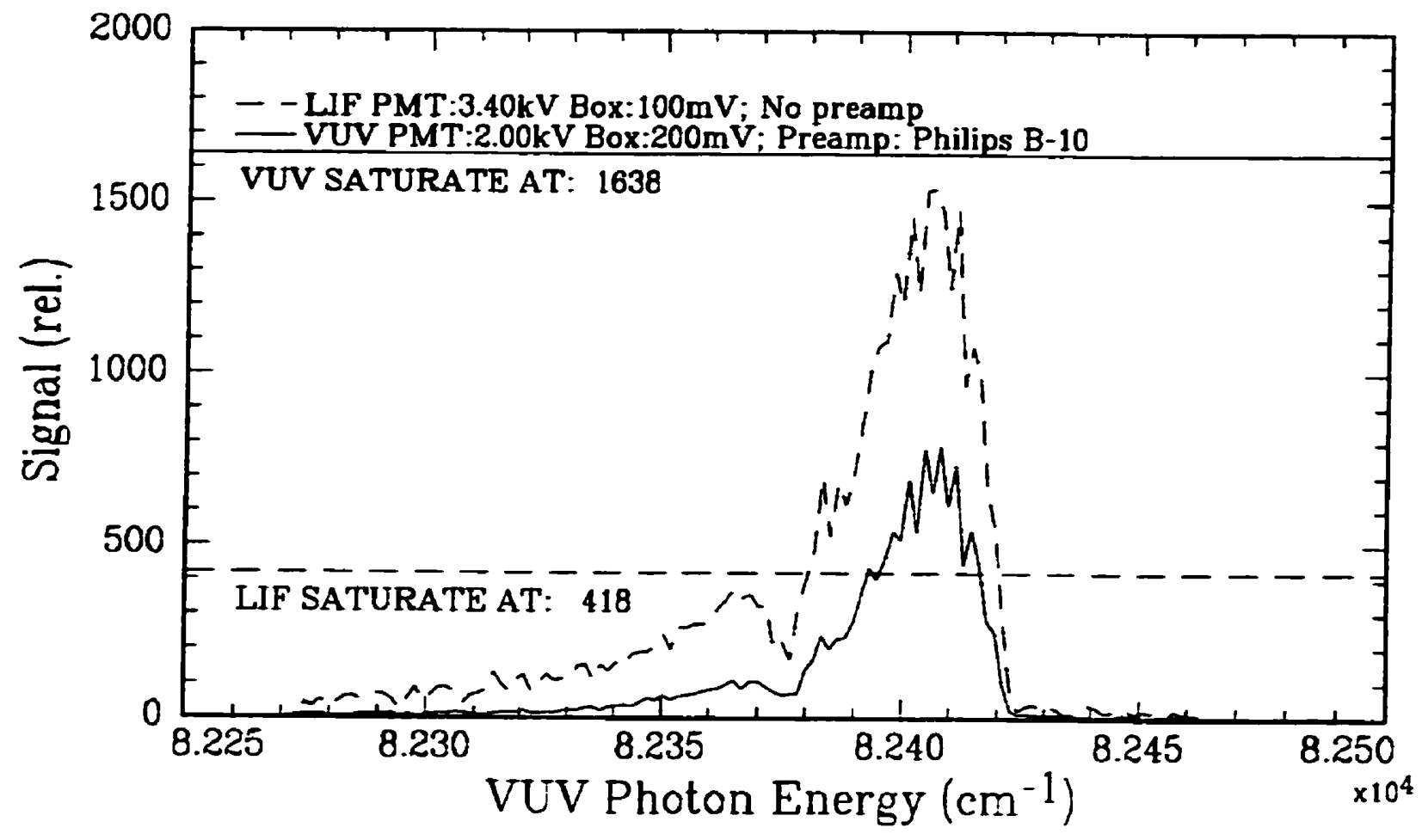

Figure E.5 Typical experimental data used to characterize the saturation process of the EMR PMT in figure E.6.

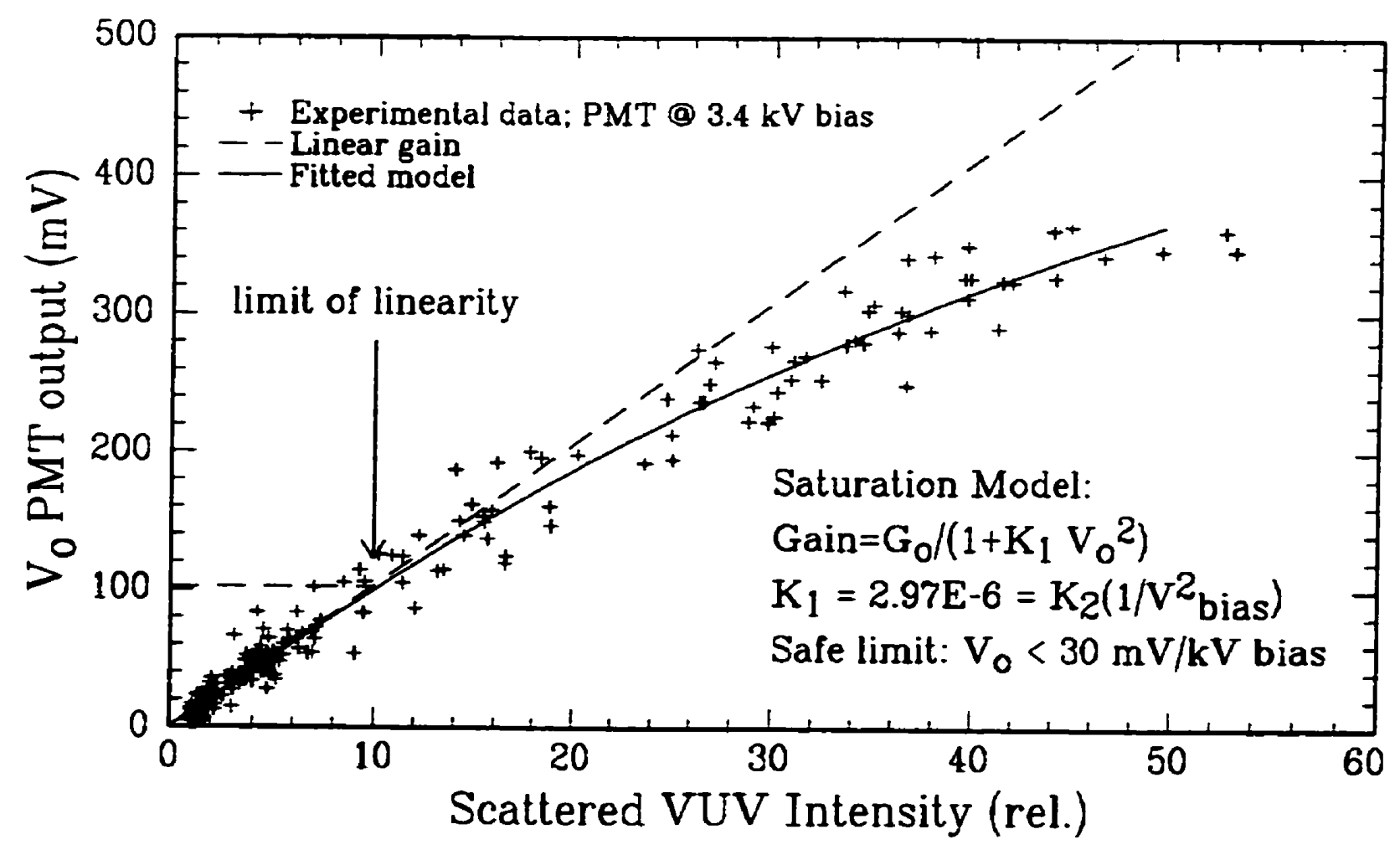

Figure E.6 Characterization of the gain saturation for the EMR PMT. 
On the other hand, if the output voltage is maintained below the threshold for gain saturation, where the relation $(E-1)$ is valid, constant gain and linear behavior is predicted and observed. The validity of equation (E-1), below the threshold of gain saturation, was illustrated through figure E.3 and E.4.

The results of these considerations provides a systematic quantification for the limit of linearity for an electron multiplier device. The assessment of this limit was found to be crucial tc discriminate among the vast amount of data acquired. This was deemed essential to obtain averaged data within the S/N ratio that was targeted by the present study. The characterization of saturation limits for PMT and preamplifier provided quantitative means for rigorous quality control of the data acquisition. By using threshold for the linearity of each component of the detection electronic to discriminate amongst the data and to reject measurements that were plagued by detector or preamplifier saturation, consistent results were obtained from the statistically averaged data.

\section{References}

1. Application notes from the catalogue of the Hamamatsu PMT.

2. Application notes for the Schlumberger EMR PMT. 


\section{please note}

Paco(s) miasing in number only; wxt follows. Finned as recoived.

\section{UMI}




\section{Appendix F Optimization beyond least mean squares}

\section{F.1. Introduction}

Statistical analysis is a crucial phase of experimental investigation, through the process of linking a given set of measurements to a parametrized model. When a large number of data can be obtained easily, the statistical analysis is trivial. However, when one has a statistically small data set $(\mathrm{N}<30)$, with well defined statistical uncertainties, the best answer and its precision are not as obvious as one would like. The specific questions that arise are: is the parametrized model appropriate, and if so, what are the precision of the parameters determined by the statistical analysis, and how does this relate to the experimental uncertainties of the data? Consequently. the rigour of the optimization process, i. $e$. the specifics of the relations between experimental uncertainties of the measurement, and the ones of the parametrized model, are of prime concern. The object of this appendix is to introduce an improvement of the optimization method, and to demonstrate its advantage compared to the customary least mean square (LMS) approach.

For the sake of the following argumentation, we sball briefly derive the essential concepts underlying the least mean square (LMS) approach to optimization. Consider a set of measurements $\left\{y_{i}\right\}$ for an observable, with statistical uncertainties characterized by one standard deviation $\left\{\sigma_{i}\right\}$, recorded against a set of values $\left\{x_{i}\right\}$ for the independent variable, on which the $\left\{y_{i}\right\}$ are understood to depend; hence the data set is represented by: $\left\{x_{i}, y_{i v}, \sigma_{i}\right\}$. A parametrized model, $y=f(x ; A, B, C)$, is assumed to afford an appropriate analytical representation of the observable dependence on the variable $x$. The goal of the optimization procedure is to determine the best estimate of the true values for the parameters $(A, B, C)$ and their uncertainties $(\triangle A, \Delta B, \Delta C)$, in addition to provide an assessment of the appropriateness of the model for the data set.

The underlying assumptions for an optimization procedure are simply that:

1- An experimental measurement, within its corresponding uncertainty, is an estimate of the true value sought.

2- The parametrized model, $f(x ; A, B, C)$, can represent the true values of the observables; consequently the optimal parameters values will be our best estimate of the true parameters values. Therefore, we want to make the best possible use of the information contained in the measurement.

3- For a given set of parameters values $(A, B, C)$, the relative probability to observe the values $y_{i}$ within an uncertainty $\sigma_{i}$, is quantified by the relation $(F-1)$.

$P_{i}(A, B, C) \propto\left(1 / \sigma_{i}\right) \cdot \exp \left\{-(1 / 2)\left[y_{i}-f\left(x_{i} ; A, B, C\right)\right]^{2} / \sigma_{i}^{2}\right\} \quad(F-1)$

In this problem, one seeks to determine a parametrized model that best represents the measurement; once the 
model has been chosen on the ground of physical rationale, it is customary to use the least mean square (LMS) method in order to achieve this goal. The LMS optimization procedure is a method characterized by the distinctive assumption that: the probability for the model to represent the whole of the measurements, is defined as the probability that it represents the point $\mathrm{i}$, and the point $\mathrm{i}+1$, and $\mathrm{i}+2$, etc. In set theory, the logical operation AND is represented by an intersection, and its mathematical implementation corresponds to a product. Therefore, the probability that a parametrized model represents all our measurements is expressed by the equation (F-2); the optimimum parameters determination consists in maximizing the product of the individual probability, as in the equation (F-2), which corresponds to the minimization of $x^{2}$, and prescribes the LMS method.

$$
\begin{aligned}
& P_{\text {tot }}^{L M S}(A, B, C) \propto P_{1}(A, B, C) * P_{2}(A, B, C) * \ldots * P_{N}(A, B, C) \quad(F-2) \\
& =\left(1 / \sigma_{1}\right) *\left(1 / \sigma_{2}\right) * \ldots *\left(1 / \sigma_{N}\right) * \exp \left(-x^{2} / 2\right) \\
& \text { where: } x^{2}=\sum_{i}\left\{(-1 / 2)\left[y_{i}-f\left(x_{i} ; A, B, C\right)\right]^{2} / \sigma^{2}\right\}
\end{aligned}
$$

Con venient mathematical characteristics have made the LMS method the dominant one, e.g. it has led to the ubiquitous polynomial regression algorithm. However practical experience has repeatedly shown that the LMS method is not very robust for small $(\mathrm{N}<30)$ data sample that contains outlier points, a situation which may be disregarded, because systematic deviation are not perceived by measurement statistics, and their occurrence may sometimes go undetected by the experimentalist, or simply for the lack of a better alternative. The issue of robustness is usually dealt with a posteriori through numerical implementation of ad hoc functions ${ }^{2}$ designated influence function, which effectively filters out outlier points through the iterative application of a least mean square algorithm; however, the arbitrariness of these influence functions leads to a fit procedure lacking the guidance provided by a physical rationale.

The fact that the LMS method is not robust can be directly appreciated from the following example. Consider the product of individual probabilities in (F-2), for which we will assumed that all the data points but one overlap with the model for the true values of parameters, and that this singular point lies more than three time its statistical uncertainty as a result of unsuspected systematic error. In that case, the probability factor for that outlier point will become much smaller than one, as a consequence of which the overall probability would be very small for the true parameter values, although the large majority of the experimental points overlap with that true parameters values. Consequently in this case, the LMS fit would produce optimal parameter values which differ from the true values by a greater quantity than the information content of the other data could afforded, would a more discriminating algorithm be available.

A Outlier points are defined as those points that lies outside the main group of a data set, with respect to a parametrized model, as a result of sporadic imperfection from the measurement process. 
An additional argument against the general appropriateness of the LMS formulation concerns the mathematical implementation of the probability statement. Posing the logical operation AND as a mathematical product is defined as such for completely independent probabilities; however, this is not the case here, as the individual probabilities share the same parametrized model.

A marked improvement in robusmess can be obtained by an optimization method which uses a more accomodating requirement for optimization: we require that the model provides an optimal statistical overlap for the experimental point $i$, or $i+1$, or ... etc. The overall probability is a sum of the individual probabilities, equation (F-3), because a logical OR corresponds to the union in set theory, and to the addition in mathematics. Note that the sum is a weighted sum, whose relative weights are normalized and proportional to the information content of each point.

It must be observed that, in the present case, the concept of weight conceptually differs from its use in LMS method. In the usual LMS method, the weight is scaling the importance of the difference between experimental and parametrized values of the dependent variable, and as such, the absolute uncertainties of the measurement suffice to quantify this model-data relation. Note also that in the LMS method. the factor in front of the exponential of the individual probabilities do not enter in the optimization.

In the novel method suggested here, the concept of relative weight signifies a very different aspect of the problem: this relative weight represents the different importance of each individual probabilities in the sum of them. The existence of the relative weight derives from the term factorizing the individual probabilities, which loose their importance in the AND formulation leading to the LMS method.

The physical meaning of the relative weight is the information content of the experimental data point, which corresponding to that individual probability; it is a quantity that weight the information content of the data point with respect to all the other data point in the data set. There is no preset definition of this quantity, but if the $S / N$ is a good measure of the information content, it could be used as a basis to quantify this relative weight, at least in appropriate case. Note that this relative weight concept is completely independent of the parametrized model. Note also that the absolute uncertainty of the data is use in the same way as in the LMS method, as its involvement in the mathematical functionality of the problem originates from the exponential function (F-1) of the individual probabilities.

\footnotetext{
A As it was arguod for the AND logical qparation. the fact that the individual probabilities are correlated indicates caution with regands to the relation between logical operator OR and mathematical product operation; in fact, as one may observe later in this work the seemingly appropriated logical statement that corresponds to this sum of correlated individual probabilities can be denoted the probability that any individual probability (as many as possible obviously!) best overlap the model.
} 
The probability statement requiring that either points will fit the model will te shown to be better suited to real experimental data. In relation to the fact that the individual probabilities are correlated by the parametrized model, and the aforementioned concerns regarding the relation between logical operation (AND and OR), and mathematical operation $\left(x_{,}+\right)$, it will be appreciated that in fact, the sum of the correlated probabilities, equation (F-3) corresponds rather to the total probability that any point statistically overlaps with the model.

$P_{\text {tot }}^{B S O}(A, B, C)=P_{1}^{B S O}(A, B, C)+P_{2}^{B S O}(A, B, C)+\ldots+P_{N}^{B S O}(A, B, C) \quad(F-3)$
where: $P_{i}^{B S O}(A, B, C)=w_{i} \exp \left\{(-1 / 2)\left[y_{i}-f\left(x_{i} ; A, B, C\right)\right]^{2} / \sigma_{i}^{2}\right\}$ and $\sum_{i} w_{i}=1$

A short glance at the formulation in the equations (F-3) indicates its robustness against outlier points: if an individual probability goes to zera, the sum in (F-3) can only differ by its respective maximum contribution. Each measurement point $\left(x_{i}, y_{i}, q\right)$ that decently corroborates the model, will contribute to the sum in proportion to the relative quality of its overlap with the model, and in proportion to its statistical weight, whence the designation of the method as best statistical overlap (BSO). If the measurement of the data set is such that the uncertaities results from statistics, the signal-to-noise $(S / N)$ ratio is equivalent to the $\left(y_{i} / \sigma_{i}\right)$ ratio, and in that case, the latter can quantify appropriately the information content, on the basis of the reasonable assumption that the $S / N$ ratio is a meaningful measure of the information content. In this case, the relative weight can be defined by the inverse of the relative uncertainty as: $w_{i} \propto\left(y_{i} / \sigma_{i}\right) \propto(S / N)$, and $1=\Sigma_{i} w_{i}$, i.e. $w_{i}$ are normalized.

In the absence of cutlier points, and for the case of an appropriate model fitted to a set of data having equivalent information content, i.e. equal weight, the LMS and BSO method become identical in the limit of the true parameters, i.e. the parameters that makes $\mathrm{P}^{\mathrm{BSO}}$ very nearly 1 . The latter statement is readily appreciated by recalling that in the limit $x->0$, the identity $\exp (-x)->1-x$ holds. Thus taking the weighted sum of $N$ terms of the form $\left(1-\delta_{1}\right)$ with equal weight $(1 / N)$, where $\delta_{i} \times 1$, one obtains $1-x^{2}$ as it is showed in expression $(F-4)$. Because to find the maximum for $1-\chi^{2}$ is equivalent to find the minimum for $\chi^{2}$, the results of BSO or LMS methods are therefore converging to the same parameters values in this case of perfect data, i.e. data deviating solely within the determined statistical uncertainties, and having identical relative information content.

Denoting: $P_{i}^{B S O}(A, B, C)=w_{i} * \exp \left(-\delta_{i}\right)$ and using $w_{i}=1 / N$,

then: $\lim \mathbf{P}_{\text {tot }}^{\mathrm{BSO}}(\mathrm{A}, \mathrm{B}, \mathrm{C})=1-\delta_{1}-\delta_{2}-\ldots-\delta_{\mathrm{N}}=1-\chi^{2} \quad(\mathrm{~F}-4)$

$$
\delta_{i} \rightarrow 0 \quad \therefore A \rightarrow A^{o p}, B \rightarrow B^{o p}, C \rightarrow C^{o p}
$$

However, the BSO formulation of optimization offers a marked advantage when it comes to deal with realistic situation, i.e. when the points have different relative information content, and where the unfortunate possibility of systematic errors cannot be dismissed; the example of section F.3 clearly illustrate this point. 
A disadvantage of the BSO formulation of optimization, compared to the LMS method, is that the calculation are slightly more involved, which make it harder to obtained closed form expression, even for simple analytical functionality such as polynomial regression. It may be suggested that the realization of this fact may historically had influenced the choice of the AND formulation of the probability in early studies, which lead to much more concise analytical formulation. However, the development of computer technology has gready reduced the difficulties of numerical calculation, which remove this limitation associated with the BSO method.

The advantage of the BSO method will be illustrated, in the demonstration of its performance, at the end of this appendix. Moreover a professional statistician ${ }^{3}$ have investigated the mathematical properties of the BSO method, and have asserted its validity for optimization.

\section{F.2. Mathematical description}

The premiss for any optimization of parametrized model is that the experimental data can be represented by an analytical model, which is characterized by adjustable parameters. The goals of the optimization procedure are: (i) to determine the optimum value of the parameters and their uncertainties, from the experimental data and their statistical uncertainties, and (ii) to assert the overall appropriateness of the model from data-model comparison.

The present BSO approach to optimization is especially suited for the case where a small statistical sample, i.e. $\mathrm{N} \leq 30$, of good data points (i.e. data points with small relative uncertainties) have been acquired, and one wishes to extract the information content in the best statistical manner. The model is an analytical function which has no points of inflexion over the range of interest, or at least 30 data points per points of inflexion, because a general statement can only be made for nearly monotonic function.

We already described the format for the data set: $\left\{x_{b}, y_{0}, q\right\}$. It must be considered as an estimate of the true values, which absolute imperfection is quantified by $\sigma_{i}$, while the relative quality of each point among the data set is quantified by $\left(y_{i} / \sigma_{i}\right)$, the latter ratio is equivalent to a $(S / N)$ ratio, and represents the information content. The BSO method has a low sensitivity for systematic errors, resulting from a fine balance between collective data versus model, and each datum compared to the collective data.

The model is represented by a function of one variable $x$, which is parametrized by $(A, B, C)$ as: $y=f(x ; A, B, C)$. The difference between model and data can be calculated for a given set of parameters values, for each data points as: $\Delta_{1}(A, B, C)=y_{i}-f\left(x_{1} ; A, B, C\right)$. The function $s=\exp \left[-\left(\Delta_{1} / q_{i}\right)^{2} / 2\right]$ for each individual probability terms can be labeled the sensitivity function; it is the function that quantifies, in the range $[0,1]$, the acceptability of a given parametrized model for each data point. 


\section{A) Relative weight}

Each experimental point may in general have a different information content, which must be quantified by the experimentalist. In many cases, it can be quantified in proportion to the relative uncertainty $\left(y_{i} / \sigma_{i}\right)$ for that specific measurement, in regards to its statistical meaning, as discussed above. We used the term information content here to emphasize the physical meaning sought rather than the specific number used: the idea is to quantify the relative importance of an experimental point amongst the data set. This relative weight $w_{i}$ is completely defined by adding the requirement that it will be normalized, i.e. $\Sigma_{i} w_{i}=1$.

\section{B) Optimizer definition}

Once these concepts are clearly established, a quantity designated by $\mathbf{O}$ for Optimizer, can be defined as the weighted sum of the sensitivity for each point, as in equation (F-5). The optimization procedure is then simply defined as the maximization of that Optimizer quantity, which in turns defined the optimum parameters: $A^{\circ}, B^{\circ}$. and $C^{\circ}$, that represents our best knowledge of the truth. In laymen's terms, this Optimizer quantity is the sum of the probabilities that the parametrized model represents each data point, each individual probability being weighted by its respective information content. The total probability $O$ is a function considered to depend on $A$. $B, C$, and it is calculated for a fixed data set: $\left\{x_{i}, y_{i}, \sigma_{i}\right\}$.

$$
O=P^{B S O}=\sum_{i} w_{i} s_{i} \quad \text { (S-S) }
$$

\section{C) Properies}

The value of $O$ varies between 0 and 1 , as a consequence of the normalized weights $\left(w_{1}\right)$, and of the same property for the individual sensitivity functions $\mathbf{s}$. The optimization procedure consists simply in scanning the parameter space, and in searching for the parameters combination that maximize $O(A, B, C)$; the optimum parameters are defined by the set of values $\left(A^{\circ}, B^{\circ}, C^{\circ}\right\}$ which corresponds to $\operatorname{Max}(O(A, B, C))=O\left(A^{\circ}, B^{\circ}, C^{\circ}\right)=O_{\max }$. The maximum value of $O$ is expected to apprach 1 very closely, while the difference to this goal can be due to either outlier points or inappropriate model. If $\mathrm{O}_{\operatorname{mex}}$ approaches 1 , the model can be deemed appropriate, if the difference from 1 can be justified in termed of the statistical uncerainties of the data, and the rare occurance of outlier points.

The uncertainties in the optimal values of the parameters are related to the uncertainties of the data points via a value of $\mathrm{O}=\mathrm{O}_{\text {uxc }}$ defined by equation (F-6), which circumvent a region of the parameter domain, using the best estimate of the parameters $\mathrm{f}^{\mathrm{pp}}=\mathrm{f}\left(\mathrm{A}^{\circ}, \mathrm{B}^{\circ}, \mathrm{C}^{\circ}\right)$ to calculate the cumulative information content; equation $(\mathrm{F}-6)$ is easily derived by recalling the conceptual correspondence between $S / N$ ratio for a given point, and reciprocal of its relative uncertainties. The extremum of the region of the parameters domain provides an estimate for the model uncertaincies. 
$\mathrm{O}_{\mathrm{UNC}}=\mathrm{O}_{\mathrm{MAX}}\left[1-\left\{\sum_{i}\left(w_{i}\right)^{2} \exp \left[\frac{-1}{2}\left(\frac{\mathrm{f}^{O P}\left(x_{i}\right)-y_{i}}{\sigma_{i}}\right)^{2}\right]\right\}^{-1 / 2}\right]$

It is intuitively expected that the finite precision of the data used to calculate the $O$ matrix results in a finite precision of the optimum parameters. A qualitative picture is afforded by considering a two parameters model optimization; there $O(A, B)$ is a surface, which has a maximum. The uncertainties in the values of the parameters are conveniendly defined by $\mathrm{O}_{m e}\left(<\mathrm{O}_{\mathrm{wax}}\right)$. The intersection of an horizontal plane at $\mathrm{Z}=\mathrm{O}_{\mathrm{mos}}$ with the $\mathrm{O}\left(\mathrm{A}_{\mathbf{v}} \mathrm{B}\right)$ surface, defines a locus in the domain of the parameters which defines their respective uncertainties. Thus the gcals set for the optimization method have been reached, i.e. the optimization procedure allows the determination of the optimum parameters and their uncertainties, and offers a quantitative estimate of the appropriateness of the model, and a transparent way to account for the effect of outlier on the overall procedure.

The distinctive advantage of the BSO method is its robustness, which results from its intrinsic ability to properly deal with outlier points using the dominant collective agreement of the data with the model.

\section{F.3. Illustrative example}

We shall now demonstrate the advantage of the aforementioned properties with a numerical example. We produced an artificial data set of 15 points, with typical relative uncertainties (2\%-6\%); the uncertainty is correctly simulated with the aid of a random number generator. Additionally an "accidental" systematic deviation is made to affect 1 point every 5 other points at random, imposing an additional deviation of 30\% on these "measured" values compared to the "true" value for these data points. Note that from the experimentalist point of view, uncertainties are obtained from measurements statistics. In the case of the present example, the physical model is perfectly known to follow a quadratic form, whose true parameters are known perfectly in this artificial case.

The data are listed in table F.I bereafter, in which the first two columns are the point's numbers (\#) and abscissa $\left(X_{i}\right)$; the third column is the true value $Y_{m}$ and the fourth and fifth columns represents the measurement $Y_{\exp }$ and the statistical uncertainties: $\Delta Y_{\text {stre }}$. Note that the weights for the BSO method are calculated as: $w_{B S O^{\infty}}$ $\left(Y_{\text {exp }} / \Delta Y_{\text {was }}\right)$, while for the LMS method it is $w_{L M S} \propto\left(1 / \Delta Y_{m e t}\right)^{2}$. The difference lies in the different meaning for weight in the two cases. As it can be seen from (F-1) to (F-4), in the case of LMS, weight originates from the denominator in the exponential function of the probability, whence the use of the absolute uncertainty from the statistics of the measurement, taken as equivalent to a standard deviation; for LMS, weights are used to scale the difference between data and model. In the case of the BSO, the absolute uncertainty is also used for the same purpose, since it originates from the same expression for individual probabilities, but it is not denominated "weight". The relative weight, $w_{k}$ in BSO, refers to the relative information content of each measurement with 
respect to the collective of the data, i.e. independently of the model. This important point is treated correctly by our rigorous BSO method: the measurements are independent statistical assessments of the true value, the quality of which depends of their respective $(\mathrm{S} / \mathrm{N})$ ratio. When taken collectively, with the aid of an appropriate model, parameters optimization is prescribed by the differences between model and data, in proportion of the corresponding absolute uncertainties. The global quality of the fit is the weighted sum of the qualities for each point; this is definition of the fit quality is a somewhat tolerant definition of quality, which has been shown by experience to be more appropriate than the requirements implied by the LMS method. The analytical function is defined as: $Y=F(X ; A, B, C)=X^{*}\left(X^{*} A+B\right)+C$, where $A, B$ and $C$ are to be determined. These simulated experimental data are used to compare the parameters obtained by using LMS and BSO optimization methods described above. In table F.2, the parameters values obtained for each methods are compared with the true values.

Table F.1 Data used for the comparative example.

\begin{tabular}{|c|c|c|c|c|}
\hline$\#$ & $X_{i}$ & $Y_{\text {rue }}$ & $Y_{\text {exp }}$ & $\Delta Y_{\text {sate }}$ \\
\hline 1 & 0.2 & 46.875 & 46.87534 & 1.1 \\
\hline 2 & 0.2857143 & 62.557 & 62.41404 & 1.3 \\
\hline 3 & 0.3714286 & 80.666 & 102.4395 & 4.7 \\
\hline 4 & 0.4571429 & 101.203 & 101.2031 & 5.8 \\
\hline 5 & 0.5428571 & 124.168 & 124.1681 & 7 \\
\hline 6 & 0.6285714 & 149.561 & 146.3987 & 8.2 \\
\hline 7 & 0.7142857 & 177.382 & 175.1848 & 4.6 \\
\hline 8 & 0.8 & 207.63 & 206.5539 & 5.6 \\
\hline 9 & 0.8857143 & 240.307 & 237.5329 & 5.5 \\
\hline 10 & 0.9714286 & 275.411 & 348.2076 & 12.7 \\
\hline 11 & 1.057143 & 312.944 & 307.46 & 15.5 \\
\hline 12 & 1.142857 & 352.904 & 352.9 & 8.5 \\
\hline 13 & 1.228571 & 395.292 & 385.77 & 10.4 \\
\hline 14 & 1.314286 & 440.108 & 433.09 & 10.5 \\
\hline 15 & 1.4 & 487.352 & 633.56 & 12.1 \\
\hline
\end{tabular}

\section{A) LMS methods}

The most common fitting method for the type of problem in which the analytical function is a second order polynomial, is to operate a quadratic regression on the data set; quadratic regression technique do not take into account the measurement uncertainties, and therefore it is better suited to the cases of very large data set $(\mathrm{N} \times 30)$, and for which statistics is usually effective in averaging out the negative effect of outliers. A more general leastmean-squares method allows to include (LMS) weights, which are based on the standard deviation for normal error distribution as a representation of the experimental uncertainties, as we have shown in the previous sections. 


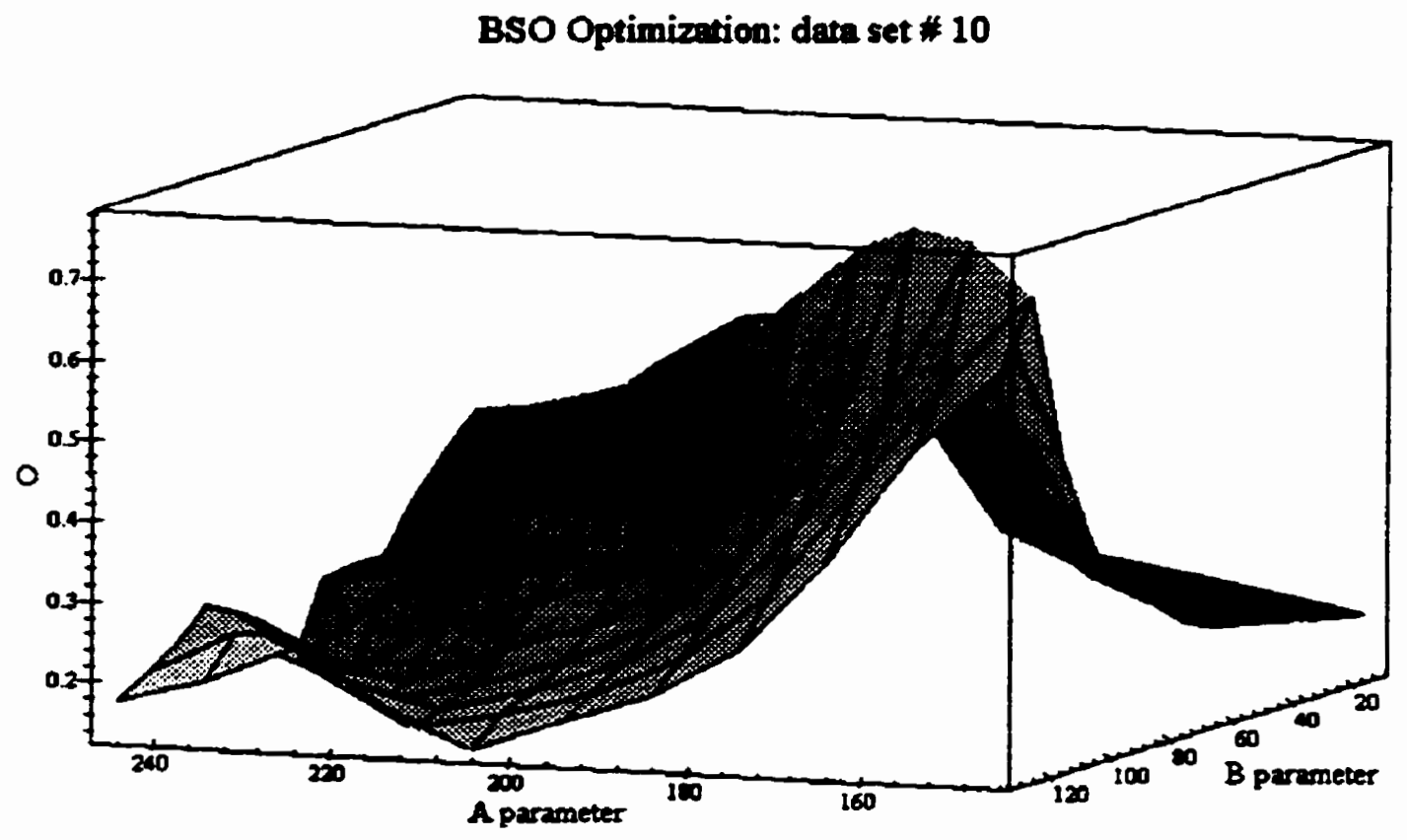

b)

\section{BSO Optimization: data set \# 11}

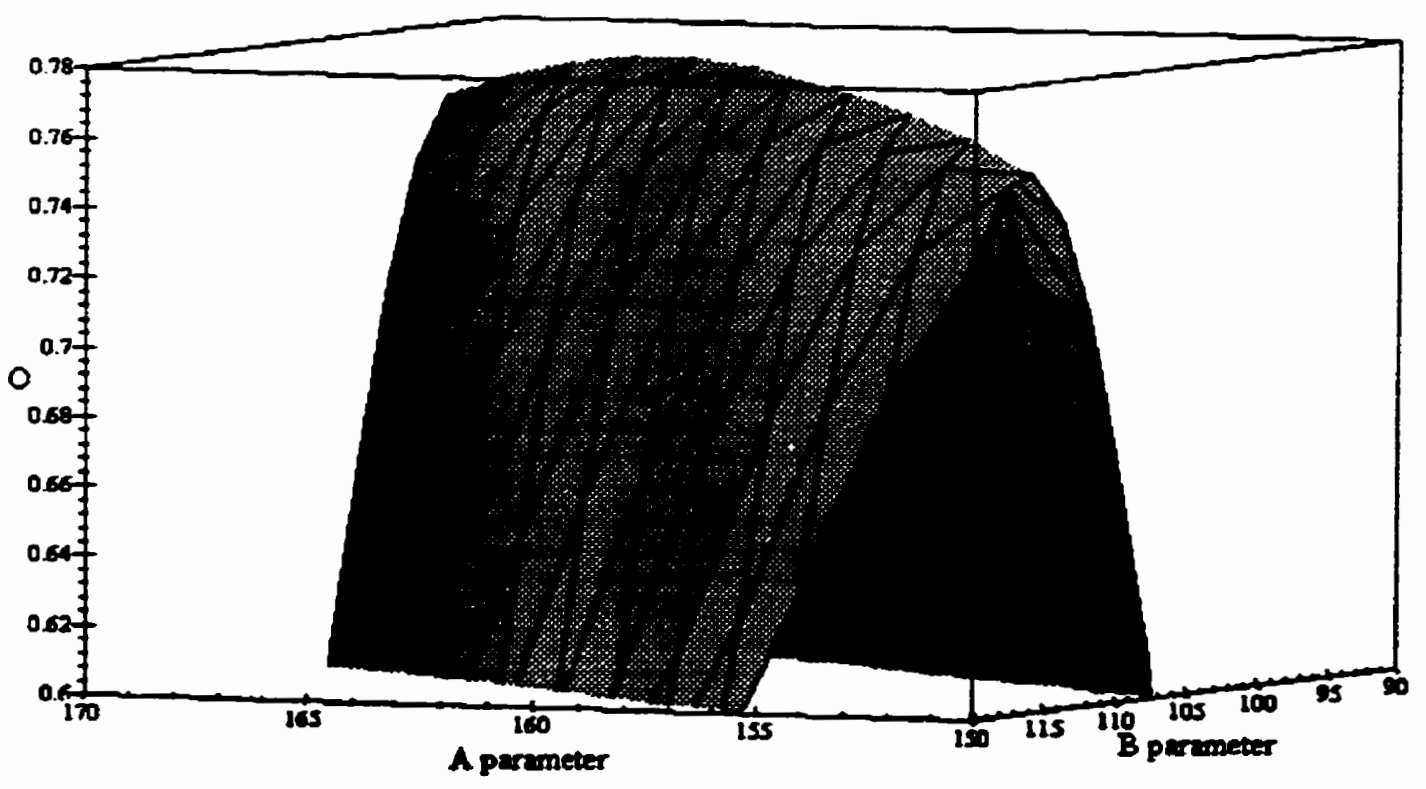

Figure F.1 Mlustration of the BSO method fo parametric model optimization; a) global convergence of the optimizer function (O-function), and b) well-defined identity of the optimum. 
In the comparison made in this section. both the LMS method and the quadratic polynomial regression were applied to the determination of the optimum unknown parameters, A, B, and C.

\section{B) BSO method}

To illustrate our BSO method, we used a brute force method of grid calculation, for the sake of simplicity and transparence of the method. The domain of the parameters A and B was made discrete onto a grid of sufficient finesse, for which each point of the grid corresponded to an entry of a discrete numerical optimizer function, or O-function. For each combination of these first two parameters, a rough estimate of $C$ is first obtained, around which a range of value is expanded, on which the Optimizer was calculated; the optimum $O$ over the $C$ range defines the $O(A, B)$ to be stored. In qualitative term, we optimized $A$ and $B$ in a parametric direction along $C$ such that $O$ is maximum. A plot of the $O$-function is shown in figure F.2 a) and the region of the optimum is magnified in figure F.2 b).

After the whole $O$-function was calculated, this discrete $O(A, B)$ function was scanned for the maximum of $O$, and the domain for the A and B parameters was readjusted to improve the definition of that maximum of O(A,B). The procedure was repeated iteratively until the maximum of $O$ was determined with certainty and the desired precision. The method to determine the uncertainties have been already discussed above. The result of the procedure is resumed in table F.2. It can be clearly appreciated that the parameters estimates and uncertainties calculated from equation (F-6) are a good approximation of the true values, i.e. that the error bars on the parameters obtained by the BSO are indeed meaningful, in that they reproduce the true values of the parameters.

Table F.2 Comparison of the parameters obtained by different optimization method

\begin{tabular}{|c|c|c|c|c|}
\hline Parameters & $\begin{array}{c}\text { Quadratic } \\
\text { regression }\end{array}$ & LMS & TRUE VALUES & BSO \\
\hline A & 146.4 & $242.3 \pm 4.8$ & 165.231 & $161 \pm 6$ \\
\hline B & 129.16 & $19.8 \pm 6.8$ & 102.694 & $105 \pm 5$ \\
\hline C & 19.137 & $37.7 \pm 1.8$ & 19.727 & $19.72 \pm 0.25$ \\
\hline
\end{tabular}

\section{F.4. Conclusion}

The goal of this comparative study was to test the BSO method, for which we proceeded in the aforementioned manner. Regarding the superiority of the BSO method, in relation to robustmess in challenging conditions, the competition is trailing far behind: the quadratic regression produces results deviating by $15 \%$ and $30 \%$ for $A$ and B respectively, the LMS is not only worse off, but it also produce wrong estimate of the uncertainties. 


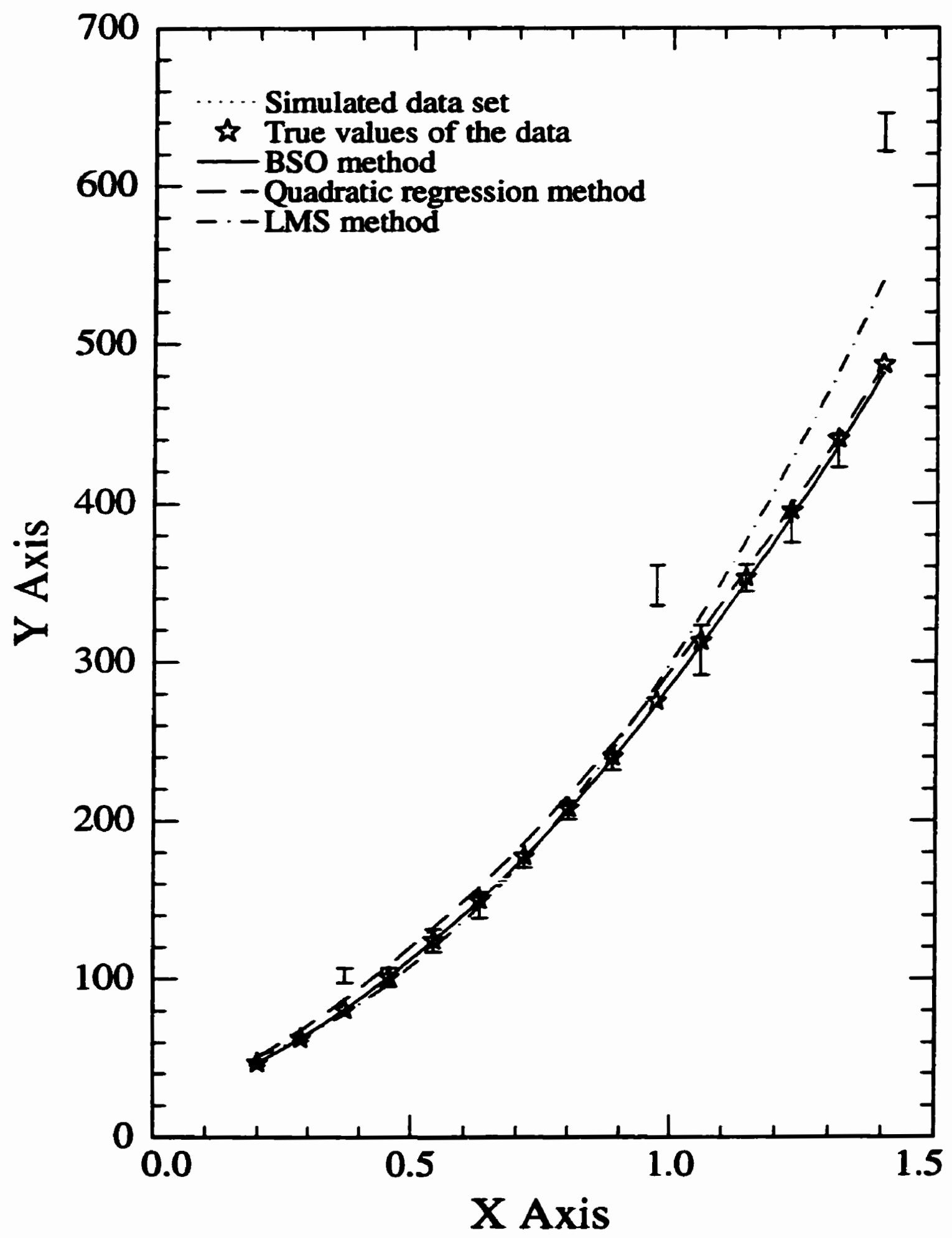

Figure F.2 Graphical comparison of the performance of each optimization method discussed in the text. The higher performance of the BSO method is amazing 
Our BSO method displays the best perfamance by offering a relatively good precision: $5 \%$ relative uncertatinties on the parameters in the worse case, while $20 \%$ of the data deviate by $30 \%$, while the statistical uncertainties of the data varies between 2-6\%. A final comparison between the estimates of the true, by these three methods, also includes the true values (stars in the graph) in figure F.3.

We have showed that the BSO method is clearly superior to the LMS in the precision afforded for the model parameters. We have discussed the rationale substantiating its superiority, and its robustness was exemplified in a typical case with simulated data having realistic properties. Therefore, it should become the preferred method for scientific studies having produced definite assessment of the statistical uncertainties of their measurements. This BSO method is a breakthrough in optimization procedure, on the basis of its advantageous properties and superior performance, in comparison to traditional LMS approaches.

\section{References}

1. Taylor, J. R. An introduction to Error Analysis, University Science Books, Mill valley, California (1982)

2. F.R. Hampel, E.M. Ronchette, P.J. Rousseeuw and W.A. Stahel, Robust statistics: The approach based on influence functions. Wiley, New York (1986)

3. Private communication, Mary Thompson, Chairwoman of department of Statistics and Actuarial Sciences, University of Waterloo, Waterloo, Ontario, Canada. 


\section{Appendix G Non-adiabatic transition in dissociation dynamics}

\section{G.1 Effect of non-crossing rule on molecular electronic structure}

\section{A) Non-crossing rule: homogeneous perturbation}

Herzberg' has shown that the electronic structure of diatomic molecules can be understood as being made up of low-order states, which may perturb each other under certain conditions, hence given rise to higher order states. The former states determine the predominant features of the structure, while the latter states take into account the modifications of the energy levels, brought about by the perturbation, which are indeed observed experimentally.

As a matter of fact, systematic understanding of peculiar spectral features observed in molecules have prompted Neumann and Wigner ${ }^{2}$ to define the non-crossing rule: for infinitely slow change of internuclear distance, two electronic states of the same species cannot cross each other. Same species means same symmetry properties or symmetry type. The avoidance of the crossing originates from a term of the Hamiltonian, that was ignored in the first approximation leading to the first-order states, and that must be treated as a perturbation, whose inclusion defines the high-order state. The non-crossing rule is the effect of a perturbation that couples the first-order states of the same symmetry, henceforth producing a repulsion of the "original" first-order energy levels.

\section{B) Diabatic and adiabatic PEC}

The typical effect of the perturbation causing the avoided crossing is illustrated in figure $c .1$. The strength of the coupling between the diabatic potential energy curves (PECs) can be seen to affect directly the width of the splitting that distinguises the adiabatic states from the diabatic ones. An important definition must be established to avoid confusion while discussing this topic. The diabatic states are the states that correspond to the first-order states. The adiabatic states are the states that results from the "non-crossing" perturbation, corresponding most closely to the observed reality. If the coupling is strong, it makes physical sense to approach nonadiabatic effects from the point of view of the adiabatic state, while if the coupling is weak, as in the case considered in the early theoretical works on this problem, it can be approached from the diabatic point of view.

\section{C) Coupling strength}

By inspection of the PEC illustrated in figure c.1, the determination of the coupling strength is obvious to the trained spectroscopist's eyes. When the coupling is quite large, as shown in the part a) of figure G.1, it may be hard to discern any effects in the adiabatic PEC, while in part b), the kinks in the PEC is quite suggestive of the avoided crossing. Depending on the internuclear region where the avoided crossing occurs, and the respective nature of the first-order states, the effect of the coupling may translate in a wide range of adiabatic PEC. Few other possibilities are shown in c) and in d). 


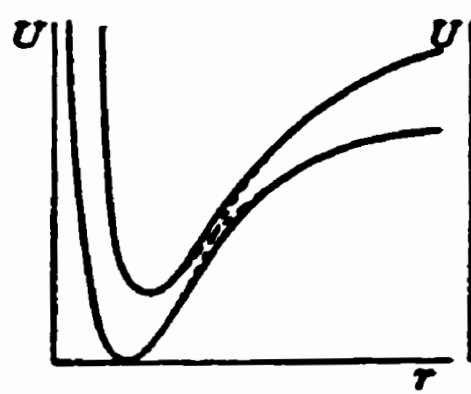

(a)

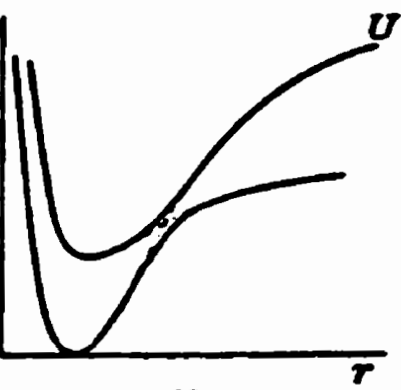

(b)

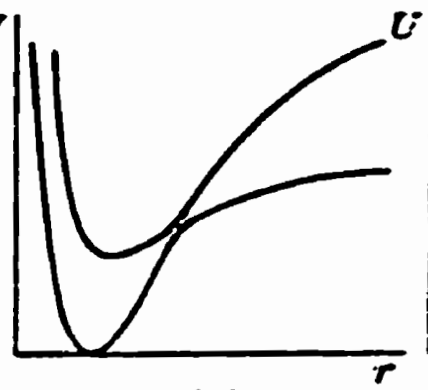

(c)

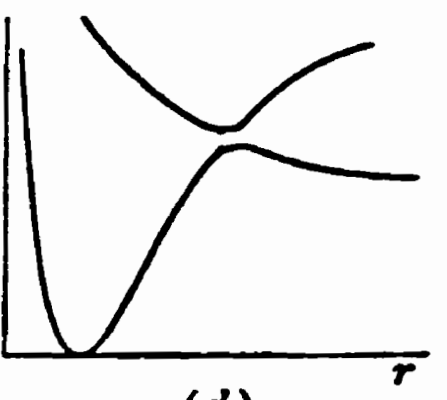

(d)

Figure G.1 Different cases of avoided crossing; a) strong coupling, b) and c) intermediate coupling strength at increasing internuclear distance, and d) coupling with a direct dissociative state (taken from ref 1).

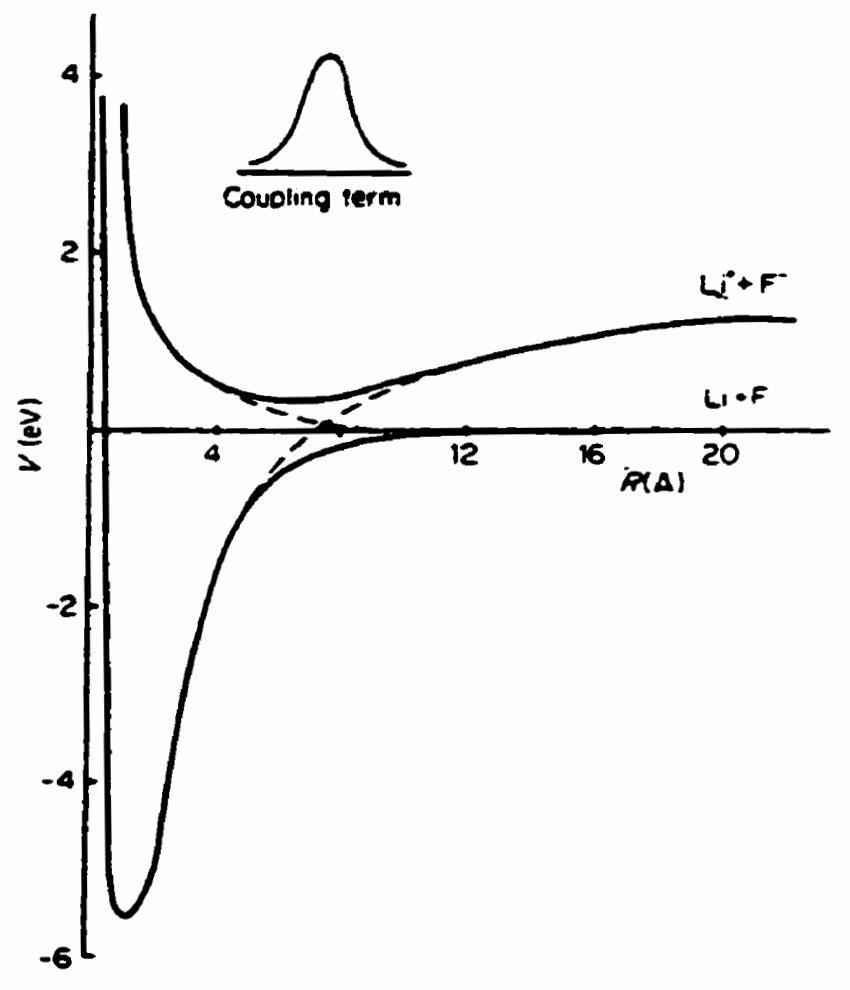

Figure G.2 Practical example: the electronic stnucture of the Lif molecule. Note the insert where the radial dependence of the coupling streagth is illustrated. The local nature of the coupling will be referred to below (taken from ref 10 ). 
A practical example of avoided crossing is provided by the electronic structure of LiF molecule shown in figure G.2. The avoided crossing results from the coupling of the ions pair potential with the lowest totally symmetric configuration of the neutral atoms combination. This system exemplifies well the nature of the perturbation. Note the radial dependence of the coupling term illustrated in the insert.

\section{G.2 Diabatic curves crossing and nonadiabatic transition}

\section{A) Background}

Many books on the theory of atomic and molecular collision have treated this crucial subject ${ }^{3.45}$. The initial works on the problem of diabatic curve crossing was developed independently by Landau ${ }^{6}$, Zener', and Stueckelberg". Historically, the topic aimed at solving the problem of charge transfer in slow collision. although analogy have been established with the equivalent problem of energy exchange, and many other generalizations since then. In the theory of collision. slow collision refers to the dynamical time scale of motion in the electronic structure. Recent treatments of the dynamical effects of curve crossing can be found in the books of Child', and Murrell and Bosanac $^{10}$. The interested reader will find a clear and concise derivation of the topic treated below in Bates ${ }^{4}$ book.

\section{B) Problem definition}

The generic problem was concerned with the probability for charge transfer during the collision between two atoms. It was recognized early that such a process had $t 0$ involve nonadiabatic transitions. The collision involved two states of the combined system, which can be described in terms of the diabatic PEC or the adiabatic PEC. which are represented respectively by the dashed and the solid curves in figure G.3. The diabatic PECs cross at a value of the internuclear radius denoted $R_{c}$, while the adiabatic PECs do not cross, because the perturbation has been taken into account for this higher order of approximation of the energy level.

The initial state of the system is taken as the asymptotically separated atoms in the lowest state denoted o in figure G.3. During the collision, the probability for the transfer translates into the probability for making a transition from the lower asymptotic state to the higher asymptotic state of the combined system. The aforesaid transition occurs predominantly in the region around $R_{c}$, where the diabatic curves cross. During the collision, this region is travelled twice: once during the approach and once during the repulsion. To assess the probability for the system to end up in the final state $f$, the two trajectories indicated in figure $G .3$ must be taken into account. In dissociation however, $R_{\varepsilon}$ is crossed only once.

At any given branching point, between the adiabatic PECs and the diabatic curves crossing, $P$ can be defined as the probability for the system to stay on the adiabatic curve, while (1-P) is the probability for the system to make a transition between the adiabatic states. The probability for the same physical situation can be equivalently 

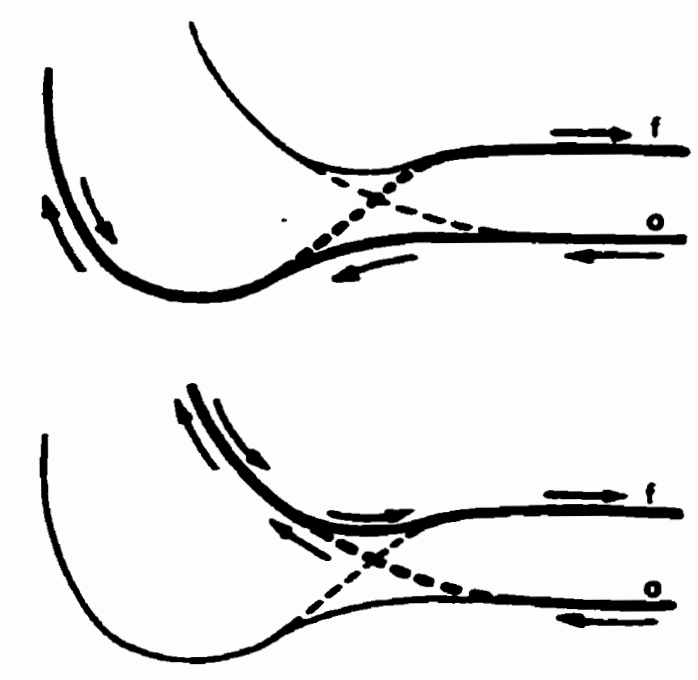

$\mathbf{R} \rightarrow \mathbf{0}$

$\mathbf{R} \rightarrow-$

Figure G.3. Ilustration of the generic problem of atomic collision for which the probability of nonadiabatic transition was originally defined. The solid lines are the adiabatic energy levels, while the dashed lines represent the diabatic states. Two events have to be considered to calculate the transition probability: (I) the transition occurs during the outward motion, whereas in (II) the transition happened during the inward motion. Arrows indicate the path of the colliding particles (taken from ref 5).

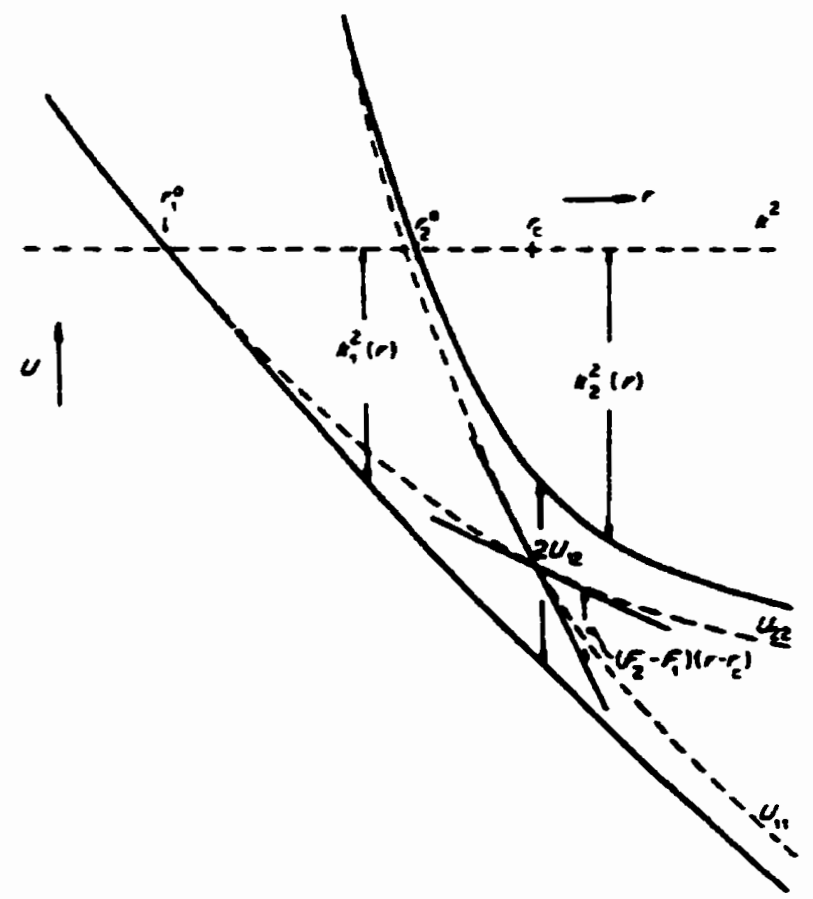

Figure G.4 The Landau-Zener curve crossing parameters. Diabatic (dasbed) and adiabatic (solid) curves are shown. $F_{1}$ and $F_{2}$ are the slopes of the diabatic curves at the crossing point (taken from ref 10). 
described in terms of the diabatic states: $P$ is then the probability for the system to make a transition between the diabatic states at the curve crossing, while (1-P) is the probability for the system to stay on the diabatic PEC curves. Diabatic states and adiabatic states are different basis states of the dymamics.

\section{C) Landau-Zener-Stueckelberg approximation}

Sweckelberg developed a detailed framework to treat such nonadiabatic interaction. He started from the Bom's approximation, and ended up with the diabatic distorted wave, characterized by a varying phase shift: the Stueckelberg phase ${ }^{3}$. While his formalism can in principle lead to precise prediction, as it has been demonstrated ${ }^{10}$, the level of precision to which the PEC are known in practice rarely warranted the application of this complex theary. Approximation on Stueckelberg formalism produces formula identical to the one derived independently by Landau and Zener, as it was first recognized by Mott and Massey ${ }^{3}$, and also discussed in Johnson". Consequently, the probability $P$ for the process described above will henceforth be referred to as the Landau-Zener-Stueckelberg (LZS) probability, denoted PLx. Figure G.4 illustrates graphically the physical meaning of the algebraic terms referred to below.

$$
\begin{array}{lll}
\mathrm{P}^{\mathrm{LZS}}=\exp \left(-\tau_{c} / \tau_{12}\right) & \text { (in terms of diabatic states) } \\
\mathrm{P}^{\mathrm{LZS}}=1-\exp \left(-\tau_{c} / \tau_{12}\right) & \text { (in terms of adiabatic states) }
\end{array}
$$

where: $\tau_{12}=\left[\frac{\pi U_{12}}{\hbar}\right]^{-1}, \tau_{c}=\frac{\delta \mathrm{r}_{\mathrm{c}}}{[\partial \mathrm{r} / \partial \mathrm{t}]_{\mathrm{r}_{\mathrm{C}}}}$,

and: $\delta r_{\mathrm{c}}=\left|2\left[\frac{U_{12}}{F_{2}-F_{1}}\right]\right|, \quad[\partial \mathrm{r} / \partial \mathrm{r}]_{\mathrm{r}_{\mathrm{C}}}=\left[\frac{\hbar\left(k_{1}+k_{2}\right)}{2 \mu}\right]_{\mathrm{r}_{\mathrm{C}}}=\mathrm{v}_{\mathrm{c}}$, where: $F_{i}=\left.\frac{\partial U_{i i}}{\partial r}\right|_{r_{C}}$

The LZS probability refers to the probability of maling a transition for a single passage across intersecting diabatic curves. Note that the original derivation and concepts definition was made in terms of the diabatic states; overloaking this very fact has been the cause of confusion in discussion on this topic. Most important is to keep in mind that the LZS formula is only an approximation, which valid in a limited range of value of the argument $\tau_{e} / \tau_{12}=\Gamma$. It will be noted that if $(v)$ is very small, $\Gamma$ is very large, and the probability for diabatic crossing predicted by the LZS formulae is very small, i.e. no transition occurs between the diabatic states for low energy encounter; this is in apparent contradiction with the non-crossing rule, which states that the adiabatic states represent the physical reality in the limit of slow dynamics. However, the contradiction is lifted by realizing that in general, the kinetic energy will differ a great deal from zero in the region of curve crossing, as illustrated by 
figure G.3. Without discussing all possible limit cases for the variables of $\Gamma$, we can simply state that the LZS formulae will give meaningful estimate for the probability of curve crossing for values of $\Gamma$ of approximately one to two orders of magnitude around 1 , or the range $[0.02,50]$.

In summary, we shall recall the following clarifications developed above: a jump between the diabatic curves is equivalent to no jump between the nonadiabatic curves, and vice-versa. Note also that, if $\mathrm{P}^{\mathrm{l} 2 \mathrm{~s}}$ is the probability to jump, (1-P(2) $)$ is the probability not to jump.

Confusion may arise out of these different points of view when one consider a real molecule, because the physical states typically determined through spectroscopic experiments are the adiabatic states, while the states considered in first approximation theory are the diabatic states. Confusion arose when that distinction is not clearly established.

\section{D) Expression for the case of weak nonadiabatic effect $=$ case of strong coupling interaction}

When the coupling between the first-order state is strong, i.e. of the same order or larger than the kinetic energy involved in the collision, it makes more sense intuitively to describe the collision dynamics in terms of adiabatic states. In the case of strong coupling, the diabatic states are well separated by $U_{12}$, and the dynamics occs preferentialiy on the adiabatic state, in the limits of very low collision energy (outside the range of validity of the LZS formula). The range of validity of the LZS formula is for collision with kinetic energy within one or two orders of magniude of the coupling energy which gave rise to the adiabatic states, i.e. it is in the limit of relatively small atomic collision velocities. In the high end of this range, $\Gamma \propto 1$, and $\exp (-\delta) \sim 1-\delta$. The term $F_{2}-F_{1}$ represents the spatial variation of the potential difference causing the nonadiabatic transition, so its physical effect will be characterized by the fundamental Fourier component of its spatial frequency. The latter spatial frequency of the potential, when experienced in the velocity of the collision, will translate into a temporal frequency, whence. energy. Therefore, the probability of nonadiabatic transition can be understood qualitatively as being due to that part of the Fourier spectrum of temporal frequencies, resulting from the combination of the diabatic potentials and collision velocities, that "resonates" with the adiabatic energy coupling

The latter term $F_{2}-F_{1}$ can be approximated by: $\left[U_{12} / \Delta r\right]$, where $\Delta r$ represents that range where the aforementioned coupling is significant. Returning to the LZS formulae for the nonadiabatic transition in terms of the adiabatic states, we can substitute the following approximation (I): $\exp (-\delta) \sim 1-\delta$ for $\Gamma \propto 1$, and the approximation (II): $\left(F_{2}-F_{1}\right)=\left[U_{12} / \Delta r\right]$, to obtain the approximation of equation $(G-4)$, for the probability of nonadiabatic effects, in terms of adiabatic states. 
The last relation can be useful in practical application where litule is known a priori for the PEC, while the value for coupling strength $\mathrm{U}_{12}$ can be estimated from spectroscopic observation of adiabatic PEC splitting, and upper limits for the value of kinetic energy and potential can be evaluated from experimental measurements. We derived and justified the approximation for nonadiabatic transition in the case of adiabatic basis states, obtained by SFB"

\section{References}

1. G. Herzberg, Molecular spectra and molecular structure, I. Spectra of diatomic structure, 2nd ed. Krieger Publishing Co., Malabar, Florida (1989)

2. J. V. Neumann, E. Wigner, Physik, Z. 30, 467 (1929)

3. N. F. Mott, H. S. W. Massey, The theory of atomic collisions, $2^{\text {pd }}$ ed., Oxford, London (1949)

4. D. R. Bates, Atomic and Molecular Processes, chap. 14, ed. by D. R. Bates, Academic Press, New York and London (1962)

5. R. E. Johnson, Introduction to alomic and molecular collisions, chap.4, Plenum Press, New York (1982)

6. Landau, Z. Phys. Sow. Un. 2, 46 (1932)

7. Zener, Proc. Roy. Soc. A, 137, 696 (1932)

8. Stueckelberg, Helv. Phys. Acta, 5, 370 (1932)

9. M. S. Child, Molecular collision theory, appendix D, Academic Press, London and New York (1974)

10. J. N. Murrell, S. D. Bosanac, Introduction to the theory of atomic and molecular collisions, Wiley (1989)

11. S. A. Singer, K. F. Freed and Y. B. Band, Adv. Chem. Phys., 61, 1 (1985) 